\title{
Biology meets drug development : the biography of a 'miracle' drug, the interferons
}

Citation for published version (APA):

Pieters, T. (1999). Biology meets drug development : the biography of a 'miracle' drug, the interferons. [Doctoral Thesis, Maastricht University]. Universiteit Maastricht. https://doi.org/10.26481/dis.19991105tp

Document status and date:

Published: 01/01/1999

DOI:

10.26481/dis.19991105tp

Document Version:

Publisher's PDF, also known as Version of record

\section{Please check the document version of this publication:}

- A submitted manuscript is the version of the article upon submission and before peer-review. There can be important differences between the submitted version and the official published version of record.

People interested in the research are advised to contact the author for the final version of the publication, or visit the DOI to the publisher's website.

- The final author version and the galley proof are versions of the publication after peer review.

- The final published version features the final layout of the paper including the volume, issue and page numbers.

Link to publication

\footnotetext{
General rights rights.

- You may freely distribute the URL identifying the publication in the public portal. please follow below link for the End User Agreement:

www.umlib.nl/taverne-license

Take down policy

If you believe that this document breaches copyright please contact us at:

repository@maastrichtuniversity.nl

providing details and we will investigate your claim.
}

Copyright and moral rights for the publications made accessible in the public portal are retained by the authors and/or other copyright owners and it is a condition of accessing publications that users recognise and abide by the legal requirements associated with these

- Users may download and print one copy of any publication from the public portal for the purpose of private study or research.

- You may not further distribute the material or use it for any profit-making activity or commercial gain

If the publication is distributed under the terms of Article $25 \mathrm{fa}$ of the Dutch Copyright Act, indicated by the "Taverne" license above, 


\title{
Biology Meets Drug Development: The Biography of a 'Miracle' Drug, the Interferons
}

\author{
PROEFSCHRIFT
}

ter verkrijging van de graad van doctor aan de Universiteit Maastricht

op gezag van de Rector Magnificus, Prof. dr. A. C. Nieuwenhuizen Kruseman volgens het besluit van het College van Dekanen, in het openbaar te verdedigen op vrijdag 5 november 1999 om 14.00 uur

door

\section{Toine Pieters}

geboren te Heerlen 


\section{Promotor}

Prof. dr. Ir. W.E. Bijker

\section{Beoordelingscommissie}

Prof. dr. R. Vos (voorzitter)

Dr. O. Amsterdamska

Prof. dr. C. A. M. V. A. Bruggeman

Prof. dr. E. S. Houwaart

Prof. dr. H. A. J. Struijker Boudier

Copyright: $\quad$ Toine Pieters 1999

Omslag Ontwerp: Zorica Vasic

Druk: $\quad$ Grafisch bedrijf Ponsen \& Looijen bv, Wageningen 
Die wahre Geschichten, die man erzählt, sind falsch; für die falschen besteht wenigstens die Chance, dass sie wahr werden könnten. ${ }^{1}$

1 E. Canetti, Nachträge aus Hampstead: Aus den Aufzeichnungen 1954-1971 (München: Carl Hanser Veriag, 1994), p. 65. 
Deze uitgave is mede mogelijk gemaakt door:

Onderzoeksschool WTMC

Stichting KNMP-Fondsen

Stichting Historia Medicinae

AHP Pharma

Biogen

Boehringer Ingelheim

Glaxo-Wellcome

F. Hoffmann-La Roche

Janssen-Cilag

Merck Sharpe \& Dohme

Parke-Davis

Schering

Schering-Plough

Serono 


\section{Contents}

Preface

Acknowledgments $\quad$ xi

Introduction:

The Making of a Therapeutic Drug; The Case of Interferon 1

Studying the Biomedical Sciences as Practice and Culture 3

The Historian's Toolbox on Display 9

$\begin{array}{ll}\text { The Biography of a 'Miracle Drug' } & 12\end{array}$

1 Setting the Scene; (re-)Modelling a Set

of Biological Phenomena

About Rabbits, Viruses, Monkeys, Mice

and Viral Antagonism

Toward Viral Interference

The Beginning of a British/Swiss Collaboration in a London Laboratory Building and Manipulating an Experimental System in Room 215 $\begin{array}{ll}\text { Writing 'Interferon' } & 73\end{array}$ 
4.1 A Partnership Originating in National Interests

Establishing a 'Collaborative' Program

for Research on Interferon

5 Managing Differences in Biomedical Research:

The Case of Standardizing Interferons

\section{Coping with Differences}





\section{Preface}

\section{Patients at work}

Thursday, June 5, 1997. "With all these uncertainties about the therapeutic effect of interferon beta I can not prevent from feeling like a potential guinea-pig." These are the words of a multiple sclerosis patient attending the meeting 'Choosing for interferon?'.

The scene of action: the 'Doctor Soer' auditorium of 'Sunny Home' in Zwolle, the Netherlands. At the right hand side of the entrance is a provisional podium flanked by two big billboards with promotion posters of the Multiple Sclerosis (MS) Society. The slogan 'Diagnosis MS, what next? immediately strikes the eye.

The local meeting is organized by the Dutch MS Society in collaboration with the Foundation for Appropriate Drug Use (DGV) as part of a national campaign to inform MS patients of the pro's and con's of treating MS with the newly registered therapeutic drug, interferon beta. Among those present are MS patients in various stages of the disease-from experienced wheelchair individuals, to less disabled 'stick' people, to the seemingly healthy group of 'greenies' who are still in the early stages of the disease. They are accompanied by their relatives and friends. In one way or another all are trying to cope with a chronic degenerative nervous disease for which there is no effective treatment available yet, other than a few drugs that provide symptomatic relief.

The speaker is a doctor representing the DGV. We learn that the DGV is an independent non-governmental organization. It aims to promote the safe and appropriate use of therapeutic drugs through interactive group meetings with clinicians and pharmacists as well as patients. In an effort to make contact with the audience the DGV representative addresses the ambiguous feelings that might have risen after reading the critical information leaflet. "Our pamphlet might have dashed your high hopes but it is not our aim to sell promises today, but to present a realistic picture of what this new therapeutic drug has to offer to you as MS patients". The DGV doctor emphasizes the problem of judging the effectiveness of therapeutic drugs in a poorly defined disease like MS. It is a therapy that is claimed to produce therapeutic effects with a less than $50 \%$ response rate. Those prepared to seize the opportunity have to accept an reduction of their quality of life, with no immediate benefits. Using interferon means coping with side-effects ranging from flu-like symptoms, painful swellings at the side of injection, up to suicidal tendencies, and learning how to fit in the storage, preparation and injection procedures into one's daily life.

"Summarizing, it might be said that while in the short-term interferon users are worse off than fellow-patients who decide not to use interferon, in the long-term the user group as a whole will be better off. However we have to keep in mind that the actual benefits will differ 
from one person to another, with a relatively large number of users who will not experience any beneficial effects at all. It certainly is not the wonder drug that the media have tried to make of it. Are there any questions so far?"

The audience seizes the opportunity to pose questions with both hands. What should I make of your rather negative image of interferon treatment with less than $10 \%$ positive and 90\% negative news"? "There seem to exist two different types of beta interferon, beta-1a and beta- $\mathrm{lb}$. Are we talking about one and the same therapeutic drug or should we regard them as two closely related but different drugs?" "Why is there a difference in dosaging between the various hospitals?" " If the history of MS is punctuated with false-dawn cures, what guarantee is there that interferon will not turn out to be the next false-hope on the list?"

In one of the last remarks before the end of the meeting the MS-patient-quoted at the beginning of my talk - indicates that with more questions than answers about the application of interferon-beta she feels subjected to medical experimentation. "In principle interferon-beta is not any different in this respect from any other therapeutic drug currently in use in clinical practice", claims the doctor in response. "The problem is that certain side-effects will not show up in the limited clinical studies which are required to qualify for a therapeutic drug license but will make themselves felt only after widespread and long-term use. For the sake of future users it is of utmost importance that clinicians as well as patients cooperate in closely monitoring the effects of therapeutic drugs, in particular the newly introduced ones. "His comments invite support from an MS patient who justifies her decision to take part in an interferon trial by pointing out the possible benefits that may result from these kind of studies for future generations of MS patients. She feels she can make a contribution, however small, to the progress of medicine.

At times the nature of the discussion between the patients and the doctor on the platform-both parties discussing similarities and differences in research results and questioning the credibility of and rationale behind claims as well as procedures-makes me feel that $\mathrm{I}$ am attending a scientific congress. As such this open-information evening questions the self-evident certainty, with which we think we know we can pinpoint who is a scientist and who is a layperson. Speaking the language of medical science clearly enables MS-patients to redraw the boundaries between the entities 'science' and the 'public' and to reconstitute the expert/lay divide. In other words this capacity empowers patients in relation to medical and scientific professionals. ${ }^{2}$

2 See, for a historical analysis of development and change in the relations between 'science' and 'the public': S. Shapin, 'Science and the Public', in R. C. Olby, G.N. Cantor, J. R. Christie and M.J. Hodge (eds.), Companion to the History of Modern Science (London: Routledge, 1990). 
Most important, the example of the MS-patients shows us that there is more to the introduction of prospective consumers to a new therapeutic drug than the transfer of readymade knowledge. The questions of quality, safety, research, evaluation and professional control are raised by prospective consumers in such a manner as to suggest that the organization of the treatment setting is negotiable. The prescription and consumption of interferon-beta appear to require the patient's belief and trust in its efficacy. Achieving this requires hard work by all the parties involved-doctors, researchers, patients, company executives and regulators. This interactive process involves not just molding the therapy routines of interferon-beta, but also the therapeutic and social context in which it is administered. The molding, however, is constrained in major ways by the cumulative impact of years of work between bench and bedside, on which the therapeutic profile of interferon-beta basically draws.

At least there is more room for negotiation about the everyday 'do's and don'ts' of interferon therapy than might have been gathered from the stringent official regulatory and delivery arrangements. The MS-patients do not seem to take for granted the official efficacy data and therapy routines of interferon-beta resulting from years of testing in the laboratory and the clinic. As such they seem to be willing to take considerably more responsibility for managing their own treatment than they are currently allowed to do.

But this book is about more than patients. It attempts to lay out the historical journey among a variety of actors-e.g. virologists, biochemists, molecular biologists, immunologists, pharmaceutical companies, journalists, regulators, doctors and patients - involved in shaping a family of therapeutic proteins, the interferons. In making manifest the inner workings of the practices by which the biomedical sciences produce and evaluate new knowledge claims and therapeutic tools, this study exposes the reader to the uncertainty and value choices involved in scientific work. It shows us that effective participation in the process of knowledge production in biomedicine not only requires the capacity to speak the technical language of the journal article and of the scientific conference, but also achieving the competence to use that language. My claim is that a thorough understanding of the practice and culture of the biomedical sciences is a sine qua non for effective public participation in biomedicine. 


\section{Acknowledgments}

The generosity and support of a collection of inspirational scholars, friends, researchers and archivists made this thesis-project possible. I have to emphasize that the project would never have been undertaken without the initial generous support of the Faculty of Arts \& Culture of Maastricht University, so I would like to thank Wiebe Bijker and Gerard de Vries first and foremost for enthusiastically backing up and restyling my crude ideas for a $\mathrm{Ph}$.D. proposal. As my thesis-supervisor Wiebe provided me with invaluable training and criticism throughout the project. I am greatly indebted to the following people, not only for their high standards but also for their warm support for the project: Huub Schellekens, for giving me an early introduction to the world of interferon research; Hans Harbers, Sjaak Swart, and Henny van der Windt for being stimulating intellectual companions; Catherine Gardiner for transforming my approximate English into the standard idiom; Brian Burgoon for editing the extravagantly long and 'meandrous' early chapters far beyond the usual limits; and to Annemarie Mol and Rein Vos for reading and advising me on several drafts of the thesis chapters.

Special appreciation goes to a small circle of friends, helpers and supporters who bolstered my spirits (in times of low spirit), reacted to my ideas and pieces of writing (however wild and incomprehensible in nature) and encouraged me to carry on (whenever they heart some muttering about having a tough time in my 'isolating cell'): Roel Otten, Patricia Faasse, Harmke Kamminga, Jo Wachelder and most precious of all my wife and dearest friend Karin Knippers.

I am also grateful to all those who aided my research and the preparation of this thesis. Staffs of archives and government offices in Britain (MRC and NIMR) and the United States (NIH, NIAID and FDA) provided friendly assistance with source materials. However, the most important input into the research came from scientists, doctors, drug company executives and policy makers involved in the development of interferon. The majority of whom were prepared to cooperate and share both their recollections and private correspondence far beyond my expectations. In particular I have to thank, Kari Cantell, Norman Finter, Leon Gauci, Ion Gresser, Sussana Isaacs Elmhirst, Jean Lindenmann, and David Tyrrell for their help. The full list of those interviewed can be found in Appendix A.

My long-lasting obsession with this biography of a wondrous family of biomolecules doubtless puzzled many friends and relatives. By asking repeatedly what it was about and why it mattered and to whom, they helped me more than might have been obvious. I doubt whether a manuscript would have emerged without their critical and sometimes sceptic questions and remarks. Special thanks to my sisters, brothers-in law and my mother, and to Marlou Banning. Petra Banning, Kostana Banovic, Gerda Bouma, Peter Drehmanns, Robert Goedegebuure, Arnout Herremans, Arjan van Hessen, Marty Huisman, Giel Janssens, Ellen Knippers, 
Jos\&Dini Knippers, Peter Post, Victor van Rijswoudt, Aalt\&Wilma Verhagen and Hein van de Wiel. 


\section{Introduction}

\section{The making of a therapeutic drug; the case of interferon}

Interferon immediately caught my imagination when first reading Mike Edelhart's popular account Interferon: The New Hope for Cancer in the early 1980's. ${ }^{3}$ I, along with a whole generation of students in the life sciences had been brought up believing that developing drugs was a difficult but relatively straight-forward industrial activity with the almighty chemists in the driver's seat. Here, however, you had a therapeutic substance that seemed different in every respect. First, chemists were of marginal importance in the development of interferon, while biomedical scientists played the leading part. Second, the greater part of the research work was done in academic settings or in small genetic engineering companies, many of which were founded in the late 1970s. Until the early 1980's the large pharmaceutical companies that have traditionally dominated modern drug research showed little interest in work on interferon. Surprisingly some of the major drug companies stepped up their efforts to develop interferon as a drug while intense public disappointment in its healing power became manifest. What was the rationale behind this seemingly odd course of industrial action? And more generally, what made interferon follow a different developmental trajectory from most other therapeutic drugs? Was it perhaps due to the fact that it lasted more than thirty years before the first interferon molecule had been isolated and chemically identified?

During my pharmacology training I had become familiar with working with substances of unknown chemical composition: in most cases identification was a question of hours or days of hard labor in a biochemical laboratory. In general when chemical analysis was not achieved within a fortnight we were commissioned to proceed with another task. I could hardly imagine scientists working for years in a row, with little more at their disposal than a biological assay, measuring the biological activity of what was only believed to be a substance of protein nature. How did they manage to cope with this rather uncertain and, in my eyes, trying situation? Of course I was aware that in the case of insulin and penicillin researchers had had similar difficulties. But wasn't that regarded the 'prehistory' of modern drug development?

Years later I came across Sandra Panem's book The Interferon Crusade. ${ }^{4}$ However interesting in its description of the political and economical aspects of interferon's history and development, it did not answer most of these questions. She had little more to say about the scientific development of interferon than what could already be found in Edelhart's account or

3 M. Edelhart, Interferon: The New Hope for Cancer (Reading: Addison-Wesley Publishing Company, 1981).

S. Panem, The Interferon Crusade (Washington, D.C.: The Brookings Institution, 1984). 
in scientific reviews. Moreover, by the time I read Panem's study interferon had just succeeded in finding a niche in clinical practice as a supplement to the armamentarium of antiviral and cancer drugs, and I was curious to find out how interferon managed to become naturalized and legitimized as part of medical practice. This appeared to be yet another question that warranted investigation. In starting to collect the published literature concerning interferon, I came to realize that the story was even more complex than I could ever have imagined.

Interferon's 'biography' appears to contain many stories in one: both success stories and failures. Unlike most other therapeutic agents in the history of medicine interferon survived successive cycles of promise and disappointment. As such it offers an ideal opportunity to do a parallel study of success and failure and to analyze what went into choices that did and did not lead into blind alleys. Furthermore, as a product of long-term work between 'bench and bedside' it sheds light on experimental practices in twentieth century biomedicine, and on how these practices, the practitioners and their products are influenced by and constitutive of social and physical environments. Moreover, the interferon story provides a historical perspective on the development, testing and use of a new generation of biosynthetic 'therapeutic molecules' in modern medicine. Finally, it may serve as a show-case of the growing interference between molecular biology and the health sciences-in an experimental world where biology meets drug development.

In describing the fascinating history of a family of 'therapeutic' proteins, this study will neither credit the current clinical usefulness of the interferons to specific individuals nor explain the more than twenty-five year delay in using these therapeutic molecules in medical practice by pointing to other individuals. Rather, the primary goal is to throw a more general light on the evolution of twentieth century biomedical practices, and on how these practices come to produce data, technologies and goods which may eventually be embedded in our modern healing culture. In addition this study seeks to present the often messy events by which the scientific and therapeutic value and use of the interferons unfolded-a process that involved conflicts and resistances as well as enrolment and acquiescence. Naturalizing interferon as part of medical practice involved establishing links and commitments between many different individuals and crganizations, including laboratory researchers, doctors, drug companies, patients and their families, and regulators. I will demonstrate how the current profile of interferon as a family of therapeutics can be regarded as a dynamic product of the history here described. 


\section{Studying the biomedical sciences as practice and culture}

More than any other product of scientific medicine, therapeutic drugs form highly sensitive tools at the frontiers of modern biomedical research and at the same time pervade daily life and clinical practice. By often providing a relatively easy, convenient, and personal means to help control health problems, they enhance the status of medicine. Drugs have acquired a special symbolic significance as an icon of the increasing healing power of modern scientific medicine, which continues to capture the public imagination. Moreover, as the 'wondrous' products of the pharmaceutical industry, they provide a promising public face for the continuing research efforts of the drug companies.

The pharmaceutical industry chose, long ago already, to let people believe that most of the twentieth century's therapeutic breakthroughs owe their origin to the industry's systematic and limitless research efforts. ${ }^{5}$ This vision of drug companies committed to research, which provide us with one innovative pill after the other to solve our health problems ranging from headaches, to infections and male impotence, has been articulated over and again in popular fiction and non-fiction literature, and in films and soap operas. ${ }^{6}$ The development of therapeutic drugs is presented as a simple and unqualified success story.

If we move from the pharmaceutical industry's image makers to social science it is surprising to see that until the 1980's, most historical and social studies of science and medicine have refrained from questioning the inadequacy of this traditional model of drug innovation-according to which the making and use of therapeutic drugs proceed linearly from bench to bedside. They either confined to describing modern medicines as products of imaginative biomedical research or decided to focus on social, policy and regulatory issues and steer away from the scientific, technological and industrial aspects. ${ }^{7}$ The notable exceptions to

5 Most recently Jordan Goodman shows in his study of the anticancer drug taxol how the pharmaceutical company Bristol-Myers Squibb, which obtained exclusive rights from the $\mathrm{NCI}$ (the original patent holder) to develop taxol for the commercial market, gradually managed to rewrite the history of taxol in company publications as a means to claim novelty and originality over the now profitable corporate product $\operatorname{Taxol}(\mathrm{R})$ : J. Goodman and Vivien Walsh. The Billion-Dollar Molecule: Tales of Taxol from the United States, France and Holland', Unpublished paper presented during the Anglo-Dutch Workshop on Remedies and Healing Cultures in Britain and the Netherlands in the Twentieth Century. London. June 1998.

6 In the case of the Dutch soap opera 'Medisch Centrum West' the pharmaceutical company Glaxo succeeded in getting advertising text for their migraine medicine, Imigran, into the script: Interview with Heiko Top who works as a doctor with the Dutch Foundation for Appropriate Drug use (DGV): See for a comprehensive examination of the media's reporting of health and medicine: A. Karpf, Doctoring the Media: The Reporting of Health and Medicine (London: Routledge, 1988).

See, for studies confined to describing modern drug development as an autonomous industrial process governed by the logic of cumulative scientific and technological advance: J. T. Mahoney. The Merchants of Life. An Account of the American Pharmaceutical Industry (New York: Harper, 1959); D. Wilson, Penicillin in Perspective (London: Faber\& Faber, 1976); D. Schwartzman, Innovation in the Pharmaceutical Industry (Baltimore: Johns Hopkins University Press, 1976); W. Sneader, Drug Discovery: the Evolution of Modern 
this apparent separation between social and scientific investigations are studies by Gladys Hobby, John Swann and Jonathan Liebenau. ${ }^{8}$ These detailed and sensitive historical accounts, have given us some idea about the intricate relations that have emerged in this century between medicine, academic research, industry, and health politics.

Hobby brings a new perspective to the history of penicillin, presenting it as one of the first major ventures in group pharmaceutical research which played an important role in the emergence of modern corporate Research\&Development organizations in the drug industry. In his study of the rise of university-industry relationships, Swann focuses on the growing importance of collaborative research between academic institutions and industry as a source of drug innovation. Liebenau, in turn, takes a broader approach by studying not only the growing cooperation between science and industry, but also the emerging links between the drug companies and the medical community.

However important their contribution to the historiography of pharmaceutical research and development in the twentieth century, these scholars have not been overly successful in opening the 'black box' of industrial and government science to critical scrutiny. For the greater part they regarded the content of drug research and development as self-explanatory without questioning the self-serving and unproblematic images of drug research and testing that have been in circulation for decades.

In the 1980's, inspired by a fast growing body of social constructivist and ethnographic approaches to the study of science and technology, some sociologists, historians and philosophers began to carve their way into the intricacies of a laboratory supported scientific medicine. ${ }^{9}$ However different their individual approach, they shared a constructivist

Medicines (Chichester: John Wiley\&Sons, 1985); M. Weatherall, In Search of a Cure (Oxford: Oxford University Press, 1990): R.P.T. DavenPort Hines and Judy Slinn, Glaxo: A History to 1962 (Cambridge: Cambridge University Press, 1992); H.C. Peyer, Roche: A Company History (Basel: Editiones Roche, 1996); A critical view of the process of drug testing and evaluation is rarely taken in these studies. At the same time more politically-challenging studies with an emphasis on controversial social and political issues related to therapeutic drug development and use, the neglect of the content and context of research within the drug companies is striking; M. Silvermann and P.R. Lee, Pills Profits \& Politics (Berkely: California Press, 1974); and P. Temin, Taking Your Medicine: Drug Regulation in the United States (Cambridge: Harvard University Press, 1980).

See, G. L. Hobby, Penicillin: Meeting the Challenge (New Haven: Yale University Press, 1985); J. Liebenau, Medical Science and Medical Industry (Baltimore: The Johns Hopkins University Press, 1987); J. P. Swann. Academic scientists and the pharmaceutical industry (Baltimore: The Johns Hopkins University Press, 1988).

See, O. Amsterdamska, Medical and Biological Constraints: Early Research on Variation in Bacteriology, Social Studies of Science, 17 (1987), 657-87: The following studies are regarded as exemplary social constructivist accounts of science and technology: D. A. Mackenzie, Statistics in Britain: 1865-1930 (Edenburgh: Edenburgh University Press, 1981); A. Pickering, Constructing Quarcks: A sociological History of Particle Physics (Chicago: Chicago University Press, 1984): H.M. Collins, Changing Order (London: Sage Publications, 1985); T. Pinch, Confronting Nature: the Sociology of Solar-Neutrino Detection (Dordrecht: D. Reidel Publishing Company, 1986); W. Bijker, T. P. Hughes and T. J. Pinch (eds), The Social Constructi- 
perspective on medical work which served as a conceptual tool to open up the 'black box' of medical knowledge - previously seen as an authoritative system of knowledge that developed independently of its social and cultural contexts. ${ }^{10}$ Within this constructivist perspective science, medicine and technology are to be treated as historically situated and social activities which can only be understood in relation to the contexts in which they occur. Experimental data in this view are just one resource among many used in social negotiations over what the correct outcome of an experiment will be-which is potentially open ended. Key assumptions are the 'theory-laden' nature of fact-statements and the underdetermination of empirical judgements by evidence, with the consequence that claims about the reality of what experiments disclose are based in consensus.

Such a position entails a form of methodological relativism that can be described in the following terms: "scientific knowledge has to be seen, not as the transparent representation of nature, but rather as knowledge relative to a particular culture". ${ }^{11}$ In my view this does not imply that all claims to knowledge are to be judged equally valid. It does mean, however, that all our understandings of 'nature' are products of human labor which have been shaped within specific cultural contexts. Insofar as scientists attribute properties to nature, it is as a consequence of scientific practice; those properties can not count as the explanation of the order of scientific work. In other words, in scientific practice distinctions between nature and society are continuously being made, unmade and remade. Empirical studies carried out within this methodological perspective have been successful in opening the 'black-box' of science, technology and medicine to examination. They have succeeded in providing us with detailed and illuminating accounts through linking social and cultural commitments of the scientific community to the content of scientific ideas, methods and procedures. Furthermore, they have

on of Technological Systems (Cambridge (MA): The MIT Press, 1987); and W. Bijker, Of Bicycles, Bakelites and Bulbs: Toward a Theory of Sociotechnical Change (Cambridge (MA): MIT Press, 1995); See, for important examples of ethnomethodological studies of science, which are based on the methodological principle of following scientists around and examine their working practices by using the notion of 'antropological strangeness' - treating scientists at work, as if they were a sort of exotic tribe with strange and puzzling customs: B. Latour and S. Woolgar, Laboratory Life (London: Sage Publications, 1979); K. KnorrCetina, The Manufacture of Knowledge (Oxford: Pergamon Press, 1981); M. Lynch, Art and Artifact in Laboratory Science: A Study of Shop Work and Talk in a Research Laboratory (London: Routledge and Kegan Paul, 1985).

10. See for comprehensive reviews of recent approaches, trends and debates in the social and cultural studies of science, medicine and technology; S. Jasanoff, G. E. Markle, J. C. Petersen and T. Pinch, Handbook of Science and Technology Studies (Thousand Oaks and London: Sage, 1995); M. Casper and M. Berg. Constructivist Perspectives on Medical Work: Medical Practices and Science and Technology Studies, Science, Technology \& Human Values, 20 (1995), 395-407; J. H. Fujimura, Crafting Science: A Sociohistory of the Quest for the Genetics of Cancer (Cambridge (MA): Harvard University Press, 1996), 237-43; and, J. Golinski, Making Natural Knowledge: Constructivism and the History of Science (Cambridge: Cambridge University Press, 1998)

A. Pickering, 'From Science as Knowledge to Science as Practice', in A. Pickering (ed) Science as Practice and Culture (Chicago: The University of Chicago Press, 1992), p. 5. 
demonstrated the importance of credibility-understood as a system of authority combining aspects of legitimation, dependence, trust and persuasion-as the backbone of the cognitive and moral order in modern scientific inquiry. ${ }^{12}$

Applied to the biomedical sciences this has shown us the important point that experimental design and results cannot be dissociated from the interests and commitments of those who frame and evaluate laboratory as well as clinical experiments. ${ }^{13}$ In addition, science and technology "not only shape the social and cultural characteristics of medicine, but are also shaped by them-redefined and transformed in their actual use in particular settings and circumstances". ${ }^{14}$ In other words one should study the development of medical practices without treating scientific and technical issues as independent of the social and cultural contexts of work. Moreover, several scholars have articulated the importance of investigating the question of how scientific practices and medical practices mutually shape and transform each other as a prerequisite for understanding scientific medicine. ${ }^{15}$

In his pioneering book Drugs Looking for Diseases Rein Vos successfully uses the constructivist perspective as a heuristic tool to study drug development practices. ${ }^{16} \mathrm{He}$ was able to show that the origin of new medicines - in his case the discovery of the beta blockers and calcium antagonists-lies in the interface between the worlds of the laboratory and the clinic. The exchange of information between bench and bedside is argued to play a central role in the generation of medical scientific knowledge that serves as a valuable input to the drug discovery process. Nelly Oudshoorn and Louis Galambos followed suit with their constructivist case-studies on twentieth-century pharmaceutical research and developmentrespectively concentrating on the development of the sex-hormones and vaccine development. ${ }^{17}$

12 See, for an extensive discussion of the credibility argument: H. Collins, and T. Pinch, The Golem: What Everyone should know about Science (Cambridge and New York: Cambridge University Press, 1993).

See, S. L. Star, Regions of the Mind: Brain Research and the Quest for Scientific Certainty (Stanford (CA): Stanford University Press, 1989): and, E. Richards, Vitamin C and Cancer: Medicine or Politics (New York: St. Martin's Press, 1991).

O. Amsterdamska, 'Social Studies of Medicine', Paper presented at the 1999 WTMC Conference, Rolduc, The Netherlands.

See, M. Casper and M. Berg, Constructivist Perspectives on Medical Work: Medical Practices and Science and Technology Studies, Science, Technology \& Human Values, 20 (1995), 395-407, p. 396.

R. Vos, Drugs Looking for Diseases. Innovative Drug research and the development of the Beta Blockers and the Calcium Antagonists (Amsterdam: Kluwer Academic Publishers, 1991).

17 N. Oudshoom. Beyond the Natural Body: An Archaeology of Sex Hormones (London: Routledge, 1994); and, L. Galambos and J. E. Sewell. Networks of innovation: Vaccine Development At Merck. Sharp \& Dohme, and Mulford 1895-1995 (Cambridge: Cambridge University Press, 1995); and, L Galambos and J. L. Sturchi, 'The Transformation of the Pharmaceutical Industry in the Twentieth Century., In J. Krige and D. Pestre (eds. ) Science in the Twentieth Century (Amsterdam: Harwood Academic Publishers, 1997). 
They both take the concept of 'actor-network'-focusing on the strategies of those actively involved in the shaping of therapeutic drugs: laboratory scientists, clinicians, pharmaceutical entrepreneurs and regulators, for building networks of linkages between these heterogeneous actors - as a model for understanding change and development of interrelationships between biomedical research, government institutions and pharmaceutical companies. Whereas Oudshoorn's major interest is to understand how a specific group of therapeutic drugs became restricted in their clinical utility (directed almost exclusively to women) during the developmental process, Galambos concentrates on how the interaction of ideas, individuals and institutions influences innovation in a science-based industry.

Unlike most other constructivist scholars both authors manage to extend the scope of analysis to include the macro-sociological dimensions of science, medicine and technology. ${ }^{18}$ In working across various social levels simultaneously-local laboratory and clinical practices, private and public organizations, and the broader national and international context of these practices-they have succeeded in broadening our understanding of the dynamics of pharmaceutical research and development. Given this outcome I decided to approach the subject-matter from a similar angle, although without adopting the 'actor-network' concept as a heuristic frame-work. I developed a constructivist perspective that does not give up 'causes' and interests' as explanatory categories and which treats the pharmaceutical playing field as more than "a field of battle where the "fact" that proves more "factual" depends on which actors succeed in enrolling allies, much as leaders enroll armies and armories and politicians enroll sponsors". ${ }^{19}$ In my view a constructivist study of biomedicine in the making-whether in the past or in the present-should explain why those involved make the choices they do. Moreover it should account for the practical successes and failures of scientific medicine, as part of a long-term process of development and change.

This book therefore focuses on the following kinds of questions: What gets studied and why; and what does not get studied and why not? What makes scientists discriminate between specific experimental features, ideas, methods or claims? What meanings are given to a research object like interferon and how do these change over time? Why do some products of

18 See, for other constructivist studies of science, medicine and technology which succeed in establishing in their accounts connections between the dynamic of scientific and technological practices and the broader context of social practices; S. Shapin and S. Schaffer, Leviathan and the Airpump; Hobbes, Boyle and the Experimental Life (Princeton: Princeton University Press, 1985); D. Mackenzie, Inventing Accuracy: A Historical Sociology of Nuclear Missile Guidance (Cambridge: The MIT Press, 1990); J. Sapp, Where the Truth Lies: Franz Moewus and the Origins of Molecular Biology (Cambridge: Cambridge University Press, 1990); S. Wright, Molecular Politics: Developing American and British Regulatory Policy for Genetic Engineering 1972-1982 (Chicago: Chicago University Press, 1994); and, T. M. Porter, Trust in Numbers: The Pursuit of Objectivity in Science and Public Life (Princeton: Princeton University Press, 1995).

J.H. Fujimura, Crafting Science: A Sociohistory of the Quest for the Genetics of Cancer (Cambridge (MA): Harvard University Press, 1996), p. 239. 
research flourish and spread around the world while others seem to languish in local obscurity? Who are involved in the evaluative processes between bench and bedside? How do biomedical scientists over time succeed (or fail) in expanding the private space of their workplaces to universalize the products of their scientific work: in other words, how do they extend their authority so as to create new kinds of therapeutic practices and life forms? $?^{20}$

In my reconstruction of 'the biography of a miracle drug, the interferons' the focus is on the evolution of material and working practices, as well as on the living and feeling people who participate in the creation of new horizons in biomedicine. To learn more about how the products of 'laboratory life' may or may not succeed in proliferating to other areas of human activity, I will account for how the biomedical sciences as practice and culture develop in symbiosis with society at large. This will be done by alternately zooming in on scientists in their workplaces and zooming out to the broader social and political context in which they operate.

Following the sociologist of science Andrew Pickering, I shall use the word 'practice' in a generic sense, around which all that follows is organized in science and medicine. In this sense it refers to the realm of realtime efforts to engineer workable and stable configurations of instrumental and interpretational procedures, phenomena, instruments and theoretical considerations through a dialectical process of "resistance and accommodation". ${ }^{21}$ The term 'culture' in turn refers to the heterogenous nature of doing science that involves establishing productive relations between the 'social' world of researchers, physicians, funding agencies, industry and medicine, and the 'material' world of facts, phenomena, laboratory tools and instruments. $^{22}$

The ultimate goal of my project is to tell an accessible and informative story about interferon, explaining a variety of issues in its wake. In producing a reconstruction, interpretation and explanation of interferon's biography I will use as little theoretical vocabulary as possible. However, the lack of theoretical terms should not be misread as

20 I fully agree with Robert Proctor that questions such as these deserve priority over the epistemologically outdated and inproductive, but ever angrier realism/positivism versus relativism/post-modernism debates. Unfortunately. in the early 1990 's both parties have decided to turn their costly skills "to raising their own exclusive fortresses, from where they rain down erudition and abuse one another" instead of joining forces and try to understand the complex enterprise science and how it succeeds in changing the 'world' around us. They even used their wisdom to start the so-called 'science wars' which have already taken their first casualties. The waiting is still for a peace agreement which acknowledges that the history of science reduces neither to the sum of the facts nor to the sum of the texts or the sum of the interests; R. Proctor, Cancer Wars: How Politics Shapes What We Know \& Don't Know About Cancer (New York: Basic Books, 1995), pp. 8-9 ; Editorial, 'You Can't Follow the Science Wars Without a Battle Map', The Economist, 13 December, 1997: C. Macilwain, 'Science Wars' Blamed for Loss of Post', Nature, 387 (1997), 325.

See, A. Pickering, The Mangle of Practice (Chicago: The University of Chicago Press, 1995), p. 4. 
indicating that this research has not been theoretically informed. The constructivist perspective as presented above, thoroughly shaped the empirical focus as well as the analytical layers in my story.

Whilst the specific socio-historical study presented in this book focuses on the interferon case, I see it as having wider relevance. It aims at furthering the understanding of the complex processes through which new remedies are created and made to work at the intersection between science, state and industry in the second half of the twentieth century. As such this historical narrative might be of interest not only to historians and sociologists of science and medicine, but also to the larger audience of scientists, doctors, patients and other interested public groups. They are all in different ways grappling with the new capabilities that 'molecular medicine' continues to yield. For instance, this book can help doctors and regulators to come to terms with the post-interferon framework for developing and evaluating new remedies. At the same time, patients and their organizations may be able to distill from the 'interferon story' pieces of information that can help them to use their growing influence in the medical arena in a more effective way.

\section{The historian's toolbox on display ${ }^{23}$}

My aim is to provide a fine-grained chronological reconstruction of the interferon story that does justice to the twists and turns of the experimental practices under survey. With most of the early work on interferon now being dismissed as "much second class research that was carried out with third class preparations slightly contaminated with interferon", it is the historian's task to recover the ways in which interferon researchers acted within a specific historical context. ${ }^{24}$ However, I have to emphasize that the historian's ambition to tell the story 'how it really was' is here subservient to the ethnographic approach of following the historical

23 The following studies helped me to compose the third part of the introduction: M.J.S. Rudwick, The Great Devonian Controversy (Chicago: The University of Chicago Press, 1985), pp. 7-9; D. Mackenzie, Inventing Accuracy; A historical Sociology of Nuclear Missile Guidance (Cambridge (MA): The MIT Press, 1990), pp. 12-14; E. Richards, Vitamin C and Cancer (New York, St. Martin's Press, 1991), p.12. L. Star, 'Power, Technology and the Phenomenology of Conventions: On Being Allergic to Onions.' In J. Law (ed.) A Sociology of Monsters: Essays on Power, Technology, and Domination (London: Routledge, 1993), 26-56; R. Kohler, Lords of the Fly: Drosophila Genetics and the Experimental Life (Chicago: Chicago University Press, 1994), 13-5; and, G. L. Geison. The Private Science of Louis Pasteur (Princeton: Princeton University Press, 1995), 11-6.

See, R. Oldham, 'Interferon a Model', In I. Gresser (ed.) Interferon 6 (London: Academic Press, 1985), p. 128. 
actors as they themselves explore and discuss every aspect of the knowledge-making process. ${ }^{25}$ In particular I focus on how these biomedical scientists went about experimenting and on how they managed to transform nebulous problems at the bench into understandable molecular entities which promised to serve the public interest. By attempting empirically to view the world in the actors' own terms, I highlight their multiple visions and means of achieving them. An important challenge posed by the rich source materials on the evolution of interferon is not only to encompass what transpired at a particular level of analysis [for example, biomedical researchers versus private and state institutions] but also to address the relations among actors and to reveal the processes that linked the various social worlds at different levels of analysis.

As a historian of twentieth century life sciences I have been able to make use of the sociologist's classic form of evidence, the 'in-depth' interview. However informative and exciting oral sources in general appear to be, oral history as a source is however inherently problematic. Recollections of scientists are as subjective and unreliable as all recollections are. This is an inevitable consequence of the continuous retrospective changes in meaning and interpretation of historical events and also of the interview as a social interaction.

In the obvious weakness of oral memory as a subjective interpretation of the past lies also its special strength. ${ }^{26}$ Interviews, even about events being recalled decades later, provided me with valuable information about issues which were hardly mentioned in written sources (with the exception of personal letters). Among other things I got to know more about driving forces, influences, the nature of relationships, 'tacit knowledge', working procedures and techniques, the character of buildings and the atmosphere in a working place, personal tragedies, even about what retrospectively are called 'mistaken' beliefs that shaped practices. Moreover, I discovered important documents like the laboratory notebooks of Alick Isaacs and Lindenmann's correspondence with Isaacs, that would have been difficult to trace otherwise.

Written or printed documents like personal letters, minutes of meetings, scientific papers, internal reports are neither trouble-free of course. They not only served specific purposes but quite often were meant to conceal rather than to reveal the twists and turns between bench and bedside. Even laboratory notebooks are incomplete traces of the laboratory work under survey; and however neatly written they often proved difficult to decipher and interpret.

In general, all information must be evaluated both in terms of internal consistency and in comparison with other sources: whether photo, film, printed or written documents, or interviews. Whenever persistent contradictions between the recollections of the participants

25. See, for a classical and best read example of studying scientific practice by using the notion of "antropological strangeness" - treating scientists at work, as if they were a sort of exotic tribe with strange and puzzling customs: B. Latour and S. Woolgar, Laboratory Life (Princeton: Princeton University Press, 1986). 
and documentary evidence in the form of letters, notebooks and manuscripts occurs I will indicate why a certain type of source has been given priority.

However rich and accessible the source materials might look at face value to historians used to study pre-modern periods and subjects, the task of historical reconstruction and analysis of events and developments in twentieth century biomedicine provides the researcher with new and genuine challenges. First of all I met with specific problems in collecting contemporaneous interferon-related documentary material. For example, institutional and governmental records in various countries have limitations on access: in the UK public records are in general subject to a privacy law that keeps them closed for at least thirty years. More significant than formal restrictions, however, is the fact that only part of the institutional or personal records make the journey into archives. Because of space constraints and frequent relocations of both individuals and institutions, loads of papers had been discarded before I could study them. Furthermore, from the 1980's onwards collections of letters between scientists, who switched to other forms of communication (telephone, fax), become a scarce commodity. ${ }^{27}$ In addition to these practical problems there is a systematic bias in the documentation, in that the fullest records are mostly those of the major actors. Since the very same 'elite group' of interferon researchers accounted for almost $75 \%$ of the production of publications and other scientific products I took this bias for granted. Having said this I have taken great care to prevent a so-called 'executive approach' by including the voices of those outside the inner-circle of 'interferonologists'.

Another difficulty that manifests itself when studying twentieth century history of science, in particular in the case of very recent developments, is that the historian is dealing with history in the making. With most of the dramatis personae of the study, their allies and opponents included, still alive and in some cases professionally active, the historian may get caught up in on-going struggles over scientific and technical knowledge claims. In principle, all participants have something at stake in the historian's interpretation and presentation of the subject matter, but for some the stakes are higher than for others. However determined to keep a safe distance from the 'battle scene', the historian cannot avoid being affected by it, if only through a lack of cooperation in obtaining access to documents and participants. Undoubtedly this will have influenced my reconstruction of the interferon story, but is this problematic in itself? My answer is 'no', as long as the historian acknowledges that the goal of achieving strictly neutral and symmetrical historical accounts "is as mythical in actual practice as the scientist's goal of neutral evaluation of competing therapies" ${ }^{28}$ In fact, in writing about interfe-

27 Despite the fact that the records available to me were not perfect and that there might be some gaps I have long since reached the point of diminishing returns. In other words I think it most unlikely that studying yet another box of documents might lead to any major change in my reconstruction of the development of the interferons. 
ron in terms of retrospect and prospect I have become part-albeit a tiny part - of the history I write about.

\section{The biography of a 'miracle drug'}

The interferon story has all the ingredients that can be found in successful Hollywood movie scripts; the heroic struggle of individual scientists to get recognition for their work, the high hopes and disappointments, the dramatic illness of one of the protagonists that brings him in an asylum and leads to an untimely death, the 'miraculous' effects of a seemingly forgotten biological compound and the hysteria surrounding interferon as a miracle drug against cancer. This biography of a therapeutic drug, however is less interested in exploiting the dramatic qualities of the story than in exploring how interferon managed to play many different roles as an evolving family of biological agents. Interferons have been regarded as research objects, miracle cures, research tools, medical commodities, conceptual part of the cellular immune system, genetically engineered proteins and patentable biological inventions. By concentrating on interferon as it proliferates in three mutually overlapping realms-laboratory science, medicine and the public domain-I tried to sort out, how, through complex lines of interaction both interferon and its 'environments' were modified. However this was far from easy. The moment I started following the things called interferon 'I lost them'; they had changed and they would continue to change during my research journey.

In the face of the complexity of the interferon story the most useful narrative thread is still a chronologically one. Each chapter deals with another episode in the biography of interferon from the 1930 's up to the present. In order to do justice to the idea of scientific work as skilled craftsmanship, practiced within a shared tradition and field of research, I will start my account with a brief sketch of the historical development of the intellectual and material framework within which the new biological factor, named 'interferon', would ultimately be shaped. In this prelude, that constitutes chapter 1, I will make explicit, as far as possible, what for the dramatis personae in chapter 2 remained largely tacit and taken for granted. The way is then free for the rest of the narrative to unfold without repeated interruption for the explanations of procedures and concepts that would otherwise be necessary.

Chapter 2 accounts for the experimental events, whereby 'interferon' as a current matter-of-fact was shaped at the National Institute of Medical Research in London between 1956 and 1957. The emphasis is on studying and analyzing how the researchers Alick Isaacs and Jeanne Lindenmann in experimenting at the laboratory bench, built and manipulated an experimental system. By the time the fleeting products of laboratory practice achieved a certain form of consistency that met with local standards, research papers were written up for publication. However publishable, the significance of the experimental claims concerning the 
new virus inhibitory factor named 'interferon' had yet to be determined.

Chapter 3 discusses 'the fierce fight' involved in transforming interferon from an obscure biological factor at the margins of viral research into a laboratory phenomenon that legitimized the 'blossoming' of a new field of research. Another point of interest is how the perceived role of medicines like penicillin as 'new wonders for the doctor's bag', as well as a national so-called 'American penicillin syndrome', triggered interest in developing interferon as a therapeutic drug in Britain.

Chapter $\mathbf{4}$ addresses the factors shaping the first successful postwar initiative to secure a formal collaboration between the British government and the drug industry, as a means to develop interferon as a therapeutic drug. The main focus is on how the various parties tried to cooperate across the domains which those involved routinely demarcated as 'science', 'policy' and 'industry'. I will describe how interferon as a product of biological research increasingly met with resistance from a chemistry-dominated pharmaceutical industry and how this gradually drove the various parties involved in the British Interferon Collaboration apart. By paying attention to the preparations for, and conduct of clinical trials with interferon, I will show that if anything is evident it is the lack of self-evidence in evaluating experimental results.

Chapter $\mathbf{5}$ studies in detail the role biological standardization played in the management of differences in interferon research. A central issue for researchers in the embryonic field of interferon research was the uncertainty resulting from the non-compliant character of research materials and organisms. This chapter is concerned with the accountability strategies used by interferon researchers to facilitate the circulation of research results and materials. I shall show, how among other factors, the intrusion of powerful spheres, such as the pharmaceutical industry, into the relatively private domain of 'laboratory life' played an important role in creating a demand for biological standardization.

Chapter 6 describes the emergence of interferon as an anticancer agent in the 1970's. Focus will be on the ways in which the major collective or individual actors-ranging from doctors, journalists, laboratory researchers, patients, politicians, regulators to drug company executives-left their mark and influenced the ways in which interferon was portrayed and perceived, primarily in the American public arena. Special attention will be payed to the role the mass media played in what has become known as the 'interferon crusade'.

Chapter 7 examines the question what ultimately constituted therapeutic success in the case of interferon. Despite having failed to live up to its public promise as a therapeutic breakthrough, interferon succeeded in finding a niche in clinical practice. The primary focus of this chapter will be the interaction between interferon and the existing biomedical culture - how this interaction both modifies the potential medical innovation and its 'environment'. Special attention will be paid to the role clinical trials have played in naturalizing the interferons as part of medical practice. 
Finally, chapter $\mathbf{8}$ is meant as an extended commentary on this biography of a 'miracle' drug. It will analyze how through the mangle of practice and culture interferon continued to change shape in its twisted existence as an evolving family of biological molecules. 


\section{Chapter 1}

\section{Setting the scene: (re-)modelling a set of biological phenomena.}

\subsection{Introduction}

In the summer of 1956 at the National Institute for Medical Research at Mill Hill near London, a visiting Swiss scientist, Jean Lindenmann, was introduced to Alick Isaacs, one of Britain's leading virus researchers. According to Lindenmann, Isaacs began to show real interest the moment the former mentioned his yet unpublished experiments dealing with a laboratory phenomenon called 'viral interference'-characterized as the inhibitory effect by one virus upon the propagation of another in a host organism. Since Isaacs had already published a series of papers on viral interference, he was eager to hear more about these experiments. Among other things it appeared that in studying viral interference Lindenmann had used a somewhat similar experimental set-up as Isaacs, with fertile hen's eggs and influenza viruses. At the end of their lively conversation Isaacs suggested they might join forces and start a collaborative study on something related to the phenomenon of viral interference...

The previous paragraph could have been the opening lines of a history of interferon research. It describes the very beginning of a collaboration which resulted in the shaping of a new laboratory factor named 'interferon'. To start a history in this way, however, would be like starting a play without having set the scene properly, thereby preventing the audience from grasping the structure and dynamics of the story-line. For instance, from what perspective should one look at Isaacs's metaphorical phrase-"Interferon was born 9 years ago of parents whose family name is viral interference and who are now 30 years old"-in the first handbook on interferons?' Was there, indeed, a parental relationship between 'development' of viral interference and the 'birth' of interferon, and in what way was interferon the fruition of the old and the beginning of the new? To shed some preliminary light on these question, I decided to write a 'prelude', which sketches the historical development of research materials, procedures and theoretical concepts that were available to Isaacs and Lindenmann in 1956.

In setting the scene for the interferon case, this chapter follows the transformation of viral antagonism among animal viruses or what became later known under the heading 'viral interference', from an object of laboratory study to a standardized laboratory tool employed in

1 A. Isaacs, 'Introduction', in N. Finter (ed.) Interferons (Amsterdam: North-Holland Publishing Company, 1966), p. X. 
viral research. ${ }^{2}$ At the same time I will explore how, by means of scientific articles, the biomedical researchers under survey communicated new knowledge claims about experimental techniques, phenomena and theoretical concepts and at the same time re-ordered and transformed already existing claims. As such, it is an attempt to trace several aspects of the process by which biomedical knowledge evolves in academic journals.

The next section deals with observations and hypotheses concerning viral antagonism in laboratory animals in European and American laboratories, and the emerging notion that the different observations of viral antagonism in animal models might be related. I shall then describe and analyze how the application of new research techniques helped to transform the study of what by the late 1940 's was generally referred to as 'viral interference'.

\subsection{About rabbits, viruses, monkeys, mice and viral antagonism.}

It was the Italian bacteriologist Flaviano Magrassi, who presented one of the first investigations leading to definite recognition of interference phenomena. In the early 1930's Magrassi had been engaged in laboratory studies dealing with herpes virus infections in rabbits at the Institute of Microbiology ('Hygienischen Institut') of the University of Basel, led by the bacteriologist Robert Doerr. ${ }^{3}$ By the time Magrassi started his viral research work in Basel, various strains of herpes virus had been isolated and tested for their pathogenic activity in pathology and bacteriology laboratories worldwide. These experiments were performed in vivo, in the rabbit. ${ }^{4}$ Over the years it had been noted by various researchers that strains of

2 In searching for relevant literature to set the scene for Isaacs's and Lindenmann's joint research efforts, I followed the route a novice to the subject of virus interference between animal viruses would have chosen in the mid-1950's. The most recent review article was often regarded as a useful starting point. Hence, I retrieved the review article 'Interference phenomena between animal viruses', published by the American physician and virus researcher Werner Henle in 1950. In the 1950's this particular review was widely considered as mandatory reading for anyone interested in research dealing with interference between animal viruses. Besides providing a comprehensive listing of published reports dealing with instances of viral antagonism among animal, plant and bacterial vinuses Henle pointed out that the first laboratory investigations leading to a definite recognition of interference between animal viruses were published in 1935. On the assumption that the literature listed by Henle is exhaustive and representative enough to mirror the total output of literature, I decided to base this chapter on literature items from Henle's review paper which were published between 1935 and 1949; W. Henle, 'Interference Phenomena Between Animal Viruses: a Review', J. Immunol. 64 (1950), 203-35.

3 My reconstruction of Magrassi's research work is largely based on the following articles and book: F. Magrassi, 'Studii sull'Infezione e sull'Immunita da Virus Erpetico. Nota r, Boll. Ist. Sieroter. Milan., 14 (1935), 773-821; F. Magrassi, 'Studii sull'Infezione e sull'Immunita da Virus Erpetico. Nota II and III', Z Hyg., 117 (1936), 501-28 and 573-620; and A. Grafe, A History of Experimental Virology (Berlin: SpringerVerlag. 1991), pp. 63-84.

4 In the 1920 's and 1930 's bacteriologists mainly depended on laboratory animals for culturing and studying animal viruses. Since the successful transmission of a herpes virus to the cornea of a rabbit in a German 
herpes virus could be differentiated according to their pathogenic (disease inducing) behavior. ${ }^{5}$

Two groups of herpes viruses were distinguished: highly pathogenic or so-called 'encephalitogenic' strains, and weakly pathogenic or so-called 'non-encephalitogenic' strains. If rabbits were inoculated with an encephalitogenic strain they typically developed 'encephalomyelitis' [i.e. an acute inflammation of the brain] and eventually died, regardless of the route of inoculation-via the skin ('intradermally'), onto the transparent membrane covering the circular part of the front of the eyeball ('onto the cornea or corneally') or into the principal part of the brain ('intracerebrally'). ${ }^{6}$

Inoculation with non-encephalitogenic strains, however, was generally followed by minor disease symptoms, such as peripheral lesions of the skin and the cornea, but not by encephalomyelitis, unless the virus preparation was injected intracerebrally. In addition it was common knowledge that rabbits which recovered from the disease in most cases were immune against subsequent reinfection with herpes virus. In other words, the rabbits did not develop any disease symptoms. This protection or immunity in rabbits, which in most cases did not last very long, was thought to result from the presence of specific, circulating, neutralizing antibodies against herpes virus.

In the course of his experiments Magrassi found that if he inoculated rabbits

laboratory in 1912, the rabbit had established itself as a suitable laboratory animal for both culturing and studying herpes viruses. In Magrassi's laboratory herpes viruses were cultured by inoculating the cornea of rabbits. Basically one started by treating the eyes of one or two healthy rabbits with a virus strain, that had been freshly isolated from its original host ('first passage'). Subsequently about 3 days later the virus was collected from the eyes of the infected rabbits and passed onto the cornea of healthy rabbits ('second passage'). This procedure was repeated every three to four days for as long as the passage was successful: See, A. Grafe, A History of Experimental Virology (Berlin: Springer-Verlag, 1991), pp. 63-84.

Ever since the word 'virus' appeared in Latin writings, the connotation of the word has undergone changes. The reasons for the gradual shift in meaning fall outside the scope of this book. Whenever necessary 1 shall give the contemporary meaning of the word virus. In the early decades of the twentieth century the word 'virus' or what in most cases was referred to by the term 'filterable virus' was used to denote any 'filterable infectious agent' which could pass through an ultrafilter that was known to retain in a reliable way bacteria. By the 1920's there was growing dissatisfaction with the significance and standardization of the procedure of filterability which was used as the main criterion for classifying infectious agents as viruses. As a result, the criterion of filtrability was no longer thought to be sufficient in itself and was supplemented with criteria like invisibility in the light microscope and non-cultivability on inert nutrient media. Viruses then became widely regarded as disease-causing agents, microscopically invisible, which could pass through an ultrafilter and were dependent on living host cells for their propagation. At the same time there was a growing tendency to use the word 'virus' in an unmodified fashion. Furthermore, researching viruses was generally considered an integral part of bacteriology, though most bacteriologists felt that the study of viruses was an esoteric and difficult subject, to be left well alone by all but a few specialists. Not until the 1950's would virology be regarded as an independent field of research. Hence, like Sally Hughes, 1 shall use in this chapter the term 'viral research' in referring to research work dealing with viruses. See, S. Hughes, The Virus: A History of the Concept (New York: Science History Publications, 1977), p. XI and pp. 109-114; T. van Helvoort, 'History of Virus Research in the Twentieth Century: The Problem of Conceptual Continuity, Hist. Sci., xxxii (1994), 185-235.

In cases where author(s) did not provide further information about the kind of animal strain that was used, i.e. whether or not it was a special bred of laboratory rabbit. 1 will use the species name. 
intradermally or corneally with a non-encephalitogenic strain of herpes virus, which was not supposed to affect the brain, after four to five days the animals appeared to be immune against a second, normally fatal injection of the same virus into the principal part of the brain. This was quite peculiar, since neither virus nor antibodies against the virus could be detected in the brain of these animals at that point in time.

Unable to explain the antagonistic phenomenon in terms of a normal immune response-the production and presence in the host of specific antibodies against the virus-Magrassi put forward the hypothesis of a special form of tissue immunity: "Lo stato non infettante-immune del cervello" (The non-infectious immune state of the brain). He thought this special kind of tissue immunity - for which there was no precedent and which would need further research-was responsible for what he called the "immunitá verso la superinfezione" (immunity against superinfection). ${ }^{7}$

After Magrassi had left Basel for Milan, the head of the 'Hygienischen Institut', Doerr, and a co-worker, Seidenberg, followed up on what they called "Phänomen von Fl. Magrassi" (Phenomenon of Fl. Magrassi) or "Konkurrenzphänomen" (Competition phenomenon). ${ }^{8}$ Doerr and Seidenberg regarded Magrassi's phenomenon and its variations as being a competition between two successive virus infections. They ascribed the experimental phenomenon to some kind of infection-related immunity, but for which no satisfying theoretical explanation seemed to be available yet. In a further comment on the competition phenomenon, Doerr drew an analogy with a study performed by Meredith Hoskins, an American bacteriologist working at the Yellow Fever Laboratory of the Rockefeller Foundation in Brasil. ${ }^{9}$

Almost simultaneously with Magrassi, Hoskins had published the following experimental results. After inoculating rhesus monkeys with a so-called viscerotropic yellow fever virus, in most cases the animals succumbed within 10 days. ${ }^{10}$ However, rhesus monkeys which had been inoculated with a mixture of the viscerotropic strain and a relatively harmless

7 See, F. Magrassi, 'Studii sull'Infezione e sull'Immunita da Virus Erpetico. Nota I, Boll. Ist. Sieroter. Milan., 14 (1936), 773-821, p. 774.; and F. Magrassi, 'Studii sull'Infezione e sull'Immunita da Virus Erpetico. Nota III, Z Hyg., 117 (1936), 573-620, p. 617.

8 "In der Absicht, die ursprüngliche Versuche nachzuprüfen und zu ergänzen": See, R. Doerr and S. Seidenberg. 'Die Konkurrenz von Virusinfektionen im Zentralnervensystem (Phänomen von Fl. Magrassi)', Z Hyg., 119 (1937a), 135-65, p. 139.

9 See, R. Doerr and M. Kon, 'Schieneninfektion, Schienenimmunisierung und Konkurrenz der Infektionen im Zentralnervensystem beim Herpesvirus', Z Hyg. 119 (1937), 679-705, pp. 702-4.

10 The term "viscerotropic virus" was used to designate a strain of yellow fever virus which was cultured or carried in the rhesus monkey ever since its isolation from a human patient. It was known to be a highly fatal strain producing lesions in the internal organs of rhesus monkeys. See, M. Hoskins, 'A Protective Action of Neurotropic Against Viscerotropic Yellow Fever Virus in Macasus Rhesus', Amer. J. Trop. Med. and Hyg. . $15(1935), 675-80$, p. 675 . 
neurotropic strain of yellow fever virus survived. ${ }^{\text {" }}$ Subsequently, it also appeared that when monkeys were inoculated successively with preparations of viscerotropic and neurotropic virus, the animals stayed alive, provided the neurotropic strain was given within 20 hours of the viscerotropic strain. Hoskins indicated that she had no adequate explanation for this apparently 'protective action' of the neurotropic virus against the viscerotropic yellow fever virus, as tests for the presence of protective substances in the virus preparations had been negative. According to Doerr, Hoskins's studies seemed to deal with a competition phenomenon between virus infections. However, he emphasized the fact that it was rather difficult to judge whether the 'phenomenon of Fl. Magrassi' bore more than superficial resemblance to the one described by Hoskins. ${ }^{12}$ Thus, Doerr did not regard either of these biological phenomena in terms of interference between viruses, or as similar phenomena.

In fact the term 'interference phenomenon' was first used by the British scientists Findlay and MacCallum, early in 1937. Upon reading Hoskins's studies in the American Journal of Tropical Medicine, both researchers, who had been involved in yellow fever virus research themselves, became seriously interested in Hoskins's so-called "curious phenomenon". Thus they decided to initiate studies in their London laboratory "to confirm the findings related by Hoskins and if possible throw some light on their causation". ${ }^{13}$

Findlay and MacCallum first made an attempt to see whether the 'protective effect' in rhesus monkeys of the neurotropic against the viscerotropic, or what they called 'pantropic' strain of yellow fever virus, would materialize in their laboratory. In doing so, they were able to confirm Hoskins's observations that monkeys inoculated successively with a pantropic and neurotropic virus preparation survived, whereas all monkeys injected with pantropic virus died. They also noted that while the neurotropic virus apparently protected monkeys against the pantropic virus, the reverse did not hold true. Subsequently, further efforts were launched to replicate the results with other laboratory animals like mice and hedgehogs. In all species under investigation they were able to show that the neurotropic virus protected against the pantropic strain, but not vice versa.

Findlay and MacCallum suggested two possible hypotheses for the observed protective

11 The term "neurotropic virus" was used to designate a strain of yellow fever virus which had been isolated from a human patient in Dakar and cultured in white mice. This strain no longer produced fatal internal lesions when inoculated in thesus monkeys. See, M. Hoskins, 'A Protective Action of Neurotropic Against Viscerotropic Yellow Fever Virus in Macasus Rhesus', Amer. J. Trop. Med. and Hyg., 15 (1935), 675-80. pp. 675-6.

12 R. Doerr and M. Kon, 'Schieneninfektion, Schienenimmunisierung und Konkurrenz der Infektionen im Zentralnervensystem beim Herpesvirus', Z Hyg., 119 (1937), 679-705, pp. 702-4.

13 G.M. Findlay and F.O. MacCallum, 'An Interference Phenomenon in Relation to Yellow Fever and Other Viruses', J. Path. and Bact., 44 (1937) 405-24, p. 405. 
effect. ${ }^{14}$ First, they speculated that a rapid immune response produced by the neurotropic virus through the formation of specific antibodies would neutralize the action of the highly pathogenic pantropic strain. Second, they also thought that a blockade of the host cells by the neurotropic virus might prevent the pantropic strain from entering cells and inducing a fatal virus infection. I will label this latter mechanism the 'blockade hypothesis'.

In spite of extensive testing in different experimental set-ups-varying, for instance, the mode of injection and the type of laboratory animal-they did not manage to obtain experimental data in support of either of the two hypotheses. Findlay and MacCallum characterized the phenomenon, for which no definite explanation was yet available, as the interference of one virus with the pathogenic action of another virus, hence giving it the name 'interference phenomenon'. ${ }^{15}$

By the time Findlay and MacCallum paper had reached the shelves of American and European university libraries, researchers at the Department of Bacteriology of Columbia University in New York had initiated experiments to see whether it would be possible to transmit poliomyelitis to laboratory mice. In the past, similar efforts had been launched in other bacteriology laboratories but all attempts to transmit the disease to laboratory animals other than monkeys had either failed or produced inconclusive results. This failure to infect animals other than monkeys had even become a standard identification test for poliomyelitis virus. However, the bacteriologists Claus Jungeblut and Murray Sanders from Columbia University had noted that a fellow scientist had recently published a report claiming that he had succeeded in performing a passage of a fresh human strain of poliomyelitis virus ('Lansing strain') from the monkey to so-called 'cotton rats', a particular rodent species. ${ }^{16}$ Apart from establishing the disease in cotton rats, he also claimed to have accomplished a subsequent successful passage to white mice.

Upon initiating efforts to replicate these results Jungeblut and Sanders made the following observations. First, the experimental data indicated that their poliomyelitis virus strain ('SK New Haven strain or SK virus') could, indeed, be transmitted from monkeys ('SK monkey virus') to mice ('SK mouse virus') by intermediary passage through cotton rats. In addition, the pathogenic nature of the SK virus appeared to change after continued passage

14 G.M. Findlay and F.O. MacCallum, 'An interference phenomenon in relation to yellow fever and other viruses'. J. Path. and Bact., 44 (1937), 405-24, p. 410.

15. They also indicated that analogous phenomena could be observed between viruses in plants and bacteria: G.M. Findlay and F.O. MacCallum. 'An interference phenomenon in relation to yellow fever and other viruses', J.Path and Bact, 44 (1937), 405-24, p. 420.

16. See, for the report on the successful monkey/rat passage of poliomyelitis virus: C. Armstrong. Passage of a Fresh Human Strain of Poliomyelitis Virus (Lansing Strain) from the Monkey to Eastern Cotton Rats (Sigmodon hispidus hispidus)'. Pub. Health Rep., U.S.P.H.S., 54 (1939), 1719-29. 
through rats and mice. ${ }^{17}$ Whereas SK monkey virus was highly pathogenic to rhesus monkeys in producing a fatal paralysis after intracerebral inoculation, SK mouse virus, which was highly pathogenic to mice and rats, induced rather mild, non-fatal, disease symptoms in monkeys. Since both viruses had been observed to invade the central nervous system of the monkeys an experiment was run to see what would happen if monkeys were inoculated intracerebrally with both SK mouse and SK monkey virus at close intervals. The subsequent experimental data showed that all monkeys which received an injection with both the poliomyelitis mouse and monkey virus, survived without producing any symptoms of paralysis.

Jungeblut and Sanders argued that the experimental design did not allow "the development of any immunity principles in the orthodox sense of the word". ${ }^{18}$ In other words, they thought the time between both inoculations far too short for activating the production of specific neutralizing antibodies against the viral agent. In their opinion the protective effect was more likely due to some kind of interference between the mode of propagation of the murine virus and the monkey virus. In spite of additional series of experiments, however, they did not succeed in giving an unambiguous explanation for their 'interference phenomenon', for the fact that the presence of the mouse virus in the monkey proved antagonistic to the propagation of the monkey virus. ${ }^{19}$

According to Jungeblut and Sanders, various analogous manifestations of viral antagonism had been reported in the literature and 'which, for want of a better term, have been loosely brought together under the name 'interference', thereby referring to Magrassi, Doerr. Hoskins, and Findlay and MacCallum. ${ }^{20}$ With regard to the modus operandi, Jungeblut and Sanders believed that these phenomena formed a rather heterogeneous group. Most likely the basic mechanism of their own interference phenomenon was very similar to those cases of interference, in which the competing viruses represented pathogenic and non-pathogenic variants of the same virus strain, or were otherwise related. Hence, it was thought to be similar to Hoskins' and Findlay and MacCallum's interference phenomena, but different to the resistance to superinfection described by Magrassi and Doerr.

17 By the term SK monkey virus the authors basically referred to a monkey poliomyelitis virus preparation or a suspension of grinded spinal cords of monkeys suffering from a fatal paralysis after inoculation intracerebrally with a fresh human SK strain of poliomyeltis virus. SK mouse vinus in turn referred to a suspension of minced brains of mice, which had been inoculated intracerebrally with SK virus, which had been harvested from cotton rats after initial passage from monkeys to rats.

C. Jungeblut and M. Sanders, 'Studies of Murine Strain of Poliomyelitis Virus in Cotton Rats and White Mice', J. Exp. Med., 72 (1940), 407-36, p.434.

C. Jungeblut and M. Sanders, 'Studies in Rodent Poliomyelitis; V. Interference between Murine and Monkey Poliomyelitis Virus', J. Exp. Med. 76 (1942), 127-42. 
Jungeblut and Sanders speculated about the possibility of an exhaustion of essential growth factors-I will label this mechanism the 'exhaustion hypothesis' - as being responsible for the interference phenomenon in their laboratory. They thought this hypothesis most plausible, though part of the experimental results seemed to suggest a another option. When the SK mouse virus was exposed to ultraviolet light, it appeared that its infective capacity was more reduced than its ability to interfere with the SK monkey virus or with one of the other poliomyelitis virus strains in rhesus monkeys. For this reason, Jungeblut and Sanders put up for discussion their original idea of the interfering principle in murine virus being identical with the infectious unit itself. Interference, so they argued, might as well be brought about by a noninfectious substance, "existing as an integral part of this unit or occurring separately in soluble form". ${ }^{21}$ According to Jungeblut and Sanders this seemed to indicate that something different from the actual infectious virus unit, one or the other inhibitory substance, might be responsible for inducing the interference. I will label this mechanism the 'inhibitor hypothesis'.

Without discussing in detail Jungeblut's subsequent research work, which he performed without the assistance of Sanders, I will show how he translated the aforementioned research problem into a new set of experiments. In order to test whether the infectious and interfering effects of the mouse virus preparation were one and the same, Jungeblut subjected the interfering SK mouse virus preparation to physical and chemical manipulations aimed at dissociating the interfering principle from the infectious virus particle. ${ }^{22}$ Among other things, Jungeblut used state of the art methods of physical separation such as the newly introduced centrifuge technique, which was performed in a so-called 'ultracentrifuge'. This sophisticated high speed spinning instrument was known to have the effect of sedimenting virus particles in a fluid at a certain speed/time ratio and was used to sort and weigh macromolecules as large as viruses. $^{23}$

However, in spite of extensive testing Jungeblut claimed that he could find no evidence for the presence in the virus material of an additional non-infectious, interfering agent. "On the contrary much of the evidence can be interpreted, that there is but a single entity", i.e. the

21 C. Jungeblut and M. Sanders, 'Studies in Rodent Poliomyelitis; V. Interference between Murine and Monkey Poliomyelitis Virus'. J.Exp.Med. 76 (1942), 127-42, p. 141.

For a detailed description of these manipulations; see, C. Jungeblut, 'Studies in Rodent Poliomyelitis; VI. Further Observations on Interference between Murine and Simian Strains of Poliomyelitis Virus', J. Exp. Med., 81 (1945), 275-94, pp. 276-83.

23. The ultracentrifuge technique had been developed in the 1920's by the professor of colloid chemistry, Theodor Svedberg, at the University of Upsala in Sweden. Up to 1937 Svedberg's research group had been the only one in the world which owned an analytical ultracentrifuge, thereby dominating the molecular study of macromolecules like proteins. Not until the late 1930's the ultracentrifuge became available to American and British researchers; see, B. Elzen, Scientists and Rotors; The Development of Biochemical Ultracentifuges (Enschede: PhD-Thesis, 1988), pp. 13-133; and L. Kay. The Molecular Vision of Life (Oxford: Oxford University Press, 1993), pp. 112-5. 
experimental data seemed to leave him no other option than to conclude that the interfering and infectious effect were induced by one and the same virus. ${ }^{24}$

In August 1944, just three months before Jungeblut submitted his latest research results for publication to the Journal of Experimental Medicine, the editors of Acta Medica Scandinavica received an article entitled, 'Tissue immunity in mouse poliomyelitis' from the Swedish bacteriologist Sven Gard for publication. In his paper Gard questioned Jungeblut and Sanders's earlier studies on what he called "a phenomenon interpreted by them as interference"..$^{25}$ Gard, who also was involved in poliomyelitis virus research, indicated that he himself had observed a similar kind of phenomenon but he could not however share the Americans' view on the mechanism of the protective effect. ${ }^{26}$ Doubtless the phenomenon of interference had been operative in analogous experiments such as performed by Magrassi, Hoskins and Findlay and MacCallum, but Gard was convinced that both his and Jungeblut and Sanders's virus inhibition phenomenon was different in nature.

Upon inoculating laboratory mice intracerebrally with a clarified extract of a highly pathogenic poliomyelitis virus strain, the animals developed symptoms of paralysis within a period of a week. However, if mice were inoculated with a mixture of the highly pathogenic virus probe and a crude brain suspension containing a rather harmless ('low virulent') poliomyelitis virus strain, Gard could not observe the usual symptoms of paralysis. Subsequently he figured out that if he used a purified rather than a crude brain suspension of the low virulent virus strain and repeated the experiment, no inhibition of paralysis symptoms could be detected. In Gard's view, this suggested the possibility that the inhibitory effect was not induced by the low virulent virus but by one or more specific inhibitors in the crude brain suspension.

In order to test this assumption, Gard then subjected the crude brain suspension of the low virulent virus to centrifugation in the ultracentrifuge as a means to separate virus and possible inhibitors. Next, the different centrifugal fractions were examined for their virus content and subsequently injected intracerebrally into the mice, together with the highly pathogenic strain, as before. The resulting animal data, as presented by Gard, indicate that mice inoculated with the centrifugal fraction that was considered to be virtually virus-free, developed substantially less paralysis symptoms after infection with the highly pathogenic strain than mice which had not been inoculated before. In Gard's opinion, the fact that the

C. Jungeblut. 'Studies in Rodent Poliomyelitis; VI. Further Observations on Interference between Murine and Simian Strains of Poliomyelitis Virus', J. Exp. Med., 81 (1945), 275-94, p. 292.

S. Gard, 'Tissue Immunity in Mouse Poliomyelitis'. Act. Med. Scand., 119 (1944), 27-46, p. 29.

26

S. Gard, 'Tissue Immunity in Mouse Poliomyelitis', Act. Med. Scand., 119 (1944),27-46, p. 43. 
virus-free fraction showed an inhibitory effect strongly suggested that the inhibitory activity was rather independent of the actual virus content of the preparations under survey. He claimed that as a result of the interaction between the first virus and the host, a specific inhibitor attached to the tissue developed which inhibited the growth of the second virus. In being distinct from the circulating antibodies of the blood this inhibitor was believed to be a demonstration in vitro of tissue immunity. ${ }^{27}$ Gard thought this tissue immunity phenomenon particularly interesting since it might offer possibilities for the development of a prophylactic therapy against poliomyelitis, a virus disease for which no cure existed as yet. ${ }^{28}$

Gard's report about Jungeblut and Sanders's presumable misinterpretation of the nature of their inhibition phenomenon was obviously taken into consideration by Jungeblut, when in 1948 he reported again on the protective phenomenon between two different viruses in monkeys — or interference as he still called it. In referring to his and Gard's earlier publications he stated: "The mechanism of this antagonism is not known even though several hypotheses have been advanced as possible explanations". ${ }^{29}$

If we analyze the first series of laboratory investigations in which viruses were largely studied at their macroscopical effect level through the notion of virus disease or virus infection, what strikes one first is the heterogeneity in terminology regarding viral antagonism: from immunity against superinfection, the phenomenon of Fl. Magrassi, competition phenomenon, protective effect, interference phenomenon to tissue immunity. This variation in terminology is easily matched by a diversity in hypotheses and explanations. Different as the explanatory ideas-from the blockade and exhaustion hypothesis up to the inhibitor hypothesis-may appear, they have in common a reference to something new and special. All the researchers involved emphasized that they were dealing with a novel host response mechanism that clearly distinguished itself from the orthodox and conventional mechanisms of humoral immunity - the production of specific neutralizing antibodies against the virus disease that circulate in the bloodstream of the host. This was instrumental in accentuating historical discontinuity.

The first series of studies under survey reveal also a rather fundamental shift in translating experimental (animal) data into textual arguments over time. Whereas Magrassi and Doerr discussed the phenomena at the level of the virus infection or disease, gradually the behavior of the virus unit in the host organism became more central to the argument. This shift is in line with Van Helvoort's proposition that the 1930's and 1940's should be considered a

\footnotetext{
27 S. Gard, Tissue Immunity in Mouse Poliomyelitis', Act. Med. Scand., 119 (1944), 27-46, p. 43.

28 S. Gard, 'Tissue Immunity in Mouse Poliomyelitis', Act. Med. Scand., 119 (1944), 27-46.

29

C. Jungeblut. 'Active and Inactive Murine Poliomyelitis Virus as Interfering Agents against Poliomyelitis Infection in Monkey's', Proc. Soc. Exper. Biol. and Med., 68 (1948), 19-22, 19.
} 
transition period in viral research, between what he calls the 'traditional concept of filterable virus' and the 'modern concept of virus'. ${ }^{30}$ Within the filtrable virus concept, viruses were essentially characterized in physical terms such as filterability and invisibility. In the 1930's this concept was increasingly criticized for its dependence on the state of the art of filtration and optical techniques, and could only be maintained because virus researchers developed their arguments at the macroscopical effect level through the notion of 'virus disease'. ${ }^{31}$ While the filtrable virus concept became the target of criticism, an alternative concept with its focus on functional properties of the virus unit emerged, which van Helvoort labeled the 'modern concept of virus'. ${ }^{32}$ This development accompanied the introduction of new analytical instruments like the electron microscope and the ultracentrifuge.

Apart from the diversity in terminology, hypotheses and arguments, I call attention to the continuous (re-)structuring of knowledge claims. With each additional account, the links and cross-overs between the laboratory studies under survey changed. Doerr was first in drawing an analogy between the phenomenon of Fl. Magrassi or competition phenomenon, which was under investigation in his laboratory, and laboratory phenomena reported by other researchers. Reasoning by analogy to other laboratory studies was a means to the end of producing order out of an obviously perplexing array of experimental results. Though, in each case the nature of the analogous features was different. For instance, in the case of Jungeblut and Sanders it was a matter of ordering the phenomena by simultaneously grouping and differentiating. Upon establishing particular knowledge connections they managed to accentuate a certain sense of continuity in research. In Gard's case, however, I argue that ordering the experimental results in relation to analogous manifestations of viral antagonism emphasized discontinuity. This clearly enabled Gard to separate both his and Jungeblut and Sanders's studies, which were considered exemplary for the phenomenon of tissue immunity, from the phenomenon of interference that had been operative in studies such as performed by

See, T. van Helvoort, 'History of Virus Research in the Twentieth Century: The Problem of Conceptual Continuity. Hist. Sci., xxxii (1994), 185-235. pp. 186.

31 With regard to the criterion of filterability it became more and more apparent that beside the problematic fact that filterability depended greatly on the filtration techniques used, the criterion applied to a continually growing range of infectious agents with diverse properties. In addition, with the introduction of new optical instrumental techniques such as UV-microscopy in the early thirties and electron microscopy in the late thirties it became possible to visualize viruses, thereby undermining the criterion of invisibility. While the relevance of these physical criteria was put up for debate, the criterion of non-cultivability became increasingly important for the characterisation of viruses. In the 1950 's, the latter would make way in turn for structural criteria based on studies with the electron microscope and ultra-centrifuge; See, S. Hughes. The Virus: A History of the Concept, (New York: Science History Publications, 1977), p. 113; and Ton van Helvoort. Research Styles in Virus Studies in the Twentieth Century: Controversies and the Formation of Consensus (Maastricht: Ph.D. diss, Univ. Limburg. 1993). pp. 189-91.

32 See, T. van Helvoort, 'History of Virus Research in the Twentieth Century: The Problem of Conceptual Continuity. Hist. Sci., xxxii (1994), 185-235, p. 186. 
Hoskins, Findlay and MacCallum and Magrassi.

In the next section we will continue to follow the evolution of this rather dynamic ground of investigations into viral antagonism phenomena, which for the greater part were performed in practice oriented public health laboratories.

\subsection{Toward viral interference}

Up to the 1940's most research regarding animal viruses had been performed in vivo, in laboratory animals. Viruses were generally known for their inability to grow on artificial media, such as agar plates, that made such comfortable homes for bacteria and molds. Animal viruses just would not grow outside the living cell. Initially, the most convenient place for growing them seemed to be live animals, such as particular strains of mice, rats, guinea-pigs or monkeys. However, the intact animal gradually lost its dominance to novel experimental models based on either 'chick embryo' or 'tissue culture' techniques.

Both kind of laboratory techniques had been introduced into the field of microbiology in the early decades of the twentieth century in an attempt to find new ways for growing or cultivating viruses. One of the reasons to search for alternatives to the use of experimental animals was that quite often viral research with laboratory animals had been hampered by ready mixing ('cross-infection') with other infectious agents which happened to be present in the host animal. The risk of cross-infection was generally considered the 'bug-bear' of the study of virus diseases in experimental animals. ${ }^{33}$ Furthermore, maintaining sick animals as a source of viruses for laboratory study was neither pleasant nor convenient, and rather time consuming and expensive.

The search for alternative experimental models based on chick embryo or tissue culture techniques was in line with a more general tendency within biology to seek less complex, uniform experimental biological systems. These were thought to enable scientists to investigate fundamentally vital phenomena at their most minimal levels. Studying animal viruses in fertile hen's eggs - or what were referred to as 'embryonated eggs' or simply as 'eggs'-and tissue cultures instead of laboratory animals was claimed to allow for more detailed study of viruses in their specific cellular environment. This in turn would help to achieve the ultimate goal of

33 F. M. Burnet, The Use of the Developing Egg in Virus Research, Special Report Series, No. 220 of the Medical Research Council (London: His Majesty's Stationery Office, 1936), p. 4. 
understanding the process of viral infection and multiplication. ${ }^{34}$

In this section I will focus primarily on how the deployment of experimental models based on chick embryo or tissue culture techniques was instrumental in transforming and articulating work on viral antagonism between animal viruses from the 1940's onwards.

\subsubsection{The new 'experimental animal'}

By the end of the 1930's techniques for growing and studying viruses in fertile hen's eggs had been adopted in most experimental laboratories involved in viral research. ${ }^{35}$ The British virus researcher Christopher Andrewes called the fertile hen's egg "the new 'experimental animal' which has yielded by far the best dividends". ${ }^{36}$ By the term 'dividends' Andrewes referred to the successful exploitation of chick embryo techniques both in viral research and in the large-scale production of vaccines against certain virus diseases to be used in the British and American armies ${ }^{37}$ In particular, work on influenza virus and the development of an influenza vaccine, which largely centered around the use of chick embryo techniques, was eagerly supported by the armed forces during World War II. The senior health officers of the British and American armies were all old enough to remember the devastating 1918 influenza pandemic, which killed thousands of soldiers awaiting discharge or transport home from the battlefields of World War I. ${ }^{38}$

Two of the protagonists of American influenza research were 'the Henles', who worked at The Joseph Stokes Jr. Research Institute that was part of the School of Medicine of the University of Pennsylvania in Philadelphia. In the early 1940's the German war refugees Gertrude and Werner Henle started research on culturing influenza viruses in fertile hens' eggs as a means to develop an influenza vaccine for humans. The chick embryo technique as it was

34 See, for the development of in vitro systems in viral research: L Kay, The Molecular Vision of Life (Oxford: Oxford University Press, 1993), p. 4; and S. Hughes, The Virus: A History of the Concept (London: Heinemann Educational Books, 1977), pp. 92-3.

35 For the purpose of this chapter it would carry to far to describe in detail the historical development of chick embryo models as laboratory devices in viral research. See, for a detailed account; A. Grafe, A History of Experimental Virology (Berlin: Springer-Verlag, 1991), pp. 65-70.

36 C, Andrewes, Virus Diseases of Man: A Review of Recent Progress', Brit. Med, Bull., 2 (1944), 265-9, p. 265 .

37 For instance, in the case of influenza virus much higher virus yields were obtained by the use of chick embryo techniques than in the pig or ferret.

38 W. Beveridge and F. Burnet, The Cultivation of Viruses and Rickettsiae in the Chick Embryo, Special Report Series, No. 256 of the Medical Research Council (London: His Majesty's Stationery Office, 1946), p. iii; and A. Grafe, A History of Experimental Virology (Berlin: Springer-Verlag. 1991). pp. 120-2. 
employed in their laboratory was basically performed as follows.

Freshly laid fertilized white Leghorn eggs were obtained from a nearby poultry farm and kept in an incubator for about 10 days at $38-39^{\circ} \mathrm{C}$. The embryonated eggs were then transilluminated in a special viewing box containing an electric light to see if the embryo had properly developed. A properly developed egg at this stage showed a large dark area representing the embryo and the yolk sac (see Fig 1). Beyond this area the lighter part was seen to be traversed by blood vessels. Subsequently the selected eggs were inoculated with influenza virus by the so-called allantoic route. The first stage of this inoculation was to cut a small groove into the egg shells with a dental drill (see Fig 2). The sterile virus preparation was then injected into the allantoic cavity with a fine needle (see Fig 3). Subsequently the groove was covered and the eggs incubated anew for varying periods of time (see Fig 4 and 5). Thereafter the eggs were opened (see Fig 6) and the allantoic fluid collected or 'harvested' both for passage to a fresh set of eggs and quantitative study of the virus content. The latter was measured either by inoculating mice with dilutions of allantoic fluid and monitoring the occurrence of pathological effects (in vivo assay) or by a particular in vitro assay, a so-called 'haemagglutination assay' - a specific quantitative biological test ('bioassay') for determining the relative rate of influenza virus multiplication. ${ }^{39}$

While figuring out the optimal conditions for culturing various strains of influenza virus in the allantoic cavity of embryonated eggs, the Henles made a chance observation early 1943. In passing allantoic fluid from embryonated eggs, which had been infected with a so-called influenza B virus strain, to a fresh set of embryonated eggs, the subsequent virus yields turned out to be unusually low. In an attempt to increase the virus yield the protocol was slightly altered, using a more concentrated virus preparation and harvesting the fluids after 4 instead of 2 days. However, far from having the intended effect, the changes in the protocol resulted in even lower virus yields. Upon initiating further trials by varying the dilution steps and incubation periods, it was noted that somehow concentrated virus preparations would give yields of virus distinctly lower than more dilute preparations, in other words the larger the dose of virus injected the less the yield of virus. Furthermore, the Henles gained the impression that the paradoxical phenomenon was more likely to be found in the case of preparations of virus infected allantoic fluids, which were harvested after prolonged incubation of the virus-infected eggs. That was after the titer of active, infectious influenza B virus and with it

My description of the chick embryo technique as it was essentially practised in the laboratory of the Henles was based on: W. Henle and G. Henle, 'Interference Between Inactive and Active Vinuses of Influenza; I. The Incidental Occurrence and Artificial Induction of the Phenomenon,' Am. J. M. Sci., 207 (1944), 705-17, pp. 706-8: W. Beveridge and F. Bumet, The Cultivation of Viruses and Rickettsiae in the Chick Embryo, Special Report Series. No, 256 of the Medical Research Council (London: His Majesty's Stationery Office, 1946): and G. Buddingh. 'Chick-Embryo Technics', in T. Rivers (ed.) Viral and Rickettsial Infections of Man (Philadelphia: J.B. Lippincott Company. 1952) pp. 109-25. 


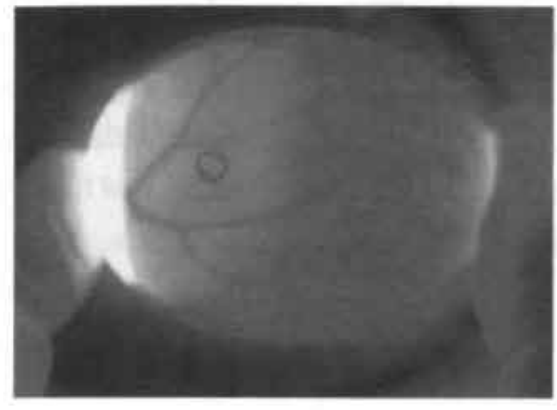

Fig. 1. Candling for allantoic inoculation at day 10.

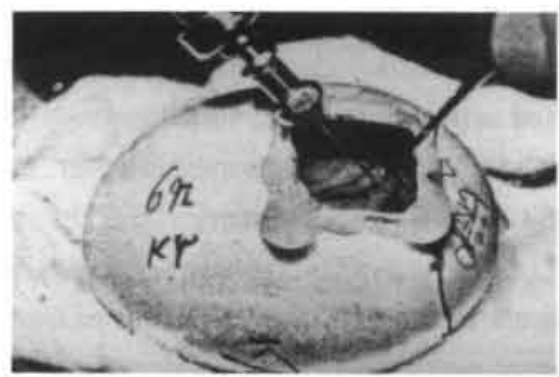

Fig. 3. The sterile virus preparation is injected into the allantoic cavity.

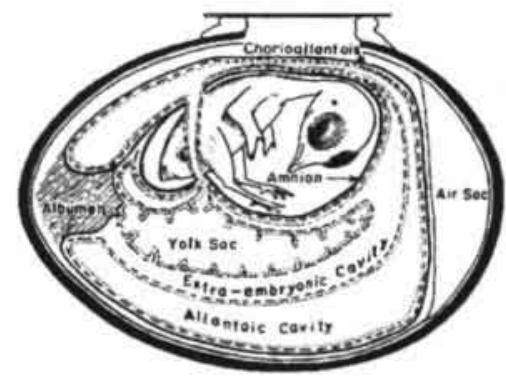

Fig. 5. Diagram of embryonated egg of 11 days' incubation showing the important structures involved in the chick-embryo technics.

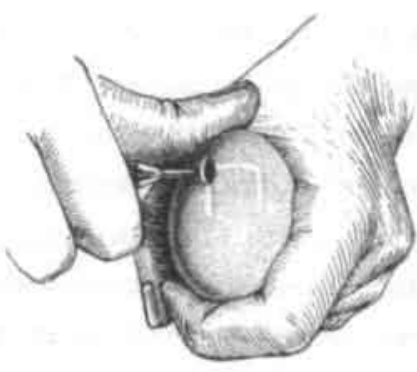

Fig. 2. Drawing illustrating method of cutting window in eggshell with dental drill.

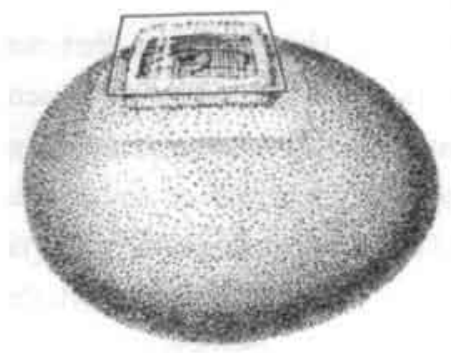

Fig. 4. Drawing illustrating closure of opening in eggshell by a cover-slip after inoculation of chick-embryo.

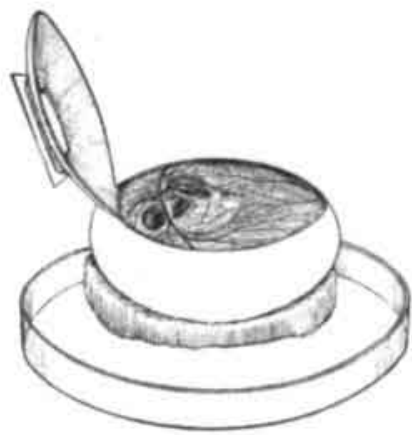

Fig. 6. Drawing illustrating the opened egg before harvesting of the allantoic fluid. 
the infectivity of the allantoic fluid was known to decrease. As a possible explanation for this 'paradoxical behavior' of the influenza B virus the Henles suggested that part of the virus particles became inactivated, in a state of non-infectivity, during prolonged incubation in embryonated eggs. On passage of the allantoic fluid to a fresh set of eggs the inactivated, noninfectious virus units were then thought to interfere with the propagation of the active, infectious virus particles. In other words, the active virus, the 'challenging virus', was hindered in its multiplication by the inactivated, the 'interfering virus'. ${ }^{40}$

In order to test this possibility, the Henles started efforts to artificially increase the amount of inactive virus in infected allantoic fluids. First they inoculated different dilutions of virus-infected allantoic fluid preparations, which had been inactivated by artificial means [through heating or ultra-violet radiation], into the allantoic cavity of embryonated eggs, and they then incubated them for a certain period of time. In accordance with previous findings the chick embryos inoculated with undiluted preparations of this partially inactivated virus showed a poor propagation rate, while normal virus multiplication rates were observed with diluted preparations. This indicated that artificially inactivated virus indeed interfered with the propagation of active virus. Subsequently, they injected active virus, either simultaneously with or shortly after inoculation of the eggs, with the inactivated virus preparations. However, even this secondary injection of active virus did not seem to affect the inhibitory effect that was claimed to be induced by inactivated virus. ${ }^{41}$

The following excerpt from the second publication dealing with these series of experiments in the American Journal of Medical Sciences, conveys the impression that the Henles believed they were on the verge of making a rather substantial contribution to influenza research:

\footnotetext{
...it becomes at once apparent that this interference by inactive virus may offer a new means of protection of susceptible cells against infection by the viruses of influenza. Not only for this reason is it worth while to study the phenomenon further, but it may offer, in addition, insight into the mechanism of infection by these agents. The use of the chick embryo for these studies is of added advantage in view of their lacking immunologic response to infection with influenza virus. ${ }^{42}$
}

The message to the reader seems to be that judging by the power of the inhibitory effect on

40 W. Henle, and G. Henle, 'Interference of Inactive Virus with the Propagation of Virus of Influenza', Science, 98 (1943), 87-9, p. 88; and W. Henle and G. Henle. The Road to Interferon: Interference by Inactivated Influenza Virus', in A. Billiau and N. Finter (eds.) Interferon I; General and Applied Aspects (Amsterdam: Elsevier, 1984), pp. 5-6.

41 W.Henle and G. Henle, Interference Between Inactive and Active Viruses of Influenza; I. The Incidental Occurrence and Artificial Induction of the Phenomenon', Am. J. M. Sci.. 207 (1944), 705-17. p. 709.

42 W.Henle and G. Henle, Interference Between Inactive and Active Viruses of Influenza; I. The Incidental Occurrence and artificial Induction of the Phenomenon' Am. J. M. Sci., 207 (1944), 705-17, p. 716. 
viral multiplication, the significance of this laboratory phenomenon might in fact be far greater than its initial incidental and local occurrence in the embryonated egg would lead one to suspect. Further study of the phenomenon not only seemed to offer a promising lead towards developing influenza vaccines but might also contribute to a better understanding of the process of viral infection.

The significance and non-local character of their phenomenon was further emphasized in the same scientific article by locating it as a new aspect of the phenomenon of interference - which was defined as the interference of one virus, the interfering virus, with the propagation of another, the challenging virus. According to the Henles this phenomenon had been described repeatedly in the literature. Subsequently, the reader is presented with a short history of interference studies.

It is worth mentioning bits and parts of this historical section as it shows us once again the ongoing transformation process of a body of laboratory studies dealing with viral antagonism phenomena, which by then was generally referred to as interference research. Among other things it shows us that by 1944 the term 'interference' had lost its provisional character.

The Henles claimed that the first instances of interference between animal viruses had "clearly" been demonstrated by Hoskins, Magrassi and Doerr, swiftly followed by Jungeblut and Sanders. In addition Findlay and MacCallum's study was referred to as an example of interference between quite unrelated viruses. The apparent non-specificity of the interference phenomenon together with the rapidity with which the protective effect was obtained was thought to set this laboratory phenomena apart from immunological reactions. ${ }^{43}$ In other words, the Henles considered viral interference to be distinct from the conventional immunological phenomena, hence requiring alternative explanations.

As possible explanations of interference among active viruses the Henles put forward the blockade, exhaustion and inhibitor hypotheses, as suggested in the literature. They also speculated about additional possibilities such as 'virucidal properties' of the interfering virus or inhibition of the growth of the challenging virus owing to pathological changes in the host caused by the interfering virus. Far from showing a preference for one or the other option to explain instances of interference among active viruses, the Henles indicated in accordance with Jungeblut and Sanders that, possibly, "interference may be caused by different mechanisms in the various instances". 44

Whatever the reason behind maintaining the multiple mechanism argument, it served

W. Henle and G. Henle, "Interference Between Inactive and Active Viruses of Influenza; I. The Incidental Occurence and artificial Induction of the Phenomenon." Am. J. M. Sci., 1944, 207:705-17. p. 705.

44 W. Henle and G. Henle. "Interference Between Inactive and Active Viruses of Influenza; I. The Incidental Occurence and artificial Induction of the Phenomenon." Am. J. M. Sci., 1944. 207:705-17. p. 706. 
well to present their experimental results as a new aspect of the interference phenomenon: the interference of inactive virus with the growth of active virus. It is worth noting that in discussing this aspect of interference, they referred rather extensively to what they believed to be analogical phenomena in studies with bacterial viruses, which were generally referred to as 'bacteriophages' or simply as 'phages'. These were published two years earlier by the life scientists and former physicists, Max Delbrück and Salvador Luria, who like the Henles were war refugees. Both men had established themselves as phage researchers respectively at Vanderbilt University in Nashville, Tennessee and at Colombia University in New York.

Upon meeting at the 1941 Cold Spring Harbor Symposium on genes and chromosomes, Delbrück and Luria had decided to work together and to spend that summer at Cold Spring Harbor Laboratory on Long Island, New York. ${ }^{45}$ They both regarded the picturesque research compound on Long Island's north shore, which occupied a few ramshackle houses, as being perfect for collaborative work on phages, for which a few petri dishes and a primitive incubator were the main equipment. Thus, while initiating phage work aimed at gaining more insight into intracellular processes of virus growth, Delbrück and Luria became involved in studies dealing with interference among bacterial viruses. ${ }^{46}$ They considered the study of interference with bacteriophages in bacterial cell cultures might be helpful for clarifying cases of interference previously described with animal and with plant viruses.

From the results of their first series of interference experiments, Delbrück and Luria concluded among other things that the interference between bacteriophages was most likely due to competition for a 'key-enzyme', which was necessary for virus reproduction and present in limited amounts in the cell. I label this mechanism the key-enzyme hypothesis. ${ }^{47}$ In their second joint publication Delbrück and Luria claimed that bacterial virus treated with a certain dose of ultra-violet irradiation lost its ability to reproduce, but was still able to produce interference with another strain of active virus. ${ }^{48}$ This experimental result not only was in line with their key-enzyme hypothesis but also suggested the possibility of rendering a host insensitive to viruses by treatment with ultraviolet irradiated virus. They firmly believed that by extending

Delbrïck and Luria made up the "social nucleus" of what would become known as the 'Phage School' with an annual phage course at Cold Spring Harbor; see, L. Kay, The Molecular Vision of Life (Oxford: Oxford University Press, 1993), p. 244; and E. Fleissner, 'Salvador Luria', in L. Levidow (ed.) Science as Politics, Radical Science Series, no. 20 (London: Free Association Books, 1986), p. 156.

46. According to Lily Kay the physicist and biological researcher Delbrück considered the experimental model based on the growth of bacteriophage in the bacterial cell as the most simple and efficient model to do studies of growth and replication with the ultimate aim to break what he called the "riddle of life"; see L. Kay. The Molecular Vision of Life (Oxford: Oxford University Press, 1993) pp. 135-36. 
this possibility to the field of animal viruses it might provide a serious lead towards the development of vaccines against virus diseases.

The Henles indicated that their research results should be regarded as an extension to the influenza viruses of Luria's and Delbrück's observations with inactivated bacterial viruses, as a more or less similar example of the interference between inactivated and active virus. However, they had far more reservations than Delbrück as to what extent these bacterial virus data might be applicable to animal viruses. The Henles regarded the key-enzyme hypothesis as just another suitable option for the explanation of their laboratory phenomenon: "As to the mechanism of this interference phenomenon, no definite explanation can be given at the moment. It is obvious that the inactivated virus is responsible for this effect" ${ }^{49}$ Thus, while leaving a specific explanation for the mechanism of action aside for the moment, they made it clear that the interfering principle was to be associated with the inactive virus particle in their case.

\subsubsection{Studying viral interference in tubes}

As I indicated in the introduction of this section, virus researchers also employed so-called 'tissue' or 'cell cultures' as a means of studying animal viruses besides experimental animals and chicken eggs. Among the first publications dealing with viral antagonism phenomena between animal viruses in tissue cultures, was Christopher Andrewes's 1942 article on interference between a so-called W.S. strain of influenza A virus ('W.S. virus') and its neurotropic variant. The latter influenza virus strain went by the name 'neuroflu' at Andrewes's Division of Bacteriology and Virus Research at the National Institute for Medical Research in London. ${ }^{50}$

Andrewes employed the following procedure for growing influenza viruses in tissue culture. Tissue fragments from embryonated eggs were obtained and minced. Subsequently minced tissue was placed in special round flat-bottomed laboratory flasks (see Fig. 7a and 7b) ), so-called 'Carrel flasks', which contained a shallow layer of liquid medium consisting of a standard salt solution, a so-called 'Tyrode solution'. In addition, the virus material was added to these tissue cultures and the flasks were put in the incubator at $37^{\circ} \mathrm{C}$. Every two to four days part of the virus containing fluid was transferred to fresh tissue cultures. Finally the presence of virus in the tissue cultures was tested for by inoculating mice intracerebrally with

W. Henle and G. Henle, 'Interference Between Inactive and Active Viruses of Influenza; II. Factors Influencing the Phenomenon', Am. J. M. Sci., 207 (1944), 717-33, p. 731.

50 C. Andrewes, 'Interference by one Virus with the Growth of Another in Tissue-culture', Brit. J. Exp. Path., 23 (1942), 214-20, p. 214. 


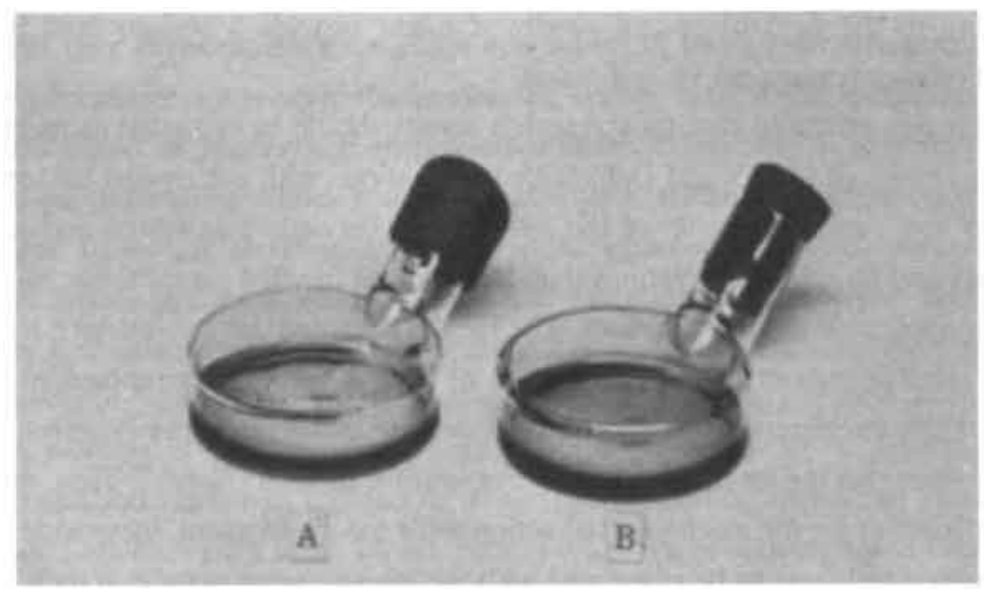

Fig. 7a. Carrel culture flasks were sometimes (A) closed temporarily with rubber caps to allow excess carbon dioxide

to escape by diffusion; otherwise (B), they

were closed with rubber stoppers and

hermetically sealed with waterproof cement. Courtesy of Paul B. Hoeber. Inc.

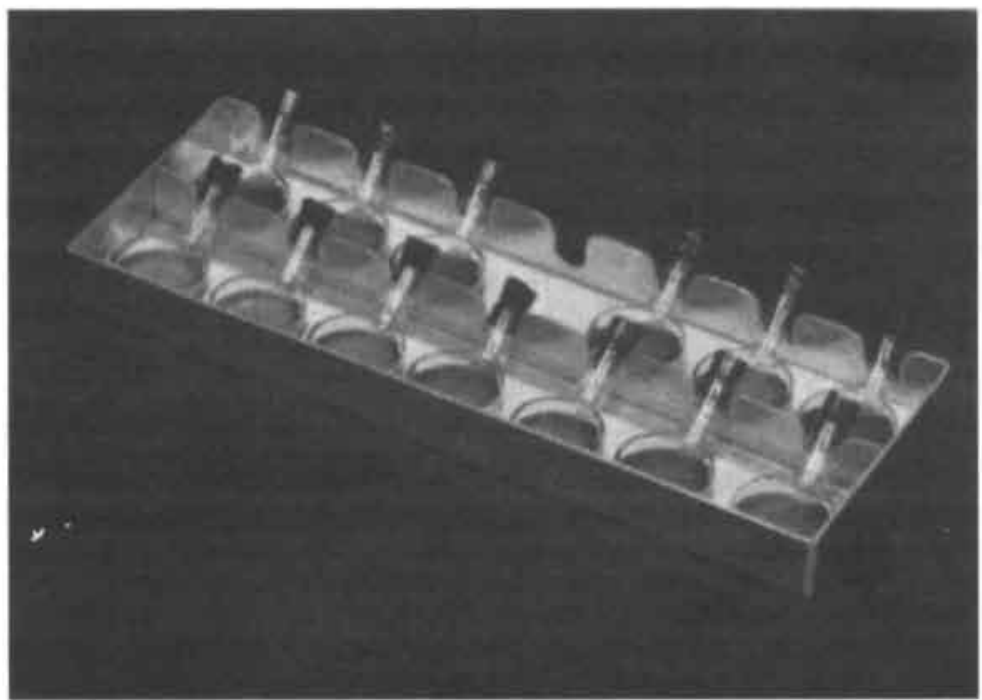

Fig. 7b. Metal rack for the accomodation of Carrel culrure flasks. 


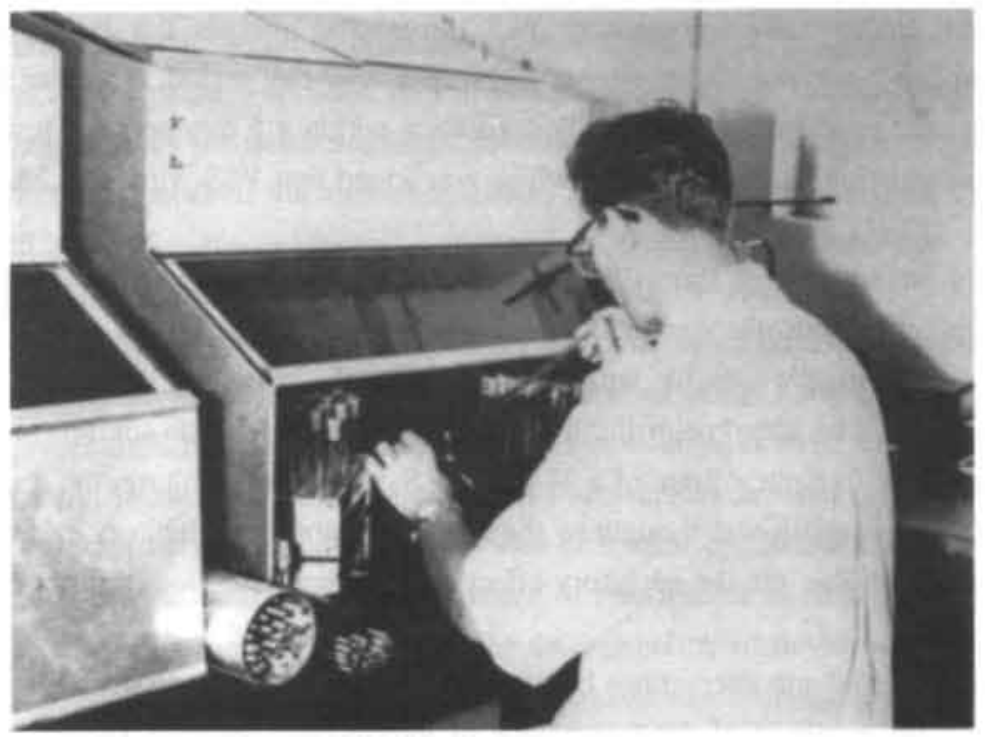

Fig, 8. Working with tissue cultures under a hood which can be sterilised by ultra-violet light when not in use. Courtesy of MRC.

tissue culture material, which was grinded up for the occasion, and monitoring the animals for the development of the corresponding disease symptoms. Since tissue cultures were known to be extremely sensitive to contamination with bacteria and other toxic agents, which disturbed both cell growth and virus multiplication, all manipulations had to be carried out under special physical conditions to prevent failures. Among other things special cleansing and sterilization procedures for laboratory equipments, and sterile working facilities were required. In Andrewes' case the manipulations were carried out in a special sterile hood ( see Fig. 8) . ${ }^{51}$

While studying possible interactions of W.S. virus and neuroflu in tissue-cultures, Andrewes claimed the following observations: When he inoculated laboratory mice intra-

51 The sensitivity to contamination and the relatively high costs of creating and maintaining the proper working conditions for successful tissue culture work, were considered a major impediment to applying tissue culture techniques by quite a few virus researchers. It was only after antibiotics like penicillin became generally available in the 1950 's that this particular technology became widely adopted in viral research. Upon preventing bacterial contamination, the antibiotics made it possible to do effective tissue culture work with nothing more elaborate than a few feet of general-purpose bench space and the sort of equipment that was usually available in any microbiology laboratory. The description of the tissue culture technique as it was basically practised in Andrewes's laboratory is based on: C. Andrewes, Interference by one Virus with the Growth of Another in Tissue-culture, Brit. I. Exp. Path., 23 (1942), 214-20, pp. 214-5; C. Andrewes, 'Virus Diseases of Man: A Review of Recent Progress', Brit. Med. Bull., 2 (1944), 265-69, p. 265; and F. Robbins, J. Enders, Tissue Culture Techniques in the Study of Animal Viruses', Am. J. Med. Sci., 220 (1950), $316-38$. pp. 316-21; R. Parker. Methods of Tissue Culture (New York: Paul B. Hoeber, Inc., 1950); and, R. Parker. Methods of Tissue Culture (New York: Paul B. Hoeber, Inc., 1962). 
cerebrally with tissue culture containing W.S. virus, the animals did not show disease symptoms and had a survival rate of almost $100 \%$ over a 12 day period. A similar inoculation with tissue culture containing neuroflu killed all mice within a 5 day period. However, if he used for the inoculation tissue cultures to which was added first W.S. virus and 24 hours later neuroflu,

the mice had a survival rate of almost $90 \%$. According to Andrewes the latter result indicated that multiplication of neuroflu was practically inhibited in cultures in which W.S. virus was already growing. In other words, the latter interfered with the growth of the former. Andrewes also found out that if he added neuroflu to cultures in which the Tyrode solution was replaced by a presumed virus-free ultrafiltrate of a 24 -hour W.S. virus tissue culture, the neuroflu grew normally. ${ }^{52}$ The latter result was thought to show that probably no inhibitory agent other than the virus was responsible for the inhibitory effect on virus growth, though further trials were required. $^{53}$

Apart from studying interactions between two strains of the same virus, Andrewes also started looking for evidence of the occurrence of interference between viruses which were unrelated. His first data directed attention to the fact that these unrelated viruses did not interfere with the propagation of the other. However, through pressure of other research work in progress in his laboratory, Andrewes had to postpone further research efforts. Subsequently, in discussing his experimental data Andrewes suggested two possible explanations for what he called "interference by one virus with the growth of another in tissue-culture" or an "in vitro interference phenomenon":

\footnotetext{
The most obvious explanation of the phenomenon is probably the correct one - that the virus first upon the scene uses up some essential foodstuff in the cells-... An alternative would be, of course, the generation within the cell of some poorly diffusible inhibitory substance."
}

Obviously Andrewes preferred a kind of exhaustion hypothesis over an inhibitor hypothesis, though he excluded neither. In both cases the phenomenon was associated with a virus/cell interaction. Andrewes added to this that by using the terms 'interference' and 'in vitro interference phenomenon', he did not wish to imply that the 'in vivo interference' of Hoskins and

52 By a virus-free ultrafiltrate of a 24-hour W.S. virus tissue culture Andrewes meant a suspension of tissue culture, which upon infection with W.S. virus, incubation for 24 hours and removal from the flasks, was passed through an ultrafilter (diameter of about $12 \mathrm{~m} \mu$ ). The latter was generally believed to block both bacteria and influenza virus particles.

53 C. Andrewes, 'Interference by one Virus with the Growth of Another in Tissue-culture', Brit. J. Exp. Path., 23 (1942), 214-20, p. 216.

54 C. Andrewes, 'Interference by one Virus with the Growth of Another in Tissue-culture', Brit. J. Exp. Path., 23 (1942), 214-20, p. 219. 
other researchers necessarily had to be explained in a similar way. The question of whether or not there was a close parallel between interference in tissue cultures (in vitro) and interference in experimental animals (in vivo), would be for future research to answer. No mention was made of differences between the various instances of in vivo interference on record, which were referred to as "Hoskins and others", and therefore it seems likely that Andrewes regarded these instances of in vivo interference as more or less similar.

The feasibility of using tissue cultures for the demonstration and study of antagonism phenomena between animal viruses was investigated further in the early 1940's by the American virus researchers Edwin Lennette and Hilary Koprowski, who were temporarily stationed at the Service for Studies and Research in Yellow Fever in Rio de Janeiro. ${ }^{55}$ Upon studying in vitro the interaction between a number of viruses in several combinations, unlike Andrewes, Lennette and Koprowski claimed the occurrence of interference both in the case of related viruses and in the case of unrelated viruses. The diverse nature of the viruses indicated that a fundamental mechanism common to all the systems studied was responsible for the interference phenomenon. They argued that with relatively harmless viruses inducing rapid protection against highly pathogenic, unrelated agents, the interference phenomenon might at least be of practical importance in the prevention of debilitating or deadly veterinary viral diseases.

As in the case of Andrewes, interference was presented as a more or less unproblematic and undifferentiated notion: "The existence of interference between animal viruses was reported in 1935 by Hoskins and Magrassi...Similar antagonistic effects in animal hosts have since been described between the viruses of...", thereby referring to Findlay and MacCallum. and Jungeblut and Sanders. ${ }^{56}$ Obviously, Lennette and Koprowski refer to all instances of antagonistic action between two viruses in a similar manner, as interference or antagonistic effects.

I argue that their portrayal of the history of virus interference research in animals as a rather unproblematical continuity of research events served to justify their argument about the existence of a fundamental mechanism common to all systems studied. Ordering in the case of Lennette and Koprowski meant accentuating historical continuity in a particular body of investigations with the aim to locate and mark their own research position.

With regard to the mechanism of action of interference, Lennette and Koprowski thought it rather unlikely that either a conventional immune response or an inhibitory agent other than the virus unit was involved in the interference phenomenon. They emphasized that whatever the nature and mechanism of this rather 'obscure' phenomenon of interference, most

55 The Service for Studies and Research in Yellow Fever in Rio de Janeiro, Brasil was jointly supported by the Brazilian government and the Rockefeller Foundation: see, E. Lennette and H. Koprowski, 'Interference Between Viruses in Tissue Culture', J. Exp. Med., 83 (1945), 195-219, p. 195.

56 E. Lennette and H. Koprowski, Interference Between Viruses in Tissue Culture', J. Exp. Med., 83 (1945), 195-219, p. 196. 
likely the phenomenon was in one way or the other associated with an interaction between virus and host cells.

In following the deployment of experimental models based on tissue culture or chick embryo techniques I have shown how their use was instrumental in transforming and articulating work on virus interference. Basically, the change in experimental work allowed for a more detailed study of the growth of animal viruses in their specific cellular environment. This in turn induced a further shift towards describing knowledge claims regarding viral antagonism phenomena in terms of the functional properties and behavior of both virus unit and host cell. However, there was a difference in idiom between the ones who employed chick embryo techniques and those who adopted the tissue culture technology. Whereas in the former case the functional properties of the interfering virus became more central to the argument, in the latter the emphasis was on the virus-cell interaction as such. The distinction became evident in the relative difference in status of the inhibitor hypothesis: on the one hand, this hypothesis was frequently tested by the 'tissue culture people'; on other hand, it received little interest from 'chick embryo people' such as the Henles.

\subsection{A standard laboratory tool for studying viruses}

In Werner Henle's review paper, which was published in 1950 and aimed at summarizing and analyzing the literature on interference among animal viruses, the inhibitor hypothesis indeed hardly received any attention. Actually, it was written off the list of suitable hypotheses for the explanation of interference. ${ }^{57}$ In this review, which will be discussed next, the term 'interference' denoted the antagonistic or inhibitory effect produced by one virus or components thereof upon the propagation of another. As on previous occasions Henle dealt separately with interference between a pair of active viruses and interference between inactivated and active viruses. However, I claim that the reason for distinguishing the two subject matters had changed over time. Drawing a basic distinction between the two types of interference, this time, no longer served to accentuate the different nature of interference involving inactivated and active virus but to emphasize that this type of interference was a more promising way to gain an understanding of the mechanism of interference. In the opinion of Henle, the chick embryo-influenza system - involving inactivated and active influenza viruses in fertilized hen's eggs — provided a

57 W. Henle, 'Interference Phenomena Between Animal Viruses: a Review', J. Immunol., 64 (1950), 203-35. p.204. 
superior laboratory tool for studying interference among animal viruses, which could be readily standardized $^{58}$

Henle pointed out that through use of the virus influenza-chick embryo system and state-of-the-art chemical and physical separation techniques, it now seemed definite that the interfering property was closely linked with the virus particle. In addition, the latest experimental data from his laboratory strongly suggested that the interfering activity was associated with what he called 'particular groupings within the virus particles' which were thought to be different from those responsible for the multiplication and toxicity of the virus. Furthermore, it appeared that for interference to occur it was necessary that the interfering agent be in direct contact with the host organism.

In contrast to his earlier publications, Henle took at this point a clear stand on the mechanism of interference, still regarding it as being entirely different from specific immunity mediated by antibodies. Basically, Henle believed that upon entrance of both the interfering and challenging virus into the cell some kind of competition for or blockade of a key element in the viral multiplication process followed, thereby inducing the interference phenomenon. This meant that he regarded the key-enzyme hypothesis as the most plausible working hypothesis for the mechanism of viral interference between animal viruses.

In discussing the practical implications of interference in the final part of his review paper Henle clearly set the agenda for further research:

\footnotetext{
The most significant contributions one might expect from continued studies of interference phenomena concern more academic problems, an increased understanding of host-virus interactions and of the nature of viral multiplication..."
}

Obviously there was less incentive to study the possibility of exploiting the interference mechanism for opening up an additional way for protection against viral disease-imparting a virus protective effect to animals and humans through infection with a live virus. That shouldn't cause any surprise since by the late 1940's several virus vaccines for humans had been tested with great success or were in the process of being tested. Moreover, the Henles had bad

58 The chick embryo-influenza system was claimed to offer the following advantages. Employing inactivated virus instead of active virus as the interfering agent was thought to stimulate fewer complicating reactions in the host organism. In addition, the chick embryo-influenza system seemed to offer advantages such as the absence of antibody formation. the ready accessibility of the host cells and the availability of simple and sensitive methods for the detection of virus. Furthermore, throughout the years this particular experimental system had provided the virology community with a wealth of information on the nature of the interfering principle and the mechanism of interference.

W, Henle, 'Interference Phenomena Between Animal Viruses: a Review'. J. Immunol. 64 (1950), 203-35, p. 230. 
experiences with testing viral interference in the clinic as a means to fight viral disease. In 1945, due to intolerable side effects they had to abort a clinical trial in which volunteers were exposed to influenza virus in the hope that this would protect them against more severe infection. ${ }^{60}$ Furthermore, after the war part of the virus researchers redirected their attention from the practicalities of the war-effort to a more academic type of work. Within this post-war academic field of virus research the process of viral multiplication or reproduction emerged as one of the central research topics. This being the case it is understandable that Henle, like most other virus researchers, no longer regarded the study of interference as an aim in itself, but as a useful vehicle for investigating the mechanism of virus multiplication.

In this respect, the phenomenon of interference in the chick embryo-influenza system meant to Henle what the interference in the bacterium-bacteriophage system was to Delbrück, a simple and efficient laboratory tool for researching host-virus interactions and the process of viral multiplication. In the years following Henle's review paper-which became widely considered as mandatory reading for anyone interested in this particular subject - the study of virus interference per se was indeed relegated to the limbo of research items of little interest to the scientific community. At the same time the phenomenon of interference became adopted as a standard tool for studying the multiplication of viruses.

\subsection{Conclusion}

Retrospectively, Henle's review paper marks a period of relative stabilization of the concept of virus interference. The phenomenon of interference became generally known as a laboratory event which could be produced regularly under definite circumstances and was instrumental in researching host-virus interactions. In order to arrive at Henle's review paper, which was regarded mandatory reading by the protagonists of the next chapter, the virus researchers Alick Isaacs and Jean Lindenmann, much hard (paper-) work was required. I traced several aspects of the process by which this specific body of biomedical knowledge evolved in academic journals.

It was a dynamic 'battle-ground' of mutually interfering laboratory practices. In the process hypotheses originally defended as universal were refuted or defended as particular and the other way around. In a similar manner the labeling of phenomena underwent transformations. For instance, what was initially considered different, for example in vitro interference versus in vivo interference or interference between active viruses versus interference between inactive and active viruses, was later on presented as similar or even as one of a kind and the

60 W. Henle, G. Henle, J. Stokes and E. Maris, Experimental Exposure of Human Subjects to the Viruses of Influenza, J. Immunol., 52 (1946), 145-65. 
other way round.

The various authors often took creative liberties, superimposing their own interpretations on the work of others in order to make convincing arguments. Far from disinterested attempts to recognize the contributions of others, the practice of citing others was instrumental in supporting a particular view. Through (re-)establishing links and crossovers between investigations researchers located and justified individual research positions. Ordering experimental results in relation to other laboratory investigations served to accentuate either novelty and historical discontinuity or coherence and historical continuity. In the process we saw how the practice of communicating knowledge claims at the level of individual investigations played an important role in the very constitution of scientific knowledge at the communal level of a subfield of research which by the 1950 's was generally referred to as the study of 'virus interference'. 


\section{Chapter 2}

\section{Interferon's Birth. ${ }^{1}$}

\subsection{Introduction}

Twenty years after Findlay and MacCallum had described virus interference its mechanism was still a mystery. How did infection of a cell by one virus prevent infection by a second?...In 1957 Jean Lindenmann and I, working at the National Institute for Medical Research in England, were investigating the action of heat-killed influenza virus when we found an unexpected handle on the problem. We found that a few hours after we had treated a cell culture with killed virus the cell-free culture medium had acquired a surprising property. When the medium was mixed with fresh cells, it made them resistant to virus infection. This resistance had all the earmarks of virus interference, since the fresh cells proved resistant not just to one virus but to many different viruses. We were soon able to isolate the active substance responsible for conferring resistance, and we named it interferon. ${ }^{2}$

Reading this 1961 portrait of what the author, the British virologist Alick Isaacs referred to as the 'discovery' of interferon, brings up several questions concerning the process of 'discovery' of interferon. What kind of research problem did Isaacs and Lindenmann start working on in the first place? Did the research problem involve elucidating the mechanism of the phenomenon of viral interference, or were Isaacs's and Lindenmann's original research questions of a different nature? How did they arrive at an "unexpected handle on their problem", and in what way was this related to the "isolation of the active substance" named "interferon"?

This chapter unravels the process whereby interferon as a current matter-of-fact was shaped in a British laboratory around 1956/57. The emphasis will be on how Isaacs and Lindenmann continually (re-)shuffled their resources in order to establish and maintain experimental systems and generate new data of some sort. The term 'experimental system' will be used to designate the manipulable aggregate of techniques, research materials, concepts, assumptions, and skills, which was shaped, reshaped or maintained by these laboratory workers at the frontier of biomedical science. With Fleck and Rheinberger, I regard an experimental system as the smallest functional unit of science which simultaneously shapes

1 A preliminary version of chapter 2 was published in Studies in History and Philosophy of Science; T.Pieters, 'Shaping a New Biological Factor. The Interferon', in Room 215 of the National Institute for Medical Research, 1956/57', Stud. Hist, Phil. Sci., 28 (1997), 27-73. 
questions and generates answers. ${ }^{3}$ The notion of 'system' emphasizes the interdependence of the constituent elements and the heterogeneous engineering involved in the evolution of experimental systems. ${ }^{4}$

After describing in the next section some of the elements that constituted the laboratory situation in which Isaacs and Lindenmann were to start their collaboration, I will then consider in detail the first two months of joint experimentation in Isaacs's laboratory. This part of the chapter pictures the intimate interplay between thought and action that took place in shaping a particular experimental system at the bench in Isaacs's laboratory. Finally in section 4 the focus will be on how Isaacs and Lindenmann came to terms with brought under surveillance a new laboratory phenomenon.

\subsection{The beginning of a British/Swiss collaboration in a London laboratory.}

On an early Monday morning in June 1956, Jean Lindenmann arrived at the gates of the National Institute for Medical Research (NIMR) at Mill Hill, London, for the first time. The Institute's enormous main entrance and seven-storeyed building, which overlooked both London's northern suburbs and the adjacent country side, made a profound impact on the young medical researcher from Switzerland (see Fig. 9). On his way to the Division of Bacteriology and Virus Research on the second floor, Lindenmann became even more impressed when he glimpsed at the interior of some of the laboratories. All imaginable equipment seemed to be available. This was confirmed later on when Lindenmann was shown around the Institute by the head of the Division of Bacteriology and Virus Research, and Deputy Director of the NIMR, Christopher Andrewes.

Andrewes belonged to the group of 'big names' in bio-medical research, which was accommodated at the NIMR, and formed part of a staff of approximately one hundred doctoral and post-doctoral workers, and an auxiliary workforce of about three hundred technicians, secretaries and others. The Institute ranked among the world's best centers for bio-medical

However interesting. Rheinberger's recent attempts to elaborate on Fleck's analysis of biomedical experimental practice, fail with respect to granting no priority to whatever aspect of experimental life. By overemphasizing the internal dynamics of experimental systems and their products, inscriptions or what he calls graphemes, Rheinberger unjustly minimizes the role in 'experimental life' of human agency and of structural constraints in departments, institutes and fields of research. Sec, L. Fleck, Genesis and Development of a Scientific Fact (Chicago: The University of Chicago Press, 1979), pp. 84-98; and H. J. Rheinberger, 'Experiment, Difference, and Writing: I. Tracing Protein Synthesis'. Stud. Hist. Phil. Sci., 23 (1992), 305-31, p. 309.

4 D. Turnbull and T. Stokes, 'Manipulable Systems and Laboratory Strategies in a Biomedical Institute', in H.E. Le Grand (ed.), Experimental Enquiries: Historical, Philosophical and Social Studies of Experimentation in Science (Dordrecht: Kluwer Academic Publishers, 1990), pp. 169-170; and J,Law,'On the Social Explanation of Technical Change: The Case of the Portugese Maritime Expansion', Technology and Culture 28 (1987), 227-52. 
research. As a result there was great interest in doing research work at the NIMR. The regular influx of visiting scientists was officially encouraged by the Director of the Institute, Sir Charles Harington. Harington believed that the resulting exchange of ideas would avoid scientific inbreeding, maintain a steady influx of new methods and techniques and keep researchers flexible in respect to incorporating new projects into their division's research repertoire. Like most other Anglo-Saxon biomedical research centers, NIMR's research agenda was primarily driven by motivations of scientific significance and impact, thereby favoring fundamental rather than applied research. At the same time the research agenda was often justified towards funding agencies for its potential medical therapeutic spin-off. ${ }^{5}$

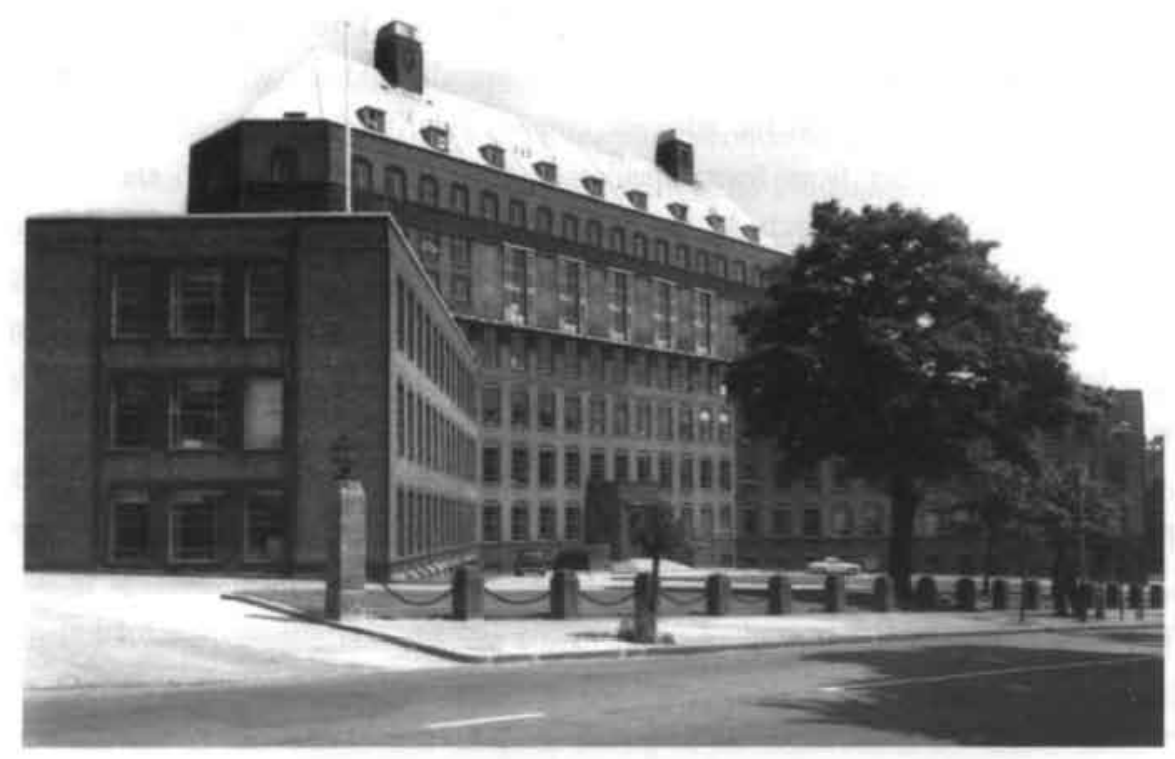

Fig. 9. Front siew of the NIMR. Courtesy of NIMR

5 J. Austoker and L. Bryder. The National Institute for Medical Research and Related Activities of the MRC, in J. Austoker and L. Bryder (eds.) Historical Perspectives on the Role of the MRC (Oxford: Oxford University Press, 1989). pp. 56-7. 
Lindenmann had never seen such a collection of sophisticated equipment like ultracentrifuges, freeze dryers, electric incubators and deepfreezes, phase contrast microscopes, and an electron microscope. The NIMR also had a special wing with large animal breeding facilities. This assured a standardized supply of healthy laboratory animals such as dogs, ferrets, rabbits, rats and mice. Furthermore there was a well organized kitchen at the heart of the Institute on the lower ground floor, which was primarily responsible for cleaning and sterilizing glassworks and preparing various standard solutions and culture media for the various research departments. The kitchen also produced special batches of media and solutions on request. This gave researchers a certain freedom to tinker with media and fluids during their experimental work. ${ }^{6}$ The kitchen was thus as much a central organ of the NIMR as the library on the fourth floor. This central storage for scientific paperwork was the last stop on their tour through the Institute.

In comparison with the rest of the Institute, the Division of Bacteriology and Virus Research had relatively few highly sophisticated laboratory instruments and facilities to their immediate disposal. However, Andrewes-or 'Cha', as he was informally known in his department - was not at all bothered by this state of affairs. On the contrary, since his research group was mainly interested in the qualitative, phenomenological aspects of virus research, there simply was not that much need for high-tech equipment. With a regular supply of fertile eggs, laboratory animals, nutritive media and glassworks, and facilities for cold and warm storage (hot and cold rooms, an electric deep freeze for biological specimens, incubators for eggs and tubes) and for sterile work (a hood for handling tissue-cultures and viruses), the division was thought to provide the standard equipment and materials for work on animal viruses and bacteria. If on occasion one needed to do some work which required facilities and equipment for complicated procedures, such as ultracentrifuges, an electron microscope, or an arrangement for electrophoresis, one could always ask the divisions of Chemistry and. Biophysics and Optics, for help. Quite often researchers in Andrewes's division would joke about their servants over there, who were so skillful in carrying out the quantitative jobs. Andrewes made no secret about the fact that he did not think a training in science of any help

6 Together with the 'melting away' of the basically selfsupporting 'nature' of biomedical institutes the role of the kitchen in biomedical laboratories has changed over the past three decades. From a major producer of laboratory materials for the greater part it has become a distributory centre for ready-made and standardized laboratorymaterials. Media, solutions, both chemical and biological substances, plastic disposables (which have displaced part of the glassworks) are purchased from commercial firms. Whenever a laboratory worker is in need of one or the other material he or she first consults one of the catalogues to see whether or not it is commercially available. Only in cases where the item is not available or appears too costly does one have to figure out a way to make it in one's own laboratory. For instance, the common laboratory practice of producing media from animal organs purchased from one of the slaughterhouses nearby is no longer common practice, apart from cases in which no standardized media are available. Hence, last minute requests for special batches of otherwise standardized materials are in general not feasible anymore. According to a number of senior laboratory workers this has diminished the impetus to tinker with media and solutions; interviews with Joseph Sonnabend, Jean Lindenmann, and Norman Finter.. 
for members of his division, who were predominantly graduates in medicine or veterinary medicine. ${ }^{7}$

Lindenmann had been working under quite different conditions in Hermann Mooser's laboratory that was part of the small institute of microbiology of the University of Zürich. The 'Hygiene-Institut', as it was called, lagged years behind its counterparts in England with its rather old-fashioned laboratories and kitchen and no access to electronmicroscopes, ultracentrifuges and the like. In a way this is remarkable, since both Swiss and English science had come through the war relatively unharmed-without being cut off from a regular supply of American journals and without a dramatic drop in research activities. According to Lindenmann, Medical Microbiology was one of the sciences which remained ossified at a prewar level until the fifties, mainly due to the intellectual isolation of the leading scientists involved. $^{8}$

Unlike the Division of Bacteriology and Virus Research at the NIMR, the 'Hygiene Institut' did not have a virology section, as it was dominated by old-fashioned bacteriologists. They still regarded viruses as ultramicrobes: living, autonomous infectious entities, which, like bacteria, multiplied autonomically by a process of binary fission. ${ }^{9}$ As such, viruses were thought to belong to the domain of bacteriology, thereby ignoring the international trend which recognized virology as an independent field of research. For the most part, the scientific staff at the 'Hygiene Institut' seemed to have missed the emerging consensus concerning the nature of viruses among American, British and French microbiologists. Viruses no longer were regarded as ultramicrobes but as infectious, potentially pathogenic, nucleic acid-containing entities of protein nature, which were reproduced from their genetic material, in casu the nucleic acid that was thought to encode the hereditary information of the virus. Unlike bacteria, viruses were considered to be dependent upon the host cell for their reproduction. ${ }^{10}$

7. This impressionistic account of NIMR's interior is based on: A. Landsborough Thomson, Half a Century of Medical Research Vol I ( London, Her Majesty's Stationary Office. 1973), pp. 108-133; D. Tyrrell, 'Personal memories of the early days', J. Interferon Res., 7 (1987), 443-444: D. Burke, 'Early Days with Interferon', J. Interferon Res., 7 (1987), 441-3: David Tyrrell, interview, Salisbury, U.K., 21 May, 1990; J. Lindenmann, 'The National Institute for Medical Research. Mill Hill: Personal Recollections from 1956/57', Arch. Virol. 140 (1995), 1687-91; J. Lindenmann to Schweiz. Akademie der Mediz. Wissenschaften, erster Semesterbericht dated December 1956. Jean Lindenmann correspondence, personal archives: interviews with Derick Burke, Jean Lindenmann and Joseph Sonnabend; and, personal visits to the NIMR in September 1991.

See, J. Lindenmann, The National Institute for Medical Research, Mill Hill: Personal Recollections from 1956/57', Arch. Virol. 140 (1995), 1687-91.

9 T. van Helvoort, Research Styles in Virus Studies in the Twentieth Century: Controversies and the Formation of Consensus (Maastricht: Ph.D. diss., Univ. Limburg. 1993), p. 186: and interview with Jean Lindenmann.

10 This characterization of viruses is more or less comparable to that given today, although the notion that the nucleic acid of the virus encoded the hereditary information of the vinus was rather vague. The mechanism of virus reproduction or replication had yet to be explored. At least there was cautious consensus that viruses 
These were anything but favorable circumstances for a young biomedical researcher with a vivid interest in the relatively new and dynamic field of animal virology - the study of viruses that prey on animals and human beings. So, after some unsatisfactory virus experiments in Zürich, Lindenmann asked permission to pursue his virus studies somewhere abroad. The head of the laboratory, the preeminent bacteriologist Mooser, was well aware of the limited possibilities for advanced virus study in the 'Hygiene Institut' and agreed to contact some British virologists whom he had met at an international microbiology meeting. One of the pioneers in the field of animal virology, Christopher Andrewes, who had acquired world-wide reputation for innovative studies of animal viruses, agreed to let Lindenmann join his research group as a 'visiting worker' for a period of one year. Subsequently, with Andrewes's letter of intent and Mooser's references, he was able to obtain a fellowship from the Swiss Academy of Medical Sciences and could start with preparations for his passage to England. ${ }^{11}$

With the intent to learn as much about animal viruses as possible, Lindenmann started working in Andrewes's division at the NIMR (see Fig. 10). ${ }^{12}$ As a relative novice to the field of virology, he was assigned to a rather low-key research job which would give him the opportunity to learn a variety of viral techniques and at the same time make a contribution to one of the division's research projects. Andrewes wanted him to work in his laboratory and do a series of experiments on the growth of polio virus in cultures of mouse and rabbit tissue. ${ }^{13}$

In quite a number of laboratories around the world polio virus was produced on a daily basis in human and monkey tissue cultures for the large scale production of vaccines and forresearch purposes. ${ }^{14}$ Since polio virus had been shown to be host-specific and not

went through an eclipse.

Interview with Jean Lindenmann.

12. Before starting his research work Lindenman had to sign an official document relinquishing in perpetuity all patent rights to whatever he might find during his stay at the NIMR to Britain's Medical Research Council; Interview with Jean Lindenmann.

13 As I already indicated in the previous chapter the practice of tissue culture technique had been plagued for years by bacterial contamination. It was not until the arrival of antibiotics like penicillin in the late fourties. that the in vitro culture of viruses in vessels with growing tissue embedded in suitable media, evolved as a major tool in viral research. By the mid-fifties this technology was known to virologists as a relatively simple and reliable means to grow a sheer endless number of viruses in their laboratories; see, F. C. Robbins and J. F. Enders, Tissue Culture Techniques in the Study of Animal Viruses', American Journal of the Medical Sciences. 220 (1950), 316-38.

Polio vinus causes poliomyelitis, an infectious, pathogenic disease that induces paralysis, mostly in infants. Until mass vaccination against polio was made possible by the advent of the Salk-vaccin in 1954, a number of devastating epidemics swept through Europe and America from the 1930's onwards, leaving a trail of paralysed and even dead people. Among those affected was president Roosevelt. He was mainly responsible for the attention that was focussed in the USA on polio as a major health threat, which after the second world war sparkled off the 'war on polio'. During this publicly led search for a cure against poliomyeltis, large sums of money were poured into virus research, boosting the newly emerging field of virology: see A. J. Levine, Viruses (New York: Scientific American Library, W.H. Freeman and Company, 1992) pp. 62-5. 
transmittable to mice or rabbits it had long been taken for granted that it would not grow in rabbit or mouse tissue culture either. However, with Donna Chaproniere, Andrewes had been able to demonstrate that myxomatosis virus did grow in guinea pig tissue cultures, ${ }^{15}$ in spite of the fact that the virus was known to be not transmittable to guinea pigs. Andrewes had picked information up at a recent meeting of the Society for General Microbiology on successful experiments concerning the growth of polio virus in cultures of rabbit tissue, despite the fact that rabbits were not prone to the disease. This suggested the possibility that the specificity of viruses for particular hosts might be lost in tissue culture. Andrewes became interested in whether polio could indeed be grown in animals not prone to the disease. As a novice scientist at the frontier of virus research, Lindenmann was asked to show that polio virus would multiply in cultures of rabbit and mouse tissue. Lindenmann's education as an M.D. and his training as a postgraduate student in diagnostic bacteriology was believed to provide him with enough resources and previous experience to tackle this new research problem. ${ }^{16}$

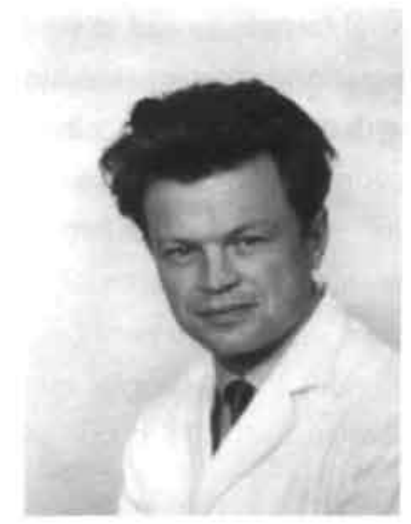

Fig 10. Jean Lindenmann (1957). Courtesy of NIMR

Myxomatosis virus was considered to be closely related to smallpox virus. In rabbits it had been shown to produce myxomatosis, a highly contagious and fatal disease, which was characterized by warts and skin tumors; see A. J. Levine, Viruses, (New York: Scientific American Library, W.H. Freeman and Company, 1992) pp. 209-10: and J. Lindenmann, The National Institute for Medical Research, Mill Hill: Personal Recollections from 1956/57', Arch. Virol. 140 (1995), 1687-91. 
Lindenmann's first two months in Mill Hill were taken up by learning through hands-on apprenticeship ('learning by doing') the craft of working with viruses as it was being practised by workers in Andrewes's division. At the same time, he initiated efforts to grow polio virus in nonspecific tissue cultures. Despite frequent changes of experimental conditions and procedures, Lindenmann was unable to find any evidence showing that polio virus multiplied in these tissues. In a first report to the Swiss Academy of Medical Sciences he stated rather optimistically:

Negative results in those experiments don't mean a lot, as only small changes in the experimental conditions can make the difference between success and failure. ${ }^{17}$

However, to the best of Lindenmann's memory the research project became increasingly frustrating. Despite numerous follow-up experiments, he still didn't manage to produce any positive results: he could only show that polio virus did not multiply in his experimental system. Andrewes strongly believed that Lindenmann's unsuccessful attempts to replicate the claimed results regarding polio virus losing its host specificity in tissue cultures were due to an as-yet undetected artifact or some uncontrolled aspect of Lindenmann's experimental set-up. ${ }^{18}$ Andrewes therefore did not think much of the idea of abandoning the project in the face of the repeated negative results. As a visitor to the Institute and novice to the field of virology, Lindenmann found it difficult to oppose Andrewes and to ask him to end the project.

Sometime during this period he was introduced to Alick Isaacs, a neighbouring researcher who had just returned from holidays. Only slightly older than Lindenmann, Isaacs at the age of thirty-five was already a distinguished virologist and an expert in influenza viruses who, like Lindenmann, had started his research career in a bacteriology department after being trained as a physician. Isaacs was in charge of the World Influenza Centre in room 215 which consisted of a large laboratory workspace with a rather small office corner for the necessary paperwork (see Fig. 11). The World Influenza Centre had been set up in 1947 by

J. Lindenmann, 'Erster Semesterbericht' dated December, 1956. Jean Lindenmann correspondence, personal archives

By the term 'artifacts' I do not mean material objects which are related to conditions of human use, such as archaeological findings, machines, designs or technical processes. In laboratory research the word 'artifact' has a different connotation. Artifacts are described by most laboratory scientists as troublesome and unintentional events, which are to distort or confuse the identifiability of 'natural' phenomena in their experimental arrangements. Such artifacts are usually attributed to flaws or inefficacies in experimental procedures. They are likely to present tricky situations and cause a great deal of uncertainty in the sense that one never knows whether an apparent artifact is a mere distortion in the observability of a 'natural' phenomenon, due to some sort of procedural flaw - a 'real' artifact or a noise-, or whether it might be a lead towards some 'hidden' phenomenon - a 'pseudo' artifact or a signal. For an analysis of artifact accounts in laboratory research, see M. Lynch, Art and Artefact in Laboratory Science (London: Routledge \& Kegan Paul. 1985). pp. $81-139$ 
Andrewes in collaboration with the United Nations' World Health Organisation (WHO) as the center of a global 'early warning' network of collaborating laboratories to study and monitor outbreaks of influenza ('flu') epidemics throughout the world. The main task of the World Influenza Centre was to gather influenza virus specimens from all over the world and collect all sorts of data concerning the outbreak and spread of flu epidemics with the aim to study the nature and epidemiology of influenza. Ultimately, the research efforts of Isaacs and his collaborators were aimed at controlling the outbreak of flu epidemics, thereby preventing a recurrence of the influenza pandemic of 1918 in which more than twenty million people had died worldwide. ${ }^{19}$

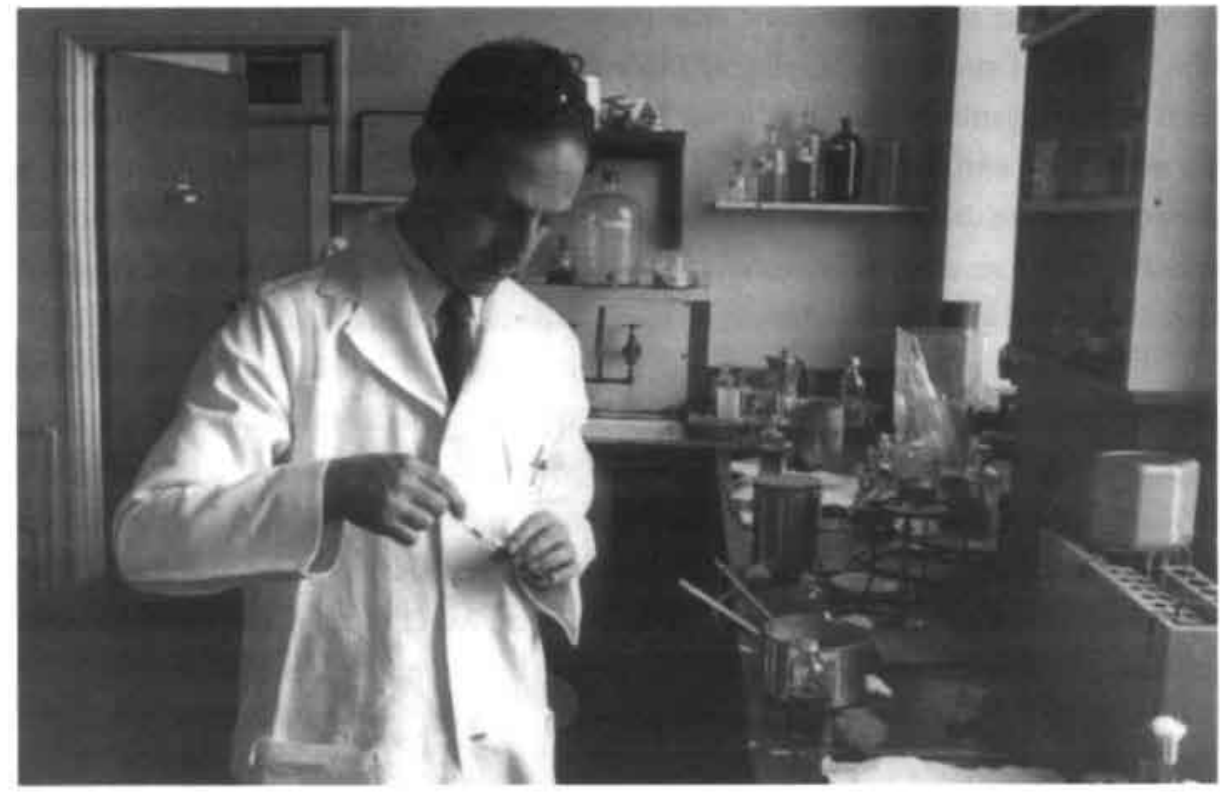

Fig. II. Alick Isaacs at work in his laboratory (1957),

Courtesy of Dr. S. Isaacs.Elmhirst.

19 C. H. Andrewes, 'Alick Isaacs', Biographical memoirs of fellows of the Royal Society. 13 (1967) 205-221: J. Austoker and L. Bryder. 'The National Institute for Medical Research and related activities of the MRC', in Historical Perspectives on the Role of the MRC, ed. J. Austoker and L. Bryder (Oxford University Press, 1989), p.43. 
During their first encounter, Isaacs asked Lindenmann about his research work in Zürich. Apparently, Isaacs began to show real interest the moment Lindenmann mentioned his yet unpublished virus interference experiments. ${ }^{20}$ Isaacs had studied for a number of years the viral interference phenomenon, which was defined as the inhibitory effect by one virus upon the propagation of another, and had published a series of papers on the subject. So he was eager to hear more about these experiments. Isaacs probably then asked Lindenmann to have lunch together. Since most researchers worked mainly within the confines of their laboratory and division, the lunch hall at the top floor was a prime market place for the exchange of information. Here researchers from all laboratories and research divisions mingled quite freely while communicating the latest shop talk and the Institute's news. ${ }^{21}$

Lindenmann told Isaacs that in May or June 1955 his boss in Zürich, Hermann Mooser, had seen a paper on the interference between strains of rickettsia, indicating that interference of one strain with the propagation or reproduction of the other was not brought about by a competition for or blockade of a receptor. ${ }^{22}$ Mooser thought it conceivable that the interference phenomenon between rickettsia might be rather similar to the interference between influenza viruses. However, to the best of his knowledge, the viral interference phenomenon was still explained in the literature as a competition for or a blockade of a cellular receptor. In the context of this view of the phenomenon of interference it seemed interesting to consider an interference experiment with influenza virus in fertile hens' eggs, mostly designated as 'embryonated eggs' or just 'eggs', which would put this 'receptor hypothesis' to a test. If Mooser had been more familiar with the viral interference literature, he would have known that by 1955 most scientists in the field had already abandoned the 'receptor hypothesis'.

In discussing his idea with Lindenmann, Mooser had proposed the following experiments: starting with the standard procedure of growing influenza virus in embryonated eggs, Mooser and Lindenmann would first try to reproduce the interference experiment between heat-inactivated, the interfering virus, and live influenza virus, the challenging virus, as described in the materials and methods section of an article in the Australian Journal of

20 The experiments were to be submitted for publication in January 1957. See, H. Mooser und J. Lindenmann. 'Homologe Interferenz Durch Hitzeinaktiviertes, an Erythrozyten Absorbiertes Influenza-B-virus', Experientia. XIII (1957), 147-8.

21 D. Tyrrell, 'Personal memories of the early days', J. Interferon Res., 7 (1987), 443-4 p. 443.

Rickettsia were considered to be insect-borne infectious agents, which at least were thought to be different from both bacteria and viruses. See, S. Smith Hughes, The Virus: a History of the Concept (London: Heinemann Educational Books, Science History Publications, 1977), pp. 93-7;and H. Mooser und J. Lindenmann, 'Homologe Interferenz. Durch Hitzeinaktiviertes, an Erythrozyten Absorbiertes Influenza-Bvirus', Experientia, XIII (1957), 148. 
Experimental Biology that Mooser had come across. ${ }^{23}$ Subsequently, they would change the experimental procedure slightly and perform a second series of experiments. They would now use heat-inactivated influenza virus stuck to red blood cells in order to see whether these viruscoated red blood cells would also be able to interfere with the reproduction of live influenza virus in eggs. Mooser believed that by sticking the inactivated virus to red blood cells, it would not be able to interact with live influenza virus. If they would be able to show that despite the physical impediment, virus-coated red blood cells inhibited the growth of live influenza virus, they could make a case for refuting the 'receptor hypothesis'.

Mooser left the work at the laboratory bench to Lindenmann, while he travelled to Africa for his annual holidays. Upon his return, Lindenmann had managed to do a number of experiments showing that inactivated influenza virus interfered with the growth of live influenza virus in the egg, even when stuck to the surface of red blood cells. Lindenmann thought that he had done a fairly good job and that the results were ready for publication. When talking over the series of experiments with the rigorous and imperious Mooser, he gradually lost his confidence. Basically, Mooser told him that the experiments had too many flaws. The most important problem was that Lindenmann could not make sure that no virus became disentangled from the red blood cells during his experiments. If virus would have got unstuck during the experiments it would imply that the interfering virus had not been fully hindered from interacting with the challenging virus and that they were most likely dealing with an artifact, in other words in that case the experiments had gone wrong. In principle it would be possible to do additional experiments but this would require state of the art instruments which were not available in the Institute. Mooser therefore decided to abandon the project and not to publish their existing results.

Lindenmann must have presented a somewhat similar account of his work at the 'Hygiene Institut' to Isaacs, who according to Lindenmann, remarked that he knew the authors of that Australian article quite well. In fact, he had published it himself in collaboration with Margaret Edney during his two-year fellowship as a visiting researcher in MacFarlane Burnet's laboratory at the Walter and Eliza Hall Institute in Melbourne. ${ }^{24}$ Isaacs had spent a great deal of his time over there extensively studying the interference phenomenon with heat-inactivated influenza virus as the interfering virus and live influenza virus as the challenging virus. In these studies, he and Edney had ascribed the interference phenomenon to the competition between

23. In this case the term 'heat-inactivated virus' refers to the process of heating the virus at $56^{\circ} \mathrm{C}$ for an hour in a buffer solution, whereupon it lost its infectious and cell destructive properties while retaining its capacity to induce viral interference in the host cell. Moreover, in the Australian paper heat-inactivated influenza virus had been shown to have superior interfering properties, while lacking enzymatic activity that would elute the virus particles from the surface of the red blood cells. For further details of the technical procedures see, A. Isaacs and M. Edney. '1. Quantitative Aspects of Interference'. Austr. J. Exp. Biol., 28 (1950). 219-30.

Lindenmann to Mooser. letter dated August 8, 1958. Jean Lindenmann Correspondence, personal archives. 
the interfering and the challenging virus for some key constituent within the cell, while rejecting a possible competition for or blockade of cellular receptors. However, he had neither been able to produce convincing experimental data in support of this hypothesis in Burnet's laboratory, nor could he on his return to the World Influenza Centre at the NIMR. ${ }^{25}$

In response to Lindenmann's reconstruction of the interference experiments performed in Mooser's laboratory, Isaacs suggested two possible explanations for Lindenmann's observation that inactivated influenza virus absorbed on red blood cells was a good interfering agent. First, in accordance with Mooser's ideas, the virus might have become detached from the red blood cells during the experiment so that it could induce interference by entering the cell as a whole. The second and rather novel assumption was that the virus coat, the 'virus haemagglutinin', might have remained firmly attached to the red cells, while only the virus content, the 'virus nucleic acid', had entered the cell and induced interference. ${ }^{26}$ Isaacs told Lindenmann that this idea was based on a rather well-known American study, which was presented at the prestigious Cold Spring Harbour Symposium, in 1952, the year before Francis Crick and James Watson published their famous Nature papers on the structure and function of DNA. ${ }^{27}$ Upon conducting a study with radioactively labeled bacterial viruses the Americans Alfred Hershey and Martha Chase claimed that during bacterial virus infection only the virus nucleic acid entered the bacterial cell, while the protein coat, the 'virus envelope', remained at the cell surface. This study provided a most compelling argument that virus nucleic acid, which could be either DNA or RNA, directed viral reproduction within the host cell. ${ }^{2 K}$

Within the field of virology there was cautious consensus about bacterial virus infections as a useful and simple experimental model for studying viral reproduction as a possible key to the elucidation of other virus-host systems. By suggesting with his second explanation that animal virus infections proceeded in a way similar to bacterial virus infections - the virus unit attached to the cell and injected its genetic information into the cell, leaving an empty protein envelope attached to the cell wall-Isaacs carried the analogy

25 S. Fazekas de St. Groth, A. Isaacs, and M. Edney, 'Multiplication of Influenza Virus under Conditions of Interference', Nature, 170 (1952), 573-4; and interview with Jean Lindenmann.

26 A. Isaacs, 'Viral interference', Symp. Soc. Gen. Microbiol., 9 (1959) 102-21, p. 108; J. Lindenmann, 'Neuere Aspekte der Virus-Interferenz', Z Hyg. Infektionskrankh., 146 (1960) 369-97, p. 383; J. Lindenmann. 'Induction of Chick Interferon: Procedures of the Original Experiments', Methods in Enzymology, 78 (I981) 181-88, p. 182 .

Interview with Jean Lindenmann.

28 S. Smith Hughes, The Virus: A History of the Concept (London: Heinemann Educational Books, Science History Publications, 1977) p.101; A. Grafe, A History of Experimental Virology (Berlin: Springer-Verlag. 1991), p. 145; L. Kay. The Molecular Vision of Life (Oxford: Oxford University Press, 1993), p.270; and interview with Jean Lindenmann. 
between animal viruses and bacterial viruses quite a bit further. This idea was rather controversial and hotly debated within the field of virology. ${ }^{29}$

Lindenmann's and Mooser's experimental system somehow seemed to provide a starting point to test Isaacs's assumption that during interference with inactivated influenza virus only the virus nucleic acid entered the host cell, while the virus coat remained outside. Most likely, this played a major role in Isaacs's decision to ask Lindenmann whether he would like to collaborate in work on viral interference, in particular, to help him figure out whether this idea, what I will label the 'nucleic acid' hypothesis, made any sense. As his poliomyelitis work was as yet far from promising, Lindenmann was pleased to hear Isaacs's research proposal. After some more talking and with Andrewes's permission, they began working together at the beginning of September $1956 .^{30}$

In the meantime, Lindenmann would continue with his poliomyelitis work, and Isaacs had to make sure that the World Influenza Laboratory was properly run and fulfilled its research obligations towards the WHO. In addition, Isaacs had to pay regular visits to the division of Biophysics and Optics, where he collaborated with the electron microscopist, Robin Valentine, in studies on the structure of influenza viruses, ${ }^{31}$

Isaacs's decision to collaborate with Lindenmann did not seem to be spurred by interests of political nature. Collaboration with the latter would bring Isaacs neither greater credibility nor would he be eligible for resources that would otherwise be missed. Furthermore, compared to Isaacs, the established influenza virus expert, Lindenmann was a relative novice at the frontier of virus research with little expertise to offer. Apparently, recruiting Lindenmann to work as a 'co-laborer' toward a common purpose was motivated by a common research interest in the phenomenon of virus interference and by the fact that Lindenmann had worked with an experimental system that seemed to offer a lead towards testing a then hotly debated idea: namely that infection by influenza virus was initiated by injection of the viral nucleic acid

29 T. van Helvoort, 'History of Virus Research in the Twentieth Century: The Problem of Conceptual Continuity', Hist. Sci., xxii (1994), 185-235, pp 213-8; and interview with Jean Lindenmann. dence, personal archives.

31 Isaacs and Valentine had just produced a series of electron microscopic images of virus preparations, which were said to show virus particles with ring-like structures inside, a kind of micro 'railway-lines' which disappeared after treatment with an RNA destroying enzyme (ribonuclease) and was believed to be RNA; see, C. H. Andrewes, 'Alick Isaacs', Biographical memoirs of fellows of the Royal Society, 13 (1967), 205-21, p. 215; and, A. Isaacs and R. Valentine, The Structure of Influenza Virus Filaments and Spheres', J. Gen. Microbiol.. 16 (1957), $195-204$. p. 195 . 
into the cell, leaving an empty virus envelope attached to the cell wall. ${ }^{32}$

\subsection{Building and manipulating an experimental system in Room 215. ${ }^{33}$}

What actions and decisions were involved in building and manipulating an experimental system in Isaacs' laboratory? How did Isaacs and Lindenmann cope with unexpected events resulting from the non-compliant nature of their experimental system? Was Isaacs's and Lindenmann's work at the laboratory bench governed by making things work in an all-or-none effort to confirm or disconfirm the 'nucleic acid' hypothesis-or-were the research questions with which the experiments were associated secondary to the events at the bench?

In attempting to answer these questions and further our understanding of the dynamics of laboratory practices I will now follow in detail the first two months of joint experimentation in Isaacs's laboratory. ${ }^{34}$ Part of the series of experiments are mapped in diagram forms. However, the reader should be aware that my retrospective experimental maps are principally meant to serve as a guide and therefore fail to capture the essentially non-linear sequences of actions and thoughts that constituted Isaacs's and Lindenmann's collaborative research work.

Isaacs's notebook has an entry for 4 September, 1956 where he and Lindenmann are said to have started with an experiment to see whether virus coated red blood cells can induce

32 The notion of co-laboring or collaborating in the sense of minimally working together toward a common product, was borrowed from Griesemer and Gerson; see, J. Griesemer and E. M. Gerson, 'Colloboration in the Museum of Vertebrate Zoology', Journal of the History of Biology, 26 (1993), 185-203, pp. 196-203.

33 This section is largely based on the following letters, articles, notebook and interview: Alick Isaacs to Herman Mooser, letter dated 18 November, 1957. Jean Lindenmann Correspondence, personal archives; Jean Lindenmann to Herman Mooser, letter dated 7 August, 1958. Jean Lindenmann Correspondence, personal archives, Zürich: Jean Lindenmann to Herman Mooser, letter dated 13 August, 1958. Jean Lindenmann Correspondence, personal archives; J. Lindenmann. 'Induction of chick interferon: procedures of the criginal experiments', Methods in Enzymology, 78 (1981), 181-88; J. Lindenmann, 'From interference to interferon: a brief historical introduction', Phil. Trans. R. Soc. Lond., series B 299 (1982), 3-5; A. Isaacs, Laboratory Notebooks, 1946-65. National Library of Medicine (Bethesda), Film number: reel 91-28, Lab Note Book, S.O. Book 135, Code 28-72-0, "Alick Isaacs Interference Expts. V",; and interview with Jean Lindenmann.

Thanks to Isaacs's carefully recorded laboratory notebooks, and Lindenmann's correspondence and retrospective descriptions of his work in Isaacs' laboratory, a day-to-day account of the laboratory work can be reconstructed. Holmes has convincingly shown that combining laboratory notebooks and other unpublished documents with published papers can yield fairly plausible accounts of investigative pathways through which researchers arrive at the knowledge which theyultimately report in published papers; See, On scientific discovery, investigative pathways and historical reconstructions, see F. Holmes, Lavoisier and the Chemistry of Life (Madison: Wisconsin Press, 1985); and F, Holmes, 'Scientific writing and scientific discovery'. Isis. 78 (1987), 220-35. 
interference in the test-tube..$^{35}$ Technically, this first collaborative experiment differed substantially from Lindenmann's experiments at the 'Hygiene Institut'. During a meeting to discuss both planning of the course of investigation and design of the first experiment, Isaacs proposed a number of changes to Lindenmann's original set-up. Instead of doing the experiment in vivo, inoculating embryonated eggs with influenza virus by the allantoic route, Isaacs preferred to employ a relatively novel in vitro technique.

This technique required the following manipulations. ${ }^{36}$ Fertile hens' eggs containing embryos 10-11 days old (but all of the same age for a particular experiment) were opened and the embryos tipped out and discarded. In addition the part of the chicken embryo membrane adhering to the egg shell was removed, cleaned ('washed') and cut up with scissors into six or seven pieces. These pieces were then pooled in a dish containing an aqueous solution and added, one at a time, to a test-tube filled with a special nutrient fluid (see Fig. 12, 13).

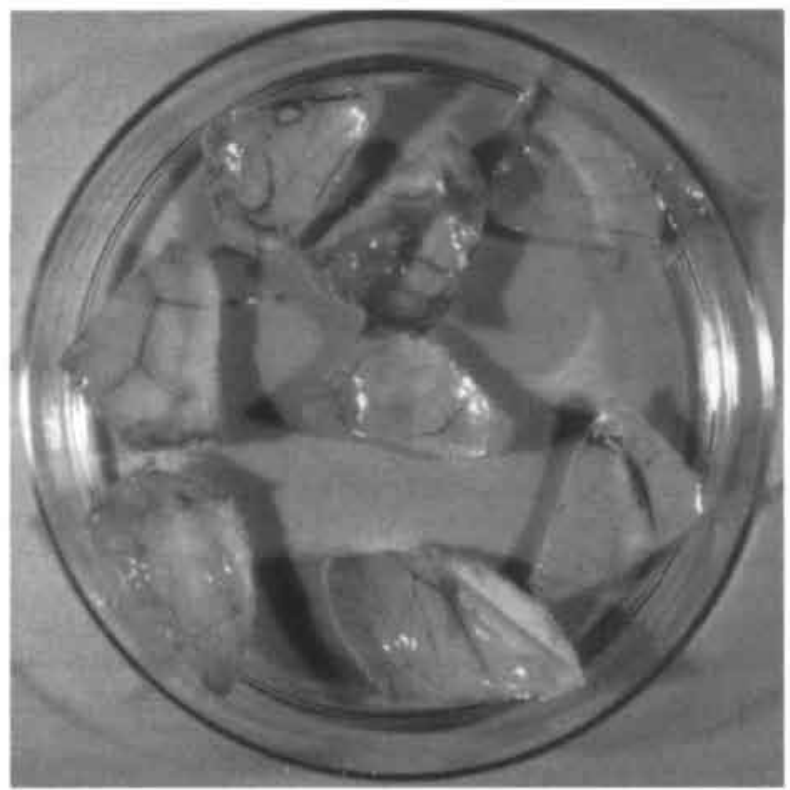

Fig. 12. Pieces of chicken embryo membrane in a petri-dish. Courtesy of Dr. D. Tyrrell

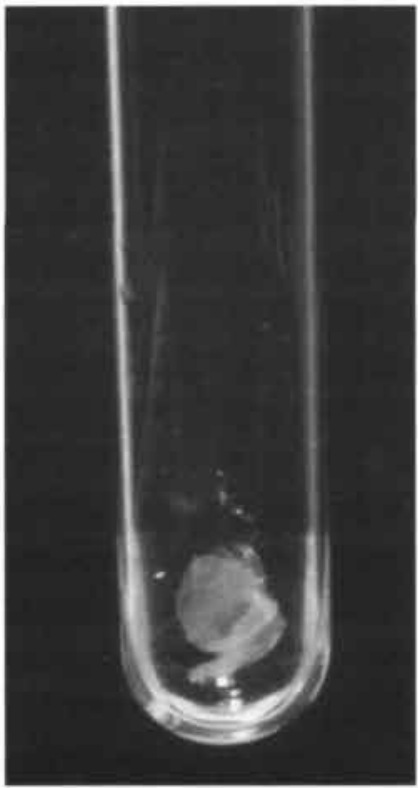

Fig. 13. Piece of chicken embryo membrane in test-tube

35 A. Isaacs, Laboratory Notebooks, 1946-65. National Library of Medicine (Bethesda), Film number: reel 91-28, Lab Note Book, S.O. Book 135, Code 28-72-0, 'Alick Isaacs Interference Expts. V', 4 September, 1956.

36 For a detailed description of this in vitro technique see, F. Fulton and P. Armitrage, 'Surviving Tissue Suspensions for Influenza Virus Titration'. J. Hyg. 49 (1951), 247-63, pp. 251-2. 


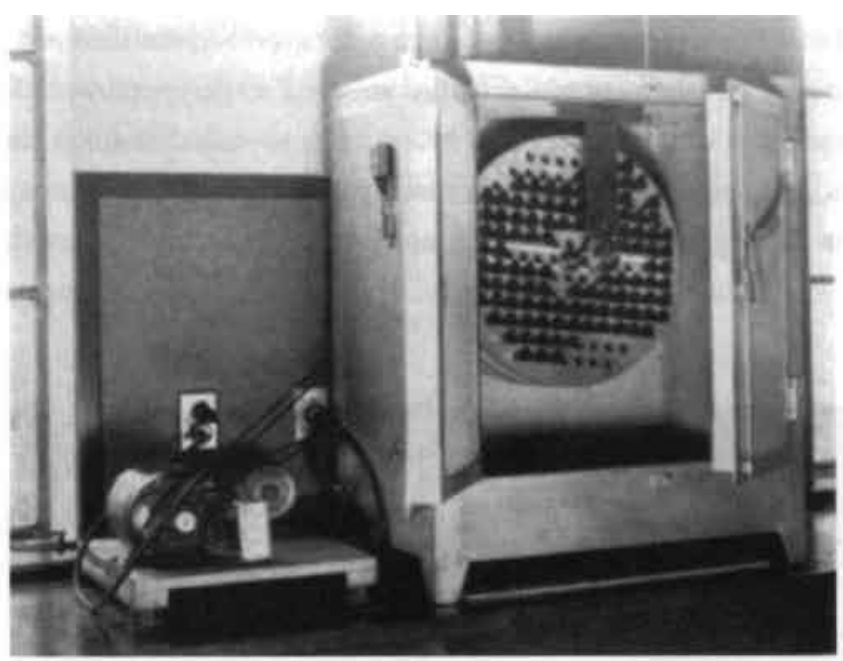

Fig. 14. Roller-tube incubator with rotator and motor assembly (1950's).

Courtesy of the NIMR).

This could be done very fast by a laboratory technician. Subsequently, the virus or the controls were injected into the test-tubes, whereupon the tubes were stored and kept in controlled conditions, at $37^{\circ} \mathrm{C}$ for 24 hours ('incubated' or 'incubation') in slowly rotating tube-containers, so-called 'roller-drums': Previous experimentation had shown that through rotation or rolling of the tubes ('roller-tube method') virus multiplication could be stimulated (see Fig. 14). The next day the fluid could be taken out of the tube and the amount of virus quantified Isaacs had applied this experimental technique successfully in studies of virus interference, which he had done together with Forrest Fulton, a fellow-virologist working at the London School of Hygiene and Tropical Medicine. ${ }^{37}$ According to Isaacs it had decisive advantages over the egg. The in vitro technique required roughly one-sixth of the usual amount of fertilized eggs, thus saving both money and space. In addition, experiments in eggs required far more skill and time than handling the pieces of membrane in the test tube.

An important incentive for the development of this in vitro technique had been the limited supply of fertilized eggs in Europe after World War II; F. Fulton and A. Isaacs, Influenza Virus Multiplication in the Chick Chorioallantoic Membrane', J. Gen. Microbiol, 9 (1953), 119-131; W. Henle and G. Henle, The Road to Interferon: Interference by inactivated Influenza Virus', In A. Billeau and N. Finter (eds.) Interferon 1: General and Applied Aspects (Amsterdam: Elsevier, 1984) pp. 3-18; Interviews with Jean Lindenmann and David Tyrrell.. 
Further, he argued that this in vitro model could be more readily quantified and manipulated. ${ }^{38}$

A second change in the procedure of the 'original' experiment would be to stick the inactivated influenza virus to haemolysed red blood cells, so-called 'red cell ghosts', instead of normal blood cells, ${ }^{39}$ By attaching influenza virus particles to red cell ghosts, Isaacs and a researcher from the Biophysics and Optics Group, Heather Donald, had managed some years before to visualize influenza virus through the use of the electron microscope (see Fig. 15). ${ }^{40}$

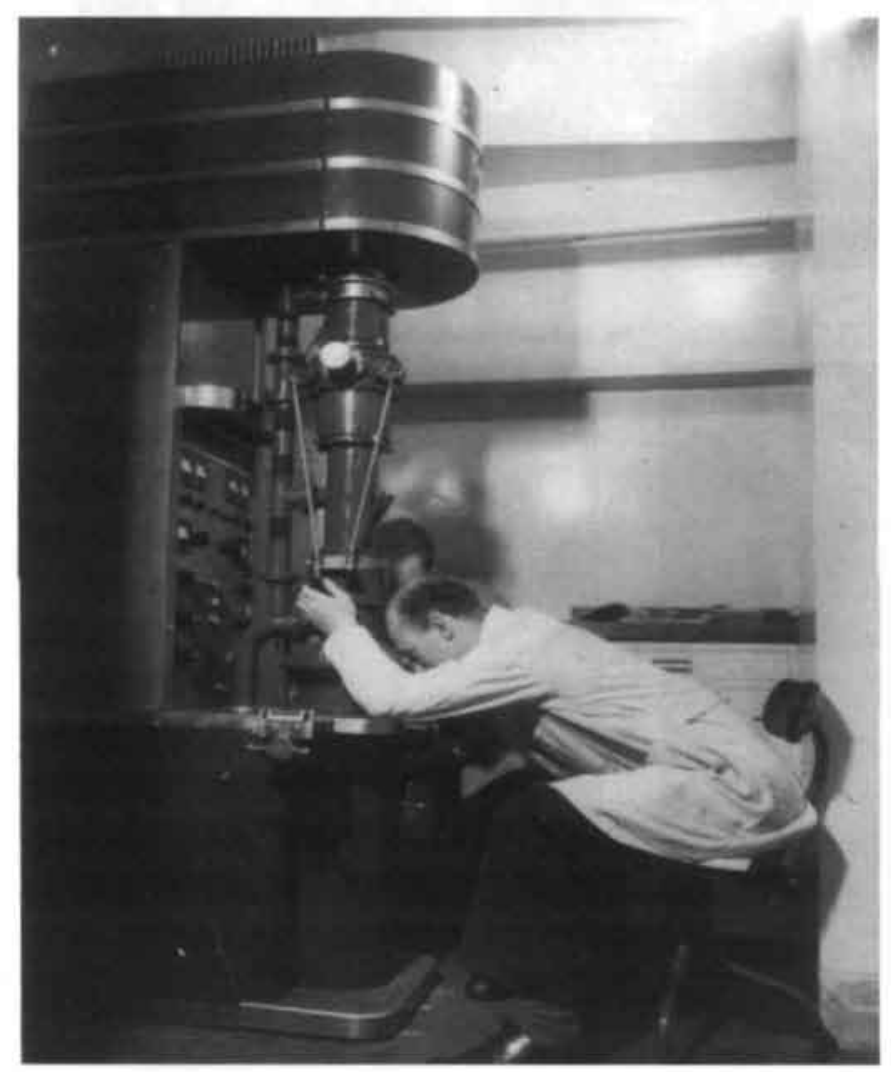

Fig. 15. Electron-microscopist at work (1950's). Courtesy of the NIMR.

38 D. Tyrrell and I. Tamm, 'Prevention of Virus Interference by 2,5-Dimethylbenzimidazole', J. Immun., 75 (1955), 43-9, p. 43; interview with Jean Lindenmann.

39 The plasma membrane of these cells had been ruptured by chemical treatment and, consequently, these red blood cells had lost their haemoglobin content (pigment).

40 H. Donald and A. Isaacs, 'Counts of Influenza Virus Particles', J. gen. Microbiol., 10 (1954), 457-64, p. 459. 
They had made a series of electron micrographs of influenza virus preparations (see Fig. 16), which were said to show red cell ghosts with distinctive little round structures on them, representing virus particles. This interpretation is clearly an accomplishment of two experts familiar with interpreting electron microscopic images of virus samples. Without Donald's and Isaacs's guidance these pictures look rather more like exotic art photographs, showing intriguing patterns of lines and dots. ${ }^{41}$
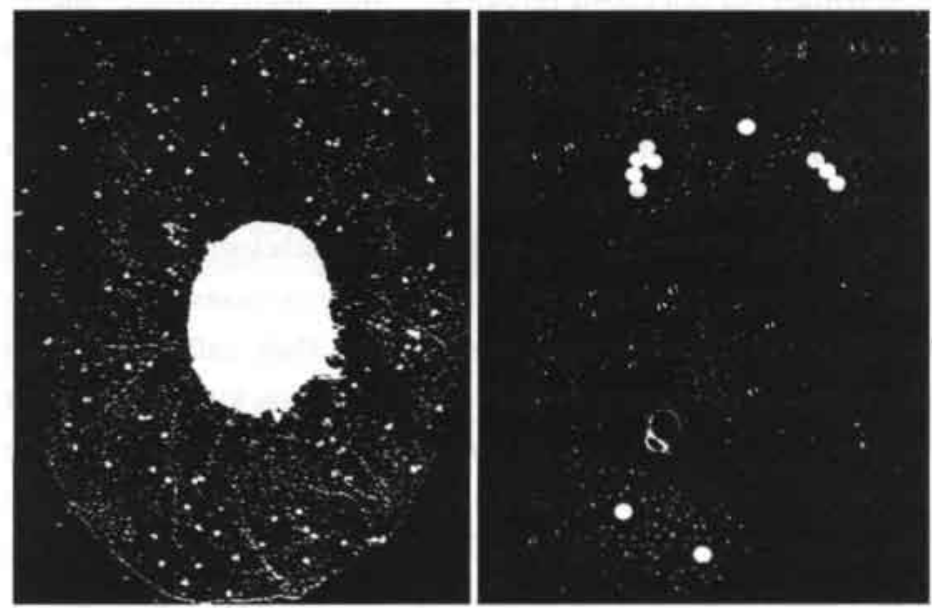

Fig. 16. Electronmicroscopic images of counts of influenza virus particles. H.B. Donald and A. Isaacs (1954). Courtesy of the Jeurnal of General Microbiology.

Isaacs thought that by sticking inactivated influenza virus to red cell ghosts it would also be possible to visualize influenza virus particles in his experiment with Lindenmann. If, Isaacs's assumption that during interference only the virus nucleic acid entered the host cell while the virus coat remained firmly attached to the red cell ghosts, was correct, then they should be able to see full round virus structures before, and collapsed structures after, interference had been induced. Furthermore, Isaacs proposed to use the same strain of influenza virus and the same method of viral inactivation, which he had used successfully in previous interference experiments. Isaacs's infectious enthusiasm made his proposals sound more than convincing and Lindenmann accepted the changes without much discussion.

41 Michael Lynch noted that the circumstantial character of the interpretative enterprise in laboratory science is a fortiori true for electron microscopy. See, M. Lynch, Art and Artefact in Laboratory Science (London: Routledge \& Kegan Paul, 1985), pp. 10-2. 
Standing face-to-face at the laboratory bench in room 215, Lindenmann, Isaacs and a laboratory technician began by opening a batch of embryonated eggs, removing and washing the chicken embryo membranes, cutting them up into pieces, heat-inactivating batches of influenza virus, preparing red cell ghosts, and sticking part of the inactivated virus to these ghosts, which were now designated as 'virus-coated ghosts'. After this preparatory work, they did a first experiment to see whether they could induce viral interference with these virus coated ghosts in vitro. One by one, pieces of membrane were put in test-tubes together with nutrient fluid and either inactivated virus stuck to red cell ghosts (group 1) or free inactivated virus (group 2) and in tubes containing nutrient fluid only (group 3) or in tubes containing nutrient fluid plus normal red cell ghosts (group 4). ${ }^{42}$ Most likely, Group 3 and 4 were to serve as 'controls'. By including 'controls' with nutrient fluid and nutrient fluid plus red cell ghosts only, Lindenmann and Isaacs wanted to check for possible unanticipated effects on the virus growth due to the nutrient fluid or the ghosts. (For a diagram of this type of experiment see Fig.17)

With the aim to control the output of data in an experimental system, biomedical researchers routinely include tests from which one or more elements are omitted and which are to be performed simultaneously with the 'main' test. They call these specific biological comparison tests which aim at controlling the experimental background 'controls'. The use of controls as a means to eliminate 'background' and to provide an experimental baseline which is instrumental in making sense of the experimental output is inextricably bound up with manipulating an experimental system. In Isaacs's and Lindenmann's experiment the controls were meant to be used both as a point of reference for normal virus reproduction and as a means to exclude errors due to experimental artifacts or 'background noise'.

The groups of test-tubes were then rotated in the roller apparatus. After 24 hours in the roller-drum the test-tube fluids were discarded, while the membranes were transferred to dishes containing an aqueous solution in order to wash off the 'old' fluid ('washing the membranes'). Subsequently, these membranes were put in test-tubes one by one together with live virus and fresh nutrient fluid, and incubated for another 24 hours in the roller-drum. Halfway through the first week, after collecting the tubes from the roller-drum, they subjected the tube fluids to a so-called 'haemagglutination titration', a specific quantitative biological test or bioassay for determining the relative rate of influenza virus multiplication.

Haemagglutination titration involved the following procedure. A series of dilutions of each tube fluid were made in cups on plastic trays and to each dilution a constant volume of fowl red blood cells was added. Upon storage of the trays at $4^{\circ} \mathrm{C}$ visible clumps were formed in some of the cups. As the haemagglutination assay was routinely performed in Isaacs's 


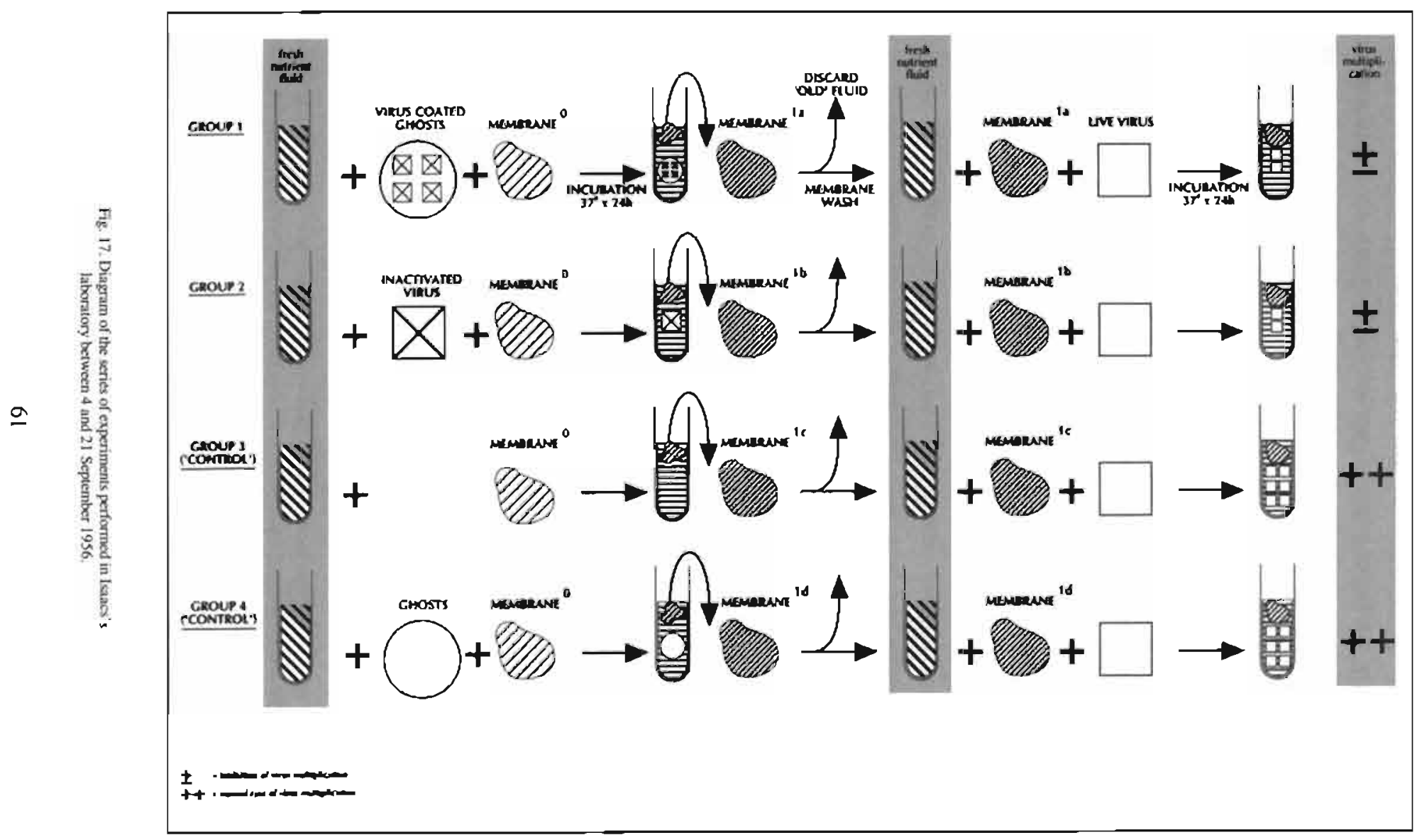


laboratory, Isaacs knew by experience that red blood cells stuck together or agglutinated proportionately to the number of influenza virus particles present. By convention, the highest dilution at which the formation of visible clumps or 'agglutination' occurred, the 'haemagglutinin titer', was taken as a relative measure for the influenza virus content of a biological fluid. ${ }^{43}$ For agglutination to occur it took hours, leaving time for discussions or other research work (see Fig. 18).

The experiments took hours to titrate....and this left time to talk. Alick was the leader in conversation, and ideas for new experiments, political discussion, or identification of snatches of opera that he would sing made the time pass quickly. Alick, too, was adept at determining where the end point was, with the aid of a hand lens, long before the rest of us could do it, and he had often planned the next experiment before the red cells had really settled.4

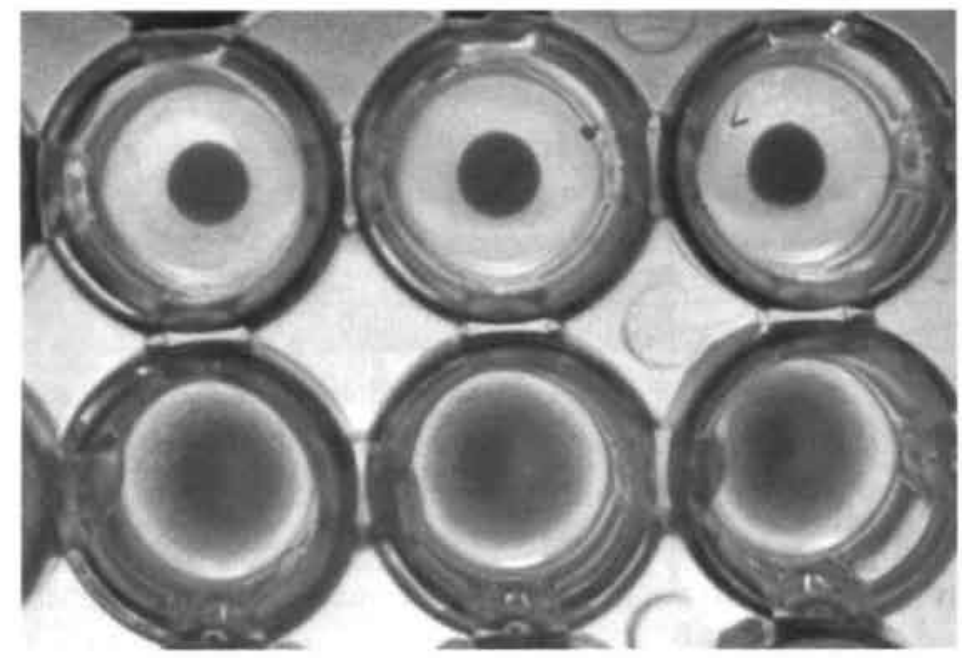

Fig. 18, Agglutination (lower row) is characterized by the formation of a pattern on the bottom of the tube. Non agglutinated blood

cells settle down to a small dot (upper row). Courtesy of Dr. D. Tyrrell.

43 A. Isaacs. Laboratory Methods Used in Investigating Influenza', Glasg. Med. J., (29) 1948, 357-61, p.359.

44 In retrospect, Derek Burke, who was to join Isaacs's and Lindenmann's researches early in 1957, gave this impressionistic account of the performance of haemagglutination titrations or tests; see, D. Burke, 'Early Days with Interferon', J. Interferon Res., 7 (1987), 441-2, p. 442. 
The operation of this haemagglutination assay involved both the transformation of matter from one state to another - the sticking together of red blood cells - and the production of data, the highest dilution at which visible clumps were formed. Laboratory configurations such as the haemagglutination assay, electron microscope, ultracentrifuge, or spectro-photometer, all produce data of some sort. The black and white shadows on an electron micrograph, the pattern of bands in a centrifugation tube, the traces in petri dishes with virus infected cell cultures or the lines on a spectrogram, are the entities of interpretation and representation. ${ }^{45}$

In the case of Isaacs and Lindenmann, it was the ordering of the data stemming from the electron microscope or the haemagglutination assay which constituted the readings in Isaacs' notebook. These readings played a major role in structuring subsequent research activities-reshaping the experimental system-thereby themselves being worked on and transformed.

The readings in the table (see Fig.19) are given as series of dilutions, named 'haemagglutinin titers'. These were the entities of interpreting and representing the degree of virus multiplication. If the end point of agglutination occurred at a 1/1 dilution the reading was taken as 1 and a $1 / 48$ dilution as 48 . The more dilutions were needed the more virus particles were present and the higher the relative rate of virus multiplication.

The data output of the first experiment was encouraging. The experimental results were similar to the results Lindenmann had obtained in Zürich: both free inactivated virus, and inactivated virus stuck to red cell ghosts ('virus coated ghosts') were able to induce viral interference and inhibit the multiplication of live virus in their 'test-tube arrangement'. After another three weeks of repeated trials to fine tune their experimental system-setting the optimal experimental conditions for the induction of interference with virus coated ghosts-Isaacs and Lindenmann decided to start efforts to visualize the virus by electron microscopy. Before and after the induction of interference in their experimental system. samples would be taken and sent to the electron microscopist who would be asked to produce electron microscopic images of red cell ghosts and virus particles. Again, if Isaacs's assumption that during interference only the virus nucleic acid entered the host cell while the virus coat remained firmly attached to the red cell ghosts was correct, then they should be able to see full

Bruno Latour and Steve Woolgar call these data-generating configurations 'inscription devices'. Hans-Jörg Rheinberger has argued that BrunoLatour's and Steve Woolgar's clearcut distinction between items of apparatus that "transform matter between one state and another" and "inscription devices" that "transform pieces of matter into written documents" is too simple. There are indeed experimental configurations like the haemagglutinin test, which are hybrids in the sense that they transform both matter between one state and another, and matter into data, thus opening a space of representation. Moreover, Latour and Woolgar oversimplify the complex transformation process of pieces of matter into written documents. The visual display of any sort in a laboratory notebook and subsequently in a scientific paper is at the far end of a complex transformation process that starts with manipulating an inscription device in onder to generate data of some kind; see, Rheinberger. 'Experiment, Difference, and Writing: II. The Laboratory Production of Transfer RNA', Stud. Hist. Phil. Sci., 23 (1992), 389-422, p. 393; and Latour and Woolgar, Laboratory Life: The Construction of Scientific Facts (Princeton: Princeton University Press, 1986). p. 51. 
round virus structures before, and collapsed structures after the induction of interference.

\section{$\underline{\text { Haemagglutinin Titers }}$}

\begin{tabular}{|c|c|c|c|c|c|}
\hline & mple & groep & groep2 & groep 3 & groep 4 \\
\hline & 1 & 8 & 3 & 96 & 192 \\
\hline & 2 & 8 & $<2$ & 128 & 80 \\
\hline & 3 & 4 & 4 & 64 & 128 \\
\hline & 4 & 4 & 8 & 160 & 80 \\
\hline & 5 & 2 & 3 & 64 & 160 \\
\hline & 6 & 8 & 6 & 32 & 128 \\
\hline
\end{tabular}

Fig. 19. Haemagglutinin titers from Isaacs's notebook

They then prepared four groups of test-tube fluids. Group 1 contained only nutrient fluid; group 2 tubes contained nutrient fluid plus virus coated red cell ghosts. Group 3 was identical to group 2, and group 4 contained nutrient fluid plus a mixture of virus coated ghosts and empty ghosts (control 3). (For a diagram of this experiment see Fig.20)

Most likely, the groups 1, 3 and 4 were to serve as internal controls. Group 3 and 4 were apparently included to verify whether or not the virus became disentangled throughout the experiment, whereas group 1 was meant to be used as a point of reference for a 'normal' rate of virus multiplication, thereby checking the induction of interference by the virus coated red cell ghosts. Thus, like before, the experiment was embedded in a series of controls in order to make sense of the data, which would hardly mean anything on their own, and to help exclude artifacts.

Samples from these four groups were taken and sent to the electron microscopist Valentine in the Division of Biophysics and Optics. Subsequently, pieces of membrane were added to group 1 and 2 test-tubes, whereupon overnight all the tubes were rolled in the rollerdrum. The next day, half way through the interference experiment, group 3 and 4 tubes together with samples from group 1 and 2 were sent to Valentine.

By special request, Valentine immediately processed electron microscopic images of the samples from Isaacs's laboratory. The pictures of the first samples, which were taken at the start of the experiment, looked promising. Clearly visible structures of red cell ghosts with 


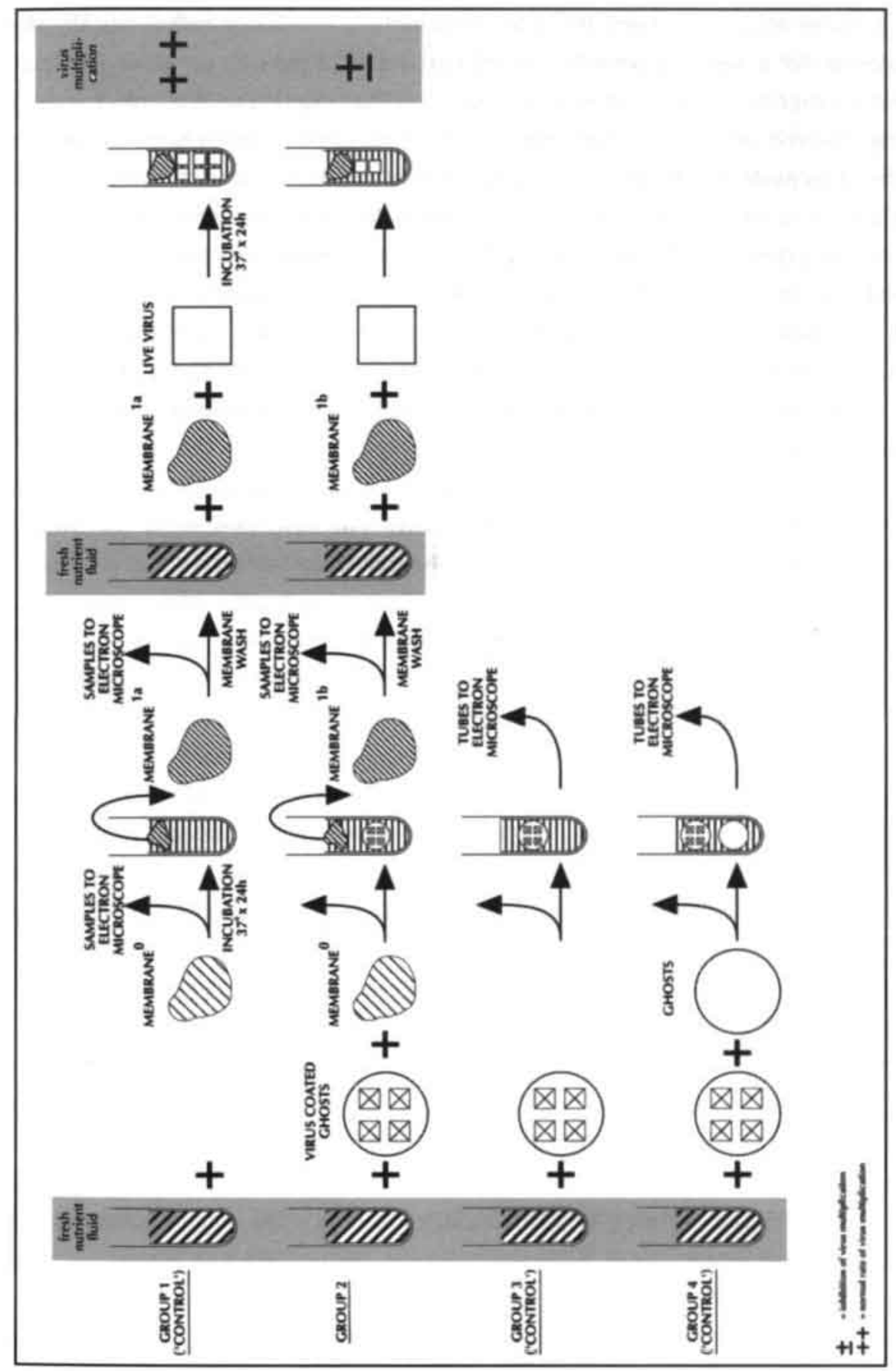

Fig. 20. Diagram of the series of experiments performed in Isaacs's taboratory between 25 September and 21 October 1956. 
virus particles attached to them could be distinguished, and Isaacs enthusiastically showed Lindenmann that it was even possible to count the number of particles per ghost. However, the electron micrographs of the other samples taken after the induction of interference were disappointing. The red cell ghosts looked rather smudgy with no more than a blurry outline of virus particles. This made the photographs hard to interpret. Neither Isaacs nor Valentine could tell the difference in structure between virus particles before and after induction of interference (half way the experiment). Furthermore, on the basis of Valentine's electron micrographs it was impossible to figure out whether in the 'control' groups without membranes (group 3 and 4), which contained either virus coated ghosts or a mixture of virus coated ghosts and empty ghosts, virus particles had become disentangled and were transferred to the empty ghosts. In Isaacs's notebook two question marks serve to emphasize the problems of interpreting the electron microscopic images. ${ }^{46}$

In the meantime, in Isaacs' laboratory, group 1 and 2 test-tube fluids were discarded, while the membranes were put in a petri dish containing an aqueous solution in order to wash off the 'old' fluid and virus coated ghosts. Next, they put these membranes in test-tubes one by one together with fresh solutions containing live virus, and, after sealing the tubes, the technician placed them in the roller-drum, which was preset at $37^{\circ} \mathrm{C}$ and 24 hours rotationtime as usual. Thereafter the tubes were collected from the roller-drum and the fluids subjected to the haemagglutination assay. In accordance with previous experiments the group of tube fluids which had contained virus coated ghosts (group 2) showed much lower rates of virus multiplication than the group without virus coated ghosts (('control') group 1). This indicated that at least they had been able to induce viral interference with the virus coated ghosts.

The kind of experiment just described was performed a couple of times, without much success. The electron microscopic images of the samples- which were taken half way through the experiment after the fluid samples with virus coated ghosts had come out of the rollerdrum-remained difficult to interpret. Analyzing the electron micrographs became a rather frustrating activity. However little, eventually they did get something out of these efforts. According to Valentine there were at least hints that in the mixture of virus coated ghosts and empty ghosts a transfer of virus particles from one to the other had taken place.

Following the successive efforts to visualize the virus-coated ghosts and the failure to produce any workable results except for the indication, albeit vague, that virus could become detached from the ghosts during the experiment, resulted in a first demonstrable reshaping of their experimental system.

In discussing the recent disappointing experimental events Isaacs and Lindenmann agreed that if they would take the hints seriously that some virus particles had gotten

46 A. Isaacs, Laboratory Notebooks, 1946-65. National Library of Medicine (Bethesda), Film number: reel 91-28. Lab Note Book, S.O. Book 135, Code 28-72-0, 'Alick Isaacs Interference Expts. V'.. 25 September, 1956. 
disentangled during the experiment, it would undermine their efforts to test Isaacs's idea - that only the virus nucleic acid entered the cell and induced interference, leaving the empty virus envelope outside. Without dropping this 'nucleic acid' hypothesis though, an additional research question was raised. Could the interference induced by virus coated red cell ghosts be due to virus units which became detached from the ghosts during the experiment? It was the same kind of question that had bothered Lindenmann in Zürich. They subsequently developed the following argument: 'let us assume this to be the case and suppose that the few virus particles that become disentangled really suffice to induce interference, then it might be possible to induce interference with the same virus coated ghosts more than once.'

In order to test for this possibility too, they did the following experiment, which differed slightly from the preceding series of experiments. Four groups of test-tube preparations were prepared and incubated in the roller-drum overnight (stage 1). ${ }^{47}$ Group A contained nutrient fluid with virus coated ghosts ('control'), group B contained a tenfold dilution of group A ('control'), group $\mathrm{C}$ was composed of nutrient fluid, virus coated ghosts and a piece of membrane, and group D contained fluid plus a piece of membrane ('control'). (For a diagram of this experiment see Fig. 21)

Since only group $\mathrm{C}$ contained membranes plus virus coated ghosts, I assume that these were the only tubes in which interference was meant to occur. Apparently, group A and B were included to verify whether virus became disentangled throughout the experiment, whereas group D was meant to be used as a point of reference for 'normal' virus multiplication, thereby checking the induction of interference by the virus coated red cell ghosts.

The next day Isaacs sent samples from group A, B and C to Valentine, in a further attempt to visualize virus coated ghosts and to see whether or not virus had become disentangled during the 24 hours period in the roller-drum. In addition, fresh pieces of membrane were added to group A and B tubes ('controls'). At the same time the 'old' membranes were removed from group $\mathrm{C}$ tubes, washed and transferred to fresh test-tube fluids (group E), while fresh membrane fragments were added to the 'old' group C test-tube fluids. Group D was left unchanged, while a new group $\mathrm{F}$ with fresh nutrient fluid plus membrane was included ('control'). Once again all tubes were stored in the roller-drum overnight (stage 2).

It is highly likely that during this second stage of the experiment they intended to check whether or not the interfering power of the virus coated ghosts had become exhausted after the first interference attempt. Now, group A and B tubes were meant to serve as a point of reference for 'normal' viral interference, while group D and F would be used as a measure

\footnotetext{
47

The notion of stage 1,2 and 3 is introduced as a didactic aid and has no significance at all with regard to the actual research process.
} 


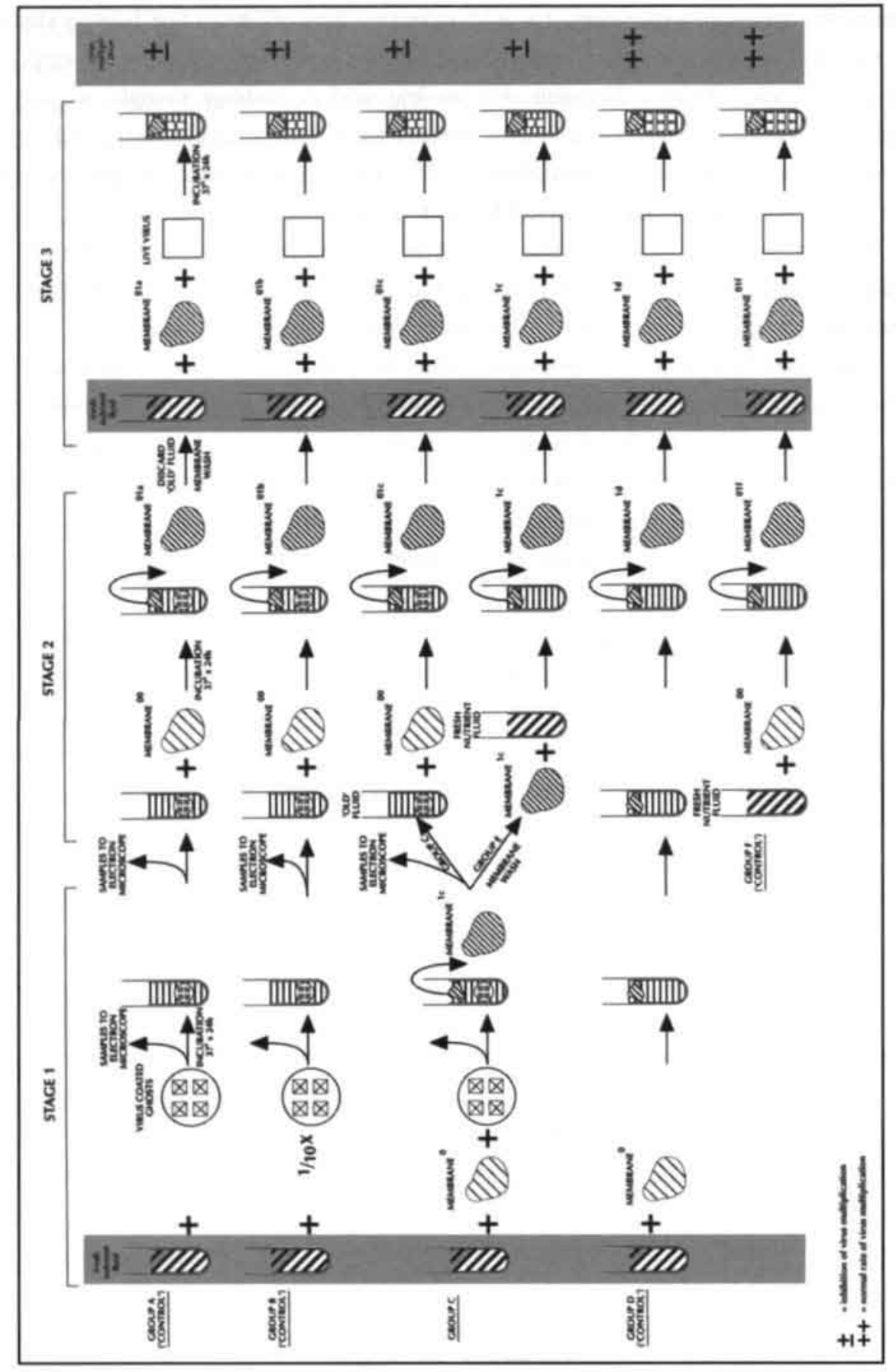

Fig. 21. Diagram of experiments performed in Isaacs's laboratory between 24 and 29 October 1956 
for 'normal' virus multiplication.

After 24 hours in the roller-drum all fluids were discarded, while the membranes were washed and put in tubes filled with fresh solutions containing live virus. Once more the tubes were transferred to the roller-drum and incubated for another 24 hours (stage 3). Finally all tube fluids were subjected to a haemagglutination test to measure the relative rate of virus multiplication in each tube. The data were listed in Isaacs's notebook (see Fig. 22). ${ }^{48}$

The measurements indicated that it was possible to induce interference with the virus ghost solution twice (stage 1 and stage 2), with no loss of interfering activity. Furthermore, there were additional hints from Valentine's photographs, which once again were difficult to interpret, that possibly some virus particles had become disentangled during the experiment. Thus, while once more failing to produce any workable experimental data with respect to the 'nucleic acid' hypothesis, their latest experiment seemed to lend support to the idea that the viral interference in their experimental arrangement was due to virus particles that became detached from the ghosts.

\section{Haemagglutin in Titers}

\begin{tabular}{|rrrcccc|}
\hline Sample & group a & group b & group c & group d & group e & group f \\
No. 1 & 1 & 32 & $<1$ & 96 & 4 & 128 \\
2 & 2 & 48 & 1 & 48 & 3 & 32 \\
3 & 3 & 40 & $<1$ & 128 & 2 & 32 \\
4 & 32 & 128 & 2 & 128 & $<1$ & 128 \\
5 & 4 & 32 & $<1$ & 128 & 2 & 64 \\
6 & 3 & 24 & $<1$ & 128 & 1 & 128 \\
\hline
\end{tabular}

Fig. 22. Haemagglutinin titers from Isaacs's notebook.

However, there was more. After reading over the experimental data in his laboratory notebook for a second time, Isaacs got the feeling that there was something unusual about the figures. Isaacs knew by experience from his previous interference studies, that during incubation at $37^{\circ} \mathrm{C}$ (the temperature at which they incubated the tubes in the roller-drum) the interfering activity of inactivated influenza virus (the same virus strain as was employed in their current experiment) decreased with time. Thus, Isaacs expected to see at least a slight drop in

48 A. Isaacs, Laboratory Notebooks, 1946-65. National Library of Medicine (Bethesda), Film number: reel 91-28, Lab Note Book, S.O. Book 135, Code 28-72-0, 'Alick Isaacs Interference Expts. V', 24-7 October, 1956. 
interfering activity, while reusing the virus coated ghost fluid in a second interference attempt (stage 2). But, surprisingly, the interfering capacity of the virus coated ghost fluid remained virtually unchanged (group $\mathrm{C}$ versus group $\mathrm{E}$ ). He discussed the matter at length with Lindenmann. Were they dealing with an artifact due to, for instance, the large excess of interfering power which was associated with the ghosts or was there more to say about the fact that the expected drop in interfering activity failed to occur? They decided that most likely they were dealing with an artifact. According to Lindenmann, up to this point neither Isaacs nor he made any suggestions relating to the notion that a factor different from the virus might be involved in interference.

In a further effort both to check for the possibility that the inhibition of virus growth was due to virus particles that became detached from the red cell ghosts and to visualize the virus coated ghost after induction of interference, yet another experiment was performed. (For a diagram of part of this experiment see Fig. 23) This time, after the first interference attempt (stage 1) and removal of the membranes, the tubes with virus coated ghosts containing fluid were transferred to the centrifuge and spinned at high speed to bring down the ghosts in the tubes, thereby separating ghosts from fluid. This separation procedure was included to find out whether or not there was any interfering activity left in the nutrient fluid due to inactivated virus particles, which were believed to have become detached from the ghosts in stage 1 .

Thereupon fresh nutrient fluid was added to the debris of virus coated ghosts together with a freshly prepared membrane fragment, and the tubes were incubated anew in the rollerdrum. Simultaneously, they transferred the supernatant fluids to a new batch of test-tubes, added fresh pieces of membrane and stored them in the roller-drum for a second interference attempt as well (stage 2$)^{49}$

Next, in stage 3 the membranes were routinely washed, put in tubes containing fresh nutrient fluid and live virus, where after the whole was incubated in the roller-drum. The subsequent measurement of the relative rate of virus multiplication in the tubes showed them once again that virtually no interfering activity was lost when re-using the virus coated ghosts in a second interference attempt. However, upon thoroughly analyzing the tables in his laboratory notebook Isaacs came up with a far more intriguing experimental feature.

The residual interfering activity in the supernatant fluid that had been separated from the virus coated ghosts, was far higher than Isaacs would have expected, judging by his extensive research experience with inactivated influenza virus as an interfering agent. Without the 1965. 


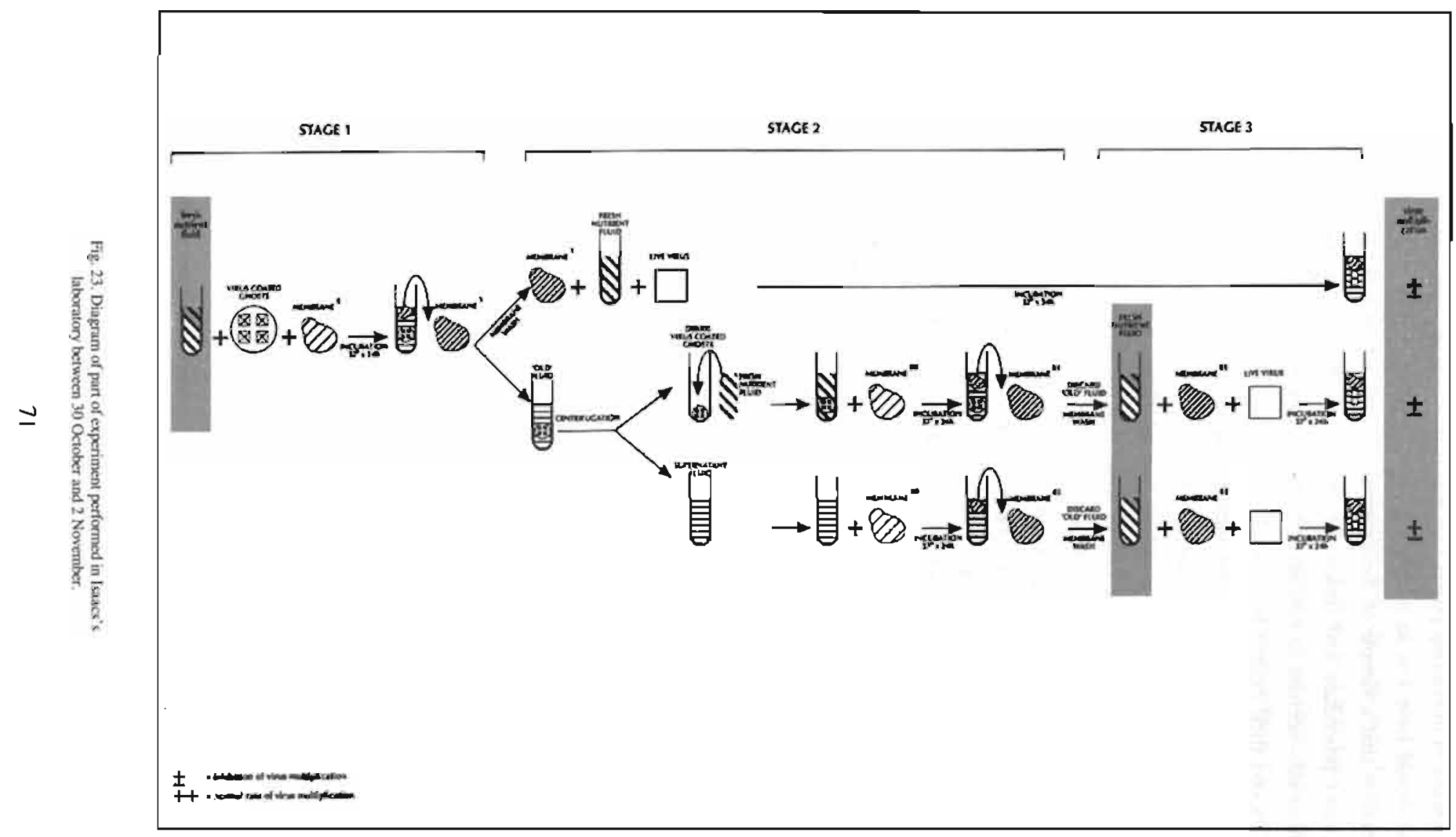


large excess of interfering power that was believed to be associated with the ghosts, the supernatant should have lost its interfering capacity more rapidly with time. Even more surprising observation that a sample of the supernatant fluid, which was taken off immediately after the separation procedure and tested for its virus content-inactivated virus that had become disentangled-seemed to contain only minute amounts of virus. The following question must have racked their brains: how could the inhibition of virus growth have come about if the inactivated virus as the interfering agent had apparently been absent?

While discussing the readings in his notebook with Lindenmann, Isaacs apparently suggested the possibility that somehow new interfering activity had been generated. According to Lindenmann Isaacs was the first to admit that the observed effect could just as well be due to a change in $\mathrm{pH}$ or to the exhaustion of nutrients in the test tube fluid, as suggested by Lindenmann. Isaacs also indicated that he knew by experience that the presence of virus in the supernatant fluid could easily have been masked by a certain membrane substance, which was known to interfere with the type of virus measurement they had used. Eventually, they had to concede that on the whole their experiments had produced more questions than they had resolved.

However, despite the fact that both researchers seemed to agree that the data present were not much to go on, each judged the experimental situation differently. Apparently, it was a perplexing experience. ${ }^{50}$ Whereas Isaacs, primarily, became fascinated by the idea that, somehow, new interfering activity had been produced in their experimental system, Lindenmann had a more cautious attitude and thought more in terms of possible artifacts that might have disturbed the expected course of events. Once again they would bring the matter up for discussion. As a joke, during one of these sessions, Lindenmann started calling Isaacs's mysterious interfering activity by the name of 'interferon'. 'Before studying medicine, I had studied physics for a while. Of course in physics terms ending in '-on' were very popular, like electron, positron, muon etc. Because of this old love for physics I believe I invented the term 'interferon'...It was not to be taken serious'. ${ }^{51}$

The unexpected handle on their initial research problem or what I would designate an 'experimental anomaly', resulted recognizably from a repeated contradiction of expectations. In other words, the anomaly was an accomplishment that had to be achieved. The relative robustness of the anomaly was going to change the order of events in Isaacs's laboratory by sparking a new line of research, thereby rendering the situation different for later data production.

\footnotetext{
50 In principle it is impossible to recapture the irreproducable 'initial' observation which was constitutive in rendering the events anomalous.

51 Interview with Jean Lindenmann.
} 
Isaacs's notebook has an entry for 6 November, 1956, starting with the sentence: 'In search of an interferon'. ${ }^{52}$ Probably, these words marked the decision to putting to a test Isaacs's vague idea about some sort of new interfering activity that was generated in their experimental system. This did not mean that the starting point of the first series of experiments, the question whether or not the virus injected its nucleic acid content into the cell ('nucleic acid' hypothesis) during virus infection was abandoned. Simultaneously they pursued the electron microscopy work. This could be easily organized because research on the question whether and how new interfering activity was generated could be done while waiting for their turn on the microscope and for pictures to be developed.

In order to find out whether or not the experimental anomaly was due to the red cell ghosts only, they decided to include test-tubes with free inactivated virus and pieces of membrane, but without ghosts. (For a diagram form of the newly included part of this experiment see Fig. 24) After they had rolled the tubes overnight in a first interference attempt, the membranes were removed and a second interference trial was carried out with the test-tube fluids and fresh membrane fragments. In order to determine the degree of viral interference that had been induced both times, the first and second set of membranes were routinely incubated with live virus, followed by a haemagglutination titration to determine the relative rate of virus multiplication. The readings in Isaacs's laboratory notebook indicate that after the first interference attempt (stage 1) the interfering activity had only been slightly reduced, both in fluids with free inactivated virus and in tubes with inactivated virus stuck to red cell ghosts. ${ }^{53}$

Apparently, the expected fall in interfering activity due to thermal degradation or adsorption of virus by the membranes had also failed to materialize in the case of tubes containing free inactivated virus instead of virus coated ghosts. Most likely Isaacs and Lindenmann then decided that in their search for new interfering activity that was possibly generated during the experiment, they might just as well leave out the red cell ghosts. Presumably the decision was motivated by a pragmatic desire to reduce the complexity of their experimental system: 'It was difficult to analyze the situation with virus absorbed on red cells' ${ }^{54}$

A. Isaacs, Laboratory Notebooks, 1946-65. National Library of Medicine (Bethesda), Film number: reel 91-28. Lab Note Book, S.O. Book 135, Code 28-72-0, 'Alick Isaacs Interference Expts. V', 6 November, 1956.

53

A. Isaacs, Laboratory Notebooks, 1946-65. National Library of Medicine (Bethesda), Film number, reel 91-28. Lab Note Book, S.O. Book 135, Code 28-72-0, 'Alick Isaacs Interference Expts. V', 10 November, 1965.

Alick Isaacs to Herman Mooser, letter dated 18 November 1957, Jean Lindenmann Correspondence, personal archives, Zürich. 


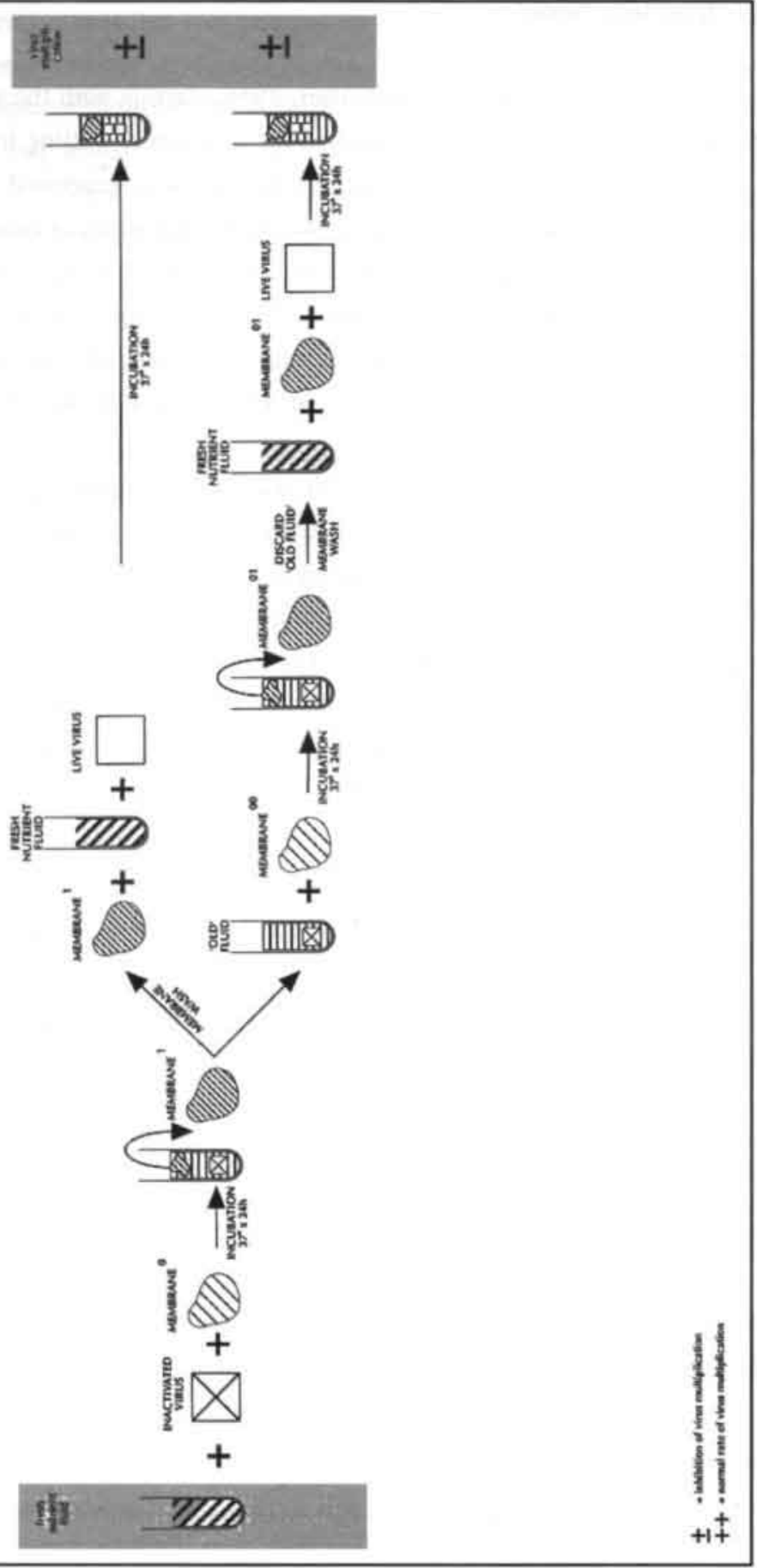

Fig, 24. Diagram of part of experiment performed in Isaacs's taboratory between 6 and 10 November 1956. 
In their next experiment they in fact dropped the red cell ghosts as constitutive elements of their experimental system. Initially, they intended to compare the interfering capacity between, on the one hand, fluids with free inactivated virus to be incubated twice with two sets of membranes in succession, and on the other hand fluids with free inactivated virus also to be rolled twice but the first time without adding a piece of membrane. However, the deletions in Isaacs's laboratory notebook suggest that Isaacs and Lindenmann made a last minute change with regard to the design of the experiment. ${ }^{55}$ In this concrete situation, there was clearly an ad-hoc manner in which the experiment was assembled.

Instead of rolling all tubes twice at $37^{\circ} \mathrm{C}$ for 24 hours in the roller-drum in the standard way, a small group of tubes was to be treated differently (For a diagram of this part of the experiment see Fig.25). A number of test-tube fluids containing inactivated virus and membrane would be incubated for two hours, after which the tubes would be taken out of the roller-drum, the membranes routinely washed and transferred to tubes with fresh nutrient fluid and put back in the roller-drum for another 22 hours. Subsequently, this group was to be treated in the usual way, removing the membranes and rolling the fluids with a second set of membranes for another 24 hours. Finally, both sets of membranes would be put into tubes containing live influenza virus, incubated and thereupon subjected to the haemagglutination assay to determine the relative rate of virus multiplication.

I do not know what made them change the procedure, but Isaacs noted that the experiment had got them 'nearer to the interferon'. ${ }^{56}$ In accordance with previous experiments, the readings in Isaacs's notebook show that the interfering capacity of the fluids had only reduced slightly after bathing the first set of membranes. Furthermore, it made almost no difference whether two sets of membranes were added, or only one after the first incubation period. In itself that would have been nothing new, had it not been for the small number of tubes that were treated differently. In this additional test group, the first set of membranes had been transferred after 2 hours to tubes with fresh nutrient fluid. Despite the initial absence of inactivated virus as an interfering principle in these fluids, roughly the same degree of interference was induced in this test group as in comparable test groups with no transfer procedure.

The researchers agreed that somehow interfering activity must have been released in

A. Isaacs, Laboratory Notebooks, 1946-65. National Library of Medicine (Bethesda), Film number: reel 91-28, Lab Note Book, S.O. Book 135, Code 28-72-0, 'Alick Isaacs Interference Expts. V', November, 1956. 


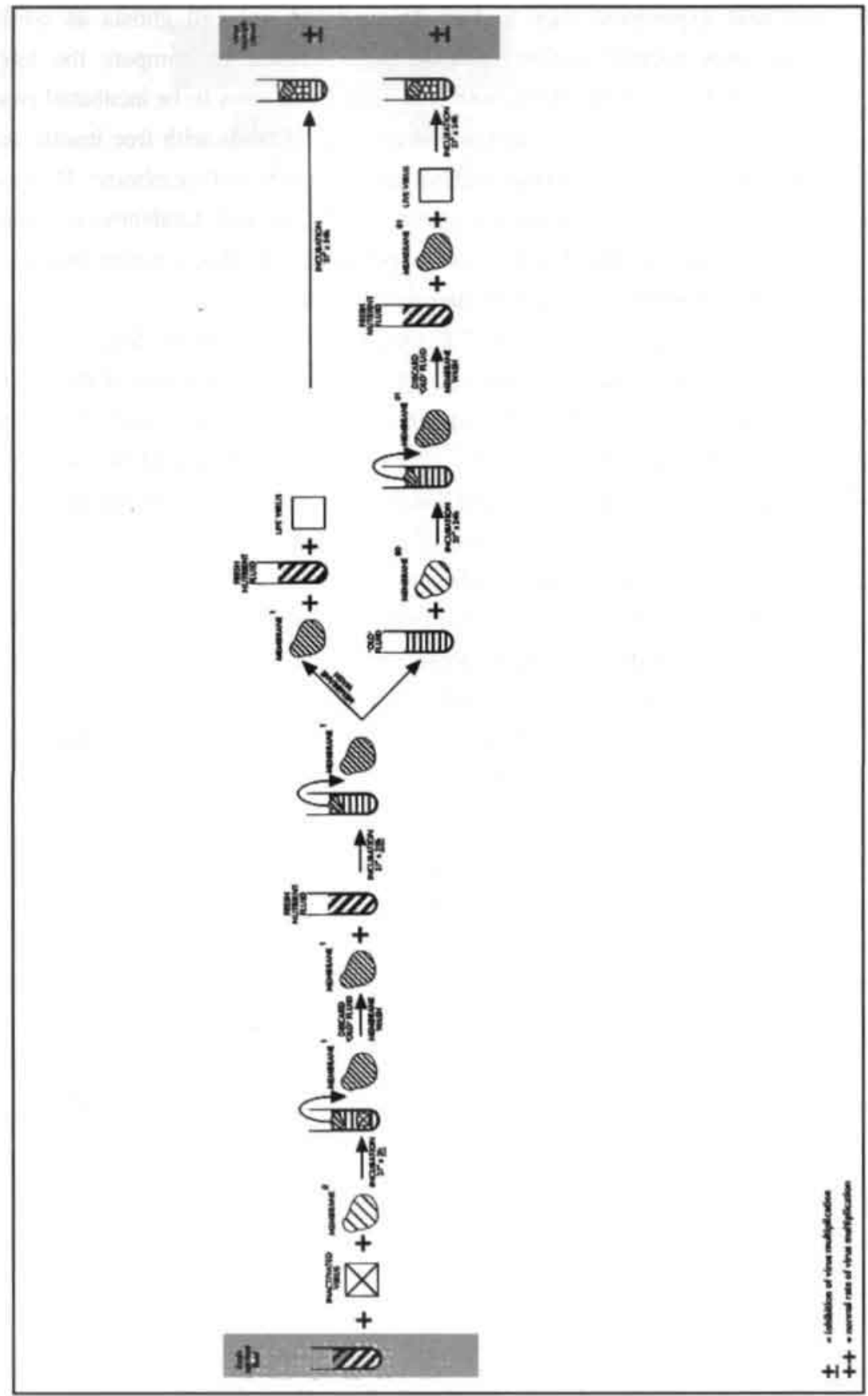

Fig 25. Diagram of part of experiments performed in Isaacs's laboratory between 13 and 17 November 1956. 
the additional test group between hours 2 and 24 , after transfer of the first set of membranes to tubes with fresh nutrient fluid and during the subsequent incubation in the roller-drum and, at least, before the second set of membranes was added. Isaacs was strongly in favour of the idea that one way or the other new interfering activity had been generated. However, Lindenmann remained more sceptical and played the devil's advocate by arguing that it could just as well be due to inactivated virus, which had been taken up by the membrane the first two hours and resecreted into the test tube fluid after the transfer procedure. For a further investigation and interpretation of the phenomenon it was agreed that they would need to elaborate on the transfer procedure, thereby reshaping their experimental system once again.

It is difficult to make sense of the subsequent series of experiments. Isaacs and Lindenmann varied their experimental set-up in an attempt to eliminate all possible background effects they could think of. One of the things they did was to check for the possibility that the 'new' interfering activity was due to the presence of inactivated virus that might be resecreted into the tubes, as Lindenmann had suggested. Thus, membranes were incubated with inactivated virus for 2 hours, washed and transferred to a fresh medium. After another 22 hours in the roller-drum, the first set of membranes was removed and before the second set of membranes was added, some tube fluids were processed in the ultra-centrifuge - this highspeed spinning instrument was known to have the effect of sedimenting the influenza virus in the tube at a certain speed/time ratio. Despite this rigorous virus separation step, the supernatant fluid, supposedly cleared of inactivated virus, turned out to contain only slightly less interfering activity than fluid samples which had not been centrifuged. According to Lindenmann, in the process they became more and more convinced that there was something, some factor in the biological soup that was responsible for the phenomenon, but they did not know what it was.

Most likely, the apparent consistency of the phenomenon made them believe that 'Isaacs's interferon' might indeed be an interesting new experimental feature, a signal instead of a noise, which was worth pursuing. In an attempt to characterize their elusive factor, they then initiated a series of 'properties of interferon' experiments, leaving their experimental system largely unchanged. It may look as if it was a rather straightforward and unproblematic series of experimental trials but again and again experiments went wrong for no decernible reasons and Isaacs and Lindenmann had a difficult time in making the experimental system work in a reproducible way, in other words, to produce workable amounts of the viral interference activity containing fluids which they referred to as 'interferon preparations'.

They basically employed the following procedure. Freshly prepared chicken embryo membranes were cut into pieces and these were put in bulk in a bottle together with inactivated virus and nutrient fluid, and incubated in a roller-drum for 2 hours. Subsequently the membranes were removed from the fluid and washed, and then each membrane placed in a separate test-tube containing fresh fluid, and rolled for another 22 hours. Thereafter the membranes 
were discarded and the remaining biological soup was referred to as 'interferon preparation'.57 Subsequently these crude preparations were subjected to a similar series of physical and biochemical manipulations as Isaacs had used some years before to identify the inactivated influenza virus as interfering agent. ${ }^{58}$

Isaacs and Lindenmann tested the stability of their interferon preparations at different temperatures, by heating at $60^{\circ} \mathrm{C}$ for one hour (result: the interfering capacity decreased sharply) or at $37^{\circ} \mathrm{C}$ for 24 hours (result: the interfering capacity remained stable) and by storing at $2^{\circ} \mathrm{C}$ (result: the interfering activity remained stable for at least 14 days). They made dilutions and subsequently compared the interfering activity of these dilutions (result: the activity decreased with dilution), or subjected interferon preparations to haemagglutination tests to see whether their putative factor would be able to agglutinate red blood cells like inactivated influenza virus did (result: no agglutination could be observed).

In addition they tried to neutralize the interfering activity with specific viral antiserum, which was to dissolve the interfering capacity of inactivated influenza virus (result: the interferon fluids turned out to be resistant against this viral antiserum). The researchers also ran filtration and centrifugation trials (result: the interfering activity of an inactivated virus preparation completely settled down in the test-tube after centrifugation, whereas the activity of an interferon preparation did not; there was no clear-cut difference with regard to filtration behaviour). ${ }^{59}$ Furthermore the viral interfering activity of interferon preparations was tested with a number of different viruses, which had also been shown to be susceptible to inactivated influenza virus as interfering agent (result: the interferon preparations showed an inhibitory activity against all viruses tested, such as the pox virus, 'vaccinia')).

It is worth noting that in between these series of in vitro trials an isolated attempt was made to see if the viral inhibitory effect of interferon preparations on influenza virus could also be demonstrated in mice (in vivo). However, no inhibitory effect could be observed. Unfortunately Isaacs's notebook does not give any hint with regard to the motives behind this

A. Isaacs, Laboratory Notebooks, 1946-65. National Library of Medicine (Bethesda), Film number: reel 91-28, Lab Note Book, S.O. Book 321, Code 28-321, "Alick Isaacs Interference Expts. VI," 10-12-1956/21-2-1957.

About half a year later lsaacs would write to Lindenmann that their filtration experiments had gone wrong. "I have a shock for you. Emst Fulton told me our filtration results were of no value since we had not used Broth. I had forgotten that Elford always insisted on Broth. We repeated filtrations in Broth and it goes through a 0.1 $\mu$ membrane and an $0.048 \mu$ membrane!!! (previously both the virus and interferon activity had been held back by a $0.6 \mu$ membrane) Results are being confirmed at the moment but I am afraid we will have to publish a disclaimer soon. Of course this now fits with the centrifugation results but I feel rather foolish and very sorry to have misled you": Alick Isaacs to Jean Lindenmann, letter dated 9 December 1957. Lindenmann Correspondence, Personal Archives. 
ad hoc in vivo trial. ${ }^{60}$

These kinds of experimental trials were performed over a two month period. They involved the most practical kind of skilled laboratory craftsmanship, a sort of cookbook benchwork, using various recipes for identifying interfering agents. As such it was part of a slow and laborious process of gaining experience and learning to read the data concerning a new laboratory factor. Despite attempts to reproduce experiments in detail, a certain variability in results was common. At times when experiments had produced no workable data, they would sit down and discuss the possibility that after all it was not the presence of something but the lack of something that kept them busy. Maybe a nutrient was being used up, thus preventing viruses from growing.

However, through the steady accumulation and superposition of data stemming from the physical and biochemical manipulations at the bench, the idea that the 'interferon' was a transitory factor, an artifact or noise, disappeared. Instead, interferon as a signal, a new kind of interfering agent, was articulated in accommodation to a series of non-trivial, data generating experimental events. These were regarded as direct indicators of the factor under study. In particular, the consistency of the interferon phenomenon in the face of widely differing physical conditions was instrumental in solidifying their sense of having found a novel biological factor. The anomaly' became increasingly transparent and self-evident and in the process a primary profile of Isaacs's and Lindenmann's elusive factor or activity was shaped. Apparently a kind of 'soluble interfering factor' was produced in their experimental set-up, which displayed properties different from other interfering agents such as the inactivated influenza virus. In a way they had ordered chaos by acquiring skills in the manipulation, interpretation and representation of a phenomenon which in November had still been perplexing.

By the end of February 1957, in consultation with Andrewes, it was decided that the experimental data looked consistent enough to argue against the interpretation of their 'interferon' as an artifact and to warrant writing up their research work for publication. ${ }^{61}$ Andrewes offered his support as a Fellow of the Royal Society in submitting the final papers to the highly prestigious Proceedings of the Royal Society. ${ }^{62}$ In this series of two articles, which were published in October 1957, Isaacs and Lindenmann claimed to have found a new interference-

\footnotetext{
60 This list of trials and results is based on the following documents: A. Isaacs, Laboratory Notebooks, 1946-65. National Library of Medicine (Bethesda), Film number: reel 91-28, Lab Note Book. S.O. Book 321. Code 28321, 'Alick Isaacs Interference Expts. VI, 10-12-1956/21-2-1957; and A. Isaacs, J. Lindenmann and R. Valentine, 'Virus interference. II. Some properties of interferon', Proc. R. Soc., 147 (1957). 268-73.

Since Alick Isaacs's personal laboratory archives were destroyed after his death in 1967. I was unable to recapture the process of drafting the early interferon papers and follow in detail the way both part of the continuous web of investigations was sealed off and ideas concerning this new interfering factor, 'interferon', further evolved.
} 
inducing factor named 'interferon' during a study of the interference phenomenon. ${ }^{63}$ The interference-inducing factor was said to be released in a test-tube fluid following the incubation of inactivated influenza virus with a piece of chicken embryo membrane. The presence of interferon in the fluid could be recognized by its ability to induce an inhibitory effect upon the growth of a number of different viruses. In addition, 'interferon proved stable at $2^{\circ} \mathrm{C}$ for 2 weeks. Marked inactivation took place after $1 \mathrm{~h}$ at $60^{\circ} \mathrm{C}$. Interferon was not measurably sedimented by centrifugation at $100000 \mathrm{~g}$ for $1 / 2 \mathrm{~h}$. It was held back by gradocol filters ... ${ }^{64}$ With reference to these properties they discussed the possible nature of their factor. Since the data 'did not allow even the crudest guess as to interferon's nature-whether it should be regarded a part of the inactivated virus, a reaction-product of the cell or an abortive product of virus multiplication-they resorted to reasoning by means of comparisons and analogies. They argued that because of observed analogies between interferon and virus production they would provisionally consider interferon as an abortive product of virus multiplication which was to block the synthesis of live virus and had many different properties from those of the inactivated influenza virus - the other interfering agent present in their arrangement.

Interferon cannot be identical with the original heated virus, the most conspicuous differences between the two being the interferon's inability to agglutinate red (blood) cells, its resistance to the neutralizing action of viral antiserum and its lower sedimentation rate...A tempting hypothesis is the following: the heat-degraded virus feeds into the cell insufficient information for orderly virus synthesis to proceed, but sufficient for some kind of ill-organized reaction chain to start, leading sooner or later to...piling up of distorted virus intermediates...It is conceivable that large amounts of such substances - interferon - could block synthesis of infective virus (live influenza virus). ${ }^{65}$ (Italics are mine)

The authors' description reflects the intricate packaging of material conditions and the emerging scientific object, 'interferon'. At the same time the description meant a divorce from the phenomenon's laborious production process at the laboratory bench. Laboratory tools and techniques, and human agency (to the extent that it was discernible) are presented as practical means to the end of claiming the finding of a new biological factor. Thus the significance and identity of materials and methods had changed retrospectively in the presence of a solution: the discovery of the substance named 'interferon'.

63 A. Isaacs and J. Lindenmann, 'Virus Interference. I. The Interferon', Proc. R. Soc., 147 (1957), 258-267; and A. Isaacs, J. Lindenmann and R. Valentine, 'Virus Interference. II. Some Properties of Interferon', Proc. R. Soc., 147 (1957), 268-73.

64

A. Isaacs, J. Lindenmann and R. Valentine, 'Virus interference. II. Some properties of interferon', Proc, R. Soc., 147 (1957). 268-73, p. 268.

65 A. Isaacs, J. Lindenmann and R. Valentine, 'Virus Interference. II. Some Properties of Interferon', Proc. R. Soc., 147 (1957), 268-73, pp. 272-3. 


\subsection{Isaacs's interferon kitchen}

Around the time when final drafts of the first two papers on interferon were being submitted to the Proceedings of the Royal Society, Isaacs was approached by Derek Burke a post-doctoral worker from the Chemistry Division, two floors down on the ground floor of the NIMR. Isaacs had known Burke for nearly two years as an assistant to James Walker, a senior organic chemist with whom Isaacs had been working on the possible effects of various synthetic organic compounds on influenza viruses. Burke, who had spent two years as a post-doctoral fellow at Yale University doing 'natural products chemistry', was responsible for studying the possible effects of the chemical agents, synthesized by Walker, on the nucleic acids of influenza viruses. ${ }^{66}$ Upon getting familiar with the basic techniques used in experimental virology, such as growing and harvesting influenza viruses in fertile hen's eggs and determining virus titers, and testing the first series of chemical agents, Burke had become seriously interested in studying the biochemistry of viruses. At the same time he gradually began to lose interest in Walker's and Isaacs's collaborative research project.

For some time, Burke had had an idea for a project involving the biochemistry of the influenza virus infection, and he wanted to talk it over with Isaacs. However, when asked for his opinion Isaacs did not seem much interested, and, instead of carefully listening to Burke's proposals, he started talking about his favorite research project. Together with Lindenmann he had been working for some time on a rather new and interesting viral interfering agent, which they were in the process of characterizing. However, he and Lindenmann were rather amateurs when it came to the task of identifying, purifying and isolating biological substances.

Both Isaacs and Lindenmann had been trained in medicine rather than in science with a rather basic knowledge of chemistry. Moreover, neither of them thought much of the chemical work, which they considered a necessary evil to characterize their putative biological factor: such work, they felt, kept them from working on the more interesting and rewarding fundamental biological research questions such as the elucidation of the mechanism of action. They would be far better off, they reasoned, if they handed over the chemical work to one of the PhD's in the NIMR's divisions of Chemistry or Biochemistry. ${ }^{67}$

This in mind Isaacs asked Burke to help with the biochemical work. Despite the poor hearing he had had from Isaacs for his own plans, the young scientist Burke considered it an honour to be asked for help by a highly regarded senior researcher like Isaacs. ${ }^{68}$ Thus, by the end of March 1957 the biochemist Burke joined Isaacs's and Lindenmann's investigations into

\footnotetext{
66. Interviews with Derek Burke,.Norman Finter and Joseph Sonnabend

67 Ibid.

68. Ibid
} 
the 'interference inducing factor', named 'interferon'. Burke's laboratory records indicate that he mainly worked on the characterization of interferon, subjecting interferon preparations to various chemical manipulations. Most of these were routine in biochemistry to identify natural substances-for example, testing the effect of organic solvents like ether, testing the effect of protein degrading and nucleic acid degrading compounds or testing the $\mathrm{pH}$-stability, i.e. acid and base stability. ${ }^{69}$ First, however, Burke enlisted the help of Isaacs's laboratory technician to familiarize himself with the production of interferon through replicating the material realization, or performance, of the interferon system. ${ }^{70}$

With the help of Burke's biochemical toolkit the list of biochemical and physical properties ascribed to interferon grew steadily. 'Interferon was stable in fairly strong acid solution...Shaking with an equal volume of ether caused complete loss of activity...Trypsin considerably reduced its activity. ${ }^{71}$ According to Burke the sensitivity to the proteindegrading enzyme, trypsin, suggested that interferon might contain protein, but the other experimental properties did not allow any further conclusions regarding interferon's chemical nature. ${ }^{72}$ It seemed unlikely that they would learn much more about its chemical composition until interferon could be prepared in a purified form. Burke also helped scaling up the experimental system in order to prepare interferon in bulk, as the production of interferon was lagging behind the increased research demand.

Isaacs and Lindenmann - who coined Burke the name 'Calamity Joe' because whenever he handled glass-ware, there was a fair chance that he would break it-in turn, shifted their attention to the phenomenological aspects of interferon research, such as the mode of production and the mode of action of interferon in vitro, in test-tubes each containing at least one piece of chick embryo membrane. ${ }^{73}$ Through this research work unexpected differences emerged between interferon and virus. For example, membrane preparations could be induced repeatedly to produce the same amounts of interferon, whereas at the same time the preparations showed a greatly reduced ability to support virus multiplication. These

D. Burke, Laboratory Notebook, S.O. Book 321. Code 28-321, Interferon Book I. 4-3/26-9-1957. Personal Archives.

70 In order for the replication of the material realization of an experiment successful to be called successful it is required that "the same actions are performed and the same experimental situations produced from the point of view of the daily language description of the material realization of the experiment". See, H. Radder, 'Experimental Reproducibility and the Experimenter's Regress', in D. Hull. M. Forbes and K. Okruklik (eds.) PSA, Volume I (East Lansing, Michigan: Philosophy of Science Association, 1992), p. 65.

J. Lindenmann, D. Burke and A. Isaacs, 'Studies on the Production, Mode of Action and Properties of Interferon', Brit. J. exp. Path., 38 (1957), 551-62, p. 559.

72 Interview with Derek Burke.

Interview with Jean Lindenmann. 
experimental results were thought to suggest that only part of the 'complex machinery' that was required for viral synthesis was required for the production of interferon. ${ }^{74}$ The results strengthened Isaacs's and Lindenmann's beliefs that interferon was most likely an abortive product of virus multiplication, which passed through only part of the viral reproductive process. As such, interferon was regarded as an abnormal viral entity that played a major role in mediating viral interference. ${ }^{75}$

Apart from studying the mode of action of interferon in vitro, Isaacs also initiated a series of experiments to test the effect of interferon preparations in vivo. According to Lindenmann as soon as Isaacs learned that interferon preparations were capable of inducing viral interference against vaccinia virus, in vitro, in the test-tube, he began suggesting plans to test interferon's action on vaccinia in vivo, on laboratory rabbits. Such experiments were argued to be perfectly in line with trials Isaacs had performed in his laboratory about three years earlier. In the autumn of 1953, he succeeded in showing that inactivated influenza virus interfered with the development of vaccinia virus not only in ovo, in fertile hen's eggs, but also in laboratory rabbits. He figured out that if he injected rabbits intradermally-into the skin-with inactivated influenza virus preparations, and one day later made injections with vaccinia virus preparations in the same areas, the development of skin lesions, normally expected after a single vaccinia virus inoculation, was inhibited significantly. ${ }^{76}$ Since interferon was considered to be an interfering agent with a viral interfering activity similar to inactivated influenza virus, Isaacs expected to see a similar effect when substituting inactivated influenza virus for interferon in aforementioned rabbit experiment. ${ }^{77}$ However, substituting inactivated influenza virus for interferon did not initially meet with Isaacs's expectations. Unlike inactivated influenza virus preparations, interferon fluids did not seem to have any effect on the development of vaccinial lesions in the rabbit skin. Only after many unsuccessful trials, changing the experimental conditions a number of times, did Isaacs finally manage to observe a minor inhibition of lesions with one of his laboratory rabbits. Subsequently a number of other test series were required to substantiate the observed effect, though the overall inhibitory effect of interferon appeared to be far less significant compared with similar experiments using inactivated influenza virus. Isaacs was somewhat puzzled by the apparent difference in the

74 J. Lindenmann, D. Burke and A. Isaacs, Studies on the Production, Mode of Action and Properties of Interferon', Brit. J. exp. Path., 38 (1957), 551-62, pp. 561.

75

Interview with Jean Lindenmann.

76 R. Depoux and A. Isaacs, 'Interference between Influenza and Vaccinia Viruses', Brit, J. exp. Path. , 35 (1954), 415-18, p. 418.

77 J. Lindenmann, D. Burke and A. Isaacs, "Studies on the Production, Mode of Action and Properties of Interferon." Brit. J. exp. Path., 38 (1957), 551-62, p.558. 
protective effect between interferon and inactivated influenza virus. After all, the viral interfering effect of the latter was thought to be mediated through interferon, which was thought to be released as a result of the virus-host interaction. Isaacs and Lindenmann discussed the rabbit experiments over and over again, but they could not think of an explanation other than that far more interferon was present in the skin of rabbits treated with inactivated influenza virus. ${ }^{78}$

The persistency with which the animal experiments were performed suggests that the idea of the practical medical application of interferon preparations to virus disease must have crossed Isaacs's and Lindenmann's medically trained minds. In principle the laboratory rabbit is as much a model of the 'real' situation as the test-tube, but biomedical researchers have a 'natural' propensity for linking the experimental output of in vivo experiments, more easily than in vitro experiments, to practical medical applications. ${ }^{79}$

Most likely the in vivo experiments with laboratory rabbits were the first instances in which interferon's effects were translated into practical medical terms. As I will show in the next chapter medical concerns would continue to play a role in structuring interferon research, exploring the interferon phenomenon in terms of its possible value as a tool to be used in clinical practice. The fact that animal virology was institutionalized predominantly as part of the medical field of microbiology, whose major professional goal was the prevention, diagnosis and treatment of viral diseases, played an important role in this respect. ${ }^{80}$

\subsection{Conclusion}

In following the evolution of particular passages of experimental work in room 215 of the NIMR between June 1956 and June 1957, this chapter addressed several aspects of the process of scientific inquiry that are not visible in scientific communications to learned journals. First, my reconstruction of the investigative pathways which led to the production of a new laboratory factor, 'the interferon', reveals how the research setting of the NIMR was strongly

78 See the annual report from the Bacteriology and Virus Research Group of the NIMR, 10, 1956/'57, held at the NIMR Library Archives; and A. Isaacs, Laboratory Notebooks, 1946-65. National Library of Medicine (Bethesda), Film number: reel 91-28, Lab Note Book, S.O. Book 321, Code 28-321. "Alick Isaacs Interference Expts. V1,"21-3-/10-7-1957; and interview with Jean Lindenmann.

79 The 'real' in vivo situation is a chimaera or something one cannot gain access to via the artificial and constructive nature of laboratory practice.

80 This observation is in line with Olga Amsterdamska's claim that "the selection and formulation of problems in mission-oriented sciences must be considered within the wider contexts of their disciplinary goals, which are closely linked to the social contexts of practice": O. Amsterdamska, Medical and Biological Constraints: Early Research on Variation in Bacteriology'. Soc. Stud. Sci., 17 (1987), 657-87, p. 659. 
conducive to innovative collaboration between the two biomedical researchers, Isaacs and Lindenmann. I pointed out that the NIMR provided a state-of-the-art research environment aimed at stimulating internationally competitive research projects at the frontier of fundamental biomedical sciences. The distribution of and access to material and conceptual resources in these surroundings served as relatively fixed points of laboratory practice which affected the actions and decisions at the bench-but without necessarily determining courses of action.

We then saw how, on the basis of Isaacs's and Lindenmann's common objective to test a particular hypothesis and produce specific kinds of data, an experimental system was set up. The net result was an experimental system made up of elements stemming from the local context of the division and institute, from research projects Isaacs and Lindenmann had worked on in the past and from simultaneous projects. Manipulating their experimental system and performing the series of experiments under survey in this chapter involved a continuous interplay between researchers' assumptions and expectations, material procedures, and data production.

In principle any particular run of an experiment was a unique accomplishment, an event that created a situation that was different from the one before it. ${ }^{81}$ At the same time every second run depended on an experimental system encompassing earlier trials, decisions and expectations. Repeated failure to fit the 'old' and the 'new' - when the data repeatedly failed to meet prior assumptions and expectations due to resistances arising from the non-compliant nature of the instruments or materials at hand-was instrumental in inducing changes in the experimental system. The repeated failure to visualize the virus coated ghosts and the unexpected stability of the interfering activity in their arrangement, are all examples of series of inconsistent experimental events that made Isaacs and Lindenmann reshape their experimental system.

As a result certain research problems, ideas, materials and procedures were relegated to being peripheral whereas there was a growing commitment toward other ones. I will illustrate this argument on the basis of the events following Isaacs's remarks regarding an unexpected stability of interfering activity in their experimental set-up. Initially the unexpected data output did not change the course of actions and was explained away by associating it with one of the material elements they had relatively little experience with, the virus coated ghosts. However, when the expected fall in interfering activity failed to materialize repeatedly, the phenomenon acquired anomalous status. Incorporating the resulting 'experimental anomaly', as I called it, into their experimental system created a perplexing situation which was accommodated by ad hoc changes in the experimental system. The starting point of the first series of experiments,

81 See, Bruno Latour, The Force and Reason of Experiment', In H. E. Le Grand (ed.), Experimental Enquiries: Historical, Philosophical and Social Studies of Experimentation in Science (Dordrecht: Kluwer Academic Publishers, 1990) p.65. 
the 'nucleic acid' hypothesis, which had neither been falsified nor corroborated, was marginalized. Instead there was a growing commitment to the question whether or not the anomaly was related to some sort of new biological activity that was generated in their system as part of the phenomenon of viral interference. This refocusing also applied to the physical elements of the experimental system. Gradually the electron microscope and the virus coated ghosts also lost their position as constitutive elements of the experimental system.

The actions and decisions involved in adding yet another line of research clearly show that the work at the laboratory bench was far from an all or nothing effort to confirm or refute hypotheses. Isaacs and Lindenmann did not change course because one hypothesis seemed to make more sense than the other. Instead, the range and priority of hypotheses and research questions with which the experiments were associated was subsidiary to and dependent on the events at the laboratory bench. The thing that turned the biological researchers on was the prospect of experimenting with some kind of novel biological activity that would increase the range of manipulative options at their bench.

At the time of Lindenmann's introduction of the name 'interferon' neither he nor Isaacs had the slightest idea about the nature of this phenomenon they called 'interferon'. Something was involved in the experimental anomaly, but what was it? Another viral interfering factor or factors, or something quite different? It was a slow and laborious process of gaining experience and learning to read the data with regard to this 'interferon' thing. The indicators of the experiments were far from clear, and time and again Isaacs and Lindenmann discussed the dangers of 'chasing a red herring', an anomaly of artifactual nature.

Within this experimental situation, their status of novice and expert were relative to eachother. I initially called Lindenmann a 'scientist novice at the frontier of viral research', and Isaacs, who was widely known as a distinguished virus researcher, a 'scientist expert'. However, we saw that when exploring and manipulating their experimental system, both Isaacs and Lindenmann were, in a certain sense, novices. The two men had yet to discover how to go about experimenting and what experimental success meant in their case. In modeling the experimental system on a permanent basis they accumulated manipulative skills that would bring the new and unexpected under control. ${ }^{82}$ As such, both Isaacs and Lindenmann were 'tinkering' craftsmen, who, through building and manipulating an experimental system, were constantly engaged in producing, mastering ('domesticating as it were') and communicating new procedures, ideas and phenomena. ${ }^{83}$

82 On the relative nature of expertise in science see also; T. Nickles, 'Justification and Experiment', in D. Gooding. T. Pinch and S. Schaffer (eds.) The Uses of Experiment (Cambridge University Press, 1989) pp, 300-301; and D. Gooding. Experiment and the Making of Meaning (Dordrecht: Kluwer Academic Publishers, 1990), pp. $29-69$.

83. The use of the term tinkering was not to connote unskilled work but to emphasize the essential contingent nature of work at the frontier of science; see, K. D. Knorr-Cetina. The Manufacture of Knowledge: An Essay on the Constructivist and Contextual Nature of Science (Oxford: Pergamon Press, 1981) pp. 34; and F. L. Holmes, 
I called, their subsequent attempts to characterize the elusive laboratory factor, a most practical kind of skilled laboratory craftsmanship, a sort of 'cookbook' benchwork. Through the steady accumulation and superposition of data the idea of 'interferon' as a transitory factor, an artifact or noise, faded. Gradually a primary profile of Isaacs's and Lindenmann's elusive factor was shaped.

The main upshot of the series of data-generating and data-transforming events were a couple of scientific articles in highly prestigious journals. Ends and means had been reversed, in these papers, taking the phenomenon of interference and interferon as the explanatory ground for the collaborative research work. Apart from the intricate packaging of material conditions and the object of study 'interferon', the knowledge claims in the resulting scientific articles bear relatively few marks of their generative production process. The twists and turns at the bench in Isaacs's laboratory and the flaws the researchers had experienced in manipulating their experimental system were omitted from the final papers. Instead, the occurrence of what was called, alternately, the factor or substance 'interferon' had been caught in a web of mostly posthoc reasonings. This is in line with a change in meaning of the novel object under study: from interferon as a transitory factor, a 'mysterious' kind of biological activity, to interferon as a new interference inducing factor, possibly of practical medical significance. In the next chapter we will see that the meanings attributed to interferon continued to change over time.

'Manometers, Tissue Slices, and Intermediary Metabolism', in A. Clarke and J. Fujimura (eds.) The Right Tools for the Job (Princeton: Princeton Univ. Press, 1992), pp. 169-70 


\section{Chapter 3.}

\section{Shaping a new field of research: investigating interferon(s).}

\subsection{Introduction}

In the previous chapter I outlined how the virus researchers Isaacs and Lindenmann working on a particular problem, built and manipulated a specific experimental system. In attempting to forge a problem-related set of data they stumbled across an intriguing but separate problem which made them produce and bring under surveillance a new biological phenomenon. My analysis focused primarily on how explanatory ideas and hypotheses and the objects with which they were concerned, as well as those materials used to elaborate them, were 'co-produced'. However, by concentrating on the evolution of an experimental system and the dynamics of shaping 'the interferon' in NIMR's room 215, I have illuminated only part of the generative processes of knowledge production. Relatively little has been said about the great diversity of knowledge-making activities displayed in biomedical laboratory practices. Apart from establishing and maintaining experimental systems, and elaborating representations of the 'phenomena' under investigation, laboratory researchers also communicate, negotiate and modify knowledge claims, interfere with each others' experimental work, and seek funding in order to proceed with a particular research project. None of these aspects have been discussed in detail thus far.

At the end of the previous chapter I indicated how work on interferon gradually extended beyond the physical space of Isaacs's laboratory. I showed how the operations at the laboratory bench began to interact with the job of mobilizing local interest and support, writing up the experimental data for publication and getting the papers published in a major scientific journal. In this chapter, I shall describe how interferon evolved from an obscure biological phenomenon at the margins of viral research to a phenomenon that legitimized the 'blossoming' of a new subfield of research-depicting the evolution of interferon in time and space. To this end I shall investigate the ways in which work on interferon was constituted and modified through actions inside and out of Isaacs's laboratory exploring both the private spaces of laboratories and the public spaces in which interferon met its audiences.

A first point of interest is how interferon shaped the expectations and interests of the various human agents both as individuals and as social groups and directed both their activities and those of the institutions they represented. I shall show how different sets of expectations about interferon came into being and developed in the adjudicating community. In shaping judgements about, for instance, the novelty, validity and possible practical implications ('utility') of knowledge claims, these varying expectations were again open to change. In this 
process of transformation, the meanings attributed to interferon in terms of what it signified and what its biomedical significance was, altered and multiplied.

A second point of interest concerns the modes of interchange and influence between various researchers working on interferon: between those who claimed to have command of a novel laboratory procedure enabling them to produce a hitherto unknown experimental feature, and those who still had to include and integrate the new feature into their laboratory practices. This shows that the process of validating interferon and forming it into a basis for consensus involved not only evaluating claims in light of the perceived research opportunities and of negotiating consensus, but also a collective process of communicating, manipulating, interpreting and elaborating a common assembly of research objects.

In order to grasp these various issues, the chapter begins with a description of how Isaacs and Lindenmann communicated the discovery of interferon to various audiences, thereby gradually extending expectations regarding interferon beyond the walls of the NIMR. In analyzing this extension process I will consider the material, social and cognitive conditions that were instrumental in shaping expectations. I shall then examine the ways in which other research groups arrived at similar claims about the characterization of interferon-like factors. The emerging cross-links between these 'others' and Isaacs's research group, I will show, mark a turning point, as work on interferon no longer exclusively meant activity in Isaacs's 'interferon kitchen'. The latter part of this chapter portrays how interferon, upon changing the order of events in a growing body of laboratory practices, came to embody a network of practices with researchers, who shared a common set of research problems, materials, and objectives.

\subsection{Interferon going public}

Early in June 1957 Lindenmann's term as a visiting researcher came to an end and he returned to Switzerland. Before resuming work in Mooser's laboratory at the 'Hygiene Institut' in Zürich, Lindenmann was expected to present a paper on his foreign research work at the annual meeting of the Swiss Society of Microbiology. It was interferon's first official and extramural appearance.

In his paper presentation entitled 'trials on virus-interference' Lindenmann claimed to have found a product of influenza viral interference, named 'interferon', which induced an interfering effect upon the multiplication of live influenza virus in the test-tube. He indicated that little was known about the mode of production and action of interferon and about its chemical composition. His lecture gave rise to a rather lively debate among the members of the audience. Prof. Hallauer, one of Switzerland's leading bacteriologists and virus experts, started the discussion by asking Lindenmann: 'What kind of theoretical interpretation should we give 
to this experimental feature?' and 'Do we have to question the fact that viral interference is induced by the virus itself?' Lindenmann replied that it was the virus that was at the centre of their experimentation too, and that he believed interferon to be an intermediary product of the virus interference reaction. 'This answer did not satisfy Hallauer, who retorted that 'as this [interferon] was utterly new and contrary to all he had read, it must be rubbish'.2 Hallauer's fierce critique met the approval of most of the scientists present. They all considered viral interference an inter-viral competition phenomenon, due to the competition between interfering and challenging virus for a key element of the viral reproductive process, which was present in limited amounts in the host cell. This was in general agreement with the concept of interference as presented in the latest international viral handbook. ${ }^{3}$ The idea of an autonomous viral interfering factor, whether or not of viral origin, just did not seem to fit into the textbook-concept of viral interference, and it was therefore rejected.

One of the few people who did not share the general negative verdict on Lindenmann's paper presentation was the virologist Dr. Kradolfer from the drug company Ciba. In March 1957, Ciba observers in London had sent a promising report to Ciba's headquarters in Basel, which associated interferon's workings in the laboratory with potential therapeutic effects in the clinic. Upon assessment of the report Kradolfer was assigned to the job of exploring interferon's therapeutic potential. Kradolfer's interferon project was a product of Ciba's firm belief in their own strategical observation and screening system, which aimed to signal and establish links between fundamental biomedical research and clinical problems as early as possible, as a means to the end of bridging the gap between the laboratory and the clinic. ${ }^{4}$

In a corridor chat with Lindenmann during the Swiss meeting, Kradolfer indicated that he had been working on interferon for about three months and that he wanted to exchange information with Lindenmann on this possible lead toward antiviral therapy. Lindenmann expressed his willingness to do so, though he was far from impressed by Kradolfer's research efforts. According to Lindenmann, Kradolfer could confirm the appearance of new interfering activity by using a similar experimental system that he and Isaacs had used, but only as a transient phenomenon. ${ }^{5}$ Lindenmann, in turn, suggested the possibility that the transient nature of

I J. Lindenmann und A. Isaacs, 'Versuche über Virus-Interferenz', Schweiz, Z Path. Bakt., 20 (1957), 640-6, p. 646.

2 Jean Lindenmann to Alick Isaacs, letter dated 28 June, 1957. Jean Lindenmann Correspondence, Personal Archives.

3 F. M. Burnet, Principles of Animal Virology (New York, Academic Press, 1955)

4 Interview with John Beale.

Jean Lindenmann to Alick Isaacs, letter dated 28 June, 1957. Jean Lindenmann Correspondence, Personal Archives. 
the phenomenon might be due to a difference in the strain of virus, which was employed in Kradolfer's experiment. In a letter to Isaacs, Lindenmann wrote about his contacts with the Ciba researcher and asked him whether he was against exchanging information with Kradolfer. ${ }^{6}$ Isaacs's reply was quite explicit: 'I think it is a very bad idea to show our manuscript to anyone in a commercial firm. I would be very much against your doing so in future'. 7 On first thoughts this answer seems to reflect a general tendency amongst British academic workers in the 1940's and 1950's to view collaborative research with an industrial firm as an intrusion upon their scientific work. But there was more to it, as the following excerpt from Isaacs's next letter illustrates: ${ }^{8}$

\begin{abstract}
In this country we are all very sensitive about the fact that penicillin is a British discovery, but that we have to pay royalties to American commercial firms for every gram of penicillin we use. I am not suggesting that interferon is in the same class as penicillin but on general grounds I don't think it is a good thing to deal with commercial firms. It is much better that they should wait until our information is published and then they can compete on even terms.
\end{abstract}

The way Isaacs associated interferon with penicillin in this second response to Lindenmann not only shows rather high hopes for interferon's possible medical applications, but also reveals a cultural condition that was instrumental in shaping Isaacs's policy toward interferon. There was a general feeling amongst government and academic workers in Britain that, because of an inability to control the patents, penicillin had been given free of charge to commercial drug houses and, in particular, American commercial firms. Even worse, royalties or licensing fees had to be paid to American drug companies for using penicillin in British medical practice, while Britain's dollar deficit was a constant source of political concern and irritation. ${ }^{10}$ To

Jean Lindenmann to Alick Isaacs, letter dated 28 June, 1957. Jean Lindenmann Correspondence, Personal Archives.

Alick Isaacs to Jean Lindenmann, letter dated 1 July, 1957. Jean Lindenmann Correspondence, Personal Archives.

According to a 1941 article on pharmaceutical research there was a "tendency amongst academic workers to view with apprehension whole-hearted alliance with the research of any one firm". See, J. Liebenau, The British Success with Penicillin', Soc. Stud. Sci., 17 (1987), 69-86, p. 72.

9 Alick Isaacs to Jean Lindenman. letter dated 7 July, 1957. Jean Lindenmann Correspondence. Personal Archives.

10 See, for a detailed analysis of how the story of penicillin was intertwined with great issues in post-WOII British culture: "Pride over technological prowess, resentment over the loss of opportunity, jealousy of American Success. The national Health Service and the emergence of the modern pharmaceutical industry": R. Bud, 'Penicillin and the new Elizabethans', Br. J. Hist. Sci., 31 (1998), 305-33; In 1957 Britain was still recovering from its third major payments crisis after the Second World War; see, D. Cantor, Cortisone and the Politics of Drama, 1949-55, in J.V. Pickstone (Ed.), Medical Innovations in Historical Perspective (Hampshire: Macmillan Distribution, 1992), pp. 175-6. 
avoid another penicillin-like 'patents affair', novel scientific findings were handled, at least within Britain's national research institutes, with exceptional caution until results had been published and patents had been filed. In particular within the field of bio-medicine, developments were closely monitored by government officials and taken up actively at the very first sign of a potential application in practical medicine. Moreover, as an additional and legal safeguard to secure the development of inventions resulting from public research in Britain, an entirely new type of government agency, the National Research Development Corporation (NRDC), had been established under the British Industries Act of 1948. The NRDC's task was to help to obtain patents and to provide financial support for the development of inventions up to the stage where industry was prepared to support the project completely. In facilitating the commercial development of state-aided research, the new agency tried to meet everyone's wishes to make better use of patenting in the medical field. "

Almost simultaneously with interferon's public appearance in Switzerland, Isaacs's laboratory was visited by a group of senior officers from the Medical Research Council (MRC), who were on their yearly inspection visit to the NIMR. ${ }^{12}$ On these occasions researchers were expected to give information about the current state of research affairs. ${ }^{13}$ Isaacs had prepared a demonstration of interferon's effects to convince the official visitors that he and Lindenmann had recently discovered a biological substance with distinctive antiviral properties. Several experimental set-ups were on display to show that interferon preparations had an inhibitory effect on a number of viruses both in vitro and in vivo. While showing the MRC officers around, Isaacs enthusiastically speculated about the possibility that this interferon might offer a potential new approach to the medical treatment of virus infections. ${ }^{14}$

Most likely, Isaacs's speculation about interferon's clinical potential was more than a mere expression of his professional interest as a medical doctor in practical problem-solving. He must have been aware of the fact that within the MRC there was a tendency to favor research projects with practical medical promise, even though MRC's official policies were

II D. Wilson, Penicillin in Perspective (London: Faber\&Faber, 1976), p.245; and L. G. Matthews, History of Pharmacy in Britain (London: E.\& S. Livingstone, 1962), pp. 330-3.

12 The publicly funded Medical Research Council played and still plays an important role in supporting and undertaking biomedical and clinical research in Britain. The NIMR was set up as the MRC's central institute for medical research in 1914. In 1960 up to twenty percent of its research funds were allocated to finance the NIMR and the rest to finance research projects throughout the country. See, for the role of the MRC in shaping a national system of Medical research in Britain: J. Austoker and L. Bryder (eds.) Historical Perspectives on the Role of the MRC (Oxford: Oxford University Press, 1989).

Interview with John Porterfield.

Interview with Derek Burke. 
directed more to basic than applied research. ${ }^{15}$ It seems that the Secretary of the MRC, Sir Harold Himsworth, as a former practicing clinician, emphasized the need to link basic research to clinical problems. The penicillin story was Himsworth's favorite case to convince his staff of the necessity to be on alert for claims linking fundamental research with practical medicine. ${ }^{16}$

The subsequent news of Ciba's interest in interferon as a lead towards antiviral therapy undoubtedly must have strengthened Isaacs's feeling that, however premature, he was justified in making the claim to the MRC that interferon could have therapeutic potential. In one of his letters to Lindenmann Isaacs emphasized the fact that the MRC had been very impressed with the demonstration. ${ }^{17}$ Thus, while keeping the Swiss drugmaker at a distance, Isaacs actively tried to draw the MRC into the interferon project. Obviously Isaacs was concerned to safeguard British interests and at the same time win wider interest in and public support for further research on interferon.

In the meantime Lindenmann was defending his scientific existence. From the first moment he resumed work at the 'Hygiene Institut' his boss, Mooser began behaving in a peculiar way. On the one hand, Mooser emphasized the fact that he did not think much of Lindenmann's efforts to continue research on interferon by saddling him with so much other work that Lindenmann had to conduct his interferon research in the evening. Furthermore, he told Lindenmann right away that if he intended to use large numbers of laboratory eggs on a regular basis he had to pay the expenses out of his own pocket. ${ }^{18}$ On the other hand, Mooser repeatedly entered the laboratory and asked Lindenmann to give yet another detailed account of his interferon experiments with Isaacs at the NIMR. The fact that Lindenmann had difficulty in repeating the interferon experiments he had worked on in London only complicated matters.

The situation took a turn for the worse after the reprints of the Proceedings of the Royal Society papers arrived. Mooser immediately sent for Lindenmann and asked him why he was not quoted in either of the two publications. Mooser had taken great offence as he firmly believed that the idea of an interference inducing factor had originated from his experiments with Lindenmann in 1955. He indicated that he deserved at least as much credit for his pioneering laboratory work as Lindenmann himself. In a way, he even believed he was a codiscoverer of this 'interferon', and despite Lindenmann's efforts to provide evidence to the con-

15 L. Bryder, 'Public Health Research and the MRC', in J. Austoker and L. Bryder (eds.) Historical Perspectives on the Role of the MRC (Oxford: Oxford University Press, 1989), p. 81.

16

C. C. Booth, 'Clinical Research', in J. Austoker and L. Bryder (eds.) Historical Perspectives on the Role of the MRC (Oxford: Oxford University Press, 1989) pp. 234-9.

17 Alick Isaacs to Jean Lindenman, letter dated 26 June, 1957. Jean Lindenmann Correspondence, Personal Archives.

18 Jean Lindenmann to Alick Isaacs, letter dated 28 June 1957, Lindenmann Correspondence, Personal Archives; and interview with Jean Lindenmann. 
trary, Mooser persisted in this belief. Eventually the situation became unbearable and Lindenmann had to leave his position at the 'Hygiene Institut' and accept a job as a microbiologist at the Federal Health Department in Berne. ${ }^{19}$

\subsubsection{The anti-viral penicillin}

While Lindenmann was fighting a losing battle to keep his university position, most of Isaacs's time was consumed by running the World Influenza Centre. ${ }^{20}$ Nevertheless, with Andrewes's support and with both Burke and a technician at his side, Isaacs managed both to keep research on interferon going and to involve himself in operations to raise the factual status of interferon in- and outside the walls of the NIMR. He organized an Institute colloquium on interferon, was responsible for submitting another two articles dealing with studies on interferon, and he also sent out reprints of interferon publications to all influential virologists he could think of. ${ }^{21}$ Furthermore Isaacs prepared a short paper on the discovery of the substance 'interferon' for the MRC Annual Report, in agreement with the demonstration for MRC officers.

In this report attention was primarily focused on interferon's effects against viruses both in the test-tube and the rabbit model. Furthermore, interferon's workings in the laboratory were associated with potential therapeutic effects in the clinic: interferon was presented as a possible new approach to the problem of finding suitable remedies against virus diseases. It was emphasized that hitherto all chemotherapeutic substances which had been found to inhibit virus growth had been toxic to the host, thus implying that this new biological substance, interferon, might offer a non-toxic alternative. In the test-tube, at least, it had been shown to be innocuous. 22

In 1964 Lindenmann would return to the University of Zürich as an associate professor at the Institute for medical Microbiology. By then he had already left the field of interferon research. See, Jean Lindenmann to Alick Isaacs, letter dated 19 May 1964, Lindenmann Correspondence, Personal Archives; and interview with Jean Lindenmann.

20 Together with other WHO centres Isaacs's laboratory was tied up with characterizing an apparently new influenzavirus strain, which was held responsible for a world-wide flu epidemic ('Asian flu') in 1957. See Alick Isaacs to Jean Lindenmann, letter dated 9 September 1957, Lindenmann Correspondence, Personal Archives; and Alfred Grafe, A History of Experimental Virology (Berlin, Springer-Verlag, 1991), p. 252.

21 Alick Isaacs to Jean Lindenmann, letters dated 17 October and 9 December 1957, Lindenmann Correspondence, Personal Archives; J. Lindenmann, D.C. Burke and A. Isaacs, 'Studies on the Production, Mode of Action and Properties of Interferon', Brit. J. exp. Path., 38 (1957), 551-62; and, D.C. Burke and A. Isaacs, 'Further Studies on Interferon', Brit. J. exp. Path., 39 (1958), 78-84.

22 See the annual report from the Bacteriology and Virus Research Group of the NIMR, 10, 1956/57, held at the NIMR Library Archives. 
The MRC Annual Report, together with the series of two articles in the Proceedings of the Royal Society and further annotations in the Lancet and British Medical Journal, drew the attention of the science correspondent of the Daily Express. ${ }^{23}$ The latter immediately phoned Isaacs with a request for additional information. Despite Isaacs's refusal to provide the extra information an article was published at the end of October 1957 in the Daily Express on the isolation of an antiviral substance, named 'interferon'. ${ }^{24}$ Following this newspaper article, the pharmaceutical companies Glaxo and Benger Laboratories wrote to the MRC, urging for a change of name of Isaacs's substance. Since both had got Registered Trade Marks for products with names similar to interferon, they anticipated difficulties if it was to be developed as a drug in the future. ${ }^{25}$ However, far from leading to a change of name, these minor events led the MRC, which had been impressed by Isaacs's private demonstration and were closely following the latest developments on interferon, to consider more urgently the patentability of Isaacs's discovery in order to protect the Council's interests. ${ }^{26}$ As I argued before, the MRC was on the alert to actively take up research projects with potential links to practical medicine in order to prevent missing out on a penicillin-like medical breakthrough. Furthermore, the MRC wanted to keep up a high profile toward the Treasury, and as a means to this end the MRC was always looking for promising research results produced by one of its research units which would make for a big story. ${ }^{27}$

The MRC consulted their patent adviser at the NRDC to see whether or not the subject-matter was patentable, with the result that in May 1958, applications were made for patents in the United States, Canada and Germany. However, they were told by the NRDC that it was impossible to obtain a British patent because more than six months had elapsed since the first public report on interferon had been published. Obviously the MRC had little experience in patent matters. ${ }^{28}$

23 See, Editorials, 'A lead towards virus chemotherapy?', Lancet, i (1957), 931-2; and 'Interferon', Br, Med. J., i (1957), 1102.

Alick Isaacs to Jean Lindenmann, letter dated 23 October 1957, Lindenmann Correspondence, Personal Archives.

Glaxo to MRC, letter dated 1 November, 1957. MRC Archives, File No. A814/17: and Benger Laboratories to MRC, letter dated 28 November, 1957, MRC Archives, File No. A814/17.

MRC to Sir Charles Harrington, letters dated 6, 29 November. 5 December, 1957, MRC Archives, File No. A814/17; and Sir Charles Harrington to MRC. letters dated 13 November, 7. 10 December, 1957, MRC Archives, File No. A814/17.

Statement of a senior MRC-official during the discussion following the presentation of a preliminary version of this chapterto the Twentieth Medical History Group of the Wellcome Trust, held at the Royal College of Physicians, 11 February, 1992. 
At about the same time that the MRC and NRDC began to regard interferon as a patentable commodity and started to file patent applications, Isaacs and Burke gave a public demonstration of interferon's workings at the Annual Conversazione of the Royal Society. Probably a similar kind of experimental set-up to the one shown earlier to MRC officers was on display at the Royal Society in London on 15 May 1958 - thereby extending Isaacs's laboratory space beyond the doors of the NIMR. The catalogue of the exhibits to be seen at the Royal Society contained the following description:

\begin{abstract}
INTERFERON: AN INHIBITOR OF VIRUS GROWTH
It has been known for many years that one virus may interfere with the growth of a second. Recently it was found that this interference is mediated through a substance which has been named 'interferon'. Active preparations of interferon can be easily produced by the liter without special equipment, and the activity can be readily concentrated. Such preparations are potent inhibitors of the growth of influenza and related viruses in vitro, and preparations which have been concentrated 10-fold inhibit the growth of vaccinia virus on the chick chorioallantoic membrane or in the rabbit skin. $^{29}$
\end{abstract}

According to a correspondent of the Daily Telegraph, a Fellow of the Royal Society had said at a press preview: 'I find enormous excitement in the promise of interferon'. ${ }^{30}$ The next day, the Daily Telegraph, among other newspapers, reported the discovery of a substance named interferon which might one day play an important part in the fight against viruses. In a comment on the exhibits at the Royal Society by the British Medical Journal 'interferon' was said to break new grounds in medicine as a revolutionary therapeutic tool against viral disease appropriate to the satellite age, 'while the latest and largest sputnik made its first orbit over Great Britain'. ${ }^{31}$ Isaacs was even asked to repeat the demonstration on television, in the program 'Eye on Research'. ${ }^{32}$

The sudden national press coverage of interferon as a kind of new 'antiviral penicillin' in newspapers, magazines, in the scientific press, and on British television had an impact (see Fig 26.). Translating interferon's viral inhibitory effect at the laboratory bench into therapeutic

in the U.S.A. under No.734106 on May 9. 1958.", MRC Archives, File No. A813/104; Elkington and Fife, consulting chemists and chartered patent agents, to the MRC, letter dated 20 December, 1957. MRC Archives, File No. A813/104.

29

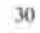

31

32

See the invitation to the Annual Conversazione of the Royal Society on 15 May 1958. The Annals of the Royal Society, 1 (1958), p.10.

30 See newspaper article, "Interferon' May Aid Fight Against Flu', The Daily Telegraph, 16 May 1958.

Anonymous, 'Royal Society Conversazione', Br. Med. J., i (1958), 1229.

Alick Isaacs to Jean Lindenmann, letter dated 5 June 1958, Lindenman Correspondence, Personal Archives. 

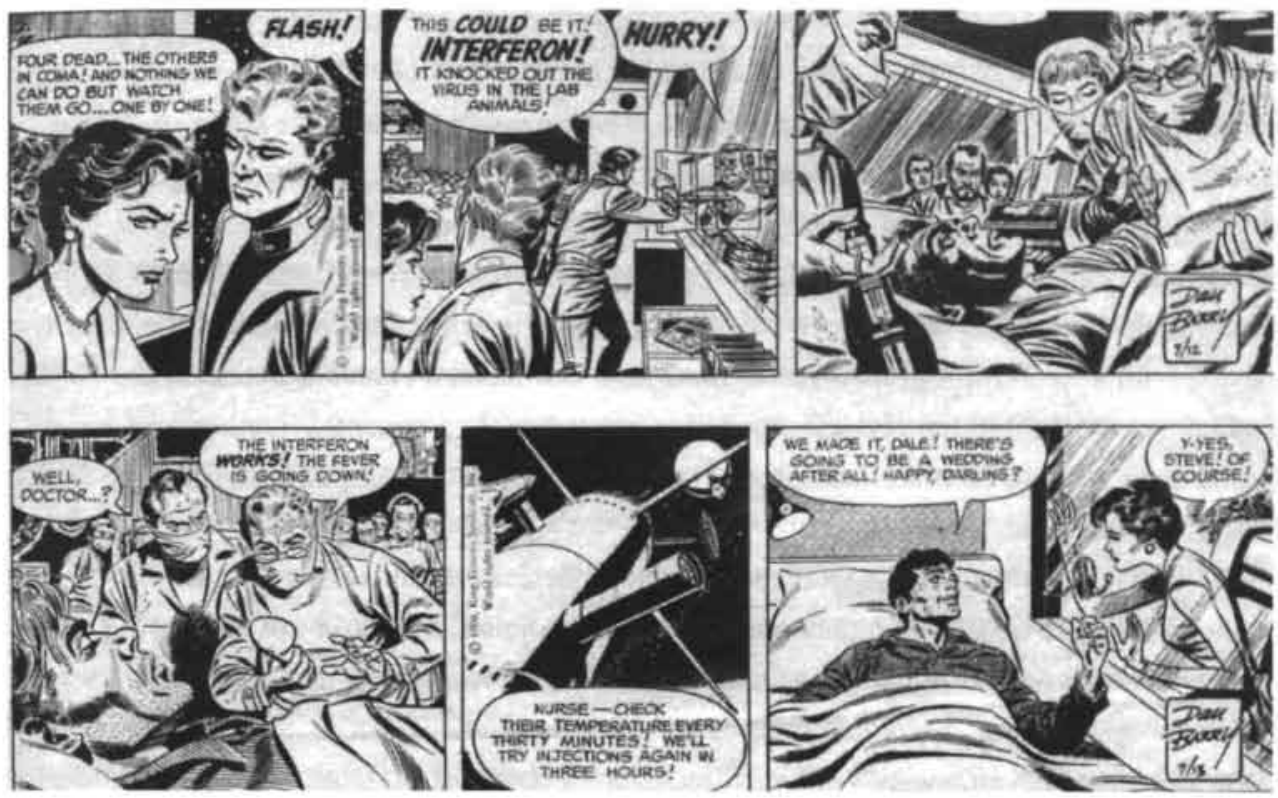

Fig. 26. The first human trial of interferon was 'performed' in this Flash Gordon strip (dated early 1960).

terms brought interferon to the public stage. Penicillin served as a powerful cultural symbol for interferon's legitimacy as a promising lead toward antiviral therapy. The introduction of 'miracle-drugs' like penicillin and cortisone in the 1940's had provided physicians with a curing power never seen before. Their spectacular clinical potential of specific and truly curative drugs, so-called 'magic bullets', and the often dramatic portrayals of beneficial effects in the clinic, had raised hopes regarding scientific medicine's ability to come up with yet another 'wonder' for the doctor's bag. ${ }^{33}$ It effected a firm belief among doctors, administrators and the public in therapeutic breakthroughs as a dominant feature of a laboratory-supported scientific medicine. ${ }^{34}$ Together with the British 'penicillin trauma', or what was generally referred to as 'the American penicillin syndrome', this set the stage for interferon's dramatic reception in Britain and the subsequent efforts to develop interferon as a drug. ${ }^{35}$

33 D. Cantor, 'Cortisone and the Politics of Drama, 1949-55', in J. V. Pickstone (ed.) Medical Innovations in Historical Perspective (Hampshire: Macmillan Distribution. 1992), p. 173.

34

H. M. Marks, 'Cortisone, 1949: A Year in the Political Life of a Drug, Bull. Hist. Med., 66 (1992), 419-439.

35 The same 'penicillin syndrome' played a most vital part in the concurrent British efforts to develop cephalosporin C as a drug; see, D. Wilson, Penicillin (London, Faber\&Faber. 1976), pp. 252-253. 


\subsubsection{Misinterpreton}

Interferon mattered in a rather different way to the international audience of animal virologists, thereby yielding a different set of expectations. Instead of immediately assessing interferon's potential benefits to practical medicine, virus researchers judged Isaacs's and Lindenmann's knowledge claims in a different light: firstly, the association of those claims with the predominant theoretical framework concerning viral interference; secondly, the expected relevance for research programs that figured high on national research agendas; thirdly, the practicality of integrating Isaacs's and Lindenmann's experimental features in existing experimental systems in other laboratories; and fourthly, the success or failure of replicating these experimental features.

The international virology community was far less receptive than Isaacs had hoped. Despite a series of articles in highly prestigious British journals and the established reputation of both Isaacs and the NIMR, Isaacs nevertheless had to engage in a quest for scientific interest and credibility regarding interferon. In particular, the cool reception within the powerful community of animal virologists in America concerned Isaacs. Although claiming the existence of a factor associated with viral interference, supposedly of viral origin, did no harm to the basic premise that interference was mediated by a virus, some researchers argued that it did infringe upon the consensus of viral interference as an interviral competition phenomenon. For others, the mere association with a laboratory phenomenon that had been relegated to the shadows of phenomena with relatively little research priority prevented them from becoming interested: viral interference was just not 'sexy' enough to capture their 'imagination'. ${ }^{36}$ In addition, Isaacs's and Lindenmann's publications were criticized for their vagueness as to the nature and mode of production of the novel factor. Moreover, those who tried to implement Isaacs's and Lindenmann's experimental features in their own experimental systems, found it extremely difficult to reproduce even part of the material results reported in Isaacs's and Lindenmann's publications. Whatever viral inhibitory activity was present in their experimental systems, it could not be linked with the experimental features claimed to be specific to interferon. A growing number of animal virologists in America began to question the validity of Isaacs's and Lindenmann's interferon claims, and a rumor spread that interferon was most likely a noise due to small amounts of inactivated virus coming through during the experiment. For this reason, influential American virus researchers like Harry Rubin and Howard Temin

More rewarding and fashionable research topics for animal virologists in America in the late 1950's were the study of the biochemistry and genetics of animal viruses, quantitative tissue-culture studies and the characterization of new viruses, and the study of animal tumor viruses in conjunction with the possible virus etiology of human cancer; A. Grafe, A History of Experimental Virology (Berlin: Springer-Verlag, 1991), pp. 164-97; M. Pollard (ed.) Perspectives in Virology: A Symposium, (Texas: The University of Texas Medical Branch, 1958). 
started nicknaming interferon 'misinterpreton'. ${ }^{37}$

With the great expectations building up over interferon in Britain and the same time the 'misinterpreton' rumours coming in from the USA, Burke witnessed how during the summer of 1958 Isaacs began to appear increasingly stressed. Gradually Isaacs fell victim to doubts about the validity of the interferon findings himself. Part of the assumptions and practices that had been buried in the process of publishing the claims were opened to criticism in the open again. This continued to the point that the claims about interferon as a product and mediator of viral interference were threatened with complete dissolution.

More and more frequently, Isaacs would come into his laboratory in a depressive mood and do nothing but talk with Burke about the possibility that the observed antiviral effects of interferon might after all be due to the presence of traces of inactivated virus or to the absence of one or the other nutrient that prevented the virus from multiplying. Would they not be better off by adding interferon to the list of noteworthy laboratory artifacts? The situation had a paralysing influence on interferon research and a damaging effect on Isaacs's mental health. In the fall of 1958 Isaacs had a nervous breakdown and went on sick leave, suffering from a severe bout of depression which lasted several months. Thus Burke was left in the laboratory on his own. ${ }^{38}$

With technical advice from a protein chemist in the Biochemistry Division on the third floor of the NIMR, Burke had been working for more than half a year on the purification of interferon preparations with the aim to isolate interferon, which he and Isaacs believed to be protein in nature. Just about the time of Isaacs's mental breakdown Burke had the feeling that he was on the verge of a major breakthrough in his purification studies. However, confronted with the 'credibility crisis' - the factual status of interferon being seriously called into question Burke decided to put his purification studies on the backburner and instead concentrate on the 'misinterpreton' issue.

Eventually Burke thought it necessary to invest his resources in the activity of replicating a great deal of the experimental results as obtained by Isaacs and Lindenmann during their original series of interferon experiments. It was not a matter of exactly reproducing the original experiments, as Burke performed a principally different set of experiments. In order to check

37. The researchers Temin and Rubin were closely associated with one of the protagonists of American animal virology, Renato Dulbecco, See, Grafe, A History of Experimental Virology (Berlin: Springer-Verlag. 1991). pp. 164-197; M. Pollard (ed.) Perspectives in Virology: A Symposium, (Texas, The University of Texas Medical Branch, 1958): Interviews with Sam Baron, Robert Wagner, Norman Finter, David Tyrrell, Derek Burke.

I agree with Stephen hall that it is impossible to reconstruct the story of interferon without taking into account Isaacs' manic-depressive disorder that manifested itself for the first time in the fall of 1958; S. S. Hall. A Commotion in the Blood; Life, Death and the Immune System (New York: Henry Holt and Company, 1997). 
for the presence of virus and to prevent virus from coming through and 'contaminating' the interferon preparations, he built in all kinds of additional procedures. What he basically did was to try to replicate a particular number of material results which were claimed to be characteristic for the presence of interferon under experimental conditions that were thought to exclude specific sources of artifacts. ${ }^{39}$ It was principally a matter of producing a new series of experimental events in order to check for the reproducibility of an 'old' set of experimental results and, as such, of linking the past to the future. ${ }^{40}$

The crisis of faith in interferon in Isaacs's 'interferon kitchen' and the American 'misinterpreton' rumors do not seem to have affected the high expectations in Britain with regard to interferon as a possible new 'antiviral penicillin'. At about the same time the MRC decided to start efforts to develop interferon as a drug and seek collaboration with British drug companies. In addition, drug companies had begun to approach the MRC about interferon. ${ }^{4 I}$

These circumstances which involved the high hopes regarding interferon as a potential therapeutic breakthrough widely shared in British society, made MRC's development plans relatively immune to both the late developments in Isaacs's laboratory and the misinterpreton rumours. They were instrumental in creating a niche, in other words a relatively stable and safe environment, for subsequent efforts to develop interferon as an antiviral drug in Britain. ${ }^{42}$

39 The notion of 'material realization' of an experiment was borrowed from Hans Radder. See, H. Radder, 'Experimental Reproducibility and the Experimenter's Regress', in D. Hull, M. Forbes and K. Okruklik (eds.) PSA, Volume I (East Lansing, Michigan: Philosophy of Science Association, 1992), 63-73, p. 64.

D. Burke, Laboratory Notebooks, S.O. Book 321. Code 28-321, Interferon Book III, March/Sept 1958 and 'Interferon Book IV', Sept 1958/March 1959, Personal Archives.

41 Christopher Andrewes to Jean Lindenmann, letter dated 10 November, 1958. Jean Lindenmann Correspondence, Personal Archives: interviews with Susanna Isaacs-Elmhirst, Derick Burke, and David Tyrrell.

42 See, for an in-depth analysis of the role of expectations and niches in technological developments $\mathrm{H}$. van Lente, Promising Technology: The Dynamics of Expectations in Technological Developments (Delft, Ph.D. diss., Eburon, 1993). 


\subsection{From the Brink to Revival: Meeting The Others}

The second part of this chapter chronicles a gradual reversal of the balance of forces in Isaacs's quest for scientific credibility and interest regarding interferon. The events in John Enders's laboratory at American's East-coast were key to this reversal and the subsequent formation of an international body of laboratory practices with shared commitments in the pursuit of a common set of research problems and objects. As it was exemplary for the changing relation between interferon and 'room 215', the growing interference between Isaacs's 'interferon kitchen' and Enders's laboratory deserves further attention.

\subsubsection{Viral Inhibitory Factor (VIF) vis-á-vis Interferon}

By the time Isaacs returned to work in the course of January 1959, Burke had finished his series of 'virus trials'. The data in Burke's laboratory notebook convinced Isaacs that despite extensive testing, his collaborator had not been able to find any indications for the alleged presence of virus particles in their interferon preparation. At least within the confines of Isaacs's laboratory the factual status of interferon was no longer up for discussion and, Isaacs concluded, the interferon studies were to be pursued with renewed effort. The very procedure of replicating the original set of experimental results by means of a different set of experiments not only was instrumental in regaining control and restoring belief in the validity of the original experimental results but also added to the robustness of interferon as a new biological entity and promising object for research.

Burke recalled the day that Isaacs received a letter from the American virologist John Enders, sometime around June 1957, as one of euphoria. Isaacs immediately showed the letter to Burke. Apart from requesting a sample of interferon, Enders informed Isaacs of the fact that together with one of his postdocs, Monto Ho, they had been working for some time now on a so-called 'viral inhibitory factor (VIF)' which seemed to have properties similar to interferon. Here, finally, were American researchers who had found what seemed to be interferon. ${ }^{43}$ We shall now leave Isaacs's laboratory and the substance interferon, and instead zoom in on John Enders and his postdoctoral fellow, Monto Ho, and closely follow how they found their way to their so-called 'viral inhibitory factor'.

One day, early in 1957 in Boston's Chinatown, Monto Ho was having dinner with his friend Bob Chang, who worked as a microbiologist at the Harvard School of Public Health. As usual they informed each other of the ongoing research activities over dinner. Ho might have told 
him his latest experimental findings with regard to the observed protective effect of some components of rat blood against the lethal action of bacterial endotoxins, i.e. toxins retained within certain bacteria. According to Ho, the conversation turned to future plans for research. Ho was about to finish a fellowship year as a researcher in the Bacteriology Division of the Thorndike Memorial Laboratory in Boston. He told his friend that he was not sure yet what to do next, but that he would like to pursue his endotoxin research. Chang argued that further endotoxin research did not seem to pay off much and suggested another more promising option. It might be worthwhile, he suggested, to consider the possibility of entering the relatively new and promising field of animal virology - the study of viruses that prey on animals and human beings. After all, one of the most prominent animal virologists and recent Noble Prize winner, John Enders, was working almost next-door as the head of a small research group at Boston Children's Hospital.

At first Ho was surprised at this proposal. The thought had never entered my mind, as the study of virology was thought to be a quixotic pursuit of a few pediatricians and I was trained in adult medicine'. ${ }^{44}$ According to Ho it must have been the attraction of the unknown and the close vicinity of a rather famous virologist like Enders that made him decide to set up an appointment with the latter. The subsequent interview made such an impact on Ho that almost immediately afterwards he applied for a job as a research fellow with Enders's research group. ${ }^{45}$ Ho got the job and by the end of September 1957 he started work in Enders's department. As a novice to the field of animal virology, he first had to acquire basic skill in working with viruses. While being shown around by one of Enders's assistants he learned that tissue cultures, particularly so-called 'monolayer cultures', represented the favorite system for growing and studying viruses in Enders's laboratory. As the name implies, these monolayer cultures consisted of a single layer of cells growing on the glass surface of closed containers, such as test tubes with screw top, or flatsided flasks or bottles (see Fig. 27 a, b, c). ${ }^{46}$ It appeared to be work on the multiplication of poliomyelitis virus in vitro in this type of tissue culture, which had proven crucial for the development of a polio-vaccine, that had earned

44 Monto Ho, 'An Early Interferon: "Viral Inhibitory Factor"', J. Interferon Res., 7 (1987), 455-7, p. 455; Up to the 1950's American medical training programs did not seem to pay much attention to virology. Of course polio was a big issue but in general virus diseases hardly featured at all in medical school; Monto Ho. interview; Sam Baron, interview; and Ion Gresser, interview.

Monto Ho, interview.

46

See, for the method for preparing monolayer culture as it was basically practiced in Enders's laboratory; R. C. Parker, Methods of Tissue Culture (New York: Hoeber Medical Division, Harper \& Row. Publishers, 1950); T. M. Bell, An Introduction to General Virology (London: William Heinemann Medical Books, 1965) pp. 26-36; J. M. Hoskins, Virological Procedures (London: Butterworths, 1967) pp. 3-73, 120-31; D. Balducci and G. Penso, Tissue Cultures in Biological Research (Amsterdam: Elsevier Publishing Company, 1963) pp. 29-138. 


\section{Enders the Nobel Prize in $1954 .^{47}$}

The enormous effort put in the meticulous cleaning and sterilizing of the glasswork and other laboratory tools in the glassware section, which was referred to as the 'kitchen', almost immediately struck Ho. He was told that after basic design the kitchen was next in order of importance for the operation of the virus laboratory. The thorough cleaning and sterilizing process in the kitchen, which employed four workers, was considered the key to successful work on viruses in tissue or cell culture. Since cultures of cells had been found to be extremely vulnerable to contaminants, everything possible was done to prevent contamination with any other particle or agent. Enders insisted on the working rule that all glasswork and other tools be regarded as chemically and biologically dirty unless known to be otherwise. Ho was told that a laboratory worker who was prepared to take a chance in this respect was a hazard to himself and even more so to his associates. Providing he followed the established procedures, he was told, the preparation of tissue cultures was rather straight forward. Soon Ho would find out that the term 'straight forward' was rather inappropriate to denote the esoteric craft of preparing tissue cultures and studying viruses in these cultures. According to Ho, the gist of it was learning this craft through hands-on apprenticeship. Actual practice in Enders's laboratory turned out to be a mixture of instructions in a methodology book on tissue cultures, and local idiosyncrasies. For example, it was standard practice in Enders's laboratory to use double distilled water when culturing cells, instead of 'normal' distilled water. ${ }^{48}$ As part of this training on the job Ho learned to cope with the frequent 'whims' of tissue cultures leading to difficulties in interpretation of the experimental data. Most common was a spontaneous degeneration of the cells, which occurred for reasons which were not always apparent: it might be due to a poor viability of the cells, a lack of essential nutrients from the medium, or the introduction of toxic agents. Furthermore there was always the danger of artifacts resulting from bacterial and fungal contamination or from the presence of unknown viruses so-called 'wild viruses' in the original tissue material.

During Ho's introductory period Enders came forward with a suggestion for research which would offer Ho ample opportunity to learn the ins and outs of virus work. Quite recently Enders had become interested in the exceptional behavior of a particular strain of poliovirus, named 'RMC virus'. Unlike all other strains of poliovirus it had almost no celldamaging effects when added to a culture of human kidney cells. However, when introduced in a culture of human amnion cells, RMC virus proved to be highly cytopathogenic. 


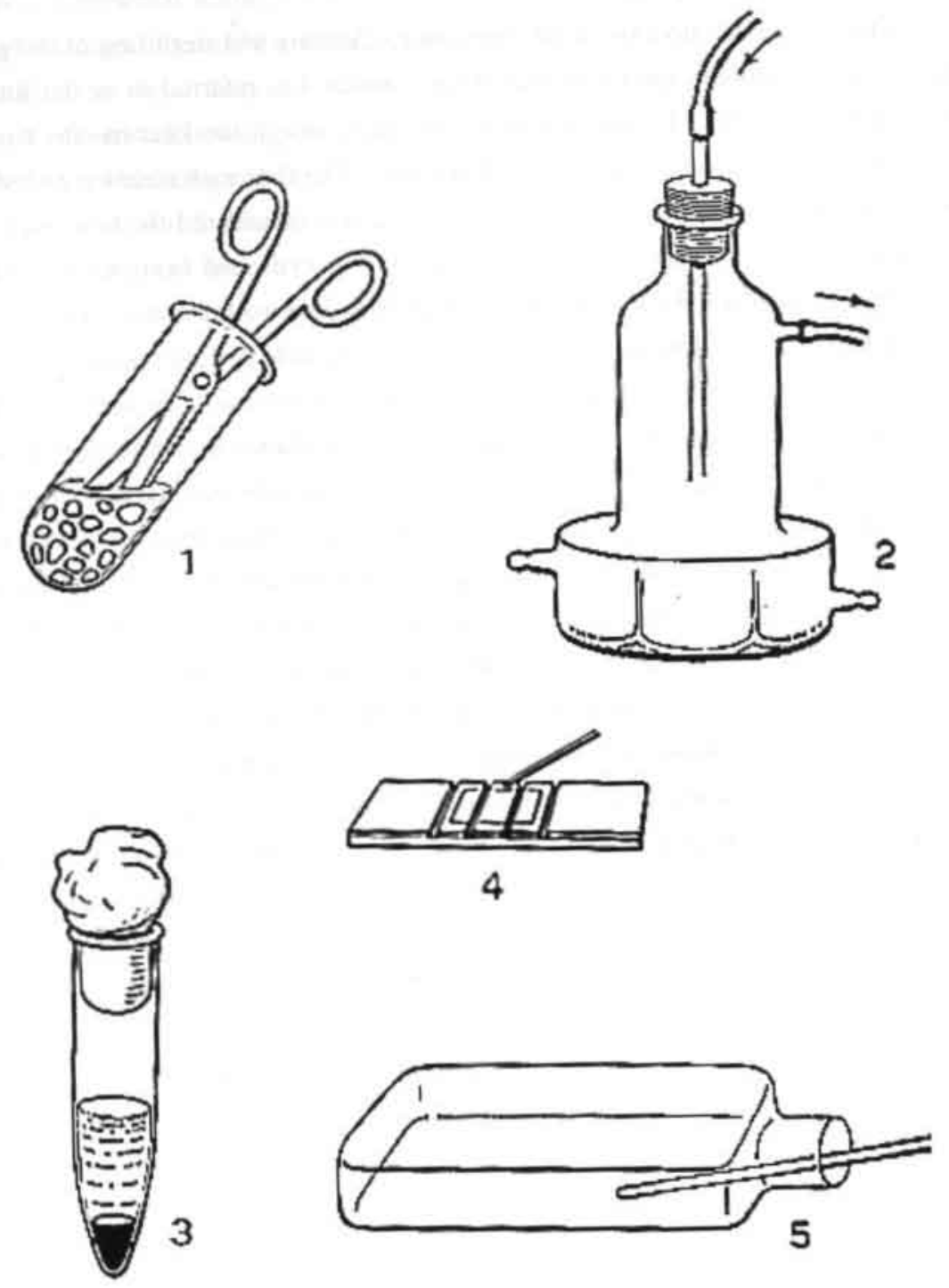

Fig 27a. Diagram form of preparation of tissue culture (1950's):

(1) fragmentation of tissue:

(2) trypsinization of the fragments;

(3) Sedimentation of the cells:

(4) Copunting of the viable cells:

(5) Distribution of cells suspended in growth medium.

Courtesy of MRC 

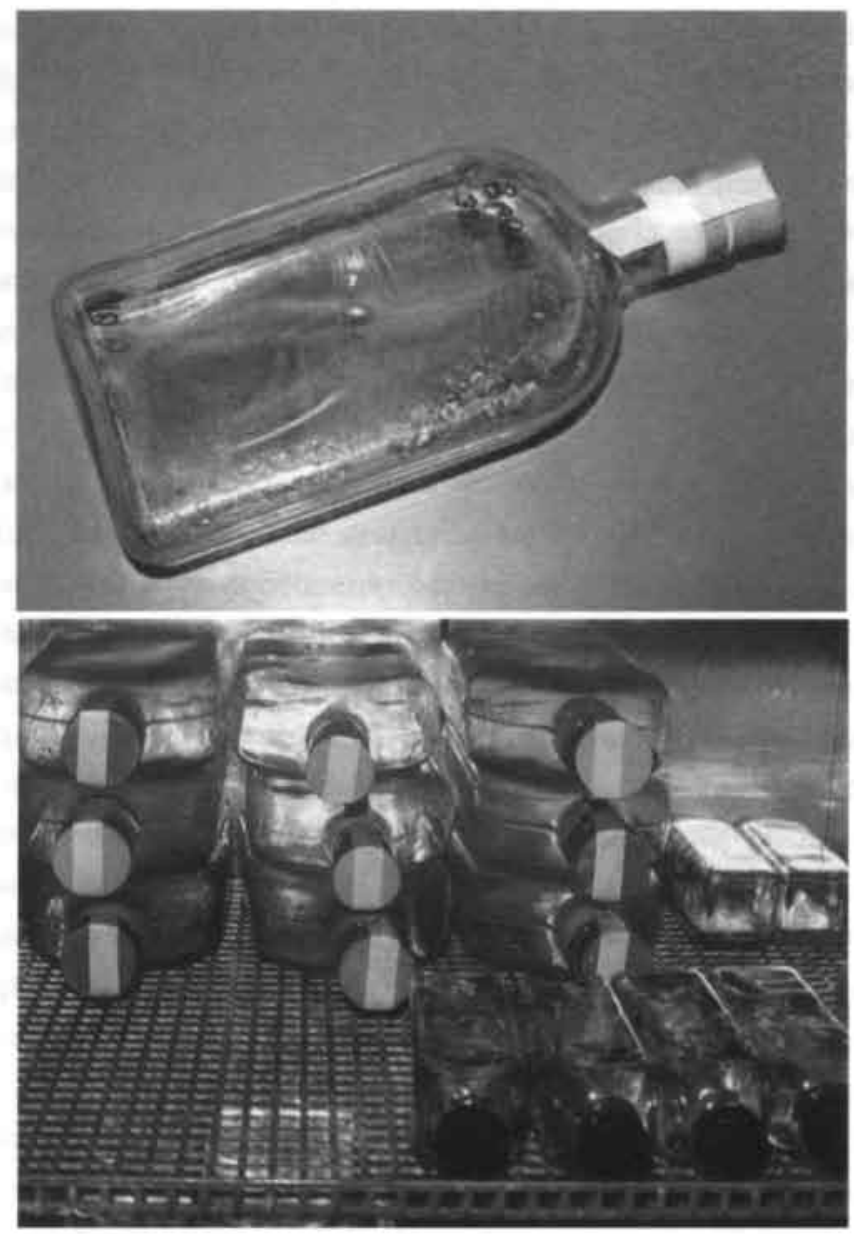

Fig. 27b, c. Large surfaces of cells are produced in rubber stoppered pyrex lass 'Roux' flasks which are stored in an incubator. Courtesy of J. Venteeg.

Moreover, this strain of poliovirus had the rather unusual ability to multiply in chick embryos. ${ }^{49}$

Ho received a stock of RMC virus, which had been grown in embryonated eggs, and was asked to see whether he could find out more about the deviant behavior of this biological agent. This provided the impetus for Ho to build and manipulate his own experimental system. While doing so he ran into resistances: the observed viral effects were, and even more impor-

49 Monto Ho, interview; and J. Enders, 'A Consideration of the Mechanisms of Resistance to Viral Infection Based on Recent Studies of the Agents of Measles and Poliomyelitis', Trans. Coll. Physcns. Philad., 28 (1960), 68-79, pp. 75-6. 
tantly, remained inconsistent with the expected effects. He indeed managed to observe that, when added to his test-tubes with human cell cultures, RMC virus had a highly pathogenic effect on amnion cells and almost no effect on kidney cells, although evidence of virus multiplication could be obtained in both cases. However, Ho was surprised to find that when he collected fluids from tubes containing either RMV virus infected human kidney or amnion cell cultures, and subsequently used these fluids to infect a fresh series of test-tubes with human amnion cell cultures, the pathogenic effect did not occur at all. If, instead, he first diluted these fluids and then added them to fresh amnion cell cultures, he could observe the usual destructive effect on these embryonic cells. It was a perplexing situation.

Eventually Ho suggested the possibility that the anomalous effect might be due to one or the other factor present in the medium. Of course, Ho was aware of the likelihood that some artifact was involved, but the effect could also be due to an as yet unknown biological factor. The novel factor might be responsible for inhibiting the pathogenic effect of the virus, a factor analogous with the inhibiting components in rat serum which Ho had studied during his fellowship year at Thorndike Memorial Laboratory. When Enders heard of his postdoc's latest findings, he seemed to have favored an alternative explanation.

In Enders's view what had happened in Ho's experimental system was very similar to a phenomenon that had been described extensively in the literature, the auto-interference phenomenon. It basically amounted to the claim that the infectivity and pathogenicity of concentrated virus preparations tended to be lower than that of diluted preparations. This paradoxical phenomenon was thought to be due to the high content of non-infective virus particles in undiluted fluids, which interfered with the multiplication of infective virus particles. Upon discussing the experimental events over and over again, the two researchers agreed that further experiments were required. ${ }^{50}$

Thus, both Ho and Enders were building their explanations upon perceived similarities between the actual, unknown experimental events and known phenomena, which had either been described in the literature or experienced in another unrelated research project. It was a similar kind of analogical reasoning - utilizing the knowledge one has of a familiar case to a new, unclear and problematic situation-as Isaacs and Lindenmann practiced once and again in shaping the interferon factor in room 215 of the NIMR. ${ }^{51}$

One of the experiments Ho did next was to see whether there would be a difference in effect if 'normal' RMC virus from the chicken-grown stock was added to fresh amnion cell

J. Enders, 'A Consideration of the Mechanisms of Resistance to Viral Infection Based on Recent Studies of the Agents of Measles and Poliomyelitis', Transactions \& Studies of the College of Physicians of Philadelphia, 28 (1960), 68-79, pp. 75-6; E. Lennette, 'Interference Between Animal Viruses'. Ann. Rev. Microb., 5 (1951), 277-94, pp. 284-85; and interview with Monto Ho.

51 See, for an analysis of the scientist as an analogical reasoner; K. Knorr. The Manufacture of Knowledge: An Essay on the Constructivist and Contextual Nature of Science (Oxford: Pergamon Press, 1981), pp. 49-66. 
cultures, or to amnion cell cultures which had been pretreated with medium collected from RMC virus infected human kidney cell cultures. Like before, the chick-grown RMC virus had clearly visible pathogenic effects on fresh amnion cell cultures.

However, far more interesting to Ho was the observation that it hardly had any effect on the pretreated amnion cell cultures. Ho immediately informed Enders of his latest finding, which he believed to point in favor of the idea that the undiluted infected fluids contained a factor capable of inhibiting the pathogenic effect of RMC virus on embryonic cells. In addition, he asked Enders what he thought about calling his as yet hypothetical factor 'viral inhibitory factor' or simply 'VIF'. Enders cautioned him by saying that 'viral inhibitory fluid' would be a more appropriate terminology. It was more appropriate because it would be impossible for them to determine whether they were dealing with a biological factor related to the virus, a non-viral factor or with a tissue culture artifact due to a deficiency of some nutrient from the medium that was essential in supporting cell and viral growth.

Somewhat reluctantly Ho then admitted that he was proceeding too fast with his inferences, although he thought that Enders was a bit overcautious in using the term fluid instead of factor. Ho agreed with Enders that further experiments were required to determine whether the observed inhibitory effect of VIF was related to the presence of virus in the medium or to some other factor, and to see whether or not Ho's VIF inhibited the pathogenic effect of other viral agents than RMC virus. ${ }^{52}$ First, however, Ho had to scale up the production of VIF, as such experiments would require relatively large quantities of VIF. Instead of the original test-tubes, Enders told him to use the bigger chemically neutral Pyrextype flatsided 'Roux' bottles, which were routinely used in his laboratory to grow larger quantities of virus, which he did. The change from tube to bottle monolayer cultures of human kidney cells required some time. Ho had to figure out the optimum conditions for the preparation of VIF in Roux bottles as he had previously when using the smaller tubes. ${ }^{53}$

The exact sequence of events can no longer be traced, but it is most likely that sometime during the spring of 1958, Ho and Enders must have become aware of Isaacs's and Lindenmann's articles on interferon. Presumably it was Enders who found out first about interferon during one of his informal meetings with the animal virologist Werner Henle. According to Ho, one morning Enders popped into the laboratory and told him that he had discussed their 'VIF experiments with Henle. Apparently, Henle seemed to have been quite

M. Ho, 'An Early Interferon: "Viral Inhibitory Factor"', J. Interferon Res., 7 (1987), 455-7; and interview with Monto Ho.

M. Ho and J. Enders, 'An Inhibitor of Viral Activity Appearing in Infected Cell Cultures', Proc. N. A. S., 45 (1959), 385-9, p.385; M. Ho, 'An Early Interferon: "Viral Inhibitory Factor"', J. Interferon Res., 7 (1987), 455-7, p.456; J. Enders, 'A Consideration of the Mechanisms of Resistance to Viral Infection Based on Recent Studies of the Agents of Measles and Poliomyelitis', Transactions \& Studies of the College of Physicians of Philadelphia, 28 (1960), 68-79, pp. 76; and interview with Monto Ho.. 
skeptical of Enders's report on VIF, which the former to some extent believed to be similar to recent work published by the British animal virologist, Alick Isaacs. The latter claimed to have found a factor called 'interferon', presumably a small viral component which escaped detection by conventional virological techniques and interfered with the growth of viruses in their host/virus system. Upon reading the interferon articles, Henle and his associates had almost immediately started efforts to see whether a similar inhibitory factor might be responsible for the observed resistance of chronically virus infected tissue cultures against unrelated viruses. ${ }^{54}$ However, despite extensive testing, they had not been able to demonstrate the presence of an interferon-like factor in their experimental system. Henle had concluded that it was most likely that the observed virus resistance, which was said to fulfil the criteria for viral interference, had nothing to do with interferon and he even doubted the validity of Isaacs's experimental claims. These claims did not correspond with Henle's concept of viral interference as an inter-viral competition phenomenon that was not considered to be transmissible. As Enders's report on 'VIF' sounded similar to what Henle had read about interferon, Henle did not think much of it either. Far from discouraging Enders and Ho, the meeting with Henle was an incentive to carry on and to get hold of the English interferon articles. ${ }^{55}$

What were for Henle experimental findings that resisted replication and violated the basic criteria for viral interference, were for Enders results that not only sounded interestingly familiar but might also be instrumental in furthering work on VIF. It is highly questionable that Enders considered the subsequent situation in these terms, but retrospectively it looks like the transfer of knowledge regarding interferon changed the order of events in Enders's laboratory, rendering the situation different for work on VIF. The fact is that the subsequent series of experiments that were performed to forge a primary profile of VIF bore a close resemblance to trials Isaacs and Lindenmann had run in the case of interferon, a year earlier.

Ho and Enders realized that they should be careful to rule out the possibility that the observed virus inhibitory effects in their experimental system might after all be due to the direct effect of interfering virus particles. Since interference by virus particles had already been known for years, the presence of virus particles in their VIF preparations would relegate their phenomenon to the limbo of ordinary laboratory events. Hence, all available techniques and procedures that were known to exclude the presence of virus were applied to see whether or

54 Henle et al were already in the process of studying host cell-vinus interactions in continuous lines of human cells, of malignant origin (e.g. human bone marrow cells derived from bone marrow of a leukemic patient, socalled 'MCN cells'). which were chronically infected with virus (e.g. an avian virus that went by the name New Castle disease virus); G. Henle, F. Deinhardt, V. Bergs and W. Henle, 'Studies on Persistent Infections of Tissue Cultures: I. General Aspects of the System', J. Exptl. Med., 108 (1958), 537-60.

55 F. Deinhardt, V. Berghs, G. Henle and W. Henle, 'Studies on Persistent Infections of Tissue Cultures: III. Some Quantitative Aspects of Host Cell-Virus Interactions'. J. Exptl. Med., 108 (1958), 573-89; interviews with Monto Ho, Ion Gresser, Norman Finter, and Kari Cantell. 
not the virus inhibitory activity of VIF would disappear. For example, VIF preparations were centrifuged at a certain speed that was known to separate fluid and virus particles. After removing the 'virus sediment' the presumably virus-free supernatant fluid was tested for its virus inhibitory capacity (result: the supernatant fluid still showed a considerable virus inhibitory activity). In addition to the centrifugation experiment they tried to figure out-like Isaacs and Lindenmann had done before-whether treating the virus specimen with a specific viral antiserum would affect the viral inhibitory activity of their VIF preparations (result: it did not affect the virus inhibitory activity). Furthermore Ho tested the effect of VIF preparations against a number of different viruses, among others vaccinia virus (result: in all cases under investigation a considerable reduction in cytopathogenicity could be monitored) and subjected to various physical manipulations (result: for example heating at $56^{\circ} \mathrm{C}$ resulted in partial loss of inhibitory capacity).

Moreover, some efforts were devoted to studying the mechanism whereby the inhibition was brought about. The first data indicated that VIF neither acted directly on the virus nor interfered with absorption of virus particles on cells, but presumably affected virus multiplication within the cells. These kinds of trials were run primarily by Ho over a period of eight months, thereby forging a primary profile of the elusive factor which he and Enders thought to be present in their experimental system. ${ }^{56}$

Since there were still many questions concerning the nature of the factor which might be responsible for the inhibitory effects, Enders was in favor of maintaining the term 'viral inhibitory fluid' in their first joint publication which was submitted to the Proceedings of the National Academy of Science on 7 January, 1959..$^{57}$ In this article they claimed to have discovered that fluids from certain human cell cultures, which were infected with a strain of poliovirus, inhibited the cytopathogenic effect and probably multiplication of several viruses. This property was associated with the presence of a factor that could be separated from the virus and was not inactivated by specific viral antiserum. In addition, the following reference was made to interferon: 'In certain respects this inhibitor is comparable to 'interferon', a factor appearing in chick embryo tissues exposed to influenza virus. ${ }^{58}$ However, no reference whatsoever was made to the phenomenon of interference, nor was there any further speculation as to the nature of their factor.

The rather short reference to interferon in the aforementioned publication did not prevent $\mathrm{Ho}$ and Enders from having frequent discussions in Enders's room about the growing

56

M. Ho and J. Enders, 'An Inhibitor of Viral Activity Appearing in Infected Cell Cultures', Proc. N. A. S., 45 (1959), 385-9.

57

Ibid.

M. Ho and J. Enders, 'An Inhibitor of Viral Activity Appearing in Infected Cell Cultures', Proc. N. A. S., 45 (1959), 385-9, p.389. 
list of similarities between VIF and interferon, and from speculating once and again on the nature of the relationship between VIF and interferon. It became a matter of sorting out differences and similarities. With a steady growth in the number of perceived similarities between VIF and interferon, the novelty of VIF was clearly challenged. The issue began gradually to stalemate research on VIF.

At the end of May 1959, Enders decided that in order to put an end to all guesswork it would be best to ask Isaacs to send them a sample of interferon in order to test its effects in his Boston laboratory. Enders's decision to ask Isaacs to ship off tubes with interferon in order to arrange for a 'real-time and real-space meeting' of VIF and interferon-in other words, for an experiment to compare the workings of both inhibitory factors in his Boston laboratory - was a final attempt at clearing up the rather confusing and uncertain laboratory situation by practical means.

According to Ho he was more than relieved to find that the specimens of interferon, which Isaacs had arranged for them to receive by air in a special paper bag container, showed no effects at all in Ho's 'VIF-system'. After all, more than a year of manipulating and producing at the bench had made Ho develop a major commitment to his VIF studies. Ho considered the test pivotal in the sense that it reestablished his, and to a lesser extent Enders's belief that they were dealing with something different and novel. ${ }^{59}$ It is questionable whether Ho thought of the test in these terms, but I would argue that at least to Ho the testing of Isaacs's interferon alongside VIF was a crucial event. Before that VIFs status as a novel laboratory factor had been in jeopardy. After the experimental event, with Isaacs's interferon having shown no activity whatsoever in Ho's hands, a difference between VIF and interferon seemed self-evident to Ho, thereby reinforcing his commitment to VIF.

Further research on VIF was discussed at great length in a second article, which was accepted for publication in Virology about half a year later. As in the case of interferon, material conditions and the emerging scientific object, VIF, are strongly interwoven:

The factor (or factors) responsible for this inhibition is inactivated by treatment with trypsin and is not dialysable. It cannot be neutralized with type 2 poliovirus antiserum nor sedimented with infectious virus. Attempts to identify it either with ribonucleic acid or ribonuclease have failed... ${ }^{*}$

Another striking feature is the use of the term 'VIF, which is far from consistent. Despite the statement of the authors that 'VIF' stands for 'viral inhibitory fluid', more than once VIF seems to connote viral inhibitory factor (Ho's favorite translation), as in the following case: '...may

\footnotetext{
59 Interview with Monto Ho.
}

60 M. Ho and J. Enders, 'Further Studies on an Inhibitor of Viral Activity Appearing in Infected Cell Cultures and Its Role in Chronic Viral Infections', Virology, 9 (1959), 446-77, p. 446. 
invoke an inhibitory factor analogous to $\mathrm{VIF}^{1}{ }^{61}$ In addition, it is interesting to see that on this occasion VIF and interferon are compared quite extensively:

\begin{abstract}
The chemical and physical properties of the two substances [interferon and VIF], as far as these have been determined, are similar. On the other hand, certain differences have been defined: (1) VIF is optimally produced with active and interferon with inactive virus.(2) VIF absorbs poorly if at all to cells, whereas interferon is readily bound. (3) VIF acts most effectively if it is continuously present in the systems, whereas interferon after acting on cells to render them resistant may then be removed. (4) Interferon prepared in chick embryo tissues, as tested in our hands, does not inhibit type 1 polio virus infection in amnion cultures. These differences may be resolved in the course of further studies. But at the moment, it appears more probable that there may exist several different substances inhibitory to viral infections that are incidental products of viruscell interactions. Such substances may not be identical in chemical composition and may process varying degrees of biological specificity. ${ }^{2}$
\end{abstract}

In retrospect Ho explained that as Isaacs's interferon preparations had not produced any effects in his experimental system he then strongly believed that VIF and interferon were neither biologically nor chemically the same. Of course there were striking similarities, and some sort of relationship was to be expected, but in essence he considered VIF and interferon to be different. ${ }^{63}$

It is worth noting that in the very same article Ho and Enders discussed the possibility of VIF being a product of the virus infected cell. ${ }^{64}$ Instead of provisionally considering their factor as an 'abnormal' viral product, as Isaacs and Lindenmann had done two years earlier in the case of interferon, Ho and Enders considered VIF an 'abnormal' cellular product. In retrospect it looks like this conceptual difference is related to a fundamental difference in the nature of their respective experimental systems. Whereas in Ho's experimental system the host cell figured prominently, in Isaacs's case the host cell played a secondary role. For instance, the starting point of Ho's investigation was to study differences in pathogenic effects on different cell types of a particular virus, while Isaacs's starting point was to study a possible difference in virus structure before and after the induction of viral interference. In Ho's work the phenomenon of viral interference hardly played a role. Furthermore, whereas in Ho's case the host cell was directly involved in the production of experimental data, in Isaacs's system the host cell

61 M. Ho and J. Enders, 'Further Studies on an Inhibitor of Viral Activity Appearing in Infected Cell Cultures and Its Role in Chronic Viral Infections', Virology, 9 (1959), 446-77, p. 475.

62 M. Ho and J. Enders. 'Further Studies on an Inhibitor of Viral Activity Appearing in Infected Cell Cultures and Its Role in Chronic Viral Infections', Virology, 9 (1959), 446-77. pp. 475-6.

63 Interview with Monto Ho,

64 M. Ho and J. Enders, 'Further Studies on an Inhibitor of Viral Activity Appearing in Infected Cell Cultures and Its Role in Chronic Viral Infections'. Virology, 9 (1959), 446-77, pp. 475. 
was only a means to the end of producing data.

\subsubsection{Debating interferon's mode of operation: the specificity effect}

We will proceed by concentrating again on the events and occurrences related to 'Isaacs's interferon kitchen'. At about the time Ho and Enders submitted their second article to Virology, Henle sent personal word across the Atlantic to Isaacs about the characterization of what seemed to be an interferon-like factor. Apparently, the same kind of experimental system which had previously resisted all attempts to detect interferon-like substances and had fueled Henle's scepticism regarding interferon and VIF, was now said to produce signs indicating that an observed resistance against viruses was most likely due to the presence of an interferon. It would have been interesting to know more about the sequence of events in Henle's laboratory that tipped the balance in favor of interferon. This was not of great concern to Isaacs, however, who seemed to be more interested in the bare fact that his ideas about the existence of a viral interfering substance were gaining some ground among American animal virologists. $^{65}$

As I already mentioned, Isaacs was informed about Ho and Enders's research on VIF. However, in contrast to the research team in Boston, the association of VIF with interferon encouraged rather than discouraged Isaacs in his research work. In Isaacs's case, the perceived similarities between interferon and VIF were important. These were considered instrumental in putting an end to all doubts with regard to the isolated character of the interferon phenomenon. From the very first moment that Isaacs had heard from Enders, Isaacs believed VIF to be interferon produced by polio virus in a culture of human amnion cells. At once the possibility of producing and manipulating an interferon in any laboratory no longer seemed purely speculative. Neither Ho's failure to produce an effect with Isaacs's interferon specimens, nor Isaacs's failure to prepare interferon in cultures of human amnion cells, could reverse the situation. Isaacs also knew that it was possible to produce interferon in in vitro systems other than the original chick embryo system, as he and Burke had succeeded in producing interferon in cultures of monkey kidney cells. ${ }^{66}$ Concurrent events in the laboratory of his friend and colleague David Tyrrell, who worked as an animal virologist at the Common Cold

65 Alick Isaacs to Jean Lindenmann, letters dated 17, 24 July and I September, 1959. Jean Lindenmann, Correspondence, Personal Archives; and interview with Derek Burke..

66 D. Burke and A. Isaacs, 'Some Factors Affecting the Production of Interferon', Brit. J. exp. Path., 39 (1958). 452-8, p. 456. 

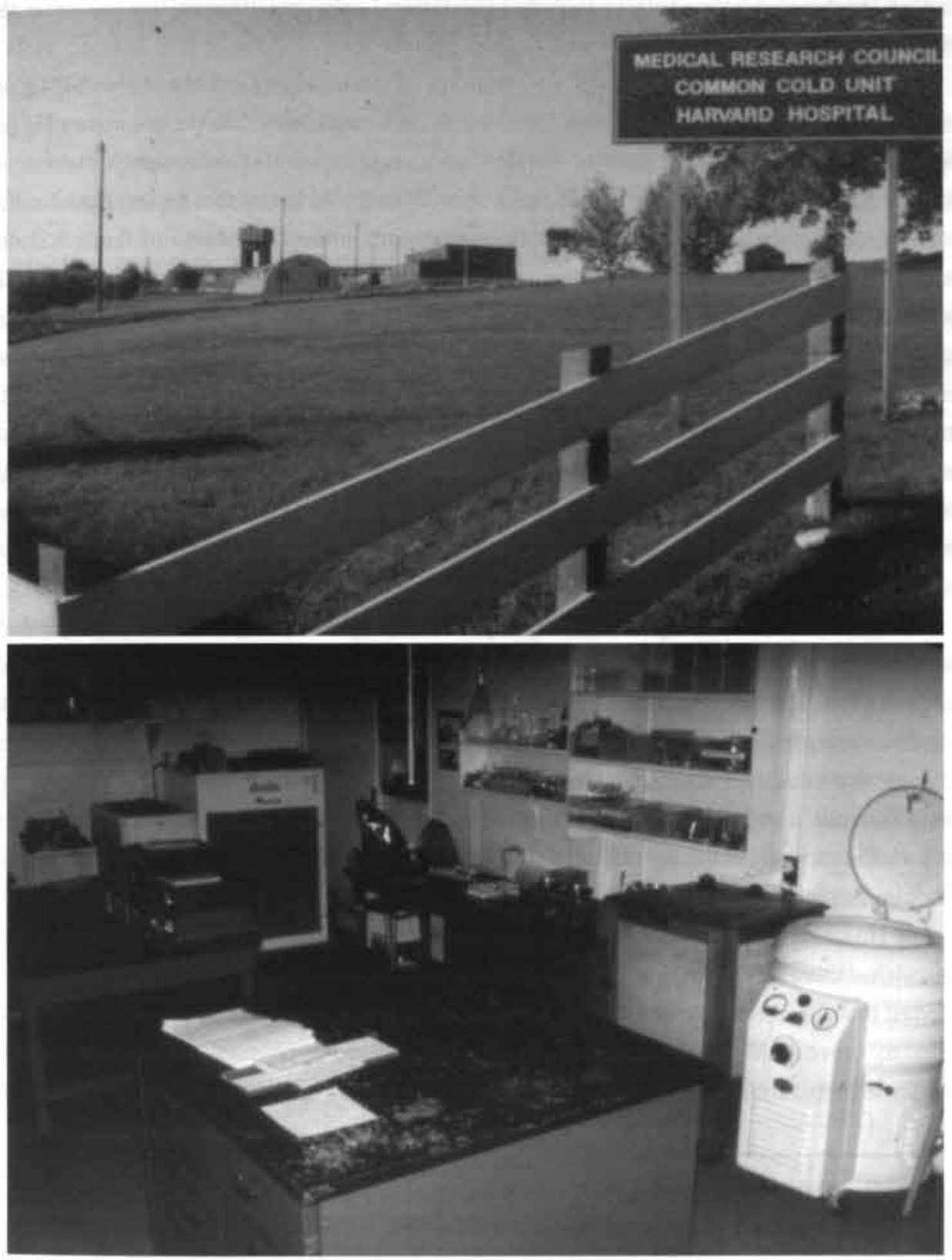

Fig. 28a,b. The Common Cold Unit was housed in a former American army field hospital. Tyrrell's laboratory was located in one of the central barracks. Courtesy of D. Tyrrell 
Unit on the downs to the south of the city of Salisbury, only strengthened Isaacs's belief that VIF was an interferon.

The Common Cold Unit was an extension of Andrewes's Division at the NIMR and was regarded as a sort of field station for research on viruses related to the common cold (see Fig 28a). ${ }^{67}$ Tyrrell would go over to London quite regularly to discuss research matters and visit the NIMR's library. During one of these visits Tyrrell told Isaacs that he had found indications suggesting the presence of an inhibitory factor resembling interferon in fluids collected from monolayer cultures of calf kidney cells which were infected with virus-a so-called 'Sendai' virus strain of the subgroup of Parainfluenza viruses. ${ }^{68}$ Tyrrell emphasized the preliminary status of his observations. He had only just started physical and chemical tests to characterize his virus interfering factor and, moreover, his experimental system was different from the chick embryo membrane system Isaacs used to produce interferon. Tyrrell agreed with Isaacs that it would be an interesting option to take a frozen sample of Isaacs's interferon to the Common Cold Unit and test its effects on his Sendai virus/calf kidney cell system. Communicating the interferon-like experimental features to Isaacs thus led to the transfer of interferon related expertise and research materials from Isaacs's to Tyrrell's laboratory (see Fig. 28b).

Back in his laboratory in Salisbury, Tyrrell then started an experiment to see if Isaacs's interferon preparation would induce the same effects as his own viral interfering fluid. Surprisingly it did not. The tube cultures of calf kidney cells which were treated with the interferon sample showed an almost normal level of cytopathogenicity-that was regarded as a measure for virus reproduction-after the introduction of Sendai virus. Conversely Tyrrell could measure a reduction in cytopathogenicity in cultures which had been treated with his own viral interfering fluid. Tyrrell did not know what to make of the absence of an inhibitory effect in the case of interferon. He immediately initiated another attempt.

However, the second set of data were almost identical. Since Tyrrell had heard from Isaacs that there were frequent problems with the stability of the interferon preparations, he inferred that the interferon sample most likely had lost its virus inhibitory activity over time. In order to prevent this from happening again he considered it necessary to work with fresh interferon preparations in future experiments. The logistics involved in getting a regular supply

The Common Cold Unit was set up after WO II in the buildings of the former American Red Cross Hospital on the initiative of Andrewes and with support of the MRC and Ministry of Health as a centre for systematic studies on the aetiology and spread of common cold inducing viruses such as the rhino and influenza viruses. See, D. Tyrrell, The Common Cold Unit 1946-1990: Farewell to a Much-Loved British Institution', PHLS Microbiology Digest, 6 (1991), 74-6.

Parainfluenza viruses were considered a subgroup of the Influenza viruses. These in tum were and are still counted as part of the Myxoviruses. Contrary to the influenza viruses the Parainfluenza subgroup did not appear to grow well in the chick embryo. The Sendai virus strain was hold responsible for inducing pneumonia in infants; T. M. Bell, An Introduction to General Virology (London. William Heinemann Medical Books, 1965) pp. 129-43. 
of fresh interferon preparations from Isaacs's laboratory would be quite demanding. Tyrrell therefore decided first to see if by reproducing Isaacs's interferon system it would be possible to prepare interferon in his own laboratory.

Tyrrell was quite confident that he would succeed in his efforts, as Isaacs's experimental system for the greater part resembled the in vitro system Tyrrell had used to study viral interference between influenza viruses as a postdoc at the Rockefeller Institute for Medical Research in New York some years before. Furthermore, on a number of occasions, he had closely watched Isaacs manipulating his interferon system. It took him some time to figure things out, but eventually he was able to prepare a batch of interferon by inoculating tubes containing pieces of chicken embryo membrane and nutrient fluid with inactivated influenza virus.

Remarkably however, his 'fresh' interferon preparation, which clearly showed a virus inhibitory effect in the chicken embryo membrane system, had hardly any antiviral activity when applied to the calf kidney cell system. Likewise the viral inhibitory fluid which had been collected from the calf kidney cell system was much more active in this system than in the chicken embryo membrane system. Yet there did not appear to be a difference in physical and chemical properties between the two preparations. It was a puzzling result. ${ }^{69}$

Tyrrell believed that in both cases he was dealing with more or less the same chemical substance, 'interferon', but there seemed to be a difference in biological activity. According to Tyrrell, the only thing he could think of at that time was that some sort of cellular specificity effect might be involved. ${ }^{70}$ In response to virus infection both his calf cell and chick membrane system produced interferon. However, in one way or another, in both cases, the interferon shared specific features with the cell type from which it was formed, such that the chick cell interferon was less active in calf cells and the same was true for calf cell interferon in chick cells. $^{71}$

Sometime early in 1959, Tyrrell talked about his findings with Isaacs and Burke. The first reaction was one of disbelief:

Well it had never occurred to us at all that interferon might show host cell specificity. Viruses don't, antibo-

D. Tyrrell, 'Interferon Produced by Cultures of Calf Kidney Cells', Nature, 184 (1959). 452-3, p. 453; and interviews with David Tyrrell. James Porterfield, and Derek Burke.

70 Robert Wagner is definitely wrong when he retrospectively claims that the animal species specificity of interferon action was 'discovered' in his laboratory. He confuses 'discovering' with re-examining the hypothesis of species specificity as stated in his 1961 article in Virology; See, R.R. Wagner, 'Biological Studies of Interferon', Virology, 13 (1961), 323-37, p. 334; and. R.R. Wagner. 'Reminiscences of a virologist wandering in Serendip', Arch. Virol., 141 (1996), 787-97.

D. Tyrrell, "Interferon Produced by Cultures of Calf Kidney cells," Nature, 184 (1959), 452-3: interview David Tyrrell, and John Porterfield. 
However, they both knew Tyrrell as a careful experimenter, and the data seemed to be quite clear. It was Isaacs who first acted on Tyrrell's idea that interferon might share specific features with the cell type from which it was formed. Tyrrell's experimental results seemed to offer a possible explanation for the inconsistencies Isaacs had experienced in testing the effect of both interferon and inactivated influenza virus on laboratory rabbits more than a year ago. At that time Isaacs had been surprised to see that inactivated influenza virus regularly protected rabbits against infection of the skin with vaccinia virus whereas rather variable results were obtained with interferon. This was thought to be inconsistent with his idea that the protective effect of inactivated influenza virus was mediated through interferon. Now, with Tyrrell's experimental results at hand, the rabbit experiments no longer seemed problematic to Isaacs. The perceived inconsistency could be explained away. The most likely conclusion was that, following injection with inactivated influenza virus into the skin of laboratory rabbits, interferon was locally produced. Upon taking into account a cellular specificity effect it was only to be expected that this rabbit-skin interferon would protect rabbits more effectively against infection with vaccinia virus than was the case with rabbits that had received an injection with chick interferon. Isaacs, Tyrrell and Burke agreed that it would be worthwhile to examine this cellular specificity effect more closely.

By bringing interferon into his laboratory and gradually incorporating it into one of his own experimental systems Tyrrell created a new situation: the primarily one-way traffic between Isaacs's and Tyrrell's laboratory became two-way traffic. Basically it meant that in manipulating and producing at his laboratory bench in Salisbury Tyrrell managed to change the order of events in Isaacs's 'interferon kitchen'. Tyrrell, firstly, failed to produce any effects with Isaacs's interferon, which was ascribed to the instability of the interferon probe; secondly, he started reproducing the material realization of Isaacs's interferon system; and thirdly, Tyrrell brought this reproduced system face to face with his own original Sendai virus/calf kidney cell system. Tyrrell's claim that most likely a host cell specificity was involved in the action of interferon not only made Isaacs rethink and resume his earlier rabbit experiments, but was also instrumental in reshaping Isaacs's interferon system as a whole: virus-oriented procedures and models were modified to make the experimental system fit a more cell-centered research perspective.

With regard to Isaacs's laboratory the specificity issue had both practical and theoretical consequences, and resulted in a rather substantial reshaping of research on interferon. The production of interferon as a 'blind alley' of virus production no longer seemed to make sense, once the specificity effect had been confirmed in Isaacs's laboratory. The host

\footnotetext{
72 Interview with Derek Burke.
} 
specificity was thought to indicate differences between interferons from cells of different animal species. One and the same virus could induce different interferons depending on the type of host cell, and this simply did not seem to fit the idea of interferon as an abortive product of virus multiplication. Apparently the process of virus multiplication and the mode of production of interferon had to be independent. Isaacs began to associate the production of interferon with a cellular defense mechanism against viruses. According to Burke, it was no longer the virus, but the cell, that was at the center of their thoughts. ${ }^{73}$ Isaacs confirmed this at a symposium on virus diseases in September 1959 in London, where he proposed the following line of thought with regard to the nature of the interferon phenomenon:

It may be that interferon is concerned in a natural mechanism of cellular resistance to virus infections. For example, the fact that people recover from colds in the absence of any clear evidence of specific immunity [resisting infection by the presence of antibodies in the blood] suggests that local cellular immunity may play a part. ${ }^{74}$

From this point of view interferon was considered to be a product of the cell, which might be involved in the natural defense of cells against viruses.

Obviously the virus was losing ground to the cell. This transformation of ideas concerning the nature and mechanism of action of interferon coincided with other changes in the experimental system. Basically, virus-oriented procedures were modified to make the experimental system fit a more cell-centered research perspective. In studying the mechanism of interferon's activity, attention was now focused on cellular metabolism, as such, rather than on viral multiplication and viral interference. It meant doing different experiments. The emphasis shifted gradually from experimental virology to a kind of cellular biochemistry, which Lindenmann dubbed jokingly 'cytochemistry'. ${ }^{75}$ Isaacs moved to the molecular level of analysis only when he needed to translate his experimental work into terms of role and function of interferon in the cell when it was feasible to do so. As a biological phenomenologist-showing a preference for studying and analyzing viral behavior-Isaacs was not interested in biochemistry and a molecular interpretation of virus-cell interaction per se. Nor was he very familiar with the subject matter. However, only a basic kind of biochemistry was needed and Isaacs could easily overcome his 'handicap' by asking a biochemically trained scientist outside the

Interview with Derek Burke.

74 A. Isaacs, 'Interferon: The Prospects', The Practitioner, 183 (1959), 601-5, p. 605.

75

Jean Lindenmann to Alick Isaacs, letter dated 2 December, 1959, Jean Lindenmann Correspondence, personal archives. 
department for assistance. ${ }^{76}$

As the viral point of view gave way to a cellular research perspective, there was a shift in emphasis from experimental virology to a more interdisciplinary, molecular biology type of research. It meant employing whatever tools and techniques the problem at hand seemed to demand to explain interferon's role as part of the cellular defense mechanism in terms of fundamental biochemical and molecular processes. Changes in the experimental system and research perspective not only meant doing different experiments but also reshaping interferon's identity as a research object. The ideas regarding interferon as a deviant product of the cell closely associated with virus multiplication were abandoned in favor of interferon as an essential product of the process of cellular resistance to viral infections. The events in Ho and Enders's and Tyrrell's laboratories played an active part in this transformation process, as did the practical context in which knowledge of interferon was claimed by Isaacs to be applicable to the problem of antiviral therapy. In other words, work on interferon no longer meant work on Isaacs's experimental system only-meeting the 'others' resulted in 'working forward' with the 'others'.

\subsection{Researching interferon(s).}

In the rest of this chapter I shall describe how interferon emerged as a central concept to a network of laboratory practices within the field of animal virology. These practices were particular and local in nature, each with its own ways of doing things and of presenting the manipulations at the laboratory bench. Through the interaction of their activities, the heterogeneous material and conceptual resources of these particular practices were fine-tuned, leading to new ways of doing and representing things, independent of the immediate local experimental situations. We will see that out of this adaptive behavior a new subfield of research emerged in which a group of experimentalists learned to manipulate, communicate about and work on a common set of research problems and laboratory phenomena.

\subsubsection{What's in a name?}

In the fall of 1959 additional foreign reports reached Isaacs's desk concerning work on substances which seemed to have properties similar to interferon. Among these was a French paper by two Japanese animal virologists, Yasuiti Nagano and Yasuhiko Kojima from the

76 A. Isaacs, 'Metabolic Effects of Interferon on Chick Fibroblasts', Virology (letter to the editor), 10 (1960), 144-5; and interview with Joseph Sonnabend. 
Kitasato Institute in Tokyo. In their article they described the production by vaccinia virus of a virus-inhibitory factor, named 'facteur inhibiteur', which was separable from the virus and was able to inhibit vaccinia virus infection in the rabbit skin. Their factor was said to be relatively heat resistant, unlike vaccinia virus not sedimented by centrifugation at $100.000 \mathrm{~g}$ for two hours and resistant against ultraviolet radiation. However, no further pronouncements were made as to the nature of their factor. ${ }^{77}$ Upon reading the article Isaacs became convinced that the Japanese had been working with interferon. 'I have just seen a paper by two Japanese... who have found interferon in vaccinia virus but don't know it'. ${ }^{78}$

Isaacs then wrote a letter to Nagano to communicate his findings. Nagano answered by return post that this 'facteur inhibiteur' was indeed very much like interferon, and included a 1954 article indicating that they had already pointed in their experimental system to the presence of a virus-inhibiting factor, years before Isaacs and Lindenmann published their interferon data. Since Isaacs thought Nagano's 1954 article even less clear regarding the nature and effect of this factor, he did not pay much attention to Nagano's remark about the early date of his research. For the moment he let things go at that and instead focused on yet another American report concerning an interferon-like substance. ${ }^{79}$

Isaacs had received information through Andrewes that an American colleague of theirs at John Hopkins Medical School, Robert Wagner, who in the early fifties had worked as a research fellow in Andrewes's department for one year, had an interferon-like substance under investigation in his virus laboratory. Apparently the substance was referred to as 'interferon B' and was said to be distinct from Isaacs's interferon. As with Nagano, Isaacs immediately got in touch with Wagner. ${ }^{80}$

He learned that Wagner had become seriously interested in the interferon phenomenon when he repeatedly managed to induce an inhibitory effect on the growth in chick cell cultures of a highly pathogenic virus, a so-called Eastern Equine Encephalitis (EEE) virus under investigation in his laboratory. ${ }^{81}$ Like interferon, Wagner's inhibitory factor appeared to be

77 Y. Nagano and Y. Kojima, 'Inhibition de L'infection Vaccinale par un Facteur Liquide dans le Tissu Infecté par le Virus Homologue', Compt. Rend. Soc. Biol. Filiales, 152 (1958), 1627-29.

78 Alick Isaacs to Jean Lindenmann, letter dated 10 September, 1959. Jean Lindenmann Correspondence. personal archives.

79. Minutes of the third meeting of the Scientific Committee on interferon, dated 26 October, 1959, MRC Archives File No. $788 / 2 / 1$.

80 Unfortunately I was not given access to Wagner's correspondence and consequently the following section is a reconstruction largely based on: interviews with Roben Wagner. Derek Burke and Norman Finter, Minutes of the third meeting of the Scientific committee on interferon, dated 26 October, 1959, MRC Archives File No. 788/2/1; and R.R. Wagner, 'Viral Interference', Bacteriol. Rev., 24 (1960), 151-66, pp. 157-61.

81 Interview with Robert Wagner. 
susceptible to treatment with protein degrading enzyme (result: its biological activity was destroyed) and its biological activity could be precipitated by ammonium sulphate. However, the data indicated that Wagner's preparation was more stable in relation to heat than the interferon preparation described by Isaacs. Wagner's factor appeared to be completely stable at $70^{\circ} \mathrm{c}$ for one hour, whereas in the case of interferon marked inactivation took place after 1 hour at $60^{\circ} \mathrm{c}$. However, Wagner's factor seemed to be less stable in strong acid solution: stable at $\mathrm{pH}$ 3-11, as compared to 1-11 in Isaacs's case. As in the case of Enders and Ho, Wagner let the differences outweigh the similarities. Because of these differences to Isaacs's interferon, Wagner called his virus inhibitory factor 'interferon B'.

In response to Wagner's claims regarding 'interferon B', Isaacs argued that the differences between 'interferon' and 'interferon B' did not seem to be significant. Most likely the difference in heat stability could easily be reduced to a difference in $\mathrm{pH}$ of their respective preparations. With regard to the $\mathrm{pH}$-stability Isaacs pointed out that in accordance with Wagner's data they too had noted some loss in activity over the $\mathrm{pH}$-range from 1 to 3 , but regardless of this decrease in activity they had decided in favor of the stability range 1 to 11 . If one took into account the fact that most biological substances were completely destroyed between $\mathrm{pH} 1$ and 3 , it seemed justifiable to say that interferon was rather stable over this range. In addition Isaacs pointed out that Wagner's experimental system seemed to differ only in its use of the whole chicken egg as a means to produce preparations of interferon instead of the chicken embryo membrane system employed in Isaacs's laboratory. Isaacs therefore thought it highly unlikely that they were dealing with different substances.

On his part, Wagner, who had digested Isaacs's remarks, admitted that the decision to use the name 'interferon B' was based on a comparison of their experimental data with mostly preliminary information on the physico-chemical properties of interferon, which had been available to him at the time. However, upon assessing Isaacs's latest information he saw no further need to make a difference between 'interferon' and 'interferon B', and decided to withdraw the latter term. According to Wagner this decision was not based on any tests with Isaacs's interferon in his laboratory at John Hopkins. It should be emphasized that Wagner's 'conversion to interferon' does not necessarily mean that Isaacs and Wagner both identified interferon-B as interferon in a similar way. At least they agreed on identifying interferon B with interferon biologically, but it is far from clear if Wagner shared Isaacs's belief that both were chemically identical too.

In the process of discussing the nature of their respective virus-inhibiting factors both researchers informally agreed on a provisional set of experimental criteria as to what laboratory material they both would call an interferon. The production of this set of criteria added up to listing the biological, chemical and physical properties the individual research objects had in common, and choosing from this list the most exceptional ones-exceptional in the sense that it would distinguish their factor from other known biological factors. For 
instance, one of the informal criteria was $\mathrm{pH}$-resistance as both factors shared a resistance to acid conditions. 'Fairly early on we had criteria among ourselves as to what we would call an interferon. One of the very important things was pH-resistance. There are not too many proteins with that property. ${ }^{82}$

In the meantime, word got around within the animal virology community that an increasing number of laboratories were reporting work on interferon-like factors. Even fierce critics like the American virologist Harry Rubin began to pay some attention to these 'interferon stories', although the issue still remained controversial. The published experimental data were still considered circumstantial, as none of the laboratories involved could present more than biological activities and a limited number of physico-chemical properties. For instance, the claim that one was dealing with a protein was based on little more than experiments showing that the virus inhibitory activity of preparations was destroyed by trypsin, which was known to inactivate proteins, and precipitated by saturation with ammoniumsulphate like most other proteins. Was it really a protein though? One of the things that disturbed people was that hardly any other proteins were known to resist both heat and acid conditions in a similar way. Moreover, assuming that it was a protein, some wondered how you could know that it was a cellular protein and not a viral protein? Of course, different viruses reportedly induced the same kind of inhibitory activity in one particular type of cells, whereas one and the same virus could induce different kinds of interfering principles in cells from different animal species. But what other proof was there to say that one was actually dealing with a primary product of the cell? Repeatedly, researchers like Ho, Enders and Wagner had to respond to such questions from their fellow scientists. ${ }^{83}$

While attending a meeting of the American Cancer Society in New York in November 1959. Andrewes noticed the growing interest in interferon. He was even approached by one of the members of the Program Committee of the prestigious Gustav Stern Symposium on Perspectives in Virology to be held early January 1960, with a last minute request to present a paper on interferon. On his return to Mill Hill, it was agreed that Isaacs would deliver a paper with his latest data and ideas regarding the nature and function of interferon. ${ }^{84}$ The latter immediately dropped Lindenmann a note of the news:

I am going to America early in January to speak at a meeting on Perspectives in Virology and will take the opportunity to meet all the interferonologists there, viz. Schlesinger, Henle, Wagner and the senior interfero-

82 Interview with Robert Wagner.

83 R.R. Wagner and A. Levy, 'Interferon as a Chemical Intermediary in Viral Interference', Ann. N.Y. Acad. Sci. 88 (1960), 1308-18, p. 1310: and interviews with Robert Wagner, Sam Baron. Monto Ho, and Hilton Levy.

84 Minutes of the fourth meeting of the Scientific Committee on interferon, dated 26 November, 1959, MRC Archives File No. 788/2/1. 
In response, Lindenmann, who was aware of the growing number of claims regarding interferon-like substances, congratulated Isaacs on his success, but at the same time expressed his concerns about a possible confusion in the field. He thought it important to try to give at least some direction as to what antiviral factors should be called interferon:

It is probably premature to undertake this, but on the other hand there is the danger that as more workers pop into the field different names might be proposed for the same thing. It would be a good policy to reach a provisional agreement as to what points are to be considered relevant for inclusion of a new antiviral factor in the interferon group. ${ }^{\mathrm{s6}}$

Isaacs, for his part, thought that Lindenmann went a bit too far in his suggestions, and told him that they could not stop 'other people from describing whatever they like and calling it whatever they like. ${ }^{187}$

Isaacs was in favor of a more subtle approach to the problem. He believed that it might be possible to prevent people from using different names for substances similar to interferon in the future by adopting a fairly broad description of interferon. To persuade other researchers to adopt the term 'interferon' for biological factors similar to interferon, Isaacs proposed to use the following 'tailor-made' definition at the 'Perspectives in Virology' meeting in January 1960:

\begin{abstract}
A protein, slightly smaller than antibody globulin, produced by cells of different animal species following inoculation with inactivated or live virus of many different kinds and capable of inhibiting intracellularly the growth of a variety of viruses in cells of the same animal species, in doses which are not obviously toxic for cells. ${ }^{.8}$
\end{abstract}

Isaacs told Lindenmann that he did not think it easy for them to be dogmatic in saying that a substance with these properties should be called an interferon. "It is usually much better to let someone go his own course and see what happens in time...I have already transformed Wagner

85 Alick Isaacs to Jean Lindenmann, letter dated 25 November, 1959, Jean Lindenmann Correspondence. personal archives.

86 Jean Lindenmann to Alick Isaacs, letter dated 2 December 1959, Jean Lindenmann Correspondence, personal archives.

87 Alick Isaacs to Jean Lindenmann, letter dated 4 December, 1959, Jean Lindenmann Correspondence, personal archives.

88

Ibid. 
and hope to work on Enders in January". ${ }^{89}$ Isaacs's strategy appeared to be successful. At the Perspectives in Virology meeting, Enders, who acted as chairman of Isaacs's session, made a public statement, saying in effect that VIF might be considered an interferon:

\begin{abstract}
The more recent data that Dr. Isaacs and his colleagues have assembled, together with those of others, interest me because we have been working with an analogous factor, which we have called viral inhibitory factor (VIF). We have noted certain differences between interferon and the principle we have dealt with, but these more recent observations have, I think, explained most if not all these differences, and I now believe that we are all dealing with fundamentally similar, closely related factors. ${ }^{00}$
\end{abstract}

Enders made his announcement at the end of the discussion following Isaacs's paper presentation on the nature and function of interferon. In adopting the term 'interferon' for his and Ho's viral inhibitory factor, Enders, like Wagner before him, seemed to agree with Isaacs on a basic set of experimental criteria as to what kind of biological factors would be qualified to bear the name interferon. Enders's formal announcement had an impact among those present and was instrumental in gradually attuning the research activities of a growing number of laboratories in America, Britain, Japan and on the Continent, to one another.

\title{
3.3.2 The blossoming of a new subfield
}

By common usage, the term 'interferon' came to refer to the active antiviral substance(s) in particular culture fluids. When used in journal articles, the word 'interferon' was considered a generic term for all laboratory factors which complied with a specific set of experimental criteria. ${ }^{97}$ These criteria had been shaped and reshaped and modifications were constantly being made. Since a generally accepted definition and categorization of interferons was not yet available, these 'interferon criteria' functioned as a means of communication for investigators, who each used their favorite experimental system. The range of these various virus-cell systems was wide. This shared set of experimental criteria was formulated as follows by

Alick Isaacs to Jean Lindenmann, letter dated 4 December, 1959, Jean Lindenmann Correspondence. personal archives.

Alick Isaacs, 'Nature and function of interferon'. in Perspectives in Virology. ed. Morris Pollard, (Minneapolis: Burgess Publishing, 1960) p. 123.

91 M. Ho, 'Interferons', New Engl. J. Med., 266 (1962), 1258-64; A. Isaacs, 'Interferon', Advance. Virus Res, 10 (1963). 1-39; and R.R. Wagner, 'The Interferons: Cellular Inhibitors of Viral Infection', Ann. Rev. Microbiol., 17 (1963), 285-94. 


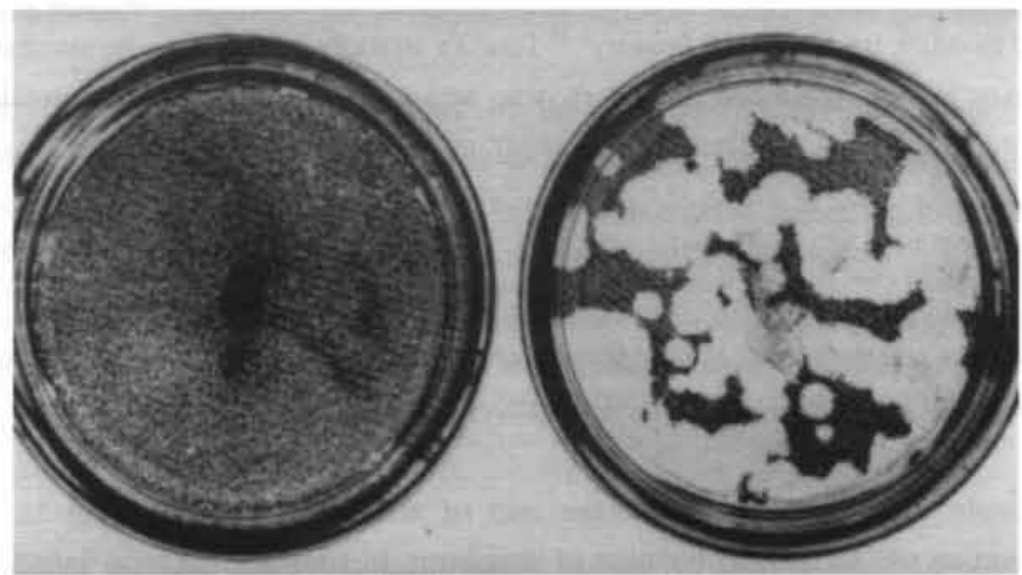

Fig. 29. Interferon plaque assay.: The two Petri dishes have been seeded with mouse cells and infected with sufficient influenza virus to destroy all cells.

The left-hand plate contained interferon and shows no visible destruction of the cells (live cells show up dark). The right hand plate contained no interferon and shows large zones of dead cells (show up light). Courtesy of R. Wagner.

Robert Wagner in one of the first review articles on what he called 'interferons' or 'cellular inhibitors of viral infection':

The first criterion is production in cell cultures or host tissues in response to infection with active or inactivated viruses...To qualify as an interferon, a culture fluid or cell extract must be capable, on transfer to test cultures of inhibiting the multiplication or cytopathic effect of a challenge virus. All current bioassay methods are based on this principle...Additional criteria for identifying interferons are based on physicochemical properties. Interferons are not sedimented by centrifugation at $100,000 \mathrm{~g}$ for $4 \mathrm{hrs}$, resist prolonged exposure to $\mathrm{pH} 2$ at $4^{\circ} \mathrm{C} . .^{22}$

These shared criteria added up to a set of identity conditions, with which research objects had to comply in order to deserve the label 'interferon'. The bioassay played a central role in shaping these criteria, which investigators used to qualify a certain culture fluid or cell extract as an interferon preparation.

Quite different ways of assaying these biological substances were employed. The process of tuning in the activities of the various laboratory practices to one another and rendering them compatible resulted in the use of similar types of bioassays based on the so-

92 R.R. Wagner, The Interferons: Cellular Inhibitors of Viral Infection', Ann. Rev. Microbiol., 17 (1963), p. $285-6$. 
called 'plaque inhibition' method (see Fig 29).${ }^{93}$ However, this was far from a straightforward process. Isaacs's attitude is exemplary in this respect. Despite his knowledge that the plaque assay technique was most likely a superior technique, Isaacs demonstrated a reluctance toward change. He had worked for so long with the haemagglutination assay, that he was rather hesitant to choose in favor of a new kind of bioassay. Switching to another assay technique required reorganizing his experimental system, and thereby running the risk of losing the subtle control over his interferon system. Not until Isaacs's restraint toward introduction of a new assay technique threatened to isolate his laboratory practice was he prepared to change course. Far from replacing the haemagglutination assay as a standard tool in Isaacs's laboratory, the plaque assay was employed as an additional laboratory device in his interferon work. ${ }^{94}$

The process of validating interferon and forming it into a basis for consensus involved, besides the recurrent elements of evaluating claims in terms of the perceived research opportunities and of negotiating consensus, a collective process of engineering mutual ways of communicating, manipulating, interpreting and elaborating on a common assembly of research objects. The lead on interferon in the May 1961 issue of the Scientific American symbolized a turnabout with regard to the scientific status of interferon (see Fig. 30)..$^{95}$

The controversy over the significance of the original knowledge claims regarding interferon had died down. This was consonant with a shift in status of scientific statements regarding interferon. Whereas up to 1960 journal articles contained modalities with reference to interferon such as The data presented are too scanty to allow even the crudest guess as to the nature of...' or 'It therefore seems reasonable to postulate that...' or 'It is necessary to state from the outset that the evidence is purely circumstantial...', by 1962 these kind of modalities

93. The 'plaque inhibition' technique had been largely developed by the prominent American animal virologist, Renato Dulbecco, in the early fifties at the California Institute of Technology. It was basically performed as follows. Monolayers of living cells were attached to the surface of petri dishes. Subsequently a normal maintenance medium was mixed with a red stain and a gel forming extract, so-called 'agar', dissolved in water. After inoculation with virus this mixture was then allowed to solidify over the cell sheet. Plates treated in this way were placed in the incubator. The period of incubation varied with the virus used. The red stain was included to distinguish between normal living cells, which were known to acquire a reddish colour through absorbtion of the stain, and dead cells that had lost their colour through rupture of the cell wall. Colourless areas were seen on the plate where cells had been destroyed. As viruses were known to induce cell rupture or lysis after viral infection and multiplication, each of these colourless areas or 'plaques' as they were called was claimed to indicate the presence of virus or to be more specific each plaque was said to represent the production of a single original viral particle and its progeny; R. Dulbecco, Production of Plaques in Monolayer Tissue Cultures by Single Particles of an Animal Virus', Proc. Nat. Acad. Sci. U.S.A., 38 (1952), 747-52. See, for a detailed description of the historical development of the virus plaque assay by Renato Dulbecco, who was a member of Max Delbruick's imminent research group at Caltech: D. J. Kevles, 'Renato Dulbecco and the New Animal Virology: Medicine, Methods, and Molecules', J. Hist. Biol., 26 (1993), 409. 42 .

Interview with Burke. 


\section{SCIENTIFIC AMERICAN}

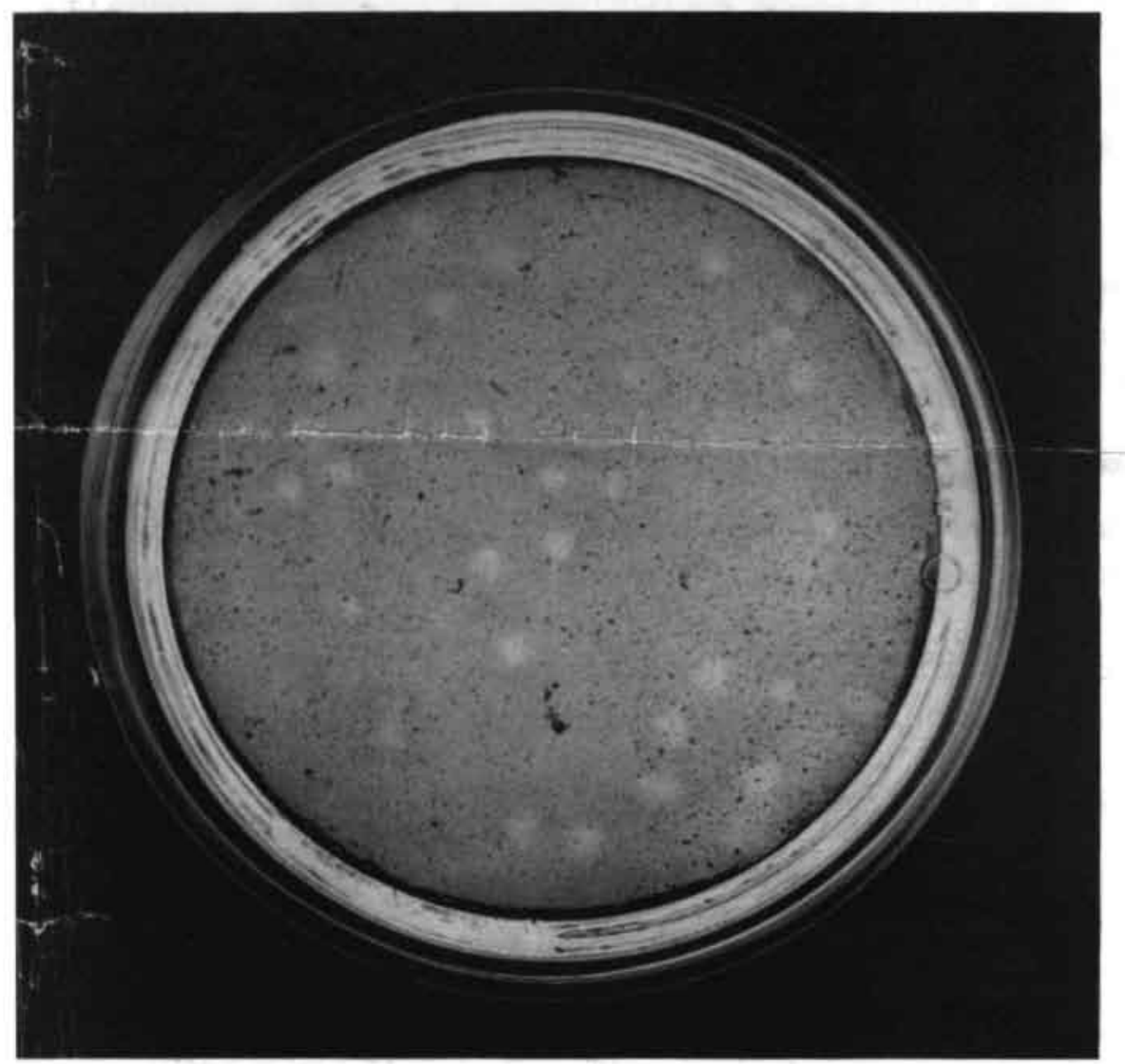

INIIIBITED VIRUSES

FIPTV CEITS

Ollay 1961

Fig. 30, Cover of the Scientific American, May,1961.

Reproduced with kind permission from the publisters. 
were mostly dropped. ${ }^{96}$ Statements such as 'In 1957 Isaacs and Lindenmann observed the formation of a virus interfering substance, to which they gave the name interferon...' or 'The most notable breakthrough in the general area of host resistance to viruses was the discovery in '57 by Isaacs and Lindenmann of a substance called interferon...' became common place. ${ }^{97}$ As such, the provisional state of interferon was transformed into scientific fact. Interferon became widely regarded as a product of the process of cellular/host resistance to viral infection. ${ }^{98}$ At least from a biological point of view, the existence of interferon as a specific biological entity was beyond dispute. However, in chemical terms interferon was still more a putative substance, the active fraction of an impure biological preparation, or what often was dubbed a 'protein soup', which showed some activity in a particular bioassay. ${ }^{99}$ Without a chemical structure, interferon's chemical 'reality' was still up for debate.

In the process, 'misinterpreton' rumors gave way to talking about the pros and cons of joining what some individuals now jokingly called an 'interferon bandwagon'. ${ }^{100}$ The number of laboratories and researchers working on research projects associated with the term 'interferon' was indeed on the rise. However, for assessment of figure 31, which shows the number

A. Isaacs, J. Lindenmann and R. Valentine, 'Virus Interference. II. Some Properties of Interferon', Proc. $R$. Soc, 147 (1957), 268-73; A. Isaacs, 'Viral Interference', Symp. Soc. gen. Microbiol.. No. 9, 102-12I, p. 109; and R.R. Wagner and A. H. Levy, 'Interferon as a Chemical Intermediary in Viral Interference', Ann. N.Y. Acad. Sci. 88 (1960), 1308-18, p. 1308.

97 D. C. Burke, The Purification of Interferon', Biochem. J., 78 (1961), 556-64, p. 556; and M. R. Hilleman, 'Interferon in Prospect and Perspective', J. cell. comp. Physiol., 62 (1963), 337-53, p. 337.

98 In claiming that he and Emanuel Heller simultaneously 'discovered' that interferon was a cellular product and not a product of the virus by showing in 1963 that the antibiotic actinomycin D (a known inhibitor of DNA. directed RNA synthesis of cellular proteins) inhibited the production of interferon, Robert Wagner not only disregards the work of his female fellow-scientist Joyce Taylor, but even more important the lengthy and complex transformation process through which interferon's identity as a cellular inhibitor of viral infection was shaped. Wagner's, Heller's and Taylor's experiments were neither more nor less than constitutive of interferon's genesis as a cellular protein: R.R. Wagner, Reminiscences of a Virologist Wandering in Serendip, Arch. Virol., 141 (1996), 779-788, p. 783; E. Heller, 'Enhancement of Chikungunya Virus Replication and Inhibition of Interferon Production by Actinomycin D', Virology, 21 (1963), 652-56; R.R. Wagner. 'Inhibition of Interferon Biosynthesis by Actinomycin D', Nature, 204 (1964), 49-51; and J. Taylor, 'Inhibition of Interferon Action by Actinomycin', Biochem. Biophys. Res. Comm., 14 (1964), 447-51.

The point of modalities which are constantly added, modified and dropped in the transformation of a provisional claim into a scientific fact was first noticed by Fleck and developed in greater detail by Latour and Woolgar, L. Fleck, Genesis and Development of a Scientific Fact (Chicago: The University of Chicago Press, 1979), p. 118; Latour and Woolgar, Laboratory Life: The Construction of Scientific Facts (Princeton: Princeton University Press, 1986), 151-86. 


\begin{tabular}{|c|c|c|c|c|c|c|c|c|c|c|c|}
\hline $1957-1967$ & 57 & 58 & 59 & 60 & 61 & 62 & 63 & 64 & 65 & 66 & 67 \\
\hline NATURE & & 1 & 2 & 1 & 3 & 2 & 9 & 8 & 7 & 2 & 7 \\
\hline SCIENCE & & & & & & & 3 & 4 & 1 & 2 & 4 \\
\hline LANCET & & & 2 & 3 & & 2 & 2 & 1 & 2 & & \\
\hline $\begin{array}{l}\text { N.ENG.J. } \\
\text { MED. }\end{array}$ & & & & & & 1 & & 1 & 3 & & 1 \\
\hline VIROLOGY & & 1 & 1 & 1 & 8 & 15 & 11 & 6 & 8 & 8 & 5 \\
\hline $\begin{array}{l}\text { J. } \\
\text { IMMUNOL } \\
\text { OGY }\end{array}$ & & & & & & 2 & 1 & 1 & 2 & 4 & 6 \\
\hline PSEBM & & & 1 & & 4 & 1 & 6 & 8 & 10 & 20 & 14 \\
\hline $\begin{array}{l}\text { Other Pu- } \\
\text { blications } \\
\text { Britain }\end{array}$ & 3 & 3 & 2 & 1 & 3 & 4 & 1 & 5 & 3 & 5 & 6 \\
\hline $\begin{array}{l}\text { Other Pu- } \\
\text { blications } \\
\text { USA, CAN }\end{array}$ & & 1 & 1 & 2 & 3 & 6 & 16 & 15 & 13 & 17 & 31 \\
\hline $\begin{array}{l}\text { Other Pu- } \\
\text { blications } \\
\text { Europe, } \\
\text { Russia }\end{array}$ & & & & 4 & 6 & 3 & 5 & 8 & 15 & 16 & 21 \\
\hline $\begin{array}{l}\text { Other Pu- } \\
\text { blications } \\
\text { Asia, Aust. }\end{array}$ & & 1 & & 2 & 1 & & & & 7 & & 2 \\
\hline Total & 3 & 7 & 9 & 14 & 28 & 36 & 54 & 57 & 71 & 73 & 97 \\
\hline $\begin{array}{l}\text { Number of } \\
\text { different } \\
\text { authors or } \\
\text { groups of } \\
\text { authors }\end{array}$ & 1 & 3 & 5 & 8 & 12 & 21 & 31 & 38 & 45 & 51 & 50 \\
\hline
\end{tabular}


of papers relating to interferon appearing in any of the given categories of scientific journals, it is necessary to take into account the fact that the expansion of interest in interferon studies after 1960 was associated with a rapid growth of biomedical research in general. ${ }^{101}$ Seen in this perspective, the growth of scientific interest then attached to interferon as a research topic and the investment of resources in it was less spectacular than the numbers suggest. ${ }^{102}$ By the end of 1963, about 30 laboratories and 80 researchers worldwide had become involved in interferon research, but far from all of them pursued interferon research to the exclusion of all other research problems. The researchers who contributed to the study of interferon were mostly animal virologists with a training in medicine and, in a few cases, biochemists with a training in science.

Gradually the outlines of a new subfield of research emerged, which was reflected by the organization of an official symposium on interference and interferon at the 8th International Congress for Microbiology in Montreal in 1962 and subsequently the organization of the first formal interferon meeting by Vilcek in the castle of Smolenice near Bratislava, Czechoslovakia, in 1964 (see Fig. 32). ${ }^{103}$ Finally, it is interesting to see that once interferon was gaining some momentum in the international biomedical research community informal priority disputes concerning the discovery of interferon began to surface. In the corridor it was increasingly rumored either that the American researcher Koprowski had worked with interferon long before Isaacs and Lindenmann, or that the Japanese researcher Nagano had first discovered interferon, what he called 'facteur inhibiteur'. According to Burke, finishing

101 The table is based on author indexes and references from: M. Ho, 'Interferons,' N. Eng. J. Med., 266 (1962), 1258-1264; A. Isaacs, 'Interferon,' Advanc. Virus Res., 10 (1963), 1-39; N.B. Finter (ed.), Interferons (Amsterdam, North-Holland Publishing Company, 1966); and N.B. Finter (ed.), Interferons and Interferon Inducers (Amsterdam, North-Holland Publishing Company, 1973); See for the exponential growth in biomedical research in America in the 1960's, J. T. Patterson, The Dread Disease (Cambridge: Harvard University Press, 1987), p. 245.

102 C. Chany, 'Interferon-like Inhibitor of Viral Multiplication from Malignant Cells', Virology, 13 (1961), 485. 92; E. De Maeyer and J. Enders, 'An Interferon Appearing In Cell Cultures Infected with Measles Virus'. Proc. Soc. Exper. Biol. \& Med., 107 (1961), 573-78: L. Glasgow and K. Habel, 'Role of Interferon in Vaccinia Virus Infection of Mouse Embryo Tissue Culture', J. Exper. Med., 115 (1962), 503-12; and M. Ho. 'Kinetic Considerations of the Inhibitory Action of an Interferon Produced in Chick Cultures Infected with Sindbis Virus', Virology, 17 (1962), 262-75.

J. Lindenmann, 'L'Interferon'. Med. et Hyg, 19 (1961), 945-46, p. 945; N. Gibbons (ed.), Recent Progress in Microbiology. Proceedings of the VIII International Congress for Microbiology. Montreal 1962 (Toronto, University of Toronto Press, 1963), pp. 419-457; The first formal gathering of interferon researchers was held at the Home of Scientists in the wire-tapped castle of Smolenice in the woods near the town of Bratislava. Out of fear of being tapped by the secret police, all informal talking occurred in the surrounding woods. Word has it that Vilcek's flight to the West was the most important achievement of the four day meeting. My impression is, although none of those involved wanted to speak about the subject, that he was smuggled out of the country in the car of two of the participants to the conference. What I do know is that due to Vilcek's flight to the West, the publication of the proceedings of the meeting in the form of a monograph by the Publishing House of the Czechoslovak Academy of Sciences, was banned; and interviews with Jan Vilcek, Edward De Maeyer, and Kari Cantell. 
off these rumors was one of the primary incentives for Isaacs to put his mind to writing a review on interferon. The introductory section of this review paper, published in Advances in Virus Research in 1963, shows that Isaacs wanted to set things straight regarding who deserved priority over the very first interferon claims:

Interferon derived its name from virus interference, since it was first isolated and characterized during a study of this phenomenon (Isaacs and Lindenmann, 1957). However, similar substances were previously observed, although they were not characterized. ${ }^{104}$

By placing the work of others like Lennette and Koprowski, and Nagano in a particular order of relations with one another he succeeded in making a convincing argument for justifying his and Lindenmann's priority position. In doing so he superimposed new meaning on past scientific work, thereby providing directions in research activities both retrospectively and prospectively, as I will show in the next chapters. ${ }^{105}$

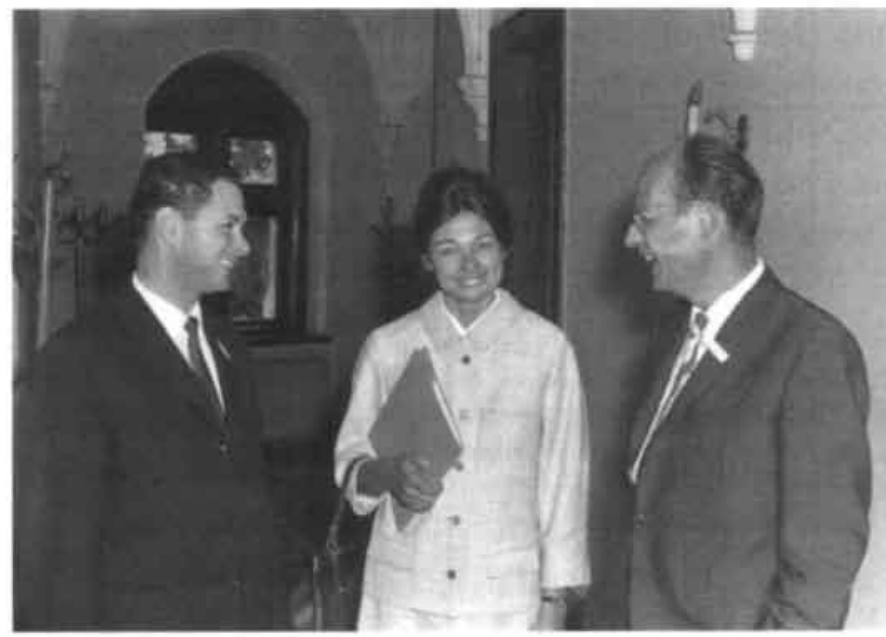

Fig. 32, From left to the right, Jan Vilcek, Jacqueline De MacyerGuignard and Edward De Maeyer in the corridors of the Smolenice meeting. Countesy of Jan Vilcek.

A. Isaacs, 'Interferon', Advan. Virus. Res. 10 (1963), 1-39, p. 2.

105 However, Isaacs' attempt to justify his and Lindenmann's priority claim regarding interferon could not prevent the American patent authorities from claiming in 1965 that the American researchers Lennette and Koprowski published a paper in the Journal of Experimental Medicine in 1946 which might have anticipated the work of Isaacs and Lindenmann. As such it threatened to undermine the possibility of a valid patent in the U.S.. although eventually in March 1966 after lengthy negotiations between the NRDC and the American patent agency the basic U.S. patent application on interferon was accepted; Meeting of the G.N.R.D. Patent Holdings Lid., 18 February 1965. MRC Archives. File No. A 812/5; and, Minutes of the 33th meeting of the Scientific Committee on Interferon, dated 7 April 1966. MRC Archives File No. S788/2/4. 


\subsection{Conclusion}

This priority dispute provides a clear illustration of the fact that the knowledge claims regarding interferon had been weaned from their matrix of origin and gained acceptance among fellow scientists-largely independent of Isaacs's 'interferon kitchen'. Only about six years earlier, while being 'hammered out' in the local context of Isaacs's laboratory, interferon seemed largely inseparable from its 'home ground'. By moving rather easily between the 'world' and his laboratory, Isaacs played a major role in linking interferon to a growing number of events and people 'outdoors' and thereby in accomplishing a separation that mobilized interferon beyond the physical space of room 215. Lindenmann's paper presentation in Switzerland and Isaacs's demonstration for MRC officers were the first extramural extensions of interferon research. A closer look at this mobilization process will gain us a clear insight into how interferon developed as the organizing principle for a new line of investigation within the international field of animal virology and more in general into the early stages of the evolution of a scientific subfield.

First, I call attention to how different sets of expectations regarding interferon came into being and provided a certain impetus to judge and act for a growing number of human agents-both as individuals and as social groups. Depending on the audience the knowledge claims regarding interferon aroused certain expectations. This was consistent with differences in the audiences' description and handling of interferon.

Penicillin served in two ways as a powerful cultural symbol that steered judgments and activities relating to interferon in Britain. First, a national so-called 'penicillin syndrome'-the British desire to prevent at all costs missing out on a penicillin-like success story - was at stake here and was typical for the British situation in the 1950 's. Second, penicillin played a vital part in legitimizing and strengthening the image of interferon as a promising lead toward anti-viral therapy. The introduction of 'wonder drugs' like penicillin had effected a widely shared belief in therapeutic breakthroughs as a dominant feature of a laboratory supported scientific medicine. Together, these two cultural conditions played a pivotal role in yielding intense excitement for what became publicly known in Britain as a possible new 'antiviral penicillin'. As I will show in the chapter the interlocking of expectations of various social groups in Britain created a niche for subsequent efforts to develop interferon as an antiviral drug.

$I$ indicated that interferon mattered, in a different way, to members of the international community of microbiologists. Instead of immediately focusing on the practical context in which work on interferon might be applied to the solution of medical problems-assessing interferon in the light of its potential benefits to practical medicine-Isaacs's and Lindenmann's experimentally derived knowledge claims were validated in their own right. This meant that the expectations and judgments were structured jointly by theoretical, methodological, practical and strategical concerns. 
For the scientists involved it was a matter of judging Isaacs's and Lindenmann's claims according to several criteria: First, the nature of the association of the claims with prior findings and the prevailing theoretical framework, with the question being how the existence of a biological entity like interferon fit into the existing body of knowledge; second the expected 'return on research investment', assessing the relevance of interferon research for biomedical research programs that figured high on national research agendas; and third, the practicality of integrating Isaacs's and Lindenmann's experimental features in existing experimental systems in other laboratories.

The judgments made by experts varied from individual to individual and laboratory to laboratory. ${ }^{106}$ What was for Hallauer an insupportable inconsistency with accepted theories, was for Nagano an immediate cause to start a priority dispute. For some, the mere association with viral interference, a laboratory phenomenon that had been relegated to the limbo of obscure phenomena of little biological significance, prevented them from becoming interested. For still others the main reason to doubt the reliability of the claims was the inability to replicate even part of the material results of Isaacs's and Lindenmann's experiments.

I should emphasize the important role replicability or reproducibility played in extending work on interferon beyond the physical space of Room 215. Basically two types of reproducibility can be distinguished in this chapter. The first type of replication was the reproducibility of the material realization, or performance, of experiments, as happened in the case of Tyrrell for instance. The successful replication in this cases implied that the same actions were performed and the same experimental situations were produced as part of routine laboratory practice in Isaacs's laboratory. This did not necessarily require agreement on the interpretation of the results. This type of replication predominantly played a role in the transfer of skills and the stabilization of a particular experimental system. ${ }^{107}$

Most common, however, was replicability of the material results of a particular set of experiments, with investigators who each used their own favorite experimental system or procedure to replicate the results. The evaluation of these attempts was the upshot both of a non-trivial process of producing a particular set of material results at the bench and a rather

Unfortunately my source materials did not allow for specifying how all these different evaluative modes were weighed against each other in individual cases.

Hans Radder has shown that there are more types of replicatication imaginable than the two presented here. In exploring the scope and role of reproducibility in various case studies dealing with experimental practice, by distinguishing as he calls it "between reproduction 'of what'-reproducibility of the material realization, reproducibility of the theoretical interpretation and reproducibility of the result of the result of the experiment - and reproduction 'by whom' (by any scientist or any human being, by contemporary scientists, by the original experimenter and by the lay performers of the experiment)". Radder was able to draw a finely grained map with up to 12 different kinds of reproducibility. See, H. Radder, Experimental Reproducibility and the Experimenter's Regress', in D. Hull, M. Forbes and K. Okruklik (eds.) PSA, Volume I (East Lansing, Michigan: Philosophy of Science Association, 1992), pp. 63-73. 
trivial process of judging the nature of the results in question and deciding how to go about the research problem under investigation. ${ }^{108}$ Failure to replicate was instrumental in bringing about the 'misinterpreton rumours', and the subsequent claims about successful replication helped reverse the balance of forces in favor of interferon. It clearly shows that both success and failure in replication were used instrumentally to turn the activities of different laboratory practices to one another. In other words, replication of the material results of experiments fuelled the spread of interferon to other laboratories and countries.

Gradually the significance of experiments and experiences relating to interferon was being transformed from a local into a trans-national issue as part of a new international line of investigation within the field of animal virology. Work on interferon no longer meant work in Isaacs's 'interferon kitchen' only. Through circulation, comparison and combination of written and printed texts (letters, papers, articles, text-books, charts), research materials and skills, interferon research became increasingly dependent on events outside the NIMR. It was a matter of doing different experiments in a different time/space proportion. Beside Isaacs's 'interferon kitchen', 'others' began to take an active part in describing and defining the nature and meaning of interferon in complementary ways.

In order to bring about a transformation of interferon's scientific status from a possible 'misinterpreton' to a new locus for professional commitment within the field of animal virology, researchers like Isaacs, Wagner and, Ho and Enders, had had to engineer mutual ways of communicating, manipulating, interpreting and elaborating on a common assembly of research objects in an effort to describe other substances that complied with the agreed upon criteria in terms of an interferon. I showed that this required quite some coordinating and coaching on the part of Isaacs. This implied, among other things, helping to identify and eliminate factors which prevented researchers in other laboratories from reproducing and manipulating an interferon, organizing private experimental performances for fellow scientists in his London laboratory, giving frequent talks on interferon, making suggestions for the publication of interferon related experimental work, and stimulating further efforts to establish a common set of experimental criteria as to what biological factors should bear the name interferon.

By accomplishing changes in the order of events in a growing number of laboratory practices outside the NIMR, interferon increasingly gained independence from its initial site of production. Isaacs's laboratory and interferon were no longer inseparable. In the process, 'interferon' emerged and functioned as an organizing principle and communication device of a set of heterogeneous experimental practices-as a framework for manipulative and interpretative practice-with investigators who each used their own favorite virus-cell system. 
The range of these various experimental systems was wide and their number was on the increase. As we have seen in chapter three, the building and manipulation of a workable experimental system in Isaacs's laboratory required hard, uncertain, skilled and creative work. It was a 'real-time and real-space' struggle to make things work. To engineer an association of different laboratory practices that shared a data domain and style of language, is, then, not a small achievement that can itself be taken as the explanation of a certain degree of scientific consensus regarding the 'existence' of interferon as a specific biological entity.

Finally, with the benefit of hindsight, one may say that though in essence Isaacs's laboratory had not changed that much, Isaacs's research world had become rather different from the one he had inhabited when submitting his first interferon papers. What in 1957 was still a laboratory phenomenon of local significance that had yet to pass its referees had not only become the organizing principle for a new line of investigation-a growing group of researchers sharing mostly practical and programmatical commitments within the field of animal virology - but had also become a serious possibility for the development of an antiviral drug. Furthermore, the scientific notion of interferon, which matured in conjunction with the work of Isaacs and the 'others', had undergone a transformation. From a viral interfering factor which was provisionally considered a deviant viral entity, interferon came to refer to active antiviral substances in particular culture fluids - as a generic term-which complied with a specific set of experimental criteria and which were considered essential products of the cell that played a part in resistance to viral infection. ${ }^{109}$ However interesting this may be from a cognitive point of view, we should keep in mind that the scientists under survey did not regard the situation in terms of a shift of world view. They 'simply' manipulated, produced, and interpreted a body of laboratory phenomena in what seemed to them the most appropriate way-as a means to the end of increasing the range of manipulative options at the bench and of promoting the blossoming of a new, cutting-edge field.

109 In cognitive sciences, e.g. psychology of science, the transformation process which interferon underwent would be argued to exemplify in a gradual fashion some variant of a classical gestalt switch (quite common are the rabbit-duck, pelican-antelope and Einstein-nude reversals). See, M. De Mey, The Cognitive Paradigm (Chicago, The University of Chicago Press, 1992), pp. XVI-XVII, 89-93, 173-6. 


\section{Chapter 4}

\section{Interferon on Trial $^{1}$}

\subsection{Introduction}

In the previous chapter I described the formation of an international body of laboratory practices with shared commitments in the pursuit of a common set of research problems. This involved assembling heterogeneous collections of people and objects, without regard for the walls of the laboratory. In being an essentially heterogeneous activity, collective work on interferon required cooperation in order to create common understandings and produce knowledge which retained its integrity across time and space. We saw how those engaged in interferon research constantly faced uncertainty and difficulty. This was related to the problems involved in operating essentially different experimental systems and the same time making efforts to exchange and compare research materials and data. Moreover we saw how the products of research yielded and shaped different meanings and expectations in different social realms. I showed that it was not laboratory research alone, but the perceived role of medicines like penicillin as 'new wonders for the doctor's bag' as well as a national so-called 'American penicillin syndrome', which triggered interest in developing interferon as a therapeutic drug in Britain.

In this chapter I shall pursue my investigation of the emerging links between interferon researchers, government administrators and entrepreneurs from several drug companies in the British context. I will address the factors shaping the first successful postwar initiative to secure a collaboration between the British government and the drug industry as a means to develop interferon as a drug. ${ }^{2}$ The main point of interest is how these various parties tried to cooperate across the domains which those involved routinely demarcated as 'science', 'policy' and 'industry'. To what extent did they succeed or fail in bridging the differences between industrial and academic 'life'? Furthermore I shall describe and analyze how interferon resisted attempts at being integrated into conventional drug research and testing practices and how the various parties reacted through a change of expectations and research agendas to these

1 A shortened version of chapter 4 was published in Medical History: T. Pieters, 'Interferon and Its First Clinical Trial': Looking Behind the Scenes', Medical History, 37 (1993), 270-95.

In 1955 the MRC and NRDC also initiated efforts to seek collaboration with British drug companies on the development of the possibly interesting antibiotic, cephalosporin. However, despite initial mutual enthusiasm because of slow research progress most companies soon lost interest and it never came to a formal Collaboration Agreement; See, D. Wilson Penicillin in perspective (London: Faber \& Faber, 1976), p. 251. 
resistances. The intention here is also to point out how simultaneously the modes of perceiving interferon as a therapeutic substance changed and differentiated.

The chapter begins with describing how upon deciding to start efforts to develop interferon as a drug, the Medical Research Council (MRC) sought collaboration with British drug companies to bring in the necessary expertise and resources. Attention focuses on the symbiotic kind of relationship which develops between Isaacs, the MRC and drug companies. I shall examine efforts to develop and carry out a collaborative research program by setting up an Executive Body and a separate Scientific Committee on Interferon under the chairmanship of Isaacs. In closely following the wheeling and dealing of this committee, I will show how in the absence of a standard system for the testing of potential therapeutic drugs in the 1960's in Britain, those involved decided on what tests should be done on the experimental substance, interferon, to show if it might be safe and effective in the treatment of viral disease in humans. We will see that in the process the expectations and commitments of the various parties began to diverge. By focusing attention on the dynamics of the transitions between in vitro tests, in vivo tests and tests on humans I will illustrate how through the consecutive experimental sequences the objects of research as well as research agendas and commitments underwent such changes as to drive the various parties gradually apart.

\subsection{A partnership originating in national interests.}

Following the extensive press coverage of interferon in which it was presented as a possible 'magic bullet' against viral disease in Britain in May 1958, the MRC received a letter from the pharmaceutical company Johnson \& Johnson. The American drug firm had noted the publicity surrounding interferon in the UK and was most interested in receiving additional information on this possible new lead to anti-viral therapy. In particular they wanted to know whether any results from the administration of interferon to humans were available. ${ }^{3}$ The American request was passed on to Isaacs for reply. In response Isaacs provided Johnson \& Johnson with references of the latest publications on interferon while emphasizing that the NIMR and the MRC had not yet "contemplated any experiments in man and even if present experiments go favorably it is unlikely that we would do so for some time". ${ }^{4}$ Isaacs responded in a similar way when contacted informally by Maurice Hilleman, the head of Virus and Cell Biology Research

3 The American pharmaceutical company Johnson \& Johnson to the MRC, letter dated 25 June 1958, MRC Archives, File No. A813/104.

4 Alick Isaacs to Johnson \& Johnson. New Brunswick. New Jersey, U.S., letter dated 25 June 1958, MRC Archives , File No. A813/104. 
of the American drug firm Merck Sharpe \& Dohme ('Merck').

At about the same time, the Secretary of the MRC, Sir Harold Himsworth, was informally approached by Glaxo's top executive, Sir Henry Tizard, about the patent position on interferon. ${ }^{6}$ Together with the American requests for research information, this event provided an important incentive for the Secretary of the MRC to consult the director of the NIMR, Sir Charles Harington, about further action on interferon. Both parties realized that in order to develop interferon as a drug some kind of collaboration with the British drug industry would be needed. Harington indicated that he would welcome cooperation with one of the UK's leaders in biologicals if it took the form of supplying interferon in large amounts, but he would wait for Glaxo to take the initiative. However, Himsworth was in favor of a different approach.

The MRC's reluctance and restraint in co-ordinating things with regard to the industrial development of penicillin during the second World War and the subsequent 'British failure' was still fresh in everybody's memory. ${ }^{7}$ It had an important influence on the MRC's attitude toward interferon. If interferon would prove to be the new 'antiviral penicillin', the opportunity should not be lost to develop it in Britain. ${ }^{8}$ Taking into consideration the American interest and a possible drift of knowledge on interferon across the Atlantic where it might be developed into a commercially successful product, Himsworth was in favor of taking determined policy steps. ${ }^{9}$ By actively seeking collaboration with British commercial firms only, they could best serve the national interest. ${ }^{10}$

Under the specific circumstances of British post-war science policy with the 'penicillinsyndrome' as one of its guiding principles, the National Research Development Corporation (NRDC) was entrusted with the task of making proposals for a form of cooperation. As a result of the British "failure" to exploit penicillin, the National Research Development

5 Alick Isaacs to Jean Lindenmann, letters dated 19 March, I3 May 1958, Jean Lindenmann Correspondence, personal archives.

Internal Note MRC, dated 4 November 1958, MRC Archives, File NO. A814/17.

7 Liebenau pointed out how as a result of difficulties experienced when trying to co-ordinate things for insulin in the 1920's, the MRC decided to keep its distance in future dealings with drug companies as can be seen in the case of penicillin. Another factor that may have played a role in the MRC's reservations in the case of pennicillin was the failed attempt in 1920's to unite a number of prominent British firms-among others Boots, British Drug Houses and British Dyestuffs-in The Pharmaceutical Corporation': see, J. Liebenau, The MRC and the Pharmaceutical Industry: the Model of Insulin', in J. Austoker and L. Bryder (eds.). Historical Perspectives on the Role of the MRC (Oxford: Oxford University Press, 1989), pp. 179-80.

Interviews with Derek Burke, Norman Finter and David Tyrrell.

9 In the 1940's the image of international competition (with American pharmaceutical companies) also helped British academic workers to overcome part of their restraint towards British drug firms and start collaborating on the development of penicillin as a drug; see, J. Liebenau, 'The British Success with Penicillin', Soc. Stud. Sci, (17) 1987, 69-86, p. 75.

Internal Note MRC, dated 6 November 1958, MRC Archives, File No. A814/17. 
Corporation (NRDC) was set up under the British Industries Act of 1948 to secure the development of inventions resulting from public research and to obtain patents for these inventions. One of the first research projects in the field of biomedicine the NRDC took an interest in was the development of the antibiotic cephalosporin C. In accordance with its terms of reference the NRDC had been steadily patenting research results ever since the first results on cephalosporin had been published in 1951 by an Oxford research group. By the time its chemical structure had been established in 1955, the NRDC had initiated efforts to call in the help of the British industry. In exchange for certain rights over use of the developed product, several British pharmaceutical companies agreed to look at the problem. However it never really came to a formal collaboration agreement and the NRDC experienced a rapid loss in interest when progress turned out to be slower than expected. The cephalosporin experience influenced NRDC's preference for a formal multi-party collaboration arrangement in the case of interferon."

In consultation with the NRDC the MRC decided to formally invite Glaxo as well as other major British pharmaceutical firms to cooperate, by invitation to the Association of British Pharmaceutical Manufacturers (ABPM). ${ }^{12}$ In general, caution prevailed among British drug company executives. The developments with the biological substance interferon were closely followed. However, without Isaacs's claim about interferon's potential as a therapeutic drug being substantiated by more extensive laboratory studies they decided to wait and see. Even within companies like Glaxo with a long history of working with biologicals a reserved attitude toward interferon prevailed. Few company scientists seemed to know what to make of what essentially was not more than a mere protein soup with a claimed biological activity. The perception of biologicals - drugs that are made of biological source materials (e.g. vaccines or hormones like insuline) and whose potency and identity can only be determined by cumbersome and inaccurate bioassays - as profitable but troublesome and high-risk commodities, played an important role in preventing the drug companies from throwing themselves into interferon research. In being primarily oriented toward medical chemistry and pharmacology the drug companies preferred to deal with the relatively straightforward chemicals- compounds that are made of source materials whose potency and identity can be adequately tested by chemical or physical means. As far as the pharmaceutical industry was concerned, except for the vaccines, most biologicals had a problematic developmental track record. They were tricky to produce-requiring elaborate and expensive production and standardization proce-

11 See, L. G. Matthews, History of Pharmacy in Britain (London: E.\& S. Livingstone, 1962), pp. 330-3; and, D. Wilson Penicillin in perspective (London: Faber \& Faber, 1976), pp. 250-5.

12

Internal Note MRC, dated 4 November 1958, MRC Archives, File No. A813/104. 
dures, to be difficult to quantify and difficult to store. ${ }^{13}$

At the same time British drug company executives were quite familiar with the industrial potential of substances of biological origin: the unparalleled clinical success record of the biosynthetic penicillin-like antibiotics was all too visible. Their spectacular clinical activity in eradicating bacterial infections had created a firm belief in the 'Ehrlichean' chemotherapeutic research approach. Paul Ehrlich's ideal of generating chemical agents which, like 'magic bullets', seek out and destroy the enemy and injure nothing else, seemed to have materialized. ${ }^{14}$ Except for viruses most disease inducing microorganisms were known to be susceptible to antibiotics. The common view was that the chemotherapeutic research approach would eventually also provide them with some kind of effective and clinically useful anti-viral therapeutic drug. One might say that in a clinical sense there was already an established frame of thought for chemotherapeutic control of viral disease. This despite the fact that only costly research failures had been reported as a means of developing a serious lead on viral chemotherapy. Most promising leads in the test-tube had proven to be too toxic in vivo and ever increasing effort was being given to the control of viral disease by time-proven vaccination ('immunoprophylactic') principles. ${ }^{15}$

Gradually, in reaction to the surge in public enthusiasm in Britain, and in relation to the perceived clinical and economic promise of interferon as an 'anti-viral penicillin' the caution of British drug firms subsided. The promise of interferon as a potential specific (that is truly curative) and innocuous drug which seemed to fit into the conventional ('Ehrlich's') concept of chemotherapy as a sort of 'magic bullet' against virus infections was rather tempting. Moreover, from industry's point of view, risk-sharing with the MRC who offered their unique expertise in interferon research and the eventual rights in the developed product, made work on interferon look more feasible. This compensated for the uncertain manufacturing perspective of a biological like interferon. ${ }^{16}$

Nearly all major British drug companies decided to accept the invitation and sent representatives to the meeting with the MRC and NIMR. On 22 April, 1959 in one of the

L. E. Arnow, Health in a Bottle; Searching for the Drugs that Help (Philadelphia: J.B. Lippincott Company. 1970): and, W. Breckon, The Drug Makers (London: Eyre Methuen Ld, 1972); and interviews with John Beale and Norman Finter.

14. For an in-depth historical analysis of the development of Ehrlich's chemotherapy theory which was largely based on the dictum "If the law is true in chemistry that corpora non agunt nisi liquida, then for chemotherapy the principle is true that corpora non agunt nisi fixata.": see, J. Parascandola, The theoretical basis of Paul Ehrlich's chemotherapy', J. Hist. Med. 36 (1981), 19-43; and, P. Ehrlich, 'Chemotherapy'. In: The collected papers of Paul Ehrlich, (Himmelweit, F., Ed.) Vol 3 (1914), p.507, London.

D. Wilson Penicillin in perspective (London: Faber \& Faber, 1976), pp, 278-279.

D. Wilson, Penicillin (London: Faber\&Faber, 1976), pp. 278-82; and interviews with John Beale and Norman Finter. 
NIMR's conference rooms at Mill Hill, London, representatives of the companies were informed about the current state of affairs concerning interferon. Sir Charles Harington opened the meeting by pointing out American interest in interferon, but emphasized MRC's preference to first seek collaboration with British firms. Harington told the pharmaceutical companies that the NIMR needed their help in developing this possibly interesting biological. The NIMR simply lacked the expertise to tackle the production problem. In exchange he offered them eventual rights in the developed product. Subsequently, both technical and patent matters were discussed. A patent expert from the National Research Development Corporation (NRDC) helped to clarify the patent position on interferon whereas Harington would call upon Alick Isaacs, to answer technical questions. ${ }^{17}$ Isaacs was highly regarded by everyone as the discoverer of interferon. Except for Harington nobody was aware that Isaacs had only recently resumed all his duties at the World Influenza Centre (NIMR) after recovering from a severe bout of depression and that at times he could behave in a rather manic way. ${ }^{18}$

Most experimental results were argued to point in favor of the possible therapeutic value of interferon. Enthusiastically Isaacs told the audience that in his laboratory interferon had been shown to inhibit the growth of a wide range of viruses in vitro. He emphasized the fact that apart from these in vitro studies also early animal experiments were carried out which showed that interferon protected animals against virus infection. Moreover, so far all the indications were that interferon was innocuous without any obvious ill-effects in animals. Of course, Isaacs argued, his audience should take into consideration that systematic and large scale investigations had yet to start and that there were still a couple of experimental problems to solve. A major difficulty was the production of large enough amounts of active material to start systematic investigations of both interferon's effectiveness against viral infection and its toxicity in vivo. However, Isaacs expressed his confidence that with the help and expertise of some of the companies present they would be able to find satisfactory solutions to these practical problems. ${ }^{19}$

Subsequently, with the help of the NRDC's patent agent, Harington succeeded in playing down as a mere technicality the fact that the information available was considered insufficient by the American patent examiner. Mainly because of a lack of evidence on the drug's utility, the American examiner had decided that the patent application for interferon as

17 Minutes of a meeting to discuss collaboration in a program of work on interferon. held at the NIMR. 22 April, 1959, MRC Archives, File No, S788/I.

18

See, C. Andrewes, 'Alick Isaacs', Biographical Memoirs of Fellows of the Royal Society, 13 (1976), pp. 205. 21, p. 215; and interviews with Derek Burke and David Tyrrell.

19 Minutes of a meeting to discuss collaboration in a program of work on interferon, held at the NIMR, 22 April. 1959, MRC Archives, File No. S788/1. 
an antiviral agent had to be rejected until further research results could be made available. ${ }^{20} \mathrm{At}$ the end of the meeting it was agreed that each firm represented at the meeting would let Harington know as soon as possible whether or not they wished to collaborate on the development of interferon.

In the weeks following the meeting the two British leaders in biologicals BurroughsWellcome (Wellcome) and Glaxo Laboratories (Glaxo), and Imperial Chemical IndustriesPharmaceuticals (ICI) informed the MRC of their willingness to co-operate. ${ }^{21}$ Significant was the fact that Sir Harry Jephcott, the chairman and managing director of Glaxo, announced that Wellcome and Glaxo were prepared to act as one, hoping thereby to exclude ICI (who as yet had not been active in the field of biologicals) from collaboration with the MRC. ${ }^{22}$

The following two factors played a most obvious role in this respect. First of all Wellcome and Glaxo regarded biologicals as there core-business and tried to defend their position as British leaders in biologicals against a newcomer like ICI (ICI's pharmaceutical group was an off-shoot of its dyestuff division and as such had built up a strong position in chemicals). Moreover, they both had had bad experiences with ICI as an unreliable collaborator on penicillin. During the Second World War a consortium of British pharmaceutical companies (including Wellcome, Glaxo and ICI), incorporated as the Therapeutic Research Corporation (TRC), had collaborated on a penicillin development program. ${ }^{23}$ However, to the unpleasant surprise of the others, soon after the war had ended ICI had prematurely pulled out of the penicillin program. ${ }^{24}$

The matter was eventually settled at an informal meeting when the MRC made it clear to Jephcott that for public policy reasons ICI could not be arbitrarily excluded if the company wished to participate. ${ }^{25}$ Anxious not to miss the boat on what might turn out to be a promising innovative lead toward anti-viral therapy, the drug companies were eventually prepared to put up with the multi-party collaboration arrangement. As will turn out the MRC's intervention could not prevent the issue from being an early burden on the success of the collaboration.

Elkington and Fife, consulting chemists and chartered patent agents, to Alick Isaacs, letters dated 9 March, 1959, MRC Archives, File No. A813/104.

21 The pharmaceutical companies Wellcome, Glaxo and ICI to the MRC and vice versa, letters dated May 1959. MRC Archives, File No. S788/1.

22 Minutes of a meeting to discuss collaboration in a program of work on interferon, held at the NIMR, 2 June, 1959, MRC Archives, File No. S788/1.

23 J. Liebenau, 'The British Success with Penicillin', Soc. Stud. Sci., (17) 1987, 69-86, p. 83.

24 Unfortunately due to the fact that the company archives have remained closed to outsiders up to this day the knowledge about the wheeling and dealing of the companies and their policies is limited; J. Liebenau, 'The British Success with Penicillin', Soc. Stud. Sci., (17) 1987, 69-86, p. 83.

25 Internal note MRC, dated 5 June, 1959, MRC Archives, File No. S788/1. 
Further meetings were organized a couple of months later with all parties (NIMR, MRC, NRDC, Glaxo, ICI and Wellcome) to discuss in more detail the proposed collaboration and settle the outlines of an agreement for a collaborative program of further work on interferon. ${ }^{26}$ At the joint meeting which was held on 3 July 1959, the NRDC proposed to set up an Executive Body to hold property, grant licences and administer the arrangement between all the parties involved. ${ }^{27}$ They suggested that NRDC's Patent Holdings Company, which had been founded in 1953 with the aim of carrying out future collaborations between British government institutions and private industry, might be used for this purpose. In addition they proposed to set up a Scientific Committee on Interferon, which would come under the Executive Body and provide a platform for the exchange of technical information and know-how.

Following further talks between the MRC, the NRDC and the three drug firms, all parties in principle agreed to these proposals. ${ }^{28}$ The MRC patents on interferon which were currently being filed in the USA, Canada and West-Germany, as well as any further Council patents regarding interferon, were made available to the NRDC's Patent Holdings Company. The Executive Body would be run by senior managers of all the parties involved and the Scientific Committee was to consist of research workers of the industrial firms and the MRC, under the chairmanship of Isaacs. This committee was not only meant to serve as a clearing house for information but also as a kind of scientific steering committee to allocate research work to the various parties and to advise the Executive Body on the scientific aspects of further work on interferon. $^{29}$

The parties concerned only agreed to the main points of an agreement for collaboration. Further discussions and negotiations were needed to determine the exact terms of a formal agreement between the NRDC, MRC and the three pharmaceutical firms relating to interferon. In particular the differences in opinion on the issue of publication of research results would require quite a bit of talking through before they could be settled. Harington and Isaacs maintained that the results of all research work should be made available for scientific publication without limitations in the normal way in scientific journals. Only in order to ensure adequate patent protection of any discoveries would they allow some delay to occur in publication. The representatives of the drug companies, however, emphasized the need for

26 Minutes of a meeting to discuss collaboration in a program of work on interferon, held at the NIMR, 2 June, 1959, MRC Archives, File No, S788/1.

Minutes of a meeting to discuss collaboration in a program of work on interferon, held at the NIMR, 3 July, 1959, MRC Archives, File No. A812/5/1.

28. The executive body would be formalized in a GNRD Patent Holdings Ltd. with a board made up of representatives of each of the collaborating firms and the NRDC, under an independent scientific chairman. 
stringent control over publication. If necessary it should be possible to reject publication on grounds that harm might be done to current and future patent positions.

All parties concerned regarded the issue of publication as the one point on which the whole program of collaboration could easily break down. As I will show in the next two sections much effort had to be invested in preventing this from happening. All parties to the collaboration seemed to be prepared to do so. At least as long as each party remained committed to the same central idea of developing interferon as an antiviral therapeutic drug and shared similar thoughts about the constraints and the prevailing conditions under which collaborative research should be performed. ${ }^{30}$

\subsection{Establishing a 'collaborative' program for research on interferon}

While negotiations on the precise terms of a Collaboration Agreement were dragging on, Isaacs was informally given the go-ahead to start discussing a program of research as chairman of the Scientific Committee on Interferon. ${ }^{31}$ In showing their unconditional belief in the authority of Isaacs's scientific expertise the MRC and the drug companies would virtually give Isaacs carte blanche to pursue his personal research agenda regarding interferon.

In September 1959, under the vigorous leadership of Isaacs, the Scientific Committee started to review and evaluate recent work on interferon. ${ }^{32}$ Isaacs emphasized that ultimately all parties hoped to develop research on interferon to a point where it could be used therapeutically in humans and animals. This would require both basic research and research on scaling up from laboratory to manufacturing procedures. At the present stage of work it seemed that more scaling-up than basic work was needed. Hence, Isaacs was very much in favor of a provisional program for research that would focus on the development of methods for the largescale production, purification and storage of interferon. He also emphasized the need to examine thoroughly the question of whether or not interferon was a species-specific biological substance. MRC's research workers claimed that interferon prepared in chick tissues showed a much higher antiviral activity when tested in vitro in chick cells than it did in calf cells. Similarly, chick interferon when tested in vivo in rabbits was found to have a far less protective effect than rabbit interferon against viruses. Despite the fact that this species specific effect did

MRC to NRDC, letters, dated 10, 29 July 1959, MRC Archives, File No. A812/5/1.

31 The formulation of a clause on the publication of research results indeed turned out to be a major obstacle in the negotiations. It would take until April 1961 to reach a formal Collaboration Agreement relating to interferon.

32

Minutes of the first and second meeting of the Scientific Committee on interferon, 3, 9 September, 1959. MRC Archives File No. S788/2/1. 
not seem to be absolute, it could have far-reaching consequences for the testing trajectory. If it were to be substantiated by further experiments, tests on the virus-protective effect of interferon in animals, and later in humans subjects, would require samples of interferon that had been produced in cells of the same or a closely related species. The species specificity issue most notably bothered the drug company scientists. They immediately realized that this would complicate working in line with the conventional industrial new drug development trajectories with preliminary toxicity studies on at least two species of animals. ${ }^{33}$ It meant that testing interferon in mice would require the production of mouse interferon, testing it in rabbits would require the production of rabbit interferon and so on. In addition, comparing test results in different animal species would become far more difficult.

Until the spring of 1960, research on interferon was carried out, more or less, along the lines of this provisional program, which closely resembled the initial stage in the industrial development of a new drug involving a division of work and interplay between various workers and disciplines (biologists, biochemists and physicians) ${ }^{34}$. The MRC's researchers mainly worked on the development of a purification method, the problem of the speciesspecificity, and the mechanism of action. Meanwhile, researchers from the collaborating firms focused on the development of methods for the large scale production of interferon. ${ }^{35}$

In the meantime negotiations on the terms of an agreement relating to interferon between the NRDC, MRC, Wellcome, Glaxo and ICI continued. The drug firms kept on emphasizing the importance of a common obligation not to prematurely disclose relevant subject matter to any person outside the collaboration. ${ }^{36}$ Besides ensuring that patents were taken out before publication of research results, the industrial collaborators wanted an agreement that would provide for a particular clause to prevent one of the parties disclosing information coming from another party. Wellcome even wanted to go as far as to impart nonpatentable information to outsiders. This met with resistance from Harington.

The director of the NIMR thought an absolute restriction on sharing of all nonpatentable information to colleagues most objectionable. In causing unnecessary delay in publication of research results it could only harm the primarily academic interests of his research institute. The MRC told Harington not to worry too much about this additional demand. Since, as the MRC argued, this sounded fair and in these matters it would be better to

33 L. E. Amow, Health in a Bottle; Searching for the Drugs that Help (Philadelphia: J.B. Lippincott Company. 1970); W. Breckon. The Drug Makers (London: Eyre Methuen Ltd, 1972); and interview with Norman Finter. Ibid.

35 Minutes of the 3rd and 4th meeting of the Scientific Committee on Interferon, dated 26 October, 26 November, 1959. MRC Archives, File No. S788/2/1. 
wait and see how eventually the clause would be drafted. ${ }^{37}$ Ultimately all parties would agree to the NRDC approving all papers before they were submitted: every first draft of a paper would be submitted to the NRDC, who had agreed to give their approval within 14 days. ${ }^{38}$ However, this could not prevent the parties from having occasional skirmishes over the exchange of research information.

Apart from the publication issue, it is interesting to see how the definition of interferon became part of the negotiations on the terms of an agreement and changed over time. From the very beginning the industrial collaborators and patent advisers worried about the fact that interferon did not denote a chemically characterized substance but a substance whose identity could only problematically be determined by biological means. During the negotiations they emphasized the need for a 'crystal-clear' definition of the agent of the agreement in order to prevent future problems. The provisional description of interferon as "a viral interfering substance produced by the action of partially-inactivated virus on a susceptible tissue" did not satisfy this demand. ${ }^{39}$ However, it appeared to be rather difficult to find a definition that would be narrow and broad enough to satisfy the demands of patent advisers, company executives and researchers.

Eventually all parties chose in favor of a definition that was thought to serve the purpose of both limiting the field of collaboration, and of covering under the agreement as many natural virus induced substances with antiviral properties as possible: ${ }^{40}$

\footnotetext{
any substance (other than antibodies) which is produced by the interaction of a vinus and a living animal tissue and which is able to prevent, diminish or modify the multiplication of active viruses in living animal cells."
}

However, the moment this definition made its appearance in the draft agreement it was again called into question. The NIMR research workers had just claimed to have found natural virus inhibitory substances which were different from interferon in that they shared serological characters with the virus responsible for their production. In order to prevent these newly discovered substances from being included automatically in the Interferon Collaboration and

37

38 Minutes of the 8 th
File No. $5788 / 2 / 1$.

39 Minutes of a meeting to discuss the draft Agreement prepared by the NRDC between Glaxo Laboratories Ld., The Wellcome Foundation Ld., Imperial Chemical Industries Ld., the National Research Development Corporation and G.N.R.D. Patent Holdings L.d. relating to collaboration on interferon, held at The Wellcome Building, Euston road, on 27 January, 1960, MRC Archives, File No. A812/5/1.

40

41 MRC to NIMR and vice versa, letters with comments on the drafts of an interferon agreement, dated 23, 31 March, 1960. MRC Archives, File No. A812/5/1. 
offering them for free to the industrial partners, the NIMR urged for the following change in the wording of interferon's definition:

\begin{abstract}
any substance (other than antibodies) which is produced by the interaction of a virus and a living animal tissue and which, whilst it lacks the serological properties of the virus, is able to prevent, diminish or modify the multiplication of active viruses in living animal cells."
\end{abstract}

While succeeding in producing a more careful wording, gradually the complexity and length of the latest definition began to bother the participants. As soon as it turned out that this threatened to reduce the chances for obtaining official registration of the drug name interferon from the national authority in this field, the British Pharmacopoeia Commission, negotiations on a new wording started anew. The process of dropping, adding and changing the order of modalities which either narrowed or widened the meaning of the wording went on until the following definition was finally accepted by the Pharmacopoeia Commission:

a protein formed during the interaction of animal cells with viruses which is capable of conferring on animal cells resistance to virus infection. ${ }^{43}$

Once recognition at the national level was achieved, efforts were immediately launched to secure the very same definition for interferon as a therapeutic compound at the international level through adoption by the Council on Drugs of the American Medical Association and the World Health Organization (WHO) in Switzerland. The industrial collaborators were alarmed by the information that an Austrian drug firm had already started efforts to register the word 'intraferron' as an international trade mark. They regarded the definition and registration question a high priority issue, as a means to defend their future market position. ${ }^{44}$

The complex nature of the negotiations about the collaboration agreement with fierce debates down to the smallest detail took the NRDC by surprise. They obviously had not expected to see such differences in interests between the MRC and the drug companies with regard to research and information management, and, with regard to patent and market position.

At a meeting in March 1960 NRDC officers asked the other parties whether there might be a better way of effecting this kind of collaboration than the current proposal involving the use of a Patent Holdings Company. The NRDC representatives even went as far as to ask

42 MRC to NIMR and vice versa, letters with comments on the drafts of an interferon agreement, dated 23,31 March, 1960, MRC Archives, File No. A812/5/1.

Harington to MRC, letter dated 7 December, 1960, MRC Archives, File No. A814/17. 
whether it would be desirable to proceed with the present collaboration using such an arrangement. Upon discussing the matter it was agreed that, although in future cases it would be simpler "not to use a patent holdings company but to set up a contractual arrangement", the present collaboration should continue on the basis of a patent holdings company being used. ${ }^{45}$ This certainly shows that so far the NRDC staff was far from on its own in their negative assessment of the collaboration arrangement.

At about the same time, after eight months of collaborative research, the Scientific Committee suddenly revised the provisional research program quite radically. ${ }^{46}$ During the April 1960 meeting research workers from the MRC reported the following two research results. First, when testing the specificity of interferon prepared in a variety of animal tissue cultures, monkey interferon had shown activity against a range of human and simian cells. In particular, the observation that monkey interferon appeared to be active against a number of respiratory viruses in human tissue-cultures aroused interest. It was agreed that, although extrapolation from individual tissues to the complete organism was hazardous, monkey interferon might be fit for future use in humans. Moreover, Wellcome's and Glaxo's experience of many years with the production of poliomyelitis and measles vaccine in large scale cultures of monkey kidney cells was argued to be helpful in overcoming problems with the production and safety of monkey interferon.

Second, the purification work on interferon had reportedly resulted in a pure product. The prospect of producing sufficient quantities of pure monkey interferon had a profound impact on the planning of further work on interferon by the Scientific Committee. Immediately, four suggestions were made for future experiments with purified interferon in monkeys and humans: ${ }^{47}$

1) Since the MRC was in the process of testing a measles vaccine, facilities to test interferon's effect against measles would be widely available. It therefore seemed a feasible option to test its protective effect against measles infection in volunteers. However, objections were immediately raised. As measles was a systemic infection a measles trial would require considerable amounts of interferon and extensive safety tests. Furthermore, interferon's effect on measles had neither been tested in vitro nor in vivo.

2) Interferon had shown a clearcut inhibitory effect against vaccinia virus ('smallpox') in vivo and in vitro and it was proposed to test the protective effect of interferon against local infection with attenuated smallpox in volunteers. There would be enough recruits available

45 Minutes of a meeting to discuss proposals of the industrial participants relating to the Collaboration Agreement on interferon, held at NRDC offices, on 16 March 1960, MRC Archives, File No. A812/8/1.

46 Minutes of the 6th meeting of the Scientific Committee on Interferon, dated 6 April, 1960, MRC Archives File No. $788 / 2 / 1$.

Ibid. 
who had not yet been vaccinated against smallpox and were willing to volunteer in such a trial. Moreover, as a local infection it would need much less interferon than a systemic infection and require fewer safety tests.

3) The idea to test the protective effect of interferon on respiratory infections in volunteers originated from a combination of experimental results and logistics. Interferon had shown a protective effect against respiratory viruses in the laboratory and the MRC had an official site for common cold trials in the form of the Common Cold Unit in Salisbury. Moreover the common cold was a local infection which would require only small amounts of interferon and relatively limited safety testing.

4) Quite recently Andrewes's Division had succeeded in culturing trachoma virus, which caused local but potential debilitating infections in the eye and the MRC seemed interested in finding a therapy against this virus disease. ${ }^{48}$ Testing the protective effect of interferon on trachoma virus infection in monkeys was considered an excellent experiment to do first as a final check before proceeding to experiments in humans. As a local infection it would require only small amounts of interferon.

Isaacs, in particular, seems to have been rather keen on having an early demonstration of interferon's effect in humans. He felt that it would help to keep people interested and maintain the necessary pressure, because the negotiations on the Collaboration Agreement were not completed yet. ${ }^{49}$ The company scientists were less enthusiastic about deviating from the conventional drug testing trajectory. However, they too were aware of the fact that the American patent examiner had rejected interferon's patent application on utility grounds. If interferon could be shown to have an effect on humans, even in an experimental infection, this decision might be reversed. Moreover, Isaacs convincingly argued that interferon was different from most other biologically active compounds in showing unusually few toxic side-effects in human cell-cultures. According to Isaacs any substance being tried out in humans always presented hazards, but in the case of interferon it was a great comfort to realize that it was not so much a matter of administering a foreign substance as supplementing a natural mechanism of resistance to virus infection. For this reason there was little chance of interferon producing any severe adverse effects when administered to humans. Isaacs met the company scientists part of the way by indicating that for safety reasons they would carry out the trachoma experiment in monkeys first, followed by the vaccinia and common cold trial. A measles virus trial was just too demanding to carry out at the present time. All agreed that in order to be able to

48 The trachoma agent which is now considered to be part of the Chlamydiae family-a group of bacterial organisms-was still referred to as a virus in the early 1960 's; interview with a senior MRC-official who prefers not to be identified, following the presentation of a preliminary version of this chapter to the Twentieth Medical History Group of the Wellcome Trust, held at the Royal College of Physicians, 11 February, 1992.

49 The collaboration agreement was signed on 24 October, 1960. Memorandum and Articles of Association of GNRD Patent Holdings Limited. MRC Archives File No. S788/6. 
carry out the trial program an early start had to be made with work on the large-scale production, purification and storage of monkey interferon. ${ }^{50}$

As we have seen in this section various considerations played a role in guiding plans for research, most notably Isaacs's conviction that interferon was a natural non-toxic bodysubstance and the fact that the patent examiner had rejected interferon's patent application on utility grounds. Obviously work at the bench and policy-making co-evolved. Recall that when members of the Scientific Committee reported the observation that monkey interferon showed an activity against viruses in human cells in the test-tube, it was agreed that extrapolation from individual cells to the complete organism was problematic. Yet it was decided to switch attention to trials in monkeys and humans without further pursuing the legitimate question to what extent extrapolation from one organism to the other was justified. In the next section I will focus on the problems and questions that were raised during the preparations for and performance of the trials, and again analyze what elements played a major role in constituting the conditions of practice and in (re-)shaping plans for research.

\subsection{Preparations for an early trial in volunteers}

In preparing for clinical trials at its 1960 meetings, the Scientific Committee on Interferon started with general discussions about the nature and design of testing procedures. For a detailed understanding of the trial proceedings it is important to realize that in the 1960 's in Britain there was no national system for controls regulating the testing of potential remedies. Neither the Dangerous Drug Act 1951 nor the Therapeutic Substances Act 1956 provided a regulatory context for the testing of medicinal substances in human subjects. And there were no ethical committees to sanction (or otherwise) experiments on human volunteers or formal rules on how to carry out a clinical trial. ${ }^{51}$ The British Ministries of Health left the control over testing of new pharmaceutical substances for toxicity to the good sense of the researchers and pharmaceutical companies involved. ${ }^{52}$

Minutes of the 6th meeting of the Scientific Committee on Interferon, dated 6 April, 1960, MRC Archives File No. 788/2/1; A. Isaacs, 'Interferon', Scientific American, 204 (1961), 51-7, p. 57; Interviews with John Beale, Derek Burke, Norman Finter, and David Tyrrell.

Medical ethics were primarily defined by the hippocratic oath, which all newly qualified doctors were required to swear, J.S. Porterfield to T. Pieters, letter dated 9 November, 1993.

In the late 1950's in response to public anxiety in Britain about the hazards to the public health from widespread use of potent over-the-counter drugs for self medication, the system of selfregulatory control over new medicines was subjected to growing criticism. Political momentum was building up to reconsider and strengthen British medicines regulation. Governmental steps to set up a central regulatory agency on drugs safety were stimulated by what has become known as the 'Thalidomide tragedy'(1960-62). In 1957 Thalidomide was marketed in Europe as a sleep-inducing and anti-emetic drug under the brand-name Contergan. 
Except for the routine testing of immediate toxic effects on at least two species of laboratory animals for several months no standard procedures existed to evaluate the clinical safety and efficacy of new therapeutic substances. Usually after completion of the acute toxicity studies in laboratory animals British academic and commercial laboratories began with studies on healthy volunteers. ${ }^{53}$ This earliest trial of a new drug on humans involved administering a single dose or a small number of repeated doses of the pharmacological substance to a small group of about twenty volunteers. In most cases the volunteers participating in these early trials were employees of the pharmaceutical company or institute involved in the development of a specific therapeutic substance. Volunteering came with the job. ${ }^{54}$

I will show that in the absence of a standard system for the testing of potential therapeutic drugs, the knowledge and skill assembled over the years from testing biological

The drug was claimed to be far safer than the commonly used barbiturates and became a popular sleeping-pil. However, in the course of 1960 the number of reports about serious side-effects related to the use of thalidomide began to rise sharply. By 1962 its use by pregnant women in Europe had been associated with the birth of several thousand babies who exhibited serious birth defects, most notably a condition where children were born without hands or feet named phocomelia. In the wake of the thalidomide scandal politicians and the public in America and Europe called for stronger drug laws. In Britain the issue of medicines control was put at the top of the political agenda and this led to the setting up of a Committee on Safety of Drugs ("The Dunlop Committee") in June 1963. However, a new drug regulatory legislation which would force manufacturers who wanted to obtain a Product Licence for a new medicinal preparation to provide the Government with clinical evidence of safety and efficacy, had to wait until 1971 when the Medicines Act of 1968 came into force. Within the resulting regulatory system a so-called 'Medicines Commission' acted in concert with the newly established Committee on Safety of Medicines (CSM) to advise through the Medicines Division the Minister of Health (the Licensing Authority) with respect to the safety, quality or efficacy of a drug: See, W. Breckon, The Drugmakers (Plymouth: The Bowering Press, 1972), pp. 121-41; and D. G. Grahame-Smith. Problems Facing a Regulatory Authority, in J. F. Cavalla (ed.) Risk-Benefit Analysis in Drug Research (Lancaster, MTP Press Limited, 1981), pp. 51-4: and E. M. Tansey, Catterall P.P., Christic, D.A., Willhoft S.V. and Reynolds, L.A., Wellcome Witnesses to Twentieth Century Medicine Vol I (London: Wellcome Trust Occasional Publications, 1997).

In the 1950's toxicity testing required no more than LD-50's in a single species.

As soon as the results from the volunteer studies and additional animal testing indicated that there were no serious health hazards likely to be encountered, preliminary studies in patients were initiated. In practice, it meant that the new therapeutic substance was distributed among a small group of doctors who were experts in the disease for which the substance was claimed as a therapeutic agent. These clinical investigators or 'consultants' as they were called set up trials ('suck it and see trials') in their hospital wards. Neither in the case of these preliminary studies in patients nor in the early studies in healthy volunteers did the investigator have to obtain 'informed consent' from patients and volunteers or was approval required from a humansubject review board or other qualifying agency. A special code of ethics relating to early drug testing on volunteers had to wait until 1971 when the Association of the British Pharmaceutical Industry (ABPI) publicly announced that its members would observe a new code of ethics relating to early experiments with volunteers. In 1960 in Britain you got the permission of the people involved and went out and performed the trial; This description of the drug testing practice in Britain around 1960 is based on; W. Breckon, The Drugmakers (Plymouth: The Bowering Press, 1972), pp. 121-41; A. Landsborough Thomson. Half a Century of Medical Research Vol. II (London: Her Majesty's Stationary Office, 1975), 226-243; W. Sneader. Drug Development: From Laboratory to Clinic (Chichester: John Wiley\&Sons, 1986), pp. 75-86; D. Healy, The Psychopharmacologists (London: Chapman and Hall, 1996), p. 113; and, information gathered during discussions following the presentation of a preliminary version of this chapter to the Twentieth Century Medical History Group of the Wellcome Trust, held at the Royal College of Physicians, 11 February, 1992. 
products both within the companies and the MRC played an important role in establishing testing procedures. This is nicely illustrated in the discussions about the need for and nature of safety testing. Repeatedly the question would be brought up for debate whether they should adopt an already existing safety test procedure for biologicals or devise an alternative testprotocol for interferon. ${ }^{55}$

\subsubsection{Debating safety tests and interferon standards}

At the June 1960 meeting of the Scientific Committee, the Glaxo researcher Alastair Dudgeon, pleaded in favor of employing the same stringent safety test procedures as were being used throughout the whole production process of the oral poliomyelitis vaccine, including the repeated use of quarantined laboratory animals. Only by applying these kind of 'in-process' safety controls did Dudgeon think it possible to exclude the rather worrying possibility of potentially dangerous monkey viruses or other infectious microorganisms being present in the final monkey interferon product. The Wellcome representative, David Edwards, however, objected that repeated testing in quarantined animals throughout the production process would greatly increase the expense of the project. He in turn thought it sufficient to concentrate on the safety testing of the final product. The Glaxo chemist, Karl Fantes, then argued that it might be profitable to study the effect of the virus-killing chemical, formalin, on interferon. A combined treatment of interferon with acid and formalin, which were both known for their viral inactivating capacity, might greatly increase the safety of the final interferon product without having to rely on a complex and costly series of in-process controls (see Fig. 33a, b)..$^{56}$

Eventually, on the basis of these discussions, preliminary safety requirements were drafted for the production of interferon to be used in clinical trials. This included an early virus identification test as was used for poliomyelitis vaccine, subsequent treatment with both acid and formalin, and final in vitro and in vivo control tests. Moreover, an early trial in volunteers would be organized first before any large-scale clinical trial was projected. In order to receive critical feed-back on this draft the Scientific Committee proposed to send it for comment to David Evans, the Head of both the NIMR's Division of Biological Standards and Immunological Products Control Laboratory which was housed in the old building of the NIMR at

55 Minutes of the 7th, 8th and 9th meeting of the Scientific Committee on Interferon, dated 24 June, 4 October, 5 December 1960, MRC Archives, File No. S788/2/1.

56. Minutes of the 7th meeting of the Scientific Committee on Interferon, dated 24 June 1960, MRC Archives, File No, S788/2/1. 


\section{Tiasue Culture}

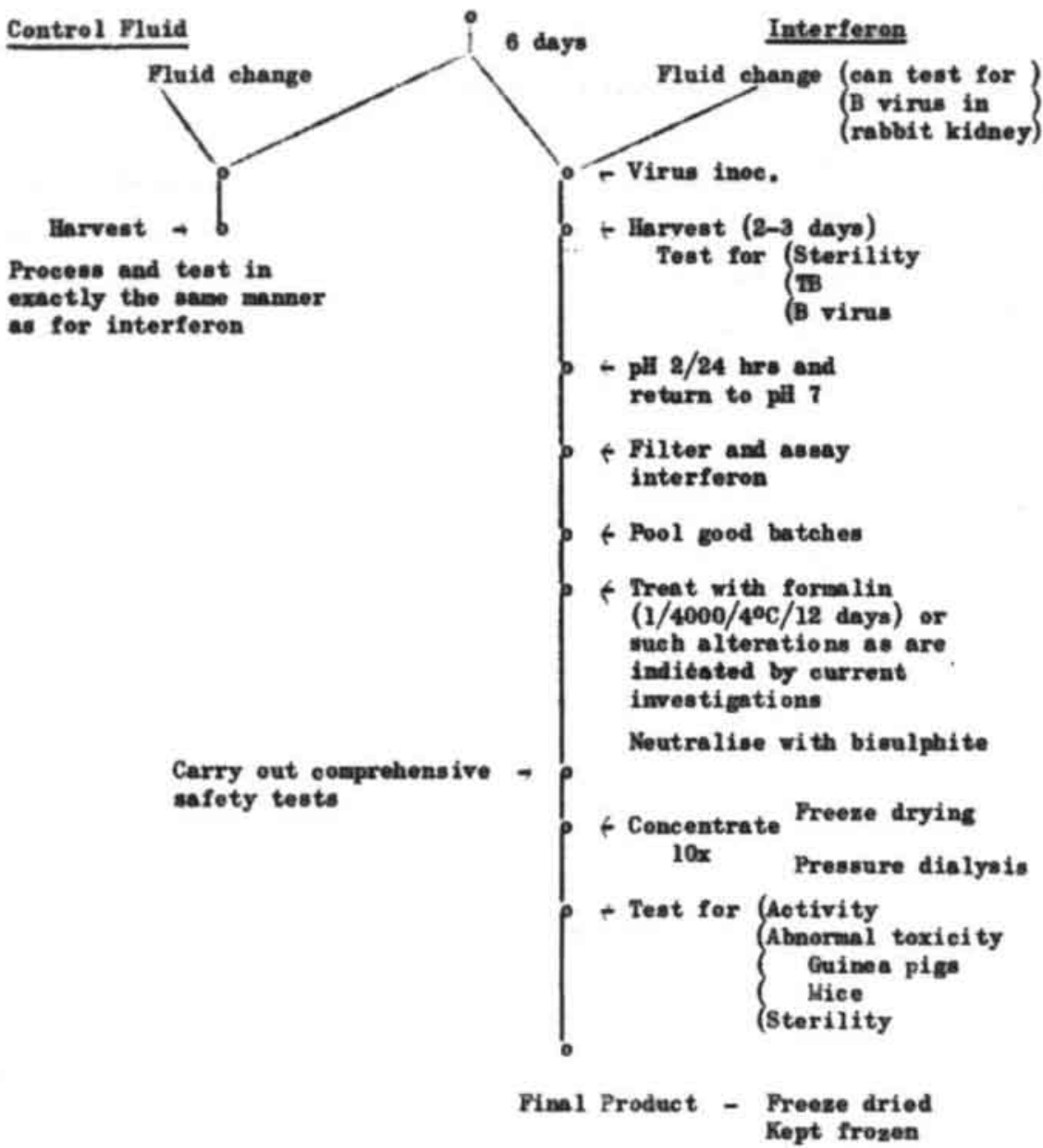

Fig. 33a. Scheme for the production of interferon for clinical trials drawn up at the loth meeting of the

Scientific Committee on Interferon held on 16 th February, 1961. 
Safety Tests

\begin{tabular}{|c|c|c|}
\hline $\begin{array}{l}\text { Stage at which Test } \\
\text { is Carried Out }\end{array}$ & Test & Purpose \\
\hline $\begin{array}{l}\text { 1. Preliminary at } \\
\text { first fluid } \\
\text { change }\end{array}$ & Rabbit kidney tissue & Virus B \\
\hline 2. Harvest & $\begin{array}{l}5 \mathrm{al} \text { (a) glueose broth } \\
\text { (b) eooked meat } \\
20 \mathrm{ml} \text { guinea pigs i.a. } \\
25 \mathrm{ml} \text { (a) monkey kidney } \\
\text { tissue } \\
\text { culture } \\
\text { (b) rabbit kidney } \\
\text { tissue } \\
\text { culture }\end{array}$ & $\begin{array}{l}\text { Bacterial sterility } \\
\text { TB } \\
\text { Virus B }\end{array}$ \\
\hline $\begin{array}{l}\text { 3. Final before } \\
\text { concentration }\end{array}$ & 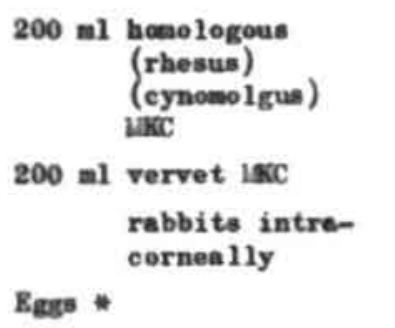 & $\begin{array}{l}\text { Sinian viruses } \\
\text { Vacuolating virus } \\
\text { Virus B } \\
\text { Residanl seed virus }\end{array}$ \\
\hline $\begin{array}{l}\text { 4. Final after } \\
\text { concentration }\end{array}$ & $\begin{array}{l}\text { Assay } \\
\text { Mice i.p. } \\
\text { Guines pigs s.c. } \\
\text { Glucose broth } \\
\text { Cooked mest }\end{array}$ & $\begin{array}{l}\text { Potency } \\
\text { A Abnormal toxicity } \\
\text { SBeterial sterility }\end{array}$ \\
\hline
\end{tabular}

Fig. 33b. Diagram of in-process safety tests to be performed during the production of monkey interferon (22-2-1961): Courtesy of NIMR. 
Hampstead about 3 miles from the Institute at Mill Hill. ${ }^{57}$ In addition they planned for a special meeting with Evans's staff of safety testing experts before finalizing a more detailed safety test protocol.

Since its establishment in 1955 as a laboratory specially aimed at exercising laboratory control for the Ministry of Health over the safety of the American (Salk) vaccine against poliomyelitis, the NIMR's Immunological Products Control Laboratory had widened its activities. Other control work for the Ministry had been added to its functions and by the early 1960 's it was involved in the safety testing of a large range of virus vaccines and other biologicals. Over the years a lot of knowledge and skill in testing the safety of biologicals had been accumulated. In asking the Hampstead people for advice the Scientific Committee on Interferon hoped to profit from this pooled experience. ${ }^{58}$ The safety test protocol that resulted from the discussions with the experts at Hampstead on Wednesday, 22nd February 1961, was based on the same 'in-process control' principle that was applied in the production of vaccines. Extensive in vitro and animal tests would be performed at several stages of the production process, so as to minimize the possibility of contamination with infectious agents. ${ }^{59}$

In the meantime the trial plans underwent changes. Isaacs proposed to cancel the trachoma experiment and concentrate on the vaccinia and common cold experiments. The latest information on trachoma was that it responded, unlike any viral disease, to some antibiotics and this casted doubt on the virus aetiology of this disease. The agent responsible for trachoma might as well be of bacterial origin and hence insensitive to the actions of interferon. Most likely, a trachoma trial in monkeys would only complicate research matters instead of contributing to the understanding of interferon's actions in vivo ${ }^{60}$ All agreed with Isaacs that taking into account the innocuous nature of interferon and the proposed extensive safety testing procedure it was justified to start with volunteer studies right away. Since a vaccinia trial seemed to require less preparatory time than a common cold trial, the former was chosen as a first experiment to test the anti-viral effect of interferon on virus infections in

57. Minutes of the 10th meeting of the Scientific Committee on Interferon, dated 16 February, 1961, MRC Archives File No. S788/2/1.

Before its move to Mill Hill in May 1950 the whole NIMR was located at the Hampstead site. Since the work on biological standards and control expanded more rapidly than expected soon it became necessary to reoccupy the old Hampstead laboratories again. Eventually in 1972 a seperate National Institute for Biological Standards and Control would be established at Hampstead; See, A. Landsborough Thomson, Half a Century of Medical Research Vol. II (London: Her Majesty's Stationary Office, 1975), pp. 250-252; and, J. Austoker and L Bryder. 'The National Institute for Medical Research and Related Activities of the MRC'. in J. Austoker and L. Bryder (eds.) Historical Perspectives on the Role of the MRC (Oxford: Oxford University Press, 1989), pp. 56-7. 
human subjects. All agreed that the first volunteers should be recruited from the staffs of the NIMR, Glaxo, Wellcome and ICI.

Apart from the nature and design of the human experiments, and the recurrent safety debate, the need for standardization was discussed at length in preparing for trials with interferon. From the very beginning the variability of assay results had been on the Scientific Committee's research agenda. Researchers reported considerable variations in the potency of interferon preparations from day to day, week to week, from researcher to researcher and laboratory to laboratory. Each time the matter came up for discussion, pleas could be heard for the standardization of assay methods and the creation of standard interferon preparations for common use. However, apart from individual efforts to set some interferon preparations aside to be used as internal standards in one's own laboratory, little was done to establish common interferon standards until the issue began to hamper the preparations for human trials.

Half-way through February 1961, the ICI researcher, Norman Finter, again raised the standardization issue. ${ }^{61} \mathrm{He}$ told the others that he was able to achieve a marked reduction in the variability of test results by expressing the potency of interferon preparations as a percentage of an arbitrary interferon standard which was assayed in parallel. By coinciding with discussions on requirements for human trials Finter's report stirred up serious interest in the standardization issue. Everyone began to realize that establishing and using a reference standard preparation was absolutely necessary to give authority to the eventual trial data, which could be appealed to under all circumstances. However, the need for standardization was also increasingly felt in the preparations for the trials. Without sharing a standard it appeared already difficult to agree on the activity of a particular preparation, let alone justifying the decision to discard a costly batch of clinical trial interferon because of its poor biological activity. Moreover, it was hardly possible to produce sound answers to important questions-like 'Does the interferon remain stable over time?', and, 'What dose of interferon is required to produce a detectable antiviral effect in humans? - in the absence of a tool to correlate and compare the potency of interferon preparations.

All these considerations underlined the immediate need for establishing a reference standard preparation for monkey interferon, to which an agreed potency would be assigned. This time things went beyond the discussion stage. The Scientific Committee decided to start effort to establish a provisional standard for monkey interferon against which all monkey interferon samples could be compared. In order to prevent the members of the Scientific Committee from describing potency in several different ways, the potency would be related to a common unit for monkey interferon. Finter indicated that, as no such unit existed, it had to be defined on an arbitrary basis once appropriate material for the standard preparation had

61 Minutes of the 10th meeting of the Scientific Committee on Interferon, dated 16 February, 1961, MRC Archives File No. S788/2/1. 
been obtained. This was the usual procedure in establishing a standard for biologicals. As the NIMR's Division of Biological Standards was responsible for establishing and supplying all British biological standards it was thought most appropriate to leave the task of keeping and distributing a provisional standard to this Division. ${ }^{62}$

The Executive Body was far less enthusiastic about the efforts to establish national interferon standards. The free circulation of standards, however practical and profitable in research terms, was perceived as troublesome in management circles. Senior company executives had just signed the final Agreement for Collaboration on Interferon and the plans with regard to establishing a provisional British standard was giving rise to concern among the Board of the Patent Holdings Company. ${ }^{63}$ It was of particular concern to them that the standard preparation would most likely be made available to workers outside the collaboration. They strongly believed that this would inevitably lead to the disclosure of valuable research information and hence to the commercial disadvantage of the collaboration.

The concerns over standardization coincided with rumors within the Executive Body that Isaacs had conveyed secret information to an outsider. Reportedly he had told Enders about his latest research results concerning the stimulation ('induction') of interferon production in cell cultures by the administration of so-called 'non-viral (foreign) nucleic acid fractions'-preparations of nucleic acid (either DNA or RNA) derived from cells not infected with viruses such as RNA from yeast cells. Moreover, reference to this highly confidential subject matter also appeared to have been made in an article that was published by Isaacs in the Scientific American. ${ }^{64}$ It was generally felt that Isaacs's unauthorized disclosures were putting efforts to establish a strong patent position for inventions that might emerge from work on the use of these foreign nucleic acids as interferon inducers in jeopardy. All agreed that not only a letter should be sent to the MRC to draw attention to the possibly damaging nature of both issues to the Company, but also a personal approach would be made to Sir Harold Himsworth. ${ }^{65}$

The affair gave rise to substantial disquiet within MRC quarters. Himsworth, who wanted at all costs to make a success out of the first formal post-war collaboration between the Government and the British pharmaceutical industry, personally demanded that Harington

62 Minutes of the 11th meeting of the Scientific Committee on Interferon, dated 19 April, 1961, MRC Archives File No. $\$ 788 / 2 / 1$.

Agreement for Collaboration on Interferon, 3 May 1961, MRC Archives, File No. A 812/5/1.

A. Isaacs, 'Interferon', Scientific American, 204 (1961), 51-7.

65

Minutes of a meeting of Directors of GNRD Patent Holdings Limited, dated 19 June, 1961, MRC Archives, File No. A $812 / 5 / 1$. 
should provide an explanation. ${ }^{66}$ Harington immediately consulted Isaacs, who unaware of having caused resentment in MRC circles, was enjoying his recent appointment as head of the Division of Bacteriology and Virus Research. Isaacs told Harington that he could not remember leaking significant information to Enders or the Scientific American. ${ }^{67}$ Isaacs was equally surprised to hear about the fuss that was made over the standardization issue. As far as he was concerned an experimental standard had been prepared for research purposes only, and it had so far only been distributed to members of the Scientific Committee. ${ }^{68}$

Through the agency of Harington, whose social skills were seriously tested, the matter was eventually settled. ${ }^{69}$ To show his good-will Isaacs formally asked approval from the NRDC to present as invited speaker his research on foreign nucleic acids as inducers of interferon to the Swedish Academy of Medicine and the Royal Society. ${ }^{70}$ As we can read in the minutes of the subsequent meeting of the Executive Body, which was held at the office of the NRDC in London on 21 September, 1961: "The Board expressed itself content with the exchanges which had taken place". This included a compromise-in-the-making about the issue of the provisional British interferon standard. Eventually the Board decided that they had no objection to the distribution of material to be used for standardization purposes only to "appropriately qualified" non-collaborating persons or parties under the control of a member of the MRC staff. ${ }^{71}$

The whole affair clearly shows the enormous energy that continued to go into bridging the differences in cultures. On the one hand, you had the government researchers like Isaacs who continued to regard the free exchange of information and laboratory materials as a precondition for fruitful research. On the other hand, virtually opposed to this position, stuck the drug company people to their perception of the same working practice as a threat to the commercial development of therapeutic drugs. But at least for the time being they were able to craft compromises.

66. Internal note MRC, dated 20 June 1961, MRC Archives, File No. A 812/5/1.

A. Isaacs, 'Interferon', Scientific American, 204 (1961), $51-7$.

Harington to NRDC, letter dated 21 June 1961, MRC Archives, File No. A 812/5/1.

69 Harington then sent a couple of letters to both the NRDC and MRC, in which he emphasized the fact that the Scientific Committee supported the agreement that the distribution of the standard would not be extended to people outside the collaborating group without prior reference to the NRDC: Harington to NRDC, letter dated 21 June 1961, MRC Archives, File No. A 812/5/1; and Harington to MRC, letter dated 10 August 1961. MRC Archives, File No. A $812 / 8 / 1$. 


\subsubsection{Handling production and safety problems}

Meanwhile, problems were forming relating to the preparations for tests in humans. The fact that the production of a large batch of monkey interferon for clinical trials took much longer and resulted in less material than was anticipated began to put the collaboration within the Scientific Committee under strain. Isaacs, in particular, was disappointed by the slow rate of progress. He was surprised to see that the scaling up of production and purification procedures took so long. The latest in a row of setbacks was the news from Glaxo Laboratories about the loss of a large batch of satisfactory monkey interferon due to one or another filtration error. Isaacs wondered whether something could be done about this rather frustrating situation and what steps were needed to speed up research work. Far too often there had been duplication of research work and, in his view, the contribution of the scientific staff of the collaborating firms could have been more substantial. The representatives of the firms agreed that progress was slow, but this was only to be expected in this stage and certainly not due to a lack of commitment. Confronted with an unexpectedly stiff opposition Isaacs gradually toned down his criticism and showed his good will by promising to pay special attention as chairman to a more efficient distribution of research work. ${ }^{72}$

Almost all of Isaacs's spare time went into keeping the wider public interested in the interferon cause. Both in lead articles in the Scientific American and the New Scientist, and on the BBC television program 'Achievement 1961' Isaacs made reference to imminent tests of interferon in humans which would show whether interferon fulfilled its promise as medicine's first really effective cure for virus infections. ${ }^{73} \mathrm{He}$ also became the first academic investigator ever to deliver a lecture at the annual British Pharmaceutical Conference (see Fig. 34).

Addressing the audience of mainly pharmacists and representatives of the British Pharmaceutical Industry he called interferon "a round unvarnish'd tale", an exciting example of how investigation in an academic field of research can lead to the practical prospect of treating virus infections in humans. Apart from informing his audience on the general state of the art of interferon research Isaacs emphasized the fact that success in reaching the stage of clinical use

72 Minutes of the 13th meeting of the Scientific Committee on interferon, dated 13 September, 1961, MRC Archives. File No. S788/2/1: Tyrrell, interview; and Finter, interview.

73 A. Isaacs, 'Interferon', Scientific American, 204 (196I), 51-7; and, S. Baron and A. Isaacs, 'Interferon and Natural Recovery from Virus Diseases', New Scientist 243 (1961), 81-2: and, Internal note MRC. MRC Archives 9 March 1962. File No. A 742/14. 
of interferon in humans largely depended on the ability of the MRC and three pharmaceutical firms to develop a productive research partnership. ${ }^{74}$ In publicly linking the success of the research venture to the success of the Collaboration Isaacs cleverly put all parties under an obligation. At the same time, by presenting the Collaboration as a crucial test-case that might be extended in the future to many other fields of research, Isaacs limited his own freedom of speech and action: neither the others nor he was supposed to prejudice its possible success.
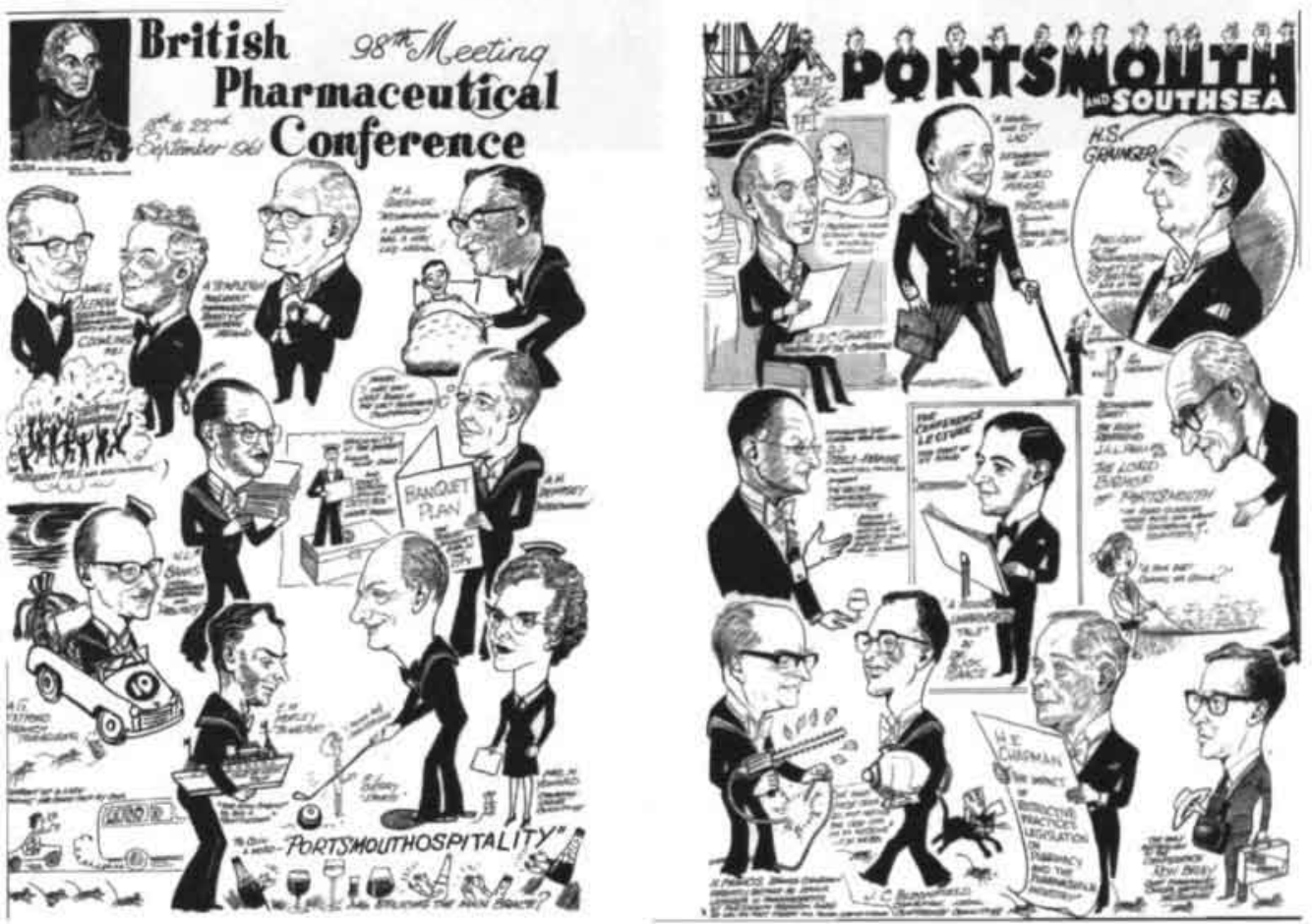

Fig. 34. A round unvarnish'd tale, Courtesy of Dr. S. Isaacs Elmhirst

74 A. Isaacs, Interferon: A Round Unvarnish'd Tale, The Journal of Pharmacy and Pharmacology Supplement, 13 (1961), pp. 57T - 61T; and, Editorial, The Chemist and Druggist, 30 September 1961, 375. 

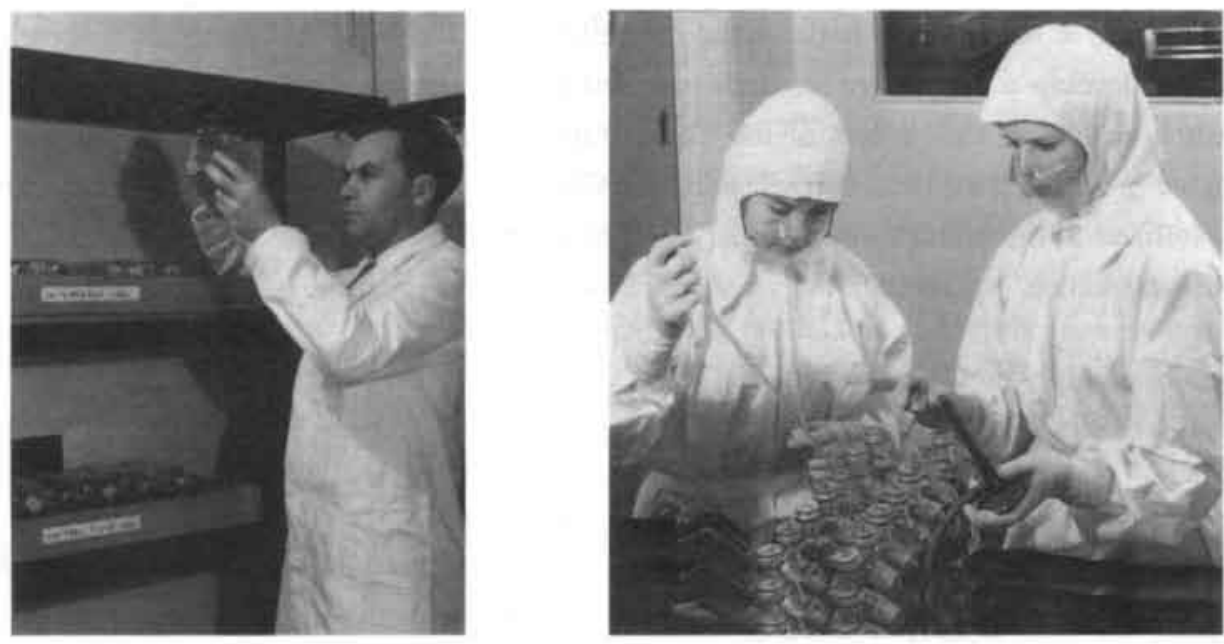

Fig 35a,b. Production of interferon at Welloome Laboratories with Dr R.F. Sellers in the left hand photo (1961),

Used with the permission of Glaxo Wellcome ple.

In November 1961, two months after the disruptive notice that a large batch of monkey interferon had been lost due to a technical failure, news came from the Wellcome Laboratories that the first three litres of clinical trial interferon and control material were ready and had proved satisfactory in both activity and sterility tests (see Fig. 35a,b). According to the agreed upon safety test protocol, the clinical trial interferon and control material had been subjected to toxicity tests in mice and guinea pigs. The toxicity test in mice went satisfactorily. However, this was not the case when the guinea pigs were used. One guinea pig out of two died three to four days after subcutaneous inoculation. Confronted with the high mortality rate in guinea pigs the Wellcome scientists immediately had decided in favor of additional tests in dogs and monkeys. The same interferon and control material had then been given to a couple of two year old dogs subcutaneously and to two rhesus monkeys intradermally, but in both cases no sign of toxicity was monitored. It was altogether a puzzling affair. What was the meaning of the high guinea pig mortality after interferon inoculation? ${ }^{75}$

The matter was brought up for discussion during a meeting of the Subcommittee on Preliminary Clinical Trials in Man at the Immunological Products Control Laboratory in Hampstead. Dr. Sellers from Wellcome Laboratories pointed out that guinea pigs were described in the literature as being extremely sensitive to penicillin. Since penicillin was used to prevent bacterial infection in the large scale culture of monkey kidney cells in which interferon

75. Interferon report by The Wellcome Research Laboratories, December 1961, MRC Archives File No. S788/2/1. 
was produced there might be enough penicillin in the interferon material to be fatal to guinea pigs. Unfortunately the lengthy debate that followed was not registered, but what I do know is that after much discussion the majority agreed that although further guinea pig tests were required to confirm their singular sensitivity to penicillin no harm would be done by proceeding at the same time with toxicity tests in human volunteers. The members of the scientific committee would perform the toxicity tests on themselves and volunteering colleagues. As a precautionary safety measure penicillin-sensitive and allergic individuals would be excluded. The proposed course of action showed the strong belief in interferon as a non-toxic natural antiviral agent in humans. Instead of waiting for the results of additional tests in guinea pigs the members of the scientific committee volunteered for self-testing. ${ }^{76}$

At the subsequent meeting of the Scientific Committee the misgivings of the past months seemed to be forgiven and forgotten with the preparations for a trial in humans moving into gear. Isaacs reported that of 5 volunteers inoculated at the NIMR only two showed an abnormal flushing of the skin after 24-hours, but only in one case was the reaction so severe as to prevent the person from being enlisted in the ultimate trial. Tyrrell had inoculated himself and three other subjects and only he himself had had an abnormal flushing of the skin. Of the five volunteers inoculated at Glaxo the members of Scientific Committee, John Beale and Robert Andrews, showed a reaction to the interferon inoculation consisting of a substantial swelling of the skin of the arm and pain but no reaction to the control. Beale argued that in having experienced a rather severe inflammatory reaction to the interferon inoculation himself, he was far less worried about the safety aspect than about the possibility that such an inflammation due to interferon might interfere with the interpretation of the experiment. In assessing the provisional results all agreed that since no alarming reactions had occurred it was justified to go ahead with the trial and start discussions on the trial design. With no standard rules how to perform a clinical trial the trial design was left to the wisdom of those involved.

Most common at the time was a so-called 'open' experiment in which the investigator was held responsible for administering the pharmaceutical substance to the volunteers or patients and monitoring their condition. On the basis of the volunteer or patient records the clinical investigator then formed impressions of the potential toxicity and the probable therapeutic value of the substance and sent a summary of the experiment together with his conclusion to the sponsor of the trial. However, the practice of testing therapeutic drugs in

Officially self-testing was and still is frowned upon and condemned as 'sloppy' science: "How can one possibly maintain objectivity in the reading of results if the experimenter and the human subject who will undergo the preliminary experiment are the same?". However, in actual laboratory practice it was the normal thing for scientists to try out any new medicines or biomedical procedures on themselves first in order to get an indication as to whether a larger field experiment was warranted. Self-testing still takes place on a much larger scale in our laboratories than anyone dares to acknowledge. Minutes of meeting of the subcommittee on preliminary clinical trials in man at the MRC, Hampstead, 4 December 1961, MRC Archives File No. S788/2/1. 
humans was slowly beginning to change in Britain. Convinced that the methods of the statistically controlled trial or so-called 'randomized controlled trial' would provide a more reliable, more objective basis for evaluating medical research and directing clinical practice, the MRC actively promoted the use of randomization in testing new therapies. ${ }^{77}$

In the most advanced type of randomized clinical trial the new agent was tested against a look-alike placebo-a harmless, inert preparation-under circumstances where neither the patient nor the doctor and nurses knew whether the remedy or the placebo was administered to particular subjects. This type of controlled experiment was usually referred to as a 'double blind' experiment. The double-blind clinic trial was the most complicated to run. Every dose of both trial medication and placebo had to be coded, and the codes had to be cryptic enough not to be cracked by the ingenious minds of the people involved. An independent third party had to keep careful records of who got what and keep those records secret until after the trial monitoring data had been gathered, counted, compared and evaluated. Among biostatisticians the double-blind trial was considered an absolute requirement if you did not want to throw the statistical validity of your test right out of the window. For instance, in a single-blind study, the 'blind' subjects might pester their physician who kept records of who got what, to tell them what they got or did not get, or the doctor might react to the condition of his patients according to their expectations about the new agent. In double-blind placebo-control studies, subjects and investigators alike were believed to be much less susceptible to psychosomatic or other personal factors which threatened to undermine the statistical validity and hence assessment of clinical trial data. ${ }^{78}$

Taking into account the central role of the MRC in the Collaboration it may not come as a surprise that the members of the Scientific Committee decided in favor of the state of the art double-blind placebo-controlled trial design. The trial would be carried out on a limited number of about forty non-allergic and unvaccinated volunteers, who would be recruited within the various collaborating laboratories. The trial procedure went as follows. ${ }^{79}$ Two sites $5 \mathrm{~cm}$ apart on the upper part of the arm of each volunteer were to be inoculated with either interferon or the control material. The ampules with interferon and control preparations would

See, for a personal account of the historical development of controlled trials in British medicine: R. Doll, 'Development of Controlled Trials in Preventive and Therapeutic Medicine', J. Biosoc Sci, 23 (1991), 365-78.

78 This description of the actual controls over new medicines in Britain around 1960 is based on: W. Breckon, The Drugmakers (Plymouth: The Bowering Press, 1972), pp. 121-41; A. Landsborough Thomson, Half a Century of Medical Research Vol. II (London: Her Majesty's Stationary Office, 1975), 226-243; W. Sneader. Drug Development: From Laboratory to Clinic (Chichester: John Wiley\&Sons, 1986), pp. 75-86; and information gathered during discussions following the presentation of a preliminary version of this chapter to the Twentieth Century Medical History Group of the Wellcome Trust, held at the Royal College of Physicians, 11 February, 1992.

79 Minutes of the 15th meeting of the Scientific Committee on interferon, dated 14 December, 1961, MRC Archives File, No. $5788 / 2 / 1$. 
be labeled either $\mathrm{X}$ or $\mathrm{Y}$ and distributed 'blind' together with special forms by a researcher from NIMR's Immunological Products Control Laboratory, who would be the only one to know the code. A so-called form A with the name of the volunteer should indicate, as decided by a random coding procedure, whether the inoculator was supposed to inoculate $\mathrm{X}$ or $\mathrm{Y}$ in the front or backside of the arm. Subsequently the inoculated areas would be marked with indelible pencil to make it possible to carry out the vaccination with attenuated cowpox virus twenty four hours later on the exact site of each of the previous inoculations. A different person, the observer, should then receive a so-called form B with only the name of the volunteer on it and spaces for daily observations for a period of 14 days as to ensure that the vaccine takes would be read 'blind'. Any possible sign of a lesion developing at the injection side would be monitored as closely as possible as inflammation due to interferon might in one way or the other affect the development of virus lesions (see Fig. $36 \mathrm{a}, \mathrm{b}, \mathrm{c}$ ). ${ }^{80}$

On Thursday the 22nd February 1962 the great day had come for Isaacs and the other members of the Scientific Committee on Interferon to exchange and discuss the results of the first trial of its kind to see whether interferon prepared in the laboratory could prevent the establishment of a virus infection in humans. Unusually large delegations of all the parties involved were assembled for the occasion in one of the conference rooms of the NIMR and waited anxiously for the chairman to open the meeting. Isaacs cheerfully announced that the trial with interferon had yielded promising results. ${ }^{81}$

At the NIMR 17 out of 23 volunteers showed a clear protection at the interferon site against cowpox infection and the development of vaccinial lesions. As far as interferon's protective effect was concerned the NIMR volunteer group had been exceptionally successful. At Wellcome Laboratories 7 out of 19, at Glaxo Laboratories 2 out of 4, at MRC Hampstead two out of seven and at the Common Cold Unit only 1 out of five volunteers showed clear protection against virus infection. Despite the considerable deviation in test results between the various volunteer groups the overall protection rate of $60 \%$ against virus infection was considered a definite success. ${ }^{82}$

All agreed that the results of the clinical trial were certainly qualified for publication in either the Lancet or the British Medical Journal. Isaacs immediately offered his services to prepare a first draft. This time even the commercial side to the Collaboration encouraged him

80 Minutes of the 15th meeting of the Scientific Committee on interferon, dated 14 December, 1961, MRC Archives File, No. S788/2/1.

81 Minutes of the 16th meeting of the Scientific Committee on interferon, dated 22 February, 1962, MRC Archives File, No. S788/2/2; and Tyrrell. interview.

Ibid. 


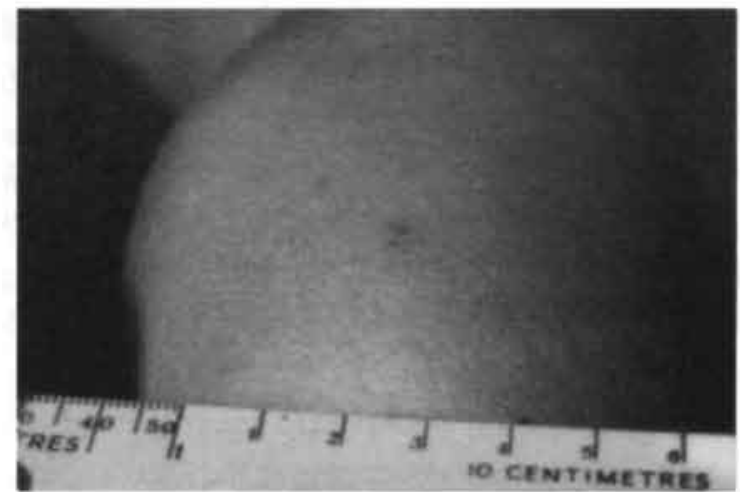

Fig $36 a$. The arm of a human volunteer before vaccination.
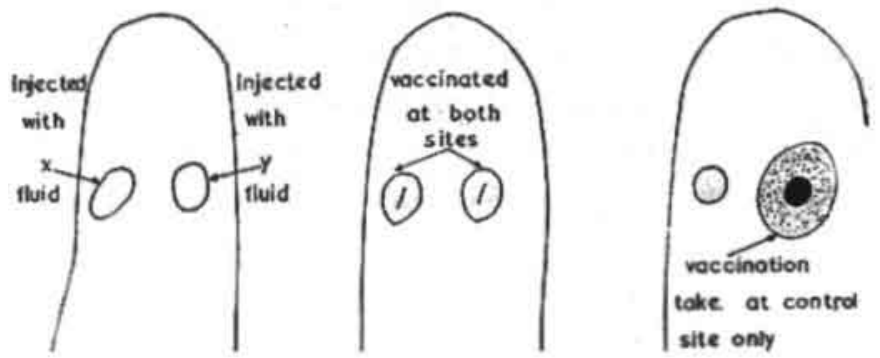

Fig 36b. Principle of the vacination experiment with interferon. The arm is injected with two fluids, ' $x$ ' being interferon and ' $y$ ' an inactive control material for comparison. Subsequently smallpox vaccinations are made at both sides.

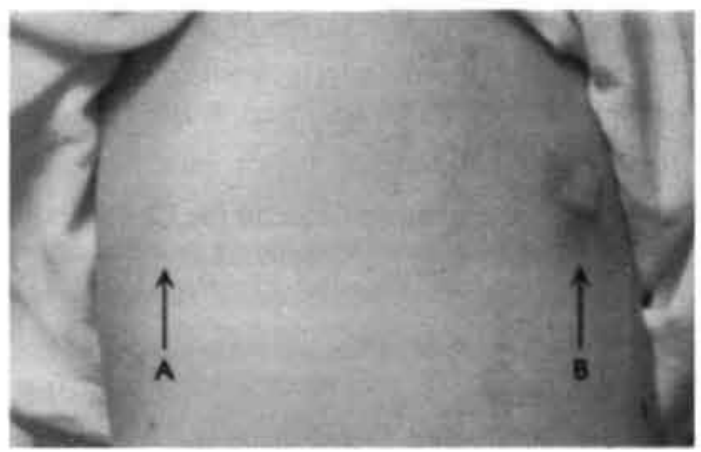

Fig 36c. The arm of a human volunteer after vaccination. The vaccine has clearly 'taken' in site B (no interferon),

but not in the other (interferon).

Courtesy of D. Tyrrell. 
to do so. All parties realized that with such a publication they would have a strong case in support of the American patent application. For once the interests with regard to the dissemination of information of both government researchers and company executives seemed to correspond.

\subsubsection{The Lancet report.}

As promised, Isaacs prepared a rough draft of the trial results and circulated it to the members of the scientific committee and to the MRC before finalising it as an official report to the MRC from the Scientific Committee on Interferon, and submitting it for publication to the Lancet. During a routine check of the trial data in Isaacs's report, the MRC's medical officer who had been present at the presentation of the preliminary results, came across oddities in the figures from the Wellcome laboratories. As far as he could remember more than the reportedly three volunteers had participated in the Wellcome trial group. Furthermore he could not avoid the impression that apart from the fact that the vaccinator and the observer had been one and the same person, this individual also seemed to have taken part as a volunteer in the trial. The medical officer immediately informed Isaacs, who confirmed that his suspicions were correct: "Dr Isaacs telephoned me this morning to say that the query I had raised in my letter to him of 2nd April about the results from the Wellcome laboratories had been very astute (more so than I had suspected)" ${ }^{83}$

Isaacs told him that after the meeting of the scientific committee he found out to his dismay that the Wellcome scientists had made a mess of things. How on earth could the monitoring of the volunteers have been blind if there was no difference between the vaccinator and the observer? They even appeared to have forgotten about the agreed upon single arm procedure and inoculated volunteers in both arms. By tampering with procedures the Wellcome laboratories obviously had broken the rules of the trial which was supposed to be double blind. Isaacs indicated that he had tried to cover up for Wellcome's sloppy data in his report by including only 3 out of the 19 volunteers tested at the Wellcome Laboratories. The issue worried him and he asked the MRC officer advice on what to do next. Would it be best to make no reference to the Wellcome data at all as suggested most recently by David Edward of Wellcome, who had been away in the United States during the time of the trial?

In response the medical officer urged Isaacs to discuss the matter with Sir Charles Harington. Eventually, in consultation with the research director of the Wellcome Laboratories, Colonel Mulligan, Harington decided that the Wellcome laboratory figures should be 
Fig 37: Results of individual vaccinations in the vaccination trial ${ }^{84}$

\begin{tabular}{|c|c|c|c|c|}
\hline Laboratory & Volunteer & Site treated with & $\begin{array}{l}\text { vith } \\
\text { Control }\end{array}$ & Interferon at \\
\hline $\begin{array}{l}\text { National Institute for Medical Research } \\
\text { Inoculations: } \\
\text { Dr. G.W. Bissett and Dr. D.H. Sproull } \\
\text { Observations: } \\
\text { Dr. A. Isaacs }\end{array}$ & $\begin{array}{l}\text { D.H. } \\
\text { K.R.P. } \\
\text { J.S.C. } \\
\text { B.B. } \\
\text { F.C.F. } \\
\text { S.S. } \\
\text { K.M.S. } \\
\text { W.J.B. } \\
\text { N.G.B. } \\
\text { J.M.G. } \\
\text { A.C. } \\
\text { R.C. } \\
\text { S.D.D. } \\
\text { B.D. } \\
\text { M.H.G. } \\
\text { T.G.J. } \\
\text { G.A. } \\
\text { S.C. } \\
\text { J.E. } \\
\text { M.G. } \\
\text { V.H. } \\
\text { J.J.S. } \\
\text { W.F.W. }\end{array}$ & $\begin{array}{l}0 \\
0 \\
++ \\
0 \\
0 \\
0 \\
0 \\
+ \\
0 \\
+ \\
0 \\
0 \\
+ \\
0 \\
0 \\
0 \\
0 \\
0 \\
0 \\
0 \\
++ \\
+ \\
0 \\
\end{array}$ & $\begin{array}{l}++ \\
++ \\
0 \\
++ \\
++ \\
++ \\
++ \\
++ \\
++ \\
++ \\
++ \\
++ \\
++ \\
++ \\
++ \\
++ \\
++ \\
++ \\
++ \\
++ \\
++ \\
++ \\
++ \\
++ \\
++\end{array}$ & $\begin{array}{l}\text { Front } \\
\text { Front } \\
\text { Back } \\
\text { Back } \\
\text { Front } \\
\text { Front } \\
\text { Back } \\
\text { Front } \\
\text { Front } \\
\text { Front } \\
\text { Back } \\
\text { Back } \\
\text { Back } \\
\text { Front } \\
\text { Front } \\
\text { Back } \\
\text { Back } \\
\text { Front } \\
\text { Back } \\
\text { Back } \\
\text { Back } \\
\text { Front } \\
\text { Back } \\
\end{array}$ \\
\hline $\begin{array}{l}\text { Common Cold Research Unit, Salisbury } \\
\text { Inoculations: } \\
\text { Dr. D.A.J. Tyrrell } \\
\text { Observations: } \\
\text { Dr. M.L. Bynoe }\end{array}$ & $\begin{array}{l}\text { V.M.B. } \\
\text { A.E.M.G.H. } \\
\text { P.K.B. }\end{array}$ & $\begin{array}{l}+ \\
0 \\
+\end{array}$ & $\begin{array}{l}++ \\
++ \\
++\end{array}$ & $\begin{array}{l}\text { Back } \\
\text { Back } \\
\text { Front }\end{array}$ \\
\hline $\begin{array}{l}\text { Immunological Products Control, } \\
\text { Hampstead } \\
\text { Inoculations: } \\
\text { Dr. M. Clarke } \\
\text { Observations: } \\
\text { Dr. M.M. Winter }\end{array}$ & $\begin{array}{l}\text { J.W. } \\
\text { D.J. } \\
\text { H.H. } \\
\text { A.P. } \\
\text { B.H. }\end{array}$ & $\begin{array}{l}++ \\
0 \\
0 \\
+ \\
0\end{array}$ & $\begin{array}{l}-1 \\
+4 \\
++ \\
++ \\
++ \\
++\end{array}$ & $\begin{array}{l}\text { Back } \\
\text { Back } \\
\text { Front } \\
\text { Front } \\
\text { Front }\end{array}$ \\
\hline $\begin{array}{l}\text { Glaxo Laboratories } \\
\text { Inoculations: } \\
\text { Dr. R.J. Andrews } \\
\text { Observations: } \\
\text { Dr. A.O. Hagger } \\
\end{array}$ & $\begin{array}{l}\text { P.L. } \\
\text { R.H. } \\
\text { A.C. } \\
\text { R.C. }\end{array}$ & $\begin{array}{l}0 \\
0 \\
++ \\
++\end{array}$ & $\begin{array}{l}+ \\
++ \\
++ \\
++ \\
++\end{array}$ & $\begin{array}{l}\text { Back } \\
\text { Front } \\
\text { Back } \\
\text { Back }\end{array}$ \\
\hline $\begin{array}{l}\text { Wellcome Laboratories } \\
\text { Inoculations: } \\
\text { Dr. T.M. Pollock and } \\
\text { Dr. W.T.W. Lawson } \\
\text { Observations: } \\
\text { Dr. T.M. Pollock }\end{array}$ & $\begin{array}{l}\text { Mrs. G. } \\
\text { Mr.W. } \\
\text { T.M.P. }\end{array}$ & $\begin{array}{l}0 \\
++ \\
+\end{array}$ & $\begin{array}{l}++ \\
++ \\
++\end{array}$ & $\begin{array}{l}\text { Front } \\
\text { Front } \\
\text { Back }\end{array}$ \\
\hline $\begin{array}{ll}++= & \text { Definite take } \\
+= & \begin{array}{l}\text { Take, but distinctly smaller than } \\
\text { the take at the other site. }\end{array} \\
0= & \text { no take }\end{array}$ & & & & \\
\hline
\end{tabular}

84 Scientific Committee on Interferon. 'Effect of Interferon on Vaccination in Volunteers', Lancet, i (1962), 873. 5. p.874. 
deleted from the text of the report. ${ }^{85}$ In the meantime the original report had been submitted to the Lancet but was being held up until the matter had been settled. With the "delete decision" in mind, it is surprising then to see that in the final Lancet report the three Wellcome volunteers can still be found. Furthermore, both in the case of the Common Cold and Hampstead figures, two volunteers are missing (see Fig. 37). ${ }^{86}$

According to the report in the Lancet-"A report to the Medical Research Council from the Scientific Committee on Interferon" - the clinical trial with interferon to study the effect of interferon on vaccination in volunteers was carried out as a result of unambiguous experiments with an antiviral substance in the laboratory. The transition from experiments in the test-tube and in laboratory animals to experiments with humans was depicted as unproblematic. In addition, the trial was said to have proceeded smoothly and unmistakably showed that interferon had an antiviral effect in humans. ${ }^{87}$

Obviously the contingencies and uncertainties which the committee members had faced in preparing for and performing the vaccination trial and the simultaneous management of uncertainty were deleted from the public record. While Isaacs proclaimed the trial a success and wrote in the New Scientist that work on interferon had "just passed a critical stage" by producing statistically highly significant results which showed that interferon protected human volunteers against infection with the vaccinia virus, the managing boards of the companies judged the efficacy data differently. ${ }^{88}$ In their opinion the resources invested in the trial efforts were in no proportion to the experimental results: interferon had only shown a protective effect in a clinically insignificant viral infection under controlled circumstances. The trial was not regarded as particularly informative as to whether or not interferon deserved the label therapeutically-interesting with regard to everyday practice. They were concerned about the already high costs of producing minute amounts of semi-purified monkey interferon, not to mention the possible production costs of human interferon.

Going by the information from their scientific staff the production process seemed to be bedeviled by technical problems too and much more basic laboratory work would be needed than expected to figure out interferon's clinical potential. The idea began to settle within management circles that it might take years before the large-scale commercial production of material suitable for clinical use might even be taken into consideration. With the growing doubts about whether interferon was indeed worth the big effort that was being put into it, the

85 Alick Isaacs to Derek Burke, letter dated 5 April, 1962, Derek Burke Correspondence, Norwich, personal archives: and Internal Note MRC, dated 4 April, 1962, MRC Archives File No. A813/127.

Unfortunately the current editorial staff of the Lancet was unable to locate the 1962 Lancet editorial files dealing with the publication of the vaccinia trial report from the Scientific Committee on Interferon; D. Sharp to T. Pieters, letter dated 25 March 1998, T. Pieters personal archives.

87 Scientific Committee on Interferon. 'Effect of Interferon on Vaccination in Volunteers', Lancet, i (1962), $873-$ 5. p.875.

A. Isaacs, 'Interferon tried in man', New Scientist, 3 May 1962. 
commitment of the three drug companies to interferon came under pressure. ${ }^{89}$

My analysis of the 1962 Lancet report shows that the consistency of an experimental study is influenced by more than the rigor of its experimental design and the statistical elaboration of the trial data; this can be seen by the way test objects are recruited, by the compliance of those involved to the agreed-upon trial procedures, and by the way in which decisions for starting, stopping and reporting a study are made. Moreover, in closely paralleling what one might call a treatment's scientific basis, the published efficacy data appeared to be of little value to those primarily interested in assessing interferon's therapeutic potential in everyday practice ('effectiveness'). I will show in the next section how following the publication of the first trial report a gradual reversal of commitments and objectives of those involved came about. Questions were raised about whether or not the development of interferon as a therapeutic drug constituted a worthwhile pursuit, and about the appropriate research approach among those working at the bench and administering the collaboration.

The dissensions which arose in the process reflected the conflicting time-horizons and conflicting ways of handling and judging research data of the various parties involved in the Collaboration. The academic scientists were foremost interested in creating new opportunities for research, advancing their scientific careers and the prestige of the institute or research organization. They regarded interferon as a somewhat unruly but promising biological substance that represented a major new stratagem of defense against infection by viruses. Sooner or later interferon would prove of use to medicine in one way or another-if not as an end in itself than as a means for stimulating people to make their own interferon. However difficult and time-consuming, this was not believed to be beyond the powers of modern pharmaceutical technology available through the pharmaceutical industry.

The executives of the drug companies, on the other hand were trained in evaluating research projects rather sooner than later in terms of their market potential, and the likely time and expenses involved in translating laboratory data and processes into practical and commercially-viable medical technologies. They took note of the published efficacy data but reacted to what they judged and perceived to be therapeutically-interesting remedies. They actively created their own profile of the treatment's effectiveness, attaching much importance to commercial-viability (research expenses compared to market potential) and social-acceptability (whether or not a provisional every-day therapeutic rational existed for its use). Their negative assessment of interferon's therapeutic potential was accompanied by mounting pressures for accountability. 


\subsection{Interferon losing its momentum}

Company scientists working on interferon sensed a shift of research priorities away from interferon. Unlike before, their research plans and efforts were assessed with the utmost rigor in competition for company research resources. It is unlikely that its development as a therapeutic drug would have been pursued much longer on the ground of purely economical motives. It is in this context that the 'American penicillin syndrome' continued to play a most vital role in sustaining interest. ${ }^{90}$

All senior company executives were aware of the fact that one of the U.S. leaders in biologicals, the large drug company Merck Sharpe \& Dohme, was putting considerable resources into interferon research. While the British Interferon Collaboration invested heavily in efforts to test the effect of interferon in humans, the American drug firm focused on biochemistry: making sure that interferon could be processed to a high degree of purity in a cost-effective way. ${ }^{91}$ In a most recent informal talk with one of the members of the Board of the Interferon Collaboration, Max Tishler, the president of Merck Sharpe \& Dohme Research Laboratories at Rahway, New Jersey, had indicated that it would only be a matter of time before interferon would be obtained in pure crystalline form. ${ }^{92}$ This kind of competitive rhetoric from the other side of the Atlantic kept the various research projects dealing with interferon within the three drug companies going, though on a much more modest level.

It did not last long before the lower rating of interferon within drug company quarters made itself felt within the Scientific Committee. Isaacs's ambitious plans to step up immediately the production of clinical trial interferon to proceed to further clinical trials met with increasing opposition from Glaxo, Wellcome and ICI scientists. Confronted with a decline in research resources and increasingly critical managements they questioned more and more frequently the aim and relevance of the Committee's research program. Instead of stepping up the costly and troublesome production of monkey interferon and expanding the clinical trial program, the representatives of the drug firms argued in favor of doing more animal work and more chemical studies on purification. As the most outspoken critic of a continued clinical trial approach, the ICI researcher Norman Finter was on a collision course with Isaacs. ${ }^{93}$

Interviews with John Beale, Derek Burke, Norman Finter, David Tyrrell and Karl Fantes. 


\subsubsection{Emerging conflicts over research approaches}

The mutual dissatisfaction over research policies began to manifest itself when the material consequences of the eroding support for the clinical trial approach were making themselves felt through a shortage of clinical trial interferon. By the end of May 1962 Isaacs was fed up with the situation. The fact that the collaborating firms continued to fail to meet the agreed-upon production requirements originally seemed to confirm his earlier fears that the firms were dragging their feet. In consultation with the MRC, who told him not to beat the big drum, Isaacs decided that he would first challenge the firms in the Scientific Committee. As chairman he felt it would be relatively easy to put the problematic supply position of interferon on the agenda. $^{94}$

At the last Committee meeting before the 1962 summer holidays Isaacs brought the matter up for discussion. In accordance with previous plans, the next step should be for Tyrrell to perform a trial at the Common Cold Unit in Salisbury in order to test the effect of interferon on the common cold in volunteers. The idea was that if the human nose and pharynx was treated repeatedly with interferon by means of nasal drops or nasal spray it might become protected against infection with viruses capable of causing colds like rhinovirus. ${ }^{95}$ The trial would be carried out in line with the general experimental routine at the Common Cold Unit-fortnightly trials with volunteers kept under strict isolation at the special volunteer flats, daily clinical examinations, a short quarantine period to exclude intercurrent infections, and a double blind assessment of symptoms. ${ }^{96}$ Isaacs emphasized that Tyrrell did not envisage having difficulties in finding appropriate volunteers for the interferon trial. The Common Cold Unit had a regular supply of volunteers from the general public, mostly students and civil servants, who were willing to participate in the Unit's ongoing common cold studies program with a nationwide reputation. The interferon trial would be run side by side the regular research on common colds. ${ }^{97}$

However, Isaacs sneered, with the current problematic supply there would be hardly any need for volunteers. ${ }^{98}$ The available clinical trial interferon only allowed for 3 instead of the

Internal note MRC, MRC Achives File No. S 788/2; and MRC to Charles Harington, letter dated 29 May, 1962, MRC Achives File No. S 788/2.

95 Minutes of the 16th meeting of the Scientific Committee on interferon, dated 22 February, 1962, MRC Archives File, No. S788/2/2

D. Tyrrell, The Common Cold Unit 1946-1990: Farewell to a Much-Loved British Institution', PHLS Microbiology Digest. 6 (1991), 74-6.

97 In order to maintain a high public profile and attract enough volunteers for its regular common cold studies program, regular press visits were organized to the Unit's laboratories and spacious centrally-heated volunteer huts: Interview with David Tyrrell.

98 This impressionistic account of the 18th meeting of the Scientific Committee on Interferon is based on: Minutes of the 18th meeting of the Scientific Committee on Interferon, dated 2 July, 1962, MRC Archives File, No. S788/22; Internal note MRC, 11 July 1962, MRC Archives File, No. S788/5; Internal note MRC, 16 August 1962, MRC Archives File, No. S788/2/2; I. Gresser, Production of Interferon by Suspensions of Human Leucocytes, P.E.S.B.M., 108 (1961) 799-803; Ion Gresser to John Enders, letter dated 26 June 1962, 
planned number of 20 volunteers per trial. With less than a handful of trial subjects one could not expect to generate statistically significant data. Isaacs was willing to accept that at Wellcome Laboratories the production of interferon was affected by organizational problems. The two scientists who had been undertaking the production and purification work at Wellcome had been transferred, and it was only to be expected that the production would fall or stop in consequence until substitutes would have been found. However, the other parties had no valid excuse for their shortcomings.

Glaxo's representatives on the scientific committee time and again promised half a liter of concentrated interferon, the last time within a month. When at last he decided to contact someone at Glaxo more immediately concerned with the matter, Isaacs learned that no such goal was set and that there were still numerous problems to overcome. The situation at ICI was even worse. Isaacs's impression was that ICI not only produced no signs of producing any clinical trial interferon, but did not even seem to be prepared to undertake any initiatives in that direction.

Finter rather boldly confirmed this position. He said that he could not envisage ICI making interferon for use in the common cold trial from (primary) monkey kidney cells and, to be honest, he thought that there was no ultimate hope for the production of interferon from any primary tissue culture. At ICI they preferred to concentrate on animal work, on the mode of action of interferon and on the production and purification of interferon. He thought it absolutely necessary to develop a more practical way of making interferon since the current procedures were commercially unacceptable.

It cost quite some time to ease the tension between Finter and Isaacs, but towards the end of the meeting the situation was looking up a little. Without succeeding to recapture his full authority as chairman of the Scientific Committee, Isaacs settled the differences by compromise: they would await the results of the common cold trial before entering into further debates about the research program.

Isaacs's disillusionment was great when at the end of September 1962 Tyrrell revealed that the trial results had been disappointingly negative. After having tested interferon against three different common cold viruses in 20 volunteers there was no clinical evidence of a protective effect (see Fig. 38). However negative his trial results looked, Tyrrell indicated that he had not given up hope. Personally he did not consider discontinuing this line of work as it would most likely only be a matter of increasing the dose of interferon. ${ }^{99}$ Tyrrell's reassurance could not prevent the company scientists from reopening the discussion on the targets for future work on interferon. The company scientists seized the opportunity with both hands to bring the research program more in line with the standard research trajectory for biologically

Ion Gresser personal archives. And interviews with John Beale, Derek Burke, Norman Finter, and David Tyrrell.

Minutes of the 19th meeting of the Scientific Committee on Interferon, dated 25 September 1962, MRC Archives File, No. S788/2/2; D. Tyrrell, Interim Report Clinical Trials with Interferon up to 24th September, 1962, MRC Archives File, No. S788/2/2; and, Internal Note MRC, 26 September 1962, MRC Archives File. No. $5788 / 2 / 2$. 


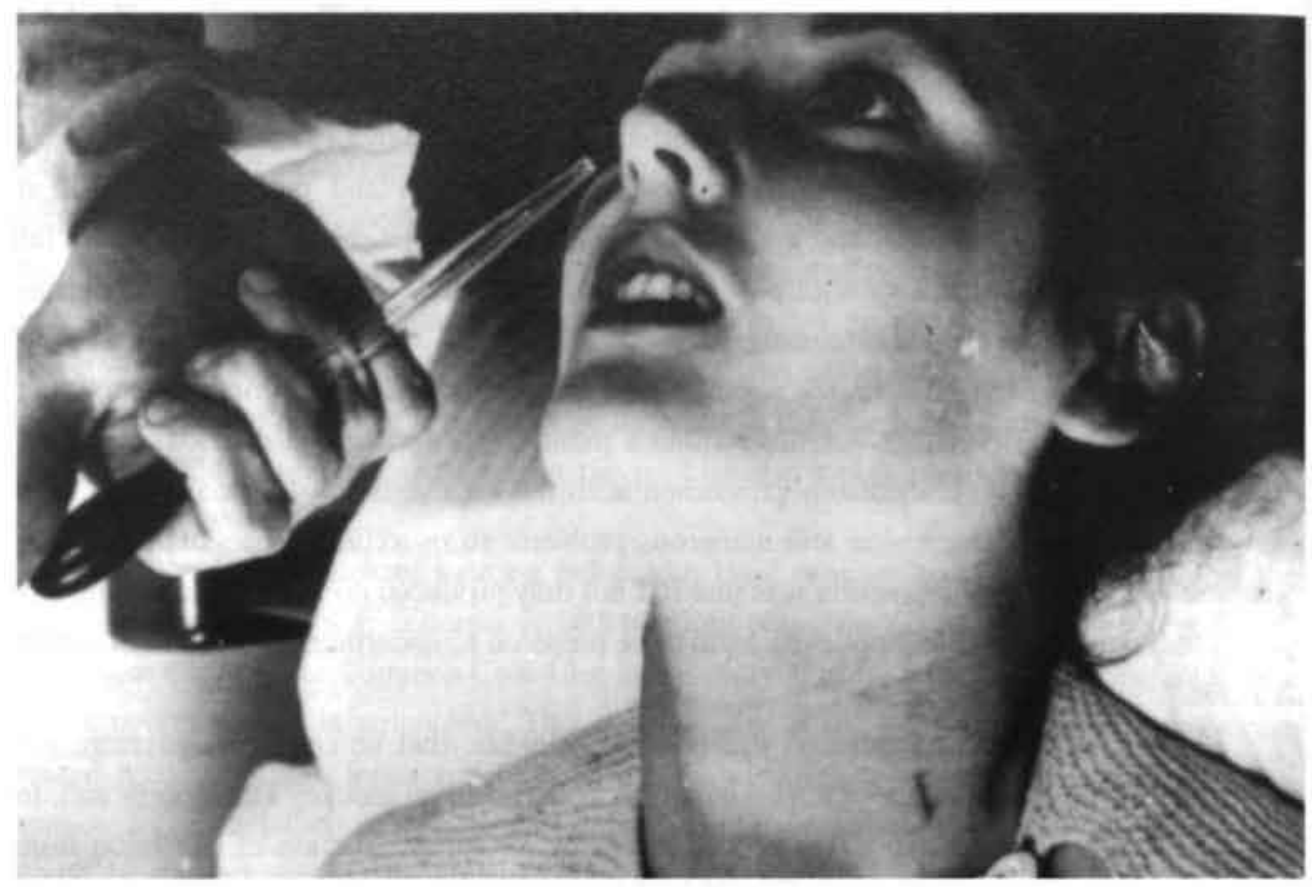

Fig. 38. After having inhaled an interferon preparation the woman volunteer (one of the 'guinea-pigs' at the Common Cold Unit at Salisbury) is now infected with common cold virus. Courtesy of David Tyrrell

active compounds in the pharmaceutical industry-focusing on animal testing and production processes first.

They pointed out that the Scientific Committee now had a considerable amount of experimental information showing that human clinical trials should wait until better methods for the production and purification of human interferon were developed. The most practical and cost-effective way to find out whether interferon did do something in infections, and to learn about dose-response relationships, definitely was the use of laboratory animals. They could then simultaneously try to develop a more practical way of making interferon for human application since the use of monkeys not only was cumbersome but also commercially unacceptable. Isaacs must have realized that the preparedness to collaborate actively and closely on a clinical trial program had just received yet another blow, although he managed to keep putting off a decision on the issue..$^{100}$

Outside the private rooms of the collaborators few seemed to know that the Interferon Collaboration went through hard times. A clear indication was the following story entitled "Whitehall men join battle on common cold: This new drug may be the answer", which

100 Minutes of the 19th meeting of the Scientific Committee on Interferon, dated 25 September 1962, MRC Archives File, No, S788/2/2. 
appeared in the Evening Standard on Friday, October fifth:

\begin{abstract}
Scores of top Civil Servants are being asked to take 10 days off to act as "guinea pigs" in the first large-scale trial of a British medical discovery which doctors hope can be used to beat many virus diseases including the common cold...scientists will inject them with a newly-discovered substance called interferon and at the same time try to infect them with thousands of cold germs. The hope is that colds will not break out...A Medical Research Council spokesman told me today: ..."Not everybody will be given interferon: some will get water or a salty solution but nobody will know who has which. We have to satisfy the statisticians that any effect on colds is not due to chance."
\end{abstract}

The final assessment 'indoors' of the carefully controlled common cold trial in December 1962 doubtless must have satisfied the statisticians, but it was disastrous for the clinical trial program. The number of volunteers which either developed disease symptoms or improved on the interferon treated group was said to differ insignificantly from the control group. It seemed pointless to pursue these clinical studies any further until more information had been obtained on dose-effect relationships and the method of administration. Even Isaacs agreed that the trials should not be proceeded with for the present, knowing that none of the collaborators was willing to continue the costly production of monkey interferon to perform further clinical trials.

Isaacs reconciled himself to the situation and decided in favor of more animal experimental work in the hope of finding a better test model. Moreover, confronted with the negative sentiments regarding the testing of the clinical potential of 'exogenous' interferon, Isaacs chose to ride his latest hobbyhorse. He told the other members of the Scientific Committee that after all he was becoming more and more convinced that interferon injected or otherwise given to patients might prove less useful than developing a means for stimulating people to make their own interferon. He pointed out that he soon hoped to clarify the use of foreign nucleic acids as a means of stimulating 'endogenous' interferon production through tests in laboratory mice. ${ }^{102}$ In his view this would make a most promising new approach for future work of the committee. However Isaacs had lost too much political capital to get a hearing for what the company scientists already had dubbed 'Isaacs's ludicrous nucleic acid story.. ${ }^{103}$ By reacting rather halfheartedly to his proposed change of research priorities the fellow-members of the Scientific Committee made him feel more and more isolated and exposed.

The December meeting of the Scientific Committee not only proved to be somewhat of a landmark inasmuch as the decision had been taken to stop the clinical trial program but also with regard to Isaacs's fragile mental health. Isaacs's continuous concern about the working of the Collaboration, the disappointing trial results, and the growing opposition to his research guidance within the Scientific Committee began to take their toll. The succession of problem-

\footnotetext{
101

Peter Fairley, Whitehall Men Join Battle on Common Cold, Evening Standard, Britain, 5 October, 1962.

102

Minutes of the 20th meeting of the Scientific Committee on Interferon, dated 4 December 1962, MRC Archives File, No. S788/2/2.

103

Interviews with Norman Finter and John Beale.
} 
atic events was instrumental in provoking a second bout of depression that was more severe in nature than the first three years earlier. He felt extremely restless and irritable most of the time with little self-confidence. This time Isaacs had to go into mental hospital where he received extensive medical treatment for a couple of months. ${ }^{104}$

Meanwhile across the Atlantic Ocean at the Merck Institute for Therapeutic Research, a paper had been cleared for publication through Merck Sharp \& Dohme's (MSD) patent office that would play a major role in the reordering of the British research agenda as well as research organization concerning interferon. Sections of this paper on the purification and characterization of interferon had already been leaked purposely to MSD's British rivals. ${ }^{105}$ In making a tentative approach early in 1962 to join the Collaboration on Interferon (which was ignored by the British) Max Tishler, the President of Merck Sharp \& Dohme Research Laboratories revealed that his researchers had prepared interferon material that was far superior to anything the British had thus far produced. Almost simultaneously Maurice Hilleman MSD's head of Virus and Cell Biology Research - a man who had a rather strong ego and was not averse to competition and throwing his competitors into confusion-let Isaacs informally know that they had prepared chick interferon that not only was 200 hundred times more pure than Burke's interferon but also had a different molecular weight. ${ }^{106}$

While these American newsleaks mainly had the effect of stimulating the sense of rivalry on the British side without really affecting work on interferon, the formal publication of MSD's studies on interferon in the prominent American journal Proceedings of the Society of Experimental Biology and Medicine had a more profound impact. ${ }^{107}$ Reading in black and white that for years British researchers had studied interferon which essentially contained only minute amounts of the interferon agent and consisted largely of extraneous material, was quite a different cup of tea. Hilleman's research team claimed to have purified interferon 4500 times with respect to initial protein content-an unprecedented degree of purity of interferon material. ${ }^{108}$ In the article the previous failures of purification were articulated by juxtaposing purity with impurity: emphasizing the marked difference in properties between their highly purified interferon and the allegedly crude interferon studied heretofore by other researchers, most notably Isaacs and his British colleagues.

Minutes of the 20th and 21th meeting of the Scientific Committee on Interferon, dated 4 December 1962 and 7 March 1963, MRC Anchives File, No. S788/2/2,3; Internal note MRC, 4 December 1962, MRC Archives File. No. S 788/1; D. Tyrrell, Experiments With Interferon, Third Draft of Interim Report to the Medical Research Council by the Scientific Committee on Interferon, MRC Archives File, No. S788/2/2.

Interview with Hilleman.

See, for a detailed social-historical description and analysis of the development of biologicals at Merck. Sharp \& Dohme between 1895-1995: L. Galambos and J. E. Sewell. Networks of Innovation (Cambridge, University of Cambridge Press, 1995).

G. Lampson, A. Tytell, M. Nemes and M. Hilleman, 'Purification and Characterixation of Chick Embryo Interferon', P.S.E.B.M., 112 (1963), 468-78.

Ibid. 
Although the disagreement was not complete it was substantial enough to throw serious doubts on the British research achievements so far and to further weaken the British patent position. To the same extent that it lent credibility to MSD's efforts, the American publication threw discredit on the work of the British collaborators. In doing so it would have an additional discouraging effect on senior British company executives to invest considerably

in interferon thereby reshaping the balance of forces both within and outside the Interferon Collaboration.

\subsubsection{Clipping Isaacs's wings}

By the time Isaacs had recovered from the severe manic-depressive episode at the end of April 1963, deputy chairman David Tyrrell had brought the American article under the attention of most members of the Scientific Committee on Interferon. Finter was first to react. ${ }^{109}$ In a series of two letters to Isaacs, he mentioned having read the studies on interferon published by Hilleman's research group and indicated that on the face of it these appeared to be an advance over anything which had been achieved within the Collaboration on Interferon. In Finter's opinion the collaboration's lack of success in purifying interferon remained a major stumbling block to further progress in the development of interferon as a practical chemotherapeutic agent. "From the scientific point of view there might therefore be very real advantages to be gained from liaison with Merck Sharp \& Dohme if this would give us access to their 'knowhow' in this field (since in all probability they have data available other than those published). ${ }^{110}$ Finter then proposed to reopen the question of whether or not MSD be invited to join the collaboration, assuming that they still wished to do so, and discuss it at the next meeting of the scientific committee.

Isaacs, however, showed himself rather reluctant to bring the matter up for discussion. "I He didn't think much of a liaison with yet another party and certainly not if it meant collaborating with Hilleman whom he suspected of being driven solely by self-interest. At least in the article, Hilleman had done everything to make the work of Isaacs, Burke and the Committee look rather silly. It was only because of Finter's persistence that eventually the question of liaison with MSD was discussed. Finter must have been disappointed to find out that the other members of the Committee did not think much of the idea either and decided to hand over the matter to the Board. However, rejecting the proposal to collaborate across the

Norman Finter to Alick Isaacs, letter dated 9 May 1963, MRC Archives File No. A812/5/2. 
Atlantic ocean did not mean ignoring the American interferon studies. ${ }^{112}$

Isaacs rapidly got the better of his initial embarrassment about losing the initiative to Hilleman's research group in a field of which he considered himself the founding father. More determined than ever before, he devoted himself to steering the scientific committee, pursuing his interferon studies and promoting his ideas. ${ }^{113} \mathrm{He}$ decided to turn the published American purification and characterization studies to his advantage. Should interferon indeed be powerfully adsorbed onto glass, as claimed in the American report, and should as a result interferon preparations stored in glass bottles lose their anti-viral activity in time, then this put the outcome of the clinical trials in another perspective. Isaacs therefore thought it worthwhile to assay some interferon bottles left over from the trials. When it indeed turned out that the antiviral activity of the clinical trial interferon remnants had dropped considerably as compared with the original material, Isaacs took the matter up with the Scientific Committee. Isaacs's story was considered very convincing, and it was unanimously agreed that since there was no guarantee that the interferon samples used were still potent at the time of the trials, the assessment of the clinical trials of interferon should be suspended-a private report should be sent to the Council but not published. ${ }^{114}$

So far so good, it seemed. However, much to Isaacs's regret, the reassessment did not take away the general reluctance to plan further trials. Even more disappointing in Isaacs's opinion was the subsequent lack of support for his proposal to discontinue the obviously disastrous purification studies and instead concentrate on the radically different approach of exploring ways to exploit the potential interferon productive capacity within a living organism. If it would be possible to find ways to fight off viruses by stimulating the production of interferon inside the body ('endogenous interferon') by non-viral means Isaacs believed that there would be no further need to continue the costly and problematic efforts to produce large amounts of exogenous interferon. His proposal for a change of course in work on interferon resembled to some extent Hilleman's simultaneous shift in attention from interferon administration to interferon induction as the most promising lead toward anti-viral therapy, although Isaacs did not share Hilleman's radical ideas about exogenous interferon as a dead end. ${ }^{115}$

112 Minutes of the 22th meeting of the Scientific Committec on Interferon, dated 21 May 1963, MRC Archives File No. $5788 / 2 / 3$.

113 I agree with Stephen Hall that "Isaacs's battle for intellectual normalcy, for a return to the wit and brilliance of his earlier career, is without doubt the saddest chapter of the interferon story"; See S. S. Hall, A Commotion in the Blood: Life, death, and the immune system (New York, Henry Holt and Company, 1997) p. 157.

114 When at the end of 1964 word came from the NIH that they were planning to repeat Tyrrell's trial against the common cold, the publication ban was lifted. Tyrrell was able to convince the other members of the Scientific Committee that because they had now further evidence that the British common cold trial had been unsuccessful due to the small dose and speed of removal of interferon inside the nose it would still be worthwhile to report the trial results to the scientific world: Minutes of the 28th meeting of the Scientific Committee on Interferon, dated 10 November 1964, MRC Archives File No. S788/2/3; and, A Report to the Medical Research Council from the Scientific Committee on Interferon, 'Experiments with Interferon in Man'. Lancet, i (1965), 505-6.

115 Minutes of the 22th meeting of the Scientific Committee on Interferon, dated 21 May 1963, MRC Archives File No, S788/2/3; and, Internal note MRC. 1 June 1963, MRC Archives File No. S788/1. 
After almost four years of interferon studies, Hilleman had come to believe that despite its remarkable non-toxicity, administering exogenous interferon had no real potential for clinical usefulness in anti-viral therapy. Interferon's prophylactic rather than therapeutic action and the expected high costs and technical problems involved in producing large amounts of high quality interferon material were instrumental in bringing about a change in research perspective. Instead of regarding interferon as a potential chemotherapeutic agent in itself, Hilleman began to see and promote interferon in terms of a research tool to study resistance to and control of viral infection. Eventually this new approach was thought to open the door to the development of chemical means for achieving viral chemotherapy. This would also fit in well with the majority of chemistry-based drug development programs within MSD. Consequently Hilleman decided to give up his purification studies. Instead he turned his research resources to assessing the possibility of engineering chemical compounds which are potent inducers of 'endogenous' interferon production and can be used to limit and preferably preventing viral infection in a host organism. Hilleman's new endogenous approach closely resembled Isaacs's ideas about the non-viral stimulation of the production of interferon in living organisms. ${ }^{116}$

Taking into consideration Hilleman's motivations to change course it comes as a surprise to see that the 'endogenous' approach did not even receive the benefit of the Glaxo, Wellcome and ICI scientists within the Scientific Committee. ${ }^{117}$ The wonder grows if we add to this Isaacs's accusation that the attitude of the company scientists within the Scientific Committee seemed to be conditioned for the greater part by what he called a "chemical habit of thought". Apparently the mere association of the endogenous approach with Isaacs's interferon inducer studies sufficed to relegate the new concept to the circular file within the Scientific Committee. As such it marked the credibility crisis concerning Isaacs's abilities to steer and coordinate research on interferon. ${ }^{118}$

Increasingly frustrated in his efforts to direct research as chairman of the Scientific Committee, Isaacs eventually decided to plead his case through other channels. At the yearly visit of medical and science correspondents to the National Institutes of Medical Research Isaacs saw his opportunity both to promote his 'foreign nucleic acid' interferon inducer research and to show his dissatisfaction with the Interferon Collaboration. On this occasion Isaacs had prepared a laboratory demonstration aimed at associating his work on foreign nucleic acids with the practical medical promise of interferon. While showing the journalists

Since Isaacs correspondence got lost and access to Hilleman's correspondence was not granted it was not possible to properly address the interesting question how both Isaacs and Hilleman developed similar ideas about the use of interferon inducers as an alternative means to exploit interferon's therapeutic potential and to what extent Hilleman's new line of research was related or influenced by Isaacs's work: M. Hilleman. Interferon in Prospect and Perspective, J. Cell. Comp. Physiol., 62 (1963), 337-53; Internal note MRC, MRC Archives, File, No. S788/1; and interview with Maurice Hilleman.
}

117 Minutes of the 22th meeting of the Scientific Committee on Interferon, dated 21 May 1963, MRC Archives File No. $\mathrm{S} 788 / 2 / 3$. 


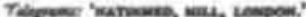

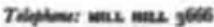

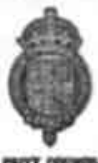

CoNITDERTAL.

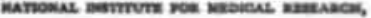
nie siberwaY, wiLl wath. Lowbow, w.w.7. $1=9-13$

Deser Foter,

At a recent pecing of the Selentirle Coentten on Interforon it vae agreed that a roport ahould be proparnd for subeland on to the Couneil en the clinfeal triala of interferon carried out until sov, and on the general progrese of the vork of the Coundttee. The report vas prepared by Jyrrell in collaboration $\mathrm{d}$ th Andreva of Glaxo and $I$ enclose a copy. the time aeens to ce to be opportune to give you in confldence $=y$ ovn personal opinion of the vork of the Coendttee.

The Comeitee 18 bow nearly 4 yeare ald. The flrat year was apent in atudying the tiasue opeciflelty of interferon and at the end of this tim it vas decided that ve ebould atvdy the action of nonicy interferon 1a man. A procranes was egreed that a batch of 11 11tros of interferom vould be prepared, concentrated 10 timea and waed as follows -

a A prelindary trial would be earried out of the effeot of thin naterial in preventing the eccurrence of prisary vaceination in ean wich would need about 1 litre of interferon.

b) If this vere evecessful the renaining 1011 tres vould be used for trials wth combon oold vruaen in Sallabury.

To ay aind 21 litres was a vodest target, but the smount of effort devoted by the pharnaceutical fl ras va poor. I.C.I. eade no acokey interferon at all, and loft this entirely to the other two firas. If the end of the second year Glaxe had male near2y 10 litres of interferon - and thes loot the entire batch by filterles it without anking aure beferehasd that the technique vas safe. Velleces Laboraterles proceeded alowy but sore effectively and 18 montha after the deciaion to anke an 11-1itre batch, Vellcoene had prepared 3 I1tres. Our Coenttee then had to decide whether to vatt unt12 the 11 Ltrea van prepared or to cut our loeses and proceed with the vacoinia trial. The latter course vas declded on and in fact the trial proved nuccesaful (1962 roport encloaed).

Having asde this effort, Wellcoee Laboratories then decided they had done enough and that it vas time Glaxo produced sone interferon. lant cuaner Giaxo had produced a feu 11 trea and Vollecese have not ande any sort. On a nubber of occadione both fira have atreesed the coet of preparine naterial of thin $\mathrm{kdnd}$, and while one canbot diapute that it hae been costly I have alvays felt that a rether nore adventurous apirit vas required of the flrae thas they have shove oo far.

The interferon prepared by $010 x 0$ has been uted for trials alth eoenos cold virusea in sallebury and triala with herpes virun infectione of the eye earried out in eollaboration with $\mathrm{Kr}$. Barrie Jones. You wil 5ee fros the enclosed roport of the Conalttee that the reaults have

large $2 y$ been negative. liowever, I think $1 t$ in worth streasing that the interferon, when teated for 1to antivial activity after the oinical trialo, ahowed a lare drop in titre. The material used by Barrie Jonea vas ment to Tyrrell and to se in coded fore and ve both found that $1 \mathrm{t}$ had lout about 90 per cent of 1ts activity. Becently a roport has appeared (Lawpoen et nl. 1963, rejrint escloeed) Indieating that purifled chiok interforos in atrencly aboorbed to glasa and thet thin can be preventad

Fig. 39. Letter Alick Isaacs to Sir Peter Medawar (1-7-1963), Courtesy of Dr. S. Isaacs-Elmhirst. 
by keopling the interferon in poly-propylene contalnera. The saterind wo uaed for olinical trials was otored in clans tubea in refriceratore

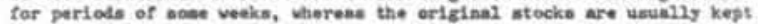
rrosen. It weene important, therefore, to investigate whether thio gould be a factor is the aefative reawis of the recent elsaseal trinla. However, the genoral comeat I would nake is that the whole acale of the effort no far bas been pitinally eanil.

A second field of activity of the Conedteo hea been to wtudy the purifleation of interferon. I have alveye felt that this ghould

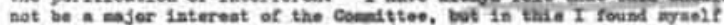
in diangreenent with the tirma and have had to bow to their opiniest. It turna out that nll throe flres have nov apent a conolderabis tins

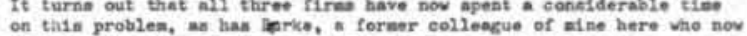
works in Aberdeen and hat remalned a nember of the Coselttee. Tet in mpite of the fnet that this has been a nejer netivity of the Coustttee It would not, I thinik, be an exaceceration to any that practiesliy nothing of note has been schleved by wa. Meankile, Merck, Bharpe

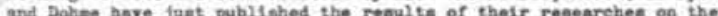
and Dahae have jaet jublished the results or their researchee on the jurification of chick isterferon (reprint encloeed) and I sa bound to admit (and I have heard the coment froe others) that it askee the wor of our Coest ttee look sily. Their material is 100 timea sore jure than the best anterlal produced by aty nember of the Comalttee, and ineldentally it ehowe interferon to have an extrenely high poteney, one unit belng between $0.0017 \mathrm{wg}$ and $0.0067 \mathrm{~kg}$ of protein.

Al1ison vas told by Dr. R11leman that Merck, Bharpe and Dohee had apent $\$ 250,000$ on this research and that they had decided not to work farther on this problea but to concentrate inetend on loeiding at wabatanese that atimilate the produetien of interferon. Fila latereated oe creatiy as tais is preelsely what I have been afrocating to the Coendtee for mene tise. Abeut 2 years age I jut forvend the theor? thet profuetion of interferos night represent the resction of celle to

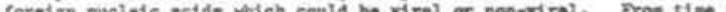
rorelga macle tise 1 bave presented to the Condittes resultin of work which tended to fuvour this hypothesis but the Coentitee has reacted lukevaraly, at lases in terns of practical resanreb. Recently our evidence in favour of thin 1des has been srowing atronger but a foeling is that the Conittee are quite happy for ee to inveatigate this and whow littie viah to join in.

In general the ateosphere at Conal ttee metinga has been fairly good. There has been very little joblouny or eecrecy betwees the firma. The fault netha to lis in the oppoaite direction, ansely that each tirie prefers to leave it to the otherk. I have been told that Jurnet has advised Glano that interferon in theoretically interestine but unlikely to be of praetical value and this say mecount in port for theis lack of enthualaen. Yot aurely. this 1o not enough to explain bev when 3 pharmaceutical 51 ras and Burke and his collenguea vere engaged on the problea of purification and had quite a start over all competitors, they were left behind by an Ameriean firm. I can only eonelude rather madiy that our three firta ere greatly lacking drive.

To $\mathrm{my}$ alded, the present machinery has been given a long enough trlel and is not ochieving what $1 t$ eet out to do. Soverel poeniblo solutions wight be coneidered:

1. In attnapt nay be ade to inject nox ilfe into the coelttoe, for exasple by bringing in frof. K. B. Chain abd bis teas. I aght aention that in the pant, Chain han whovn great intereat in thile subject.

2. If the partielpation of 3 Ara is an useound approach, 2 tirne aight be asked to vithdrav, and the third invited to incroase greatly the ocale of its offort.

3. The collaboratioe could be dissolved and an acedente body encouraged to develop this work on an alequate neale.

I would be nost happy if by nase of obe of these aolutions, or by scoe other means, the Coenitee could tarry out an offort of wileh the Counc1l could be proud.

Iner yours,<smiles>c1cnc2cc[nH]c2c1</smiles>

(Alsek Isauca) 
around in his laboratory, Isaacs told them that he believed the UK's effort on interferon to be inadequate and particularly so when compared with what was going on in the USA. ${ }^{119}$ Only a couple of days earlier Isaacs had sent a formal letter with a similar vote of non-confidence to the new director of the NIMR, the immunologist and recent Nobel prize winner Peter Medawar. ${ }^{120}$

Isaacs's personal letter to Medawar evokes an image of a scientist who feels intensely disappointed in the commitment of other parties to his life's work and launches an all-out effort to turn the tide (see Fig 39). ${ }^{121}$ However colored his analysis of the problematic state of affairs might seem-as key person in directing the research program, he himself is curiously enough not to blame-Isaacs raises legitimate questions. Why is it that in spite of having a start over all competitors, the British Interferon Collaboration was beaten in its research efforts by a single American drug company? Could it be that the formal and at the same time noncommittal and fractionary nature of the multi-party collaboration frustrated concerted efforts at the laboratory bench? Going by the course of events one, indeed, gets the impression that the Interferon Collaboration was burdened with a squandering of resources, energies and commitment. Too much effort had to be invested to keep the various parties with their specific working cultures aligned with each other.

Medawar, was informally - through his predecessor Harington-already well aware of Isaacs's dissatisfaction with the co-operation they had received from the consortium of three drug companies. And he immediately passed the letter on to Sir Harold Himsworth. The letter did not take Himsworth by surprise either. Himsworth already knew for some time that Isaacs was dissatisfied with the efforts of the industrial collaborators. At first Himsworth thought that Isaacs was too soft to be chairman of the Scientific Committee, tending to discuss possible lines of action rather than requesting that certain work should be done. Later on however he came to believe that this was not the case. The main reason for the firms' "lethargic" effort was a collective action problem in terms of the logistics involved in communication between so many different parties and the growing feeling amongst them that there was no practical value in interferon. ${ }^{122}$ After extensive consultation with his own staff and Medawar, Himsworth eventually decided that Medawar should meet the Board of the Patents Holding Company ('Executive body') to put forward and discuss all the reasons for concern about the working of the Interferon Collaboration. Medawar met the Board at their November 20th meeting. The whole atmosphere was charged with an anxiety as to whether the collaboration should be continued. Whereas Medawar was disappointed with the contribution of the industrial collaborators, the commercial partners concentrated on the negative assessment of the possible tolerance": A. M. Silverstein. A History of Immunology (San Diego, Academic Press. Inc, 1989), p. 344-345. 
practical and commercial value of interferon. They generally felt that the prospects of interferon becoming a saleable therapeutic agent had not improved since the start of the collaboration. Despite its imaginative action against a wide range of viruses, interferon did not live up to the hopes of an Ehrlich sort of "poison-arrow" specifically aimed at the virus invader. ${ }^{123}$ Worse yet, far from being the 'magic bullet' that was hoped for, interferon was known as a chemically undefined biological substance that was part of a poorly understood natural mechanism of resistance to virus infections. As such it was regarded as alien to the industry's Ehrlichean chemotherapeutic program.

Medawar's interpretation of the backwardness of the enterprise as a lack of engagement and effort of the three drug firms met with severe criticism from the commercial collaborators. Though lack of progress was not denied, the effort of the companies was said to be "genuine and wholehearted". ${ }^{124}$ The chairman of the Board of Directors of the Patent Holdings Company indicated that the industrial partners had spent about twice as much as Merck Sharpe \& Dohme-over 250.000 pounds so far. On a yearly basis this meant that about $0.75 \%$ of the annual research budget of all British-based pharmaceutical firms was spent on interferon. He clearly wondered how much further they should go. ${ }^{125}$ So little progress had been made to date that there were serious doubts as to the wisdom of continuing the Collaboration. At the same time, the companies were reluctant to write off all their efforts over the past few years.

The fact that a marketable product was no nearer than when the Collaboration started was attributed, if not to the "cussedness of nature" and to the incompetence of the scientists engaged on the work, then to the shortcomings of Isaacs as chairman of the Scientific Committee and research coordinator. ${ }^{126}$ According to the Wellcome representative, Col. Mulligan, it seemed clear that there was a difference in opinion between Isaacs and the other members of the Scientific Committee on whether priority should be given to the production of large quantities of interferon for clinical trial or whether more work should first be done on the physico-chemical properties of interferon. With reference to similar dilemmas and difficulties in the early days of insulin and penicillin development he thought it most likely that the trouble with the case of interferon had arisen because the collaboration had tried to produce interferon for clinical trials before sufficient knowledge had been collected about its properties. Mulligan doubted whether Isaacs really understood how difficult and expensive the production of large batches of clinical trial interferon was. Did Isaacs realize that the product could not be tested

123 J. Parascandola, 'The theoretical basis of Paul Ehrlich's chemotherapy', J. Hist. Med. 36 (1981), 19-43, p. 38; and interviews with John Beale and David Tyrrell.

Minute of a special meeting of the Board of G.N.R.D. Patent Holdings Ltd., dated 20 November 1963, MRC Archives File No. A812/5/2. 
on humans without the most exhaustive safety trials? ${ }^{127}$

It was generally felt that Isaacs was too deeply involved in interferon, scientifically and emotionally to continue carrying the responsibility for the scientific policy of the Collaboration. During the meeting Medawar grew increasingly uneasy about Isaacs's position. Medawar was taken aback by the views expressed by the Board in particular with regard to the general dissatisfaction with the way Isaacs had run the Scientific Committee. Without letting Isaacs down he eventually agreed with the Board that the enterprise lacked effective overall scientific direction-the consortium as he put it had no "central nervous system". As a means to tackle this problem Medawar proposed to set up a small high-powered Scientific Steering Committee with an independent chairman. ${ }^{128}$ The existing Scientific Committee could then continue in its present form with the same chairman to fulfil the essential task of acting as a forum where the bench workers could meet and exchange information and ideas. ${ }^{129}$

None of the parties appeared to be willing to take the initiative in bringing the collaboration to an end without giving it another trial period. However unanimous in their wish to continue the Collaboration for another year, it required quite some talking on the part of the MRC to reach an agreement on an arrangement for regulating the responsibility for research policy and strategy. Himsworth himself had to take diplomatic action the highest company level to convince the industrial collaborators-who did not think much of Medawar's idea of multiplying committees and were in favor of removing Isaacs from the chairmanship of the Scientific Committee- that the best thing to do was to set up a separate Scientific Steering Committee. ${ }^{130}$

Faced with a lack of imaginative research results, not only drug company executives but also the British medical community grew impatient with interferon as is interferon is succinctly illustrated by the following excerpts from a 1964 editorial in the British Medical Journal:

Immense practical problems attend any future use of interferon in man...., and even if they can be overcome the problem still remains of ensuring that the interferon reaches the cell in time to produce any therapeutic response. The use of it for controlling virus infections in man and other animals is likely, therefore, to be limited. ${ }^{13}$

Minute of a special meeting of the Board of G.N.R.D. Patent Holdings Ltd., dated 20 November 1963, MRC Archives File No, A812/5/2; Internal note MRC, dated 17 december 1963, MRC Archives File No. A812/5/2; Medawar to Himsworth, letter dated 7 January 1964, MRC Archives File No. S788/8.

Minute of a special meeting of the Board of G.N.R.D. Patent Holdings Ld., dated 20 November 1963, MRC Archives File No. A812/5/2.

Medawar to Himsworth, letter dated 7 January 1964, MRC Archives File No. S788/8.

130 Minutes of a meeting of the Directors of GNRD Patents Holdings Limited, dated 20 February 1964, MRC Archives File No. A 812/8/1; Himsworth to Medawar, letter dated 19 March 1964, MRC Archives File No. A 812/5/2: Internal note MRC, dated 29 April 1964, MRC Archives File No. A 812/5/2 and, minutes of the first meeting of the Interferon Scientific Steering Committee, dated 24 July 1964, MRC Archives File No. S $788 / 8 / 1$. 
Through this kind of high-profile vote of no confidence in the clinical potential of interferon as a therapeutic drug, interest plummeted and interferon became widely regarded as just another medical 'discovery' which had not lived up to its promises. ${ }^{132}$ The waning of public enthusiasm and the new arrangement for scientific policy within the Interferon Collaboration made itself felt in the work of the Scientific Committee. In response to the steady decline in research resources and new research directives from the Scientific Steering Committee plans for clinical trials were pushed into the background. Attention shifted to studies of the physico-chemical properties and mode of action of interferon and to assessing through animal experiments the possible therapeutic value of interferon. ${ }^{133}$

The greater part of the political jousting over his position and the change of research priorities passed Isaacs by for the greater part. On New Year's Day 1964 Isaacs suffered from a sub-arachnoid hemorrhage which kept him away from work-due to a slight paralysis and blurring of his eye-sight-for more than three months. The hemorrhage was said to be related to an abnormal tumorous blood vessel which compressed the surface of the brain, as revealed by angiography. Apparently, from its position, the tumor was out of reach of the surgeons. ${ }^{134}$ On return to the Institute Isaacs found himself relieved from his post as head of the Division of Bacteriology and Virus Research and instead he was appointed head of a small group for research on interferon - consisting of Isaacs, Joseph Sonnabend and his laboratory technician Dennis Busby - that was officially named 'The Laboratory for Research on Interferon'.

Isaacs's lamentable condition was symbolic for the paralytic state of affairs not only of British interferon research but of interferon research in general. Despite a short-lived excitement that accompanied Hilleman's research group's purification studies, the enthusiasm for interferon within the international scientific community had definitely waned. The powerful group of quantitative, molecular oriented virologists in America (with people like Delbrück, Dulbecco, Rubin and Huebner), who had tolerated what they considered as one of those shortlived 'research fashions' as long as it lasted, had an important hand in marginalizing interferon research. ${ }^{135}$

By openly displaying their serious doubts about interferon as a troublesome biological substance, which after more than five years of research still resisted chemical characterization and purification, they gave interferon researchers the 'grilling of their lives'. Interferon became widely perceived as an odd laboratory substance that had never got much further than an

Interviews with John Beale, Derek Burke, Norman Finter, and David Tyrrell.

133

For instance, at ICI Finter had initiated experiments in mice on the possible therapeutic or prophylactic use of interferon - Finter had prepared 11.5 litres of crude mouse interferon from the brains of over 8000 mice and after concentration of up to 20 -fold he was in the process of using this large batch of mouse brain interferon in a series of experiments which aimed at demonstrating protective effects in mice against systemic virus infections; Minutes of the 25th meeting of the Scientific Committee on Interferon, dated 8 January 1964, MRC Archives File No. S788/2/3.

Helio Pereira to Jean Lindenmann, letter dated 8 January 1964, Jean Lindenmann personal archives; and, Internal note MRC, dated 7 January 1964, MRC Archives File No. A. 812/5/2. 
assumed protein and had not lived up to its promise as an important lead toward viral chemotherapy - and that was unlikely to be ever of much practical value. The fact that the scientists interested in problems of interferon admitted that the agreed-upon criteria for identifying an biological factor as an interferon were not exclusive enough to determine authoritatively whether or not one was dealing with an interferon only complicated matters. ${ }^{136}$

In the corridor of scientific conferences and meetings, questions like 'Do you really think that interferon exists?', and, 'How do you know that the phenomenon which you observed in your laboratory was due to interferon and not to an impurity?' were repeated. ${ }^{137}$ Unlike in the early sixties, not 'everything' on interferon was publishable anymore by the end of 1964. Edward de Maeyer, for instance, received rejection letters from both Virology and Science on the grounds that the material under study ('interferon') was ill-defined and impure, and therefore of no great scientific interest. ${ }^{138}$ Never before had this been a reason for rejection.

Considering interferon's low scientific image as an ill-defined laboratory substance of peripheral interest, for most biomedical researchers, entering the area of interferon research just did not seem worth the effort. Why join a marginal subfield of virus research that was considered to lie well outside the mainstream of virology (viral genetics and animal-tumor virology)? $?^{139}$ Apart from image related problems there were other more technically related matters that "locked people out". ${ }^{140}$ If you wanted to start working on interferon as an outsider to the subfield of interferon research you first had to acquire specific skills to set up your own production and assay system. This was rather time consuming. Practically it meant that one had to learn through hands-on apprenticeship the production and assay techniques from established interferon researchers. This was far from a straightforward process as the interferonologists themselves were still in the process of getting to grips with their experimen-

136 This very problem was discussed during an informal gathering of 31 interferon researchers at the 1964 meeting of the American Society for Microbiology in Washington, D.C.. According to Monto Ho they could not and would not produce an inclusive definition of what more precise conditions must be met for a substance to be called interferon; See M. Ho, 'Identification and "Induction" of Interferon', Bacteriological Reviews, 28 (1964), 367-81, p. 367.

The ambiguous status of interferon is nicely illustrated by the following excerpt from Vilcek's 1969 monograph on interferon: The other day I had lunch with the Bacterial Geneticist working next door to my lab. While consuming his yoghurt(which he had for desert) he asked what I was working on all the time. So I told him all about interferon synthesis requiring mRNA to be made thus being an induced protein which induces new mRNA and another protein which by bindig to ribosomes inhibits translation of the viral mRNA but not the translation of other mRNA. "That's very interesting", said the Bacterial Geneticist while finishing his yoghurt, "but do you really think that interferon exists?"."; J. Vilcek, Interferon (New-York, SpringerVerlag, 1969), p. 111.

138 Science to Dr. Jacqueline De Maeyer-Guignard, letter dated 21 January 1965, Edward de Maeyer personal archives; E. De Maeyer, 'Interferon Twenty Years Later', Bulletin De L'institut Pasteur, 76 (1978), 303-23, p. 304: Edward De Maeyer and Jacqueline De Maeyer-Guignard, interview.

Interviews with Jan Vilcek, Jean Lindenmann, Edward De Maeyer, and Sam Baron.

140 We can see in figure 31 , chapter 3 that the number of scientists working on interferon leveled off between 1964 and 1967. 
tal systems and the biological substances that went by the name interferon. ${ }^{141}$ As we will see in the next chapter a great deal of their efforts went into managing differences.

\subsection{Conclusion}

Confronted with the pervasive scepticism that prevailed at the end of 1964 it seems hard to imagine that only four years earlier efforts were started to secure a collaboration between the British government and British drug industry. This immediately raises the question "What brought about the dramatic changes in commitments and objectives of those involved, and how did they materialize? Without trying to delineate any schematic succession of 'changes', I shall analyse the dynamics of the processes through which changes came about.

Bringing the partners together in the first place appeared to be a matter of aligning commitments and tasks. Both the MRC and the drug companies were committed to the notion of therapeutic breakthroughs as a dominant feature of a laboratory-supported scientific medicine, to the feasibility of generating medications which like 'magic bullets' seek out and destroy infectious agents, and to the need of defending British industrial interests. However promising in terms of a potential 'antiviral penicillin', British drug companies, including the UK leaders in biologicals, initially showed restraint with regard to interferon. This was due to the 'iffy' status of interferon as an undefined biological substance and the general perception of biologicals as troublesome and high-risk commodities with uncertain manufacturing prospects. From industry's point of view, risk-sharing with the MRC, who offered their unique expertise in interferon research and the eventual rights in the developed product, made work on interferon a more attractive option. Joining in with the MRC-led interferon project, without having to start from scratch a private high-risk developmental program, reduced the liability and as such made it easier to accept the many imponderables. At the same time the resources of the pharmaceutical industry matched the MRC's need for material and financial support in developing interferon as a therapeutic drug. The MRC nurtured the links with the pharmaceutical companies because it realized that the pharmaceutical industry alone had the expertise and capacity to produce large amounts of biologicals for use in humans.

I showed that just as Isaacs drew legitimation from the use of his knowledge claims in MRC's deliberations, so MRC and drug company officials were better able to legitimate their decision to participate in efforts to develop interferon as a drug by attaching to it the authority of Isaacs's expertise. Thus, the MRC, the drug companies and Isaacs had good reasons to get close to each other, but not too close. Their dilemma was to prevent choices and futures becoming either merely 'scientific', 'political' or 'commercial' beyond the grasp and thus control of the other.

In order to fit their own programmatic goals and maintain the integrity of mutual interests there was a continual need to maintain accountability to one another with each party 
working hard to re-interpret and translate the concerns of the others. Differences became manifest in conflicts over research agendas and publication rules. While the commercial partners felt bound to stringent control over publication and relatively fixed developmental trajectories, MRC workers like Isaacs were used to freedom of publication as means to the end of advancing scientific reputations and to 'follow their nose' in exploring research problems. From the beginning much energy and resources went into bridging the differences between industrial and academic cultures. As long as each party was committed to the same central idea of developing interferon as an anti-viral drug, and had similar thoughts about the constraints and the prevailing conditions under which collaborative research should be performed, they were prepared to put up with this efficiency loss. However, their perception of the Collaboration changed dramatically when it appeared that they were scored off by the research efforts of a single American drug company. To the same extent that it lent credibility to MSD's efforts, it threw discredit not only on the work of the British collaborators but also on their form of research organization.

I agree with Isaacs's assessment of the situation that the noncommittal and factionary nature of the multi-party collaboration arrangement frustrated concerted efforts at the laboratory bench-although he as chairman of the Scientific Committee deserved some of the blame, too. His increasingly tiresome and even frantic behavior was not particularly helpful to keep the various parties with their specific working cultures aligned with each other.

As work at the bench and policy-making co-evolved plans for research and the objects of research underwent changes. Initially the plans for research closely resembled the initial stage of conventional industrial new drug development trajectories with an emphasis on animal testing and the development of production techniques. However, from the beginning the difference between the restraint of the company scientists and the drive of the academic scientist Isaacs to bridge the worlds of the laboratory and the clinic, comes to the fore. Isaacs's dominance within the Scientific Committee early on is reflected by the premature shift in attention to trials on human volunteers. In succeeding to define interferon in terms of a natural non toxic-agent, which was different from most other existing pharmaceutical substances, Isaacs created latitude to follow an alternative testing trajectory. He received unwitting support in his rush to human testing from the American patent-examiner, who rejected interferon's patent application on utility grounds. It made the industrial partners realize that having an early demonstration of a clinical effect in humans was needed in order to reverse this judgment and safeguard their future market position. Similar concerns led to early efforts to create a common and publicly acknowledged definition for interferon that would be narrow yet broad enough to satisfy the demands of patent advisers, company executives, researchers and drug registration authorities.

Zooming in on the question whether or not interferon could induce a clinical effect in humans did not result in a whole-sale shift in research work but in a reshuffling of research priorities. There was no such thing as laboratory oriented research giving way to clinically oriented research-as either or alternatives. As can be expected from 'exploring uncharted territory' the scientists under survey in this chapter worked in a 'zigzag' fashion; as soon as any 
progress was perceived toward tackling one problem, they would simultaneously begin work on the next problem regardless of whether this involved a transition from in vitro to humans or from in vivo to in vitro and back to humans. However this did not mean that in the preparations for and performance of the clinical trials there was no guidance of any sort. Whenever possible those involved tried to create landmarks by linking up their trial efforts with already existing testing practices. This immediately raises the question, "What points of reference played a major role in constituting the conditions of practice in the case of testing interferon?"

In the absence of a standard system for controls regulating the testing of potential new drugs in Britain, the members of the Scientific Committee tried to fall back on existing testing practices for biologicals and other therapeutic drugs within the companies and the MRC. Among other things this resulted in the adoption of the in-process safety control concept, and the MRC promoted statistically controlled trial procedure. Due to the non-compliant nature of the subject matter or resistance arising from the continuous interplay between the various parties, adjustments had to be made over and again. Deciding on how to go about problematic and uncertain situations and cope with the 'mangle' of practice involved ad hoc judgments and assumptions. This is not to say that they necessarily proceeded arbitrarily, but that given the specific problem context they collectively weighed the perceived pros and cons of proposed solutions derived from the material, social and cognitive resources at hand. ${ }^{142}$

As a final point I would like to specify how the modes of perceiving interferon in terms of a therapeutic substance changed. The Interferon Collaboration started from the common understanding and perception of interferon as a potential 'anti-viral penicillin'. As a non-toxic agent interferon seemed to come close to Paul Ehrlich's ideal of generating medications which (like 'magic bullets') seek-out and put infectious agents out of action without harming the host organism - in other words a most important lead toward viral chemotherapy. With accumulating production and purification problems, the perceived lack of effectiveness and the changing ideas about the mechanism of action of interferon, questions were raised about the practicality and rationality of developing interferon as a chemo-therapeutic agent along the lines of what I dubbed the 'exogenous approach' Interferon did not seem to be the 'magic bullet' against viruses that was hoped for, but a chemically undefined substance with a rather ambiguous mechanism of action that was quite alien to the Ehrlich's concept of chemotherapy - different in other words from conventional chemotherapeutic substances.

We saw how both Maurice Hilleman at Merck Sharp \& Dohme and Alick Isaacs at the NIMR - in efforts to come to terms with the practical limitations of the 'exogenous approach'-almost simultaneously began to promote an alternative therapeutic concept for interferon, which I designated the 'endogenous approach'. While Isaacs considered it as just another option for research, Hilleman held stronger views. In his opinion interferon should no longer be considered as a practical therapeutic agent in itself but as a research tool to develop preferably chemical means for achieving viral chemotherapy. This made a difference in the

142 Brian Wynne was first in pointing out the important role of ad hoc rules and judgments in developmental practices such as clinical trials in: B. Wynne, 'Unruly Technology: Practical Rules, Impractical Discourses and Public Understanding.' Social Studies of Science, 18 (1988), 147-67, p. 162. 
ways Hilleman went about making plans for research. Instead of concentrating on ways to tackle production and purification problems, attention shifted toward investigating ways of stimulating endogenous interferon production. The credibility crisis surrounding Isaacs seemed to have played a major role in the negative assessment of the new endogenous approach in the British context, at least temporarily.

In the next chapter I will show how these differences in 'seeing' interferon would lead to a serious controversy in the field of interferon research and paradoxically helped to keep the British Interferon Collaboration 'afloat'. 


\section{Chapter 5}

\section{Managing Differences in Biomedical Research: The Case of Standardizing Interferons. ${ }^{1}$}

\subsection{Introduction}

We have finished with the romantic period of interferon and we now have to start on the scientific period. ${ }^{2}$

In a very general sense this chapter is concerned with the topic of transferability or portability. I shall describe the difficulties which researchers in the embryonic subfield of interferon research faced in maintaining collaborations and alliances, communicating research results and ensuring the reliability and credibility of the products of their research work, both locally and trans-nationally. More specifically, what is at issue here is to show how standardization played a part in mastering and establishing differences in interferon research. ${ }^{3}$ To further our understanding of the dynamics of biological standardization, the following questions deserve further exploration. ${ }^{4}$ What were the incentives for establishing interferon standards? What efforts and resources did it take to establish, disseminate and maintain standards? And, to what extent were they instrumental in narrowing down the margins of interpretation and changing individual laboratory practices into universal practices that could be made to work elsewhere?

In chapters four and five I already indicated that a central issue for the working on

A preliminary version of chapter 5 was published in Studies in History and Philosophy of Science; T. Pieters, 'Managing Differences in Biomedical Research: The Case of Standardizing Interferons', Stud. Hist. Phil. Sci., 29 (1998), 31-79.

Quote is taken from statement by Pieter De Somer at the first session of the International Symposium on Standardization of Interferon and Interferon Inducers; See, F.T. Perkins and R.H. Regamey (eds.), Symposia Series in Immunobiological Standardization vol. 14 (Basel: S. Karger, 1970), p.5.

3 Most studies in the history and sociology of science which deal with standardization as a key dimension of portability in the biomedical sciences are confined to discussing the role and effects of standardization; See, N. Oudshoorn, The Making of the Hormonal Body (Amsterdam: Ph.D. diss., Univ. Amsterdam, 1991), pp. 72-80; J. H. Fujimura, 'Crafting Science: Standardized Packages, Boundary Objects, and "Translation"', in A. Pickering (ed.) Science as Practice and Culture (Chicago: The University of Chicago Press, 1992), pp. 168211; P. Gossel, 'A Need for Standard Methods: The case of American Bacteriology', in A. Clarke and J. Fujimura, The Right Tools for the Job (Princeton: Princeton University Press, 1992), pp. 287-311; P. Faasse, Experiments in Growth (Amsterdam: Ph.D, diss., Univ. Amsterdam, 1994), pp. 92-111.

4 With an exception to Theodore Porter's book Trust in Numbers the processes of realizing, maintaining and disseminating biological standards have received relatively little attention from science studies scholars to date; T. M. Porter, Trust in Numbers (Princeton: Princeton University Press, 1995), 29-32. 
interferon was the uncertainty resulting from the non-compliant character of research materials and organisms, and the idiosyncrasies of the myriad of experimental systems. Apart from production and purification problems, interferon researchers had a difficult time comparing experimental results. An example of this was the measurement of the antiviral activity or potency of interferon preparations. What was for some a high potency preparation was for others a preparation with a low biological activity, depending on the laboratory, the test method and the scientist involved.

This chapter will be concerned with how interferon researchers tried to cope with these kinds of differences and with the extent to which they were able to reduce uncertainties with regard to the exchange and interpretation of research data. As we follow the subsequent attempts to manage differences, we shall find that there is nothing inevitable or 'natural' about establishing and using biological standards in biomedical research. The researchers involved accomplished much by informally agreeing on exchange mechanisms-without rigorously defining common units of measurement. I shall show how, amongst other factors, the intrusion of powerful spheres, such as the pharmaceutical industry, into the relatively private domain of 'laboratory life' was instrumental in breaking down informal practices of measurement and in creating a demand for standardization. In focusing attention on the process of realizing interferon standards, I then highlight how national centers for biological standardization played a role in the initiation and coordination of standardization efforts. Simultaneously, I will describe how the mounting controversy between the proponents of interferon inducer research and the supporters of the exogenous approach not only strongly influenced the standardization efforts but also the further vicissitudes of the British Interferon Collaboration.

\subsection{Coping with differences}

In the previous chapter we saw that from the very moment the British Scientific Committee on Interferon started a collaborative research program in September 1959, as a means to the end of developing interferon as an antiviral drug, the issue of mastering differences had been on the agenda. Making comparisons between the results obtained on different occasions within any one of the collaborating laboratories was already quite demanding; far greater difficulties arose when attempts were made to compare results obtained in different laboratories. Again and again the members of the Scientific Committee reported considerable variations in experimental results from day to day, week to week, from researcher to researcher and laboratory to laboratory. For the greater part, this was thought to be due to the wide variety of methods and materials employed in the assay of interferon. It was generally believed that a standardization of procedures might be helpful in facilitating the comparison of research results. However, little was known about how to go about standardizing interferon research 
and the extent to which standardization would make the numerous variables in these biological systems more manageable.

Sheer pragmatism made them choose in favor of material standards, since standardization of methods would make far deeper inroads into laboratory life without any guarantee of success. ${ }^{5}$ Initially, the need for standardization was nourished by the mere involvement of the pharmaceutical industry, which had a practical interest in preventing the existence of a variety of different arbitrary units for expressing the potency of one and the same drug. However important as a first incentive to standardization I will show that the national centers for biological standardization played a central role in disseminating and 'materializing' the perceived needs for standards.

\subsubsection{NIMR's Division of Biological Standardization}

Shortly after the establishment of a provisional standard for monkey interferon at the NIMR's Division of Biological Standards, early in 1962, David Evans left his post as head of the NIMR's Division of Biological Standards to become Professor of Bacteriology and Immunology at the London School of Hygiene and Tropical Medicine. ${ }^{6}$ His successor, Derek Bangham, was informed about Isaacs's wish to set up a reference standard preparation for chick interferon. Partly for historical reasons-having a head-start as Isaacs's original experimental system - and partly because of the relative simplicity and low cost, most work in the field of interferon research was performed on chick systems: this made some researchers refer to chick interferon as the "working man's interferon". ${ }^{7}$ However, while the absence of a monkey interferon standard had been regarded as an immediate threat to the preparations for clinical trials by the Scientific Committee-a reference standard preparation was considered mandatory in the case of human trials, the absence of a chick interferon standard was regarded as a handicap which rendered collaborative research work more difficult but not impossible. This difference in perception of research needs was reflected by the fact that a working standard for chick interferon had yet to be established.

With Bangham in charge of the Division of Biological Standards, new priorities were established. Bangham immediately took a keen interest in the issue of setting up a working

5 Interviews with Norman Finter and David Tyrrell.

6 In 1972 the NIMR's Division of Biological Standards was transformed into what is currently known as the National Institute for Biological Standards and Control (NIBSC): See, for a short history of this British institute, A. Landsborough Thomson. Half a Century of Medical Research Vol. II (London, Her Majesty's Stationary Office, 1975), 244-54.

Interviews with Norman Finter, Robert Friedman, and David Tyrrell. 
standard for chick interferon. It fitted well with his plans to extend the scope of the Division's activities. ${ }^{8}$ In serving as a national and international center for biological standardization, the Division had primarily been engaged in establishing and providing national and international standards (defined as preparations with assigned units) and reference preparations (defined as preparations without assigned units) on behalf of the British Government and the World Health Organization (WHO) for practical medical purposes-for use in control of therapeutic biological substances like penicillin and insulin. Bangham now thought it worthwhile to start efforts to use the Division's resources for the introduction of reference standard preparations of interest to medical research. ${ }^{9}$ He firmly believed that, with the rapid growth rate of medical and biological research there were great opportunities for the application of the methods of biological standardization in biomedical research. Establishing an official British research standard for chick interferon was considered a useful pilot-project that served the Division's expansion plans. In consultation with Isaacs it was then decided that the Division of Biological Standards would start efforts to establish an official British working standard for chick interferon. $^{10}$

On 22nd March 1962 a parcel containing tubes with crude chick interferon prepared by Glaxo Laboratories as batch No. A62, was sent to the NIMR's Division of Biological Standards. The tubes were kept in crushed ice for three days, then Bangham and his staff started preparing the research standard. Basically, they followed the Division's standard production procedure for reference standard preparations. First, the batch of chick interferon was concentrated tenfold by centrifugation and the supernatant distributed by special filling equipment into hard glass ampules-approximately 0.9 grams of solution per ampule." Subsequently, the ampules were treated in the Division's automated freeze-drying system which, like the filling system (see Fig. 40), had very recently been designed, built and installed in close collaboration with the NIMR's Engineering Division. Finally, at the end of the production line, the ampules were sealed under anaerobic conditions (by inflating with the inert gas nitrogen) and received a label which designated 'MRC Research Standard A for

8 Interview with Derek Bangham.

9 In 1962 only three research institutes working on biological standards had received the WHO designation 'International Laboratory for Biological Standards': the Statens Seruminstitut, Copenhagen, the NIMR and the Central Veterinary Laboratory, Weybridge, U.K.; See, W. C. Cockburn e.a., The International Contribution to the Standardization of Biological Substances, Biologicals, 19 (1991), 257-64.

10 Annual Reports nr. 19 (1962-1963) and 20 (1963-64) of the NIMR to the MRC, NIMR Archives: and interview with Derek Bangham.

11 First draft of the Report of Collaborative Examination 1962 of Medical Research Council Standard A of Chick Interferon. Oktober 1962, MRC Archives, File No. S788/2/2. 


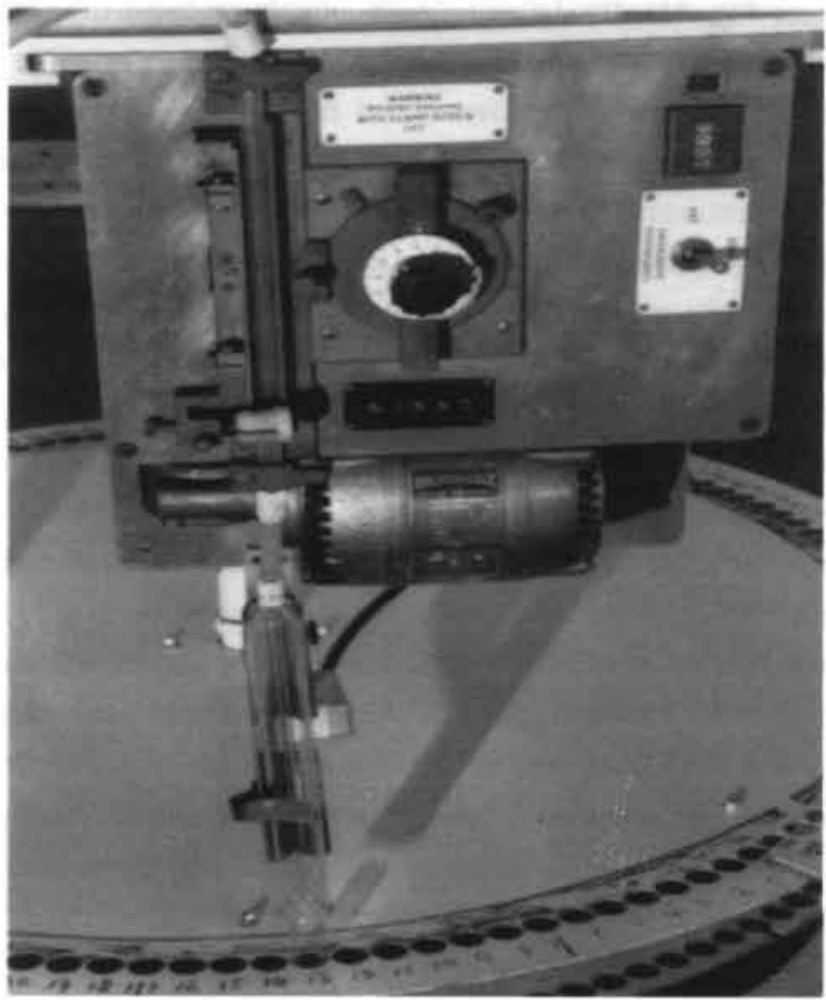

Fig. 40. Ampule filling machine in use at the NIMR's Division of Standardisation in the $1960^{\circ} \mathrm{s}$.

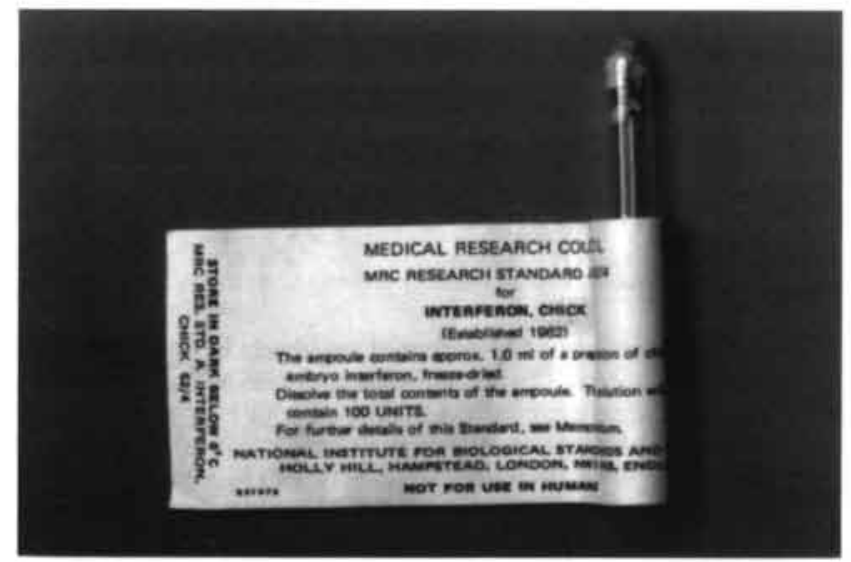

Fig. 41. MRC Research Standard A, 62/4 for Interferon, Chick. Courtesy of NBSB 
Chick Interferon' (see Fig. 41). By definition each ampule was said to contain 100 units of chick interferon. $^{12}$

It was common practice in the Division of Biological Standards to assign to each standard a unitage: it was decided that one ampule of standard chick interferon contained 100 units of activity. This was an arbitrary choice and the only thought that was given to the choice was that the unitage should be such that when the activity of interferon was expressed in units, the numbers used were of a manageable size. The unitage that was settled upon provided an enduring yardstick of chick interferon activity so that measurements made and potencies obtained could be communicated and related in time and in space. ${ }^{13}$

As part of the usual process of establishing an official reference standard preparation, a number of the British laboratories involved in interferon research were asked to assess the suitability and stability of MRC Research Standard A in a so-called 'collaborative assay'. It was known, on the basis of experience, that the successful establishment of reference standard preparations depended on the agreement of the 'user experts' that the standard was suitable. Basically, the test procedure went as follows: first of all a test protocol was designed in collaboration with the Division's statistician, then the draft protocol was sent to the participating laboratories for approval. ${ }^{14}$ Subsequently, the Research Standard and dilutions thereof were distributed to five out of the seven laboratories working on interferon within the British Collaboration on Interferon, and finally assays were carried out according to the jointly agreed protocol. The laboratories sent the 'raw data' from the actual measurements back to the NIMR for analysis. ${ }^{15}$

Processing the complete set of raw data and working out the results was time consuming. A lot of 'number crunching' had to be done with only the help of a little mechanical machine for doing mathematical computations. Furthermore, much of the time was occupied with discussion on the variation in 'precision and accuracy' between laboratories, and between experiments in individual laboratories, and on clarifying ambiguities in experimental procedures and assay reports, and finally, by obtaining agreement on the draft report. According to the final report - which was made available in December 1962 - after thorough statistical analysis of the data, the collaborative assay results were considered to be "all in the correct order and

Up to 1959 the same procedure had to be performed completely by hand and would take more than two weeks. Both technicians and scientific staff would participate in the production process which included glass blowing thousands of ampules; and interview with Derek Bangham. Interview with Derek Bangham.

14 Minutes of the 17th meeting of the Scientific Committee on Interferon, dated 18 April 1962, MRC Archives File No, $\$ 788 / 2 / 2$. Chick Interferon. Oktober 1962, MRC Archives. File No. S788/2/2; and interview with Derek Bangham. 
were all of the correct order of magnitude". ${ }^{16}$

In practice, however, it meant little more than that, on the whole, the results from the collaborative assay were considered to be encouraging. ${ }^{17}$ The most exciting aspect of working with the MRC Research Standard was generally thought to be the fact that for the first time one was able to talk about the activity of different chick interferon preparations in terms of a jointly agreed unitage. By employing laboratory 'yardstick', as Bangham and his staff used to call the research standard, all the researchers involved agreed that the comparisons of research results from one laboratory with another, and between researchers could be made more meaningful. ${ }^{18}$ In other words through the use of a common standard differences in experimental results between individual laboratories became more manageable.

So far so good, it seemed, but formal agreement on the suitability of MRC Research Standard A, for Interferon, Chick, would turn out to be a long way off from broad acceptance of its application in laboratory practice amongst those actively engaged in interferon research. For one thing, in response to concerns raised by the Board of Directors of the British Interferon Collaboration about giving away valuable information to third parties, the distribution and use of Research Standard A was not actively promoted. ${ }^{19}$ Consequently, few research workers outside the Scientific Committee on Interferon learned of the establishment of the British standard, and of the possibility of obtaining ampules of freeze-dried material free of charge on request from the NIMR's Division of Biological Standards. It is questionable, however, whether there would have been many more requests for the MRC Research Standard for Chick interferon if its application had been promoted more actively right from the beginning. This becomes apparent when compliance with the agreed upon standardization procedure by the members of the Scientific Committee is taken into account. It appears that the application of the MRC Research Standard in-every-day laboratory life-as a constitutive element of the various experimental systems - cannot be taken for granted, even for those who started the discussions of the need for standardization, and who were closely involved in

16 Final Report of Collaborative Examination 1962 of Medical Research Council Standard A of Chick Interferon, December 1962, MRC Archives, File No. S788/2/2.

17 Minutes of the 19th meeting of the Scientific Committee on Interferon, dated 25 September 1962, MRC Archives File No. S788/2/2.

18 First draft of the report on the Collaborative Study of Interferon Assays, October 1962, MRC Archives, File No. S788/2/2; Minutes of the 19th meeting of the Scientific Committee on Interferon, dated 25 September 1962. MRC Archives File, No. S788/2/2; Final Report of Collaborative Examination 1962 of Medical Research Council Standard A of Chick Interferon, December 1962, MRC Archives, File No. S788/2/2; and interviews with Derek Bangham, Norman Finter, and David Tyrrell.

19 It was of particular concern to them that the standard preparation would most likely be made available to workers outside the collaboration. They strongly believed that this would inevitably lead to the disclosure of information and hence to the commercial disadvantage of the collaboration: Minutes of a meeting of Directors of GNRD Patent Holdings Limited, dated 19 June, 1961, MRC Archives, File No. A 812/5/1. 
establishing a common reference preparation for chick interferon.

Upon assessing the use of the MRC Research Standard in 1965, about three years after its establishment, Finter had to conclude during one of the meetings of the Scientific Committee that the compliance had been rather poor. ${ }^{20}$ The disappointing performance was thought to be due to the dominance of the informal, personal kind of knowledge exchange and to the absence of sanctions or rewards which would compensate for the extra work and selfdiscipline that was required to implement the standardization procedure in one's laboratory. ${ }^{2 t}$ Whenever there were disagreements about experimental results, they mostly could be negotiated by letter or telephone, or face-to-face. If the dispute could not be settled by those involved, then they would exchange research materials and informally agree on additional tests in order to assess the nature of the variation in results. Only in the case of preparations for human trials, which required special concern for control and rigor due to a relatively high level of public responsibility that was involved, serious efforts were made to formalize the process of knowledge production. By establishing and using a research standard for monkey interferon, the Scientific Committee explicitly tried to address problems of uncertainty and variability, in order to anticipate public scrutiny and to satisfy a larger audience.

\subsubsection{Portrait of a 'gift culture'}

In general, however, researchers working on interferon operated with a high degree of informality in a relatively small and flexible network of personal contacts that was built and maintained through conferences, colloquia, sabbatical visits, postdoctoral fellowships and extensive correspondence. Basically it was a 'gift culture' based on the regular exchange of laboratory samples, techniques and skills. ${ }^{22}$ This gift culture was instrumental in facilitating the comparison of research results and the management of differences between laboratories and researchers in the field of interferon research. A case in point is the relationship of many years which existed between the Finnish virologist Kari Cantell and the American virologist Kurt Paucker. When, on his return to the Department of Virology at the State Serum Institute in Helsinki at the end of the summer of 1962, after a two-year stay as a postdoctoral fellow at the

20 Minutes of the 30 th meeting of the Scientific Committee on Interferon, dated 4 May 1965, MRC Archives File No. $5788 / 2 / 4$.

21 D. Burke and A. Buchan. 'Interferon Production in Chick Embryo Cells; I. Production by UltravioletInactivated Virus', Virology, 26 (1965), 28-35, p, 29; and interviews with Derek Burke, Norman Finter and David Tyrrell.

22 The notion of a 'gift culture' was borrowed from Theodore Porter; See, T. M. Porter, Trust in Numbers (Princeton: Princeton University Press, 1995), p. 226. 
Virology Section of the Research Department of the Children's Hospital of Philadelphia, Cantell experienced difficulties in establishing an experimental system similar to that he had worked with in America (the transfer of skills and materials had only been partly successful) he immediately fell back on his former research 'pal', Paucker. As we can see in the following excerpt from a letter by Cantell to Paucker, there was more at stake than an occasional call for assistance to reproduce a particular production system for chick interferon:

\begin{abstract}
Our work on interferon is in slow progress here (Helsinki). We produce interferon in eggs with mumps virus, but have not yet found suitable conditions to get very high titers. This is why I would like to have WS (strain of influenza virus), if we can not improve the yields with mumps virus. The interferon is assayed by a similar plaque inhibition test we had there (Philadelphia) and it works nicely. We get the Falcon plastic dishes (assay-dishes) from California. Our purpose is to start soon producing interferon on a rather large scale and to purify the stuff to some extent. One of the things I would like to do with it (the purified interferon) is to prepare good anti-IF sera. I wonder whether we could not establish some co-work or co-operation between your lab and my lab. We would supply you with antiserum against chick interferon and you us with antiserum against mouse interferon. What do you think about this? (llalics are mine) ${ }^{23}$
\end{abstract}

By emphasizing the profitability of mutual assistance Cantell obviously tried to establish a permanent connection between his Helsinki laboratory and Paucker's laboratory at the Children's Hospital of Philadelphia. As it turned out Paucker too thought it profitable to set up a kind of permanent barter with Cantell. During Cantell's stay in Philadelphia both men had learned to respect each other's abilities as laboratory researchers and they knew that they could count on each other to produce high quality data and research materials which would be useful in pursuit of one another's research. ${ }^{24}$

Both Cantell and Paucker were well aware of the problems involved in exchanging data and research materials between laboratories. They knew by experience that even when working together in the same laboratory it could be quite demanding to make comparisons between each other's data. According to Cantell, different researchers could make research materials perform differently and these differences could be even greater in the case of experiments involving biological specimens. Collaboration between the same pair of researchers in different laboratories would only complicate matters. ${ }^{25}$ Apart from occasional difficulties with customs over the contents of packages and occasional loss of material due to critical damages to containers during the journey or to contamination, most problems originated from the

23 Cantell to Paucker, letter dated 24 September 1962, Cantell Personal Archives.

24 Interview with Kari Cantell.

25

Ibid. 
changeable behaviour of biological specimens. ${ }^{26}$ However skillfully one packaged the tubes with biological materials (mostly in containers filled with dry ice and labeled as "Biological specimen, no commercial value") for shipment, the exchange of biological specimens often met with resistance. ${ }^{27}$ More often than not, strains of cells or viruses which were sent from one laboratory to the other behaved differently on arrival and it required some manipulation to make them 'adapt' to the local situation. At times, for no obvious reason at all, a virus strain or cell-line lost some of its original properties on transfer to a foreign laboratory, or even stopped growing at all. ${ }^{28}$ In general, however, the differences were manageable and laboratory organisms could be made to work in a similar fashion in both Cantell's and Paucker's laboratory. ${ }^{29}$

The regular exchange of research materials turned out to be profitable for both parties. Whenever either Cantell or Paucker experienced difficulties in working along similar lines of research, they could appeal to the other for help. Paucker called it 'the easy way out'. ${ }^{30}$ Cantell indicated that the exchange mechanism was instrumental in addressing problems of uncertainty and variability. ${ }^{31}$

In the process, they also exchanged interferon preparations in use as internal laboratory standards, with the aim of facilitating the comparison of assay results. It was far from a rigorous attempt at correlating research results. Cantell, for example, sent Paucker a number of tubes with "standard IF" with the following specifications:

This interferon has been prepared by Lee virus in chick embryo's and purified to some extent according to Tytell et al. Every tube contains $50 \mathrm{mg}$ of dry substance consisting mostly of salts because the IF was not dialysed before lyophilization. In our hands every tube contains about 10.000 IF units when assayed by the plaque production test against VSV. By the way, we use 13 day-old chick embryos for this purpose, because

26 Whenever possible, mostly on return from overseas meetings, biomedical researchers would carry the packages themselves or ask fellow scientists who happened to be in the area of a specific laboratory to pick up a package on their way home. For instance at the end of his stay in Philadelphia on his trip back to Helsinki Cantell took along with him a couple of bottles with virus strains he had worked with in Werner Henle's Department. It was regarded as a practical way to get around problems relating to logistics (customs, export licences, transport costs and damages). In particular during the summer months with a peak in congresses in both Europe and the USA there was a lively traffic of biological specimens between the continents: This observation is based on both correspondences and interviews of the various scientists under survey in this paper.

27 Interviews with Kari Cantell, and lon Gresser.

Kari Cantell to Kurt Paucker, letter dated 23 October 1962, Cantell Personal Archives. Interview with Kari Cantell. 
This description of the properties of Cantell's chick interferon standard is largely qualitative in nature with only a minimum concern for rigor. As long as interferon researchers like Cantell restricted their dealings to fellow scientists like Paucker with whom they could make frequent recourse to shared, often tacit knowledge this made perfect sense. However, when following the first formal interferon gathering in Czechoslovakia, in 1964, researchers with an interest in interferon research began to expand their networks with fellow scientists they only knew by name, the informal form of measurement began to show flaws. This is clearly illustrated by the problems involved in an exchange of samples of human interferon between Cantell and the Head of the Division of Infectious Diseases of Stanford University School of Medicine (CA), Thomas Merigan.

\subsection{Creating a breeding-ground for standardization}

After noticing in the literature in the fall of 1965 that Merigan, whom he had met once at the Smolenice meeting, like himself was involved in setting up a production system for human interferon, Cantell immediately contacted Merigan. ${ }^{33}$ With most interferon researchers using an assay system of their own, it was difficult to compare the assay data in the literature and to judge which production system was more promising in terms of the yields of human interferon. In order to get some idea about how his production and assay system compared to Merigan's, Cantell therefore proposed to exchange interferon probes. Merigan, who was keen to improve on his laboratory results with an eye toward producing a preparation suitable for limited trial in man, was receptive to the suggestion. Subsequently Cantell sent Merigan a batch of his human interferon. As per the specification the human interferon had been prepared by incubating cultures of human white blood cells ('leucocytes') with Sendai virus. The isolation and purification method employed was said to be similar to the methodology of Tytell et al. The potency ('titer') of the material was said to be '1:500' or 500 units when assayed by "plaque reduction against VSV (virus) in amnion cells". ${ }^{34}$

Whereas a similar kind of specification made perfect sense to Paucker, in the case of Merigan it gave cause for confusion. In Merigan's hands, Cantell's interferon had measured a

Kari Cantell to Kurt Paucker, Letter dated November 19, 1964, Cantell Personal Archives.

Kari Cantell to Thomas Merigan, letter dated 30 October 1965. Cantell Personal Archives. 
titer of about 1:2600 or 2600 units, but Merigan did not know what to make of the five fold difference between his and Cantell's results. ${ }^{35}$ If confirmed, it would imply on the one hand that his assay system was definitely more sensitive, but on the other hand, and less positively, it would imply that Cantell's production system for human interferon was superior to the one he employed (based on virus infected cultures of neonatal fibroblast cells). However the results were far from clear yet. Much depended on what Cantell meant exactly by indicating that the titer of the Finnish interferon was 500 units. In a letter to Cantell Merigan therefore put the matter up for discussion.

I am not clear when you say 500 units whether or not you mean per $\mathrm{ml}$ or per $4 \mathrm{ml}$ as you use as an absorption volume in your assay on $15 \times 100 \mathrm{~mm}$ plastic plates or the equivalent. If you measure your interferon in 4 $\mathrm{ml}$ and divide it by 4 to express it on a per $1 \mathrm{ml}$ basis then our results on your WBC (white blood cell) interferon would be quite similar. ${ }^{36}$

Merigan emphasized that in order to figure things out and enable a more accurate comparison of assay data, it would be necessary to know as precisely as possible the assay procedure each of them employed, as well as the mode of expressing interferon units. In his view, it was most important to know the volume the units were measured in. He told Cantell that following an exchange of chick interferon samples between his and five other American laboratories, he chose to express his units as "the reciprocal of the dilution which inhibited $50 \%$ of the VSV plaques as measured in $4 \mathrm{ml}$ aliquots". ${ }^{37}$

Judging by a follow-up letter from Merigan to Cantell, Cantell disagreed with Merigan on the relative importance of the volume aspect, but he shared Merigan's concern for more rigor in the exchange of information. They were ultimately able to settle, at least for the time being, on a fivefold difference in sensitivity of their assay systems. However, before extending this sort of comparison to a larger number of laboratories they were both in favor of establishing a common standard for human interferon. Merigan indicated that he would bring the standardization issue up for discussion at the next international interferon meeting that was planned for the spring of 1967 in London. ${ }^{38}$

For a couple of years Merigan had vocalized his support for the establishment of interferon standards among the group of American scientists interested in this biological agent. In order to assess the therapeutic potential of interferon, Merigan had chosen to work along

Thomas Merigan to Kari Cantell, letter dated 11 July 1966, Cantell Personal Archives.

36 Ibid.

37 Ibid,

38

Thomas Merigan to Kari Cantell, letter dated 17 November 1966, Cantell Personal Archives. 
the lines of exploring in a quantitative way the conditions needed for optimal protection against experimental virus infections both in vitro and in vivo. Merigan had not been discouraged by the inconclusive results of the British experiments with interferon in humans. The various published data on the broad antiviral spectrum and non-toxic nature of interferon in vitro was too promising to be put off by a single unsuccessful human trial. In his view, the British scientists had been too hasty. ${ }^{39}$ They should have at least waited until they had investigated the quantitative aspects of the interferon phenomenon more thoroughly. It did not seem to make much sense to start clinical trials without knowing more about how much interferon was needed to protect laboratory animals against experimental virus infections. Having experienced difficulties in assessing the relative merits of the various published methods for production and purification, when starting his interferon research in 1963, and later on in correlating his research data with those of others, he became increasingly concerned with standardization.

However, he learned that for a majority of workers in the field standardization was not a major issue. A large portion of the work done was qualitatively descriptive in nature and usually considered to stand on its own merits. In general these researchers felt less need to be able to correlate published results from different laboratories. As long as the variation in research results did not make a qualitative difference, the matter was not taken very seriously. ${ }^{40}$ As a subject, standardization was considered rather boring. ${ }^{41}$ According to Finter, as far as most interferon researchers were concerned, if they got an answer than that was the answer. Should somebody else then come with a tenfold difference in research results, for instance, mostly the reaction would be in the following terms: "Okay if you say so, most likely this means that your assay is more sensitive then mine". ${ }^{42}$

Merigan was pleased to hear during an informal gathering of interferon researchers at the 1964 meeting of the American Society for Microbiology in Washington, D.C., that the established interferon researchers Sam Baron and Monto Ho shared his concern about the need for common units of measure. They agreed with Merigan that with a growing number of scientists interested in research problems relating to interferon, and a gradual shift away from qualitative descriptive to a more quantitative oriented type of work, the usual way of correlating one's results with those of others through the exchange of laboratory samples would become increasingly time-consuming. In the long-term, it might therefore pay off to set up interferon standards as a means to even the variation between laboratories and to facilitate the comparison of research results. Following the discussion of this subject, Baron, Ho and

Interview with Thomas Merigan.

40

Ibid.

41 Jean Lindenmann to Alick Isaacs, letter dated 19 May 1964, Lindenmann personal archives.

42 Interview with Norman Finter.
} 
Merigan announced that they would request financial and material support from the National Institutes of Health $(\mathrm{NIH})$ for establishing and distributing reference standard preparations for chick and mouse interferon, which were most widely used among American interferon researchers, ${ }^{43}$

\subsubsection{An experiment in scientific communication}

Without much trouble, the NIH granted the request. In his capacity of Medical Director of the National Institute of Allergy and Infectious Diseases (NIAID)—one of the institutes that make up the NIH - and as former Head of the Virology Section of the Division of Biological Standards, Baron knew his way around the NIH bureaucracy. Mainly through Baron's offices the NIH administrators gave the undertaking to supporting efforts to establish reference reagents for both mouse and chick interferon. This basically meant that the NIH acquired - through the efforts of Baron and Merigan-batches of partially purified mouse and chick interferon, where after the Reference Reagents Branch of the NIH was given the job to prepare thousand ampules filled with one $\mathrm{ml}$ freeze-dried samples of what would be designated 'NIH Reference Reagent' for chick and mouse interferon respectively. ${ }^{44}$

While the preparations for research standards were under way, Baron learnt through his NIH network that NIH administrators were looking for research areas that might benefit from experimenting with a new form of communication, the so-called 'information exchange group'. In studying ways to speed up the development of the biomedical sciences, the NIH had engaged in an experiment in communication which aimed at improving and speeding up communication between scientists. It was argued that because of the inevitably long delays in publication and the distaste of editors for polemics, there was no longer any space left for real discussion in journals. Setting up information exchange groups with the means of dispatching communications, without any editorial restrictions, within a matter of days, free of any charge to all the members of a group, was expected to restore the role of argument as a public instrument of scientific progress. As Baron understood it, the working of this new form of information exchange would enable anyone working on interferon to communicate research findings or other scientific information in record time to all the others in the same area of research throughout the world. This seemed just the kind of boost the embryonic field of interferon research needed. Baron decided overnight to take the initiative and ask the NIH Information Exchange Group Office for support to set up an information exchange group to

\footnotetext{
43 Interviews with Sam Baron, Monto Ho, and Thomas Merigan, and George Galasso. 
provide better communication among scientists actively interested in problems of interferon. ${ }^{45}$ "I went to the administrator and said, "Here is an area that is not fully accepted with a few struggling laboratories around the world. If we can keep them in constant communication they are likely to accelerate the development of this biological substance that will be of ultimate use as a therapeutic drug. "I indicated that it would be catalytic to set up an information exchange. Would they be willing to try it and they said "yes". I wrote to Isaacs and he agreed too. In fact he was chairman". ${ }^{46}$

Whereas most interferon researchers in America and overseas welcomed the initiative and immediately applied for membership of the NIH sponsored Information Exchange Group no. 6 ('IEG 6') for research on interferon, its reception within the British Scientific Committee on Interferon was mixed. The letter of invitation which was signed by both Baron and Isaacs took the members of the Scientific Committee on Interferon by surprise and immediately caused some unease. Apart from the fact that they had not been informed about the American initiative by Isaacs, they were irritated with the idea of losing the lead in the field to the Americans. ${ }^{47}$ Furthermore, when on reflection the members of the Scientific Committee realized that there was every advantage to be gained from joining this group, they became worried about possible resistance within the collaboration. Participation in the exchange group was thought to conflict with the policy of stringent control over publication of work on interferon. ${ }^{48}$ Their suspicions proved to be correct.

The industrial collaborators, in particular, were horrified by the thought of members of the Scientific Committee freely exchanging information with anyone actively working in the field. The invitation letter spoke about communications that would include not only manuscripts but also discussions, unpublished research findings, technical suggestions, unofficial notes of meetings or any other original communications. The drug company executives took the view that to accept this would be to destroy the basis of collaboration. ${ }^{49}$ Eventually, following extensive correspondence between the Scientific Committee and the Board of Directors of the Interferon Collaboration it was decided that on the condition that only manuscripts would be circulated which were already intended for publication and had

D. E. Green, 'An Experiment in Communication: The Information Exchange Group', Science, 143 (1964), 308-9; Anonymous, 'Information Exchange Group', Nature, 204 (1964), 627; Interviews with Sam Baron and George Galasso. Interview with Sam Baron.

47 Minutes of the 28th meeting of the Scientific Committee on Interferon, dated 10 November 1964, MRC Archives File No. S788/2/3. Internal note MRC, dated 10 November 1964, MRC Archives File No. S788/2/3.

49 Minutes GNRD Patent Holdings Ltd. meeting, 10 December 1964, MRC Archives File No. A812/8/2; and Internal note MRC, dated 10 December 1964, MRC Archives File No. S788/9. 
been cleared by the patent adviser, members of the collaboration were allowed to participate in the exchange group..$^{50}$

By the end of January 1965, 109 biomedical researchers from 17 countries had been enlisted in IEG $6 .^{51}$ In its first two years the interferon exchange group distributed over 220 different communications of its members. ${ }^{52}$ For the greater part these consisted of preprints of articles in press and reprints of talks given at symposia, but also included unofficial notes of meetings, technical suggestions, notes of unpublished research findings, and discussions of research problems such as difficulties in reaching some decision on what one ought to call an 'interferon', or difficulties with correlating research results from different laboratories. ${ }^{53}$ Generally the information communicated by the IEG 6 preceded journal publication by six to nine months. It was this gain in time that appealed most to the members of the exchange group. According to Baron once you had your laboratory results processed you could have them out in two weeks time. "This allowed for the following kind of dialogue: in response to so and so's item of last month I want to say that I did the same experiment but it did not come out quite the same way and I therefore think that the interpretation should be different". ${ }^{54}$

However valued by its members the new communication system began to face increasing opposition from the editors of the biochemical journals. The editors heavily criticized the fact that a large part of the communications appeared to consist of preprints of full articles. As such the communications were in effect publications and could no longer be considered to be an equivalent to a society newsletter. They threatened to ban from publication anything that had first gone through an IEG. In order to meet the objections of the journals IEG 6 was modified and transformed into 'Interferon Scientific Memorandum' in the course of 1967 (see Fig. 42). However, the change of name and sponsorship-from the NIH IEG office to the NIAID Policy Council-and the new rule that only abstracts of work being prepared for publication, plus the tables and figures would be accepted for circulation, did not affect the principle of the communication mechanism.

50 Sonnabend to Medawar, letter dated 9 November 1964, MRC Archives File No. S788/9; Medawar to NRDC, letter dated 9 November 1964. MRC Archives File No. S788/9; NRDC to Medawar, letter dated 10 November 1964. MRC Archives File No. S788/9: and Minutes GNRD Patent Holdings Ltd, meeting, 10 December 1964. MRC Archives File No. A812/8/2

51 IEG 6 membership list, dated 18 January 1965, Kari Cantell personal archives.

52 Sam Baron to members of IEG 6, letter dated 2 November 1966, Ion Gresser personal archives.

33 IEG 6. communications 1-220, Kari Cantell personal archives.

54 Interview with Sam Baron. 


\section{Interferon Scientific Memoranda}

February 1968

The present systew for the rapid and informal exchange of information among researchers in the interferon fleld is being modified and improved, as was announced in the January 1968 issue of Interferon Scientific Menoranda (ISM). The first issue in the new forat, still enticled Interferon Scientific Memoranda, will be matled in March.

\section{Synopais of New System}

The purpose of the ISM is to bring scientists engaged in interferon research around the world into closer contact for a mutually beneficial exchange of findings and opinions. The modified aysten, hovever, will perait the exchange of tables, graphs, and photos as well as aotes and abstracts.

\section{What Kind of Material to Subwit}

Contributions way include;

1. Progress notes, abstracts or suamaries of unpublished research findings, along with tables, figures, and photos which clarify the text.

2. Discussions and criticiams,

3. Notes of bfbliographic onissions.

4. Technical suggentions.

5. Unofficial notes of meetings.

6. Any other original contributions relating to interferon research.

Material will be printed in the language aubicted, along with an English cranslation if included.

Complete manuscripts cannot be accepted. As in the past, the Interferon Combittee vill play an advisory role in setting policy and making operational decisions.

As a recipient of privileged, unpublished inforation, each participant agrees on his own behalf and on behalf of any other person with whom he shares the information that research findings communicated via the ISM w11 be treated as "personal conmunications" from professional colleagues, and that they will be given credit as such in any situation where questions of priority might arise. Reference to material in the ISM should be cited only as "personal communication," with the date of ISM isaue (e.g., "Smith, J., Personal Communfcation, Jan. 1968").

Fig. 42. February 1968 new policy announcement on ISM by Gerald Bidlack, project manager. Courtesy of NIAID. 
Like IEG 6 before it, the Interferon Scientific Memorandum functioned as a central clearing house for communications on interferon. ${ }^{55}$ Sharing a formal mechanism for communication that was meant to accelerate the dissemination of information required sharing and shaping common denominators that allowed for dialogue and enabled participants to share research problems and cope with differences. As such the NIH sponsored international information exchange system was instrumental in creating needs for standards, At the same time, by bringing out difficulties with correlating research results obtained in different laboratories, the IEG and Scientific Memorandum contributed to a growing awareness of and attention to this issue. The gradual rise in interest in the standardization issue manifested itself in American and subsequently British efforts to establish common units of measurement.

\subsubsection{Safeguarding British interests}

The news that the NIH had obtained batches of chick and mouse interferon from Merigan and were distributing freeze dried samples of this material as provisional reference standard preparations stirred a renewed interest in the standardization question in Britain. Before the news about the existence of American interferon standards, there had been occasional calls for more attention to the use of standards by the NIMR's Division of Biological Standards, but without much effect. ${ }^{56}$ However, the fact that the Americans seemed to treat the matter very seriously led to an immediate reappraisal of the subject. ${ }^{57}$

Most members of the Scientific Committee had mixed feelings about the American standardization efforts. In particular, the claim that the NIH chick standard was made of the purest chick interferon material yet produced, was regarded with suspicion. The existence of another reference standard preparation of chick interferon beside the MRC research standard gave reason for concern, all the more so since there were indications that the MRC standard contained an impurity that was responsible for non-specific protection. Neither the fact that the biochemists on the Committee were convinced that Merigan's two-step purification procedure did not justify the American claim to purity, nor recent studies suggesting that highly purified chick interferon was far less stable than partially purified interferon, could dispel their

G.E. Wolstenholme and M. O'Connor (eds.), Interferon (1967, Ciba Foundation Symposium).(London, J\&A Churchill, 1968), pp. 260-1.

56

Minutes of the 26th, 27th, 28th and 29th meeting of the Scientific Committee on Interferon, dated 21 April, 7 July. 10 November 1964 and 25 January 1965, MRC Archives File No. S788/2/3.

57 Minutes of the 30 th and 31th meeting of the Scientific Committee on Interferon, dated 4 May and 21 September 1965, MRC Archives File No. S788/2/4; and, Internal notes MRC, dated 5 May and 29 september 1965, MRC Archives File No. 788/2/4. 
uneasiness about the state of affairs. ${ }^{58}$

On behalf of the Division of Biological Standards of the NIMR, Frank Perkins urged the necessity of active participation of the Scientific Committee in drawing up international units of measure. By experience Perkins knew that if at one of the future meetings of the Expert Committee on Biological Standardization (ECBS) of the World Health Organization (WHO) the need for international standards for interferon would be raised, its members would certainly want to know what the best preparations were and what the best ways of assaying them were. In his view, the Scientific Committee had much to contribute to the American standardization efforts on these two points. The normal procedure of the WHO in setting up an international standard was to relate the new international unit to that of the first wellrecognized national standard prepared. In order to safeguard British interests and prevent the MRC research standard for chick interferon losing to the American standard, it would not only be helpful but desirable to keep up with American efforts. In the short term, the best thing to do would probably be to establish an additional semi-purified chick standard that was species specific, and a suitable mouse standard, and try and reach an agreement with the NIH on these reference standards. This might be accomplished by arranging a collaborative assay to be carried out by workers in both countries. Furthermore, he suggested that the committee should undertake to circulate a report on the MRC research standard through the information exchange group. In the meantime, Perkins would ask Bangham to bring up for discussion at the ECBS of the WHO the need for international standards for interferon. ${ }^{59}$ Following Perkins's plea for action, Glaxo scientists promised to supply the NIMR's Division of Biological Standards with a large batch of semi-purified, species-specific chick interferon. This material could then be used to prepare a new chick interferon standard that would stand a better chance of being accepted by the WHO as an international standard than the currently available provisional MRC Research Standard A for chick interferon. ${ }^{60}$

In order to keep the question of standardization on the Committee's agenda on a permanent basis, Finter added a little extra. With the very different units of measure used by

Ibid.

59 In November 1966, through the agency of Bangham at a meeting of the Directors of the WHO Respiratory Virus and Enterovirus Reference Centres, it was decided that it was advisable to establish reference material to be used in the calibration of interferon assay systems. Subsequently, the ECBS of the WHO agreed that 'further information was necessary on the suitability of various materials and the quantities that would be needed for particular purposes and requested the National Institute for Medical Research, London, in collaboration with the WHO Seccretariat, to collect such information'; Report on Human Interferon. The British Research Standard A, Division of Biological Standards, dated August 1971. MRC Archives File No. S788/15/2.

60

Minutes of the 30 th and 31 th meeting of the Scientific Committee on Interferon, dated 4 May and 21 September 1965, MRC Archives File No. S788/2/4: and. Internal notes MRC, dated 5 May and 29 september 1965, MRC Archives File No, 788/2/4. 
various workers, Finter thought it hardly possible to evaluate quantitative data from different laboratories in the field of interferon research. How should he correlate his interferon data with those of Baron at the $\mathrm{NIH}$, who like him was involved in studies on the possible use of exogenous interferon to control virus infections in mice? Of course, as before he could try to correlate his results with those of Baron by arranging informally for an exchange of internal laboratory standards. However, even with people like Baron, whom he knew fairly well, this procedure would increasingly take up his research time. How then should he judge recent reports by American workers, whom he knew only by name, that high levels of interferon-like activity could be induced in cell cultures by adding a substance known as Statolon, which supposedly was a highly molecular carbohydrate? ${ }^{61}$ Did this new approach, which seemed to be very much in line with Isaacs's ideas about artificially stimulating organisms to produce endogenous interferon through the use of specific foreign substances, justify opening up a new line of research? Because of the very different potencies of the interferon units used by different workers, Finter thought it rather difficult to make a comparison between this endogenous approach, and his exogenous approach. In Finter's opinion these latest developments only emphasized the need for international reference standards for interferons. ${ }^{62}$

Subsequently, Finter asked and was given permission to exchange samples of his laboratory reference preparation of mouse interferon-which, provided it would pass future stability studies, might serve as a basis for a provisional British standard-with Merigan and Baron. This was considered to be the first systematic attempt at correlating the unit used for a preparation of mouse interferon by different workers in both Europe and America. ${ }^{63}$

61 W.J. Kleinsmidt, J.C. Cline and E.B. Murphy, 'Interferon Production Induced by Statolon'. Proc. Natl. Acad. of Sci. U.S. 52 (1964), 741-4.

62 Professor Ernst Chain, who had received the Nobel Prize for the discovery of penicillin and its therapeutic effects in various infectious diseases, would not wait for Finter's assessments. On hearing from Isaacs that the interferon inducing substance Statolon was produced by a penicillium mould he immediately decided to start looking for other active interferon inducing agents in penicillium moulds, which were available in abundance in his laboratory at the Imperial College of Science and Technology in London. Both Chain and Isaacs hoped that the penicillium molds might lead the way into the field of chemotherapy against viruses just as they did into chemotherapy against bacteria.Minutes of the 30th and 31th meeting of the Scientific Committee on Interferon, dated 4 May and 21 September 1965, MRC Archives File No. S788/2/4; and, Internal notes MRC, dated 5 May and 29 september 1965, MRC Archives File No. 788/2/4; Minutes of the Scientific Steering Committee, dated 9 March, 1965, MRC Archives File No. S788/8/1; and interview with Norman Finter.

63 There was also general agreement that Finter should accept the invitation from the North Holland Publishing Company, which was trying to extend its interests into the biological sciences, to edit a monograph on interferon. It was felt to be a good idea to invite a number of British and American interferon experts to contribute to a state-of-the-art textbook on interferons, before the field expanded further and became unwieldy. All agreed that a textbook approach would not only be helpful to make the subject more accessible and encourage scientists to refer in their publications specifically to interferon but also to bring coherence to the field.Internal note MRC, dated 12 May 1965, MRC Archives File No. S788/2/4: The textbook book on interferons would be published at the end of 1966, N. B. Finter, Interferons (Amsterdam, North Holland 
In the process of exchanging laboratory samples of mouse interferon with Merigan and performing comparative assays, it turned out that Merigan's pure material was only as strong as the ICI crude material. Finter was pleasantly surprised not only to see that correlating his results with those of Merigan, with whom he only had a "nodding acquaintance", went rather smoothly, but also to find out that the mouse unit used in both laboratories differed only slightly. ${ }^{64}$ Conversely, correlating the units used in his and Baron's laboratory put his patience to the test. The seemingly unremitting variance in the potencies obtained for a particular preparation became a source of irritation on both sides of the Atlantic. Initially it was mainly Baron who struggled to suppress the variation in his laboratory and Finter blaming him for not sufficiently perfecting his series of measurements. However, the situation became confusing when for no obvious reason the potencies in Finter's laboratory began to drift too. ${ }^{65}$ Eventually they decided to come to grips with the problems by systematically studying the differences internal to and between their laboratories; each series of measurements would be performed by using the same reagents and assay method.

While Finter and Baron were still very much in the process of studying the nature of the differences in results and agreeing on a conversion factor to correlate the work of both laboratories, word came from Isaacs - who after a series of manic-depressive episodes with periodic hospitalizations and occasional medication was having one of his rare good periods $^{66}$ - that the virologist and interferon researcher Charles Chany in his laboratory at the Hôpital St. Vincent-de-Paul, Paris, had succeeded in setting up a production system that seemed feasible for the eventual 'mass production' of human interferon. Chany claimed to have produced the largest batch of partially purified human interferon available anywhere in the world. The news struck home as two months earlier it had still been difficult to envisage the production of large amounts of human interferon that would be needed to perform clinical trials. $^{67}$

At the October 1966 session of the Scientific Committee on Interferon Isaacs raised the question as to whether or not efforts should be made to join forces with the French in instituting clinical trials. Isaacs argued that establishing such a liaison would be in the interest

Publishing Company, 1966).

64

Minutes of the 31 th meeting of the Scientific Committee on Interferon, dated 24 September I965. MRC Archives File No. S788/2/4.

N.B. Finter, 'Interferon Assays: Sensitivity and Other Aspects', in G. Rita (ed., 1967, June, Siena Symposium) The Interferons (New York, Academic Press, 1968), p. 211: and, Finter, interview.

Unbeknownst to his colleagues, he attempted to take his own life on at least two occasions; interview with Susanna Isaacs-Elmhirst.

Internal note MRC, dated 14 October 1966, MRC Archives File No. S788/2/4; and N. B. Finter, Interferons (Amsterdam, North Holland Publishing Company, 1966), p. 264. 
of both parties. As the three participating firms were clearly lagging behind in the production of human interferon, he believed that the committee might take advantage of Chany's expertise with the large-scale production of human interferon. While giving Chany and his collaborators the credits for their production skills, there was reserve with regard to French clinical methodology: the French were generally considered hopeless at arranging clinical trials. Judging by Chany's interest in supplying human interferon for a clinical trial in the UK, the French, in Isaacs opinion, were well aware that they could benefit from the Committee's experience of conducting clinical trials with interferon and from the availability of testing facilities at the Common Cold Research Unit. Isaacs then asked if the other members of the committee thought the idea of arranging a clinical test of human interferon acceptable. ${ }^{68}$

Isaacs's question stirred a discussion within the Scientific Committee about the desirability of clinical trials as opposed to more basic in vitro and animal studies of interferon. Eventually the majority agreed that they should give it another try four years after the negatively assessed clinical trial program had been aborted. Most members considered Finter's mouse studies and the prospect of having access to a production source for human interferon too promising to allow the opportunity of a test in humans to pass. ${ }^{69}$

They agreed after much debate that it was most important not to go too fast. It was emphasized that before any arrangement was made with Chany the work which might be involved must be carefully considered. The chairman should therefore sent a letter to Chany asking him for a quantity of human interferon and only suggesting the possibility of a joint trial. ${ }^{70}$ Finter thought it terribly important first to correlate the units used for human interferon in different laboratories. Without correspondence between units used by different workers, it was difficult to compare Chany's interferon with human interferon that was produced in a similar fashion by Cantell in Helsinki and Merigan at Stanford. Finter indicated that he would be able to discuss the idea for a comparative study in detail with all three workers at the Second International Symposium of Applied Virology and Medicine held in Fort Lauderdale (Florida) in December 1966. ${ }^{11}$

Minutes of the 34th meeting of the Scientific Committee on Interferon, dated 20 October 1966, MRC Archives File No. S788/2/4.

Minutes of the 34th meeting of the Scientific Committee on Interferon, dated 20 October 1966, MRC Archives File No. S788/2/4; and, Internal note MRC, dated 27 October, 27 October 1966, MRC Archives File No. S788/2/4; N.B. Finter. Interferon as an Antiviral Agent In Vivo: Quantative and Temporal Aspects of the Protection of Mice against Semliki Forest Virus', Brit. J. Exp. Pathol., 47 (1966), 361-70.

7 Minutes of the 34th meeting of the Scientific Committee on Interferon, dated 20 October 1966, MRC Archives File No. S788/2/4; and, Internal note MRC, dated 27 October, 27 October 1966, MRC Archives File No. S788/2/4. 


\subsubsection{The 'interferon' versus 'inducer' people}

In the corridors of the Florida meeting there were rumors buzzing that interest in interferon was picking up momentum. No less than two international interferon meetings were scheduled for 1967 and after years of radio silence the field of interferon research seemed to produce results that were newsworthy. Beside news about French as well as Finnish and Russian investigators having developed production methods for human interferon and plans to use this interferon in clinical trials, word spread about promising results with the use of synthetic interferon stimulating agents in protecting against viral infection. ${ }^{72}$

It had been known for some time that the endogenous production of interferon could be stimulated by extracts of living material such as the fungal product Statolon. However, the complex and uncertain nature of these impure materials-Statolon initially was thought to be a complex polysacharide, but now was claimed to be either a fungal virus or a double-stranded ribonucleic acid-obtained from natural sources raised doubts about their practicality in laboratory and clinical research. Most researchers were reluctant to work with natural materials of undefined composition, which made their research completely dependent upon biological test procedures with their inherent limitations of accuracy and their heavy cost in material and skill. The impurity argument had been used before to call into question the practicality of developing interferon as a therapeutic substance against clinical viral infection. ${ }^{73}$

The news that Maurice Hilleman at the Merck Institute for Therapeutic Research at Rahway, New Jersey, and William Regelson at the Medical College of Virginia had both successfully explored the possibility of preparing synthetic materials of known composition in their laboratories which were capable of stimulating endogenous interferon activity was snapped up. It was most exciting that endogenous interferon production could be stimulated and studied in vivo by the use of easy to make and inexpensive chemicals without having to worry about impurities. Basically, working along these lines meant that interferon was no longer perceived in terms of a practical therapeutic agent but as a research tool to develop preferably chemical means - in other words synthesizing chemical compounds-for achieving viral chemotherapy. The dominant view was that if interferon was ever going to come to

72 E.R. Falcoff, R. Falcoff, F. Fournier, and C. Chany, 'Production en Masse, Purification Partielle et Caractérisation d'un Interféron Destiné a des Essais Therapeutiques Humains', Ann. Inst. Pasteur, 111 (1966), 562-84; A.K. Field, A. A. Tytell, G.P. Lampson, G.P. and M. Hilleman, Inducers of Interferon and Host Resistance: II. Multistranded Synthetic Polynucleotide Complexes', Proc. Nat. Acad. Sci. U.S.A., 58 (1967), 1004-10; W. Regelson, 'Prevention and treatment of Friend Leukemia Virus (FLV) Infection by InterferonInducing Synthetic Polyanions', Advan. Exp. Med. Biol., I (1967), 316-32; and interviews with Kari Cantell, Maurice Hilleman, and Thomas Merigan.

M. Sanders and E. H. Lennette, Medical and Applied Virology, Proc, Soc, Int, Symp. (St. Louis: Warren H. Green Inc., 1968); and interviews with Sam Baron. Kari Cantell. Norman Finter. Maurice Hilleman, and Thomas Merigan. 
something clinically useful, the synthetic interferon inducers showed most promise. ${ }^{74}$

Isaacs was not to get a great deal of pleasure out of the reappraisal of the field of interferon research. Following a fatal hemorrhage Isaacs died on 26 January 1967 in the University Hospital, at the age forty-five. However shocking the news of his untimely death was, it did hardly affect the course of events. Due to his illness he was already quite some time a mere shadow of his former self. ${ }^{75}$

Through the rapidly expanding work on interferon inducers new needs were being created for common denominators. The research question that began to dominate the research agenda was whether the endogenous approach offered a fruitful alternative to the exogenous approach. Gradually, a division emerged between those who favored working along the lines of the endogenous approach - the 'inducer people'-and others opting for holding on to the exogenous approach - the 'interferon people'. ${ }^{76}$ However different their modes of perceiving interferon as an object for research, both groups shared a growing interest in standardization and establishing common yardsticks as a means to the end of comparing and criticizing another one's research data.

The higher rating of the subject found expression in the fact that during the general discussion of the April 1967 Interferon meeting in London, special attention was paid to ongoing attempts to prepare standard interferon preparations and two months later at a followup conference in the beautiful setting of Siena (Italy), Finter-to his own amazement-was invited to give a lecture on standardization. ${ }^{77}$ According to Finter, he did not think at the time

74 While it had been agreed in 1965 under pressure of the three pharmaceutical companies, who preferred to work independently in the area of interferon inducers, to leave this topic outside the Interferon collaboration, some MRC officers could be heart saying that it might be more advantageous to join forces on the study of inducers too; Internal note MRC, dated 11 September 1967, MRC Archives, File No. A812/17/1.

Tyrrell had been de facto chairman of the Scientific Committee on Interferon for the past few years and in practice Isaacs's scientific assistant, the virologist, Joseph Sonnabend, ran Isaacs's Laboratory for Research on Interferon at the NIMR. Medawar, the director of the NIMR, who had been thinking of disbanding the unproductive Laboratory for Research on Interferon for quite some time, seized the opportunity to reassimilate the Interferon Laboratory into what had been renamed the "Division of Virology". Medawar met hardly any opposition as he could convince worried MRC officers that this would not mean that all work at the NIMR on interferon would come to an end. On the contrary, he assured that the NIMR's efforts in the field of interferon research would be continued "at not less than its present intensity". Medawar enforced his claim by pointing out that the most promising research work on interferon at the NIMR, studying how interferon acted at the molecular level, was currently performed by one of the staff members of the Biochemistry Division, the biochemist, Martin Kerr.Internal Note MRC dated 9 February 1967, MRC Archives, File No. A812/8/2; The MRC to Sir Peter Medawar, letter dated 14 February 1967, MRC Archives, File No. S788/13; and Sir Peter Medawar to the MRC, letter dated 17 February 1967, MRC Archives, File No. A812/8/2.

Interviews with Sam Baron, Kari Cantell, Norman Finter, Robert Friedman, Ion Gresser, Maurice Hilleman, and Jan Vilcek.

The London meeting held 19th-21st April 1967 in London, which was organized on behalf of the Ciba Foundation, was the first meeting after Smolinice completely devoted to the subject of interferon. With the idea of getting some new input from an expert-outsider, Francis Crick was specially invited as a participant to 
that his presentation made very much impact except for the fact that it was instrumental in emphasizing once again the need for standards and in initiating discussions in the corridors concerning British and American efforts to set up interferon standards. ${ }^{78}$

Following the Siena symposium the standardization issue as well as the organization of clinical trials figured prominently on the Scientific Committee's agenda. In consultation with the NIMR's Division of Biological Standards, plans for organizing international collaborative assays of chick, mouse and human interferon were made. One of the researchers in Bangham's Division, Gray Anderson, was already in the process of enlist on behalf of the ECBS of the WHO seven laboratories in Britain, the U.S. and Finland which were prepared to participate in an international collaborative assay of chick embryo interferons. The participants were told that the assay was to be carried out in order to assign potencies to both a new British and American chick interferon standard in terms of the existing British Research Standard. This was said to enable researchers in the future to assign potencies on a common unitage basis. ${ }^{79}$

What Anderson did not tell the participants was that in the first place the assay was meant to produce a replacement of the MRC Research Standard A by a new standard to be offered to the WHO, which was expected to stand a good chance of being accepted as an international reference preparation. At the same time, the opportunity was taken to include in the assay the NIH chick interferon standard, so that in the future British and U.S. standards could be assigned potencies on a common unitage basis. Since the usual practice of the WHO on setting up an international standard was to relate the new international unit to that of the first well-recognized national standard prepared, the British hoped that the Americans would agree to assay their standard in terms of the MRC Standard $\mathrm{A}^{80}$ This did work out rather well. Baron agreed to assay the NIH standard in terms of the MRC Standard A while the members of the Scientific Committee did their share by adding to future papers a reference to the British standard used. ${ }^{81}$

The Division of Biological Standards had hoped to start simultaneously with an international collaborative assay of human interferon. This assay was to be carried out with the primary intention of investigating the suitability of Chany's material to serve as a research

the conference; see, G.E. Wolstenholme and M. O'Connor (eds., 1967, April, London Symposium) Interferon (London, J\&A. Churchill LTD., 1968). pp. 71-4.

Minutes of the 36th meeting of the Scientific Committee on Interferon, dated 28 June 1967, MRC Archives, No. S788/2/5; and interview with Norman Finter.

Gray Anderson to Kari Cantell, letter dated 19 September 1967, Cantell personal archives.

Minutes of the 36th meeting of the Scientific Committee on Interferon, dated 28 June 1967. MRC Archives, No. $5788 / 2 / 5$.

81

Minutes of the 37th meeting of the Scientific Committee on Interferon, dated 17 Oktober 1967, MRC Archives File No. S788/2/5. 
standard to be used in clinical trials. If successful, the MRC would be the first to offer a standard for human interferon, and it was hoped that such a standard would eventually prove suitable for submission to the WHO for consideration as an international standard. ${ }^{82}$

However, it would be a long time before Anderson started organizing an international collaborative assay of human interferon. From the very beginning events were against the establishment of a human interferon standard. The complications began with the presentation of the results of the comparative study of human interferon from four different laboratories at the March 1967 meeting of the Scientific Committee. ${ }^{83}$ Rather unexpectedly, the results of the comparative assays showed that Chany's material was not particularly potent. It turned out that Cantell's interferon was approximately 130 times more potent than Chany's sample which was of average potency. The members of the Scientific Committee were in a scrape, because they already had officially arranged for a meeting with Chany during the London Interferon Symposium to discuss a proposal to carry out a clinical trial with Chany's interferon in MRC facilities. They did not feel like putting the friendly relation with the influential French interferon researcher Chany on the line. That could only put at risk the growing coherence in the field of interferon research, and that was in nobody's interest. Eventually it was decided to tell Chany that the Scientific Committee could not but perform clinical trials with the most potent material available-Cantell's interferon, but that Chany's material could well serve the purpose of setting up a standard. It was a political compromise that would leave its mark over the years. Although, Initially, everything went smoothly. Chany accepted the offer and at the beginning of May 1967 sent five liters of human interferon preparation to the NIMR's Division of Biological Standards. As soon as Anderson had time free in his freeze-drying program, he began to process the material in accordance with the Division's standard production procedure for reference standard preparations. ${ }^{84}$

At the June 1967 meeting of the Scientific Committee Bangham was able to report that more than three thousand $1 \mathrm{ml}$ ampules (labeled with the code number 67/87) filled with freezedried human interferon material were in store. The proposed MRC research standard 67/87 was currently undergoing stability tests, and he hoped that Anderson could soon start arranging an international collaborative study in which this material would be compared with other preparations of human interferon. During the same meeting plans for clinical trials with

82 Minutes of the 36 th meeting of the Scientific Committee on Interferon, dated 28 June 1967, MRC Archives, No. $5788 / 2 / 5$.

83 Minute of the 35th meeting of the Scientific Committee on Interferon, dated 3 March 1967, MRC Archives File No. $\mathrm{S} 788 / 2 / 5$.

84 Interim Report on Standards for Human. Mouse and Chick Embryo Interferons, Division of Biological Standards, dated September 1967, MRC Archives File No. S 788/2/5. 
Cantell's interferon were discussed and it was decided that the action to be undertaken first was to organize an inspection visit of Cantell's laboratory. ${ }^{85}$

\subsection{A 'Finnish detour'}

On Sunday afternoon the 6th of August 1967 at Helsinki airport Kari Cantell nervously awaited the arrival of Norman Finter. It had been more than half a year since he had decided, during informal talks with Finter and Karl Fantes at the Fort Lauderdale conference, to start a collaboration with the British Scientific Committee on Interferon Research. Over a six month period Cantell had supplied Fantes with several batches of his human interferon produced in white blood cell ('leucocyte') cultures for purification and potency studies. Apart from the development of a precipitation method by which the interferon could be concentrated, little had been achieved in terms of the purification of interferon. He had therefore been pleasantly surprised early in July to receive an official request from the Scientific Committee to intensify their collaboration. The British asked for additional amounts of his interferon to carry out work which would lead to a trial in humans. However eager to participate in an ambitious international project such as this, Cantell was somewhat taken aback by the fact that he had to allow for an inspection of his laboratory by the secretary of the Immunological Products Advisory Committee of the MRC, Frank Perkins. At first he felt irritated by the apparent lack of confidence of the British in the safety of his production method: the production protocols in his laboratory were in accordance with the same stringent safety standards that were operative at the Finnish Red Cross Blood Transfusion Service nearby. Finter's personal assurances that laboratory inspections were made a compulsory part of British research projects that involved making biological material for use in humans helped to overcome his negative feelings. In talking over the matter with his Swedish research-assistant, Hans Strander-who was trained in medicine and immunology at the Karolinska Institute in Stockholm and had arranged to pursue his doctoral thesis at the State Serum Institute in Helsinki-he was further persuaded to cooperate. ${ }^{86}$

Minutes of the 36th meeting of the Scientific Committee on Interferon, dated 28 June 1967, MRC Archives File No. $5788 / 2 / 5$.

This impressionistic account of Finter's and Perkins' visit to Cantell is based on: N. Finter to J. Lindenmann. letters dated 7, 15, 28, 31 July and 11 August 1967, K. Cantell correspondence, personal archives; K. Cantell to N. Finter, letters dated 15 and 25 July and 10 August 1967, K. Cantell correspondence, personal archives; Minutes of the 36th meeting of the Scientific Committee on Interferon, dated 28 June 1967, MRC Archives File No. S788/2/5; Report of a visit made by Dr. N.B. Finter and Dr. F.T. Perkins to Helsinki to see Dr. K. Cantell, Dr. H. R. Nevanlinna and Dr. H. Strander, MRC Archives File No, S788/2/5; H. Strander and K. Cantell, 'Production of Interferon by Human Leukocytes in Vitro', Ann. Med. exp. Fenn, 44 (1966), 265-73; K. Cantell, H. Strander, Gy. Hadhazy and H, R. Nevanlinna, 'How Much Interferon Can Be Prepared in 
Hadn't they been concerned too about the risks involved in using a product stemming from leucocytes from blood donors in a trial in humans? Apart from the possibility of allergic side-reactions, the final product might be contaminated with hepatitis or other pathogenic viruses originating from the blood of infected donors. It was therefore understandable that the Scientific Committee like they themselves wanted to proceed with the utmost caution in this matter. Moreover, they both regarded the inspection visit as a sign that the British took the project very seriously. Cantell also discussed the likelihood of virus infection, in particular the hepatitis problem, with Harri Nevanlinna, the director of the Red Cross Blood Transfusion Service in Helsinki. The information he gathered was less promising than expected. Cantell learned that there could be no absolute answers. The only thing that Nevanlinna could be sure about was that so far his service's plasma products had caused very few cases of hepatitis. It made him feel a little bit uneasy about the inspection visit.

Cantell was relieved to find out that Finter would arrive one day in advance of Perkins and stay overnight at his private home. This provided them ample opportunity to have preliminary discussions about the ins and outs of the collaborative project and about the state of the art of interferon research. Over dinner it turned out that they both looked upon the rapidly growing popularity of interferon inducer research with envy and felt themselves increasingly marginalized by the 'inducer people' under the virtual command of the powerful and charismatic Maurice Hilleman. According to Hilleman there was no future for exogenous interferon in medical therapeutic terms. This made Cantell and Finter feel that they were fighting for a common cause: researching the actions of exogenous interferon without the exclusion of the interferon inducer line of investigation. They felt encouraged by reports from Vladimir Soloviev of the Virology Department of Russian Academy of Medical Sciences in Moscow-despite a shared scepticism about the state of the art of Russian interferon research-who had performed several successful trials with human leucocyte interferon in large numbers of volunteers infected with influenza virus. The best protection seemed to have been obtained when the interferon was given to humans 2-24 hours before being challenged by the virus.

By the time Perkins arrived on Monday afternoon, Cantell had already familiarized Finter with his work as head of the Virus Department at the State Serum Institute in Helsinki. Finter learned that optimizing the production of interferon by human leucocytes was a laborious and time consuming research activity governed by trial and error. The production process of interferon appeared very sensitive to disturbances and changes-for instance they experienced that for uncertain reasons using flat instead of round bottles for culturing the white blood cells appeared to affect the interferon yields in a dramatic way.

Human Leucocyte Suspensions', in G. Rita (ed.), The Interferons (New York: Academic Press, 1968), pp. 223-32: Interviews with Kari Cantell, Norman Finter, and Hans Strander. 
Keeping up the production levels already required hard and meticulous work-let alone engineering an increase in interferon yields and to scale up the production system. So far the most rapid and abundant production of interferon was achieved by incubating purified cultures of white blood cells obtained from blood donors with large doses of Sendai virus. ${ }^{87}$ After storage overnight the supernatant fluid which contained interferon was harvested, where after the interferon fluid underwent a basic purification procedure. Since both Cantell and Strander lacked the biochemical skills thought to be necessary for purifying the interferon preparations to a degree acceptable for use in humans it was quickly agreed to leave further purification and concentration work to the responsibility of Karl Fantes of Glaxo laboratories.

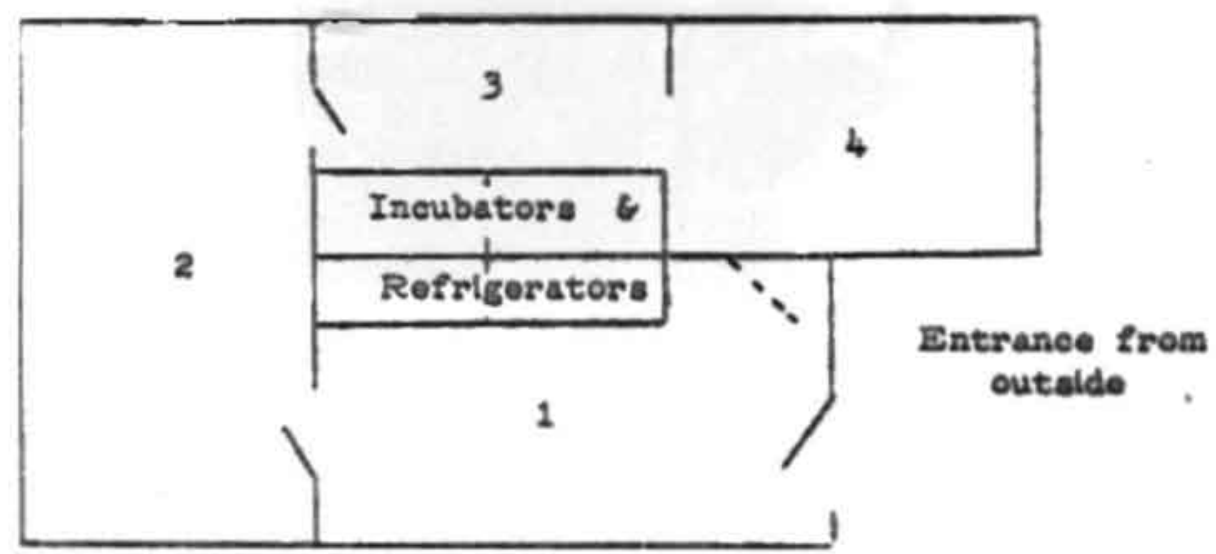

Fig. 43. A plan of Cantell's laboratory (dated 1967)

The laboratory workers entered room 1

from the outside and clanged their clothing.

They then walked round to room 4 through rooms 2

and 3 since the door from room 1 to room 4

would be kept locked. The sterile air

supply was common to the rest

of the building 100 ..

87 In experimenting with a large number of different sorts of viruses from his laboratory stock Cantell figured out that a rare Finnish strain of Sendai virus in the back of his laboratory freezer was the best inducer of interferon. 


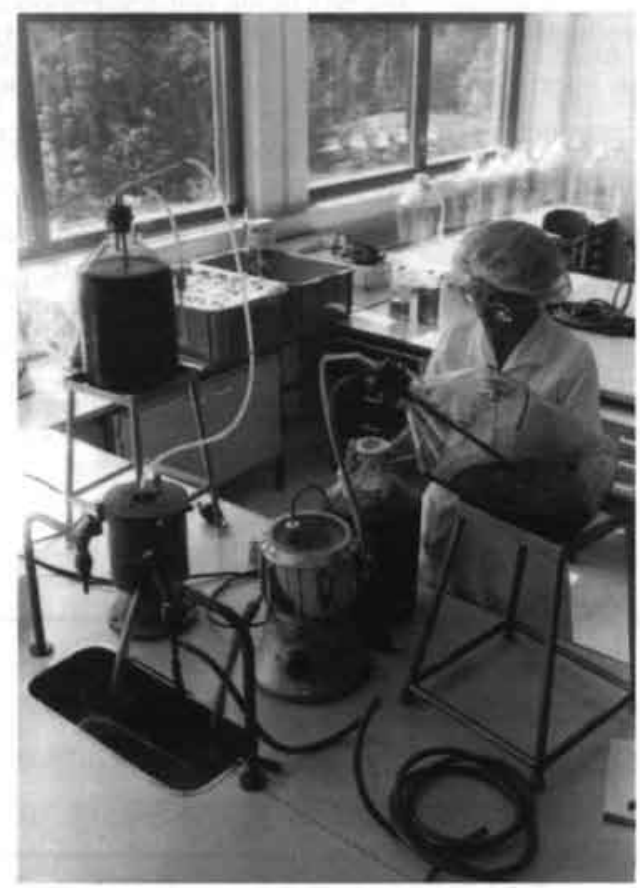

Fig. 43b. The production of leucocyte interferon in Helsinki (late 1960's/carly 1970's), Courtesy of Dr. K. Cantell.

Finter as well as Perkins were impressed by the amount of work that went into setting up and maintaining an experimental production unit for human interferon, and by the efficiency and skilfulness with which the production of interferon was dealt with. Interferon was produced in a special room that was isolated from public health work and was occasionally used for the production of vaccines. The small laboratory space for the production of interferon was continuously supplied with sterile air and before entering the production area Finter and Perkins had to change clothing in order to prevent contamination and to maintain sterile conditions during the production process (see Fig. 43a, b). This resembled very closely the stringent British vaccine production procedures.

They were also shown around the transfusion service where they learned that the preparation of interferon was not done at the expense of blood that was needed for blood transfusions. Since separate fractions of blood (plasma, packed red cells, antihaemophelic factor and platelets) were increasingly used for transfusions instead of whole blood the transfusion service was in the process of fractioning more and more of its donor blood. Of the 250.000 blood vessels collected each year about 100.000 were currently centrifuged to separate the various parts of blood. It appeared to require only little extra work to routinely 
collect the leucocytes during this procedure as a 'by-product' that was to be sent to Cantell's laboratory for further processing ${ }^{88}$

However short the actual tour through the Red Cross building, the visit itself was drawn out. Lengthy discussions took place concerning the risk of hepatitis infection of blood products. In an attempt to reassure his visitors with regard to the hepatitis problem, Cantell emphasized that Finland compared favorably with other European countries with its low incidence of infectious hepatitis and that he would as soon as possible send the latest official figures to Finter. This turned out to be far from the last word in the matter.

On return to Britain Perkins met with further objections to the use of leucocyte interferon within MRC's Immunological Products Advisory Committee that was responsible for safeguarding the clinical safety of the final product. ${ }^{89}$ Ultimately, following lengthy discussions it was decided, on the basis of Perkins's favorable report of the state of the art nature of Cantell's production facilities, to allow for limited experimental use of leucocyte interferon in localized infections in humans. ${ }^{90}$ Priority was given to a study of human leucocyte interferon against rhinovirus in volunteers, with interferon given as a nasal spray. ${ }^{91}$ One of the decisive factors in planning this trial first was that it could relatively easy be arranged in the Common Cold Unit in Salisbury through David Tyrrell. Moreover they could take advantage

88. If they spinned blood in the centrifuge, they were left with three layers. Formerly after removal of the top layer consisting of a thick, straw-colored liquid known as plasma or 'serum' they got rid of the intermediate almost colourless layer of leucocytes, the so-called 'buffy coat', where after the bags with the remaining red cells were stored for further use. Instead of discarding all buffy coats they now routinely collected the leucocytes.

Apart from the disturbing likelihood that the interferon material might be contaminated with hepatitis, one of the committee members pointed to the theoretical risk of contamination with so-called 'slow' viruses like Kuru, Scrapie and disseminated Multiple Sclerosis. Despite the fact that this latest objection was not sustained in view of the lack of practical evidence that these kind of slow viruses had ever caused problems in similar situations, it was illustrative of the negative mood within the Advisory Commmittee regarding clinical trials with Cantell's interferon.

90 First of all the final product should be tested for the presence of pathogenic viruses in vitro and in vivo, in particular extensive animal tests for hepatitis viruses were required. Second, the interferon inducing Sendai virus in Cantell's production unit should be free from leucosis (leukemia inducing) viruses. Third, it was agreed that the blood donors involved should be checked for continued freedom from clinical hepatitis for 6 months after donating their white blood cells. Fourth, monkey experiments should be carried out in advance resembling as far as possible the trials in humans. Furthermore the Safety Committe was in favour of making attempts to obtain information from neurologists and the Multiple Sclerosis Club concerning contamination problems related to blood transfusion of patients with disseminated sclerosis; First draft of the minutes of the 37th meeting of the Scientific Committee on Interferon, dated 17 October 1967, MRC Archives File No. S788/2/5; N. Finter to K. Cantell, letters dated 20 September and 1 November 1967, K. Cantell Correspondence, personal archives; and K. Cantell to N. Finter, letter dated 26 September 1967, K. Cantell Correspondence, personal archives.

91 Except for a proposal to test the inhibitory effect against viraemia produced by yellow virus vaccine in volunteers, the plan included the same kind of trials in humans as had either been performed or proposed by the Scientific Committee in the early 1960 's. 
of Tyrrell's experiences in the 1962 Common Cold trial. ${ }^{92}$

About the same time interest in interferon rose to a new height within the British drug company quarters. ${ }^{93}$ Rumors were buzzing around that at Merck and the NIH animal experiments were already in progress which showed that endogenous interferon stimulation through treatment with specific synthetic substances could cure acute viral infection in animals at a non-toxic dosage level. The drug company executives were drawn to these synthetic interferon inducers because they were easy to make chemically-fitting perfectly well their chemically oriented drug development trajectories-inexpensive and induced large quantities of interferon, whereas the production of useful, in other words potent and pure enough preparations of exogenous interferon appeared to be difficult and costly. ${ }^{94}$ In management circles everybody was particularly eager to capitalize on the clinical promise of the interferonstimulating agents. They seemed to be alerted by the tremendous drive between the NIH and the pharmaceutical industry in America in the field of interferon inducers.

\subsection{Wrestling standards}

In the meantime, national as well as international interest in establishing interferon standards continued to grow. Not only the British plans for human trials played an important role in this respect but also the frequent misunderstandings between the groups of 'inducer' and 'interferon' people about the quantitative aspects of their laboratory studies (such as the dose-yield relationships or the toxicity:therapeutic dose ratios of interferon inducers versus exogenous interferon). The fact that sometimes similar experiments appeared to produce inconsistent results, without any prospect of reconciling these differences, added to the confusion. The at times vehemently opposing parties acknowledged that, since neither of them knew how exactly interferon acted or what its precise nature was, common yardsticks at least were needed to facilitate the assessment of the relative merits of employing interferon inducers versus exogenous interferon. ${ }^{95}$ As I will show in this section the NIMR's Division of Biological Standards took advantage of this perceived need by pushing ahead their standardization efforts.

By the time Anderson started up a collaborative study of the proposed research

92 No, $\mathbf{S 7 8 8 / 1 5 / 1}$.

93

94

95

Minutes of the Human Interferon Working Party, dated 4 January and 8 february 1968, MRC Archives File

Interviews with John Beale, Karl. Fantes, and Norman Finter.

Internal notes MRC dated 9, 16 and 19 February 1968, MRC Archives File No A812/5/3.

The minority of mostly European 'interferon guys' had a hard time defending themselves against the powerful American alliance of inducer supporters headed by the charismatic Maurice Hilleman. Ion Gresser to Sidney Farber, letter dated 17 January 1969. Gresser personal archives; and interviews with Kari Cantell, Ion Gresser, Maurice Hilleman, Monto Ho, and Jan Vilcek. 
standard of human interferon named '67/87' which originated from Chany's Paris laboratory, in the fall of 1968, the Scientific Committee on Interferon began to make an issue of its relatively low potency. ${ }^{96}$ The fact that some of its members, who had been doing informal potency tests with $67 / 87$, failed to measure any interferon activity in the ampules, was of overriding importance in the growing call for a more potent standard. It was argued that to be of any use as an international reference preparation, a standard should also be suitable for those who used less sensitive assay methods. However, to prepare another standard would mean another long delay and moreover the Division of Biological Standards had already heavily invested in the proposed MRC standard 67/87. Eventually, it was decided that Anderson should begin preparations of a more potent standard that was made from human interferon to be obtained from Cantell, while continuing the arrangements for an international collaborative assay of $67 / 87 .{ }^{97}$

The parcel of Cantell's frozen leucocyte interferon arrived with flight AY 831 from Helsinki on the 29th January 1969, 9.05 am local time at Heathrow airport, and was safely transferred to Anderson's deepfreeze. ${ }^{98}$ About two months later the material was thawed, filtered, distributed into glass ampules, coded 69/19 and freeze-dried. It had taken two years to reach this stage, but except for the availability of two different proposed research standards for human interferon little progress was made. Establishing 69/19 in conjunction with 67/87 even created new disparities and therefore could only be expected to complicate the job of managing differences. At least there was a 'private' human interferon standard (a batch of 67/87) available for use by members of the Scientific Committee only, which enabled them to informally establish a common unit of measure. ${ }^{99}$ This could not be said of mouse interferon. The state of affairs here was considered depressing by the Scientific Committee on Interferon. From the very beginning things went wrong with regard to setting up a mouse interferon standard.

It began with the news that the mouse interferon material prepared by Finter was not

97 Minutes of the 40th meeting of the Scientific Committee on Interferon, dated 24 September 1968. MRC Archives File No. S788/2/5; and Minutes of the 8th meeting of the Working Party on human interferon, dated 6 November 1968, MRC Archives File No. S788/15/1.

Kari Cantell to Gray Anderson, letter dated 20 January 1969, Cantell personal archives; and Gray Anderson to Kari Cantell, letter dated 4 february 1969. Cantell personal archives.

99 By organizing an informal collaborative assay between the laboratories working within the Interferon Collaboration Finter's colleague, the ICI researcher Bob Bucknall, was able to establish the relative sensitivities of all the assay procedures, thereby facilitating the comparison of assay data: Bob Bucknall to Kari Cantell, letter dated 29 July 1968, Cantell personal Archives. 
considered by him to be sufficiently stable to be used as a standard. ${ }^{100}$ Subsequently, Anderson invited the NIH to contribute some of the same bulk material of crude mouse interferon produced by Merigan, which was used to prepare the provisional NIH standard, for collaborative study as a proposed mouse interferon standard for international use. ${ }^{10 t}$ Upon acquiring and processing this material, the ampules with freeze dried mouse interferon standard preparation were distributed by Anderson to Finter, Baron and Merigan for collaborative study. However, as soon as the first test results were assembled it appeared that something had gone wrong. There were enormous discrepancies between the assay data obtained in Finter's laboratory and in those of Baron and Merigan - for which no one seemed to have any rational explanation. After repeated series of laboratory tests, it was eventually agreed that the NIH material was unstable and lost most of its activity over time. The reasons for this loss in potency were not quite clear. Early in 1969, word came from Baron that the NIH was working hard to produce a new batch of mouse interferon material that instead of being freeze-dried would be kept in the frozen state and could serve as an interim standard. ${ }^{102}$

The problems to coordinate standardization efforts at an international level were a constant source of irritation between the British, Americans and French. In an effort to bring the parties closer to one another the Permanent Section of Microbiological Standardization of the WHO started preparations for an international conference on the subject of standardizing interferons. ${ }^{103}$

\subsubsection{The October, 1969, Standardization Conference}

In his conference address the chairman, David Evans, made an appeal to the 84 delegates from 13 countries to direct their attention mainly to reaching agreement on setting up interferon standards, on assigning a unitage to them and on the use of these standards by all those engaged in the interferon field. In Evans's opinion this was the only way to overcome the major stumbling block in the interferon field - "namely, the inability of workers to express the activity

100 Minute of the 35th meeting of the Scientific Committee on Interferon, dated 3 March 1967, MRC Archives File No. S788/2/5.

101 Minutes of the 36th meeting of the Scientific Committee on Interferon, dated 28 June 1967, MRC Archives, No. $5788 / 2 / 5$.

102 Norman Finter to Thomas Merigan, letter dated 7 August 1968, Gresser personal archives; Norman Finter to Ion Gresser, letter dated 7 August 1968, Gresser personal archives; and, Minutes of the 41 th meeting of the Scientific Committee on Interferon, dated 14 January 1969, MRC Archives File No. S788/2/5.

103 Minutes of the 10th meeting of the Human Interferon Clinical Trials Working Party, dated 25 February 1969. MRC Archives File No. S788/15/2. 
of interferons in meaningful terms". ${ }^{104}$ At the same time, in an apparent effort to reassure those who were afraid of the fuss that was felt to be associated with the standardization efforts, Evans tried as hard as he could to play down the requirements for providing all laboratories with the same yardsticks. However, setting criteria, assigning units and choosing suitable reference materials would require far greater efforts from the participants than Evans tried to make himself and the others believe.

This is aptly illustrated by the numerous and lengthy discussions which were needed to achieve consensus about the ins and outs of interferon preparations that would make most suitable research standards. For instance, Evans indicated that the standard should be distributed in a form which would ensure their stability for many years. Among other things, it should be kept at a low temperature. However, what was for some a "frozen state" that would guarantee stability was for others a freezing temperature that would still allow for degradation. It was eventually agreed that the reference standard should ideally be freeze-dried and kept at $70^{\circ} \mathrm{C}$, but that the more practical temperature of $-20^{\circ} \mathrm{C}$ was acceptable too. ${ }^{105}$ In addition, Evans's criterion that ampules containing standards should be sealed by fusion with glass threatened to exclude the United States, where it was common practice to use rubber stoppered vials, from contributing standard preparations. The issue was amicably settled by a recommendation to siliconize the rubber stoppers, which would remove most likely contaminants such as oxygen and water. Furthermore, should interferon standards be crude or purified? Crude interferon preparations were known to produce easily misleading results because of the presence of loads of impurities. While highly purified interferons would have the advantage of giving those involved less cause to rack their brains over the nasty question: "Is this phenomenon which I observe in my laboratory due to the interferon, or is it due to an impurity?", they were claimed to be rather unstable. ${ }^{106}$ The compromise was to purify them in such a way that would rid them of as many biologically active interfering impurities as possible. ${ }^{107}$

At times, an issue was judged too ambiguous to allow any conclusion, for example the significance of the claim that the active interferon itself was not a homogeneous substance. Was the claimed heterogeneity only a matter of one interferon molecule that was attached to a

104

D.G. Evans, 'Conference Address', in F.T. Perkins and R.H. Regamey (eds.), Symposia Series in Immunobiological Standardization (Basel, S. Karger, vol, 14, 1970), p. 2.

F.T. Perkins and R.H. Regamey (eds.), Symposia Series in Immunobiological Standardization (Basel. S. Karger, vol. 14, 1970), pp. 280-1

F.T. Perkins and R.H. Regamey (eds.), Symposia Series in Immunobiological Standardization (Basel, S. Karger, vol. 14, 1970), p. 297.

107 F.T. Perkins and R.H. Regamey (eds.), Symposia Series in Immunobiological Standardization (Basel, S. Karger, vol. 14, 1970), pp. 291-5. 
variety of other inert molecules, or did there indeed exist several different interferon molecules, on analogy with other biologically active proteins, and what consequences would this have for the use of standards? Quite a number of these kinds of questions had to be left unanswered.

Toward the end of the conference the nature of the discussion changed. More practical issues were discussed, such as the problem of acceptance. One of the participants stated that what kept them arguing so long about standardization was basically the fact that it meant extra work. All agreed that the investigators must be willing to do the extra work and that, without some kind of encouragement from this Symposium, very little was going to happen. It was generally felt that the timing was opportune and if they did not seize the moment they could expect not to have common units of measure for a long time. Eventually it was decided that the most practical way of achieving something was to ask a select group of interferon researchers to try to make some provisional recommendations for research standards of the various interferons. These would then be discussed during the final session of the Symposium. ${ }^{108}$

In the ensuing final discussion, whenever disagreement about the nature or status of a most suitable standard for the various interferons threatened to deadlock the decision process. Evans intervened. He repeatedly told the participants that they should not be perfectionist in this matter, as there was no such thing as a biological standard that worked ideally every time. In his view "nothing would come out in the wash" if they did not assign a unit and establish a research standard. It was basically learning by doing. Evans argued from experience that, as a result of handing around standards to people, new findings would arise that would contribute to a better understanding of both the field of research and the use of standards. Having made his point he then tried to put all the bits and pieces together, and produce, by general agreement, final recommendations of the Conference. ${ }^{109}$

The Symposium recommended the adoption of four research reference standards, to which an arbitrary number of units were assigned and that in future all results in published work would be quoted in terms of the relevant units. While it was decided that the research standards for mouse (6000 units per ampule) and rabbit interferon (3000 units per ampule) would be made available through the Reference Reagents Branch of the NIH, they agreed to adopt as the research standards for chick and human interferon the preparations that were at store at the NIMR's Division of Biological Standards.

As spokesperson - in the absence of Finter who suffered from a pneumonitis - of the Scientific Committee on Interferon, Tyrrell had tried hard to argue against adopting the proposed MRC standard 67/87 (with human interferon material obtained from Chany) and to

108 F.T. Perkins and R.H. Regamey (eds.), Symposia Series in Immunobiological Standardization (Basel, S. Karger, vol. 14, 1970), pp. 291-5.

109 F.T. Perkins and R.H. Regamey (eds.). Symposia Series in Immunobiological Standardization (Basel, S. Karger, vol. 14, 1970), pp. 296-318. 
make out a case for the more potent reference preparation $69 / 19$ (with human interferon material obtained from Cantell) during the final session of the Symposium. ${ }^{110}$ However, he came away empty handed as Evans and the others strongly favored $67 / 87$, which had already been handed around to and used by a lot of researchers without major problems being reported. The fact that it had proved to be quite reliable in terms of stability was valued more highly than its relatively low potency. ${ }^{111}$

I do not know what went on behind the scenes in the weeks after the meeting, but what I do know is that the recommendations had changed by 18 November, when the conclusions of the Standardization Conference were sent to all participants. ${ }^{112}$ The British Conference Secretary, Frank Perkins, indicated in an accompanying letter that in consultation with Evans he had decided to include in the recommendations that the more potent preparation $69 / 19$ instead of $67 / 87$ should be adopted as the research standard for human interferon.

\subsection{2 'Having to haul down one's colors'}

On receipt of Perkins's letter and the enclosed conclusions of the Symposium, Charles Chany was, to say the least, surprised to see the changes and immediately sent back a letter to Perkins to tell him that he had misquoted the recommendations. ${ }^{113}$ Critical reactions from American and other quarters followed swiftly, and within ten days of the first letter Perkins was forced to send around a revised statement on the section concerning human interferon. ${ }^{114}$ It said that the Conference agreed to adopt $67 / 87$ as the research standard for human interferon and that the Conference was also informed that a more potent preparation $69 / 19$ would be made available to serve as a working standard for special use in insensitive assay systems. The section ended by giving the advice-which eventually would not be published in the Conference Proceedings - that $67 / 87$ should be replaced with $69 / 19$ sooner rather than later. Perkins had done his best to find a compromise that was thought to suit either side, but in doing so he all but helped to clarify the situation.

110 Norman Finter to Kari Cantell, letter dated 10 November 1969, Cantell personal archives.

111 F.T. Perkins and R.H. Regamey (eds.), Symposia Series in Immunobiological Standardization (Basel, S. Karger, vol. 14, 1970), p. 311.

112 Frank Perkins to Kari Cantell, letter dated 18 November 1969, Cantell personal archives.

113 Interview with Charles Chany.

114 First draft of the Minutes of the 14th meeting of the Human Interferon Working Party, dated 25 November 1969, MRC Archives File No. S788/15/2; Frank Perkins to Kari Cantell, letter dated 28 November 1969. Cantell personal archives; and Frank Perkins to Charles Chany, letter dated 28 November 1969, MRC Archives File No. S788/15/2. 
At the 'laboratory bench', the state of affairs with regard to the standardization of human interferon was regarded as messy. ${ }^{115}$ Instead of facilitating the comparison of results, the provision of different human interferon standards seemed to complicate their correlation, as is apparent from the following excerpt from a letter from Finter to Cantell. "Unfortunately and shamefully there is major confusion over standards, and Karl (Fantes) has used four different ones on different occasions..... if one cross-correlates all the results as far as is possible, the position seems to be (subject to re-checking all the arithmetic) that the pool has a potency of about 100.000 International Units". ${ }^{116}$ Poor compliance with quoting results in terms of the unitage of the agreed upon research standard ('international units') was not considered particularly helpful either. ${ }^{117}$

Despite or, more likely, due to a series of efforts by the NIMR's Division of Biological Standards to set things right with regard to human interferon standards, the situation became even more confusing in the fall of 1970 . This is aptly illustrated by the ad-hoc changes in the reporting of the Division's collaborative assay exercises on human interferon. In the first draft report, Gray Anderson concluded that owing to the relatively low potency of $67 / 87$ it did not seem advisable to recommend $67 / 87$ as a research standard. ${ }^{118}$ The second and third draft reports suggested that $69 / 19$ was about fifty times as potent as $67 / 87$, but there turned out to be a considerable variation in the estimates of potency both within laboratories and between laboratories. If taken seriously, this meant that in the present situation the usefulness of either preparation as a standard was debatable. ${ }^{119}$

However uncertain and ambiguous the interpretation of the results in the reports, the nature of reactions which the reports elicited from their readers was far from ambiguous. Finter, who still had strong feelings about the adoption of $67 / 87$ at the Standardization Symposium immediately reacted to the draft reports. ${ }^{120} \mathrm{He}$ wrote to Anderson to tell him that he did not agree with the general tenor of the reports. "At the Standardization meeting last October, it was generally agreed that there was much to be gained by establishing systems of

115 Kari Cantell to Hans Strander, letter dated 21 January 1970, Kari Cantell personal archives.

116 Norman Finter to Kari Cantell, letter dated 8 December 1969, Cantell personal archives.

117 Norman Finter to members of the Human Interferon Working Party, letter dated 12 December 1969, MRC Archives File No. S788/15/2.

118 Draft of a Report concerning a collaborative study of a proposed research standard (67/87), dated 1 October 1970. MRC Archives File No. S788/15/2.

119 Draft of a Report concerning a first collaborative assay of $69 / 19$ in terms of $67 / 87$, dated 30 October 1970 . MRC Archives File No. S788/15/2.

120 Norman Finter to George Galasso (Head of the NIAID Antiviral Substances Programme), dated 19 November, 1970, MRC Archives File No. S788/15/2. 
units quickly, and little to be gained by being perfectionist. A year later we seem no further forward in relation to human interferon. Now is the time for taking the bull by the horns!". ${ }^{121}$ In Finter's opinion, it was a reasonable estimate to say that $69 / 19$ was about 50 times more potent than $67 / 87$. It therefore seemed to him that a great deal could be gained, and very little lost, by designating $69 / 19$ as the reference standard, with a potency of about 5000 units per ampule. $^{122}$

The Scientific Committee on Interferon was eventually able to talk Anderson into changing the overall conclusion of the three collaborative studies. Anderson recommended, in a fourth report which was intended to be a summary of the whole project, that preparation 69/19 be established as the British Research Standard A for Human Interferon, and that it be assigned a unitage "so that are for all practical purposes 5000 units of activity in each ampoule". In practical terms it meant that Anderson proposed to abandon 67/87, make 69/19 the primary standard and judge the suitability of $69 / 19$ by its performance in action. ${ }^{123}$

The summary report which was sent to all the participants in the collaborative studies caused quite some upheaval in French quarters. Chany was baffled by the fact that, regardless of the admitted inadequacies in the test results, a decision seemed to have been made regarding the suitability of his and Cantell's material to serve as a research standard. Had not 67/87 and $69 / 19$ both been found sufficiently stable to be employed for longer periods? How could the Finnish material be established as a research standard without being able to produce a reliable estimate of 69/19 in terms of 67/87? As far as Chany knew it had been agreed upon at the Standardization Conference that all future reference materials would be adjusted to $67 / 87 .{ }^{124}$ The British not only did not seem to feel bound to abide by the recommendations of the Standardization Conference, but on top of that they proposed to fly the British flag over what was supposed to function as an international research standard. ${ }^{125}$

Chany immediately decided to take action. He began by sending his grievances in diplomatic terms both to Anderson and the participants in the collaborative studies. ${ }^{126} \mathrm{He}$ realized that he had the odds stacked against him. In the January 1971 issue of the Interferon Scientific Memorandum a joint statement from the NIMR and the NIH had been published to

\footnotetext{
121 Norman Finter to Gray Anderson, letter dated 14 October 1970, MRC Archives, File No. S788/15/2.

122 Ibid.

123 Summary of reports of three collaborative studies, dated I December 1970, MRC Archives File No. S788/15/2.

124 Charles Chany to Gray Anderson, letter dated 22 December 1970, Cantell personal Archives.

125 Interview with Charles Chany.

126 Charles Chany to Gray Anderson, letters dated 22 December 1970 and 7 May 1971, Cantell personal archives.
} 
formally acquaint the workers in the field with some modifications of the recommendations of the Standardization Conference. The position regarding the provision of reference materials was said to be that $67 / 87$ was no longer regarded as the research standard for human interferon but that, depending on their suitability, either $67 / 87$ or $69 / 19$ would soon become established as a research standard. ${ }^{127}$ In Chany's view this was already a first formal step toward abandoning 67/87, and unless he succeeded in gaining the support of the Americans he was fighting for a lost cause. So, when Anderson did not show any response to his adverse comments and let him know that most participants were in agreement with the use of $69 / 19$ as reference material and with establishing it as the British Research Standard for human interferon. Chany chose a more aggressive approach. ${ }^{128}$ Beside pleading his case in the corridors of the September 1971 International Colloquium on Interferon that was being held at the University of Leuven, Belgium, in January 1972 Chany paid a visit to the NIH in Bethesda, Maryland, to win support from those directly involved in the standardization efforts. ${ }^{129}$ At the same time he requested that the Division of Biological Standards return to him the material that he had given to them as a possible interferon standard. He told them that he was planning to distribute it on his own initiative as the original interferon standard.

Eventually, by the end of May 1972, through the intervention of the Americans, a compromise was reached that offered a way out of the confused situation. Basically it meant that $67 / 87$ was retained as the original reference reagent available only on special request and used as the basis for assigning unitage to any subsequent reference material, while $69 / 18$ would be distributed routinely as the standard for general use. Furthermore, to satisfy the American demand that "nationalism be removed from the reagents", the NIMR's Division of Biological Standards eliminated the word 'British' from the label and, renamed 67/87 into 'Research Standard $\mathrm{A}^{\prime}$ and $69 / 19$ into 'Research Standard B' for human interferon. ${ }^{130}$

The political jousting hardly affected the work at the laboratory bench. Insofar as

George Galasso to David Tyrrell, letter dated 24 December 1970, MRC Archives File No. S788/15/2.

Gray Anderson to Charles Chany, letters dated 19 April and 12 May 1971, MRC Archives File No. S788/15/2: and interview with Charles Chany.

129 George Galasso to Gray Anderson, letter dated 11 February 1972, MRC Archives File No, S788/15/2.

130 George Galasso to Gray Anderson, letters dated 11 February and 12 April 1972, MRC Archives File No. S788/15/2; Gray Anderson to George Galasso, letter dated 15 March 1972, MRC Archives File No. S788/15/2. 


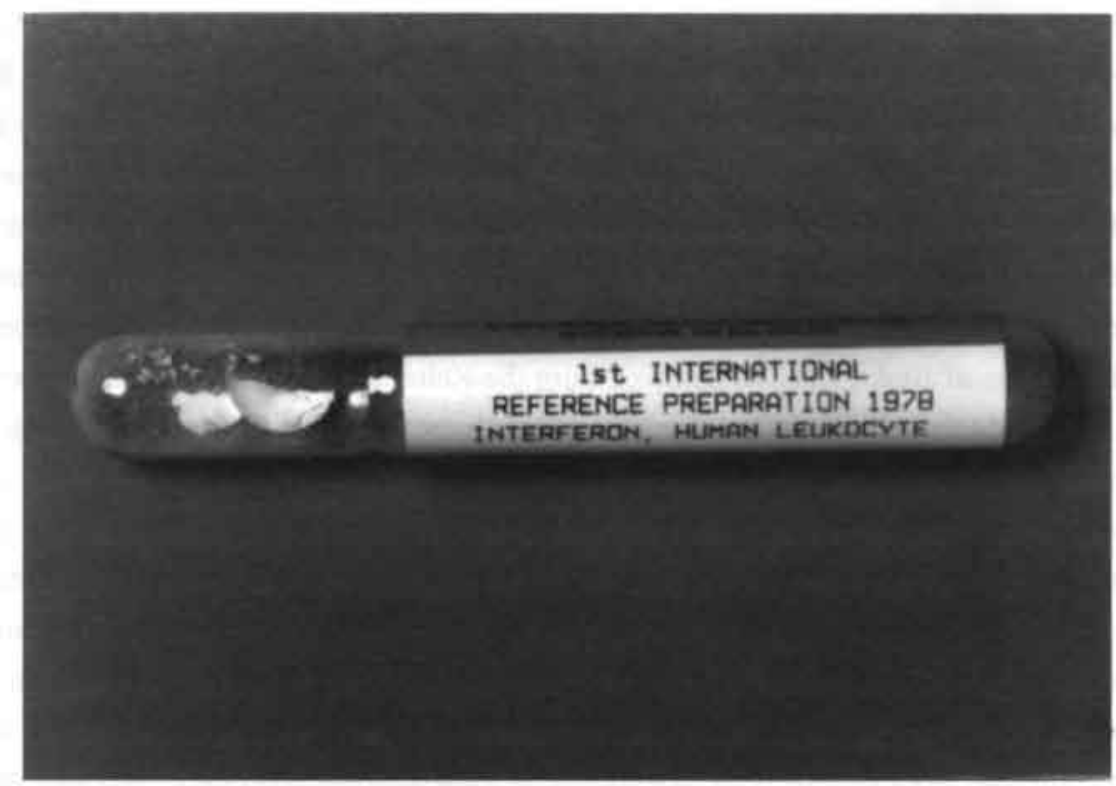

Fig. 44. The Expert Committee on Biological Standardization of WHO considered data reiating to $69 / 19$ and finally in 1978 establisted pan of the stuck of preparation 69/19 (MRC Research Standard B) as the International Reference Preparartion of Interferon, Human Leukocyte, Courtesy of NBSB.

researchers had been involved in the collaborative studies of human interferon they (apart from Chany) already showed a strong preference for using the more potent preparation $69 / 19$ as a working standard. Eventually this preparation became regarded as the base line for comparing work on human interferon (see Fig. 44). This was already the case in the report of the common cold trial performed by the British Scientific Committee on Interferon at the end of March $1970 .{ }^{131}$ With reference to the standard $69 / 19$ the poor results were claimed to be due to the use of insufficient interferon, to the inadequate dosage and to an unexplained 10 fold decline in biological activity between the time of preparation and administration. ${ }^{132}$ This explanation could not prevent the respiratory trial from leaving its marks on the Interferon Collaboration per se. It played an instrumental role in Glaxo's and ICI's decision to abandon work on

131 Minute of the 17th meeting of the Human Interferon Clinical Trials Working Party, dated 14 April 1970. MRC Archives File No. S788/15/2.

132 Minute of the 17th meeting of the Human Interferon Clinical Trials Working Party, dated 14 April 1970. MRC Archives File No. S788/15/2; and, Scientific Committee on Interferon, 'Experiments with Interferon in Man', Lancet, i (1965), 505-6. 
interferon, leaving only Burroughs Wellcome and the MRC in interferon research. ${ }^{133}$

However little this chemically putative natural substance still meant to most British drug makers interferon had managed to establish itself as an object of laboratory study for an international field of researchers. They mainly worked with chick, mouse and rabbit interferons. By 1972 most major laboratories were in the process of including the internationally recognized research standards into their laboratory routines by calibrating their internal laboratory standard against the appropriate research standard obtained free of charge from the NIMR's Division of Biological Standards or from the Reference Reagents Branch of the NIH. Increasingly, editors of pertinent scientific journals requested that papers dealing with interferons should include results in terms of the internationally accepted unitage, but also in informal contacts a growing number of researchers started to communicate in terms of the appropriate MRC or NIH unit. ${ }^{134}$ The researchers involved were still in the process of learning how to handle common units of measurement and disciplining themselves into specific routines and actions. The local variances were still considerable, and time and again problems arose with interpreting unexpected high variation in laboratory results. Had the activity of the standard gone off, was it related to flaws in the standardization procedure, was one dealing with yet unknown biological characteristics of interferon or even with a different type of interferon $?^{135}$

By the time a second international workshop on standardization was being held at Woodstock, in September 1978, the use of specific research standards had become embodied in daily laboratory practice in the field of interferon research. It was widely considered to constitute a necessary condition for performing research on interferons. The worldwide distribution and use of a fixed set of research standards not only was generally thought to have

133 Whereas the formal collaboration on interferon terminated on 2 May 1973, all parties agreed that the GNRD as the Patent Holdings Company should be continued in order to protect the property held-e.g. U.S. interferon patent; Internal note MRC, dated 13 May 1971. MRC Archives File No. A 812/8 (ii); and, Minutes of the 46th meeting of the Scientific committee on Interferon, dated 22 February, 1973, MRC Archives File No. S788/2/6; Internal note MRC, 22 April 1973, MRC Archives File A812/2/1.

134 For instance, in Paris, upon receiving a sample of mouse interferon from Bill Stewart, who temporarily worked as a post-doctoral worker at the Rega Institute in Louvain, Belgium, Ion Gresser immediately wrote him a note to ask him whether the units indicated on the label were NIH units. "The titer I gave you was in PDD-VSV units, which was about 1/5 of an NIH unit in Delaware but seems to be about the same (within 2-fold) as an NIH unit here in Leuven" Ion Gresser to William E. Stewart II, letter dated 28 February 1972, Gresser personal archives; and William E. Stewart II to Ion Gresser, letter dated 2 March 1972, Gresser personal archives.

Interviews with Sam Baron, Norman Finter, Robert Friedman, Ion Gresser, Joseph Sonnabend; In 1965 Frederick Wheelock of Western Reserve University in Ohio had published a highly controversial article in Science claiming that he had identified a new interferon-like inhibitor in cultures of human white blood cells, thus implicating that not only different species produced different interferons but also that the same species produced different types of interferon. Without proper standards he had difficulty in substantiating. Wheelock had to wait a couple of years before his study was taken serious in the field of interferon research; $E$. F. Wheelock, 'Interferon-like Vinus-Inhibitor Induced in Human Leukocytes by Phytohemagglutinin', Science, 149 (1965), pp, 319-21; and interview with Frederick Wheelock. 
made the comparison of results from different laboratories more interpretable but also to have played an instrumental role in redefining the nature and functions of interferons. ${ }^{136}$ It was no longer a question whether interferon was heterogeneous in nature and not only different species but also the same species produced different interferons-two different types of human interferon were officially distinguished: so-called 'classical' (acid-stable) type I interferon and 'immune' (acid-labile) type II interferon-but what the nature and function of these newly established differences were. Thus, in attempting to cope with and master differences, interferon researchers had created and as I will show in the next chapter would continue to create new differences, albeit of a different nature.

\subsection{Conclusion}

With the constructed character of the standards no longer visible at the end of this chapter it seems hard to imagine that the distribution and use of common units of measurement was not the inevitable result of the evolving interaction between those involved in interferon research. At the same time, what makes a lasting impression is that it took more than 15 years and enormous efforts to reach this stage: from occasionally invoked devices to reduce extreme variations between laboratories and researchers, to the use of standards as a prerequisite to get research results published. In a most general sense, the large time span between the first discussions about standardization and the routine use of at least three different international research standards as stable and unquestioned parts of interferon research shows that discussing standards was as important to the emerging field of interferon research as their actual establishment and use. My claim is that the standardization discourse in itself played an instrumental role in disciplining interferon researchers into specific routines and actions, and thereby coordinated their research practices.

Ultimately the introduction and routine use of international research standards was little more than the tangible result of a collective effort to create a common frame of reference, as a means to the end of welding and keeping together the field of interferon research. Moreover, this chapter shows that the perceived need for standardization was dependent on the nature and purpose of research, and on institutional and national as well as field interests. What, then, were the incentives and impediments for standardization? And, what efforts and resources did it take to establish, disseminate and maintain standards?

As long as the efforts to tune in the research activities of a growing body of laboratory

At the workshop all the recommendations of the 1969 Conference were reinforced by recommending the same set of research standards for acceptance as WHO International Reference Standards Internal memorandum on the occasion of an International Workshop on Interferon Standards held at Woodstock, Illinois, USA, dated September 1978. MRC Archives File No, S805/7. 
practices to one another and to engineer a new field of research were not seriously constrained or threatened by the ability of experimentalists to choose their own favorite experimental system and to provide their own interpretations of the objects under study, there was hardly any incentive for standardization. Of course, the idiosyncrasies of the myriad of experimental systems posed certain problems. For instance, what was to some a potent interferon preparation could become in the hands of others material with a low biological activity. However, by informal means, through circulating, comparing and combining research materials in a relatively small and flexible network of personal contacts, researchers managed to reduce extreme variations of experimental results and were able to narrow down the margins of interpretation to workable levels. As I argued before, it was a 'gift culture' based on the regular exchange of laboratory samples, techniques and skills. This gift culture was instrumental in facilitating the comparison of research results and the management of differences between laboratories and researchers.

Collaboration within the British Scientific Committee on Interferon between researchers from different domains - industrial and government laboratories - as a means to the end of developing interferon as an antiviral drug put the issue of standardization on the agenda for the first time. With the intrusion of a higher order of interests (company, governmental and public interests) into the relatively private domain of laboratory life, the informal kind of measurement which interferon researchers normally practiced fell short of what was required. Performing clinical trials meant passing judgments on the actions of experimental substances within certain limits related to human health. Due to the high level of public responsibility that was involved in experiments with humans, there was special concern for quantitative control and rigor with regard to issues like effectiveness, toxicity and stability. Establishing and using standards was considered a necessary operation to give authority to trial data, which could be appealed to in the future.

The premature ending of the clinical trial program put standardization in another perspective: from a necessary condition for work on interferon it became perceived by members of the Scientific Committee as a course of action to manage differences, and one that meant extra work. Without some extra encouragement, for instance in the form of editors of the major journals demanding the use of common units of measure or a reappraisal of the clinical trial program, there was little inclination to use standards at the bench. With an uncertain return on investment, most chose in favor of the proven informal exchange mechanism.

Not until the informal form of measurement began to show flaws, with the expansion of the field of research and the shift away from a qualitative descriptive to a more quantitative oriented type of research work, did a more general and permanent need for standardization began to manifest itself. In conjunction with both the creation of a NIH-sponsored formal information exchange mechanism, and an emerging controversy between the so-called 'inducer 
people' (endogenous approach) and 'interferon people' (exogenous approach) over the possibility for application of their different research approaches in human medicine, this provided a breeding ground for standardizing interferons. Standards were at least considered helpful tools in resolving disputes, and in performing the balancing act between conflict and appeasement, or between plasticity and coherence, that prevented the field from becoming unwieldy. Finally and more generally those involved tried hard to diffuse escalating conflict for the sake of the coherence of the field.

National centers for biological standardization played a central role in disseminating and 'materializing' the perceived needs for standards. However, there was a marked contrast between the nature of the involvement of the NIH Reference Reagents Branch and the involvement of the NIMR's Division of Biological Standards. Whereas the American laboratory for biological standardization played a relatively passive role as a provider of standards, its British counterpart functioned both as a provider and facilitator, by initiating and coordinating standardization efforts. Apart from the fact that establishing interferon standards fitted rather well with the Division of Biological Standards' plans to extend the scope of its activities into the area of medical research, this was mainly a result of historical contingencies.

Ever since the responsibility for establishing and safeguarding international standards was divided between the State Serum Institute in Copenhagen and the National Institute for Medical Research in 1924, the Medical Research Council had regarded biological standardization as one of the spearheads of its activities. In practice this meant that research work associated with biological standardization was actively promoted and that the Division of Biological Standards was encouraged to pursue an active policy in the international field of standardization, aimed at maintaining its unique position as custodian of standards for the whole world. It was considered a matter of national interest. ${ }^{137}$ American biomedical research policy, on the other hand, had always been rather indifferent with regard to international standardization, as is evident from the fact that until 1950 the USA maintained their own national standardization system. By the 1960's international standardization began to receive more attention from NIH officials, but it never became a priority issue. ${ }^{138}$

We saw that, initially, the establishment and provision of standards only seemed to complicate matters at the bench by adding to the uncertainty and variability of the test results. Considerable efforts, in terms of complex negotiation and decision-making processes, were required to achieve consensus about the specifications of interferon preparations that would make most suitable research standards. The value and power of interferon standards as

A. Landsborough Thomson, Half a Century of Medical Research Vol. II (London, Her Majesty's Stationary Office, 1975), 244-54.

W. C. Cockburn, 'The International Contribution to the Standardization of Biological Substances. I. Biological standards and the League of Nations 1921-1946', Biologicals 19 (1991), 161-9. 
common frames of reference or 'laboratory yardsticks', which in being mobile, and conveying relatively stable information, transform individual laboratory practices into universal practices, appeared largely dependent on the extent to which they became embodied in actual research practice. I should emphasize the instrumental role the statisticians played in this respect. In their unremitting efforts to help the laboratories for biological standardization improve their performance in terms of production of statistically reliable units of measurement, they were largely responsible for designing the kind of collaborative standardization experiments that involved a large number laboratories and researchers.

Ultimately as a result of handing around and learning to use standards, the interferon researchers achieved far more than resolving research problems. In shaping and defining the outcome of experiments, interferon standards came to serve as a constitutive part of interferon research. As such, the use of standards not only helped researchers to discriminate between and compare outcomes of experiments, but was also instrumental in creating differences and redefining problems of research: in a collective effort to master differences those involved had established new differences of such a nature that these multiplied their options for research. In the next chapter we will see how the creation of new options between bench and bedside in the 1970 's and 1980's once again resulted in the public promotion of interferon. 


\section{Chapter 6}

\section{Interferon, Audiences and Cancer: A 'Quest' for Miracle Cures. ${ }^{1}$}

\subsection{Introduction}

Every so often, in the past forty years, scientific claims of therapeutic accomplishment in the treatment of cancer have entered the public domain in western countries like the USA, Britain and the Netherlands. ${ }^{2}$ The biographies of most of these experimental cancer therapies seem to have a familiar pattern in common: implied claims for a cancer cure based on preliminary tests, exaggerated coverage in the media, high public expectations, widespread disappointment and loss of interest when the claims failed to materialize in large scale trials, researchers and administrators forced on the defensive, and finally there seems hardly any reason for continuation of research or for clinical application.

In the late 1970 s, following scientific claims about interferon having an inhibitory effect on human tumors, interferon made a come back as a promising therapeutic substance. As might be anticipated, the media gave enthusiastic coverage to the implied claims for a cancer cure. The question then deserves scrutiny whether a similar sequence of events unrolled in the case of interferon?

In an effort to understand how the cycle of euphoria and disappointment manifested itself, this chapter explores the complex events shaping interferon's biography as a new type of cancer remedy. Focus will be on the ways in which the major collective or individual actors-ranging from doctors, journalists, laboratory researchers, patients, politicians, and regulators, to drug company executives-left their mark and influenced the ways in which interferon was portrayed and perceived, primarily in the American public arena. ${ }^{3}$ Special attention will be paid to the role the mass media played in what has become known as the "interferon crusade". ${ }^{4}$ They functioned as intermediaries between the scientific and medical

1 A preliminary version of chapter 6 was published in Clinical Applications of the Interferons: T. Pieters, History of the Development of the Interferons: From Test-tube to Patient, in R. Stuart-Harris and R. Penny (eds.) The Clinical Applications of Interferons, (London: Chapman\&Hall, 1997), pp. 1-19.

2 See, for exemplary historical cases: J. T. Patterson, The Dread Disease (Cambridge (MA): Harvard University Press, 1987); and, R. W. Moss, The Cancer Industry (New York: Paragon House, 1989).

The actors to be analyzed are not only those individually and collectively 'present', articulate and committed to action but also those implicated by the actions of others. As we will see the cancer patients as potential users of interferon are the implicated actors examined in this chapter.

4 See, S. Panem, The Imterferon Crusade (Washington, D.C.: The Brookings Institution, 1984). 
community, the pharmaceutical industry, government and the lay public, each using their own specific arguments, images, facts and figures. I show how the media stressed and added certain aspects and thereby contributed to the continuous process of reshaping the meaning of interferon.

This chapter begins by laying out the events that helped to establish links between interferon and cancer at the bench and at the bedside. The heart of the chapter examines how interferon became a major issue in the American cancer arena and how the various engaged social groups dealt with this natural substance and product of a laboratory-supported scientific medicine. The similarities and differences between these groups' understanding and knowledge of interferon will be highlighted. In addition I will pay attention to the ways in which each group tried to convince others of the validity of their own perspectives, to create alliances and further their own divergent interests. In the fourth and fifth section I will concentrate on the genesis and dynamics of the 'interferon crusade'. In the conclusion, we return to the analysis of the cycle of euphoria and disappointment and the important role played by the media.

\subsection{The emergence of interferon as an anti-cancer agent}

In the USA in early 1970, Mary Lasker the philanthropist and notorious lobbyist of American medical research had assembled a panel of consultants. This group, largely consisting of friends and influential associates also known as the "Laskerites or Mary's little lambs", was to advise the American Senate on legislation involving a "moonshot" approach for cancer that held out the promise of major progress in the officially declared "war on cancer". ${ }^{5}$ One of the scientific panel members was Mathilde Krim, a Swiss-born geneticist and Tumor virologist at New York's Memorial Sloan Kettering Cancer Center, whose husband Arthur Krim was influential in the Democratic party. Krim helped draft working papers on the progress of cancer research, which became part of the technical portion of the Panel's report entitled National Program for the Conquest of Cancer. This was presented to the Senate Committee on Labor and Public Welfare in December $1970 .^{6}$

After presentation of the report to the Senate, Krim remained closely involved with the prelegislative agenda setting activities of Mrs Lasker up to the enactment by President Richard Nixon of the National Cancer Act in December 1971. This event marked the start of an expanded National Cancer Program and was the result of more than a year of political struggle

R. A. Rettig, Cancer Crusade. (Princeton: Princeton University Press, 1977), p. 18.

6 See, R. A. Rettig. Cancer Crusade, (Princeton: Princeton University Press, 1977), pp. 77-115. 281-315: and interview with Mathilde Krim. 
and compromise in which Mathilde Krim became familiar with the ins and outs of American cancer politics. According to Krim it was Lasker who taught her the political groundwork of how to use power and influence in order to secure one's ends. ${ }^{7}$

Krim did her share of the work in searching the literature for promising areas of cancer research that deserved more attention. In doing so she ran across the interferon literature and learned that there had been some noteworthy developments since she had attended Isaacs's paper presentation on interferon at the prestigious Gustav Stern Symposium on Perspectives in Virology in January $1960 .^{8}$ Among other items, studies had been published claiming that interferon and interferon inducers, by means of their antiviral activity, had an inhibitory effect on tumor viruses and virus induced tumors in mice and rats. More recently, mouse studies had been published by the American virologist Ion Gresser. His studies suggested that interferon might also have an inhibitory effect on tumors that had no obvious relationship to viral infections. As a tumor virologist, Krim firmly believed that tumor viruses played a major role in the etiology of malignancies not only in animals but also in humans and that interferon might have an effect against both virally induced mouse tumors and mouse tumors not directly induced by virus. She therefore regarded both interferon and interferon inducers as potential antitumor agents for use in humans and insisted on including interferon research in the Panel's report as a promising area that needed intensive further study. ${ }^{9}$

Before following up the further wheeling and dealing of 'Mary's most eager lamb', and interferon promoter, Mathilde Krim, I will first describe how the notion of the antitumor effect of interferon came into being.

\subsubsection{Interfering with cancer}

The action of interferon on tumor viruses had first been singled out for study and speculation by two French virus researchers, Charles Chany and Pascu Atanasiu in the early 1960's. They claimed that interferon inhibited the growth of polyoma virus, a specific strain of tumor virus, in hamsters. ${ }^{10}$ Initially scepticism prevailed among fellow scientists, but after a couple of

7. S. Panem, The Interferon Crusade (Washington, D.C.: Brookings Institution, 1984), p. 16; and Krim. interview.

8

M. Pollard (ed.), Perspectives in Virology (Minneapolis: Burgess Publishing, 1960)

9

S. Panem, The Interferon Crusade (Washington, D.C.: Brookings Institution, 1984), p.16; and Krim, interview.

10

P. Atanasiu and C. Chany, 'Action d'un Interféron Provenant de Cellules Malignes sur L'infection Expérimental du Hamster Nouveau-né par le Virus du Polyoma', Comptes Rendus Hebdomadaires Séances de L'Académie de Sciences, 251 (1960), 1687-9; and, C. Chany. 'An Interferon-like Inhibitor of Viral Multipli- 
British and American publications confirmed that interferon seemed to have an inhibitory effect on certain strains of tumor viruses in vivo the subject matter was taken more seriously. ${ }^{11}$ At about the same time several articles were published including one in Science, suggesting that leukemia in humans was a viral disease. ${ }^{12}$ Since viruses which were considered to induce leukemia had already been isolated in mice and chickens, the claims were in general taken seriously by biomedical scientists. ${ }^{13}$ Consequently the 'hunt' for a human leukemia virus was intensified and a growing number of biomedical researchers got involved in the field of leukemia research. As did the American virologist Ion Gresser.

Having accepted Chany's invitation to set up his own laboratory of viral oncology at the Institut de Recherches Scientifiques sur le Cancer in Villejuif in the outskirts of Paris, Gresser was looking for research projects that would satisfy his interests in interferon research, fit into the research profile of a cancer research institute, and would be promising and daring at

cation from Malignant Cells (the viral autoinhibition phenomena)', Virology, 13 (1961), 485-92.

11 J. Bader, 'Production of Interferon by Chick Embryo Cells Exposed to Rous Sarcoma Virus', Virology 16 (1962), 436-43; R. Friedman, A. S. Rabson, W. Kirkham, Variation in interferon Production by Polyoma Virus Strains Differing Oncogenicity, Proc. Soc. Exp. Biol. Med., 112 (1963), 347-51: 'NCI Scientists Publish Data on Interferon as Cancer-Inhibitor. The NIH Record, XVI, 17 (1964), p. 8; See, the session on viruses and interferon in Viruses, Nucleic Acids and Cancer [A Collection of Papers Presented at the Seventeenth Annual Symposium on Fundamental Cancer Research, 1963]. (Baltimore: The Williams and Wilkins Company, 1963), pp. 429-84.

12 J.D. Almeida, R.C. Hasselback and A.W. Ham, 'Virus-like Particles in Blood of Two Acute Leukemia Patients', Science, 142 (1963). 1487-9; and, E. J. Freireich and E. Frei, III, 'Recent Advances in Acute Leukemia', in Tocantins, L. M. (ed.) Progress in Hematology, Vol IV (New York: Grune and Stratton, 1964), pp. 187-202.

13 Not until the late fifties did research into the possible viral etiology of cancer gain more respectability. For more than fourty years the biomedical research community had persisted in the belief that mammalian cancer was not contagious, thereby relegating occasional claims about viruses that could cause tumors in mammals to the fringes of scientific research. The following developments played an important role in the change in attitude towards the idea of viruses causing cancer. First, after a virtual stand-still in the reporting of viruses that were thought to cause cancer in mammals, a number of scientists from highly regarded research centers like the NIH came up with claims about the isolation of viruses that caused leukemia and other cancers in laboratory animals like the rabbit, mice and rat. Second, virology as a whole had grown to maturity and was increasingly recognized as a major scientific discipline. The isolation of the polio virus and the successful production of effective vaccines against the widely feared disease polio should also be mentioned. This medical breakthrough earned virologists a lot of credit both in and outside the scientific community. Third, it was noticed that prominent virus researchers like Jonas Salk, who played a major role in the development of a vaccine against polio virus, joined the club of supporters of the theory on the viral etiology of cancer. Hence, by the early 1960 's the idea that viruses could cause cancers at least in animals, had gained a firm foothold; R.E. Shope, 'Evolutionary Episodes in the Concept of Viral Oncogenesis', Perspectives on Biological Medicine 9 (1966), pp. 258-74; M. B. Shimkin, Contrary to Nature (Washington D.C.: U.S. Department of Health, Education, and Welfare, National Institutes of Health, 1979), pp 213-23; K. Studer and D. Chubin. The Cancer Mission: Social Contexts of Biomedical Research (Beverly Hills: Sage publications, 1980), pp. 19-21; J. Patterson. The Dread Disease (Cambridge (MA): Harvard University Press, 1987), pp. 59, 98, 186. 
the same time. ${ }^{14}$ By coming close to these conditions he chose to organize his research around the problem of leukemia and viruses. ${ }^{15}$ To Gresser's best knowledge there was no published report of interferon's value or lack of activity in mouse leukemia. ${ }^{16}$ More still, he knew of only a small number of other interferon researchers who thought it worthwhile to invest their resources in studying interferon's effects in vivo.

The consensus among interferon researchers was that with the available techniques you could never produce enough interferon to make a difference in animals, let alone man. ${ }^{17}$ Moreover interferon had only been shown to prevent the development of virus diseases (as a 'prophylactic') and was without effect once the infection had established itself. Gresser, however, did not want to take this for granted as long as nobody had made a serious attempt at administering high doses of interferon in virus infected animals for longer periods of time. ${ }^{18}$

Gresser could afford to plunge himself into a problem which entailed a break from mainstream interferon as well as tumor virus research. The research expenses in his Paris laboratory were for the greater part covered by a generous unconditional grant provided for by the Nestor of American Cancer Research, Sidney Farber, who worked as Director of the wealthy Childrens Cancer Research Foundation in Boston, the largest clinical center for the study and treatment of child leukemia in the U.S.. ${ }^{19}$ This enabled him to take a relatively independent research position and choose an area of research which most fellow-researchers shunned.

Gresser's first objective was to find a method to produce large amounts of highly active mouse interferon. From the literature Gresser knew that Norman Finter had developed a

14 The reason to give up a potential bright research career at the world-famous Harvard Medical School for a relatively uncertain living in what was known as the French biomedical 'snake pit' was private in nature. During his one year stay in 1961 as a postdoctoral research fellow at Charles Chany's virology laboratory at Hopital St-Vincent-De-Paul in Paris he had fallen in love with both the city and a particular Parisian; interview with Ion Gresser.

I. Gresser to S. Farber, progress report period June-December 1965, dated 10 December 1965, I. Gresser personal archives; Gresser, interview.

16 He only knew of one recent American study by the virologist Frederick Wheelock in the New England Journal of Medicine claiming that repeated administration of viruses to a patient with acute leukemia had a temporary inhibitory effect on the disease process; F. E. Wheelock and J.H. Dingle, 'Observations on the Repeated Administration of Viruses to a Patient with Acute Leukemia', New. Eng. J. Med., 271 (1964) 64551.

R.R. Wagner, 'Interferon; A Review and Analysis of Recent Observations'. American Journal of Medicine, 38 (1965), 726-737. personal archives. 
production method-obtaining interferon from the brains of mice after infection with so-called 'West Nile' virus. However, the methodology was rather demanding because of the large number of mice needed and the laborious isolation procedure. ${ }^{20}$ But, with a limitless supply of laboratory mice at his disposal and the help offered by Chany and his team-who were also interested in interferon as a possible approach to anti-leukemia therapy-Gresser decided to give it a try. ${ }^{21}$

By the end of November they routinely injected on Mondays about 500 mice with West Nile virus and on Fridays it was 'butcher's day'. Standing all together along large tables they mechanically opened and emptied the hundreds of mouse skulls and threw the brains in ice chilled beakers. ${ }^{22}$ At the end of Friday afternoon the brains were mashed up in an electric mixer where after the resulting mouse brain suspension was taken for further biochemical processing, including centrifugation. The final product was the equivalent of soup broth after all solids have been strained out and was designated 'mouse interferon'. ${ }^{23}$

These preparations of mouse interferon were employed to figure out what effect interferon had on the pathogenesis of murine leukemia. It meant that a fixed number of mice were treated with mouse interferon and subsequently inoculated with Friend mouse leukemia virus ('Friend virus') which he had obtained from the American tumor virologist Charlotte Friend. Daily administration of interferon was continued for 2 to 5 weeks. Simultaneously a control group was only inoculated with the virus. The course of the leukemic disease process in the infected interferon treated and infected untreated mice were compared. This entailed daily monitoring of the condition of the mice until the animals were sacrificed between the second and fifth week. ${ }^{24}$

For more than four months they seemed to go absolutely nowhere. Analysis of data in

N. Finter, 'A Rich Source of Mouse Interferon, Nature, 204 (1964), pp. 1114-5; N. Finter, 'Protection of Mice by Interferon Against Systemic Virus Infections', Brit. Med. J., 2 (1964), 981-85.

In 1963 in an desperate effort to save the life of his best friend's daughter, who suffered from acute leukemia, Chany - as a strong believer in the vinus ethiology of cancer-treated the girl with crude human interferon preparations. Since the patient with about 6 months life expectancy lived for another 1.5 years, he believed that the administration of interferon might have been beneficial; and interviews with Charles Chany Ernesto Falcoff, and Ion Gresser.

22 E. Falcoff, R. Falcoff, F. Fournier and C. Chany, 'Production en Masse, Purification Partielle et Caractérisation d'un Interfgeron Destiné a des Essais Thérapeutiques Humains', Annales de I'Institut Pasteur, (5) 1966. 562-84; and interviews with Charles Chany, Ernesto Falcoff, and Ion Gresser.

23 I. Gresser to N. Finter, letter dated 8 September, 1965, Gresser Correspondence, personal archives; N. Finter to I. Gresser, letter dated 14 September 1965; Gresser Correspondence, personal archives; I. Gresser to S. Farber, progress report period June-December 1965, dated 10 December 1965. I. Gresser personal archives; Finter, interview; Gresser, interview.

24 1. Gresser to S. Farber, progress report period June-December 1965, dated 10 December 1965, 1. Gresser personal archives 
the lab notebooks were disappointing since no statistically significant differences could be determined between treated and control group. Over and over again the experimental conditions were changed until they were able to demonstrate by May 1966 that interferon, provided that extra concentrated preparations were given, showed an virus inhibitory effect in mice. While immediately initiating further experiments Gresser put himself to the job of writing up the first results for publication. Ultimately in August 1966 a series of two articles were submitted to the Proceedings of the Society of Experimental Biology and Medicine. ${ }^{25}$

The referee reports were received with mixed feelings by Gresser and his team. They were credited for showing that mouse leukemia could be inhibited by biological means, but the data indicating that interferon was responsible for the inhibitory effects were considered unconvincing. The identification of an antiviral substance as an interferon was known to be fraught with pitfalls implicit in reliance on bioassay procedures. This was certainly the case when using almost crude preparations. The inhibitory action might as well be due to residual interfering virus or to mechanisms as yet undetermined. They insisted on Gresser employing the term 'preparations of interferon' instead of interferon, since there was not sufficient proof that the inhibitory effect was mediated by interferon. It was sheer pragmatism that made Gresser, who himself was convinced that interferon was the decisive element in his experiments, adapt his text. At least the incident underlined the importance of investing part of his resources in attempts to further purify and concentrate his interferon preparations. ${ }^{26}$ The data showing that purified preparations of mouse interferon were as effective as crude interferon preparations, were used instrumentally in subsequent publications and seminars in both Europe and America to argue in favor of interferon being responsible for the inhibitory effects. ${ }^{27}$

At about the same time the discussions during the Friday 'slaughter' sessions in Gresser's laboratory centered around the mechanism of the observed inhibitory effects. Gresser

I. Gresser to S. Farber, progress report period December 1965- June 1966, dated 2 September 1966, I. Gresser personal archives

I. Gresser, J. Coppey, E. Falcoff and D. Fontaine, 'Action Inhibitrice de L'interféron brut sur le Développement de la Leucémie de Friend Chez la Souris', C.R. Acad. Sci. Paris, 263 (1966), 586-88; 1. Gresser, Coppey, J.. E. Falcoff and D. Fontaine, Interferon and Murine Leukemia. - 1. Inhibitory effect of Interferon Preparations on the Development of Friend Leukemia in Mice, P.S.E.B.M., 124 (1966), 84-91; and, I. Gresser, D. Fontaine, J. Coppey, R. Falcoff and E. Falcoff, Interferon and Murine Leukemia. II. Factors Related to the Inhibitory Effect of Interferon Preparations on the Development of Friend Leukemia in Mice, P.S.E.B.M., 124 (1966), 91-4; and interview with Ion Gresser.

27 I. Gresser, J. Coppey, J. Falcoff, D. Fontaine, 'Action Inhibitrice de L'interferon Brut dur le Développement de la Leucémie de friend Chez la Souris, C.R. Acad. Sci. Paris, 263 (1966) 586-88; 1. Gresser, J. Coppey, D. Fontaine-Brouty-Boyé, R. Falcoff, Interferon and Murine Leukemia. III: Efficacy of Interferon Preparations Administered after Inoculation of Friend Vinus', Nature, 215 (1967), 174-5; I. Gresser, R. Falcoff, D. Fontaine-Brouty-Boyé, F. Zajdela, J. Coppey, E. Falcoff, Interferon and Murine Leukemia. IV. Further Studies on the Efficacy of Interferon Preparations Administered after Inoculation of Friend Virus', P.S.E.B.M., 126 (1967), 791-97; and, I. Gresser to S. Farber, letter dated 19 January 1967, I. Gresser Correspondence, personal archives. 
would usually bring the topic up for discussion in the following way: "Given the dogma in the field of interferon research that interferon inhibits virus growth we automatically seem to accept that it acts by repressing viral multiplication and thus protecting normal cells from infection and subsequent malignant behavior. But what evidence is there to exclude the theoretical possibility that also some kind of direct inhibitory action of interferon on virustransformed malignant cells is involved?". ${ }^{28}$

Started as a kind of funny thought experiment, it gradually became one of Gresser's favorite topics for discussion both in and outside his laboratory. Most interferon researchers laughed his theoretical speculations about interferon having additional biological effects off. ${ }^{29}$ They were not prepared to give up on the generally accepted concept of interferon as an essential part of a particular cellular defense mechanism against viruses. The fact that no other research group had succeeded to inhibit Friend leukemia disease in mice by the administration of interferon did not add to Gresser's credibility. ${ }^{30}$

Far from discouraging him the critics made Gresser more stubborn about his mouse leukemia studies. ${ }^{31}$ In the process he was able to show that daily administration of mouse interferon was also effective if treatments were started two and even as long as 7 days after the mice had been infected with Friend virus. To his knowledge, these results represented the first demonstration that interferon exerted a significant antiviral effect after infection had been well established in the animal host.

At the end of 1967 Gresser learnt that a fellow-researcher in his institute happened to be in the process of establishing a so-called 'RC19' tumor cell line which originated from mice infected with Rauscher leukemia virus. The available tumor cell line suggested the opportunity to test the idea about interferon having a direct effect on mouse leukemia that was different in nature from inhibition of viral multiplication. ${ }^{32}$ It did not seem much extra work to do some

Interviews with Charles Chany, Ion Gresser, and Ernesto Falcoff.

1. Gressser et al (1967, April, Ciba Foundation Symposium) The Effect of Interferon Preparations on Friend Leukemia in Mice, in G. E. Wolstenholme and M. O'Connor (eds), Interferon (London: J\&A. Churchill LTD, 1967) pp. 240-8; Interviews with Sam Baron, Edward De Maeyer, Robert Friedman, Joseph Sonnabend, and Robert Wagner.

M. VandePutte, J. Delafonteyne, J. Billeau and P de Somer, 'Influence and Production of Interferon in Rauscher Virus Infected Mice', Arch. Ges. Virusforsch., 20 (1967), 235-45; and, E. F. Wheelock, Effect of Statolon on Friend Virus Leukemia in Mice, Proc. Soc. Exp. Biol. Med., 124 (1967), 855-8; E. F. Wheelock. and R. P. B. Larke, Efficacy of Interferon in the Treatment of Mice with Established Friend Virus Leukemia, P.S.E.B.M., 127 (1968), 230-8; Interviews with lon Gresser and Frederick Wheelock.

31 I. Gresser to J. Enders, letter dated 18 January 1968, I. Gresser Correspondence, personal archives.

I. Gresser, C, Bourali, J.P. Lévy, D. Fontaine-Brouty-Boyé, M. Thomas, Cancérologie. Prolongation de la Survie des Souris Inoculées avec des Cellules Tumorales et Traitées avec des Préparations d'Interferon, C.R. Acad. Sci., 268 (1969), 994-7: I. Gresser, C. Bourali. J.P. Lévy, D. Fontaine-Brouty-Boyé, M. Thomas, Increased Survival in Mice Inoculated with Tumor cells and Treated with Interferon Preparations, Proc. Natl. 
experiments with the 'RC19' tumor cells, since a couple of changes in the experimental design of the Rauscher virus experiment would suffice. Gresser left the bench work to his experienced laboratory technician Chantal Bourali, who started with the actual mouse trials early in 1968. Instead of inoculating mice with Rauscher virus she injected Rauscher leukemic cells and wait for 24 hours for the leukemia to establish itself. Then she split the animals up into two groups. One control group received nothing and the other the usual daily administration of interferon. Bourali ear-tagged all the animals with a metal ring, wrote down the number of the mouse and the treatment it received. When after two weeks Bourali told Gresser that the first mice in the control were dying while the interferon treated mice were only having minor disease manifestations he responded coolly that it was best to wait and see what happened next. ${ }^{33}$

Two weeks later in reaction to Bourali's presentation of the preliminary results of her experiment Gresser was much more attentive. Whereas most of the control mice were reported dead more than $90 \%$ of the interferon mice were still alive. Though initially excited he feared the dramatic result might not be repeatable. He read Bourali's laboratory notes over and over again. He suspected Bourali of having made a major mistake, but he could not figure out what had gone wrong except for the fact that she had forgotten to inject the tumor cells into the interferon group. He asked her to repeat the experiment. When by the end of April Bourali again reported similar results Gresser was perplexed. He was still reluctant to accept the results at face value and opted for yet another repeat experiment, this time together with Bourali. However, the student riots in Paris - bringing public life to a stand still for more than two months-put their patience to the test. ${ }^{34}$

By the time the international interferon conference was held in Lyon early in January 1969, Gresser had enough confidence in the consistency and persuasiveness of the laboratory data to come out with the claim that continued administration of mouse interferon increased the survival of mice inoculated with tumor cells. However, most interferon researchers continued to smile at the suggestion that interferon might have a direct inhibitory effect on the tumor cells-implicating that interferon was a multi-functional agent. They argued that the antitumor effect was most likely due to non-interferon contaminants in Gresser's preparations. ${ }^{35}$ Moreover, another paper presentation by the NIH researcher Hilton Levy, who worked along similar lines using synthetic interferon inducers instead of mouse interferon, was

Acad. Sci. USA, 63 (1969), 51-7; I. Gresser to F. Farber, progress report, period January-June 1968 (dated I July 1968), Gresser personal archives; and, interview with Ion Gresser.

33

34

35

Ibid.

Ibid.

I. Gresser, 'Prolongation de la Survie des Souris Inoculées avec des Cellules Tumorales et Traitées avec des Préparation Brutes d'Interferon'. Symposium International sur L'interféron, Lyon, Janvier 1969; Interviews with Ion Gresser, Kari Cantell. Charles Chany, Sam Baron, and Robert Friedman. 
not particularly supportive of Gresser's claim. ${ }^{36}$ Levy indicated that he did not think that the observed antitumor action of his chemical compound was directly related to its interferon inducing capacity, particularly since only two of the tumors tested were of viral origin. He believed that something different was involved in the chemical's antitumor action and this made him very doubtful about the possibility that he and Gresser were dealing with the same phenomenon. In Levy's opinion interferon was in neither case at the basis of the observed effects. $^{37}$

Despite the controversy about the nature of the reported antitumor actions, there was general consensus about the practical relevance of both studies. Supporters and critics alike agreed that Gresser's and Levy's animal data were fascinating in their own right by suggesting new therapeutic possibilities in the treatment of cancer. ${ }^{38}$

\subsubsection{Hoping for patients to behave like laboratory mice}

Like most other interferon researchers present at the Lyon meeting, the Finnish interferon researcher Kari Cantell had never paid special attention to the series of articles from Gresser's research group showing that interferon was effective in treating virus induced leukemias in mice. It never occurred to Cantell that Gresser was exploring "different territory". ${ }^{39}$ His experiments seemed to confirm interferon's potential as a broad spectrum anti-viral agent but this never made Cantell think about a serious therapeutic link between interferon and cancer. However, hearing Gresser talk about increased survival of mice inoculated with tumor cells and treated with interferon preparations made a difference to the physician Cantell. Whatever the mechanism of the effects the exciting thing in his view was that interferon seemed to show definite activity against a variety of tumors. ${ }^{40}$

1. Gresser, 'Prolongation de la Survie des Souris Inoculées avec des Cellules Tumorales et Traitées avec des Préparation Brutes d'Interferon'. Symposium International sur L'interféron, Lyon, Janvier 1969; and, H. Levy, L Law and A. Rabson. 'Inhibition of Tumor Growth by Polyinosinic-Polycytidylic acid', Proc. Nat. Acad. Sci. U.S.A., 62 (1996), 357-63; Interviews with Hilton Levy, Ion Gresser, and Kari Cantell.

37 'Antitumor Action of Chemical on Mice Suggests Possible New Cancer Weapon', The NIH Record, XXI, 2 (1969), pp, 1, 7; and, I. Gresser to S. Farber, letter dated 17 January 1969, Ion Gresser Correspondence. personal archives.

L. Gresser to S. Farber, letter dated 17 January 1969. Ion Gresser Correspondence, personal archives: M. Hilleman. Double-stranded RNAs (PolyI:C) in the Prevention of Viral Infections', Arch. Intern. Med.. 126 (1970). 109-24; Interviews with Hilton Levy, Kari Cantell, and Ion Gresser.

40 'NIAID Study Secks to Explain role of Interferon system in Viral Resistance', The NIH Record, XX, 21 (1968), p. 1.7. 
Gresser's animal studies in combination with the interest of the National Institutes of Health (through its Antiviral Substances Program that was established in 1969) in his interferon production facilities encouraged Cantell to initiate trials in humans on his own. ${ }^{4 t}$ Collaboration with the Americans was attractive, because of the high payments they offered for the production of human leucocyte interferon. ${ }^{42}$ This enabled Cantell to employ more staff and obtaining more sophisticated equipment needed to expand his production capacity as well as research activities. ${ }^{43}$

While visiting one of the pediatricians at the University Hospital in Helsinki to discuss plans for a leukemia trial in children in October 1969, Cantell learned that a 15 year old boy was suffering from a rare complication of a measles infection ('SSPE') - a severe and ultimately fatal subacute sclerosing panencephalitis (inflammation of the brain)-without any hope for recovery. Apart from an immediate professional interest in the case as a virologist, Cantell became personally touched by seeing the child-patient fighting a losing battle against the virus disease. In consultation with the responsible pediatrician and the parents, who were willing to try anything that might help, Cantell decided to give it a try. Treating the boy seemed better than doing nothing and at least it provided a good opportunity to learn more about the pharmacology (dose-effect relationships) of his interferon preparations. ${ }^{44}$

It was a big disappointment-although no one knew what to expect-to see that the intravenous injections with the interferon material were followed by serious side-effects. In spite of the fact that a couple of hours after the injections the clinical condition seemed to improve considerably the pediatrician and Cantell decided to discontinue the treatment. They would not resume interferon treatment until Cantell would have succeeded in producing a more purified interferon preparation that was believed to cause less toxicity problems.

Two months passed before Cantell felt confident enough about his purification procedure to give it another try. As a precautionary measure the injections were no longer

41 K. Cantell to G. Galasso, dated 15 September, 1969. Cantell personal archives; and, K. Cantell to K. Paucker, letter dated 12 November, 1969, Cantell personal archives.

42 K. Paucker to K. Cantell, letter dated 12 August 1969, Cantell personal archives; and, K. Cantell to K. Paucker, letter dated 12 November, 1969, Cantell personal archives; Cantell, interview; and Finter, interview; As soon as the British got to know that the Americans were going to pay Cantell for his services they offered payment too. "I am pleased to advise you that the Council has agreed to reimburse the institute the costs of the salaries, materials and equipment detailed in your letter (3390 pounds). For administrative reasons the scientific equipment, i.e. large capacity centrifuge will have to be regarded as property of the Council on loan for an unspecified period"; MRC to K. Cantell, letter dated 10 february 1970, MRC Archives File No. S788/15/2.

43 K. Cantell to K. Fantes, letter dated 12 february 1969, K. Cantell personal archives.

44 K. Cantell to K. Paucker, letter dated 12 November, 1969. Cantell personal archives: K. Cantell to I. Gresser. letter dated 19 November, 1969, Gresser personal archives; and K. Cantell. The Story of Interferon: The Ups and Downs in the Life of a Scientist (Singapore, World Scientific, 1998). 
given directly into the veins but intramuscularly. The boy received a total of about seven injections with what Cantell called 'semi-purified material'-containing about 400.000 'international units' of interferon. ${ }^{45}$ This time only a few minor flu-like side-effects were monitored. However encouraging, the injections did not make a difference to the already bad clinical condition of the boy. After treating yet another child-patient without achieving any Favorable clinical results the pediatrician lost interest in Cantell's trial plans. ${ }^{46}$

The failure of the Helsinki Endeavor coincided with a request from Hans Strander-who after leaving Cantell's Helsinki laboratory had started residency in oncology at the Karolinska Hospital in Stockholm - to do a collaborative study with interferon in cancer patients at the cancer ward ('Radiumhemmet'). ${ }^{47}$ The culture of clinical experimentation was strongly present at the Radiumhemmet. There were historically grown links between 'cuttingedge' research and cancer therapy. It was this combination of therapy and research that attracted cancer patients from all over Sweden in the hope to benefit from the latest technological advances: experimental surgery, $x$-ray machines and experimental drugs. ${ }^{48}$

For residents like Strander it was normal practice to combine cancer therapy with clinical research. As a physician-researcher he was supposed to straddle between the bedside and the laboratory bench in an attempt to bridge the gap between the laboratory and the clinic. In other words he had to question what experiments in the test-tube and in mice meant vis-ávis human patients. Simultaneously, as a practicing physician, he had to take care of patients suffering from more or less advanced cases of cancer, mostly with a bad prognosis. This made him familiar with the ambiguous dilemma whether or not to treat patients with an experimental therapy that had not been tested sufficiently yet, in view of the general willingness of patients and doctors alike to try anything in search for a cure. ${ }^{49}$

However eager to collaborate with Strander and gear up for a test on cancer patients, Cantell realized that even without a lot of administrative hurdles setting up a proper trial would be far from easy. First of all there was only enough interferon to treat a few cases for a limited period of time, while Gresser's mouse studies indicated that it was necessary to continue treatment over a relatively long period. Furthermore the assessment of the clinical data would be difficult. Malignant diseases mostly had a variable course and due to the fact that only in

K. Cantell to K. Fantes, letter dated 27 February 1970, Cantell personal archives.

K. Cantell to K. Fantes, letter dated 25 May 1970. Cantell personal archives.

47 K. Cantell to N. Finter, letter dated 25 May 1970, Cantell personal archives; H. Strander to K. Cantell, letter dated 7 July 1970. Cantell personal archives.

48. Interviews with Kari Cantell, and with Hans Strander.

49 Interviews with Hans Strander and Emest Borden. 
very desperate cases oncologists were prepared to omit other treatments it was difficult to find proper controls.

Cantell was curious to hear from Strander what cases apart from leukemia he considered suitable for treatment. "What would you think about myeloma (or other lymphomas), fulminant melanomas or brain tumors?". ${ }^{50}$ Strander speculated on the basis of his clinical as well as research experience that interferon as a viral inhibitory substance would most likely have a beneficial effect in malignancies which were supposed to have a viral origin. Except for human leukemia Strander identified at least four possibilities: multiple myeloma and another kind of lymphoma, Hodgkin's disease (malignant tumor of lymph nodes), osteosarcoma (a rare but extremely malignant bone cancer) and melanoma (highly malignant form of skin cancer). Since there already existed an experimental treatment program for leukemia patients at the Radiumhemmet, Strander opted for performing first a preliminary trial with the other problematic malignancies such as osteosarcoma and melanoma. ${ }^{51}$

In February 1971 Strander and Cantell were given the green light with the understanding that they would only treat advanced cases of cancer who had been unresponsive to conventional treatments. Since the study was primarily meant to see whether high-dose interferon therapy would produce adverse effects when administered to humans, in other words clinically feasible, a control group did not come up. Their first patient was a 39 year old man with malignant melanoma which originated from a birth-mark in his skin and had spread all over the body. He appeared to be resistant to all existing drug therapies and had a rather poor prognosis. The patient consented to allow treatment with an 'experimental treatment' which, Strander emphasized was something entirely new and hardly tested in humans. ${ }^{52} \mathrm{By}$ injecting the semi-purified interferon preparation-containing about half a million units-directly in the blood stream ('intravenously') Strander hoped to accomplish the most optimal exposure of the tumor cells.

Initially everything seemed to go well but after a couple of hours suddenly the patient's pulse quickened and his temperature rose sharply. Subsequently he developed chills, began vomiting and was shivering severely. The patient was diagnosed as suffering from a potentially life-threatening anaphylactic shock and Strander had to intervene with an emergency cortisone injection, where after gradually the side-effects subsided. The disappointment was great. Cantell and Strander did not know what to make of the patient's severe reaction to interferon. Was the patient allergic to interferon or was something different involved? They decided to

K. Cantell to H. Strander, letter dated 12 September 1970, Cantell personal archives.

51 One of the researchers at the Tumor Institute claimed to have developed an enzyme test which could be used to identify virus related cancers. Strander's selection of four cancers had all tested positive,H. Strander to K. Cantell, letter dated 2 December 1970. Cantell personal archives. 
investigate the case thoroughly before continuing with further experiments in patients. ${ }^{53}$

Cantell launched extensive series of animal experiments in his laboratory in Helsinki in order to learn more about the toxicology-supposedly due to the impurities in the preparation-and pharmacology-next to nothing was known about the fate of interferon in the organism-of his interferon preparations. It became a puzzling affair, tests with the same interferon preparation, even when given in massive doses, produced only few side-effects-a slight fever or contraction of vessels - in the animals studied. Neither did allergic skin tests on the patient produce a reaction. In the end Cantell decided to see whether or not changing the mode of administration would make a difference. At least in rabbits intramuscular and subcutaneous injections resulted in long-lasting and stable blood interferon levels without even the slightest contraction of the ear vessels. ${ }^{54}$

Three months after the first effort Strander and Cantell decided to give it another try. This time Strander administered the interferon intra-muscularly, starting with a relatively small dose. To his great relief the patient tolerated the injection "pretty well", with only a slight and transient rise in body-temperature - a "feeling of warmth" and malaise manifested itself a few hours after the injection. ${ }^{55}$ Even much higher doses up to 1.5 million units per injection did not seem to harm the patient except for the fact that the transient malaise and fever reaction grew more severe. The flu-like symptoms lasted for about half a day. Strander thought it most likely that the dose dependent side-effects were due to contaminants in the preparations-which were known to be still far from pure-rather than interferon. As long as he could minimize the discomfort to the patient by giving Aspirin after each interferon injection he was prepared to try even higher dosages. ${ }^{56}$

By August 1971 two other patients had been enlisted in the interferon treatment program: a 46 year old woman with far advanced Hodgkin's disease and a 19 year old female with advanced osteosarcoma who had just recovered from the surgical removal of a very large tumor in her frontal bone. All received three weekly injections with interferon without further complications being monitored. Everything seemed to go well, although Strander thought it

53 H. Strander, K. Cantell, G. Cariström, and P. Jakobsson, 'Clinical and Laboratory Investigations on Man: Systemic Administration of Potent Interferon in Man', J. Nat. Cancer, Inst., 51 (1973), 733-42; and interview with Kari Cantell. Ibid.

55 K. Cantell to T. Merigan, letter dated 10 June 1971, Cantell personal archives.

56 H. Strander, K. Cantell, G. Carlström, and P. Jakobsson, 'Clinical and Laboratory Investigations on Man: Systemic Administration of Potent Interferon in Man', J. Nat. Cancer. Inst., 51 (1973), 733-42; and interview with Kari Cantell. 
too early yet to say anything about the effect on the malignancies. ${ }^{57}$ The untimely death of the two older patients at the end September only shortly affected morale and did not prevent Strander from including 5 more advanced cases of cancer in his treatment group. Strander spent a lot of time monitoring the condition of his patients at the bedside. Initially the clinical data accumulated without noticing remarkable differences, but after a couple of months Strander got the feeling that he was seeing responses to interferon therapy in a few patients.

However questionable the nature and the endurance of the responses he did not have difficulty in convincing Cantell to sent additional batches of interferon. On the contrary Cantell assured that with the NIH contract money he had been able to scale-up the production of interferon considerably. This would enable him to supply Strander with enough interferon to keep 10 patients under treatment. ${ }^{58}$ Cantell also indicated that he had succeeded in improving his purification process. He would ship by air freight a container with both the conventional interferon material, which he had marked as 'c(rude)-IF, and a few tubes labeled 'p(urified)-IF containing what he called "superinterferon". ${ }^{59}$

With the more highly purified and concentrated interferon preparations they hoped to see more definite clinical responses but also a reduction in the dose dependent side-effects. ${ }^{60}$ Their hopes did not materialize in the sense that the patients tolerated the P-IF only marginally better, although the local reaction at the injection site almost disappeared. In none of the patients was treatment with interferon followed by changes in the clinical condition that were dramatic enough to support the claim that interferon exhibited antitumor or antiviral effects. Nonetheless, Strander, relying mainly on his 'clinical touch' as a physician, became increasingly convinced that he was getting some response: the three osteosarcoma child-patients were all faring better than what might be expected from the 'average' osteosarcoma patient at the Radiumhemmet and he observed how one of lymphoma patients became well, at least temporarily, from his tumor disease. Furthermore, he was fascinated by the quick clinical recoveries from shingles in a couple of the cancer patients treated with interferon, although he was aware that spontaneous recoveries in these cases were not uncommon. ${ }^{61}$

At least the clinical data looked promising enough to justify the decision that all future osteosarcoma patients admitted to the Karolinska Hospital would be treated with interferon

57 K. Cantell to K. Kato, letter dated 6 September 1971. Cantell personal archives; H. Strander to K. Cantell, 2 September 1971, Cantell personal archives.

K. Cantell to N. Finter, letter dated 21 June 1971. Cantell personal archives.

K. Cantell to H. Strander, letter dated 29 december 1971, Cantell personal archives. 
and to extend his studies with myeloma patients. ${ }^{62}$ Strander also got himself involved in laboratory studies at the nearby Institute of Tumor Biology, testing the effect of interferon on the potentially virus-related human tumor cell-lines that were in stock. ${ }^{63}$ In doing so he organized his studies along two coexisting lines: laboratory investigations and bedside practices. Like Cantell, Strander seemed to adhere to the principle that establishing continuous links between laboratory data and clinical data would facilitate the systematic development of interferon therapy.

If we recall the series of experimental events that helped to shape the notion of interferon as a potential cancer therapy two issues appear to figure prominently. First, I would like to point at the central role the question of purity played so far in evaluating knowledge relating to interferon. Depending on the situation researchers made an appeal to the purity or impurity of interferon preparations as a means to justify and criticize experimental results and claims. This is nicely illustrated by the controversy which arose over Gresser's antitumor claim and the way in which Cantell and Strander tried to come to terms with unexpected clinical effects in patients. By providing interferon researchers with a flexible arbiter in the context of justification the 'purity' parameter would remain invariably popular in the production of evidential arguments.

Second, the coming of age of the field of interferon research began to make itself felt through the dogmatic resistance to a change of concept of interferon as part of the host defense against viral infection and the pervasiveness of the non-toxicity dogma in evaluating clinical data. In the next section I will describe how a similar kind of conflict and competition over what questions were important, what phenomena were interesting and what answers were acceptable, continued to influence the development of interferon as we follow Mathilde Krim's efforts to mobilize interest in interferon as a possible lead toward cancer therapy in the USA in the early 1970 's.

\section{2 'Mary's most eager lamb': Mathilde Krim}

Despite strenuous efforts, Mathilde Krim did not fare well in mobilizing support for research into the antitumor properties of interferon. Her applications for funding were rejected by the National Cancer Institute (NCI) and the National Institute of Allergy and Infectious Diseases

62 H. Strander, K. Cantell, G. Carlström, and P, Jakobsson, 'Clinical and Laboratory Investigations on Man: Systemic Administration of Potent Interferon in Man', J. Nat. Cancer. Inst., 51 (1973), 733-42; I. Gresser to E. Frei III. letter dated 6 November 1972, 1. Gresser personal archives: H. Strander to I. Gresser, letter dated 3 January 1973. I. Gresser personal archives.

63 H. Strander to I. Gresser, letter dated 15 November 1972, I. Gresser personal archives. 
(NIAID), which are both part of the National Institutes of Health (NIH). During a visit to the NCI's office in Bethesda, MD, Krim learned that NCI officers were not willing to support the project because that would mean having "to step on to NIAID's turf". ${ }^{64}$ The NIAID already had a formal interferon program (as part of the Antiviral Substances Program) which coordinated NIH in-house research with regard to interferon and was responsible for all interferon extramural research-spending roughly 900.000 dollars or 1,5 percent of the NIAID's annual research budget in $1970{ }^{65}$ Requests for funding of extramural interferon research should therefore be addressed to the NIAID and not the NCI.

Apart from the territorial issue, the antitumor claims of the seemingly non-toxic (in marked contrast to most other available modes of cancer therapy) virus inhibitory substance, 'interferon', had little appeal, despite the high priority of NCI's special Virus Cancer Program. ${ }^{66}$ Behind the scenes Hilton Levy had made no secret of his scepticism regarding Gresser's claim that interferon in addition to its antiviral properties might have a direct inhibitory effect on the tumor cells. ${ }^{67}$ At the same time Levy himself and the NCI researcher Arthur Levine were having a difficult time in keeping the $\mathrm{NCI}$ administrators interested in their Poly I:C antitumor testing program. Preliminary tests in cancer patients had been disappointing thus far without any indication that the interferon inducer produced an antitumor effect; in addition the compound appeared more toxic than was hoped for. ${ }^{68}$

The problematic and often conflicting reports regarding the tumor-inhibitory effects of both interferon inducers and interferon were hardly an inducement for support. Moreover, there were strong feelings within $\mathrm{NCI}$ quarters against funding research with a poorly defined and impure substance or, even worse, some sort of family of biologically active proteins. Published reports suggested a marked heterogeneity of human interferons with regard to molecular weight, electrical charge and differences in stability to heat and acid. ${ }^{69}$ However, none of these interferons had been characterized in chemical terms and the production of interferon was known to be beset with difficulties. In many ways interferon seemed to resemble the kind of ambiguous complex biological activity the NCI had experienced in the

M. Krim to L. Gresser, letter dated 30 November 1971, 1. Gresser personal archives.

In the fiscal year 197022 contract were awarded, totalling 868.811 dollar: Evaluatory report of the Antiviral Substances Program, September 1976, NIAID Archives, DMID-ARB box \# 14; and, NIAID Initiates Antiviral drug Program; Awards Contracts for Interferon Study', The NIH Record, XXII, 18 (1970), pp. 1, 4.

R. A. Rettig. Cancer Crusade (Princeton: Princeton University, 1977), p. 69.

67 Interview with Arthur Levine. 
INTERFEROM PROGRAM

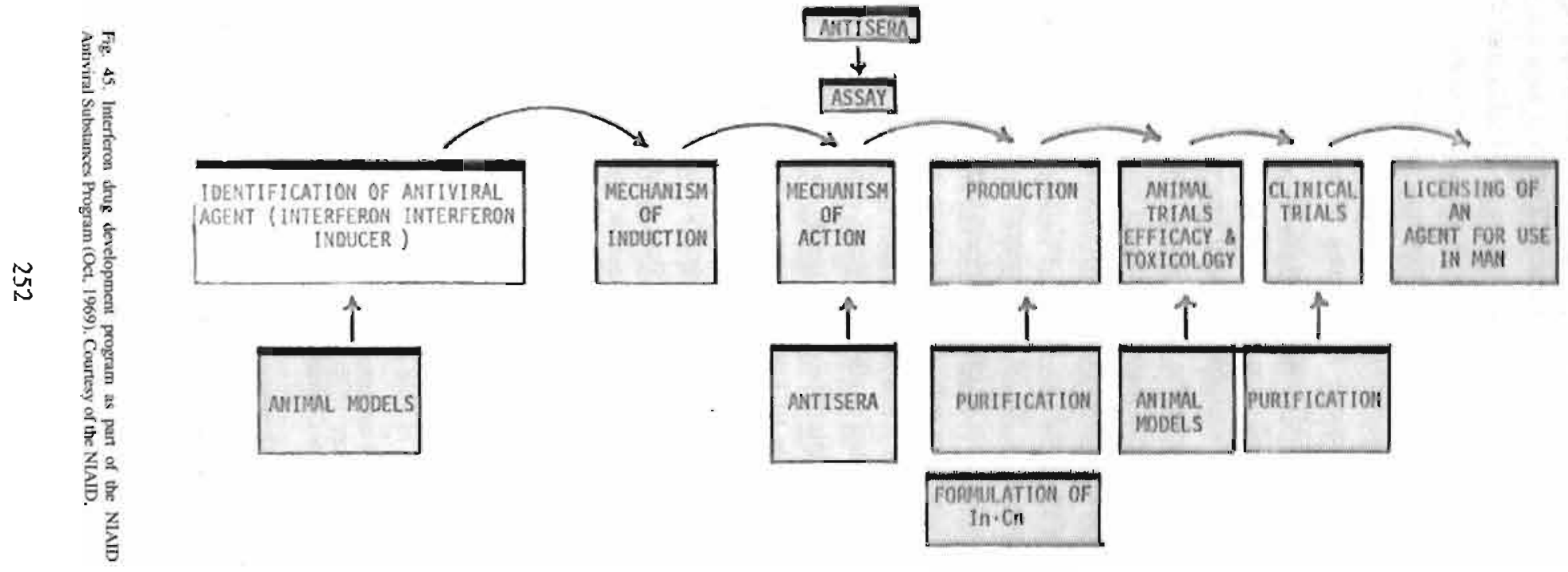

RETIDOS OF

PFEOUCT IOMI] 
past with unproven cancer therapies like 'Coley's toxins'. ${ }^{70}$

The NIAID, in turn, was not receptive to Krim's applications either. George Galasso, who headed the Antiviral Substances Program, was interested in interferon as a potential antiviral drug but was skeptical about the possibilities of interferon as an antitumor agent. ${ }^{71}$ His sceptic attitude was based on a lack of evidence that inhibition of tumor growth was mediated by interferon and not by some other molecule, given the impurity of interferon preparations. Moreover, all interferon researchers on his Program Advisory Committee resisted Gresser's idea that the action of interferon might be pleiotropic or polypractic-inhibiting the growth of viruses as well as tumor cells. They held on to the principle that cells produced interferon in response to viruses and that, after release from the cell, interferon in turn specifically reacted with cells to induce the formation of another cellular protein which mediated the actual antiviral activity. This so-called 'interferon system' was considered to be an important part of the non-immunological host defense against viral infections. As such interferon fitted in rather well with the 'biomedical model' of disease and treatment-based on the theory of specific etiology and the notion of specific therapy-on which the Antiviral Substances Program was largely based (see Fig. 45).$^{72}$

Despite the rather disappointing reception of her project proposals at the $\mathrm{NIH}$, further discouraging reactions from private foundations such as the Rockefeller Foundation, Krim did not give up her fund-raising efforts. She felt that hurdles were there to be taken and that any agent like interferon, which had shown inhibitory activity against several tumors in mice

70 R.W. Moss, The Cancer Industry (New York: Paragon House, 1989), pp. 119-31: Interviews with Mathilde Krim, Joseph Sonnabend, Arthur Levine, Thomas Merigan.

71 During a visit to Russia in the summer of 1971 Galasso had made the surprising discovery that human leucocyte interferon was already available in Moscow area pharmacies for use as a nasal spray against influenza. This encouraged him in funding a number of clinical trials to test interferon as a potential antiviral drug. For instance, the NIAID financially supported Tom Merigan's and David Tyrrell's collaborative effort to test interferon on respiratory viruses in volunteers at the Common Cold Unit in Salisbury (UK). In 1973 the latter attempt would result in claims in both the New York Times and Lancet that interferon was effective against the common cold; 'Three U.S. Scientists Visit Soviet Union; Find Antiviral research Programs Differ'. The NIH Record, XXIII, 18 (1971), p.I, 4; Minutes Antiviral Substances Workshop, NIH, dated I december 1971, NIAID archives; 'Substance in Body Said to Block Colds', New York Times, 24 March 1973; 'Scientists Report Interferon-Naturally Occurring Antiviral Protein- Effective Against Common Cold', The NIH record. 24 April 1973, p.8: T. Merigan, S. Reed, T. Hall, D. Tyrrell, Inhibition of Respiratory Virus Infection by locally applied Interferon, Lancet, i (1973), 563-7; T. Merigan to J, Gold (SKF), letter dated 4 September 1972, Tyrrell personal archives; and interview with George Galasso

S. Baron, The Defensive and Biological Roles of the Interferon System, in N. Finter (ed.), Interferon and Interferon Inducers (Amsterdam: North-Holland Publishing Company, 1973) 267-92; E. Richards, Vitamin C and Cancer: Medicine or Politics? (New York: St Martin's Press, 1991), pp. 21-2; and interviews with Sam Baron, George. Galasso, and Joseph Sonnabend. 
without any toxic side-effects, deserved further intensive investigation. ${ }^{73}$ However, more than two years elapsed before, in early 1974, Krim's applications finally met with some positive response. Representatives of the German Behringwerke Company, which had become interested in the possibilities of interferon therapy at the peak of the interferon inducer 'fashion' round about 1969, approached the Memorial Sloan-Kettering Cancer Center with an official request for a clinical trial with human interferon in cancer patients. ${ }^{74}$ The German drug company offered to supply the human interferon that was needed for the trial. The newly appointed research director of Memorial Sloan Kettering, Robert Good, was rather taken by the German interest and decided that the trial should be carried out. Since he knew about Krim's vivid interest in interferon, he asked her to act as coordinator. Krim was encouraged to involve outside experts and thus she got in touch with the most outspoken believer in interferon's antitumor activity, Ion Gresser. ${ }^{75}$

In the spring of 1974, Krim visited Gresser in his laboratory. Gresser enthusiastically informed her of his latest investigations and publications concerning interferon's antitumor effects. Krim was intrigued by Gresser's speculations about interferon as a broad spectrum cellular hormone serving to modulate cellular behavior and enhance the host defense mechanism-acting locally or at a distance, stimulating when stimulation was needed and inhibiting when inhibition was needed. ${ }^{76}$ This sounded similar to Robert Good's new approach to the treatment of cancer dubbed 'immunotherapy' aimed at the stimulation of the non-specific immune response by biological ('natural') means-involving non-specific factors that amplified immune reactions, as opposed to the specific antibody related, classical immune mechanisms. ${ }^{7}$

73 M. Krim to I. Gresser, letter dated $20 \mathrm{March}$, 1974, Gresser personal archives; and interviews with Kari Cantell and Mathilde Krim.

Interview with Mathilde Krim: I. Gresser to S. Farber, letter dated 7 November 1969, Gresser personal archives.

M. Krim to 1. Gresser, letter dated 20 March 1974. Gresser personal archives: Krim, interview; and Gresser, interview: Krim and Gresser met in the fall of 1971, when Krim was in the process of interesting the NIH in supporting work on the production of interferon for clinical trials in cancer patients: M. Krim to L. Gresser, letter dated 30 November 1971. Gresser personal archives.

1. Gresser (Unpublished manuscript, 1973), 'Cancer and Immunology or the Resurrection of Sir Colenso Ridgeon'; Gresser loved to quote his assistant Pernilla Lindahl who defined interferon as a substance "that does what you want it to do": I. Gresser, 'Interferon Therapy: Obvious and not so Obvious Applications', Acta Medica Scandinavica, 197 (1975), 49-53, p. 52: and interviews with lon Gresser, and Mathilde Krim.

From the 1920 s up to the 1960 s immunologists would describe their specialty in terms of the study of specific defense mechanisms. The concept of antibody-related specificity as Stephen Hall aptly expressed it "remained magnetic north to all immunologists, the orientation against which everyone set their research compasses". Those who pioneered non-specific mechanisms were hardly taken serious by mainstream immunologists: Stephen Hall, A Commotion in the Blood; Life, Death and the Immune System (New York: Henry Holt and Company, 1997), p. 170; The growing emphasis on local defensive processes and substances was not unique to immunotherapy but was also visible in other areas of pharmacotherapy. For instance in the early 1970 's 
As a result of extensive media coverage immunotherapy had a high visibility in the USA in the early 1970 's. ${ }^{78}$ Treatment with immunostimulating agents such as BCG (Baccile Calmette Guérin, a bacterium related to the tuberculosis bacillus) was presented in the media as a promising 'fourth modality of cancer treatment'-in addition to surgery, radiotherapy and chemotherapy. The clinical oncologists who were involved in conducting the immunotherapy trials viewed the new approach as potentially supplementing conventional cancer therapies, with a preference for a combination with cancer chemotherapy. ${ }^{79}$

Hearing Gresser argue that interferon's antitumor effects were most likely based on enhancing a variety of mechanisms of defense available to the host, Krim immediately saw possibilities to align interferon with the increasingly fashionable immunologic approach to cancer therapy. Gresser's advise to contact Hans Strander a physician at the Karolinska Hospital in Stockholm who was performing experiments with interferon in cancer patients, did not fall on deaf ears. Krim immediately booked a flight to Stockholm. ${ }^{80}$

In his room at the Radiumhemmet, Strander told Krim that over the years he had treated more than thirty cancer patients with interferon without noticing serious toxic effects. He considered the provisional results encouraging. In particular the osteosarcoma trial looked promising. ${ }^{81}$ As a routine way to deal with trials concerning advanced cancer patients-involving high and predictable mortality - he worked with historical controls: that is, Strander compared his interferon-treated patients with the medical histories of osteosarcoma patients treated by conventional means at the Karolinska Hospital. The historical record showed that about 80 percent of the osteosarcoma patients developed metastatic tumors in the lungs within 12 months that were invariably fatal, following conventional surgery and radiotherapy.

pharmacologists began to talk about aspirin as an opponent of defensive local hormone(s), so-called prostaglandines; See, H. O. J. Collier, 'The Story of Aspirin', in M. J. Parnham and J. Bruinvels (eds), Selections from Discoveries in Pharmacology (Amsterdam: Elsevier Science Publishers, 1987).

Robert Teitelman notes that with Robert Good's arrival in New York, he and immunotherapy became famous overnight. "Time put him on the cover, and The New York Times Magazine published a long admiring profile. "Today almost every puzzling disease in the medical handbook has become the target of the new immunological weapons," said the magazine"; R. Teitelman, Gene Dreams: Wall Street, Academia and the Rise of Biotechnology (New York: Basic Books, 1989), p. 31.

I. Löwy, 'Between Bench and Bedside' (Cambridge (Mass): Harvard University Press, 1996), p. 110.

Interviews with Ion Gresser and Mathilde Krim.

H. Strander to K. Cantell, letters dated 8 and 13 March, 12 April and 22 September 1973, Cantell personal archives; H. Strander to L. Gresser, letters dated 8 August, 22 September, 8 October, 29 December 1973 , Gresser personal archives; M. Krim, 'Interferon as an Antiviral and Anticancer Agent', Clinical Bulletin, 5 (1975), pp. 34-6; H. Strander, P. Jakobsson, G. Carlström and K. Cantell, Administration of Potent Interferon to Patients with Malignant Diseases, Cancer Cytology. 13 (1974). pp. 18-9; and interviews with Kari Cantell, Mathilde Krim, and Hans Strander. 
Strander believed that a doctor should always choose the treatment, based on the doctor's best judgment and knowledge of the particular patient. He argued that a retrospective study - study that looks back into case histories - could supply the data needed to judge the effectiveness of a given therapy. Furthermore he did not think it ethically acceptable to tell a fatally ill patient, "I have several treatments, but I am not sure which one is best. To find out I'll pick your treatment by a randomized procedure similar to flipping a coin. Is that okay with you?". 82

Strander's attitude is illustrative of the physician's conflict between loyalty to the perceived needs of the patients and to the objectives of research. Moreover, his course of action was in line with the culture of therapeutic evaluation in clinical oncology in the early 1970 's. The spread of randomization was slow compared with other medical specialties and the presentation of research results based on the accurate observation of single cases and on comparison with historical cases was still widely practiced. According to the historian of medicine, Ilana Löwy, the quasi-monopoly of oncologists over their patients-with little interest from other medical specialties in the treatment of the mostly hopeless cancer cases-might have contributed to the persistence of historical controls in clinical oncology. ${ }^{83}$ As we will see in the next sections, what thus began as an unproblematic decision became later a matter of controversy and policy.

\subsubsection{Staging a workshop on interferon in the treatment of cancer}

Krim learned that up until the time of her visit to Strander of the ten osteosarcoma patients that had been enlisted in the treatment group, only two had shown signs of tumor progression after more than a year of being free of symptoms. ${ }^{84}$ In addition, the data collected on lymphoma patients indicated that in single cases Strander was getting a response, at least temporarily. Strander emphasized the preliminary status of his studies and thought it premature to draw any firm conclusions. Yet the preliminary results of the Swedish trial sufficed too strengthen Krim's belief in interferon as a potential non-toxic therapeutic agent. ${ }^{85}$ Krim decided to renew her efforts to interest the NCI in supporting research into the possible antitumor effects of interferon. With Strander's osteosarcoma trial as a vehicle to build an agenda for her cause, she Interviews with Hans Strander and Kari Cantell. Mathilde. Krim and Hans Strander. 
was hopeful that NCI's Virus Cancer Program would make some funds available to interferon research. $^{86}$

Krim's immediate aim was raising consciousness. As a 'woman of action' she wanted to show people in senior positions within the American cancer establishment how much potential there was and the best she could think of was to stage an international conference on interferon and cancer. In developing funding for such a meeting she met with quite some resistance, in particular from NIH quarters. As I mentioned before, there was a basic mistrust of reports concerning interferon's antitumor properties. In addition there were strong feelings about the possibility that Mathilde Krim, an ambitious and politically well-connected outsider and woman, would interfere with the current distribution of federal funds and might gain too much control over the future course of interferon research. Moreover, the established interferon researcher Tom Merigan-who played a central role in the clinical studies part of the Antiviral Substances Program with his trials involving hepatitis patients and cancer patients suffering from viral infections - had already approached the NIH with plans for the organization of an international interferon symposium on the clinical potential of interferon as an antiviral. ${ }^{87}$ Despite the fact that Krim's meeting had a rather different objective, interferon and cancer, there were concerns that her workshop might upstage the NIH meeting. ${ }^{88} \mathrm{Krim}$, however, persisted and finally in the fall of 1974 she received an invitation from the head of NCIs brand new Molecular Control Program, Timothy O'Connor, who was to advise the Director of the $\mathrm{NCI}$, the tumor virologist Frank Rauscher, on new directions in molecular biology with great potential in the fight against cancer. ${ }^{89}$

On 12 December, 1974, Krim in the company of Strander-who had been asked by Krim to work up his trial data into a presentable form and come over for the occasion-paid a

M. Krim to D. Habif, letter dated 19 July 1974, Cantell personal archives; and interview with Mathilde Krim.

Merigan, an infectious disease specialist, developed his plans for trials with interferon in cancer patients suffering from disseminated virus infection (e.g. shingles) simultaneously with Cantell in 1971. Only after Merigan had obtained a high visibility with his promising common cold trial in Salisbury (UK) did he receive sufficient financial support from the NIAID for realizing his trial plans at Stanford (CA). In the course of 1975 he would ask permission for a trial involving human hepatitis infection, that was very common among the large gay community in the San Francisco area. After initial problems about the costs of the interferon required-the costs for the interferon were estimated at little less than 100.000 dollars for 8 patients - the green light was given in June 1975; T. Merigan to G. Galasso, letters dated 16 April, 10 May 1974 and 21 March 1975, NIAID Archives, DMID-ARB box \# 14; G. Galasso to T. Merigan, letters dated 22 April and 4 June 1975, NIAID Archives, DMID-ARB box \# 14.

Interviews with Kari Cantell, George Galasso, Mathilde Krim, Arthur Levine, Thomas Merigan, Joseph Sonnabend.

As director of the NCI Rauscher was part of what was dubbed the 'college of cardinals' which used to meet twice a month-consisting of the directors of the various institutes of the NIH. They heid in their hands space, positions and budget. Individually and collectively they were a very influential group: and interview with Frank Rauscher. 
visit to O'Connor at the NCI. O'Connor was impressed with Strander's latest data based on a group of 11 interferon treated osteosarcoma patients. Strander told him that all these patients had been followed up for at least six months, during which time none had developed tumor progression. The data were judged as being increasingly exciting as they approached a statistical significance between the interferon-treated group and the historical control group consisting of 33 osteosarcoma patients treated between 1952 and 1972 at the same hospital by conventional means. Strander suggested that the preliminary observations were worthy of further expanded study. O'Connor agreed that interferon deserved further study and he was prepared to advocate funding within NCI quarters. Shortly after this meeting, Krim received an invitation to submit an application for a Planning Grant to support an international workshop that would aim at evaluating the state-of-the-art in interferon research and formulate recommendations for further research concerning interferon and cancer. The application would be reviewed during a scheduled meeting of the Molecular Control Working Group on 7 January, $1975 .{ }^{90}$

O'Connor's working group voted unanimously to approve Krim's subsequent grant proposal on behalf of the Memorial Sloan Kettering. However, another month was needed to overcome resistance within other parts of the NCI and Krim's application had to be resubmitted to go through a second review. Finally at the end of February 1975 Krim was informed that the Division of Cancer treatment of the NCI would co-sponsor the meeting. In the meantime Krim had succeeded in getting additional funds from the Rockefeller Foundation, the Sloan Kettering Institute for Cancer Research itself and a number of American pharmaceutical companies. ${ }^{91}$ There was enough money available to do the unusual and offer each participant a free trip to New York City, plus accommodations at the Westbury Hotel and even a small honorarium. ${ }^{92}$

On Sunday 30 March about half of the participants gathered for an informal 'open house' reception in Krim's grand home at East 69th Street in New York. Krim felt that this would provide a good opportunity for getting acquainted and meeting old friends in a relaxed atmosphere before the start of the International Workshop on Interferon in the Treatment of Cancer', which was held at the Rockefeller Institute nearby. ${ }^{93}$ Krim had succeeded in bringing

See the report of the International Workshop on Interferon in the Treatment of Cancer, held in New York. March 31, April 1 and 2, 1975. Sonnabend personal archives; H. Strander and K. Cantell to Timothy O'Connor, telegram dated 13 January 1975, Cantell personal archives; M. Krim to J. Sonnabend, letter dated 15 January 1975. Sonnabend personal archives; S. Panem, The Interferon Crusade (Washington, D.C.: Brookings Institution. 1984), p.17: and interviews with Mathilde Krim and Hans Strander.

91 M. Krim to J. Sonnabend, letter dated 15 January 1975, J. Sonnabend personal archives.

92 Interview with Jan Vilcek.

93 M. Krim to K. Cantell, letter dated 13 March 1975, K. Cantell personal archives. 
together for the first time virologists, oncologists, immunologists, and representatives from the $\mathrm{NIH}, \mathrm{NCI}$ and pharmaceutical industry, who submitted, discussed, evaluated and simply listened to a host of papers mainly concerning the antitumor effects of interferon. The size of the meeting, with about 200 invitees, was not unusually large. However, for quite a number of those present, the meeting represented their first educational experience with a field of research, they had either never paid serious attention to or never heard of.

In spite of Krim's warm welcome, and the careful staging of the workshop, she could not prevent that at times the atmosphere became tense and the meeting threatened to turn into a battleground with believers and non-believers, Krim herself and $\mathrm{NIH}$-interest groups fighting each other in their common drive for power and access to federal funds. At the reception, for example, rumors were spread that Strander's osteosarcoma study, which would be presented the next day, was flawed. Detailed information about the trial and the clinical pictures of each of the 14 osteosarcoma patients, which Strander had submitted to Alan Rabson, director of the Division of Cancer Biology and Diagnosis at the NCI shortly before the meeting to independent review, was said to disclose that most of the tumors were not osteosarcomas and that the Swedish pathologist's diagnosis had been wrong. Furthermore the trial was not a formally randomized comparison but relied on comparison with a historical group treated conventionally. Moreover, the results of Strander's trial did not seem to differ significantly from the recently published clinical results with a U.S. tested new chemotherapy regime of high dose methotrexate, a cytotoxic chemical compound produced by the American Lederle company. ${ }^{94}$

Cantell among others got worried and rather annoyed about this premature judgment of research data, which had yet to be presented by Strander. "A surprisingly malicious, suspicious and negative attitude seemed to breathe towards the research of Hans and mine..." ${ }^{\text {95 }}$ Cantell had the gut feeling that the trial might become the focus of a political struggle. He therefore contacted his friend Merigan, whom he knew was part of the NIH establishment and had a reserved attitude towards Krim's meeting too. "I told my worries to Tom and flashed the threat that I would terminate my collaboration with U.S. scientists, if Hans's study would be publicly decried on unjustified grounds". ${ }^{96}$ Merigan, whose clinical studies depended largely on the continued supply of Cantell's interferon, took the threat seriously. He phoned Rabson in

I. Gresser to M. Krim, letter dated 16 April 1975, Ion Gresser personal archives; Krim, interview; Sonnabend, interview; Levine, interview; Cantell, interview; Gresser, interview; and, G. Rosen et all, 'High Dose Methotrexate with Citrovorum Factor and Rescue and Adriamycin in Childhood Osteogenic Sarcoma', Cancer, 33 (1974), 1151-63.

Kari, Cantell, Interferonin Tarina. Tiedemiehen Elämäa- Iloja Ja Suruja. (The Interferon Story: The Ups and Downs of the Life of a Scientist) (Helsinki: Finland, WSOY, 1993), p. 131.

96 Kari, Cantell, Interferonin Tarina. Tiedemiehen Elämää- Iloja Ja Suruja. (The Interferon Story: The Ups and Downs of the Life of a Scientist) (Helsinki: Finland, WSOY, 1993), p. 133. 
Washington, who would arrive the next day to review Strander's patient records. Rabson reassured both Merigan and Cantell that there were some differences in interpretation between the Swedisch pathologists and his research group, but the majority of the cases indeed represented classical osteosarcoma. Cantell then let things go at that. ${ }^{97}$

The actual session termed 'treatment of human osteosarcoma with interferon' satisfied all parties. On the one hand Strander was given credit for having started a trial with interferon in cancer patients, which had produced some potentially interesting results, but on the other hand most agreed that the experimental sample size was rather small and that the diagnosis of osteosarcoma was questionable in a number of cases. Hence, it was too early to draw any conclusions. Further extended study should be awaited, with the proviso that, prior to the entry of new patients in the study, the diagnosis of osteosarcoma should be confirmed by two independent pathologists. The same kind of compromise was reached in other sessions dedicated to in vitro and animal studies with interferon: the data were regarded as being promising but not more than that. None of the interferon researchers present recalled having heard any dramatically new in scientific terms at the workshop. ${ }^{98}$

Far from being a novel aspect of interferon related research it is remarkable to see that a complete session was dedicated to interferon's interactions with immune defense functions and cellular regulatory mechanisms. In the past there had been occasional paper presentations, most prominently by Gresser and his team and the research couple Edward and Jacqueline de Maeyer-Guignard from the Fondation Curie-institut du Radium (Université de Paris-Sud, Orsay), pointing at interactions between the interferon- and immune system. In general these claims did not meet with general approval and were ascribed to impurities in the interferon preparations. Most interferon researchers supported the view of the interferon system as part of the non-immunological host-defense against viruses. In discussions of host resistance the 'interferon system versus immune system' type of argument monopolized the conversation (see Fig. 46a) ${ }^{99}$

Now for the first time the possible pleiotropic action of interferon as a kind of immune defense modifier was on the agenda (see fig $46 \mathrm{~b}, \mathrm{c}$ ). The paper presentations at hand were far from uni vocal regarding the nature of the interactions with the immune system. On the one hand there were claims regarding an immuno-suppressive activity of interferon preparations as witnessed by a slowing down of the rejection of transplanted grafts in mice. This would

Interviews with Kari Cantell and Hans Strander.
}

98. See report of the International Workshop on Interferon in the Treatment of Cancer, held in New York, March 31. April I and 2, 1975, Sonnabend personal archives; and interviews with Kari Cantell, Mathilde Krim. Thomas Merigan and Joseph Sonnabend. 


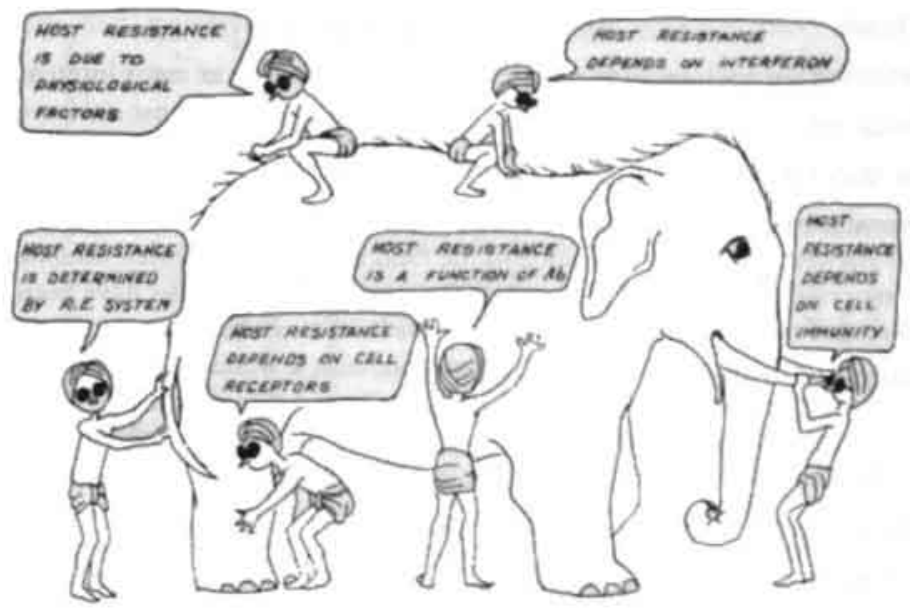

Fig 46a. Lowel Glasgow used this picture in 1970 to support his controversial claim that host resistance against viruses was multifactorial with multiple interrelationships

of the interferon system and the immune system. Reproduced with kind permission from the publisher.

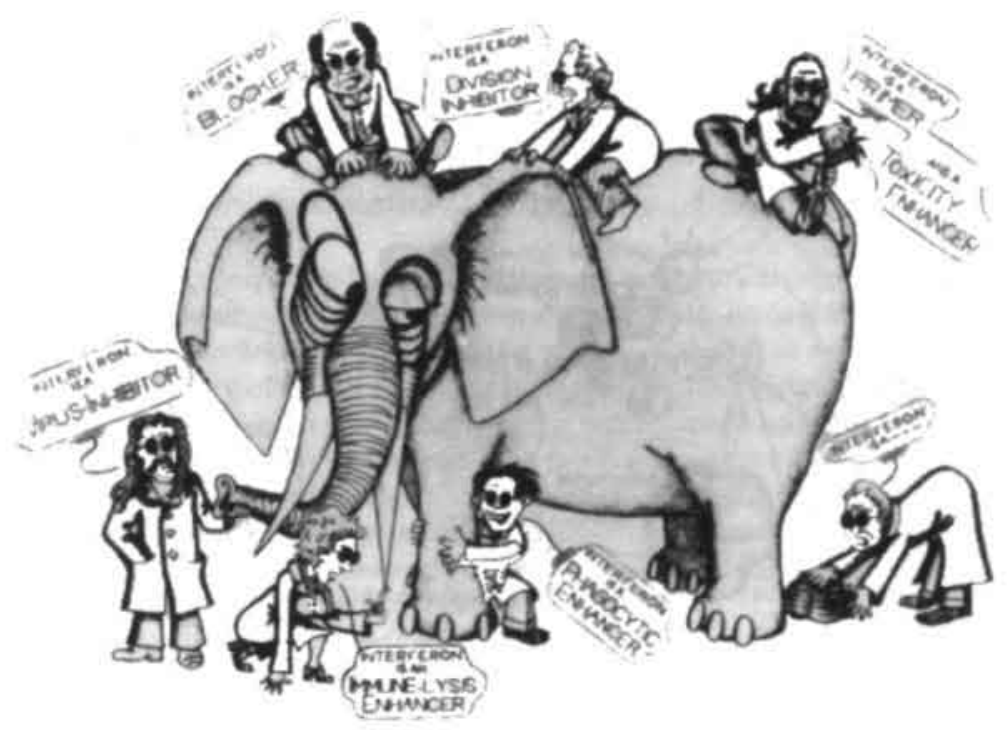

Fig 46b. Around 1976 William Stewart II used the same allegory of the seven blind men and the elephant.

to produce a caricature of several interferon workers who all argue in favour of their own favourite hypothesis. The drawing served as a means

to convince the audience that the action of interferon is pleiotropic Reproduced with kind permission from the publisher. 


\section{The Blind Men and the Elephant}

It was six men of Indostan

To Learning much inclined

Who went to see the Elephant

(Though all of them were blind),

that each by observation

might satisfy his mind.

The First Approached the Elephant.

And happening to fall

Against his broad and sturdy side.

At once began to bawl:

'God bless me! But the Elephant

Is very like a wall!

The Second, feeling of the tusk.

Cried, 'Ho! what have we here

So very round and smooth and Sharp?

To me 'tis mighty clear

This wonder of an Elephant

I very like a spear!"

The Sixth, no sooner had begun

About the beast to grope.

Than, seizing on the swinging tail

That fell within his scope,

'I see' quoth he 'the Elephant

I very like a rope!

And so these man of Indostan

Disputed loud and long

Each in his own opinion

Exceeding stiff and strong.

Though each was partly right.

And all were in the wrong!

\section{Moral}

So oft in scientific wars the disputants, I ween.

Rail on in utter ignorance Of what each other mean.

And prate about an elephant

Not one of them has seen! 
contradict the claim that interferon had an antitumor effect. On the other hand there were observations which could be interpreted as saying that interferon had an immune enhancing effect that might be related to its tumor inhibitory activity. Despite the conflicting and ambiguous nature of the experimental data, this session played an important role in mobilizing the interest of medical researchers working in the field of immunotherapy of cancer. In pioneering various ways of stimulating the cells of the immune system to inhibit tumor growth, with the aim to develop immunotherapies of cancer, they had become accustomed to the apparent paradox that non-specific biological agents with immunoregulating properties could have both immunoenhancing and immunosuppressive effects. ${ }^{100}$

The 1975 symposium was choreographed by a hopeful Krim as a watershed event. The conference was indeed instrumental in bridging the gap between the test-tube and the patient, by linking laboratory data regarding interferon's actions at the cellular and molecular levels, however insufficiently, with clinical data obtained at the bedside. Basically, it was the first major public attempt at translating interferon's antitumor effects into scientific terms that fitted both fundamental laboratory researches and clinical researches. However, Krim's grand objectives were to gain approval and support from the scientific community, funding and cooperation from the drug industry and to get enough funding from the NCI to expand interferon research - a house of cards based almost entirely on Strander's fourteen cancer patients. But, neither scientists nor the representatives of government, private funding agencies and pharmaceutical companies, who attended the meeting, seemed impressed with the experimental data presented over the three day period. The press all but ignored the event. Despite the fact that Krim succeeded in raising consciousness, the workshop as such did not suffice to gain interferon official recognition as a promising antitumor agent. ${ }^{10 t}$

100 See the report of the International Workshop on Interferon in the Treatment of Cancer, held in New York, March 31, April 1 and 2, 1975. Sonnabend personal archives; E and J. De Maeyer-Guignard, interview; and Gresser, interview.

101 Few American pharmaceutical companies expressed interest in working on interferon, for the greater part because of profitability assessments. Profitability of a drug like interferon was often examined from four standpoints: The patentability of the compound (The British had obtained most of the interferon patents), the developmental costs (Producing small quantities of semi-purified interferon was already beset with difficulties and immensely costly), the size of the market and the likely prize of the product (treating one patient for a couple of months already seemed to cost thousands of dollars) and the difficulty and expense of acquiring FDA approval (going by past experience with products from human cell produced products a lot of work was expected): M. Hilleman, interview. 


\subsubsection{Attempts to put interferon on the cancer map}

As a member of the panel of consultants, Krim had experienced the political importance of producing an accessible scientific report with recommendations for further research. After the workshop she asked the interferon researchers Merigan and Jan Vilcek and the NCI tumor virologist, Arthur Levine, to help her produce a report of the workshop. ${ }^{102}$ While the complete report was being prepared, Krim was asked by Nature to write a summary for their News and Views column. Krim immediately put together a short report and sent copies to members of the organizing committee and the Division of Cancer Treatment of the NCI.

Krim's version of the workshop caused a little row at the NCI. In her drive to put interferon on the research map, Krim had left out accounts of dissenting voices, such as the statement by Richard Adamson of the NCI that he had been unable to demonstrate an antitumor effect of interferon in his mouse leukemia system. ${ }^{103}$ This policy was criticized severely within NCI quarters and Krim was told that unless she retracted the paper and included what amounted almost to a disclaimer, detailing the shortcomings of Strander's study, immediately an official dissenting opinion would be published in Nature. Krim decided to take the threat seriously. She agreed to add Adamson's comments and to share authorship with the other members of the editing committee of the official workshop report (see Fig. 47). ${ }^{104}$ The outcome was a most carefully-worded communication, which pointed at the possibility that interferon might exert its apparent antitumor effects both directly on the tumor cells and through a modification of components of the immune system as a kind of immune defense modifier. $^{105}$

In the meantime, Krim exerted further pressure on the NCI to support interferon research. Shortly after the workshop she sent Rauscher, who to her great indignation had not attended the last day of the conference, a personal and confidential letter summarizing the final recommendations he had missed and an indication of the level of support she expected the NCI to provide. ${ }^{106}$ Reluctantly, Rauscher submitted to Krim's pressure and invited her for a meeting with senior NCI officials on 15 April, 1975. At this meeting the edited conclusions and recommendations of the workshop were presented by Krim. Following her presentation it was pointed out that the NCI was not prepared to take any action until the formal report was made

M. Krim to I. Gresser, letter dated 20 May 1975, Ion Gresser personal archives,

103 M. Krim, 'Interfering with cancer', first draft for Nature, News and Views (April, 1975), J. Sonnabend, personal archives.

104

M. Krim to 1. Gresser, letter dated 20 May 1975, Gresser personal archives.

105

M. Krim, A. S. Levine, T. Merigan, and J. Vilcek, 'Interfering with Cancer', Nature, 255 (1975), 372-4.

106

M. Krim to I. Gresser, letter dated 23 April 1975, Gresser personal archives. 


\title{
International Workshop on INTERFERON in the Treatment of Cancer
}

\author{
New York, March 31, April 1 and 2, 1975
}

\section{Co-Sponsors:}

\section{Memorial Sloan-Kettering Cancer Center}

Dr. Lewis Thomas, President
National Cancer Institute

Division of Cancer Treatment

Dr. Vincent T. DeVita, Jr., Director

\author{
Cheir: \\ Mathilde Krim, Ph.D. \\ Memorial Stoan-Kettering Cancer Center \\ New York
}

\section{REPORT}

Editing Committee:

Mathulde Krim, Ph.D.

Memorial Sloen-Kettering Cancer Center

Arthur S. Levine, M.O.

National Cancer Institute

Thoman C. Merigan, M.D.

Stanford University Medical Center

Jan Vilcék, M.D.

New York University Medical Center

Fig. 47. Front page of original workshop report.

Courtesy of Dr. E. De Macyer 
available and the newly established NCI Interferon Working Group under the chairmanship of Alan Rabson would have reviewed the report. ${ }^{107}$

By July, Rabson's interferon group had the extensive formal workshop report in hand and finally, in the autumn of 1975, the Working Group's recommendations were sent to Rauscher and the Director of the Division of Cancer Treatment, Vincent DeVita for further consideration. The group recommended that 4 million dollars be allocated for interferon research to support clinical trials, basic research and the production of interferon. In addition clinical trials should be conducted under the supervision of the NCI's Division of Cancer treatment. ${ }^{108}$ However, within the Division of Cancer Treatment, the recommendations were received with mixed feelings, and DeVita decided to bring the matter up for discussion at the November meeting of the advisory committee to his division, the Board of Scientific Counselors. ${ }^{109}$

The meeting of the Board on 11 November 1975 began with a presentation by Mathilde Krim on the use of interferon in the treatment of cancer. Right from the start Krim felt that she would have the odds stacked against her. Her report, its conclusions and recommendations were indeed severely criticized, especially Strander's clinical trial. The tumor data were questioned, the number of cases was thought to be too small and it was suggested that it was unlikely that biological activity could be obtained in a mixture containing one in thousand active molecules. Others stated that the clinical trial was difficult to interpret, even if expanded, because of the use of a historical control group. One should at least include a concurrent control group in order to be able to evaluate the present data. ${ }^{110}$

The critics within the NCI Board were, at least, biased in their accusation of Strander for non-conforming or dissenting behavior regarding the design of his trial. In 1975 of the 47 cancer trials published in the New England Journal of Medicine 34\% was uncontrolled, 13 historically controlled, 13 percent concurrent controls and $40 \%$ was randomized." ${ }^{\prime \prime}$ Clinical oncologists who claimed that randomization of cancer trials was neither ethical nor necessary, prevailed and at least half of the NCI-sponsored clinical trials were still non-randomized. In addition, the widely praised American chemotherapy studies in osteosarcoma patients which interfered with Strander's interferon claims were non-randomized either. Criticizing the design

107 M. Krim to K. Cantell, letter dated 11 April, 1975, Cantell personal archives.

108 Minutes of the meeting of the National Cancer Institute's National Cancer Advisory Board, 17-18 November, 1975. NCAB/NCl Archives.

Interview with Arthur Levine.

110 Minutes of the meeting of the National Cancer Institute's Division of Cancer Treatment Board of Scientific Counselors, 10, 11 November, 1975, Board of Scientific Counselors/NCI Archives.

111 G. Kolata, 'Dilemma in Cancer Treatment', Science, 209 (1980), pp. 792-4. 
of Strander's trial must have served the interests of the Randomized Clinical Trial lobby within the NCI, who were pushing the randomized clinical trial as the ultimate means of applying the scientific method to the practice of oncology. However, they could not have acted without the support of the powerful chemotherapy lobby within the NCI. Only after the NCI would begin to scale down its chemotherapy program in the late 1970's NCI statisticians began to question the usefulness of chemotherapy in osteosarcoma. ${ }^{112}$

An air of hostility developed toward Krim during the meeting. Her strenuous efforts to bring interferon to the attention of NCI officials and the recommendations made by the Interferon Working Group were heavily criticized. ${ }^{113}$ At the end of the meeting Krim was astonished to find that the board recommended that there was no compelling reason for establishing a special NCI research program, to study interferon in humans. The majority position was that before large-scale clinical testing of interferon was to begin, it would be more fruitful first to concentrate on basic in vitro and in vivo studies with interferon. The work could not be funded simply on the basis of Krim's say-so. The NCI was advised to provide adequate funding by contract and grant mechanisms only. ${ }^{114}$

The same month, Krim's report was also presented to the National Cancer Advisory Board (NCAB). According to Richard Rettig "it is appropriate to think of the NCAB as the 'board of directors' of the National Cancer Program" with rather far reaching powers regarding NCI's research agenda. ${ }^{115}$ Part of the NCAB were former members of the panel of consultants, like Mary Lasker herself. Krim might have known that, despite the negative recommendations of the Board of Scientific Counselors of the Division of Cancer Treatment with the support of the 'Laskerites', something positive would emerge.

Eventually, during the NCAB meeting, the differences in opinion between on the one hand Rabson's Interferon Working Group and Krim, and, on the other hand DeVita's Division of Cancer Treatment and Rauscher, over the kind of support that should be dedicated to interferon research, were settled in a compromise. ${ }^{116}$ The NCI would make 1 million dollars available - which amounted to little more than 0,15 percent of NCI's annual budget- through

112 T.C. Chalmers, The Clinical Trial', Milbank Memorial Fund Quarterly/ Health and Society, 41 (1972), $753-$ 8; E. Gehan and E. Freireich, Non-randomized controls in Cancer Clinical Trials', New England Journal of Medicine, 290 (1974), 198-203; and, 1. Löwy, 'Between Bench and Bedside' (Cambridge (Mass): Harvard University Press, 1996), p.59.

113 Minutes of the meeting of the National Cancer Institute's Division of Cancer Treatment Board of Scientific Counselors, 10, 11 November, 1975, Board of Scientific Counselors/NCI Archives.

114 Ibid.

115 R. A. Rettig, Cancer Crusade, (Princeton: Princeton University Press, 1977), p. 297.

116 Minutes of the meeting of the National Cancer Institute's National Cancer Advisory Board, 17-18 November. 1975, NCAB/NCI Archives. 
NIAID's Interferon Program to purchase human interferon for investigation of interferon as a possible antitumor agent. ${ }^{117}$ At the same time DeVita was granted approval to organize a site visit by a blue-ribbon panel of experts - consisting of two oncologists, two pathologists and a statistician — chaired by Arthur Levine, to evaluate Strander's data at the Karolinska Hospital in Stockholm. ${ }^{118}$ Apparently Krim had succeeded in overcoming part of the resistance within the $\mathrm{NCI}$ against supporting interferon research. It was not the massive funding Krim had asked for and the NIAID had succeeded in maintaining its key position as interferon's funding authority, but interferon for the first time had gained official recognition as a potential antitumor agent. ${ }^{119}$

In May 1976 the panel visited the Radiumhemmet. It was at Levine's insistence that Strander added to his interferon-treated patients and the historical controls, a group of contemporary ('concurrent') controls consisting of osteosarcoma patients that had been treated simultaneously by conventional means in other Swedish Hospitals. In Levine's view by using historical controls Strander ran the risk of "comparing apples and oranges". ${ }^{120}$ Levine did not appreciate Strander's defense that it was customary all over the world to employ historical controls in studies on osteosarcoma. ${ }^{121}$ The eventual site visit report was a great blow to Strander. ${ }^{122}$ The NCI panel concluded that by using a contemporary instead of a historical control group to evaluate the trial data no evidence had as yet be found that interferon treatment "significantly altered the present natural history of osteosarcoma of the long bones". ${ }^{123}$

On behalf of the taxpaying public Rauscher congratulated Levine for his potentially money-saving investigative efforts. ${ }^{124}$ In practice, however, the report hardly affected NCI's policy regarding interferon-the decision to earmark 1 million dollars for interferon research

117 A committee consisting of both NCI and NIAID officials, under the chairmanship of Alan Rabson, was installed to regulate the distribution of the $\$ 1$ million worth of interferon for use in clinical studies. Among others Tom Merigan was to receive a portion for a prospective randomized trial in patients with lymphomas suffering from shingles; Minutes of the March 8-9 and October 25-26 meetings of the Board of Scientific Counselors of the Division of Cancer Treatment of NCl, NCl Archives; 'Interferon Working Group', The Cancer Letter, 2, 49 (1976), 1; and interview with Thomas Merigan.

Minutes of the meeting of the National Cancer Institute's National Cancer Advisory Board, 17-18 November. 1975, NCAB/NCl Archives.

M. Krim to K. Cantell, letter dated 2 March 1976. Cantell personal archives.

V. DeVita to H. Strander, letter dated 15 April 1976, Strander personal archives. Karolinska Hospital Stockholm, dated 14 July 1976, Strander personal archives.

124 S. Panem and J. Vilcek, 'Will Interferon Ever Cure Cancer?'. The Atlantic. December 1982. 
was carried through. ${ }^{125}$ As it turns out, Rauscher overestimated the influence of the essentially negative site visit report and underestimated the impact of Krim's interferon lobby in combination with anecdotal media reports of the treatment's effectiveness in cancer therapy. ${ }^{126}$ In the next section I will describe how interferon succeeded in forming part of the American cancer agenda.

\subsection{Capitalizing on a growing demand for unorthodox cancer remedies.}

Following further political groundwork by Krim, a growing number of scientists and influential laymen in America lent their support to the interferon cause. Apart from the intrinsic lure to scientists and laymen alike of a natural substance that was reportedly devoid of the severe sideeffects associated with most other anti-cancer drugs and that had emerged from the mainstream of biomedical research, each social group or individual had private motives for their 'jump on the interferon bandwagon'. ${ }^{127}$

The group of American 'interferonologists' came to realize that Krim's lobbying efforts proved effective in bringing in additional research money. Due to the growing disappointment within NIAID quarters about interferon's therapeutic potential as an antiviral agent and the seemingly permanent inability to purify interferon, the field of interferon research had been facing a serious cutback in recent NIAID funding. ${ }^{128}$ Together with the increasing costs of doing research this made them shift attention to interferon-related problems for which funds seemed more readily available-interferon and cancer. ${ }^{129}$ They were swiftly followed by advocates of immunotherapy like the oncologist Jordan Gutterman, who worked at the Department of Developmental Therapeutics of the M.D. Anderson Hospital and Tumor Institute in Houston, Texas. Gutterman would become one of interferon's most avid promoters.

125 Minutes of the meeting of the National Cancer Institute's Division of Cancer Treatment Board of Scientific Counselors, 25-26 Oktober, 1976, Board of Scientific Counselors/NCI Archives; At least the site visit report might have played a role in Strander never being able to get his osteosarcoma data published in an international refereed medical journal.

S. Panem. The Interferon Crusade (Washington, D.C.: Brookings Institution, 1984), p. 21: and interview with Frank Rauscher.

S. Panem, The Interferon Crusade (Washington, D.C.: Brookings Institution, 1984).

E. De Maeyer, 'Interferon Twenty Years Later, Bull. Inst. Pasteur, 76 (1978), 303-23, p. 316.; R. Friedman, 'Interferon Research in the Red Queen's Kingdom', Arch. Pathol. 98 (1974), 73-6; R.Friedman, 'Guest Editorial: Interferon and Cancer', J. Natl. Cancer, 60 (1978), 1191-4; and interviews with Derek Burke, Joseph Sonnabend, Kari. Cantell, and George Galasso. 
Immunotherapy of cancer received considerable attention in the early seventies with claims of immuno-stimulating biological agents such as BCG. It was the promise of a cancer therapy which claimed to exert its antitumor effects naturally through a stimulation of the body's own immune system as opposed to the use of the highly toxic chemical drugs employed in conventional cancer therapy. As such it was a therapy well suited to the culturally unsettled climate of the 1970's which was characterized by the malaise associated with Vietnam, Watergate and the oil crisis, and by the growing number of Americans who began to question the nation's institutional policies regarding environment, medicine and, science and technology. The flourishing social reform movements, in particular the environmentalists and health-care reform groups were united both in their belief that man-made chemicals were harming the environment and responsible for the perceived cancer epidemic, and in their growing doubts about the Cancer Establishment and the effectiveness of the war on cancer. The members of the diverse but increasingly powerful cancer counter-culture favored unorthodox 'natural' cancer remedies. Immunotherapy seemed to fulfil their needs. ${ }^{130}$

Immunotherapy was simultaneously legitimized scientifically through both an array of laboratory researches and fundamental breakthroughs in immunological knowledge showing the importance of the cellular (non-specific) mechanisms underlying the immune responses. With the help of new immunological laboratory tools (e.g. lymphocytes) studies were performed resulting in the claim that the immune-responses of cancer patients were suppressed and should therefore be stimulated. However, by the mid-seventies, as negative clinical trial data with immuno-stimulating agents like BCG accumulated-immunotherapy and chemotherapy were not found to be superior to chemotherapy alone-interest in immunotherapy dwindled. ${ }^{131}$ As a promising antitumor substance which was claimed to be involved in one way or another in the non-specific immune response of the body, interferon was readily assimilable into a research program that was in urgent need of a new impetus. ${ }^{132}$

Introduced to interferon at Krim's workshop, Gutterman became intrigued by the possibility of using the supposedly non-toxic biological substance in cancer patients. ${ }^{133}$ Confronted with BCG's repeated failure to show a significant prolongation of remission and survival Gutterman decided to give interferon a try, although he continued to apply BCG as a

130 A. Elzinga and A. Jamison, 'Changing Policy Agendas in Science and Technology', in S. Jasanoff et all (eds.). Handbook of Science and Technology Studies (London: Sage Publications, 1995), 588-9; J. Patterson, The Dread Disease (Cambridge (MS): Harvard University Press, 1987), 256-7.

131 1. Löwy, 'Between Bench and Bedside' (Cambridge (Mass): Harvard University Press, 1996), p. 112.

132 R. Teitelman, Gene Dreams: Wallstreet, Academia and the Rise of Biotechnology (New York: Basic Books, 1989), p. 29; and, E. Richards, Vitamin C and Cancer: Medicine or Politics? (New York: St. Martin's Press. 1991), p. 207.

Interview with Jordan Gutterman. 
last experimental resort for patients unable to benefit from conventional therapy. ${ }^{134}$ Given his own expertise with the experimental combination of immunotherapy and chemotherapy of advanced breast cancer, he sent a request for interferon to the NCI to be used in a breast cancer study. ${ }^{135}$ The NCI supply, however, appeared to be limited and had to be spread among several clinical centers. Like all other applicants Gutterman was granted only a small part of what he had asked for-hardly enough to treat a mere handful of patients for a short period.

In complaining about the frustrating state of affairs to Krim he learned that she was increasingly successful in getting influential lay persons actively interested in interferon. Three prominent public figures: Mary Lasker, the 'patron-saint' of American cancer research, Laurence Rockefeller, the conservationist and member of the NCAB, and Congressman Claude Pepper, Democrat of Florida, lent their support to the interferon effort. Krim told Gutterman to inform Lasker personally of his problems. ${ }^{136}$

The commitment of these patrons of American cancer research to interferon was influenced by the growing public criticism on the National Cancer Program. Despite the additional investment of more than one billion dollars of tax-payers's money in cancer research since 1972, the American cancer establishment could only claim credit for minor progress in their self-proclaimed 'war on cancer'. This poor research performance did not mesh with the public announcements by politicians, nor with the claims of senior NCI and American Cancer Society (ACS) officials that they were about to discover a cure against cancer. More and more critics emphasized the gaps in knowledge between laboratory and bedside and aired doubts about the prospects for any sudden breakthroughs in the field of cancer therapy. ${ }^{137}$ Organized elements of the cancer counter-culture like the International Association of Cancer Victims

J. Gutterman, Immunotherapy for Recurrent Malignant Melanoma: The Efficacy of BCG in Prolonging the Postoperative Disease-free Interval and Survival, In M. Sela (ed.), The Role of Non-Specific Immunity in the Prevention and Treatment of Cancer (Rome: Pontificia Academia Scientiarum, 1977), pp. 57-65; Ilana Löwy argues that the low therapeutic efficacy of most new anticancer drugs emphasized the need for agreement on what should count as success in a clinical trial of a cancer therapy. Such agreement was achieved through the development of standardized criteria ('markers') of therapeutic success such as prolongation of life and remissions or regressions in terms of reduction of cancer volume or perceptible shrinkage of tumor mass: Ilana Löwy, 'Between Bench and Bedside' (Cambridge (Mass): Harvard University Press, 1996), pp. 61-2.

Report of the International Workshop on Interferon in the Treatment of Cancer, March 31. April 1 and 21975 New York, Sonnabend personal archives; J. U. Gutterman et al, 'Chemoimmunotherapy of Advanced Breast Cancer: Prolongation of Remission and Survival with BCG', Br. Med. J., 2 (1976) 774-7. with Mathilde Krim and Jordan Gutterman.

With the American defeat in South-East Asia, the metaphors of war began to turn against the American Cancer Establishment. "By comparison with the fight against polio", claimed a former FDA commisioner in 1978, "the war on cancer is a medical Vietnam".: Donald Kennedy quoted in J. C. Petersen and G. E. Markle, 'Expansion of conflict in cancer controversies', in L. Kriesberg (ed.), Research in Social Movements, Conflicts and Change, 4, (1981) pp. 151-69, see p. 152. 
disseminated information about alternative 'suppressed' cancer therapies and provided a platform for dissident cancer voices. They openly accused the NCI, the ACS, and virtually the entire American medical community of systematic opposition to alternative therapies, and emphasized the failures of the orthodox biomedical model and conventional cancer treatments in particular. ${ }^{138}$

The American cancer establishment was thus seriously under attack and needed a boost in the fight against cancer to satisfy public demands. As a product of scientific medicine and with its claimed naturalistic basis interferon seemed to offer the opportunity to silence the growing opposition. Moreover, individual experiences with the limitations of conventional cancer therapy played a role in their eagerness to encourage new approaches. Pepper's wife and close associates of both Rockefeller and Lasker suffered from the 'dread disease' and were not responding to conventional cancer chemotherapy. ${ }^{139}$

With the idea of fueling extra pressure for a major interferon campaign, Mary Lasker provided 1 million dollars through her Foundation for the purchase of additional interferon for Gutterman's clinical program. ${ }^{140}$ Without further delay, in October 1977, Gutterman, who had planned to attend a immunotherapy meeting in Rome, changed his travel plans and flew to Finland to make arrangements for buying interferon from the Finnish Red Cross through Cantell. Gutterman knew by experience (the previous year his request for purchasing interferon for clinical work in cancer patients had been turned down) that the relatively scarce and precious interferon material was not for sale simply to the highest bidder. ${ }^{141}$ In order to gain the maximum scientific and public impact for his human leucocyte interferon, Cantell cautiously controlled its distribution. Since the Finnish Red Cross was the only major producer of human interferon, Gutterman had no alternative (see Fig. 48) ${ }^{142}$ Not until Gutterman had given a full account of his research plans was Cantell prepared to start negotiations on the terms of a contract for the supply of interferon. They agreed on a price of 25.000 dollars by the billion units (daily dose between 1-10 million units a day) of partially purified interferon (PIF). ${ }^{143}$

138 Eveleen Richards, 'Vitamin C and Cancer; Medicine or Politics' (New York: St. Martin's Press, 1991), pp. 46-47: R. Moss, The Cancer Industry (New York: Paragon House, 1989); and, R. Proctor, Cancer Wars (New york: BasicBooks, 1995), and J. Patterson, The Dread Disease: Cancer and Modern American Culture, (Cambridge (MA): Harvard University Press, 1987), pp. 256-268.

Interview with Mathilde Krim.

S. Panem, The Interferon Crusade (Washington, D.C.: Brookings Institution, 1984), pp. 21-22.

141 J. Gutterman to K. Cantell, letter dated 8 June 1976, Cantell personal archives; K. Cantell to J. Gutterman, letter dated 26 August 1976, Cantell personal archives.

Interview with Kari Cantell.

143 In 1976 the annual Finnish production was about 100 billion units of crude interferon (C-IF) or 50 billion units (with $50 \%$ recovery) of partially purified preparations (P-IF); Report on the production of human 
In early 1978 Gutterman was able to begin treatment of patients with inoperable and recurrent malignancies. Like most American oncologists, Gutterman felt that it was vital to be aggressive in order to get a response-cancer chemotherapy was synonymous with the use of maximum-tolerated and often highly toxic doses of drugs. ${ }^{144} \mathrm{He}$ would usually push his patients to the limit of what they could bear. However, he had only just started to treat his first breast cancer patients with the primary aim to yield information on the highest tolerated dose when he and his colleagues began to witness spectacular, partial regressions of mammary tumors in five of nine treated woman for whom all treatment possibilities had been exhausted. Gutterman was elated at the response, although he realized that his study was small, lacking proper controls, and too short in duration to determine if the therapy really made a difference in prolonging life. Sudden and seemingly miraculous improvements in cancer patients did occasionally occur sometimes simply as a result of their doctors paying extra attention to them when testing a brand-new remedy. ${ }^{145}$ This could not prevent Gutterman from presenting an anecdotal report of his promising preliminary results at the March 1978 Workshop on Clinical Trials with Interferon organized by the $\mathrm{NIH}^{146}$

At the NIH meeting anecdotal reports presenting interferon as a lead in cancer therapy came from a couple of other researchers as well. Tom Merigan from Stanford University, reported encouraging shrinkage of tumors in two out of five patients with advanced nonHodgkin lymphoma and David Habif from Columbia University, claimed marked tumor regression in six out of seven women with inoperable or recurrent breast carcinoma. In accordance with Gutterman's clinical experiment both studies were short in duration and lacking a control. ${ }^{147}$ However anecdotal, the simultaneous presentation of three different

leukocyte interferon, dated 21 September 1977, Cantell personal archives: T. Merigan to K. Cantell, letter dated 8 September 1979, Cantell personal archives; and interviews with Kari Cantell and Jordan Gutterman.

In the 1950's the NCI developed guidelines for the screening of chemical agents of potential value for chemotherapy of cancer. Among other things compounds were considered active in animals when minimum standards of activity were met at the maximum-tolerated dose. According to Löwy this approach was later transferred to the clinics; Ilana Löwy, 'Between Bench and Bedside' (Cambridge (Mass): Harvard University Press, 1996), p. 55.

M. Edelhart, Interferon: The New Hope for Cancer (Reading (MA), Addison-Wesley Publishing Company. 1981), p. 36. the NIH, DMID-ARB box \# 14 .

147 In terms of formal clinical trial methodology in oncology these exploratory studies were considered 'Phase I trials. Basically these were toxicity screening studies to determine the maximum tolerated dose and a first step in the established testing trajectory for testing potential new cancer drugs (followed by the larger, longer and stricter 'Phase II' trials - screen for clinical activity - and 'Phase III' trials-determination of relative efficacy). Phase I trials also served the purpose of getting a first indication whether or not a new compound was of any clinical value in cancer therapy: Report of the NIAID/NCI workshop on Clinical Trials with Interferon, dated March 21, 22 and 23, 1978 at the NIH, DMID-ARB box \# 14: interview with Arthur 


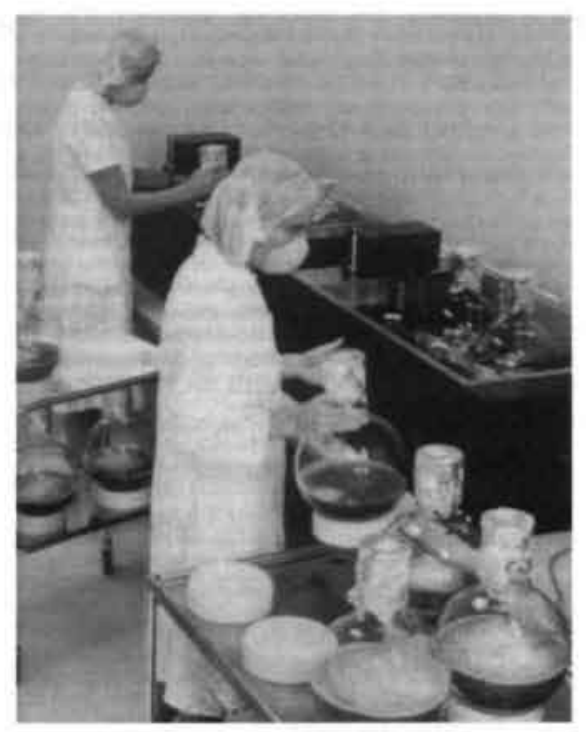

Fig. 48. The large scale production of interferon at the Finnish Red Cross Transfusion Service in the late 1970\%s. Courtesy of Dr. K. Cantell

American studies claiming that interferon produced encouraging clinical responses had an impact on the small international audience of invited interferon researchers and NIAID, NCI, and Food and Drug Administration (FDA) officials. Reduction in the size of malignant growths per se was not an unusual therapeutic effect, but interferon's ability to induce a shrinkage of advanced solid human tumors that resisted all conventional therapies was a rather uncommon phenomenon in clinical oncology.

The optimism about interferon's therapeutic potential was also shared by those involved in studying the possible efficacy of interferon as an antiviral therapy. In particular, Merigan's placebo-controlled, randomized double blind study showing that daily treatment with interferon diminished both the spread and the pain of shingles in patients with cancer, was reason for optimism. This indicated that interferon might at least turn out to be useful in controlling serious viral infections in immuno-suppressed cancer patients. There were also anecdotal reports about beneficial effects in children suffering from recurrent laryngeal papilloma-virus-induced warts that fill up the whole larynx so that the child can barely speak or breathe and can be life-threatening - for whom the only treatment available was repeated surgery. At the same time there were also less promising reports.

Levine; M. Buyse, M. Staquet and R. Sylvester (eds.), Cancer Clinical Trials (Oxford: Oxford University Press, 1984). 
The clinical data presented by Pieter de Somer from the Rega Institute in Belgium, and Huub Schellekens from the Erasmus Hospital in the Netherlands, called into question the efficacy of interferon in chronic hepatitis as claimed by Merigan's research group in $1976 .{ }^{148}$ Because it had been performed under controlled conditions, the Dutch-Belgian study would, in formal terms, have an advantage over Merigan's uncontrolled observations. However, unlike Merigan, they had used human fibroblast interferon-produced from large scale virus-infected cultures of human cells obtained from fetal tissue or the foreskin of circumcised baby boys. Since it had been established that fibroblast interferon differed from leucocyte interferon in its physico-chemical properties, it might be possible that the two types of human interferon differed in their clinical activity too. ${ }^{149}$ To Merigan and most other researchers present, giving up on what was considered one of the most promising clinical antiviral effects observed so far was not considered as being an option-hepatitis was a rather common virus infection that was held responsible for severe liver damages and for which no treatment was available. ${ }^{150}$ They argued that apparently leucocyte interferon held more promise for treatment of hepatitis than did fibroblast interferon. ${ }^{151}$

When De Somer brought up next the question of side-effects the atmosphere threatened to become rather tense. The year before at the international interferon meeting in Israel De Somer had caused a wave of indignation among his fellow interferonologists by openly criticizing the non-toxicity dogma. ${ }^{152}$ Although one of the principal virtues of interferon

148 H. Greenberg, M. Richard, R. Pollard, L Lutwick, P. Gregory, W. Robinson and T. Merigan, 'Effect of Human Leucocyte Interferon on Hepatitis B Virus Infection in Patients with Chronic Active Hepatitis', The New England Journal of Medicine, 295 (1976), 517-22.

K. Fantes, 'Purification and Physico-chemical Properties of Interferons', in N. Finter (ed.), Interferons and Interferon Inducers (Amsterdam, North Holland Publishing Company, 1973), pp. 171-99; and, J. O'Malley and W. A. Carter. Human Interferons: Characterization of the Major Molecular Components', Journal of the ReticuloEndothelial Society, 23 (1978) 299-305.

US federal researchers estimated that there were approximately 200.000 hepatitis carriers in the United States and worldwide some 100 million people. With no cure available it was considered an important research area by NIAID officials, although the escalating hepatitis epidemic among gay men was still non-existent as far as the NIAID was concerned; L. Garrett, The Coming Plague (New York: Farrar, Straus and Giroux, 1994), p. 273; and, D. Burke, 'The Status of Interferon', Scientific American, 236 (1977), 42-50.

Report of the NIAID/NCI workshop on Clinical Trials with Interferon, dated March 21, 22 and 23, 1978 at the NIH, DMID-ARB box \# 14 .

S. Panem. The Interferon Crusade (Washington, D.C.: Brookings Institution, 1984), p. 30; interview with Alfons Billiau, interview; In 1976 Gresser already submitted an article to Science concerning the question of kidney liver and spleen toxicity and disease causation by interferon in mice. The paper was rejected because of insufficient evidence that the toxic effects were related to interferon and not to impurities in the preparations. "...without data to associate a specific material in the preparation with the progressive glomerulonephritis (kidney toxicity), the findings presented are really only a starting point to isolate the cause...": 1 . Gresser to K. Cantell, letter with enclosed Science referee comment dated 22 September 1976, K. Cantell personal archives. 
was claimed to be its lack of toxicity, all interferon preparations tested so far were known to produce side-effects. Most patients receiving interferon treatment developed transient flu-like symptoms such as fever, chills, fatigue and loss of appetite, and in certain cases after prolonged administration, some hair loss. The reversible side-effects were never taken very serious as most of the clinical researchers involved were used to the much more severe toxic side-effects arising from most other forms of viral and cancer chemotherapy. Moreover, since it appeared that the effects diminished when using more purified preparations, the consensus was that the side-effects were not due to the interferon molecule but rather to one or more impurities.

Few dissenters could be heard until 1977 when De Somer reported the observation of serious side-effects following administration of interferon in a Belgian patient. In particular, the fact that De Somer indicated that the side-effects may well be related to the interferon molecule itself, was difficult to swallow for his audience. His remarks did more than violate the non-toxicity dogma in the field of interferon research. The response to this news "by some was that such negative results should not be made generally public because they might dampen popular enthusiasm for interferon and stall the interferon crusade just as it was gaining momentum". ${ }^{153}$

To the relief of the 1978 NIH workshop invitees, De Somer did not show up with yet another dramatic patient history. ${ }^{154}$ The side-effect issue hardly affected the final assessment of interferon as a promising therapeutic substance that justified the testing on larger numbers of patients under controlled conditions. However, obtaining interferon for a major study required millions of dollars and, with the risk of ending up with just another bogus cancer treatment, $\mathrm{NCI}$ officials remained hesitant to commit themselves to interferon. Gutterman did not want to wait for a change in NCI policy to come about and turned to the wealthy American Cancer Society (ACS) for help. The ACS was known to administer a special fund of 5 million dollar for cancer research aimed at financing "best bets" that might produce benefits to the cancer patient as quickly as possible. ${ }^{155}$ Encouraged by Lasker, Gutterman wrote up a daring proposal for a large test program on more than 100 patients with a variety of tumors, including metastatic breast cancer.

Once again Frank Rauscher, who had resigned as director of the NCI to become the ACS's senior vice-president for research, was confronted with a request for massive funding of

153 S. Panem, The Interferon Crusade (Washington, D.C.: Brookings Institution, 1984), p. 30.

154 Report of the NIAID/NCI workshop on Clinical Trials with Interferon, dated March 21, 22 and 23, 1978 at the NIH, DMID-ARB box \# 14 .

155. The ACS is one of the biggest fund-raising organizations in the US, which by relying on 2.5 million volunteers raised $\$ 85$ million in 1979, plus an additional \$35 million from legacies; J. Patterson. The Dread Disease; Cancer and Modern Culture (Cambridge (MA), Harvard University Press, 1987), pp. 268-9; and interview with Frank Rauscher. 
interferon research. In his previous post at the NCI Rauscher had opposed to a similar kind of request but that was in 1975 when Krim's interferon crusade still had to gain momentum. Since then political support for a major interferon campaign had been building up rapidly. Furthermore, the managing staff of the American Cancer Society believed they were making not enough progress in the treatment of cancer to satisfy public demand. This played a role in Rauscher reconsidering the matter. ${ }^{156}$

Late in July 1978 a scientific advisory panel, consisting for the greater part of interferon supporters reviewed the application. On the panel's recommendation Rauscher and the ACS Board of Directors quickly approved a 2 million dollar grant for clinical testing of interferon. Rauscher immediately appointed an ad-hoc committee of clinical researchers under the co-chairmanship of Gutterman and Merigan to work out protocol details, such as the selection of research institutions, patients and types of cancer. Scarcity and costs of the interferon material was believed to limit the trials to no more than 150 advanced cancer patients, who for reasons of validity would be randomly selected. The committee sent the experimental protocols for approval to the FDA. ${ }^{157}$

On 30 August the ACS publicly announced its plans to spend for the clinical testing of interferon the largest sum ever committed by the Society to a single project. This fact was obviously not lost on the media as the press release triggered a wave of interferon related publicity with headlines such as 'Interferon: The cancer drug we have ignored' or 'New cancer weapon?' and 'Interferon: No miracles without more molecules'. ${ }^{158}$ Together with additional interest shown by radio and television networks this brought, as we will see in the next section, interferon out of the relative seclusion of the laboratory into the limelight of public attention.

\subsection{Interferon, scientists and the media}

The portrait conveyed most often and most vividly in the mass media showed interferon as a somewhat mysterious, clinically unharnessed, non-toxic natural body substance, that was claimed to be the hottest, though long ignored-by a conservative cancer establishment-line of biomedical research currently being followed. The idea generated by the media was, that if only enough of this extremely scarce and expensive naturally occurring protein could be made

Interviews with Mathilde Krim, Jordan Gutterman and Frank Rauscher.

K. Krim to I. Gresser, letter dated 31 August 1978, I. Gresser personal archives; M. Edelhart, Interferon: The New Hope for Cancer (Reading (MA): Addison Wesley, 1981), 38-9.

'New Cancer Weapon?, Newsweek, 18 September 1978, pp. 90-1; J. Hixson, 'Interferon: The Cancer Drug We Have Ignored', The New Yorker, 4 September 1978, pp 59-64; K. White, Interferon: No Miracles Without More Molecules', Medical Tribune, 18 October 1978. 
available by scientists, a kind of miracle cure for everything from the dread disease cancer to the common cold was at hand. The double framing of interferon as a natural solution to a dread disease and as the product of 'cutting-edge' biomedical research was reinforced by the illustrations employed in the media. A case in point was a photograph in Newsweek with the subscript "searching for the natural key to interferon" showing scientists in laboratory coats staring hopeful at a sophisticated laboratory set-up composed of a tangle of wires, tubes, retorts and graduated cylinders filled with fluids, seeing things only they were supposed to recognize (see Fig. 49). ${ }^{159}$

Except for the excessive elements in the public media, the public image of interferon as a promising product of a laboratory-supported scientific medicine was not dissimilar to the associations and legitimations presented by interferon researchers to their scientific audience - continually linking state-of-the-art basic research with achieving future cures at the bedside. ${ }^{160}$ Moreover, the growing interaction between the field of interferon research and the fields of immunology (with the conceptualization of the body as a complex system) and molecular biology (with the conceptualization of the body as a system of networks that process information), helped to establish and elaborate on the image of interferon as part of a complex system held together by communication and feedback. ${ }^{161}$ This is nicely illustrated in the epilogue of the textbook The interferon system where the interferon researcher William Stewart II described the action of interferon in the following terms: "Interferon is only one of a large number of products by which cells communicate. It is as though we have learned only one of their words and pretend to speak their language". ${ }^{162}$

Similar type of information metaphors were also increasingly employed to picture interferon in the media, mostly in combination with the usual military metaphors (like bullet, hunt, war and weapon). As such the media reinforced the interferon as-part-of-a-network image. For instance, the journalist Joseph Hixson depicted in the trendy New Yorker, the body as a regulatory communications network that is continuously in the process of distinguishing between what "is 'self' and what is 'foreign' and thus to be destroyed" such as viruses and

'New Cancer Weapon?', Newsweek, 18 September 1978, pp. 90-I.

160

Discussing the interleukine-2 hype in the mid 1980's Ilana Löwy noted similar connections: 1. Löwy, 'Between Bench and Bedside' (Cambridge (Mass): Harvard University Press, 1996). p. 158.

See for extensive analyses of the rise of the information metaphor in molecular biology, immunology and medicine; E. Fox-Keller, Refiguring Life (New York: Columbia Universituy Press, 1995); and, E. Martin. Flexible Bodies (Boston: Beacon Press, 1994). 
tumors. ${ }^{16.3}$ Interferon was presented as one of the intercellular messengers (using a superman visual image) which emerge whenever what is foreign manifests itself and trigger the bodily defense forces (see Fig. 50a, b). About the same time, the reporter of the Medical Tribune noted that interferon was exemplary for a new generation of therapeutic drugs which speak to the body in their "native biochemical language". Simultaneously she pictured interferon as a sophisticated product of modern science: "The difference between interferon and less refined medical approaches is the difference between the demanding precision of Obi-Wan Kenobe's high-energy laser sword, and the clumsy but often effective brute force of a tank". ${ }^{164}$

Frequently linking interferon with advanced science and technology directly shaped arguments concerning the question of interferon's exploitation. The deployment of the new genetic engineering technology as a means to deal with the problems of interferon production and purification became a vital rhetorical strategy in the interferon crusade. The high public visibility and the scarcity of interferon as a potential new miracle drug in turn whetted the appetite of the newly established genetic engineering firms which were looking for feasible demonstration projects to lure public investments.

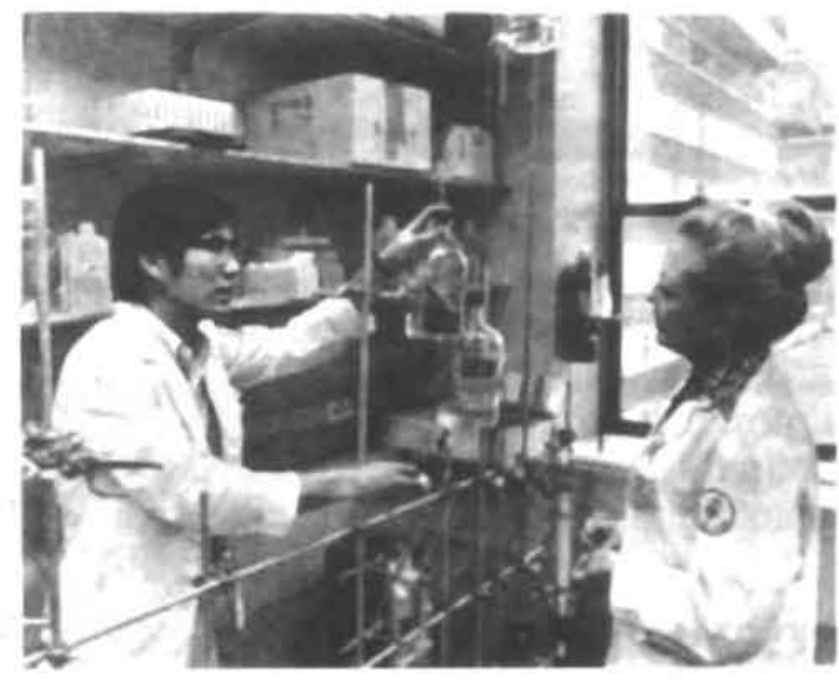

Fig. 49. Mathilde Krim and co-worker in their Laboratory at New York's

Memorial Sloan-Kettering Cancer Center (1978).

Reproduced with kind permission frpm the publisher.

163 J. Hixson, 'Interferon: The Cancer Drug We Have Ignored', The New Yorker, 4 September 1978, p. 59.

164 K. White, 'Interferon's Future: For Common Colds. Transplants?, Medical Tribune (US), 10 November 1978. 


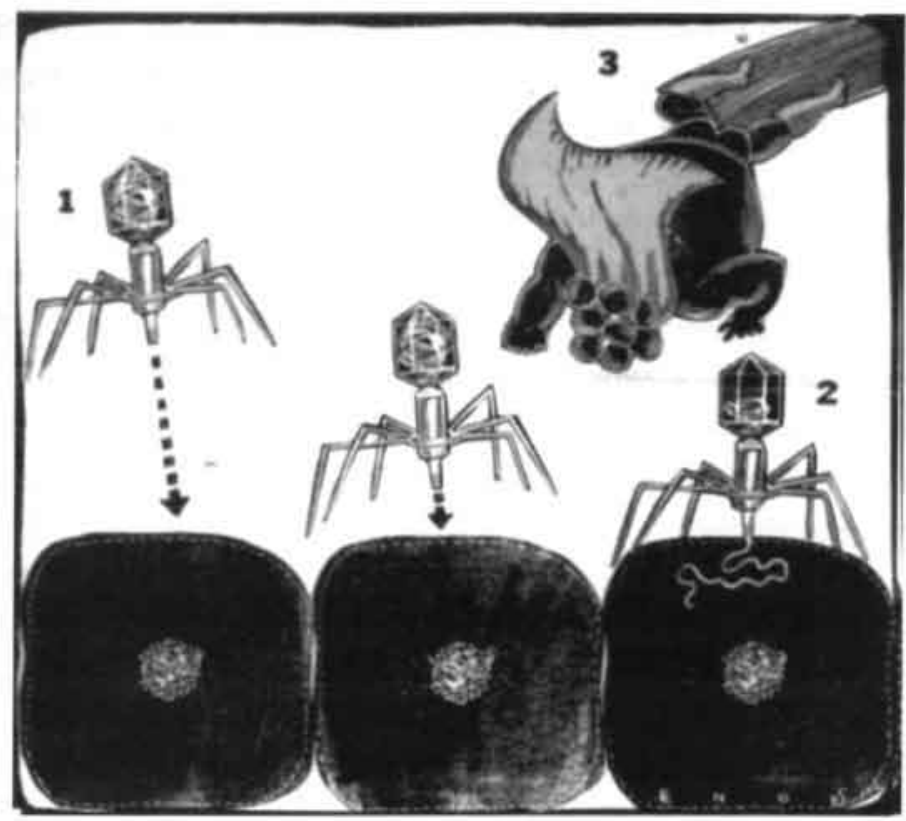

Fig. 50a. "The alert: Invading viruses (1) approach and release nucleic acid-a viral replicative blueprint-into

the cells (2). The body responds

by producing intercellular messengers,

giant protein molecules called 'interferon' (3)."

Reproduced with kind permission of the Publisher.

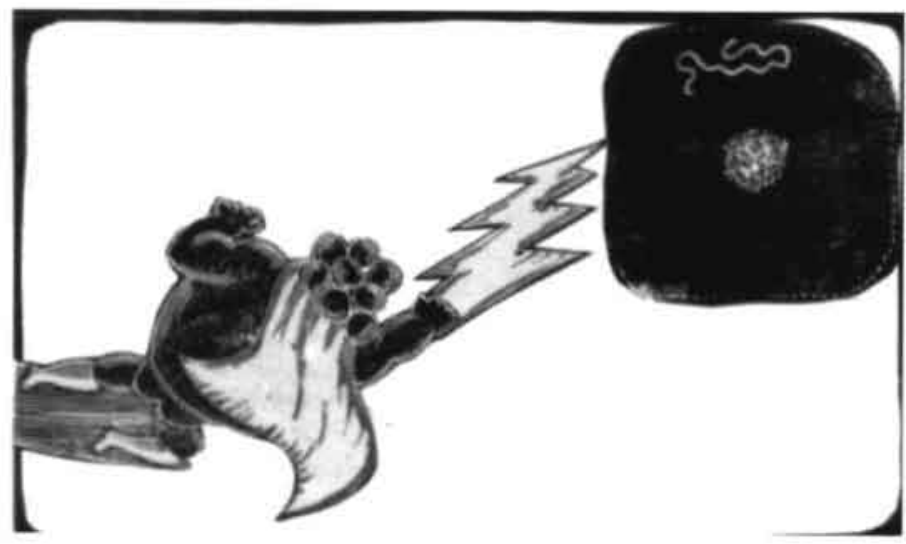

Fig 50b. "All systems go: Interferon triggers a cellular anti-viral mechanism. Reproduced with kind permission of the Publisher. 
The genetic engineers used techniques (known as 'recombinant-DNA' or 'rDNA' technology) developed in the late 1960 's and early 1970 's which allowed them to copy or 'clone' human genes by slipping them into bacteria or other micro-organisms. Around 1977-as soon as the first signs were there that the heyday of public DNA furore was over and the regulatory climate was slowly improving - the cloning and expression of genes became the object of intense research activity in the United States and Europe. The perceived allure of the new technology was that medically important, but mostly scarce and costly, human and animal proteins could be produced by the genetically engineered bacteria in unlimited quantities at modest cost. ${ }^{165}$

Confirmation of the feasibility of the commercial applications of rDNA techniques came in the fall of 1977 when American scientists achieved the first complete bacterial synthesis of an animal protein, the small peptide hormone, somatostatin, produced in the human brain. Following the somatostatin experiment a growing number of genetic boutiques were established by venture capitalists anticipating new industrial applications of rDNA techniques with the active participation of a new generation of entrepreneurial scientists in academia who were at the forefront of molecular biology. By 1978, several private companies had been formed in the field of genetic engineering. The new firms, including Cetus, Genentech (in California) and Genex (in Maryland), and the Swiss-based Biogen, were eager to prove the commercial worth of the gene-splicing technology.

There were several reasons why the cloning of interferon was considered as an attractive demonstration project that could serve this purpose. ${ }^{166}$ First, with public expectations running high, Wallstreet's capital investers would be eagerly waiting to pour money into interferon related commercial projects. Second, the production of interferon by conventional means was still beset with enormous problems after twenty years of hard labour: the scant amounts of interferon that were produced worldwide were impure and costly. It was the most expensive experimental drug ever used in clinical trials in the USA. Once you had succeeded in isolating the human interferon gene and splicing it into bacteria, the microbes were claimed to do two jobs for the price of one: they produced human interferon in large quantities and in a form (as a single protein product) that could be purified with far greater ease than for instance

See, for detailed social histories of the recombinant DNA debate, the development of international regulatory policy for genetic engineering and the subsequent rise of industrial genetics; S. Krimsky, Genetic Alchemy (Cambridge (MA): The MIT Press, 1982); S. Wright, Molecular Politics (Chicago: The University of Chicago Press, 1994): S. Krimsky, Biotechnics and Society (New York: Praeger, 1991); R. Teitelman, Gene Dreams (New York: BasicBooks, 1989).

According to Panem only a few other proteins like insuline, growth hormone and several proteins identified for use as human vaccines, qualified for a demonstration project; S. Panem. The Interferon Crusade (Washington, D.C.: Brookings Institution, 1984), p 26. 
Cantell's protein soup. ${ }^{167}$ Third, there was the technical challenge of isolating and cloning a gene coding for a protein that still awaited chemical identification. ${ }^{168}$

The claims by genetic engineering firms and leading molecular biologists about the imminent possibility of making available, in large quantities, at modest cost, substances that were expensive or difficult to make such as interferon, and the resulting imaginative race to clone the first human interferon gene, intensified public interest. ${ }^{169}$ Krim seized the opportunity and worked to use the Second International Workshop on Interferons, which like the first, was held at the Sloan Kettering Memorial Cancer Center in New York, as a podium to the outside world: she made it a media event. ${ }^{170}$

\subsubsection{Gene dreams and the inflation of expectations}

Beside the 'old' group of interferonologists and representatives of the ACS, NCI, FDA, the press, the by-invitation-only gathering was attended by about 300 molecular geneticists, immunologists, biochemists and clinicians, ranging from infectious-disease specialists, clinical oncologists to transplant surgeons. In addition more than fifty representatives of the genetic boutiques and the large established pharmaceutical companies were present. Besides Burroughs Wellcome and Merck Sharpe \& Dohme, also relative newcomers showed interest like Hoffmann-La Roche ('Roche'), the Schering-Plough Corporation ('Schering-Plough') and Bristol-Myers Company. The latter had entered into commercial contracts with respectively Genentech, Biogen and Genex not only as a means to develop interferon as a therapeutic drug but also to acquire new technical expertise that could pay off even if in the end interferon itself might never find a big commercial market. ${ }^{171}$ The growing involvement of the drug industry

S. Hall, A Commotion in the Blood (New York: Henty Hold and Company, 1997), p. 184.

Weissman, interview; See, for a detailed personal account of the cloning of the gene for leucocyte interferon, C. Weissman, The Cloning of Interferon and Other Mistakes', in I. Gresser (ed.), Interferon 3 (London, Academic Press, 1981), pp. 101-134.

The public announcement, early in September 1978, of the cloning of the first medically significant human gene to make human insulin hit the headlines and was said to open up a new and most exciting era in biology, only added to the public interest for interferon: Victor Cohn, 'Scientists in California Create Gene to Make Human Insuline', 7 September 1978, The Washington Post.

Interviews with Mathilde Krim, Joseph Sonnabend and Thomas Merigan.

171 The large drug companies had begun investing in genetic technology with the objective of protecting products and markets from being undermined by the new technology and out of economical need for new growth and above-average returns on investment; See, for a detailed account of dynamics of the industrial interest in interferon: S. Panem, The Interferon Crusade (Washington, D.C.: Brookings Institution, 1984), pp. 25-8, 5874. 
and the major molecular biology laboratories that became manifest at this meeting marked the move of interferon research to 'big science', involving large-scale research programs. The 'small fish' (interferonologists) who had taken on this line of investigation were increasingly eclipsed by the team-led efforts of the leading industrial and academic research centers. Furthermore, the intense industrial interest in interferon began to affect the relatively free exchange of research information and materials in the field of interferon research. Certain previously accepted norms of openness and exchange were no longer matter-of-course with the development of commercially motivated forms of secrecy within the research community. ${ }^{172}$

In contrast to the 1975 Krim meeting, her second workshop went largely without dissenting voices being heard and had the effect desired by Krim. It amplified the wave of public enthusiasm regarding interferon. Without going into particulars, it is worth looking at some of the latest data and concepts which were being presented, discussed or rumored over the three-day period in New York and were particularly helpful in stirring the imagination of the media and public at large. ${ }^{173}$

After more than twenty years of scientific 'go slow' regarding the purification and characterization of interferon, spectacular advances were being reported by biochemists employing new sophisticated laboratory techniques like high-performance liquid chromatography (HPLC) and the so-called 'SDS polyacrylamide' gel electrophoresis. In fierce competition a number of research groups not only claimed to have purified several different human interferons to homogeneity but also to have determined their amino acid composition. However, the chemical identity (or what many scientists still used to call "lack of identity") of these proteins in terms of amino acid sequence was still the subject of considerable speculation. ${ }^{174}$ At least the biochemical data strongly suggested the possibility that they were

172 See, for a thorough analysis of the changing nature of the flow of research information and materials in interferon research due to the growing interference between academic and industrial research activities: $\mathbf{S}$. Panem, The Interferon Crusade (Washington, D.C.: The Brookings Institution, 1984), pp. 75-81.

173 The following impressionistic account of the Second International Workshop on Interferons, April 22-24, 1979 at the Sloan Kettering Memorial Cancer Center in New York is based on: Anonymous, Can interferons cure cancer? Lancet, i (1979), 1171-72; T. Merigan, Human Interferon as a Therapeutic Agent, New. Engl. J. Med., 300 (1979), 42-3; K. Cantell, 'Why is Interferon not in Clinical Use Today?', in I. Gresser (ed.), Interferon, (London: Academic Press, 1979), pp. 2-28; J. L. Marx. Interferon (I): On the Threshold of Clinical Application, Science, 204 (1979), 1183-6; J.L. Marx, Interferon (II): Learning About How it Works, Science, 204 (1979), 1293-95; A. Rosenfeld, 'If IF works it could...' July, 1979, Life, 55-62; J. Gutterman, e.a. Leucocyte-interferon-induced regression in human metastatic breast cancer, multiple myeloma and malignant lymphoma. Ann. Int. Med. 93 (1980), 399-406; F. Balkwill. What Future for the Interferons?. New Scientist. (1980), 230-2; and interviews with Sam Baron, Kari Cantell, Norman Finter, Robert Friedman, Mathilde Krim, and Frank Rauscher.

174. The fact that interferon's chemical formula and structure had remained a mystery for more than 20 years is not exceptional in the history biochemistry. We only have to compare it with the more than 30 years it cost to produce insulin's or penicillin's chemical structure; M. Bliss. The Discovery of Insulin (London: Faber and Faber, 1982); G. L. Hobby, Penicillin: Meeting the Challenge (New Haven: Yale University Press, 1985). 
investigating a family of proteins. More than three different molecular forms of human interferon had reportedly been characterized. This, in combination with the fear of the 'oldtimers' of interferon research to lose control of a field of research that was overwhelmed by an invasion of immunologists, molecular biologists, tumor biologists and clinicians unfamiliar with their means of communicating interferon research, provided an important incentive to devise a new system for their nomenclature. ${ }^{175}$

As of March 1980 the interferon research community would decide to abolish the old terminology of Type I ('leucocyte' and 'fibroblast') and Type II ('immune') interferons in favor of the type designations alpha, beta and gamma. Alpha and beta corresponded to what always had been called Type I interferons, but helped to make explicit the knowledge that leucocyte and fibroblast cells could each produce different sorts of interferons. The new naming also enabled them to react swiftly to future claims of new interferon-like factors that were different from current types by adding sequentially a delta, epsilon etc. ${ }^{176}$

Optimism was also expressed with regard to meeting the challenge of producing more and cheaper interferon. ${ }^{177}$ Rumors abounded that several 'gene splicing' groups were on the verge of cloning and expressing the human interferon gene in the bacterium Escherichia coli ('E-coli'). ${ }^{178}$ If successful, this was thought to open the way for producing unlimited quantities of relatively inexpensive human interferon. At the same time, researchers from Wellcome Research Laboratories had reportedly succeeded in piloting an industrial process based on conventional fermenter technology for the mass production of human interferon from continuous cultures of a specific line of lymphoblastoid cells, so-called Namalwa cells. ${ }^{179}$ After

Interviews with Charles Chany and Kathy Zoon.

176 Stewart et al, 'Interferon Nomenclature: Report from the Committee on Interferon Nomenclature', In Gresser (ed.) Interferon 2 (London: Academic Press, 1980), pp 97-9.

In 1979 virtually all the human interferon available worldwide was still supplied by the Finnish Red Cross. Their production facility had an annual production output of $2.5 \times 10^{11}$ standard interferon units (roughly $1 \mathrm{~g}$ of pure human leucocyte interferon), using leucocytes from 90.000 Finnish blood donors. The production costs amounted to about 5 to 10 million dollars per gram. The Finnish supply of partially purified interferon preparations (P-IF, containing 10-20 million units per milliliter) only sufficed for annually enlisting 200 patients with neoplastic or other chronic diseases (employing a daily dose of $3 \times 10^{6}$ standard interferon units) in clinical trials; Cantell, interview.

178 E-coli or 'the common lab's workhorse' was and still is widely used in laboratory experiments for bacteriological and genetic studies. The bacteria originate from the intestines of humans and many animals; and interview with Charles Weissman.

179 This particular human lymphoblastoid cell-line originated from a young Ugandan girl named Namalwa who suffered from a fatal kind of African lymphoma (a Burkitt's tumor). A piece of her tumor taken for diagnostic purposes in the early seventies was sent to and stored in George Klein's laboratory for tumor biology at the Karolinska Institute. Strander and Cantell had figured out that somehow this particular cell-line not only grew readily under laboratory conditions but also yieided high amounts of human interferon when induced with Sendai virus. With their help the Wellcome researcher Norman Finter had been able to obtain a sample of the Namalwa cell-line. Despite the knowledge that in the early 1960's the use of malignant cell lines for the 
initial concerns about product safety of this interferon prepared from a human cancer cell line, regulatory authorities in Britain and America were expected to grant permission to start limited clinical trials with this kind of interferon. ${ }^{180}$ Apparently the Wellcome interferon closely resembled the common leucocyte interferon in chemical and immunological properties.

Notwithstanding the fact that the ACS trial had only just started there were further anecdotal reports about promising results with interferon in viral and malignant disease. Warnings that none of the trials was large enough or suitably designed, and that the interferon preparations had more side-effects than initially expected, could not prevent most participants from becoming excited about the long-range possibilities of interferon both as antiviral and antitumor agent. According to one of the clinical researchers present it might turn out to be one of the first natural products for use in human medicine, with an effect potentially as great as that of the corticosteroids. The whole thing might still be dubious - iffy'-but it was a promising topic, if only one looked at the impressive number of laboratory data that had been amassed regarding the molecular biology of the interferon system.

It is interesting to note that the question whether or not the bewildering array of effects (cell growth inhibition, induction of a resistance to virus infections and regulation of immune responses) could be attributed to one molecule, no longer aroused fierce debate. The once popular argument that effects different from the antiviral activity were most likely due to contaminants in the preparation was no longer taken seriously after several 'pure' interferons had been tested in vitro and in vivo. Instead of the 'antiviral versus antitumor' type of argument, the idea was gaining ground that the many diverse effects of the interferons were related through common initial sites of action. No longer interferons were regarded as specific

production of vaccines had been forbidden for safety reasons- the risk that one or the other residual biological agent in the preparation might induce cancer was considered too high-the Wellcome team had given it a try, but only after overcoming fierce opposition from management circles. Finter was able to convince them that by employing state of the art purification and detection techniques- to get rid of all suspicious biological material and in particular genetic material-and performing extensive animal safety testing he would be able to persuade the regulatory authorities into accepting the final interferon product. The doubts about the high investments associated with the safety aspects were taken away by pointing at the fact that the lymphoblastoid cells had the great practical and commercial advantage over leukocyte cells that they could be grown in continuous cultures on a very large scale using conventional and relatively cheap fermentor technology. It turned out to be a lucky venture; Interviews with Kari Cantell Norman Finter, and John Beale.

Important in the change in attitude toward the use of transformed (tumerous) cells in the production of a biologic like interferon, was the consensus achieved at the 1978 meeting at Lake Placid sponsored by the United States Bureau of Biologics of the FDA that if such materials could be made nucleic-acid free cautious initiation of limited trial in humans was warranted. Wellcome indeed claimed their interferon material to be nucleic-acid free; and, T. Merigan, 'Human Interferon as a Therapeutic Agent, The New England Journal of Medicine, 300 (1979), 42-3. 
viral inhibitory substances but as a kind of cell mediators or regulators, thought to modify and mediate cell functions in various ways through mechanisms similar to those described for the hormones, probably as part of the non-specific host defense system.

It was becoming fashionable to speak about interferon in terms of a prototype of a 'new' class of hormone-like cell regulatory proteins, biological response modifiers, which were known to immunologists under the name 'cytokines'. In providing a rationale both for interferon's versatile action in the test-tube and the patient, this concept of interferon had an irresistible appeal to laboratory and clinical researchers alike. On the one hand, this concept fitted the regular biomedical framework of drug/receptor interaction, despite the stated difference between the polypractic interferon therapy and the 'magic bullet' oriented conventional cancer chemotherapy. On the other hand it was considered a breakthrough that promised to open up new fields of research and therapy.

The implied claims of a potential medical breakthrough regarding both virus and cancer therapy at the New York meeting signaled yet another wave of public enthusiasm regarding interferon. The popular media gave enthusiastic coverage of the potential of the 'natural drug' interferon with dramatic lead paragraphs such as "If IF works it could ...be a vital weapon in the battle against cancer, protect against viral and bacterial disease, provide a cure for shingles, rabies, chicken pox, eye infections and prevent the common cold" or "Interferon: the promising new drug against cancer". ${ }^{181}$ The interferon story had the drama of life and death, the horror of dying patients, the awesome picture of decades of obscure and difficult research, the dedication and persistence of a handful of scientists to produce minute amounts of a potential life-saver. It had all the ingredients that journalists love to write and talk about.

Encouraged by Lasker, who more than ever before was putting all her political weight behind interferon, Krim gave numerous interviews to newspapers and magazines appeared on radio and TV programs, and she testified at the hearings on new developments in cancer treatment before the House Select Committee on Aging in June 1979. ${ }^{182}$ Instead of committing herself completely to interferon she presented interferon as a prototype of a new form of cancer therapy that should be viewed as supplementing conventional treatments. This was in line with her newly acquired status as member of NCI's Subcommittee on Biological Response Modifiers that was set up to develop a comprehensive research program aimed at developing a new generation of therapeutic drugs for the treatment of cancer. This so-called 'Biological Response Modifier (BRM)' program resulted from a compromise between interferon supporters who lobbied hard for a special interferon program aimed at purchasing as much interferon as possible and study its efficacy as a cancer therapy and opponents within the NCI

181 A. Rosenfeld, If IF works it could..., Life Magazine, July 1979, p. 55; and F. Hauptfuhrer, Will interferon kill cancer? Finnish Dr. Kari Cantell is helping the world find out, People (US weekly), 2 July 1979. 
who resisted against funding a short-term interferon-only-program. ${ }^{183}$

Krim's statement in American Congress reflected her indisputable political talents:

I want to comment on how we foresee, today, interferon's place in the medicine of tomorrow. We believe that as important as it may become, it is going to be one among many other biologicals used.... We do not think that, in the treatment of cancer, interferon will be a panacea, the cure all. Rather, it is likely to be most useful in conjunction with other treatments, such as surgery, chemotherapy and radiation therapy... ${ }^{184}$

There was a risk that temporary setbacks in work on interferon might put a premature end to research support. Obviously, Krim wanted to reduce that risk by presenting work on interferon not as a purpose in itself but as part of a new therapeutic approach that would help to constitute a medicine of tomorrow. Having said this I have to add that at the same time Krim was not scrupulous about promoting interferon on its own merits in discounting undesirable effects produced by the administration of interferon. She presented them as temporary shortcomings, though she did not go as far as Gutterman who boldly stated:

Interferon opens up a new form of cancer treatment which is non-toxic. There is no nausea, no vomiting, no diarrhoea, or the other side-effects of chemotherapy...It is the most promising cancer lead we have"185

The ends seemed to justify the means. By wittingly suppressing available information on interferon's side-effects-responses (in general reversible) as observed during interferon treatment at the M.D. Anderson Hospital and Tumor Institute included fever, nausea, vomiting, lassitude, hairloss and bone marrow suppression (which, though less severe in nature, resembled those experienced during conventional chemotherapy)-Krim and Gutterman succeeded in further fueling the enthusiasm for interferon and mobilizing public and private funds. $^{186}$

183 Minutes of the October 16-17, 1978 Meeting of the NCTs Division of Cancer Treatment Board of Scientific Counselors, NCI Archives Volume 1.

Pepper Committee Hearings, The Cancer Letter, dated 29 June 1979.

Pepper Committee Hearings, The Cancer Letter, dated 29 June 1979; and, NCI Memorandum, dated 21 June 1979. NCl Archives D-7906-3418.

Gutterman should have known better considering the paragraph on toxicity in his September 1980 article dealing with interferon therapy. His non-toxicity claim is all the more remarkable when taking into account Constance McAdam's article (she was at the time associate director of nursing at the M.D. Anderson Hospital and Tumor Institute) in the American Journal of Nursing. She indicated that because of it's side-effects patients receiving interferon required serious nursing assessment and close monitoring. Krim's silence is also surprising when taking into account the following remark by Krim in a letter to Cantell. "Dr Oettgen has started treating some patients at our hospital.....Altough he started at doses 10 times less than Levy and Arthur Levine (remember?) say can be tolerated, he already found severe toxicity, particularly immediate very high fever. He had to discontinue one patient"; K. Krim to K. Cantell, letter dated 21 May 1979. Cantell 
Following the hearings, the American Congress awarded additional funds to the $\mathrm{NCI}$ in total 13.5 million dollar (about 3 percent of the annual NCI budget), specifically earmarked for the BRM program but only on the condition that it would focus attention on interferon. ${ }^{187}$ The news that the $\mathrm{NCI}$ was going to pour millions of dollars into interferon research and production, and simultaneous public claims by Gutterman that $50 \%$ of his cancer patients showed remissions after interferon treatment, snowballed the flow of interferon-related publicity: "A wonder drug in the making", "Potent protein; Medical researchers say the drug interferon holds great promise", "Interferon: The body's own wonder drug" and "Race is on for miracle drug". ${ }^{188}$

Despite the sobering facts which underlaid the bold headlines, the frequent associations of interferon with terms like 'medical breakthrough', 'cancer', 'wonder drug', and 'panacea' built the foundation for a global interferon mania. In addition, the fact that interferon was extremely hard to come by and costly, and the knowledge that no other biological substance could match interferon's extraordinary biological activity, all encouraged the popular belief that interferon must be highly effective. Most of these descriptions in the media were directly or indirectly inspired by optimistic statements by interferon researchers, though they did their best to pepper their public declarations with 'if, 'could' and 'might'.

The race to clone the interferon gene was fascinating in its own right; not because it was one of the first medically significant human genes to be cloned nor because it proved that genetic engineering had passed, as the WallStreet Journal put it, "from science-fiction fantasy to fact" ${ }^{189}$ Rather, it was the metaphor of a race in combination with both the promises of a new and wondrous production technology and of a billion-dollar miracle molecule, that made interferon so fascinating for the general public. ${ }^{190}$

personal archives; C. McAdams, Interferon; The Penicillin of the Future?, American Journal of Nursing, April 1980, pp. 714-7; J. Gutterman et al, 'Leukocyte Interferon-induced Tumore Regression in Human Metastatic Breast Cancer, Multiple Myeloma, and Malignant Lymphoma', Annals of Internal Medicine, 93 (1980), pp. 399-406, p. 402.

Congressional inquiry received and answered by phone relating to interferon, NCI Archives No. 0078032816: In 1980 and ' 81,80 percent of the BRMP funds would be allocated for studies of interferon; In Background on Interferon, ACS News Service, NCl Archives No. 0000-001171; S. Panem, The Interferon Crusade (Washington, D.C.: Brookings Institution, 1984), p. 52.

'Interferon: The Body's Own Wonder Drug', Saturday Review, 13 October 1979; 'A Wonder Drug in the Making', Business Week, 19 November 1979; Hal Lancaster, 'Potent Protein; Medical Researchers Say the Drug Interferon Holds Great Promise'. The Wallstreet Journal, 6 december, 1979; The Race is on for Miracle Drug', The Observer, 30 December 1979; 'Interferon Trial: Early Results Promising', Medical World News. 10 December 1979. p.27. Wallstreet Journal, 6 december, 1979. 
The euphoria reached a peak after Biogen and Schering Plough announced at a joint press conference at the Park Plaza Hotel in Boston on 19 January 1980-skillfully orchestrated to arouse media attention (see Fig. 51) - that through the use of genetic engineering the molecular biologist Charles Weissmann and his team were the first to succeed in getting bacteria to produce human interferon in biologically active form. While admitting that there were still a lot of questions to be answered, Nobel Prize winner, Walter Gilbert, a Harvard professor and chairman of the board of Biogen, predicted that within one or two years the mass production of interferon for use in clinical trials would be feasible. ${ }^{191}$

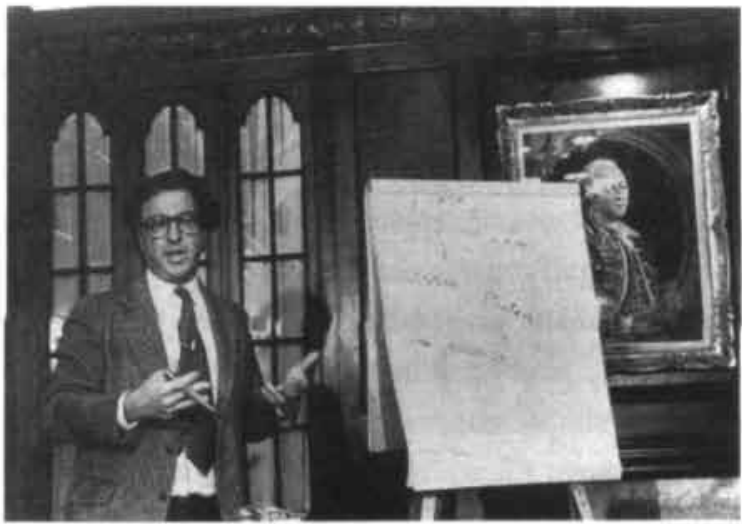

Fig. 51. Charles Weissman delivers the news of the successful cloning of the human leucocyte interferon gene and its expression in biologically active form at a press-conference held at the Boston Park Plaza Hotel. Reproduced with kind permission from the publisher.

But did this announcement really deserve the connotation 'breakthrough'? For one thing, the cloning of interferon had already been accomplished by a Japanese research team and published in the Proceedings of the Japanese Academy a few months earlier. ${ }^{192}$ In addition, no one knew whether the engineered protein would behave in the same way as the natural one

1980.

191 N. Wade, Cloning Gold Rush Turns Basic Biology into Big Business, Science, 208 (1980), pp. 688-92; S. Andreopoulos, Sounding Board; and, Gene Cloning by Press Conference. The New England Journal of Medicine, 302 (1980), 743-6.

192

T. Taniguichi, M. Sakai, Y. Fujii-Kuriyama, M. Muramatsu, S. Kobayashi, T. Sudo, Construction and Identification of a Bacterial Plasmid Containing the Human Fibroblast Interferon Gene Sequence, Proc. Jpn. Acad. 55B (1979), 46I-9. 
did. It was known that the bacterial cells were not programmed to add sugar molecules to the interferon product as the human cells would naturally do. However, hardly any knowledge was available about the biological function and effects of these missing sugar residues. Moreover, the commercial significance of the news, with several competitors in the race to produce human interferon, was far from obvious. Yet, the linkage between genetic engineering and the possibility of manufacturing a promising anti-cancer drug sufficed to make journalists report it as a 'breakthrough'. With a few exceptions they failed to ask critical questions that might have helped to place the work, that had yet to be published in the scientific literature, in a critical perspective. ${ }^{193}$

Both CBS and NBC led their evening newscasts with the latest promising news on interferon. The next days and months the story appeared on the front pages of most American newspapers and magazines, and others around the world-in France, Germany, Italy, Japan, Great Britain, the Netherlands-ran major pieces on it. ${ }^{194}$ The event was described by Nicholas Wade in Science in terms of a 'cloning gold rush' that turned molecular biology into big business-with biotechnology stocks rising to record levels at the international stock markets. Popular magazines talked about genetic engineering as the solution to the problem of producing a 'priceless miracle drug'. ${ }^{195}$ Interferon became a hype.

Thousands of telephone calls overwhelmed the NCI, NIH and ACS switchboards. Other cancer centers around the country and in Europe were similarly inundated with requests for interferon. Countless desperate patients and their families were begging hospitals, doctors, research centers and drug companies to provide them with the new wonder drug. Cantell, who like Strander was widely portrayed in the media as one of the divine heroes of interferon research, was literally besieged with requests for interferon from Europe, the US to Japan, Malaysia and India - confronting him with the horror of hundreds of fatally ill husbands, wives, relatives and children seeking a cure but also more benign cases like the following man: "I am having the biggest health problem of my life. I am having cold sores in my penis....I beg you help me... ${ }^{196}$ Even those familiar with the rage surrounding the development and testing of the

193 N. Wade, Cloning Gold Rush Turns Basic Biology into Big Business, Science, 208 (1980), pp. 688-92; S. Andreopoulos, Sounding Board; and, Gene Cloning by Press Conference, The New England Journal of Medicine, 302 (1980), 743-6.

'Medical Breakthrough Reported', Los Angeles Time, 21 january 1980; 'L'interferon: Enjeu d'une Competition Mondiale Scientifique et industrielle', Le Monde, 6 February 1980; 'Cancer Treatment Available Soon', The Guardian. 20 march 1980; The Big If-interferon'. The Listener, 29 february 1980; 'Interferon Duurste Stof ter Wereld', Telegraaf: The Making of a Miracle Drug. Newsweek, 28 Januari 1980; and interviews with Kari Cantell and Joseph Sonnabend.

195 N. Wade, Cloning Gold Rush Turns Basic Biology into Big Business, Science, 208 (1980), pp. 688-92: 'At only $\$ 100$ Million a Gram. This 'Miracle' has a Future'. Science Digest, April 1980;

For privacy reasons I can only refer to the letter files I have studied in Cantell's personal archives. 
'miracle' drug cortisone in the early 1950's had not seen anything like this happen on this scale and with such vehemence. ${ }^{197}$

The interferon mania only added to the media interest: "Wonder drug hope for miss Anelli: Amazing case book of the wonder drug doctor", "Dad's wonder drug plea" and "Drug brings hope for tumor boy 'Daniel'". ${ }^{198}$ Personification of scientific medicine and disease-projecting the inherent benevolence of medical science on individual scientists, doctors and patients-was a powerful rhetorical tool that journalists routinely employed but which helped to maintain and intensify the interferon furore. Imagery replaced content in most cases. Journalists eagerly reproduced the analogy popular among interferonologists comparing interferon's move from the laboratory into the clinic with the long, obstacle filled road that penicillin had had to travel from Fleming's laboratory to the pharmacist's shelf (see Fig, 52). ${ }^{199}$

Between personal accounts and metaphor-the media continued to describe interferon's versatile actions in the diseased body with the highly imaginative mixture of militaristic and information metaphors (see Fig. 53), a balanced view of the pros and cons of the actual technological-medical development and use of interferon was almost absent in the story-telling. The main message was that of scientific progress and faith in the promise of a medical breakthrough offered by current and future biomedical research.

All segments of society had a part in the hype. As Sandra Panem aptly portrayed the situation: "scientists who genuinely believed that they were on the right track and that money solicited at the expense of candour would be wisely used; investors and the public who wanted interferon to be a wonder drug and did not choose to ask whether the claims might be overstated; and those representatives of the media who reported anecdotes with unbridled enthusiasm". ${ }^{200}$ Until the Biogen announcement, the dramatic portrayal of interferon seemed to work to the advantage of all, with enormous amounts of energy and money pouring into efforts related to interferon in times of otherwise sharp financial cutbacks. However by the spring of 1980 the interferon wonder began to show signs of decay. Confronted with the stormy public reactions doctors, researchers and the medical authorities began to regard the

See, for a detailed account of the cortisone case; D. Cantor, Cortisone and the Politics of Drama, 1949-55, in J. V. Pickstone (ed.), Medical Innovations in Historical Perspective (Houndmills: MacMillan, 1992), pp. 165-99; and interview with Norman Finter. Mirror, 8 June 1980; and "Drug Brings Hope for Tumor Boy 'Daniel'. The Daily Telegraph, 12 April 1980.

K. Cantell. 'Why is Interferon not in Clinical Use Today?', in I. Gresser (ed.). Interferon, (London: Academic Press, 1979), 2-28, p.3. 


\section{INTERFERON The Penicillin of the Future?}

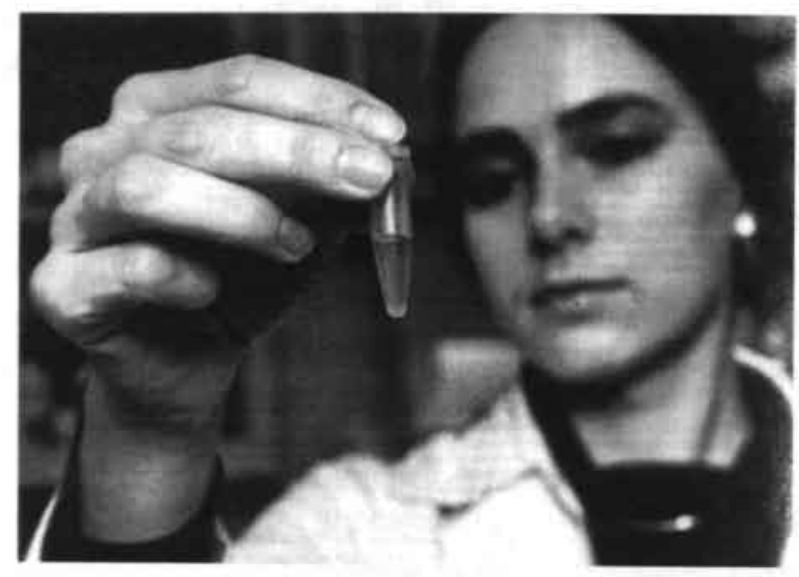

Fig. 52. Lead article in the American Journal of Nursing /April 1980. Reproduced with permission of the publisher.

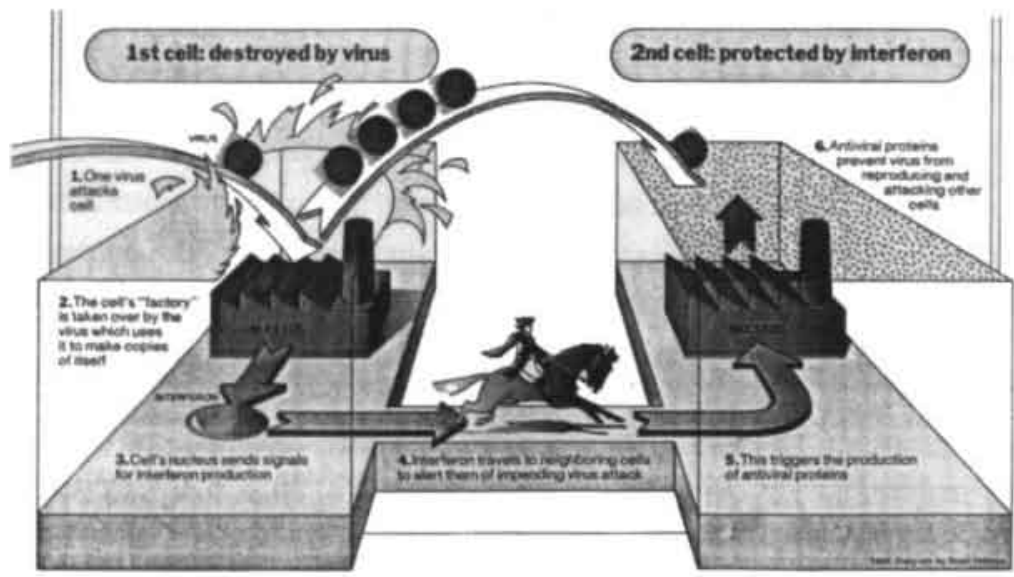

Fig. 53. This diagram filled with an imaginative combination of militaristic and information methaphors in the Time cover story on interferon (March 31, 1980) was largely based on Mathilde Krim's portrayal of interferon as a kind of chemical Paul Revere.

Reproduced with kind permission of the publishers. 
hype as disturbing and problematic.

Every patient suffering from cancer or a severe virus disease wanted to have access to a drug that was in short supply and had yet to be tested. A lot of distress was involved. In their face-to-face contact with patients, doctors found themselves besieged by demands for a drug that they could not supply, and frustrated by hopes of a cure they could not deliver. ${ }^{201}$ At centers where interferon was being tested everyone wanted to be in the trial and doctors had a hard time explaining that nobody could or would be favored in their selection of trial candidates. In order to determine whether interferon had any 'real' efficacy they had to work in accordance with stringent testing protocols which implied that only patients meeting the highly specific trial requirements would be allowed to participate. In addition, the doctors involved in testing interferon feared that the high hopes of 'the chosen' could affect the scientific validity of clinical trials - their test subjects's own enthusiasm for interferon and feelings after its administration might work against what was defined as 'objective benefit'.

Despite the fact that the excitements and motives of its own members had played an obvious part in the hype, the medical profession swiftly left the media to carry the blame. Once defined as a threat to the practice of medicine and professional autonomy, physicians on both sides of the Atlantic started making public appeals for a moratorium on publicity about interferon and in particular about ongoing clinical trials with interferon. They were openly supported by the interferon community in their efforts to blame the journalists for raising patient's hopes through irresponsible reporting. ${ }^{202}$ The primary concern of the interferonologists was that the continued media frenzy, which had initially worked to the professional advantage of interferon research, might in the end rebound to the discredit of interferon research itself. ${ }^{203}$

At the same time, medical research organizations like the NIH and the British MRC and the Imperial Cancer Research Fund (ICRF), drug regulatory authorities such as the FDA, and health administrators, saw the public demands for interferon as a challenge to their authority of drug evaluation, registration and supply procedures. ${ }^{204}$ There were worries that private funding bodies and manufacturers would yield to public pressures to provide interferon outside the formal testing channels and, even worse, that a black market for

201 'Publicity on Interferon has Caused Great Distress', The Times, 10 June 1980.

Editorial, 'What not to Say about Interferon', Nature, 285 (1980), pp. 603-4.

M. Edelhart, Interferon: The New Hope for Cancer (Reading (MA), Addison-Wesley Publishing Company, 1981), pp. 1-9; and, 'Interferon-The Hopes and the Reality', BMA News Review, September 1980, 18-22.

Department of Education and Science to Sir John Eden (MP/House of Commons), letter dated 12 June 1980, MRC Archives File No. S806/5. 


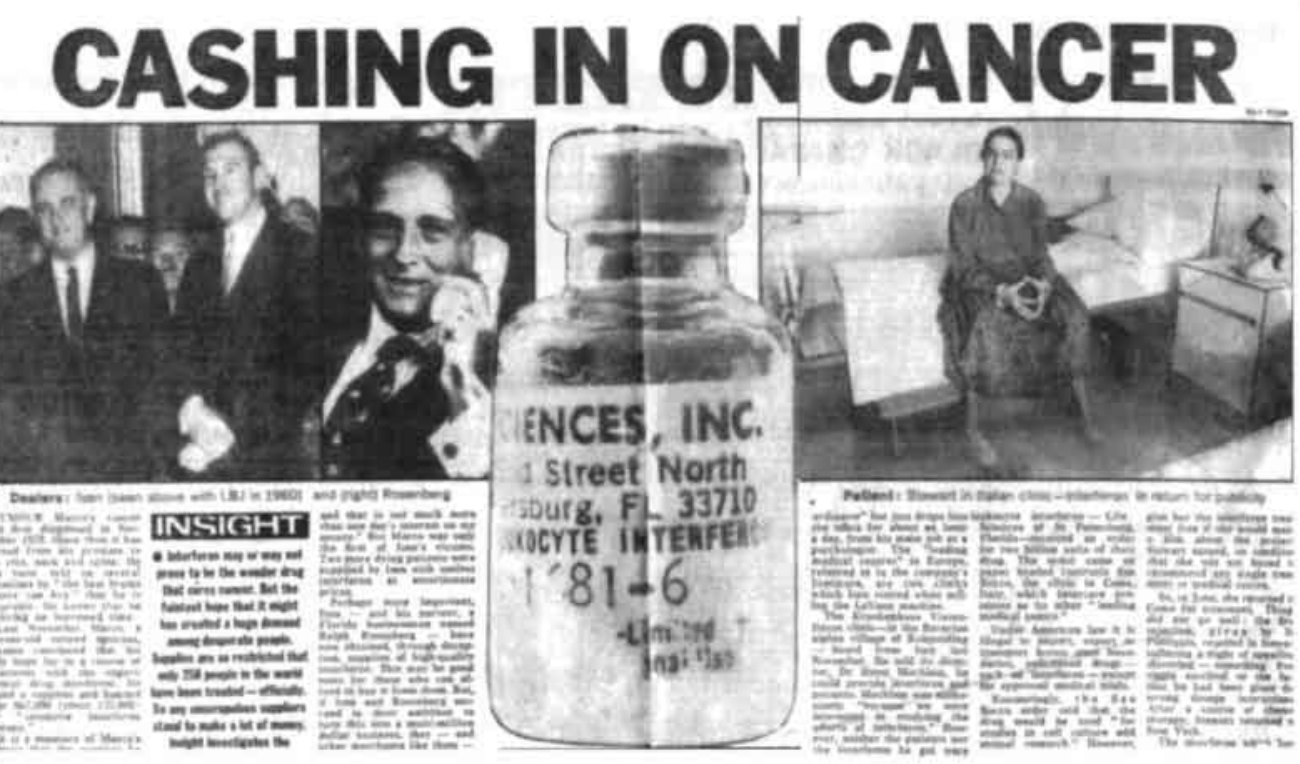

Fig. 54. Report on interferon black market in the Sunday Times

(November, 8 1981). Reproduced with

permission from the publishers

interferon might develop (See Fig. 54). ${ }^{205}$ The authorities realized that it was hard to explain to patients suffering from cancer that there might be a more effective treatment in the pipeline, but that it could not be generally available for some years until license procedures would be completed, by which time he or she might be dead. ${ }^{206}$

Distribution of the unlicenced drug interferon outside the approved medical trials was not only believed to undermine the ongoing drug evaluation process but also the whole state regulated drug testing practice — established in the 1960's to maintain certain quality and safety standards. ${ }^{207}$ With the prospect of improving supplies it seemed even more difficult to resist the public's disinclination to wait and make sure that all available material would be channeled into the official trajectory of controlled clinical studies, required for the proper evaluation and licensing of new drugs. ${ }^{208}$ Confronted with the desperate efforts of countless families and

205 A flourishing black market rapidly developed in often dubious interferon samples, fuelled by those rich. famous and desperate enough to try anything like the dying film hero John Wayne and the exiled Shah of Iran.

MRC to ICRF, letter dated 23 June 1980, MRC Research Archives File No. Di009/40. 
friends of cancer sufferers to obtain supplies of interferon at any price, the British Government and the major funding bodies for cancer research in the UK decided to issue a joint press notice in May 1980, cautioning against over-optimism and attempts at by-passing the formal drug evaluation route. ${ }^{209}$

It can be no accident that, while doctors and authorities were struggling to cope with what they regarded as mass hysteria about interferon, preliminary, unexciting results of the American Cancer Society sponsored interferon clinical trials were announced at the annual meeting of the American Association for Cancer Research. Whereas in February 1980 the consensus of the chief investigators associated with the ACS study was still optimistic and Gutterman's 50 percent response rate claim spurred on the ACS to allocate another 3.4 million dollars for further study, by the end of May things had changed. ${ }^{210}$

The information made available cooled expectations by suggesting that interferon was no more active than other available chemotherapeutic agents in treating breast cancer and multiple myeloma. Moreover, contrary to what was hoped for (in particular by outsiders who were sold on interferon as a non-toxic agent), side-effects were reported similar to those of other cancer medications; the patient who responded best was said to suffer the most serious side-effects such as abnormal liver function and even cardiac toxicity, and therapy had to be interrupted. ${ }^{211}$ These results placed interferon in perspective, just like any of the many substances being tested for antitumor activity.

Immediately following the premature release of the first ACS trial results the American Society of Clinical Oncology, with the prominent oncologist Charles Moertel of the Mayo Clinic as its immediate past president and spokesperson, took the unprecedented step of issuing a strong warning against overexpectations for interferon. ${ }^{212}$ In a personal statement to journalists Moertel emphasized that, while the jury was still out on interferon, cancer patients should realize that cancer treatment was a complicated matter and the best thing a patient could do was "get in the hands of knowledgeable cancer specialists and let them call the

209 Internal note MRC, dated 27 June 1980, MRC Archive File No. D1009/40; Press notice, dated 13 May 1980. MRC Archives File No. D1009/40.

210 Interferon Results "Promising." ACS will Commit Additional \$3.4 Million. The Cancer Letter, 22 February 1980.

211 'Interferon Results Cool Expectations', The Cancer Letter, 6 June 1980.

212 'Interferon "Hysteria out of Hand", Asco Warns Against Expecting Benefit', The Cancer Letter, 20 June 1980; This public warning on interferon by the American Society of Clinical Oncology should not be mistaken, as Evelleen Richards did, for an organized opposition of established oncologists against interferon as just another dubious immunetherapy; See, E. Richards, 'Vitamin C and Cancer: Medicine or Politics' (New York: St. Martin's Press, 1991), p. 207. 
shots". ${ }^{213}$ In saying so Moertel articulated the worries of doctors about the eventual damaging effects of public 'enthusiasm' on professional autonomy.

The redefinition of interferon as a potentially harmful experimental treatment with uncertain therapeutic benefits proved effective in undermining its public image as a wonder drug and put a lid on what Rauscher described as his continuing nightmare: "the unquenchable desire for interferon by cancer victims". ${ }^{214}$ The media immediately picked up the message with headlines such as "Interferon: Studies put cancer use in doubt", "Is it a wonder cure for cancer or the most expensive flop in history?". ${ }^{215}$ Unqualified optimism quickly shifted to the other extreme by casting doubt on interferon's potential.

\subsection{Conclusion}

My intention here is to discuss the most essential features of the interferon crusade. The first point that deserves scrutiny is the nature of opposition that Mathilde Krim, as self-appointed lobbyist, initially faced in finding an audience in the U.S. receptive to interferon as an antitumor agent, and the way she managed to overcome the resistance. Sandra Panem has already identified some of the factors that worked against Krim: apart from being a public woman in 'a man's world', Panem noted that Krim was regarded an outsider to the field of interferon research, neither knowledgeable enough nor sufficiently critical about interferon. ${ }^{216}$ Among interferon researchers and the NIH bureaucracy there was anxiety that Krim, as Mary Lasker's most dedicated disciple, might gain too much control over the course of interferon research.

Another factor that figures prominently in my story is the tenaciously held view in the field of interferon research that interferon was exclusively part of a nonimmunological host defense system against viruses ('the interferon system'), thereby excluding the possibility of a direct tumor-inhibitory effect. The idea that the action of interferon might be pleiotropic did not seem to fit either the 'interferon system' consensus concept or the dominant 'biomedical model' of specific etiology of disease. Nor did it fit the notion of specific therapy on which most therapeutic drug research programs were based. Moreover, there were concerns about

213 V. Cohn, 'Leading U.S. Cancer Doctors Agree to Issue Warnings on Interferon', The Washington Post, 15 June 1980.

214 M. Edelhart, Interferon: The New Hope for Cancer (Reading (MA), Addison-Wesley Publishing Company, 1981), p. 6.

215 H. M. Schmeck, 'Interferon: Studies Put Cancer Use in Doubt'. New York Times. 27 May 1980; 'Is it a Wonder Cure for Cancer or the Most Expensive Flop in History?, The Daily Star, 19 June, 1980.

216 S. Panem, The Interferon Crusade (Washington. The Brookings Institution, 1984). 
funding research with a poorly defined and impure substance for which a production and purification technology had yet to be developed.

Krim nevertheless succeeded in managing the opposition effectively by involving herself in a hybrid activity that combined elements of scientific evidence and reasoning with large doses of social and political judgment. Without trying to reach agreement on the controversial issues, she tried to address the common interests and characteristics of the various parties involved. On the one hand she emphasized the mysterious elegance of using a naturally occurring non-toxic substance, and on the other hand she underlined the fact that an impressive body of laboratory studies legitimated the potential value of interferon in the treatment of cancer. She properly valued the importance of linking basic research to clinical problems: convincing scientists and policy-makers alike that correlating laboratory findings with clinical findings seemed a perfectly straightforward goal in the case of interferon and cancer. However her attempts at linking laboratory with clinical data were beset with difficulties. In particular, the dispute arising over the exemplary status of Strander's trial data and the way it was managed deserve further attention.

The controversy over Strander's trial data illustrates that acceptance of experimental therapies in American clinical oncology hinges mainly on their evaluation by NCI-endorsed evaluators and methods. Moreover, it unequivocally shows that treatment evaluations shift and change in response to fluctuations in the balances of power in the context of evaluation. For instance, whereas initially Strander's trial data were well received within NCI quarters, gradually the mood changed with growing criticism of Strander for non-conforming behavior regarding the design of the trial. I indicated with reference to the NIH trial-design statistics that the critics were biased in their accusations. In my view, criticizing the Strander trial served the interests of the increasingly powerful randomized controlled trial lobby within the NCI. The outsider, Strander made a good target to plead their case and push the randomized clinical trial as the ultimate means of applying the scientific method and the objective kind of evaluation it supposedly entails to the practice of oncology.

However, as it turned out they underestimated the impact of Krim's consciousnessraising efforts. Krim understood the importance of persistent political pressure and of the transfer of negotiations about the value of interferon in the treatment of cancer increasingly to public arenas outside the established biomedical order in America. By mobilizing the politically powerful group of the 'Laskerites', Krim succeeded in upsetting the balance of forces for the benefit of her 'interferon crusade'.

Once support was building in American cancer circles for interferon as a lead towards anticancer therapy, it was coopted by a growing number of social groups. Apart from shared expectations about interferon as the natural science-based solution to cancer, each group had specific motives to jump on the interferon bandwagon: from the 'interferonologists' and the advocates of immunotherapy, who came to realize that Krim's interferon crusade might be 
helpful in giving a new impetus to their research fields; to the genetic engineers, who were looking for suitable demonstration projects to prove the commercial worth of the rDNA technology. Moreover, the American Cancer Establishment was seriously under attack and needed a scientific promise in their self-proclaimed 'war on cancer' to satisfy the growing public demands for better (more effective and less 'debilitatingly' toxic) cancer treatments. These political and professional interests, as well as consumer demands, thereby introduced additional pressures that affected professional judgments of the effectiveness and risk of interferon therapy. The interferon research community even went as far as to turn a blind eye to the emerging issue of side-effects following interferon treatment in order not to stall the interferon crusade.

An important institutional link in the network of relationships which developed around interferon is the role played by the media. My analysis focuses on the active role the mass media played as intermediaries between the scientific and medical community, the pharmaceutical industry, government and the lay public. Examining interferon stories in the British and American media, there is essentially one major script centering around the theme of 'promise and hope'.

Initially the media highlighted the dramatic curative potential of interferon against the

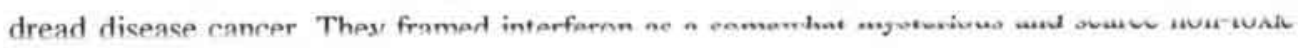
body substance which exerted its antitumor effects in a natural way, and at the same time as the latest promising product of a laboratory-supported scientific medicine. Subsequently, attention shifted to interferon as the most promising demonstration project of a revolutionary production technology, genetic engineering. I described how, by blowing up the imaginative combination of two wondrous products of modern science and medicine, the media helped to trigger an international interferon mania. However, I would like to stress that, except for a general tendency to reinforce and overstate interferon's promise, the media coverage of interferon was for the greater part a mirror of the expectations, legitimations and opinions circulating within the biomedical and public realm.

The intermediary role of the media is well illustrated by the drastic turn in the nature of reporting in the course of 1980 . For fear of the loss of control of the unexpectedly strong public reaction to the interferon crusade and of the possible future backlash in the form of a loss of authority and autonomy the international biomedical establishment launched its own counter campaign. While local doctors and authorities worldwide were struggling to cope with the interferon mania among patients and their families and friends, special news conferences were orchestrated as a means to dampen public enthusiasm. The message basically was that interferon as a non-toxic cancer wonder-drug was a myth created by the media, unsustainable in view of the available scientific test results. Whereas before the side-effects associated with interferon treatment had always been played down, now the very same side-effects were emphasized to show that interferon was hardly any different in its toxic effects from other 
available cancer treatments. In a similar fashion as biomedical researchers had been overly successful in seeking a favorable press, they managed to reshape the nature of the media coverage of interferon. Following the dissemination of information specially designed to lower public expectations, the nature of the stories in the press rapidly shifted from medical zealotry in promoting interferon's benefits as a miracle drug to the other end of the spectrum: disillusionment about a failed promise.

However similar to the cycle of promise and disappointment pictured in the introduction, the course of events in the case of interferon turned out to be far less straightforward. Moreover, as I will show in the next chapter the most common dead-end scenario of experimental cancer remedies would not materialize. 


\section{Chapter 7}

\section{The Final Twist: Marketing Interferon as a Helpful Neighbor ${ }^{\prime}$}

"When it comes to clinical trials, few issues are simple. And many are controversial" wrote the Science correspondent Gary Taubes in $1995 .^{2}$ Taubes's dictum seems to be at odds with the public model of controlled clinical trials as a most helpful tool to relieve medical practice of that most feared element known to scientists and regulators: subjectivity. Are most doctors and regulators who firmly believe in randomized controlled trials as the key to an 'evidence based medicine' mistaken? Given an ideal world without social, professional and economic interests affecting judgments of the efficacy and risks of medical therapies one might have answered 'no'. It is impossible, however, to conceive of such a trial taking place in a human vacuum. Conducting clinical trials involves establishing links and commitments between many different individuals and organizations, including clinicians, laboratory researchers, patients and their families, regulators and last but not the least the drug companies. In being shaped by the specific context of medical practice, clinical trials-even the most sophisticated randomized controlled trials-as I already have shown in chapters five and seven, are not value free measuring devices that objectively evaluate the efficacy of new therapies. Like any other medical device associated with our daily lives randomized clinical trials incorporate the beliefs and ideas of the persons and organizations who developed them and then are molded by those implementing the methodology. ${ }^{3}$

The euphoria surrounding interferon as a possible cure for cancer appeared short-lived

A preliminary version of chapter 7 was published in the British Medical Journal: T. Pieters. Marketing Medicines Through Randomised Controlled Trials: the Case of Interferon, BMJ, 317 (1998), 1231-3.

See, G. Taubes, Use of Placebo Controls in Clinical Trials is Disputed, Science, 267 (1995), p. 25.

3 A number of case studies dealing with post-war therapeutic research and development in medicine, which were published in the 1990 's, support the view that therapeutic evaluation is an inherently social and cultural process: See, E. Richards, Vitamin C and Cancer: Medicine or Politics (New York: St. Martin's Press. 1991): R. Vos, Drugs Looking for Diseases. Innovative Drug Research and the Development of the Beta Blockers and the Calcium Antagonists (Amsterdam: Kluwer Academic Publishers, 1991); A. Clarke and T. Montini, The Many Faces of RU486: Tales of Situated Knowledges and Technological Contestations, Science, Technology and Human Values, 18 (1993), 42-78; N. Oudshoorn, Beyond the Natural Body: Archeology of Sex Hormones (New York: Routledge, 1994); I. Löwy, Between Bench and Bedside (Cambridge (Mass): Harvard University Press, 1996); and, H. M. Marks, The Progress of Experiment; Science and Therapeutic Reform in the United States, 1900-1990 (Cambridge: Cambridge University Press, $1997)$. 
and faded when it turned out that both interferon's performance in large scale cancer trials had been disappointing and often produced side-effects in patients. Given intense disappointment with what by 1983 became dubbed 'the miracle drug looking for a disease', it might come as a surprise to hear that interferon would ultimately succeed in finding a niche in clinical practice. This then brings us to the question what gave interferon's biography a different turn from most other therapeutic promises in the treatment of cancer (which ended up on the laboratory shelf) and how did it become naturalized as part of medical practice?

In an effort to understand the processes by which new medical therapies are evaluated and put into practice, this chapter explores the complex events shaping the therapeutic value and clinical use of interferon as a new type of immunotherapeutic drug. The final part of the book's narrative provides an exemplary case to illustrate the complexities surrounding the application of clinical trials. I shall focus on how clinical trials make for a dialectical process of knowledge production-with the pharmaceutical industry playing center field.

In response to the drastic turn in the nature of reporting, Mathilde Krim and her staff wrote a letter to the New York Times, warning against the negative effects such qualified reporting might have on the public support of a field of research that sooner or later might provide society with a new immunotherapeutic approach of human cancer. As I will show in the first part of this chapter the core argument in this letter-emphasizing the unique property of interferons to enhance the activity of the body's own defense mechanisms-can be understood as an attempt at developing alternative strategies to legitimate work on interferon: picturing interferon as part of a promising new but experimental therapeutic approach in the future treatment of cancer and viral infection. ${ }^{4}$

\subsection{Beyond Interferon}

At the first annual International Congress for Interferon Research in Washington, DC, in December 1980 - organized by an American advertising and marketing company on behalf of the publishers of the Journal of Interferon Research in part to publicize the inauguration of the journal- the achievements of the molecular biologists aroused most excitement. ${ }^{5}$ While several teams of biochemists were working day and night to purify interferon to homogeneity and produce its chemical formula and structure, they were, as the American biochemist, Pete

4 Krim and her scientific staff's letter in the New York Times was a direct reply to Harold Schmeck's article of 24 August in the very same newspaper: See, M. Krim, W. E. Stewart II, F. Sanders, L Lin. 'Interferon Therapy', New York Times, 17 June 1980, p. C5.

According to a JAMA reporter the conference had an air of "upstage scientific excitement and backstage financial intrigue"; R. Johnson, 'Interferon: Cloudy but Intriguing Future', JAMA, 245 (1981), 109-16, p.109. 
Knight, expressed it "run over from behind by the cloners". ${ }^{6}$

An indirect research strategy to determine the structure of natural proteins commonly employed by biochemists was to try synthesizing the entity from pure amino acids on the basis of available structural data. When a substance was synthesized that matched the biological activity of the purified natural entity the structure of the natural protein might then be matched (though a tremendously sophisticated series of additional experiments would still be required to reach final agreement on its chemical structure). However, interferon appeared too complex a molecule for this approach to be successful.

What the several competing teams of molecular biologists basically had done was to beat the biochemists by genetically engineering micro-organisms that did the synthesizing job for them. In addition, the newly developed laboratory tools to engineer and decode DNA molecules provided the genetic engineers with the technical means to "read and write in the language" of the genes. ${ }^{8}$ It was a laboratory exercise that helped to reconstruct the protein structure from the DNA sequence of what was considered the gene coding for human interferon.

At the Washington meeting there were numerous displays with sequences of interferon DNA and derived amino acid structures. Together with the presentation by the molecular biologist Charles Weissmann of a slide with the first photograph ever shown of-what had always been considered a vain hope - highly purified crystals of alpha interferon, this promoted interferon to the tangible world of chemical facts (see Fig 55). The prospect of the mass circulation of unproblematic samples of crystalline interferons was expected to incite a considerably larger scientific public to use these molecules as a fresh starting point for their research. However appealing, the excitement was to a greater extent related to the claims of Biogen and its competitor Genentech that they both had succeeded in creating new, hybrid interferons by cleaving and rejoining ('recombining') pieces of two different interferon genes. According to Weissmann this was the first time that proteins had been engineered in this way, resulting in a new kind of biosynthetic interferons with unique biological properties.

6 E. Knight, 'Purification and Characterization of Interferons', in I. Gresser (ed.). Interferon 2 (London: Academic Press, 1980), 1-12, p. 2.

7 Having succeeded in this effort by creating interferon-producing bacteria, the cloners began isolating and sequencing the 'interferon DNA' with its genetic message encoded in permutations of four nucleotides bases (the bases Adenine, Thymine, Cytosine, and Guanine). Reconstruction and analysis of the A-T-C-G sequence of the 'interferon DNA' was thought to allow for the deduction of the sequence of amino acids in the protein for which it coded and thus interferon's chemical structure - each amino acid was known to be coded for by a triplet of bases. In practice the interpretation of the sequencing data-deriving the protein structure from the nucleotide sequences-was far from straightforward. See for the historical reconstruction of the cloning of interferon: C. Weissmann, 'The Cloning of Interferon and Other Mistakes', in 1. Gresser (ed.), Interferon 3 (London: Academic Press, 1981), pp. 101-34; and S. Hall, A Commotion in the Blood (New York: Henry Holt and Company, 1997), pp. 178-208. 


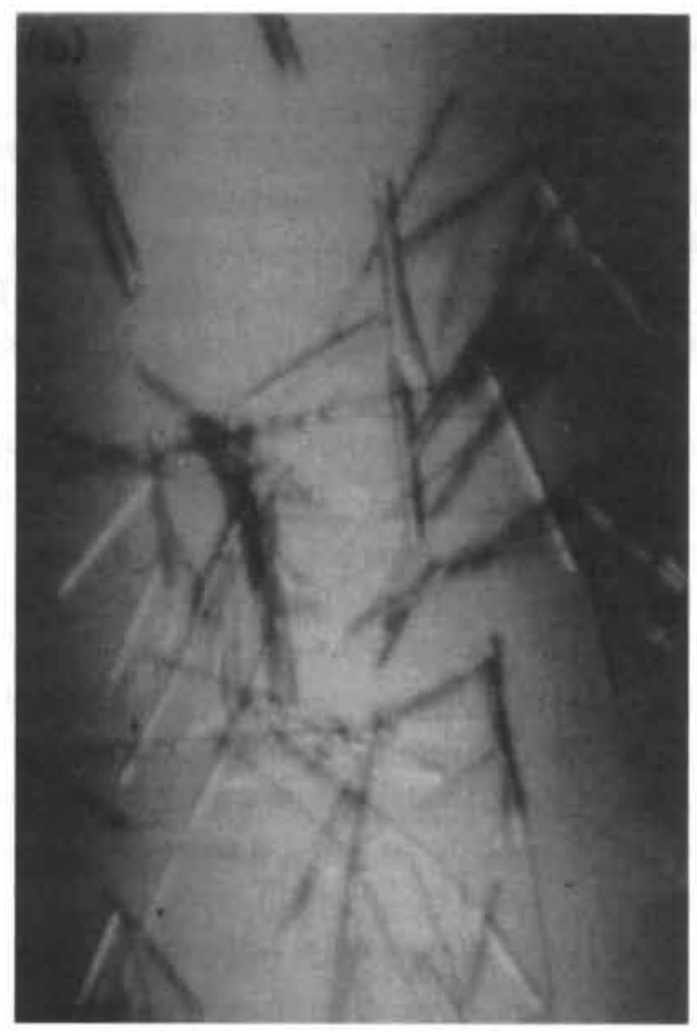

Fig. 55, Photomicrograph of interferon $\propto 2$ crystals.

Courtesy of Dr. C. Weissmann

Genetic engineering, so he argued, could in this way be used to "create an unending array of 'unnatural' interferon molecules".'

The claims of the molecular biologists can be understood as an attempt at showing the drug makers and the public that there was more to interferon than just a demonstration project of a promising new technology. It should be viewed not only as a test case of a new production technology but also as a pilot project for a new approach to drug development. In a similar way as the chemists were routinely involved in engineering an endless array of pharmacologically active chemicals, the molecular biologists would offer their expertise to drug makers to synthesize, modify and test new biosynthetic molecules. While the representatives of biotechnology firms began 'advertising' interferon as a means to establish an additional method of drug development, other interferon advocates increasingly legitimated 
work on interferon as part of a promising new multi-modality approach, in the treatment of cancer.

Most interferon researchers emphasized that cancer was too complex a disease to allow for a comparison between the search for a cancer cure and the development of wonder cures against polio or bacterial disease. In the case of a highly differentiated illness as cancer it would be better not to think in terms of overall cures, but rather of treatments. The consensus view was that though as a single agent interferon might turn out to be medically more useful in treating viral infection, ultimately it might prove most valuable as part of the increasingly popular multi-modality approach in cancer treatment. They claimed that attaining this end and learning how to use interferon, would require a further expansion of basic and preclinical investigations as well as clinical studies. ${ }^{10}$

Clinicians and laboratory researchers alike conveyed the impression that with more questions than answers they were just beginning to explore the potential of interferon, not as a single substance but as a large family of biologicals. So far more than ten different interferon molecules were claimed to circulate worldwide. The diversity of interferons with distinct and complementary biological activities seemed to grow every day. ${ }^{\text {" }}$ According to the interferon researcher Frances Balkwill in a 1982 progress report in the popular British science magazine 'The New Scientist': "It is clearly going to be difficult to know which interferon to use for any particular treatment or what combinations of "cloned" alpha interferons will be most effective". 12

Interferons were said to make up a new modality of cancer treatment in acting not as chemical toxins, directly on the tumor cells, but as biologicals through activation or modulation of key elements of the body's immunology system. They could be used as biological enhancers-helping to increasing the host's own response against the tumor-in conjunction

10 S. Krown, 'Prospects for the Treatment of Cancer with Interferon', in J. Burchenal and H. Oettgen (eds.), Cancer; Achievements, Challenges, and Prospects for the 1980's (New York: Grune \& Stratton, 1981), 367 79; R. Johnson, 'Interferon: Cloudy but Intriguing Future', JAMA, 245 (1981), 109-16; P. Newmark, Interferon: Decline and Stall, Nature, 291 (1981), 105-6; M. Sun. Interferon: No Magic Bullet Against Cancer, Science, 212 (1981), 141-2.

11 Biological Response Modifiers Program Review during the February 1981 meeting of NCI's Board of Scientific Counselors, minutes of the Board, dated 12-13 Febnuary, NCl Archives; R. Johnson, Interferon: Cloudy but Intriguing Future', JAMA, 245 (1981), 109-16; P. Newmark, 'Interferon: Decline and Stall'. Nanure, 291 (1981), 105-6; M. Sun, 'Interferon: No Magic Bullet Against Cancer'. Science, 212 (1981), 1412; Due to the high degree of species specificity of interferon, the drug industry depended on monkeys for preclinical studies of the biosynthetic human interferons (basically aimed at proving that a preparation was active and non-toxic in vivo). As head of the Dutch Centre for Primate Research, Huub Schellekens played a central role in the preclinical testing of the first r-DNA produced human interferon preparations: $H$. Schellekens et al, 'Comparative Antiviral Efficiency of Leukocyte and Bacterially Produced Human Alpha Interferon in Rhesus Monkeys', Nature, 292 (1981), 775-6.

12 F. Balkwill, 'Interferon: A Progress Report', New Scientist, 25 March 1982, 783-5, p. 784. 
with the three main cancer therapies: surgery, chemotherapy, and radiation. The NCI publicly singled out its program on interferon and other biological response modifiers (BRM) as "most exciting" in terms of benefits to be expected for future cancer treatment. ${ }^{13}$

In the opinion of the immunologist Louis Epstein of the University of California, San Francisco, the success of interferon therapy ultimately depended on the ability to forge further links between the laboratory and the clinic. She claimed to have developed a methodology in her laboratory which, if further elaborated on, could separate out those people whose cancers had the best chance of responding to a particular interferon. Her approach might make it possible in the future to individualize cancer treatment. She envisioned clinical oncologists sending tumor samples to her laboratory in order for her to figure out the susceptibility of a specific tumor to interferons and other compounds. This would enable the doctors to account for the problematic individual variations among their cancer patients. ${ }^{14}$

The overall message, was as a science reporter of the Washington Post aptly expressed it in his headline, "Beyond Interferon". ${ }^{15}$ By creating an image of interferon as a prototype of a promising, new, but still poorly understood, area of cancer therapy known as immunotherapy - one that was going to have an important role in future cancer practices- the promoters of interferon had succeeded in establishing a more permanent base for support.

Despite the growing public scepticism in the light of emerging stories about the iatrogenic consequences of interferon treatment and the rather disappointing response rates in the ACS-sponsored trials using Cantell interferon, the interest in interferon as part of a fourth modality of cancer therapy, known as 'immunotherapy', was on the rise. American cancer treatment centers that aspired to maintain an image of being at the cutting edge of the field of clinical oncology could not afford not to study an experimental therapy that was closely linked with the latest developments in tumor biology and molecular biology. ${ }^{16}$ In these centers great pressures were often exerted on practitioners to develop close relationships with the laboratory, to conduct preclinical and clinical research, and to enrol as many patients as possible in clinical trials. ${ }^{17}$ Clinical oncologists were used to side-effects and overall low

13 National Cancer Institute Program on Interferon and Other Biological Response Modifiers, dated October 1980, NCl Archives File No. AR-8000-007320; The Cancer Newsletter, 24 April 1981, p.I.

14 M. Edelhart, Interferon: The New Hope for Cancer (Reading (MA): Addison-Wesley Publishing Company, 1981), p. 49; L. Epstein. Interferon as Model Lymphokine, Fed. Proc., 40 (1981), pp. 56-61: B. Feder. Technology: Complexities Cloud Interferon, The New York Times, 23 April 1981; Epstein, interview.

C. Fenyvesi, 'Beyond Interferon'. The Washington Post, 14 June 1981, p.28.

Interview with Ernest Borden.

17 See, for a detailed account of the development of what Ilana Löwy dubbed the 'trialist ethos' in clinical oncology; Ilana Löwy, 'Between Bench and Bedside' (Cambridge (Mass): Harvard University Press, 1996), pp. 36-83. 
response rates in their day-to-day therapeutic practices. The mere knowledge that interferon as a scientifically endorsed experimental therapy reportedly produced clinically significant antitumor activity in a limited number of patients suffering from a variety of highly problematic tumors, sufficed to give it the benefit of the doubt. Stephen Sherwin, an American clinical oncologist, put his colleagues feelings aptly by stating: "Interferon may not be the wonder drug it was expected to be, but it may reduce tumors in specific cases. We have to give it a try". ${ }^{18}$

Only the relative scarcity and impurity of interferon preparations withheld them from pursuing interferon research more actively. However, as soon as the more pure and homogeneous rDNA produced interferons became more widely available in the course of 1982 (see Fig. 56 a,b,c), a growing number of American and European clinical oncologists got themselves involved in preclinical and clinical testing. ${ }^{19}$ Their testing 'zealotry' was hardly affected by the news in November 1982 that four cancer patients out of eleven in a Paris Hospital had died from a heart attack while being treated with French alpha-interferon. ${ }^{20}$ Following the French in their decision to stop only the trial in question, Robert Oldham, head of NCI's BRM Program, played down the significance of the tragic event. Thus far in American studies there was no evidence that interferon had ill effects on the heart. Most likely the fatal-incident was due to a problem with the manufacture of the French interferon or it might also be possible that the patients involved already had serious heart conditions before the trials began. ${ }^{21}$

At the January 1983 meeting of NCI's Board of Scientific Counselors Oldham did issue a warning that although biologicals like interferon were natural products this did not necessarily mean that they were non-toxic. In the same breath he called interferon one of the more active new agents tested in the last several years, perhaps just below the level of the most successful chemotherapeutic agents and recommended further clinical testing. Even if interferon treatment had been officially associated with cardiac toxicity it is unlikely that the clinical testing would have stopped. Going by the reaction to cardio-toxicity problems involving experimental cancer drugs in the early 1980 's, the NCI would have confined itself to sending letters to doctors across the country warning of the heart toxicities of interferon and

18 C. Fenyvesi, 'Beyond Interferon', The Washington Post, 14 June 1981, p. 28.

19 Minutes of American Society of Clinical Oncology, 18th Annual Meeting, St.Louis, Missouri, April 25-27. 1982, NCl Archives No. 8204-001971: K. Sikora and H. Smedley. 'Interferon and Cancer', British Medical Journal, 286 (1983), 739-40.

20 R. Walgate, 'Side Effect Scare Hits French Trials, Nature, 300 (1982), 97-8; According to a February 1983 ACS press release interferon was one of the most studied natural substances in medicine, "as researchers explore its benefits, potentials and limitations"; Interferon Update ACS, dated 11 february 1983, NCI Archives DC-8301-006691; See, on overzealous treatment practices:'Cancer a Progress Report', Newsweek. 2 November, 1981.

21 'France Halts Interferon Study', The New York Times, 4 November 1982. 


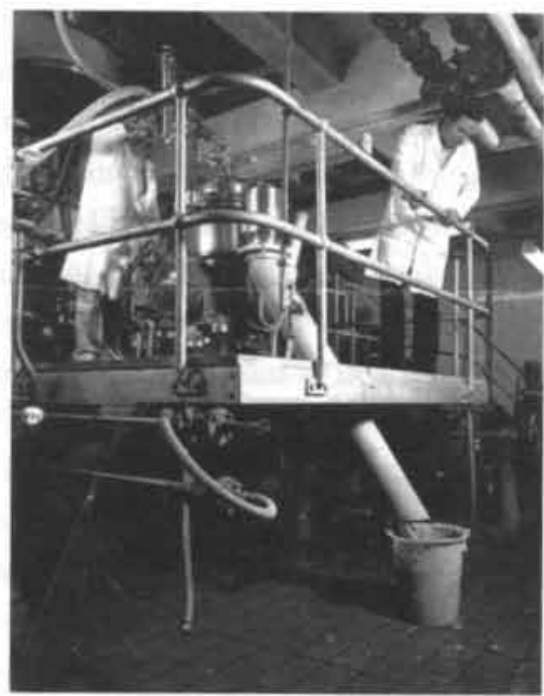

Fig. 56a. A pilot plant for the 'mass production' of interferon by genetically-modified bacteria (1982).

Reproduced with permission from the publisher.

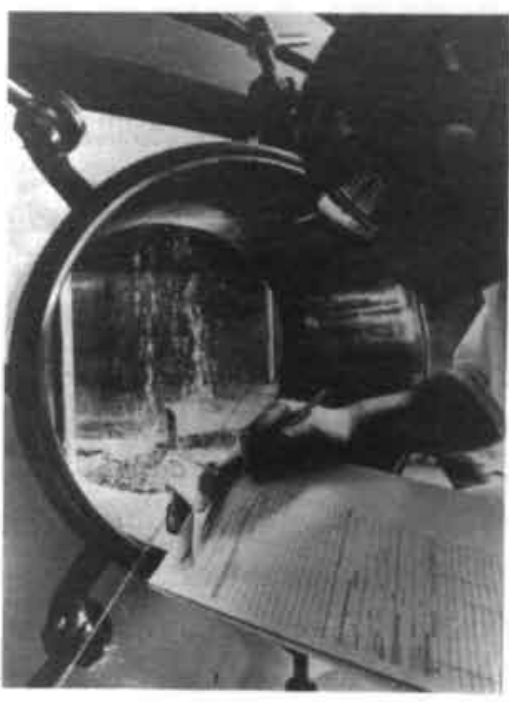

Fig. 56b. Interferon is routinely harvested from genetically-modified bacteria in vast tanks (1984). Reproduced with permission from the publisher.

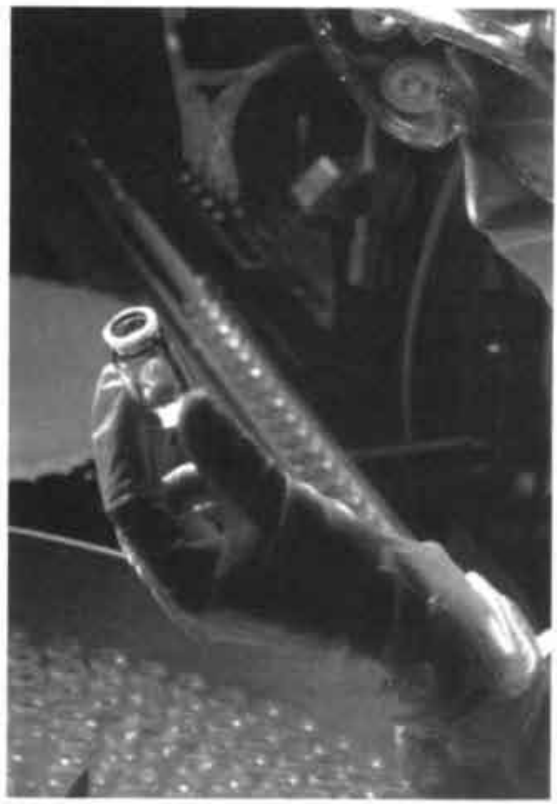

Fig. 56c. The production-line for clinical trial interferon at Biogen. Reproduced with permission from the publisher. 
advising limited use in patients with a history of heart problems. ${ }^{22}$

High risk:benefit ratios were and still are part and parcel of the culture of cancer treatment. Severe side-effects from experimental drugs had become an accepted part of life in most American and European cancer treatment centers. The treatment-related mortality rates ran from as high as 10 percent for experimental regimens combining for instance radiation and aggressive chemotherapy to between 2 and 5 percent in established, non-experimental chemotherapy regimens. ${ }^{23}$ To fully understand the situation, we should take a glimpse at the world of cancer patients and doctors. According to the 1979 ACS' statistics only 33 percent of Americans who got cancer would be alive five years after diagnosis and treatment. ${ }^{24}$ This meant that for the majority of cancer patients death was inevitable. In this world, doctors, trained to healing and frustrated by the frequent experience of being unable to deliver a cure, were prepared to go to any extreme if patients, for whom no other hope seemed to exist, allowed them to. At the same time most patients, in a desperate effort to avert their 'death sentence', were willing to try almost anything in the hope of a cure or at least a postponement of their death. In this context it is hardly surprising that institutes for cancer research like the $\mathrm{NCI}$ or cancer treatment canters placed treatment side-effects second to potential benefits.

Potential for beneficial effect was considered as sufficient basis by NCIs Board of Scientific Counselors to agree to an expansion of clinical studies and to pursuing the combined modality therapeutic approach in September $1983 .^{25}$ The apparently unremitting optimism within NCI quarters contrasted with the growing scepticism about interferon's potential among senior executives in pharmaceutical companies. Of the twenty or more firms which had announced their intention of investing in interferon development back in 1979, a growing number ceased work on interferon over the period of one year, namely during 1983. They had either lost out in the 'cloning race' or management had negatively assessed interferon's potential both as a therapeutic drug and as a means to attract capital investment from the stock markets. ${ }^{26}$ Apart from the disappointing cancer trial data there were also growing doubts about interferon's potential as an antiviral agent. The expectations-fueled by the latest reports from the Common Cold Unit at Salisbury that nasal sprays of alpha interferons markedly reduced the occurrence of colds in volunteers - about opening up the commercially interesting niche

22 T. Gup and J. Neumann, 'Experimental Drugs Cause Pain, Deaths', The Boston Sunday Globe, 18 October 1981.

See, S, Rosenberg. The Transformed Cell (London; Phoenix, 1992), pp. 236-7.

24

See, R. W. Moss, The Cancer Industry (New York: Paragon House, 1991), p. 22.

25

Minutes of NCl's Board of Scientific Counsellors meeting, dated 27-28 January 1983, NCl Archives.

26

T. Powledge, 'Interferon on Trial', Bio/Technology, 2 (1984), 214-28. 
market of common cold prevention were dashed with the latest news about its side-effects. ${ }^{27}$ Even at low-dosage levels interferon appeared to induce side-effects similar to the symptoms responsible for most of the miseries of the very same cold that it was supposed to prevent. "A single high dose of zinc may do just as good a job at substantially lower cost", was the scathing judgment of the science correspondent, Joseph Alper, in The New York Times. ${ }^{28}$

In the boardrooms of the three 'interferon champions' that had most heavily invested in the therapeutic substance-Wellcome and, most notably, Roche and Schering-Plough, interferon's lackluster clinical performance in treating tumors as well as virus infections was reason for growing concern. ${ }^{29}$ The company officials responsible for investment in interferon had a hard time defending the costly interferon research and development (R\&D) programs. However, they succeeded in convincing the sceptics in the managements that although interferon might not be a 'magic bullet' in itself, it had enormous potential as a prototype of a new generation of custom designed biosynthetic drugs that was expected to generate a therapeutic revolution. They presented it in terms of the unique possibility of learning to tap an entire medicine chest of new drugs from natural substances within the human body. Unlike most chemical drugs these genetically engineered biologicals could be viewed in their 'natural' (physiological) role as regulators or modifiers of a variety of pharmacologically interesting biological mechanisms. They argued that in order to develop these biosynthetic molecules as therapeutics, new testing methods and procedures would be needed to effectively bring them to the clinic. For instance, since human interferons were only active in humans and monkeys researchers were forced to do the unusual and skip most of the preclinical animal testing and go straight into humans to determine a toxicological and pharmacological profile.

However different, work on biologicals at the same time was argued to be easily integrated into the current system for drug development based on tinkering with molecules as a means to create variants with most optimal pharmacological characteristics in terms of risk:benefit ratios. The promise of scientific and therapeutic innovation appealed most to senior drug company executives who were less worried about today's profits than about tomorrow's prospects. With patents on most of the top prescription pills expiring by 1990 and with an unprecedented small range of chemical compounds in various stages of development in their laboratories, ready to take over as money-making ('blockbuster') products, drug companies had come to recognize that a new wave of innovation was needed to position themselves for long-

27 'Million Dollar Cold Cure', The Sunday Times, 3 October 1982, pp. 22-30; and, G. Scott et al, 'Prevention of Rhinovirus Colds by Human Interferon Alpha-2 from Escherichia Coli'. Lancet ii (1982), 186-7.

J. Alper, 'First there was Interferon', The New York Times, 18 November 1984, F, 13.

In 1983 Hoffmann-La Roche and Schering-Plough reportedly allocated 15 percent of their research budgets-more than 40 million dollars each-to interferon; T. Powledge, 'Interferon on Trial', Bio/Technology. March 1984, 214-28; and inteviews with Norman Finter and Leon Gauci. 
term survival. ${ }^{30}$

However, those responsible for the interferon related R\&D programs were not able to get round the iron business rule of profit generation. The pressure for return on investments in interferon research and in production facilities for the first genetically engineered interferon products (Schering-Plough's homogeneous interferon alpha-2 and differing by only one single amino-acid, Roche's homogeneous interferon alpha-A preparation) and Wellcome's 'natural' interferon product - containing the full spectrum of alpha interferons produced by cultured human cells-did force the interferon advocates within the companies to adapt their R\&D strategies. In line with government supported research programs, the pharmaceutical industry focused on interferon as part of a new kind of disease management: immunotherapy within a multi-modality treatment framework. Apparently the drug companies recognized the strategic and commercial importance of taking advantage of the more general move across medicine towards combination therapy. ${ }^{31}$

\subsection{Naturalizing interferons as biological response modifiers and cytokines.}

However, the drug regulatory authorities found the combined-modality approach difficult to assess as their evaluative practice and standards were still governed by a single-agent therapeutic philosophy. For interferon to be considered legally as a new therapeutic drug, it had to be officially evaluated as a single agent. This implied that before licensing procedures could be taken into consideration, the companies had to look for a disease, rare though it might be, that justified a need for interferon. Moreover, under FDA regulations each interferon variant was considered a new therapeutic drug and should be independently evaluated. ${ }^{32}$

In search of suitable diseases as candidates for interferon as a treatment, the drug companies actively supported clinical trials to evaluate the effects of interferon on as wide a variety of diseases as possible (see Fig. 57). They offered clinical investigators worldwide - free of charge-large quantities of their interferon products. Interferons were tested

30 T. Powledge, 'Interferon on Trial', Bio/Technology, March 1984, 214-28; A. Wycke, 'Molecules and Markets', The Economist, 7 February 1987.; and interviews with Leon Gauci and J. P. Warbeer.

31 Interviews with John Petricciani and Leon Gauci.

32 Interviews with John Petricciani, and Leon Gauci: See, for a similar cases of drugs looking for diseases: R. Vos, Drugs Looking for Diseases. Innovative Drug research and the Development of the Beta Blockers and the Calcium Antagonists (Amsterdam: Kluwer Academic Publishers, 1991): N. Oudshoorn, Beyond the Natural Body: Archeology of Sex Hormones (New York: Routledge, 1994). 


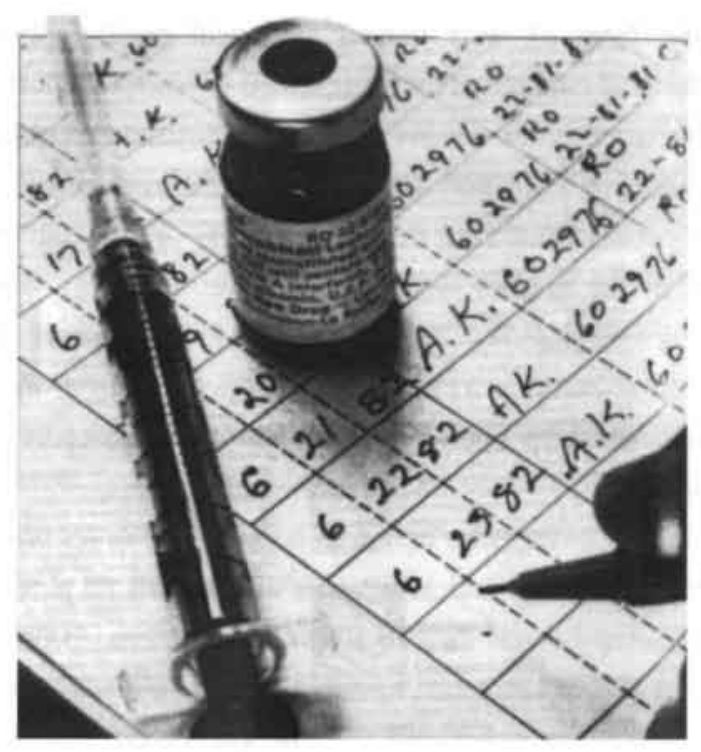

Fig. 58. Record from clinical trial with Hoffmann-La Roche's recombinant alpha-A interferon, showing a patients blood levels of interferon. Reproduced with permission from the Publisher.

for hepatitis B, various lymphomas, colds, breast cancer, prostatic cancer, multiple sclerosis, herpes keratitis, malaria, AIDS (as soon as the epidemic began to manifest itself around 1982) and many other diseases related to cancer and viruses. The drug companies mounted one of the most intensive clinical trial programs ever set up to evaluate a new pharmaceutical agent. In the first three years (1982-1984) the recombinant interferons alone were tested in over 4000 patients. ${ }^{33}$

By the end of 1983 the large-scale testing efforts finally showed signs of paying off. Spurred by Strander's chance observations at the Karolinska Hospital in Stockholm, alpha interferons were also tested on patients suffering from papilloma virus associated noncancerous tumors ('warts'). The warts ranged from being a cosmetic problem or undergoing spontaneous regression to being life-threatening. The greatest interest in the use of interferon had been in connection with genital warts ('Condomyloma Acuminata') and a less common but

33 'Interferon May Help AIDS Victims', New Scientist, 3 November 1983; 'Interferon Tested on Sclerosis', The New York Times, 21 November, 1981; and, N. Finter and R. K. Oldham (eds.), Interferon (vol4); In Vivo and Clinical Studies (Amsterdam: Elsevier. 1985); 'Update on Interferon', News Service American Cancer Society, dated 11 February 1983, NCI Archives File No. DC8301-00669I; and, 'Intron A', press information video tape produced by Schering-Plough in 1986 on the occasion of the market introduction of their interferon product. 
potentially life-threatening form of wart named 'laryngeal papillomatosis', located in the respiratory tract, particularly of children. This represented a serious clinical problem due to the tendency of the wart to recur and obstruct breathing. Some patients required surgery as often as every 2 months. Responses to interferon treatment were claimed to be of long duration in at least fifty percent of the patients suffering from these warts and so far the risk:benefit ratio looked promising. None of the studies was controlled yet but large controlled trials were underway. ${ }^{34}$ However encouraging for the patients and the doctors involved, the drug companies paid initially more attention to the research news coming from the field of clinical oncology. As this was more likely to open a window into a commercially interesting family of disorders and medical specialty. ${ }^{35}$

Drug company officials swung into action as soon as the news broke in the autumn of 1983 that Gutterman's research group had achieved a higher than 80 percent trial response to 'natural' alpha interferon therapy (with Finnish Red Cross material produced by Cantell) in patients with a rare form of chronic leukemia known as 'hairy-cell leukemia' (so-called because of the 'hairy' appearance of the malignant cells). ${ }^{36}$ The knowledge that there was hardly any viable treatment for this form of cancer with estimated mortality rates of about 15 percent per year, sufficed for those looking for a suitable disease indication. ${ }^{37}$ They seemed to take for granted the fact that among clinical oncologists scepticism prevailed about the overwhelming efficacy claim by Gutterman and his team, who had earned themselves a name for overoptimistic interpretation of their interferon trial data. ${ }^{38}$ Since the successful hairy cell leukemia trial was performed with the relatively impure (less than $1 \%$ pure) and therefore pharmaceutically problematic Finnish alpha interferon product, the companies's first priority was to demonstrate that the dramatic clinical effect could be reproduced with their own 'pure' interferon products. Following extensive clinical testing they ultimately succeeded in their efforts. Consequently licence applications for what both Schering-Plough and Roche dubbed "the world's first anticancer interferon" were sent for evaluation to among other drug regula-

B. G. Leventhal, 'Treatment of Virus-associated Tumors and Papillomas with Interferons', in N. Finter and R. Oldham (eds.), Interferon (vol4); In Vivo and Clinical Studies (Amsterdam: Elsevier, 1985), 326-35; and interviews with Kari Cantell and Hans Strander.

S. Hall, A Commotion in the Blood (New York: Henry Holt and Company, 1997), 203-5.

J. Quesada, E. Hersh and J. Gutterman, 'Hairy Cell Leukemia: Induction of Remission with Alpha Interferon', Blood, 62 (1983), $207 \mathrm{a}$.

J. Quesada, J. Reuben, J. Manning, E. Hersh and J. Gutterman, 'Alpha Interferon for Induction of Remission in Hairy Cell Leukemia, N. Engl. J. Med., 310 (1984), 15-8; and interviews with Leon Gauci, and. John Petricciani. 
tory agencies the FDA. ${ }^{39}$

In having closely monitored and guided the proceeding of interferon trials throughout the years the FDA's Office of Biologics Research and Review (OBRR) had built up considerable expertise in evaluating both 'natural' and biosynthetic interferons. The questions of identity, purity, potency and safety featured very prominently in the frequent interactions and negotiations between the OBRR and all those interested in obtaining the license "Notice of Claimed Investigational Exemption for a New Drug" (IND) that allowed for clinical trials with a particular interferon. First, the question of identity was related to the taken for granted concept of interferon as a family of proteins and corresponding genes. Interferon was made up of at least three different types, each being made up of multiple subtypes, each of which might have different effects on the body. Every interferon product made by a different method, manufacturer, organism or interferon clone could in principle be different from one another. Each biosynthetic variant, even if it differed from another interferon by only one amino acid was considered a distinct protein and likewise had to be evaluated independently under FDA regulations. There were more questions than answers to this complicated issue. Second, the question of purity was hardly less problematic. For instance, genetically engineered interferon products which were claimed to come close to 100 percent purity presented additional purification concerns such as the presence of unwanted bacterial contaminants or of altered or aggregated interferon species (due to gene mutations) which may be either non-functional or antigenic. Compared to this, the third question of interferon potency was a relatively unproblematic matter which was dealt with on a consensual basis during the successive standardization conferences in the late 1970's. Finally, the safety question was coped with in terms of a benefit:risk ratio assessment on a case-by-case basis. Since, as OBRR senior staff member Petricciani argued there was nothing absolute to say about safety of experimental drugs in the clinical research setting. ${ }^{40}$

The test procedures required by the OBRR before any experimental clinical use had evolved in parallel with the development of new interferons and new production methods. Changes in the procedures represented the emergence of a new consensus on what was

39 'Anticancer Interferon Available Soon', Hospital Doctor, 22 September, 1983; In contrast to interferon products which were still in the investigational stage and limited in their clinical use (phase 1,II,III testing). licensed products which would be available for general commercial distribution and use by the medical profession must meet a variety of extra regulatory requirements, at least in the United States, as prescribed by the Good Manufacturing Practice (GMP) provisions and the General Provisions for Licensed Biologicals (GPLB) Code of Federal Regulations; J. Petricciani, E. Esber, H. Hopps and A. Attallah, Manufacture and Safety of Interferons in Clinical Research', in P. Came and W. Carter (eds.). Interferons and their Applications (Berlin: Springer-Verlag, 1984), 357-70, p. 359.

A. Hecht, 'Interferon: Trying to Live Up to its Press', FDA Consumer, June 1981; and, H. Hopps, K. Zoon, J. Djeu and J. Petricciani, 'Interferons for Clinical Use: Purity, Potency and Safety', In N. Finter and R. Oldham (eds.), Interferon (vol4); In Vivo and Clinical Studies (Amsterdam: Elsevier, 1985), 121-33. 
acceptable and counted as "state-of-the-art testing". ${ }^{41}$ To facilitate the learning process and secure the interferon researchers's cooperation, Kathy Zoon who had an important part in the work that led to purification of the human interferons was assigned as interferon expert to the OBRR and subsequently to the interferon licensing committees. She played a key role in evaluating the preclinical and clinical volumes of data on the various alpha interferon preparations presented by the respective companies and in reaching a judgment on whether each individual product reached an acceptable level of safety, quality and efficacy. The FDA was able to secure approval for the company's product licence applications for hairy cell leukaemia within only one year of their initial submissions in the spring of 1985 . According to FDA Commissioner Frank Young the direct involvement of "bench scientists" like Zoon had proven to shorten the official review and approval process by at least half a year. ${ }^{42}$ However, there is more to say to the relatively short review time then only crediting the close involvement of expert scientists for this achievement.

By the time the interferon applications were filed the unconditional support of FDA's mandate on drug regulation legislatively set by the Kefauver-Harris Amendments of 1962 following the thalidomide tragedy, was eroding. The FDA was confronted with an increasingly intense lobby for relaxation and fastening of its drug approval procedures by a novel coalition of the pharmaceutical industry and consumer groups. Historically the organized consumer movement in America had always played an important role in the further tightening of regulatory requirements and product liability laws as a means to safeguard the public from injudicious trial and marketing of new pharmaceutical products. However, in the context of the American AIDS epidemic consumers showed that their support of stringent regulatory arrangements was not unconditional. In particular, if they perceived the regulations as slowing down the availability of promising experimental treatments for urgent disease conditions such as AIDS. The industry in turn had always sought to protect its position by politically highlighting the problem of stifling innovation through what they considered as an overrigorous bureaucratic system of controls. ${ }^{43}$ In an effort to silence the critics reduction of the average review time-which in 1977 still averaged more than two years-was given priority status within FDA quarters. ${ }^{44}$ Thus, interferon came to serve as a high visibility show-case of

41 Interviews with John Petricciani, and Kathy Zoon, and, H. Hopps, K. Zoon, J. Djeu and J. Petricciani. 'Interferons for Clinical Use: Purity, Potency and Safety', In N. Finter and R. Oldham (eds.). Interferon (vol4); In Vivo and Clinical Studies (Amsterdam; Elsevier, 1985), 121-33.

42 U.S. Department of Health and Human Services, news announcement, dated 4 June 1986, NCI Archive No. 8606-001949; Editorial, 'Interferon Drugs Join Cancer Fight', Clinical Pharmacy, 15-3-1986.

43 See, P. Temin, Taking Your Medicine (Cambridge (MS): Harvard University Press, 1980); E. Richards, Vitamin C and Cancer: Medicine or Politics (New York: St. Martins Press, 1991), pp. 58-9 and 224-5.

See, P. Tenin, Taking Your Medicine (Cambridge (MS): Harvard University Press, 1980), p. 143. 
the FDA's increased efficiency and its decisiveness to act in the case of a medical 'urgency'.

On June 4, 1986, the FDA and the U.S. Department of Health and Human Services staged a joint press-conference to announce the swift licensing of biologically engineered alpha interferons to treat a rare leukemia for which there had been no effective therapy. The event showed that the FDA too had learned to capitalize on the power of the media. The FDA's spokesperson Young hailed the development and approval of interferon as "a milestone, a promise of what will be coming in the future". ${ }^{45}$ Thus Young showed that interferon had also become a priority test-case of what he subsequently referred to as "a second revolution of pharmaceutical innovation, akin to the discovery of antibiotics in the 1940 's" ${ }^{46}$ By investing heavily in establishing state-of-the-art testing procedures for biosynthetic biologicals like interferon, FDA officials tried to anticipate on an expected explosion of biosynthetic therapeutic agents emanating from the rapidly expanding bioengineering R\&D programs in the international drug industry. ${ }^{47}$

It is interesting to see that Wellcome with its non-recombinant 'natural' IFN product fell by the wayside. In most western countries like the USA Wellcome's natural alpha IFN 'cocktail' (with the brand name Wellferon) met with difficulties from the regulatory authorities except for Britain, the home market for Wellcome products. Asiatic countries - which historically do not oppose heterogenous pharmaceutical preparations because of a different therapeutic drug culture - had hardly any problem accepting the IFN cocktail. By the time the FDA put aside its doubts about Wellferon, Wellcome decided that with the market already flooded with Roche's and Schering-Plough's rDNA produced IFNs it was no longer commercially interesting to apply for a product license. ${ }^{48}$

In the meantime the therapeutic development of interferon had helped to spawn a spectacular investigative enterprise. This included numerous academic, federal and industrial research programs, the Journal of Interferon Research and the formation of the International Society of Interferon Research. Going by the growth in the number of papers published each year with the word 'interferon' in their titles from about 800 in 1980 to more than 3000 in the year 1987, the field experienced an information explosion-in terms of new knowledge of cellular mechanisms of immunity and of new immunological explanations of the biological

'Interferon Licensed to Treat Hairy Cell Leukaemia', New Scientist, 12 June 1986.

F, Young, The Reality Behind the Headlines', In FDA Anonymous, New Drug Development in the United States (Rockville (MD), 1990).

Interview with John Petricciani.

48 Interview with a Dutch Glaxo-Wellcome worker who prefers not to be identified; Kari Cantell's Finnish leucocyte interferon 'cocktail' met with the same difficulties from the drug regularity authorities: H. Hage. 'Controlerende Instaties Moeten Soepeler Worden met Interferon', Toegepaste Wetenschap TNO, oktober 1986 . 
effects of interferon. ${ }^{49}$

The fact that the accumulation of knowledge based on laboratory data was of relatively little practical help to clinicians using interferon treatment at the bed side was not regarded as a failure but instead was used to advertise interferon's unwieldy and complex nature as part of an intricate system of checks and balances. This so-called cytokine network, was said to constitute a key part of the body's natural defenses. The complexity of the cytokine interactions was argued to complicate interferon's study in the test tube as well as its clinical application. Researchers maintained that additional knowledge of these interactions was needed to improve or optimize the use of biological response modifiers or cytokines like interferon. The questions piled up but there were few satisfactory answers. Did all interferons have the same or different functions within this network? Which interferons should be tested first clinically, on what diseases and how? Was it justified to start from maximum tolerated dosage regimens knowing that more not always appeared to be better with these substances? Would combination treatment indeed add up to more than the sum of its parts? Interferon advocates called them difficult substances to work with, far more complex than traditional therapeutic drugs. As a justification for their research efforts they kept emphasizing that interferon generated intriguing research horizons. It was claimed that work on interferon provided a window both onto biological processes at the molecular level and new approaches to the treatment of disease. ${ }^{30}$

Laboratory and clinical researchers alike stressed that the clinical potential of interferons would be enhanced by further investigation and understanding of the molecular mechanisms underlying the effects of interferon in patients. The solidarity implicit in both their emphasis on the importance of linking biomedical research at the cellular and molecular levels to clinical interventions as part of what became known as 'molecular medicine', stemmed from shared interests in the maintenance of credibility and funding. ${ }^{51}$ Disappointing as well as successful clinical results could be used by bench scientists to claim that further basic research was mandated. At the same time clinical researchers were able to defend their poor results by referring to the slow progress in the understanding of the underlying mechanisms by which interferons exerted their biological effects both in vitro and in vivo. Achieving occasionally high response rates at the bedside, however rare the disease condition, seemed to justify the

49 See, for the annual number and titles of interferon-related publications the Dindi data base, Cologne.

so S. Baron, F. Dianzani, G. Stanton and W. Fleischmann (eds.). The Interferon System: A Current Review to 1987 (Austin, TX: The University of Texas Press, 1987); S. Baron et al, Interferon: Principles and Medical Applications (Austin. TX: The University of Texas Press, 1992); C. Wallis, 'What's become of Interferon'. Time, 1 June 1985; F. Balkwill, Interferons: From Common Colds to Cancer, New Scientist, 14 March 1985. pp. 26-8; F. Balkwill, Cytokines in Cancer Therapy (Oxford: Oxford University Press, 1989).

51 See, for a focus on what constitutes today's molecular medicine the website http://www.isinet.com/prodserv/focus_on/fo_mmfrm.html. 
further expansion of both preclinical and clinical research. Nonetheless the actual expansion of interferon related activities was largely dependent on the support and coordination of the pharmaceutical industry.

During the transition to the large-scale production of genetically engineered interferons laboratory scientists and clinicians had become increasingly dependent on the industrial infrastructure. By possessing the key to the material resources and research materials the companies began to play a central role in structuring the development of interferon. ${ }^{52}$ Laboratory scientists were encouraged to deploy the new techniques of molecular biology, cellular physiology and biochemistry in the search for additional natural occurring molecules that might provide genetic blueprints for the development of these future therapeutic drugs. ${ }^{53}$ Moreover, by providing a new powerful research tool, unlimited quantities of pure crystalline interferon, the research community was stimulated in efforts to investigate the underlying molecular mechanisms of interferon's actions. ${ }^{54}$ At the same time clinical investigators were encouraged to start experimenting in humans with this first representative of a rapidly growing family of clinically active biologicals, the so-called 'biological response modifiers' or 'cytokines'. The impression was conveyed that participating clinicians would stand out as pioneers of a new era of disease treatment. ${ }^{55}$

In 1986, once the FDA, swiftly followed by other regulatory agencies, licensed Roche's and Schering-Plough's recombinant alpha IFN products for the treatment of hairy cell leukemia, the marketing branches of the companies worked hard to create a need for interferon. ${ }^{56}$ In order to interest health care professionals in the new area of interferon therapy and to convince them of its scientific character, Hoffmann-La Roche and Schering-Plough established freely accessible electronic data bases (e.g. Schering-Plough's ICON), specific journals (e.g. Roche's Progress in Oncology, Progress in Virology and Interferons Today and Tomorrow), and financed medical book publications (e.g. Schering-Plough sponsored the

Interview with Leon Gauci; and, L. Gauci, 'Interferon Drug Development: A History Truely Consistent with the Discovery Process', paper presentation at the International Meeting From Clone to Clinic, March 1990, Amsterdam; R. K. Oldham, 'Interferon: A Model', in I. Gresser (ed.), Interferon 6 (London: Academic Press, 1985), pp. 127-41; D. Barnes, 'Biologics Gain Influence in Expanding NCI Program', Science, 237 (1987), 848-50.

A. Foerstner, 'How We Make Our Own Wonder Drugs', Chicago Tribune, 20 January 1985. 1983 to clinical investigators all over the world. 
publication of Interferons in Cancer Treatment) ${ }^{57}$ The way in which the companies presented interferon by means of a combination of visuals and narrative to doctors, decision-makers and journalists in the specialist-press drew upon the same range of images that had influenced the public perception of interferon through the mass electronic and print media; framing interferon both as a natural occurring substance in the body and as a state-of-the-art product of modern science and technology.

Optimizing response rates seemed to be the explicit aim of virtually any clinical research project dealing with interferon. ${ }^{58}$ Except for hairy cell leukemia (response rate higher than 80 percent), clinical researchers involved in testing interferons claimed response rates anywhere between 10 and 50 percent. The problem and advantage of using percentages was that success appeared to be a highly ambiguous term. Overall response rates ('efficacy') resulting from clinical studies under controlled circumstances might look promising, even when it remained unclear what this actually meant for individual chances of success and how well a treatment might perform in everyday clinical practice ('effectiveness') ${ }^{59}$ Regardless of interpretation, however, response rates remained invariably low in most diseases, suggesting that it could help only some of the patients some of the time.

Under normal disease conditions this kind of negative scientific assessment would dissuade doctors from applying a therapeutic drug. But, as I mentioned before, in circumstances where there is no hope for a cure, the rules of the game appear to be different for doctors and patients alike. In diseases in which successful treatment is rare, seeking treatment through medical intervention is a gamble which can have few winners. Gambling is an alluring analogy for all parties as it turns poor clinical results into acceptable chances,

57 H.C. Thomas, F. Cavalli, and M. Talpaz (eds.) 'Thirty Years of Interferon', Interferons Today and Tomorrow, 5 (1987) (This journal is published by Mediscript, London); H. Kirchner ( ed.). 'Update on Interferons'. Progress in Oncology, 2 (1986) (Abstracting journal published by Mediscript, London); H. Kirchner (ed.), 'Update on Interferons', Progress in Virology, 1987; 1 (Abstracting journal published by Mediscript. London); H. K. Silver (ed.), Interferons in Cancer Treatment (Mississauga: Medical Education Services (Canada) INC, 1986); ICON or Interferon Communication Network was established in 1980 by ScheringPlough. In 1988 Icon contained about 15000 items related to interferon which had been selected by a group of 5 physicians payed for by Schering. The Schering team produced their own abstracts of the papers and on special request customers could obtain the original publications; and interview with $\mathrm{C}$. v. Helsen.

58 P. E. Came and W.A. Carter (ed.), Interferons and their Applications (Berlin: Springer-Verlag, 1984); C. Pinsky (ed.), 'Biological Response Modifiers', Seminars in Oncology, 13 (1986), pp. 131-227; D. Parkinson (ed.). The Expanding Role of Interferon-Alfa in the Treatment of Cancer', Seminars in Oncology, 21 (1994), $1-37$.

59 My distinction between effectiveness and efficacy is derived from clinical epidemiology; See, S. Pocock. Clinical Trials (Chichester: John Wiley \& Sons, 1983). 


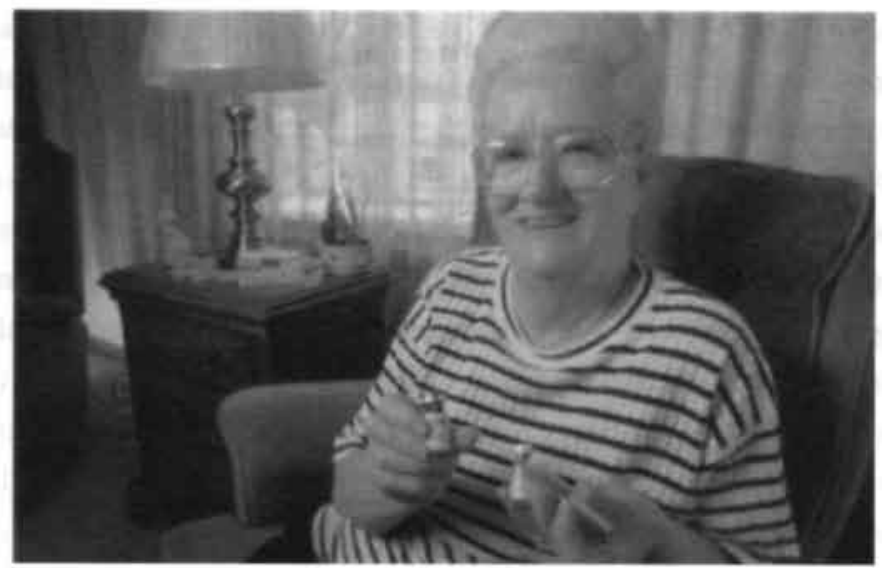

Fig. 59. The following subscript was added to this picture in the Scientific American (May 1994): "Lela Rector of Marion, was a winner in an unusual lottery last year. After a form of interferon-beta gained approval for treating multiple sclerosis in July 1993, demand far exceeded supply. So the distributor. Berlex Laboratories, held a lottery for some 67,000 qualified patients. Rector, who drew number 109, was among some 30.000 pepole reportedly in treatment by March 1, 1994. The other registrants are expected to have access to the drug by the end of this year".

allotting responsibility for failure to bad luck rather than medical or other capacities (see Fig. $59)$.

In 1986 the Dutch interferon researcher Huub Schellekens predicted that interferon would be used more widely in ten years time than might be expected on the basis of the approved list of indications because of the psychology of interferon as a potential 'multi-drug' from common colds to cancer. ${ }^{61}$ Schering-Plough and Roche of course fully endorsed this perceived mechanism and were keen to turn this into a self-fulfilling prophesy. They hoped that if they were able to show that interferon as a cytokine enhanced-even if marginally-the efficacy of routine and semi-routine therapies for problematic cancers and chronic virus diseases, the molecule could become a commercial success as an auxiliary immunotherapeutic drug. They therefore actively promoted trials and investigator's meetings that would evaluate the possible synergism between interferons and various conventional therapies. At the same time they began marketing interferon as part of the comprehensible though open-ended (in terms of number of factors involved and nature of interactions) cytokine network (see Roche illustration). In the process the Roche sponsored journal Interferons Today and Tomorrow

See, for a similar kind of phenomenon in the treatment of reproductive disorders in women: J. Van Dyck, Manufacturing Babies and Public Consent (New York: New York University Press, 1995), p. 124. 1986 on the occasion of the market introduction of their interferon product. 
changed its name into Interferons and Cytokines, while The International Society for Interferon Research became The International Society for Interferon and Cytokine Research, and the official journal of this society became the Journal of Interferon and Cytokine Research by $1995 .^{62}$

With the relentless support of the drug industry and patients in desperate need for a cure, and through the combination of scientific drive and professional ambition, most notably clinical oncologists and infectious disease specialists continued to work on the trial design. They tried different combinations (e.g. in combination with cytotoxics or a combination of various biological response modifiers) and different routes and durations of administration. ${ }^{63}$ In doing so, they ultimately tinkered toward success in terms of establishing new therapeutic drug practices for interferon and actively working on the treatment's effectiveness. Although superior treatments for the treatment of hairy cell leukemia became available - and have largely replaced the use of interferon for this indication-interferon as an adjunct to other therapeutic modalities became part of the routine treatments of a growing number of major malignancies such as non-Hodgkin's lymphoma, multiple myeloma and kidney cancer. In the 1997 Cancer Chemotherapy Handbook interferon can be found under the separate heading 'Biological Response Modifiers' (though in actual clinical oncology practice the term 'cytokine' prevails) with emphasis on the differences (in administration, dosage regimens and mechanisms of action as immunomodulating agents) from other cytotoxic therapeutic drugs in use in clinical oncology and on combination treatment as the greatest promise for successful therapeutic application. ${ }^{64}$ Moreover, by 1997 interferons as therapeutics had been approved in the U.S. for use against eight different diseases, including genital warts, Kaposi's sarcoma, several forms of hepatitis, malignant melanoma, and multiple sclerosis. The latest approval, which occurred on 11 June 1997 for use in conjunction with chemotherapy in patients with follicular lymphoma, shows that over the years the FDA had changed its attitude toward combination therapy. ${ }^{65}$

Interferon, almost given up on in terms of having a role to play in medicine by business analysts, the press, the public, scientists and doctors alike in the early 1980s, has become

62 According to an editorial by Ronald Penny the new journal title reflected the real breadth of focus in this ever expanding field. In his view the number of factors involved grew "almost at the rate of action of the cytokines themselves": R. Penny, Interferons and Cytokines, 15 (1990), 3.

63 C. Pinsky (ed.), 'Biological Response Modifiers', Seminars in Oncology, 13 (1986), pp. 131-227; D. Parkinson (ed.). The Expanding Role of Interferon-Alfa in the Treatment of Cancer'. Seminars in Oncology. 21 (1994), 1-37; R. Stuart Harris and R. Penny, Clinical Applications of the Interferons (London: Chapman\&Hall Medical. 1997).

D. Fischer et al. The Cancer Chemotherapy Handbook (fifth edition) (St. Louis: Mosby, 1997).

65 See, for FDA product approval information on biologics the FDA Web site: www.FDA.gov/cber/efoi/approve.htm and www.FDA.gov/cber/establish.htm. 
exemplary not only for a new generation of biosynthetic drugs but also for the health benefits that result from advances in molecular medicine. Fueled by a potent combination of scientific drive, professional ambition, marketing efforts and a lot of hard work between 'clone and clinic', interferon was transformed from a so-called 'orphan drug' for a rare terminal disease into a billion-dollar molecule that was used in the treatment of a wide-variety of ailments. The world-wide market for interferons - the whole spectrum of alpha, beta and gamma type interferon products - expanded from \$13.6 million in 1986, to \$751 million in 1992, to \$1.57 billion in 1993, down to $\$ 928$ million in 1995 and up to an estimated $\$ 1.2$ billion in 1998 (see Fig. 60) ${ }^{66}$

The widespread application of interferon as an effective medical commodity seems almost beyond dispute; the need for it appears to be a premise, rather than a contestable assumption. As a consequence, opposition to interferon therapy currently revolves less around questions of need than around questions of cost or economic feasibility, which increasingly dominate the political agenda of 'marketplace' medicine. As the chapter on economic aspects of interferon in the 1997 book The Clinical Applications of the Interferons shows, economic

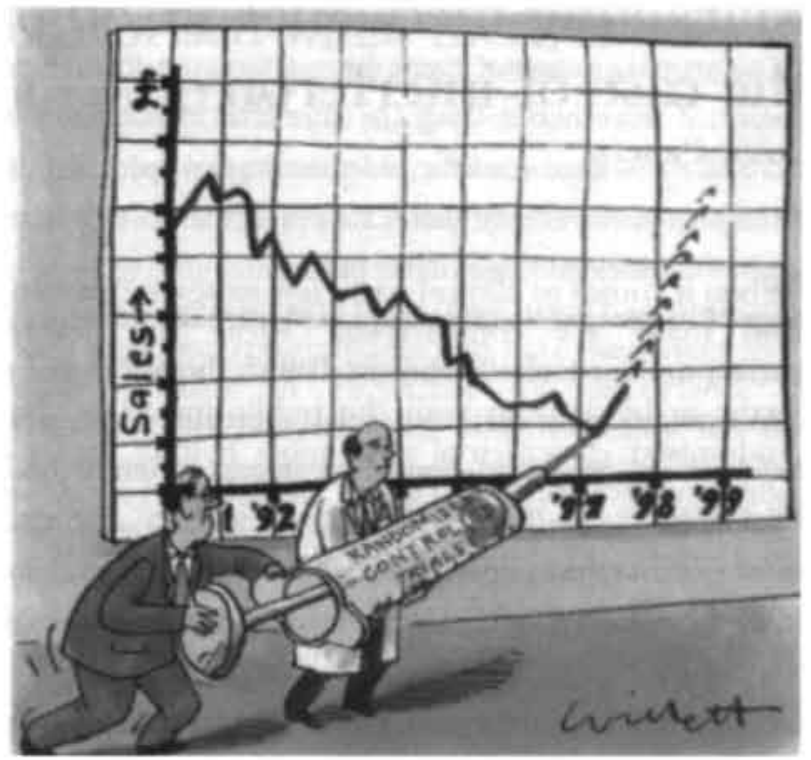

Fig. 60. Reproduced with permission from the publisher. es/Trends). 
and commercial considerations, are easily linked with medical discourse and therefore are difficult to separate from medical arguments showing the necessity for interferon treatment. ${ }^{6}$

\subsection{Conclusion}

The last paragraph on economics nicely illustrates that shaping therapeutic drug practices is not exclusively a medical or scientific affair. To think so would deny the multiple links between science, medicine and society which have been made manifest in my description of the development and dissemination of interferon as part of a new therapeutic approach in the treatment of cancer and viral disease. Of course, in order to become part of the therapeutic armament of doctors, interferon had to meet scientific standards of what constituted legitimate evidence for efficacy. But does meeting those standards suffice to explain the exponential growth in the clinical use of a variety of interferons?

As far as the spokespersons of the pharmaceutical companies are concerned it does. The growing market for interferon treatment is claimed to be a 'natural consequence' of a growing need of doctors and patients ('consumers') who apparently regard the new drug as a valuable asset. The growing range of therapeutic effects, so they argue, reflect the need for interferon. However, if there is one thing the interferon crusade can teach us, it is that the equation of need and the widening of the pharmacological spectrum, is a logical fallacy. The need for interferon was never 'simply' there. Likewise, the development and dissemination of interferon, was never a self-evident, inevitable process.

This brings us to the question how did interferon become naturalized and legitimized as part of medical practice? First of all, confronted with the erosion of public support for the interferon crusade, attempts were made to develop alternative strategies to legitimate work on interferon. Instead of the presumed non-toxic nature of interferon, its perceived unique capacity as a biological to act through the immune system was used to attract attention. Moreover, interferon was presented as having an important advantage over conventional therapies: it linked the clinics to advanced laboratory research in tumor biology, molecular biology and immunology. I dubbed the rhetorical strategy that proved effective in establishing a more permanent base for support 'beyond interferon': picturing interferon as a first step in the development of immunotherapy - a promising new therapeutic approach in the treatment of cancer, that was going to constitute a medicine of tomorrow.

Second, regardless of whether optimism or the opposite extreme prevailed, the media paid homage to the perceived mysterious polypractic healing power of this natural body

67 A. Shiell and G. Salkeld, The Economic Aspects of Interferon', In R. Stuart-Harris and R. Penny (eds.), The Clinical Applications of the Interferons (London: Chapman\&Hall, 1997), pp. 376-90. 
substance. The panacea image that was attached to interferon continued to appeal equally to defenders of medical orthodoxy and advocates of unorthodox cancer remedies. However alien to a medical practice that was dominated by the theory of specific etiology and the notion of specific therapy, interferon had an irresistible appeal to laboratory researchers and clinicians alike. It was the promise of a new flexible immunological approach to disease which might make it possible in the future to individualize disease treatment through forging further links between the bench and the bedside. I argued that speaking about interferon as a prototype of a new class of hormone-like cell regulatory proteins with the unique property to enhance the body's own defense mechanism, the so-called 'biological response modifiers', provided a fruitful scientific rationale not only for interferon's versatile actions but also for NCI's decision to set up a special Biological Response Modifier (BRM) research program.

Third, by 1980 strong links had formed between interferon research, the booming field of molecular biology and private industry. Powerful commercial, institutional and professional interests had become aligned with what was considered a major show case of the newly developing biotechnology industry and its revolutionary rDNA technology. During the subsequent successful transition to large-scale production of interferon, the international pharmaceutical industry, which was seeking to position itself for long-term survival through scientific and therapeutic innovation, became the most dynamic and strategic actor of all those involved in work on interferon. In achieving a key-position in the distribution of research resources and materials, the pharmaceutical industry increasingly dictated the development of interferon. However dominant there role in structuring interferon's biography, for the profiling and marketing of interferon the drug companies were largely dependent on their ability to enter into cross-fertilizing relationships with laboratory scientists, clinicians and regulatory bodies.

By picking up and refining the broadly based cytokine network concept in such a way that it served the needs of all those involved, the drug makers anticipated the successful integration of interferon into medical practice. However promising in scientific and commercial terms, the combined-modality therapeutic approach of interferon as a cytokine met with resistance from drug regulatory authorities. As I pointed out, the official international drug regulation practice was still largely governed by a substance thinking dominated therapeutic philosophy.

In order to legally consider a pharmacologically active compound as a new therapeutic drug it had to be officially evaluated as a single agent. This implied that before licensing procedures could be taken into consideration the companies had to look for a disease that justified a need for interferon. With most trial responses to single-agent interferon therapy comparing unfavourably with drugs already available in the clinic, the industrial interferon program managers faced the seemingly Herculean task of establishing an unambiguous justification for clinical use.

As a final point, I will analyze how the search for suitable disease candidates through 
clinical trials became a growth industry in its own right, which played a central role in transforming the interferons into the therapeutics as we know them today. In the previous chapter I showed that by being simultaneously sites of scientific research and medical care clinical trials do more than just evaluating the safety and benefit of new drugs. In serving as bridges between bench and bedside they connect a diverse assortment of actors: biomedical researchers, physicians, cancer foundations, pharmaceutical companies, patients and regulators. These diverse parties face the difficult task of participating in scientific research and at the same time making decisions in an environment where the most basic actions are intertwined with matters of sickness and health and where no one is able to draw sharp boundaries among scientific, clinical, political and economic questions. ${ }^{68}$

In the case of interferon setting up trials as a scientifically endorsed experimental therapy linking the laboratory and the clinic was compatible with professional interests in the field of clinical oncology. In addition, the conspicuous lack of efficacy in clinical studies did not stop the pharmaceutical industry from investing additional money and resources in the clinical testing of interferon. Too many resources had already been invested to reconcile them to the situation and accept an untimely failure. In my view the industrial persistence in providing interferon free of charge to all those who were willing to test interferon in the laboratory and the clinic also illustrates the high strategic value attached to interferon within the companies involved, as a protected demonstration project of a new drug development technology.

In functioning as 'hinge joints' between the laboratory, the clinic and the society at large, the clinical trials made for a dialectical process of knowledge production that tightened the links between, among others, the pharmaceutical industry and the regulators. Through many years' monitoring of the interferon trials, the drug regulatory authorities not only built up expertise in evaluating interferons, but became also increasingly committed to their development. In the U.S. the attachment of social and political significance to interferon as a high visibility show-case of the FDA's ability to combine the dual role of regulator-containing safety and efficacy - and of innovation manager - encouraging product innovation - eventually resulted in its swift licensing. The licensing of interferon as a therapeutic drug, however limited its FDA-approved indication range, provided a further incentive for the companies to expand the clinical testing.

In achieving a key position in the distribution of research resources and materials needed to set up clinical trials the pharmaceutical industry increasingly dictated development and clinical use of interferon. Highlighting success instead of failure in industry-sponsored

See, for a detailed account of the increasingly important social and cognitive role of clinical trials in twentieth century scientific medicine; H. M. Marks. The Progress of Experiment; Science and Therapeutic Reform in the United States, 1900-1990 (Cambridge, Cambridge University Press, 1997). 
research publications, without denying current limitations, came to form the implicit justification for the further growth of the clinical trial 'enterprise'. By using the 'logic' of medical evidence presented in the form of value-free results of randomized clinical trials and the power of metaphors in combination with the almost unlimited provision of research materials, the pharmaceutical companies secured the medical profession's cooperation. The drug industry was well aware that it was highly dependent on the cooperation of clinicians, foremost the clinical oncologists - who were highly skilled in the art of bringing laboratory compounds to the clinic and shaping drug profiles-to define additional clinical situations in which the interferons might be applied. In line with the culture of experimentation in clinical oncology the message was optimizing response rates through modulating the maximum tolerated dosage regimens, varying modes of administration and trying different combinations of therapies.

As the most dynamic and strategic actor involved the pharmaceutical companies were able to profit most from the very dialectical nature of the clinical trial enterprise. In positioning interferon as a helpful neighbor, compatible with and supportive of existing treatment practices, they succeeded in having interferon relatively quickly absorbed by the medical infrastructure, requiring increasingly large amounts of money for its use. 


\section{Chapter 8}

\section{Interferons in Retrospect and Prospect}

Let us assume that a worldwide survey of science journalists was conducted. If asked to name the most newsworthy medical substances of the past forty years, a majority of respondents would most likely list 'interferon'. This book's narrative shows how and why. Every so often between the 1960's and 1990's, substances that go by the proprietary name of 'interferon' entered the headlines of our newspapers. As one might expect each surge of media interest whetted the public's appetite for information on interferon. Among others, the editors of Scientific American capitalized on each successive rise in public interest by inviting prominent interferon researchers to provide their readers with a state-of-the-art account of interferon.' All these papers have historical sections in which the authors portray the origin of interferon research. They agree on several important points: time and place of the events, persons and name of the substance under examination. However similar in their efforts to legitimate work on interferon by reference to the origin of interferon research, in each case the discovery account turns out to be different. In dealing with different research horizons, every consecutive historical account of the discovery of interferon takes on a new and different form and meaning. These different ways of framing 'the story' could earn scientists easily the label 'bad history'.

Should scientists worry about earning this label? I think not. On the contrary, 'bad history' helps them to relate the work of others to one's own research problems; to tune in to actual scientific debates that ought to interest them. It is an important part of the repertoire of resources which scientists use to argue their case. Whereas historians of science focus on developmental processes and on what it is that the various workplaces and scientific events connect, surround and situate in time and in place, working scientists are primarily interested in publicly justifying their own scientific achievements or current and future scientific endeavors.

The phenomenon of "good science as bad history" proves very helpful in capturing historical development and change in a particular field of research. ${ }^{2}$ Throughout the book we

1 A. Isaacs. 'Interferon', Scientific American, 204 (1961), 51-7, p. 51; D. Burke, 'The Status of Interferon', Scientific American, 236 (1977), 42-50, p. 42; and. H. Johnson, F. Bazer, B. Szente and M. Jarpe, 'How Interferons Fight Disease', Scientific American, May (1994), 40-7.

2 With all due respect, Thomas Nickles's phrase "good science as bad history" also applies to Jean Lindenmann's critical response 'On Toine Pieters' 'Shaping a New Biological Factor'. In accordance with fellowscientists the microbiologist and interferon researcher Lindenmann finds it difficult to escape from the fallacy of framing his historical analysis in such a way as to write an implicit teleology into the evolution of the field of interferon research. Lindenmann's retrospective comments show us once again that working scientists and 
can see that in building on earlier work, later research transforms these foregoing products of science. The biomedical researchers under survey continuously reshaped problems, questions and practices they regarded as exemplary so as to energize their current work at the frontier of science. Communicating these changes resulted in a continuous but rather creeping process of reevaluating and revision that brought about changes in the currency of the products of the research practices.

The widespread application and availability of biological expertise and technologies in the pharmaceutical industry are at the end of the 1990's assumed matter-of-factly and especially investments in the area of genetics are regarded as of paramount importance to the development of successful therapeutic innovations in the 21 st-century. ${ }^{3}$ However, as the interferon story shows the situation in the 1960's and 1970's was rather different. Except for a small number of companies specialized in the production of biologicals (e.g. vaccines and blood products), biological research played a subordinate role in the postwar pharmaceutical industry.

Within a research environment oriented toward medical chemistry and pharmacology, it appeared rather difficult to follow up a biological research object that continued to resist existing industrial research practices. As a chemically undefined biological substance that was part of a poorly understood natural mechanism of resistance to virus infections, it was regarded as alien to the overly successful Ehrlichean chemotherapeutic industrial program. In failing to extend the work conditions of the biological laboratory into the world of commercial drug development, the early efforts to develop interferon as a drug remained fruitless. As a result the greater part of work on interferon in the 1960's and 1970's did not take place in the research departments of the commercially motivated drug companies but in academic research settings.

My aim in the first section of this concluding chapter is to analyze the material, social and cognitive characteristics of these research practices. What gets studied and why?; and what does not get studied and why not? What makes scientists discriminate between specific experimental features, ideas, methods or claims? What meanings are given to a research object like interferon and how do these change over time? How do biomedical scientists over time succeed (or fail) in expanding the private space of their workplaces to universalize the product$s$ of their scientific work? In an effort to capture the nuances of material culture and way of life

historians of science differ in their study and analysis of laboratory work, whether in the past or in the present: J. Lindenmann, 'On Toine Pieters' 'Shaping a New Biological Factor', Stud. Hist. Phil. Sci., 29 (1998), 113-6; and, T. Nickles, 'Good Science as Bad History: From order of Knowing to Order of Being', in E. McMullin (ed.), The Social Dimensions of Science (Indiana: The University of Notre Dame Press, 1992), p. 85 .

See, for an social-economic assessment of the biological turn in the pharmaceutical industry: G. Carr, The Pharmaceutical Industry', The Economist (21-2-1998). 
between bench and bedside, I will relate work on interferon to the aims, choices and interests of its practitioners as well as to the constraints and resources within which they operated and on which they thrived. Subsequently, I will analyze how the laboratory scientists and clinicians involved tried to align their research activities with broader elements of society, such as scientific institutions, governments, the public, pharmaceutical companies and regulatory bodies. This will be done by comparing the successive cycles of promise and disappointment in Britain and the USA. Finally, attention will be paid to the process of therapeutic evaluation of interferon within the context of the molecularization of medicine.

\section{Crafting interferon(-s)}

The book's narrative has led us deep into the particulars of science as practice and culture in a particular field of biomedicine in the second half of the twentieth century. To conceive of laboratory science as mere experimentation to test hypotheses and work on theoretically relevant problems appears to be as one-sided as picturing 'laboratory life' as the opportunistic management of interests and investments that allow researchers to do experiments. Neither description alone is sufficient to describe the full scope of scientific activity within the emerging field of interferon research-in other words to capture the notion of co-production of heterogeneous sets of conceptual, material and social elements which most appropriately exemplify the dynamics of researching interferon(-s). ${ }^{4}$

Basically the work at the bench can be understood as acts of:

a. learning through active experiment and intervention, engineering experimental systems with the aim of generating data of some sort and ultimately bring under surveillance new biological phenomena;

b. accounting for the sheer endless production of experimental data in a culturally meaningful way which makes them serve specific purposes-with control of the molecular processes of life at the heart of the enterprise.

A major part of the work went into fine-tuning the experimental systems so that experiments yielded results that were reproducibly usable - in other words either bear directly on the questions in hand or could be used to generate new questions. The range and priority of hypotheses and research questions with which the experiments were associated were continuously subject to changes in response to various constraints. On one occasion research questions might be adjusted because of practical limitations in the ability to manipulate the

4 The notion of 'co-production' is borrowed from Andrew Pickering, who in turn borrowed it from Bruno Latour. Finally the latter is said to be inspired by Marx; See, Pickering. 'From Science as Knowledge to Science as Practice', in A. Pickering (ed.) Science as Practice and Culture (Chicago: The University of Chicago Press, 1992) p. 14; and I. Hacking. The Self-vindication of the Laboratory Sciences', Ibid. p. 31. 
experimental set-up or because of the occurrence of unexpectedly interesting experimental events at the bench. On another, a change in research policy or a change in the status of a research approach forced researchers to reconsider their research questions. For instance, when a specific theoretical idea or concept acquired paradigmatic status in the field of interferon research, the flexible behavior at the bench changed dramatically. In those cases, the experimental events came to play a subsidiary role in the interpretation of the experimental data. Quite some perseverance was required on the part of those researchers willing to challenge specific dogmas.

Just as cognitive conditions shaped scientific work, the organization of research materials and the development of instrumentation and techniques had consequences for research practices. For instance, in chapter two I showed how the move in virus research from experimental animals to novel experimental models based on tissue culture techniques went together with a move towards describing phenomena at the levels of cells and molecules. In this sense techniques and research materials in the biomedical sciences can be understood as important means for projecting meaning on the world by acting upon it. When the biomedical researchers studied a virus sample or a viral inhibitory factor, they gave the object under study qualities that it did not have before. It gained an identity because of these qualitative and quantitative laboratory manipulations and measures.

On a more general level, at each point in time, interferon's properties were defined through the complete set of treatments and trials which interferon had undergone. By being dependent on trials-which in principle are open-ended events-for its qualifications, interferon's identity was necessarily unstable. In principle interferon changed with every new trial. Over time the scientific notion of interferon underwent a gradual transformation. First, as a viral interfering factor it was provisionally considered a deviant viral entity. By the mid1960 's 'interferon' came to refer to active antiviral substances in particular culture fluids-as a generic term-which complied with a specific set of experimental criteria and which were considered essential products of the cell that played a part in resistance to viral infection. More than thirty years later interferon is still regarded a generic term, but of yet a different kind; It refers to a specific part of the body's natural defenses consisting of a group of closely related immunomodulatory and regulatory proteins.

\section{Managing differences in a 'gift culture'}

An important window onto the nature of development and change in work on interferon is provided by cross-local and cross-national comparison. I showed that laboratories as workplaces had each their own characteristics, infrastructure and resources. The local distribution of and access to, as well as the familiarity with, particular materials and techniques 
did much to shape the pace, direction and hence content of the laboratory work. Seemingly small details of experimental techniques and small differences in experimental protocols, purity of reagents, and source or nature of biological organisms, could make major differences to the outcome of experiments. It might not come as a surprise, therefore, that a central issue for those involved in work on interferon was the uncertainty resulting from the idiosyncrasies of experimental systems and the noncompliant character of research materials and organisms.

Some major sources of contingencies were and still are biological organisms. When different organisms were used to investigate the very same question, they yielded systematically different results. Even when using the same organism in different laboratories the results hardly ever matched. Small differences in the features of the organism employed in the same laboratory already altered the nature of experimental results.

I agree with the science historian Richard Burian that an important problem of doing research with biological organisms is that at every level of biological organization it is an open question how general the findings are. ${ }^{5}$ This makes application of the basic biological sciences to clinical practice such an extraordinarily difficult and unpredictable endeavor. New knowledge of disease processes at the cellular and molecular level does not necessarily yield medical applications and simultaneously effective treatments are developed without appropriate understanding of the underlying pathological processes. In principle all laboratory systems for identifying and testing new therapeutic agents are faulty, as there is no way to mimic exactly the way the human body works - neither laboratory animals carrying human tumors nor human cells in culture seem to be faring any better in this respect. ${ }^{6}$ Yet as the interferon case shows the problem of biological contingencies and differences hardly seems to discourage the everyday practice in biomedicine of extrapolating in vitro and animal experiments and producing bold therapeutic claims based on that work.

To what extent and how did researchers in the field of interferon research cope with intra- and cross-local differences in research? I showed how initially the researchers involved managed differences by informally agreeing on exchange mechanisms, without rigorously defining common units of measurement. Through circulating, comparing and combining research materials, techniques and skills in a relatively small and flexible network of personal contacts, researchers managed to reduce extreme variations of experimental results. This enabled them to narrow down the margins of interpretation to workable levels.

Not until the informal exchange mechanism within this 'gift culture' began to show

5 See, R. M. Burian, 'How the Choice of Experimental Organism Matters: Epistemologiocal Reflections on an Aspect of Biological Practice'. Journal of the History of Biology, 26 (1993), 351-67, p. 365.

6 As recently as 1997 the executive director for cancer research at Merck Research Laboratories, Alan Oliff. had to admit that "the fundamental problem in drug discovery for cancer is that the model systems are not predictive at all": See, Trisha Gura, 'Systems for Identifying New Drugs Are Often Faulty', Science, 278 (1997). 1041-2. 
flaws - with expansion of the field of research, the shift away from a qualitative to quantitative research and the intrusion of the pharmaceutical industry into the relatively private domain of 'laboratory life'- did a need for formal standardization began to manifest itself. As I showed in chapter six, national centers for biological standardization played a central role in coordinating subsequent efforts to establish national and international interferon standards.

At least until the early 1980 's the gift culture remained largely intact. But in the late 1970 's the emerging profit potential in molecular biology, in casu the commercial prospect of mass-producing interferon with the help of genetic engineering technology, began to strain and rupture the informal traditions of scientific exchange. In the 1990's the once relatively open exchange of biological materials has become more and more formalized and selective. Increasingly research materials and techniques are regarded as commodities and therefore no longer freely exchanged as part of a gift culture but rather formally acquired for money.

\section{Seeking 'magic bullets'}

Throughout the history of the development of interferon as a therapeutic drug we have seen how claims and judgments regarding laboratory and clinical data were presented, contested, translated and negotiated by a variety of groups with competing interests and diverging backgrounds. Earlier on in the British context, and subsequently in America in the seventies, interferon came to be perceived as a potential medical breakthrough. Yet, in both cases interferon faced quite different circumstances.

This book shows that there is no simple, one-dimensional explanation of the subsequent rise and fall of interferon in Britain in the early 1960's and in the USA in the late 1970's. Neither technical arguments nor the interest of a specific social group can explain the cycle of public euphoria and disappointment in each period and country. Laboratory scientists, clinicians, industrialists and government bureaucrats operated in a complex social world with historical depth that cannot be assumed to be passive. They pursued their interests with respect to developing interferon as a therapeutic drug under social and cultural conditions that were not theirs to choose. This does not mean that these conditions were permanent fixtures in the global landscape or that the interests and policies are determined by them. Nevertheless they were experienced as important constraints or incentives and are therefore worth examining. To begin with I shall examine the initial success in mobilizing the British government and the drug industry for interferon.

The scientist fundamental to the early development of interferon Alick Isaacs exemplified a culture of and identity for biomedical research that is not conventionally associated with biomedical scientists in Britain in this epoch. Isaacs was a public man: the champion of persuasive public demonstrations, the strong competitor for public credibility, the 
self-proclaimed leader of a new field of research and the scientist-entrepreneur who boldly risks everything on what he believes is a promising phenomenon, hypothesis or potential medicine. As a public scientist Isaacs, unlike most of his British academic colleagues in biomedicine and despite his fragile state of health, made his work into a resource for scientific, political and industrial action. Isaacs was an example of a scientific way of life that came into prominence most visibly in the United States in the 1950's.

In analyzing Isaacs's success at connecting his private science with public issues of disease and cure I located in chapter four a public desire for and belief in emulating the success of penicillin, streptomycin and cortisone. These so-called 'miracle drugs' had raised hopes regarding scientific medicine's ability to come up with yet another cure. Of course this belief, far from being unique to the British, was shared by Americans and others alike. Especially the conjunction in the British context between the firm belief in breakthroughs as a dominant feature of a scientific medicine and the post-war 'penicillin trauma' (which nourished the British-American rivalry) resulted in interferon being touted publicly. This placed interferon high on the public policy agenda in Britain.

In carrying the greater part of the blame for the failure to develop penicillin in Britain, the Medical Research Council (MRC) in particular took an interest in interferon. The MRC chose interferon as a high-visibility showcase to convince the British public of their preparedness to invest in and pursue potential 'homegrown' medical innovations from the very first moment. Since the MRC lacked the expertise and resources to develop interferon as a therapeutic drug, they sought collaboration with the drug industry. Setting up a collaborative program with British pharmaceutical companies provided an additional opportunity for the MRC to present itself as a public policy stronghold in the defense of national interests.

However promising as a potential 'antiviral penicillin', British drug companies initially showed restraint with regard to interferon. This was due to the dominance of the Ehrlichean chemotherapeutic research approach in the pharmaceutical industry. Within this framework biological substances were perceived as troublesome and high-risk commodities with uncertain manufacturing prospects. MRC's offer to join forces made a difference though. From industry's point of view, risk-sharing with the MRC, which offered their unique expertise in interferon research and the eventual rights in the developed product, made work on interferon a far more attractive option. Moreover, they shared the determination of the MRC to prevent British research products with penicillin-like commercial potential from ending up with the label "made in the USA". The combination of these historically and culturally specific conditions led

7 The American researchers Jonas Salk and Maurice Hilleman serve as most notable examples of this new generation of scientific entrepreneurs in biomedicine: See, L. Galambos and J. E. Sewell, Networks of innovation: Vaccine Development At Merck, Sharp \& Dohme, and Mulford 1895-1995 (Cambridge: Cambridge University Press, 1995); and, J. S. Smith, Patenting the Sun; Polio and the Salk Vaccine (New York: William Morrow and Company, 1990). 
to the first successful postwar initiative to secure a collaboration between the British government and the drug industry.

In chapter five I showed how Ehrlich's 'magic bullet' concept of chemotherapy (which is intimately linked with scientific medicine's dominant theory of specific aetiology), as well as the closely related issue of chemical purity played a central role in the subsequent collaborative efforts to develop interferon as a therapeutic drug. Initially the association of interferon with Paul Ehrlich's ideal of generating therapeutic molecules which like 'magic bullets', seek out and destroy the enemy and injure nothing else, was helpful in keeping the pharmaceutical companies interested. The promise of a specific (that is truly curative) and an innocuous agent that would knock out viral disease made senior drug company executives believe that interferon, despite its problematic status as an unruly biological, would fit into their unprecedented successful Ehrlichean chemotherapeutic drug development program. Within this chemotherapeutic product development framework, though, the purification and chemical identification of the pharmacologically active part-conceptualized as a single and pure chemical compound-was considered a prerequisite for successful drug development.

So when attempts at purifying and characterizing interferon as well as efforts to translate the experimental laboratory methods into practical technologies for large-scale industrial production continued to fail, the evaluation of interferon's product potential in the light of the Ehrlichean chemotherapeutic program changed dramatically. In resisting all efforts to make it fit into conventional drug development practices, interferon rapidly lost its status as a most promising lead toward antiviral drug therapy. Without additional demonstrations of dramatic therapeutic results, industrial and public interest in interferon was granted only a short life.

\section{'Doctoring' the media as part of modern medicine's expectations trap}

The assessment and evaluation of the therapeutic potential of interferon as an antitumor agent in America in the seventies was for the greater part a different story, reflecting the preoccupations of a different society in a different epoch. The theme of the 1960's had been the firm and unconditional belief in therapeutic breakthroughs as a hallmark of scientific medicine-most vividly exemplified by American efforts to establish a National Program for the Conquest of Cancer. In the 1970's, however, with unprecedented public criticism of the technological-interventionist and reductionist orientation of scientific medicine, the search was on for the natural and organic. Cancer therapy, in particular, became the focus of the increasingly political powerful critical health movement in America, highlighting the failures and severe side effects of the conventional cancer treatments, surgery, radiation and chemotherapy. As a result the American cancer establishment was seriously under attack and needed a 
scientific promise which suited the growing public demands for effective and less toxic cancer remedies.

In achieving a high degree of compatibility between interferon as an innovative approach to cancer therapy and the specific medical culture of the 1970's in America, the interferon promoters succeeded in building support for interferon. Mathilde Krim and her fellow 'interferon crusaders' were able to capitalize on the public dissatisfaction with conventional cancer treatments, in placing a great deal of emphasis on the natural and presumed nontoxic qualities of interferon as an unorthodox but an evidence-based product of scientific medicine. As a result interferon became absorbed in the accelerating politics and economics of the American cancer scene.

To the public woman, Mathilde Krim, interferon represented as much marketing as science. She was well aware of the ever-growing importance of the mass media in generating public support and was very skillful at 'doctoring' the media in ways that would fulfil the public desire for miracle cures. She helped to create a media image of interferon as a somewhat mysterious and scarce, nontoxic natural body substance that offered a unique scientific approach to cancer, but was long ignored by a conservative cancer establishment.

In successfully mobilizing the popular media, Krim and her allies managed to reshape the agenda-setting forum dramatically from a relative private, scientific experts dominated, affair into a public policy arena-with the press blooming into the most important institutional link in the network of relationships which developed around interferon. The cancer experts in the bureaucracy and academia were forced into the open, allowing other interested parties to participate in the judgment and definition of work on interferon. Through the dual framing of interferon as a natural solution to a dread disease and the latest product of 'cutting-edge' biomedical research that promised to radically alter the expectations of life, interferon acquired 'miracle drug' status.

The expectations' boom was fueled in an interesting way by a new 'breed' of entrepreneurial molecular biologists who jumped on the interferon bandwagon-choosing interferon as a demonstration project for the industrial potential of the revolutionary genetic engineering technology. In bringing together in their claims what were widely perceived as two wondrous products of modern science and technology, the genetic engineers with the help of the media triggered an international interferon mania.

My claim is that the recurrent hailing of wonder drugs in the western world symbolizes the religious faith in a scientific medicine that promises to fulfil the quest for immortality. It is part of what Roy Porter calls the 'rising expectations trap' of modern medicine. ${ }^{8}$ However, in being intimately linked on the one hand with the almost sacrosanct belief that disease as we 
know it can be eliminated by medical technology and on the other hand with the disappointing everyday experience of incurable disease and failing remedies, the label 'wonder drug' is transitory.

As long as the public enthusiasm interferon elicited was not associated with the term 'uncontrollable', the dramatic portrayal of interferon was widely accepted as necessary for the advancement of a most promising area of biomedical research. However, confronted with a public enthusiasm for interferon which could not be met and threatened to get out of hand, the biomedical establishment began to regard the hype as problematic. Once defined as a threat to the authority over the medical realm, an international coalition of doctors, researchers and administrators resorted to a deliberate creation of public disillusionment. In a similar fashion as the interferon alliance had been overly successful in seeking a favorable press, the biomedical establishment managed to reshape the nature of the media coverage of interferon-from forceful promotion of interferons benefits to disappointment about a failed promise. ${ }^{9}$

\section{Biology meets drug development: toward molecular medicine}

The growing interference, however, between interferon research and the field of molecular biology, played a major role in bringing about a redefinition of interferon's prospects as an object of research for the pharmaceutical industry in the late 1970's and early 1980's. In particular, implementing the newly developed genetic engineering technology made an important difference. Originally deployed with the aim to make available in large quantities, at modest cost, highly purified interferon preparations, the molecular biologists, closely linked with the emerging genetic engineering industry, helped to redraw the interferon landscape beyond recognition.

In efforts to clone the interferon gene, produce the corresponding interferon protein product and reach agreement on interferon's chemical structure, the 'genetic engineers' succeeded in promoting interferon not only to the world of 'big science' and industry, but also

9 In an interesting way this interventionist scenario has a repetitious quality. In the case of other post-Second-

World war therapeutic drugs which achieved 'miracle drug' status like cortisone and streptomycin a similar kind of deliberate creation of disillusionment occurred as a means to dampen public enthusiasm and to stem the tide of public requests which could not be met. Apparently as long as the public enthusiasm it elicits can not be associated with the term 'uncontrollable', an element of optimism in advertising a new remedy is widely regarded and even promoted as necessary for the advancement of medicine. In my view the limit of acceptable, controllable enthusiasm depended in each case on the extent to which the biomedical establishment feared a loss of authority over the medical domain. See, D. Cantor, 'Cortisone and the Politics of Drama, 1949-1955', in J. Pickstone, Medical Innovations in Historical Perspective (London: MacMillan, 1992); A. Yoshioka. 'Streptomycin: Notes Toward a Cultural History of a Miracle Drug', unpublished paper presented during the Anglo-Dutch workshop on Remedies and Healing Cultures in Britain and the Netherlands in the Twentieth Century in Amsterdam, June, 1998. 
to the tangible world of chemical facts. By making attainable the 'Holy Grail' of interferon research-pure interferon-cloning put an end to the important role of chemical purity as an arbiter in disputes concerning work on interferon both in industry and academia.

However, with the intention of achieving homogeneity and progress as well as unity in the understanding of the interferon system, the molecular biologists had paradoxically created heterogeneity and new complexity by reinforcing the idea that 'interferon' represented a dynamic family of genes and corresponding proteins. In an attempt to manage the unwieldy complexity, they fell back on the concepts of 'command', 'control' and 'communication networks' which had proved successful explanatory tools in molecular biology. In doing so the molecular biologists promoted the conceptualization of interferon as part of a complex host defense system of cellular messengers, so-called 'biological response modifiers', held together by communication and feedback. This was part of a much broader shift in the biomedical realm from 'substance thinking', looking at the biochemical 'nuts and bolts' in the machinery of the body, to 'information thinking', picturing the body as a complex regulatory-communications network. ${ }^{10}$

The cloning efforts not only strengthened the idea of interferon as one of the body's regulatory defense systems, but also opened the door to an alternative approach to drug development. The genetic engineers promised to create a new horizon in pharmaceutical research. In concentrating on molecular biology (e.g. proteins and DNA) rather than on traditional chemistry, they would provide the drug companies with an entire apothecary of new biosynthetic drugs from natural substances within the body. As promoters of this new approach, they stressed novelty but at the same time they presented genetic engineering as fitting in well with the classic chemistry-based therapeutic drug development practice. With a steady decline in commercially viable and innovative therapeutic compounds from their chemistry-dependent 'pipelines', the promise of a new approach to drug development kept the pharmaceutical industry interested when the news about interferon's less-than-spectacular performance in clinical trials began to spread in the early 1980 's.

In chapter eight $\mathrm{I}$ have shown that in order to become legitimized as part of medical practice against all odds, interferon needed a therapeutic profile and treatment concept that could be integrated or combined with existing therapeutic routines. This required that the drug makers in collaboration with laboratory researchers and clinicians actively created and made available a sort of rough-and-ready 'therapeutic rationale'. Clinicians and laboratory scientists alike increasingly perceived cancer as a family of diseases in which complex cellular and molecular regulatory functions have gone awry, instead of a unitary disease characterized by the uncontrolled growth of malignant cells that spread out aggressively and destroy the body.

10 See, for a similar shift in the way immunity was dealt with in American culture: E. Martin, Flexible Bodies (Boston: Beacon Press, 1994), p. 61. 
In line with this shift, the framework in which to consider patients, cancer and its treatments could no longer be effectively and acceptably be linked with the therapeutic ideal of generating and using universal cancer medications which like 'magic bullets' seek out and destroy the enemy. In other words what used to be a socially and scientifically influential metaphor in the 1960 's and 1970's as part of the 'biochemical' paradigm in medicine, ceased to be so by the 1990 's, though without medicine becoming less target-focused. ${ }^{11}$ My claim is that magic bullets make for meaningless and potentially destructive talk in a dynamic, information thinking dominated, 'molecular medicine'. ${ }^{12}$

Talking about the "Big C" as a multi-factorial molecular disease was consistent with the emergence of immunotherapy as part of a combined-modality, laboratory-supported therapeutic framework in clinical oncology. This linked up perfectly with the efforts in the field of interferon research to redefine interferon as a means to develop a new complementary modality of cancer treatment that might be used in conjunction with conventional therapies. Redefining interferons as biological response modifiers or cytokines which could be used therapeutically to strengthen the body's self-defence as part of a laboratory-guided multi-modality therapy framework, legitimated and reinforced the new molecular perception and treatable nature of cancer. And this allowed all parties to see interferon therapy as therapeutic. To put it differently, as advertised products of molecular biology the interferons fitted in well with the 'molecularization' of medicine and this helped to make them 'work' at the bedside. I pointed to the solidarity implicit in the emphasis of both laboratory researchers and clinicians on the importance of linking through interferon bench and bedside as part of a new kind of molecular medicine. In always being able to justify that either further laboratory or clinical research was mandated, it made all parties involved relative immune to disappointing research news.

The story of how interferon managed to become part of the 'doctor's bag' clearly shows how the conduct, organization and evaluation of clinical trials and what they are capable of is dependent on the specific context of use. It reveals that the use and interpretation of clinical trials are significantly different for experimental drugs for the desperately ill than they are for research into less serious diseases like the common cold. Furthermore, in achieving a key position in the distribution of research materials and resources needed to set up preclinical and clinical studies, the pharmaceutical industry increasingly dictated the development and use of interferon. It was the industry itself that profited most from the very dialectical nature of the clinical trial "enterprise". I have shown that the 'industry' of clinical trials proved effective not

II See, for an exemplary study of the use of methaphors in Biology: E. F. Keller, Refiguring Life; Metaphors of Twentieth-Century Biology (New York: Columbia University Press, 1995), pp. 94-114.

12 See, for a detailed historical description and analysis of the rise of the molecular culture in biomedicine in the Twentieth Century: S. de Chadarevian and H. Kamminga (eds), Molecularizing Biology and Medicine (Amsterdam: Harwood Publishers, 1998). 
only in widening interferon's therapeutic profile but also in marketing the combined modality therapeutic concept that turned the interferons from orphan drugs into top-selling pharmaceuticals.

As such, this biography of a family of therapeutic drugs suggests that, rather than the therapeutic evaluation and use of pharmaceutical substances being a straightforward and strictly evidence-based process, it is a thoroughly practice-based activity which cannot be separated from the values and commitments of those involved. This is at odds with the conventional view that given sufficient methodological rigor-e.g. in the form of randomized clinical trials-'social bias' can be eliminated from medical knowledge. However, if as I have argued, social and cultural factors play a significant part in shaping the clinical profile of a drug, then it follows that the application of the interferons should continue to be scrutinized, without maintaining the illusion that 'social bias' can be eliminated. Hence, as a product of the history here described, the current practice of maximum tolerated dosage regimens for the application of interferons in cancer therapy, might perfectly fit in with the culture of oncology, but the therapeutic potential of these intriguing substances might thereby be limited.

In carefully avoiding labeling this chapter 'conclusion' I want to emphasize that the biography of the interferons is still in process. As an inseparable element of the culture of molecular medicine the interferons help to shape the goals, methods and organization of this very same culture. The final section confirms this idea. It is meant as a speculative assessment of and hint to current and future developments in the biomedical realm, although as a historian I am well aware of the risks involved in trying to interrogate the past as a means to help direct current and future policies. The unpredictable and dynamic nature of the practices, contexts and relations described makes it hazardous to predict the shape that this part of the 'marketplace' medicine will take in the future let alone suggesting new life forms. However, knowing that the application of historical knowledge to cope with current and future situations is a problematic endeavor, should not withhold us from doing so. As Thomas Nickles puts it succinctly: "the only resources available to a generation, besides its own ingenuity, are those bequeathed by its past". ${ }^{13}$

\section{Patients at risk}

So far we have seen a wide range of factors and pressures that shaped the clinical profile and use of the interferons: the disputes among scientists, the cycles of promise and disappointment, the biological turn in drug development, the 'industry' of clinical trials, the religious faith in the healing potential of scientific medicine, the marketing efforts of the pharmaceutical companies,

13 See, Thomas Nickles, 'Philosophy of Science and History of Science', Osiris, 10 (1995), 139-63, p. 154. 
the politics of regulation, the molecularization of medicine and so on. Given the complex environment in which the safety and effectiveness of this family of therapeutic molecules had to be assessed, is it any wonder that changes in this context can have unintended effects?

My report of 'patients at work' in the preface is exemplary for the profound changes the context of therapeutic evaluation has undergone since the official licensing of interferon-alpha for the treatment of a rare form of leukemia in 1986. In the traditional, that is pre-AIDS therapeutic framework, the testing and use of new therapies basically hinged on their evaluation by professionally-endorsed evaluators and professionally-endorsed methods. In the late 1980's, however, the Aids-crisis and the associated patient-activist interventions in biomedicine-which led to new liberalized drug regulatory and testing practices for the desperately ill-demonstrated compellingly the potential for change. Ever since, patient grass-root organizations have been increasingly successful in actively participating in the biomedical realm. In funding disease-related research and services, and establishing strategic alliances with academic research, industry and government, they have managed to exert a growing influence on the review and decision-making process of science and medicine.

At the end of the 1990's world-wide associations of patients like the Dutch or American Multiple Sclerosis Societies permanently monitor research and policy issues and try to convert these into information that is meaningful for individual consumers' health. Through magazines, newsletters and other publications and Internet Web-sites they keep people living with the disease, their families and healthcare providers informed about the latest disease-related news items. However, the consumer organizations in medicine make for more than clearing houses of information. In establishing and supporting counseling groups, public meetings, training workshops for healthcare professionals, scientific conferences and most lately internet news groups, they have come to serve as national and international platforms for the exchange and critical appraisal of information on medical research, care and policy.

The rapid developments in internet technology - with personal computers evolving into powerful communication tools-have played a most important role in changing the rules of the game in the market-place medicine. Through the Web, patients can freely exchange experiences with fellow-sufferers, ask treatment advise from doctors who are not theirs and may obtain the latest biomedical updates before health-care professionals. ${ }^{14}$ In principle, having unprecedented access to the world of science and medicine, should put them in an equal position of knowledge with the other parties involved.

However it remains to be seen whether patients are indeed the ones to profit most of the combination of liberalized regulatory and drug testing practices, and a freely accessible medical 'cyberworld'. The sociologist, Steven Epstein, rightly indicates that however vigilant

14 See, for MS-related sides and newsgroups: wwwnmss.org; www.mssociety.org.uk; www.mssoc.ca; and alt.support.mult-sclerosis. 
the fast growing consumer movement may seem, they underestimate the capability of science and industry to influence and redirect the consumer's interventions to their own advantage. ${ }^{15}$ The rise, fall and subsequent rise of interferon certainly reflects the considerable power of the coalition between laboratory medicine and the drug industry to monopolize the information on the safety and efficacy of therapeutic drugs. This makes it hard for consumers and certainly the desperately ill, who are dedicated to try anything that enables them to feel less than totally impotent in the face of a wasting disease, to seriously challenge knowledge claims. Paradoxically, the efforts to open up the biomedical stronghold and push medical research in directions that serve patient interests more directly, reinforce the dependence on the very same academic and industrial producers of medical products and services.

15 See, S. Epstein, 'Activism Drug Regulation, and the Politics of Therapeutic Evaluation in the Aids Era: A Case Study of ddc and the 'Surrogate Markers' Debate', Social Studies of Science, 27 (1997), 691-726. 
All persons who have been interviewed in the course of the research reported on in this thesis are listed below. Apart from Dr. all other titles (professor, etc.) are omitted. Asterisks indicate that the interview was tape-recorded.

\section{Pharmaceutical Industry:}

\begin{tabular}{|c|c|c|c|}
\hline Dr. L. Adami* & (Roche) & Basel & June 19,1990 \\
\hline Dr. John Beale* & (Wellcome) & London & May 24,1990 \\
\hline Dr. M. Fernex* & (Roche) & Basel & June 18,1990 \\
\hline Dr. Karl Fantes* & (Wellcome) & Beckenham, U.K. & May 25,1990 \\
\hline Mr. G. Farmer* & (Roche) & Basel & June 20,1990 \\
\hline Dr. Norman Finter* & (Wellcome) & Seven Oaks, U.K. & May 25,1990 \\
\hline Dr. Leon Gauci* & (Roche) & Basel & June 18,1990 \\
\hline Dr. G. Garotta* & (Roche) & Basel & June 19,1990 \\
\hline Mrs. C. v. Helsen & (Schering-Plough) & Brussels & June 23,1988 \\
\hline Dr. P. Herman & (Schering-Plough) & Brussels & June 23,1988 \\
\hline Dr. Maurice Hillema & $\mathrm{n}^{*} \quad(\mathrm{MSD})$ & West Point, PA & Oct. 10, 1992 \\
\hline Dr. P. Hunold* & (Roche) & Basel & June 18,1990 \\
\hline Dr. E. Hochuli* & (Roche) & Basel & June 21,1990 \\
\hline Dr. A. Keller* & (Roche) & Basel & June 20,1990 \\
\hline Dr. H. Leuenberger* & (Roche) & Basel & June 20,1990 \\
\hline Dr. M. Placchi* & (Roche) & Basel & June 19,1990 \\
\hline Mr. P. de Pourq & (Schering-Plough) & Brussels & June 23,1988 \\
\hline Dr. J. Ryff* & (Roche) & Basel & June 18,1990 \\
\hline Dr. David Secher* & (Cell-Tech) & London & May 23,1990 \\
\hline Dr. F. Schenker* & (Roche) & Basel & June 18,1990 \\
\hline Dr. T. Staehlin* & (Ciba-Geigy) & Basel & June 18,1990 \\
\hline Dr. J. P. Warbeer & (Schering-Plough) & Brussels & June 23,1988 \\
\hline
\end{tabular}

Academic Research and Medicine:

Dr. Frances Balkwill*

Dr. Samuel Baron*

Dr. Alfons Billiau*

Dr. Derek Burke*

Dr. Werner Boll*

Dr. Ernest Borden*

Dr. Derek Bangham*

Dr. Kari Cantell*

Dr. Charles Chany*

Dr. Lois Epstein*

Dr. Ernesto Falcoff*

Dr. Emil J. Freireich*

Dr. Robert Friedman*

\section{London}

Galveston, TX

Leuven, B.

Norwich

Zurich

Milwaukee, WI

London

Helsinki

Paris

San Francisco

Paris

Houston

Bethesda, MD
May 31, 1990

Sept. 24, 1992

Aug. 4, 1992

May 15, 1990

June 21, 1990

Oct. 12, 1992

March 3, 1992

May 5, 1992

Sept. 2, 1992

Oct. 8, 1992

Sept. 3, 1992

Sept. 23, 1992

Oct. 22, 1992 


Dr. Jordan Gutterman*
Dr. Ion Gresser*
Dr. Susanna Isaacs Elmhirst*
Dr. Monto Ho*
Dr. Pete Knight*
Dr. Mathilde Krim*
Dr. Hilton Levy*
Dr. Jean Lindenmann*
Dr. Edward de Maeyer*
Dr. Jacqueline de Maeyer*
Dr. Thomas Merigan*
Dr. Sidney Pestka*
Dr. M. Playfair*
Dr. James Porterfield
Dr. Huub Schellekens*
Dr. Gerald Sonnenfeld*
Dr. Joseph Sonnabend*
Dr. Hans Strander*
Dr. Michael Streuli*
Dr. Joyce Taylor*
Dr. David Tyrrell*
Dr. Jan Vilcek*
Dr. Charles Weissman*
Dr. Robert Wagner*
Dr. Frederick Wheelock*

Houston

Paris

London

Pittsburgh, PA

West Chester, PA

New York

Frederick, MD

Zurich

Paris

Paris

Stanford, CA

Piscataway, NJ

London

(tel. interview)

Delft, NL

Louisville, KY

New York

Stockholm

Zurich

London

Salisbury, U.K.

New York

Zurich

Charlottesville, VA

Philadelphia
Sept. 23, 1992

Sept. 1, 1992

May 28, 1990

Oct. 29, 1992

Nov. 4, 1992

Nov. 11, 1992

Oct. 10, 1992

June 16, 1990

Dec. 4, 1993

Dec. 4, 1993

Oct. 7, 1992

Oct. 27, 1992

May 29, 1990

Oct. 11, 1993

July 10,1992

Oct. 14, 1992

Nov. 12,1992

April 30, 1992

June 18, 1990

May 30, 1990

May 21, 1990

Nov. 10, 1992

June 15,1990

Oct. 13, 1992

Nov. 5,1992

\section{Government Institutions and Regulatory Bodies}

$\begin{array}{llll}\text { Dr. George Galasso* } & \text { (NIAID) } & \text { Bethesda, MD } & \text { Oct. 19, } 1992 \\ \text { Dr. Kathy Laughlin* } & \text { (NIAID) } & \text { Bethesda, MD } & \text { Oct. 23, 1992 } \\ \text { Dr. Arthur Levine* } & \text { (NCI) } & \text { Bethesda, MD } & \text { Oct. 22, 1992 } \\ \text { Dr. John Petricciani* } & \text { (FDA) } & \text { Cambridge, MA } & \text { Nov. 6, 1992 } \\ \text { Dr. Frank Rauscher* } & \text { (ACS) } & \text { Stamford, CT } & \text { Oct. 16, 1992 } \\ \text { Dr. Kathryn Zoon* } & \text { (FDA) } & \text { Bethesda, MD } & \text { Oct. 23, 1992 }\end{array}$




\section{Bibliography}

J. D. Almeida, R.C. Hasselback and A.W. Ham, 'Virus-like Particles in Blood of Two Acute Leukemia Patients', Science, 142 (1963), 1487-9

J. Alper, 'First there was Interferon', The New York Times, 18 November 1984, F. 13.

O. Amsterdamska, 'Medical and Biological Constraints: Early Research on Variation in Bacteriology', Social Studies of Science, 17 (1987), 657-87

S. Andreopoulos, Sounding Board; and, Gene Cloning by Press Conference, New. Engl.J. Med., 302 (1980), 743-6.

C, Armstrong, 'Passage of a Fresh Human Strain of Poliomyelitis Virus (Lansing Strain) from the Monkey to Eastern Cotton Rats (Sigmodon hispidus hispidus)', Pub. Health Rep., U.S.P.H.S., 54 (1939), 1719-29

P. Atanasiu and C. Chany, 'Action d'un Interféron Provenant de Cellules Malignes sur L'infection Expérimental du Hamster Nouveau-né par le Virus du Polyoma', C.R.H.S.A.S., 251 (1960), 1687-9

C. Andrewes, 'Interference by one Virus with the Growth of Another in Tissue-culture', Brit. J. Exp. Path., 23 (1942), 214-20

C. Andrewes, 'Virus Diseases of Man: A Review of Recent Progress', Brit. Med. Bull., 2 (1944), 265-9

C. H. Andrewes, 'Alick Isaacs', Biographical memoirs of fellows of the Royal Society, 13 (1967) 205-21

L. E. Amow, Health in a Bottle; Searching for the Drugs that Help (Philadelphia: J.B. Lippincott Company, 1970)

J. Austoker and L. Bryder (eds.) Historical Perspectives on the Role of the MRC (Oxford: Oxford University Press, 1989)

J. Bader, 'Production of Interferon by Chick Embryo Cells Exposed to Rous Sarcoma Virus', Virology 16 (1962), 43643

D. Balducci and G. Penso, Tissue Cultures in Biological Research (Amsterdam: Elsevier Publishing Company, 1963)

F. Balkwill, What Future for the Interferons?, New Scientist, (1980), 230-2

F. Balkwill, 'Interferon: A Progress Report', New Scientist, 25 March 1982, 783-5

F. Balkwill, Interferons: From Common Colds to Cancer, New Scientist, 14 March 1985

F. Balkwill, Cytokines in Cancer Therapy (Oxford: Oxford University Press, 1989).

S. Baron and A. Isaacs, 'Interferon and Natural Recovery from Virus Diseases'. New Scientist 243 (1961), $81-2$

S. Baron, F. Dianzani, G. Stanton and W. Fleischmann (eds.), The Interferon System: A Current Review to 1987 (Austin, TX: The University of Texas Press, 1987)

T. M. Bell, An Introduction to General Virology (London: William Heinemann Medical Books, 1965)

W. Beveridge and F. Burnet, The Cultivation of Viruses and Rickettsiae in the Chick Embryo. Special Report Series, No. 256 of the Medical Research Council (London: His Majesty's Stationery Office, 1946)

A. Billiau and N. Finter (eds.) Interferon 1; General and Applied Aspects (Amsterdam: Elsevier, 1984)

M. Bliss, The Discovery of Insulin (London: Faber and Faber, 1982) 
W. Bijker, T. P. Hughes and T. J. Pinch (eds), The Social Construction of Technological Systems (Cambridge (MA): The MIT Press, 1987)

W. Bijker, Of Bicycles, Bakelites and Bulbs: Toward a Theory of Sociotechnical Change (Cambridge (MA): MIT Press, 1995)

W. Breckon, The Drug Makers (London: Eyre Methuen Ld, 1972)

R. Bud, 'Penicillin and the new Elizabethans', British Journal of the History of Science, 31 (1998), 305-33

R. M. Burian, 'How the Choice of Experimental Organism Matters: Epistemologiocal Reflections on an Aspect of Biological Practice', Journal of the History of Biology, 26 (1993), 351-67

G. Buddingh, 'Chick-Embryo Technics', in T. Rivers (ed.) Viral and Rickettsial Infections of Man (Philadelphia: J.B. Lippincott Company, 1952)

F. M. Burnet, The Use of the Developing Egg in Virus Research, Special Report Series, No. 220 of the Medical Research Council (London: His Majesty's Stationery Office, 1936)

F. M. Burnet, Principles of Animal Virology (New York, Academic Press, 1955)

D. C. Burke and A. Isaacs, 'Studies on the Production, Mode of Action and Properties of Interferon', Brit. J. exp. Path., 38 (1957), 551-62

D. C. Burke and A. Isaacs, 'Further Studies on Interferon', Brit. J. exp. Path., 39 (1958),78-84

D. C. Burke and A. Isaacs, 'Some Factors Affecting the Production of Interferon', Brit. J. exp. Path., 39 (1958), $452-8$.

D. C. Burke, 'The Purification of Interferon', Biochem. J., 78 (1961), 556-64

D. C. Burke and A. Buchan, 'Interferon Production in Chick Embryo Cells; I. Production by Ultraviolet- Inactivated Virus', Virology, 26 (1965), 28-35

D. C. Burke, 'The Status of Interferon', Scientific American, 236 (1977), 42-50

D. C. Burke, 'Early Days with Interferon', J. Interferon Res., 7 (1987), 441-3

M. Buyse, M. Staquet and R. Sylvester (eds.), Cancer Clinical Trials (Oxford: Oxford University Press, 1984)

P. Came and W. Carter (eds.), Interferons and their Applications (Berlin: Springer-Verlag, 1984)

E. Canetti, Nachträge aus Hampstead: Aus den Aufzeichnungen 1954-1971 (München: Carl Hanser Veriag, 1994)

K. Cantell, 'Why is Interferon not in Clinical Use Today?', in I. Gresser (ed.), Interferon, (London: Academic Press, 1979), pp. 2-28

K. Cantell, The Story of Interferon: The Ups and Downs in the Life of a Scientist (Singapore, World Scientific, 1998)

D, Cantor, Cortisone and the Politics of Drama, 1949-55, in J.V. Pickstone (Ed.), Medical Innovations in Historical Perspective (Hampshire, Macmillan Distribution, 1992), pp, 175-6.

M. Casper and M. Berg, Constructivist Perspectives on Medical Work: Medical Practices and Science and Technology Studies, Science, Technology \& Human Values, 20 (1995), 395-407

G. Carr, The Pharmaceutical Industry', The Economist (21-2-1998)

A. Clarke and J. Fujimura (eds.) The Right Tools for the Job (Princeton: Princeton Univ. Press, 1992) 
A. Clarke and T. Montini, The Many Faces of RU486: Tales of Situated Knowledges and Technological Contestations, Science, Technology and Human Values, 18 (1993), 42-78

T. C. Chaimers, The Clinical Trial', Milbank Memorial Fund Quarterly/ Health and Society, 41 (1972), 753-8

C. Chany, 'An Interferon-like Inhibitor of Viral Multiplication from Malignant Cells (the viral autoinhibition phenomena)', Virology, 13 (1961), 485-92.

C. Chany, 'Interferon-like Inhibitor of Viral Multiplication from Malignant Cells', Virology, 13 (1961), 485-92

S. de Chadarevian and H. Kamminga (eds), Molecularizing Biology and Medicine (Amsterdam: Harwood Publishers, 1998)

W. C. Cockburn, The International Contribution to the Standardization of Biological Substances. I. Biological standards and the League of Nations 1921-1946', Biologicals 19 (1991), 161-9

V. Cohn, 'Leading U.S. Cancer Doctors Agree to Issue Warnings on Interferon'. The Washington Post, 15 June 1980.

H. M. Collins, Changing Order (London: Sage Publications, 1985)

H. M. Collins, and T. Pinch, The Golem: What Everyone should know about Science (Cambridge and New York: Cambridge University Press, 1993)

R. P. T. DavenPort Hines and J. Slinn, Glaxo: A History to 1962 (Cambridge: Cambridge University Press, 1992)

M. Delbrick and S. Luria, 'Interference Between Bacterial Viruses', Arch. Biochem., 1 (1943), $111-41$

R. Depoux and A. Isaacs, 'Interference between Influenza and Vaccinia Viruses', Brit. J. exp. Path. , 35 (1954), $415-18$

R. Doerr and S. Seidenberg. 'Die Konkurrenz von Virusinfektionen im Zentralnervensystem (Phainomen von Fl. Magrassi)', Z Hyg., 119 (1937a), 135-65

R. Doerr and M. Kon, 'Schieneninfektion, Schienenimmunisierung und Konkurrenz der Infektionen im Zentralnervensystem beim Herpesvirus', Z Hyg., 119 (1937), 679-705

H. Donald and A. Isaacs, 'Counts of Influenza Virus Particles', J. gen. Microbiol., 10 (1954), 457-64

R. Doll, 'Development of Controlled Trials in Preventive and Therapeutic Medicine', J. Biosoc Sci, 23 (1991), $365-78$

R. Dulbecco, Production of Plaques in Monolayer Tissue Cultures by Single Particles of an Animal Virus', Proc. Nat. Acad. Sci. U.S.A., 38 (1952), 747-52

J. Van Dyck, Manufacturing Babies and Public Consent (New York: New York University Press, 1995)

M. Edelhart, Interferon: The New Hope for Cancer (Reading: Addison-Wesley Publishing Company, 1981)

P. Ehrlich, 'Chemotherapy'. In: The collected papers of Paul Ehrlich, (Himmelweit, F., Ed.) Vol 3 (1914)

B. Elzen, Scientists and Rotors; The Development of Biochemical Ultracentifuges (Enschede: PhD-Thesis, 1988)

J. Enders, 'A Consideration of the Mechanisms of Resistance to Viral Infection Based on Recent Studies of the Agents of Measles and Poliomyelitis', Trans. Stud. Coll. Phys. Phil.. 28 (1960), 68-79

L. Epstein, Interferon as Model Lymphokine, Fed. Proc., 40 (1981), pp. 56-61

S. Epstein, 'Activism Drug Regulation, and the Politics of Therapeutic Evaluation in the Aids Era: A Case Study of ddc and the 'Surrogate Markers' Debate', Social Studies of Science, 27 (1997), 691-726. 
P. Faasse, Experiments in Growth (Amsterdam: Ph.D. diss., Univ. Amsterdam, 1994)

P. Fairley, Whitehall Men Join Battle on Common Cold, Evening Standard, Britain, 5 October, 1962.

E. Falcoff, R. Falcoff, F. Fournier and C. Chany. 'Production en Masse, Purification Partielle et Charactérisation d'un Interferon Destiné a des Essais Thérapeutiques Humains', Annales de l'Institut Pasteur, (5) 1966, 562-84

C. Fenyvesi, 'Beyond Interferon', The Washington Post, 14 June 1981

N. Finter, 'A Rich Source of Mouse Interferon, Nature, 204 (1964), pp. 1114-5

N. Finter, 'Protection of Mice by Interferon Against Systemic Virus Infections', Brit. Med. J., 2 (1964), 981-85

N. Finter (ed.) Interferons (Amsterdam: North-Holland Publishing Company, 1966)

N. Finter (ed.), Interferons and Interferon Inducers (Amsterdam: North Holland Publishing Company, 1973)

G. M. Findlay and F. O. MacCallum, 'An Interference Phenomenon in Relation to Yellow Fever and Other Vinuses', J. Path. and Bact., 44 (1937) 405-24

N. Finter and R. Oldham (eds.), Interferon (vol4); In Vivo and Clinical Studies (Amsterdam: Elsevier, 1985)

L. Fleck, Genesis and Development of a Scientific Fact (Chicago: The University of Chicago Press, 1979)

E. J. Freireich and E. Frei, III, 'Recent Advances in Acute Leukemia', in Tocantins, L M. (ed.) Progress in Hematology, Vol IV (New York: Grune and Stratton, 1964), pp. 187-202.

E. Fleissner, 'Salvador Luria', in L. Levidow (ed.) Science as Politics, Radical Science Series, no. 20 (London: Free Association Books, 1986)

R. Friedman, A. S. Rabson, W. Kirkham, Variation in interferon Production by Polyoma Virus Strains Differing Oncogenicity, Proc. Soc. Exp. Biol. Med., 112 (1963), 347-51

R. Friedman. 'Interferon Research in the Red Queen's Kingdom'. Arch. Pathol. 98 (1974), 73-6

R. Friedman, 'Guest Editorial: Interferon and Cancer', J. Natl. Cancer, 60 (1978), 1191-4

J. H. Fujimura, Crafting Science: A Sociohistory of the Quest for the Genetics of Cancer (Cambridge (MA): Harvard University Press, 1996)

F. Fulton and A. Isaacs, 'Influenza Virus Multiplication in the Chick Chorioallantoic Membrane', J. Gen. Microbiol., 9 (1953), 119-31

L. Galambos and J. E. Sewell, Networks of innovation: Vaccine Development At Merck, Sharp \& Dohme, and Mulford 1895-1995 (Cambridge: Cambridge University Press. 1995)

L. Galambos and J. L. Sturchi. The Transformation of the Pharmaceutical Industry in the Twentieth Century. In J. Krige and D. Pestre (eds. ) Science in the Twentieth Century (Amsterdam: Harwood Academic Publishers, 1997)

S. Gard, Tissue Immunity in Mouse Poliomyelitis', Act. Med. Scand., 119 (1944), 27-46

L. Garrett, The Coming Plague (New York: Farrar, Straus and Giroux, 1994)

E. Gehan and E. Freireich, Non-randomized controls in Cancer Clinical Trials', New Engl. J. Med., 290 (1974), 198203 
N. Gibbons (ed.), Recent Progress in Microbiology, Proceedings of the VIII International Congress for Microbiology. Montreal 1962 (Toronto, University of Toronto Press, 1963), pp. 419-57

L. Glasgow and K. Habel, 'Role of Interferon in Vaccinia Virus Infection of Mouse Embryo Tissue Culture', J. Exper. Med., 115 (1962), 503-12

L. Glasgow, 'Interrelationships of Interferon and Immunity During Viral Infections', J. Gen. Phys., 56 (1970), 212-27

J. Golinski, Making Natural Knowledge: Constructivism and the History of Science (Cambridge: Cambridge University Press, 1998)

D. Gooding. T. Pinch and S. Schaffer (eds.) The Uses of Experiment (Cambridge: Cambridge University Press, 1989)

D. Gooding, Experiment and the Making of Meaning (Dordrecht: Kluwer Academic Publishers, 1990)

A. Grafe, A History of Experimental Virology (Berlin: Springer-Verlag, 1991)

D. G. Grahame-Smith, Problems Facing a Regulatory Authority, in J. F. Cavalla (ed.) Risk-Benefit Analysis in Drug Research (Lancaster, MTP Press Limited, 1981)

D. E. Green, 'An Experiment in Communication: The Information Exchange Group', Science, 143 (1964), 308-9

H. Greenberg, M. Richard, R. Pollard, L. Lutwick, P. Gregory. W. Robinson and T. Merigan, 'Effect of Human Leucocyte Interferon on Hepatitis B Virus Infection in Patients with Chronic Active Hepatitis', The New England Journal of Medicine, 295 (1976), 517-22.

I. Gresser, J. Coppey, E. Falcoff and D. Fontaine, 'Action Inhibitrice de L'interféron brut sur le Développement de la Leucémie de Friend Chez la Souris', C.R. Acad. Sci. Paris, 263 (1966). 586-88

I. Gresser, Coppey, J., E. Falcoff and D. Fontaine, 'Interferon and Murine Leukemia.. I. Inhibitory effect of Interferon Preparations on the Development of Friend Leukemia in Mice, P.S.E.B.M., 124 (1966), 84-91

1. Gresser, D. Fontaine, J. Coppey, R. Falcoff and E. Falcoff, Interferon and Murine Leukemia. II. Factors Related to the Inhibitory Effect of Interferon Preparations on the Development of Friend Leukemia in Mice, P.S.E. B.M., 124 (1966), $91-4$

I. Gresser, J. Coppey, D. Fontaine-Brouty-Boyé, R. Falcoff, 'Interferon and Murine Leukemia. III: Efficacy of Interferon Preparations Administered after Inoculation of Friend Virus'. Nature, 215 (1967), 174-5

I. Gresser, R. Falcoff, D. Fontaine-Brouty-Boyé, F. Zajdela, J. Coppey, E. Falcoff, 'Interferon and Murine Leukemia. IV. Further Studies on the Efficacy of Interferon Preparations Administered after Inoculation of Friend Virus'. P.S.E.B.M., 126 (1967), 791-7

1. Gresser, C. Bourali, J.P. Lévy, D. Fontaine-Brouty-Boyé, M. Thomas, Cancérologie. Prolongation de la Survie des Souris Inoculées avec des Cellules Tumorales et Traitées avec des Préparations d'Interferon, C.R. Acad. Sci., 268 (1969), 994-7

I. Gresser, C. Bourali, J.P. Lévy, D. Fontaine-Brouty-Boyé, M. Thomas, Increased Survival in Mice Inoculated with Tumor cells and Treated with Interferon Preparations, Proc. Natl. Acad. Sci. USA, 63 (1969), 51-7

I. Gresser, C. Bourali, J.P. Lévy, D. Fontaine-Brouty-Boyé, M. Thomas, Cancérologie. Prolongation de la Survie des Souris Inoculées avec des Cellules Tumorales et Traitées avec des Préparations d'Interferon, C.R. Acad. Sci., 268 (1969), 994-7 
I. Gresser, 'Interferon Therapy: Obvious and not so Obvious Applications', Acta Medica Scandinavica, 197 (1975), 49. 53

I. Gresser (ed.) Interferon 2 (London: Academic Press, 1980)

S. E. Grossberg. The Interferons and their Inducers: Molecular and Therapeutic Considerations, New. EngL. J. Med., 287, 2 (1972), 79-85

T. Gup and J. Neumann, 'Experimental Drugs Cause Pain, Deaths', The Boston Sunday Globe, 18 October 1981

T. Gura, 'Systems for Identifying New Drugs Are Often Faulty', Science, 278 (1997), 1041-2

J. Gutterman et al, 'Chemoimmunotherapy of Advanced Breast Cancer: Prolongation of Remission and Survival with BCG', Br. Med. J., 2 (1976) 774-7.

J. Gutterman, e.a. Leucocyte-interferon-induced regression in human metastatic breast cancer, multiple myeloma and malignant lymphoma. Ann. Int. Med. 93 (1980), 399-406

1. Hacking, Representing and Intervening (Cambridge: Cambridge University Press, 1983)

S. S. Hall, A Commotion in the Blood; Life, Death and the Immune System (New York: Henry Holt and Company, 1997).

F. Hauptfuhrer, Will interferon kill cancer? Finnish Dr. Kari Cantell is helping the world find out, People (US weekly), 2 July 1979

A. Hecht, 'Interferon: Trying to Live Up to its Press', FDA Consumer, June 1981

E. Heller, 'Enhancement of Chikungunya Virus Replication and Inhibition of Interferon Production by Actinomycin D'. Virology, 21 (1963), 652-56

T. van Helvoort, Research Styles in Virus Studies in the Twentieth Century: Controversies and the Formation of Consensus (Maastricht: Ph.D. diss, Univ. Limburg, 1993)

T. van Helvoort, 'History of Virus Research in the Twentieth Century: The Problem of Conceptual Continuity', History of Science, xxxii (1994), 185-235

W. Henle, and G. Henle, 'Interference of Inactive Virus with the Propagation of Virus of Influenza', Science, 98 (1943), $87-9$

W. Henle and G. Henle, 'Interference Between Inactive and Active Viruses of Influenza; L. The Incidental Occurrence and Artificial Induction of the Phenomenon,' Am. J. M. Sci. 207 (1944), 705-17

W. Henle and G. Henle, 'Interference Between Inactive and Active Viruses of Influenza; II. Factors Influencing the Phenomenon', Am. J. M. Sci., 207 (1944), 717-33

W. Henle, G. Henle, J. Stokes and E. Maris, 'Experimental Exposure of Human Subjects to the Viruses of Influenza, J. Immunol., 52 (1946), 145-65.

W. Henle, 'Interference Phenomena Between Animal Viruses: a Review', J. Immunol. 64 (1950), 203-35

G. Henle, F. Deinhardt, V. Bergs and W. Henle, 'Studies on Persistent Infections of Tissue Cultures: I. General Aspects of the System', J. Exptl. Med., 108 (1958), 537-60

M. Hilleman, Interferon in Prospect and Perspective, J. Cell. Comp. Physiol., 62 (1963), 337-53 
M. Hilleman, 'Double-stranded RNAs (Polyl:C) in the Prevention of Viral Infections', Arch. Intern. Med., 126 (1970), 109-24

J. Hixson, 'Interferon: The Cancer Drug We Have Ignored', The New Yorker, 4 September 1978, pp 59-64

M. Ho and J. Enders, 'An Inhibitor of Viral Activity Appearing in Infected Cell Cultures', Proc. N. A. S., 45 (1959), $385-9$

M. Ho and J. Enders, Further Studies on an Inhibitor of Viral Activity Appearing in Infected Cell Cultures and Its Role in Chronic Viral Infections', Virology, 9 (1959), 446-77

M. Ho, 'Interferons', New Engl. J. Med., 266 (1962), 1258-64

M. Ho, 'Kinetic Considerations of the Inhibitory Action of an Interferon Produced in Chick Cultures Infected with Sindbis Virus', Virology, 17 (1962), 262-75

M. Ho, 'Identification and "Induction" of Interferon', Bacteriol. Rev. 28 (1964), 367-81

M. Ho, 'An Early Interferon: "Viral Inhibitory Factor"', J. Interferon Res., 7 (1987), 455-7

G. L. Hobby, Penicillin: Meeting the Challenge (New Haven: Yale University Press, 1985)

F. Holmes, Lavoisier and the Chemistry of Life (Madison: Wisconsin Press, 1985)

F. Holmes, 'Scientific writing and scientific discovery', Isis, 78 (1987), 220-35

M. Holub, Interferon, or on Theater (Ohio: The Field Translation Series 7, Oberlin College, 1982)

J. M. Hoskins, 'A Protective Action of Neurotropic Against Viscerotropic Yellow Fever Virus in Macasus Rhesus', Amer. J. Trop. Med. and Hyg., 15 (1935), 675-80

J. M. Hoskins, Virological Procedures (London: Butterworths, 1967)

S. Hughes, The Virus: A History of the Concept (New York: Science History Publications, 1977)

A. Isaacs, 'Laboratory Methods Used in Investigating Influenza', Glasg. Med. J., 29 (1948), 357-6I

A. Isaacs and M. Edney, 'I. Quantitative Aspects of Interference', Austr. J. Exp. Biol., 28 (1950)

A. Isaacs and R. Valentine, The Structure of Influenza Virus Filaments and Spheres', J. Gen. Microbiol., 16 (1957), 195-204

A. Isaacs and J. Lindenmann, 'Virus Interference. I. The Interferon', Proc. R. Soc., 147 (1957), 258-67

A. Isaacs, J. Lindenmann and R. Valentine, 'Virus interference. II. Some properties of interferon', Proc. R. Soc., 147 (1957), 268-73.

A. Isaacs, 'Viral interference', Symp. Soc. Gen. Microbiol., 9 (1959) 102-21

A. Isaacs, 'Interferon: The Prospects', The Practitioner, 183 (1959), 601-5

A. Isaacs, 'Metabolic Effects of Interferon on Chick Fibroblasts', Virology (letter to the editor), 10 (1960), $144-5$.

A. Isaacs, 'Interferon', Scientific American, 204 (1961), 51-7

A. Isaacs, 'Interferon tried in man', New Scientist, 3 May 1962. 
A. Isaacs, 'Interferon', Advance, Virus Res., 10 (1963), 1-39

S. Jasanoff, G. E. Markle, J. C. Petersen and T. Pinch, Handbook of Science and Technology Studies (Thousand Oaks and London: Sage, 1995)

R. Johnson, 'Interferon: Cloudy but Intriguing Future', JAMA, 245 (1981), 109-16

C. Jungeblut and M. Sanders, 'Studies of Murine Strain of Poliomyelitis Virus in Cotton Rats and White Mice', J. Exp. Med., 72 (1940), 407-36

C. Jungeblut and M. Sanders, 'Studies in Rodent Poliomyelitis; V. Interference between Murine and Monkey Poliomyelitis Virus', J. Exp. Med. 76 (1942), 127-42

C. Jungeblut, 'Studies in Rodent Poliomyelitis; VI. Further Observations on Interference between Murine and Simian Strains of Poliomyelitis Virus', J. Exp. Med., 81 (1945), 275-94

C. Jungeblut, 'Active and Inactive Murine Poliomyelitis Virus as Interfering Agents against Poliomyelitis Infection in Monkey's', P. S. E. B. M., 68 (1948), 19-22

A. Karpf, Doctoring the Media: The Reporting of Health and Medicine (London: Routledge, 1988)

L. Kay, The Molecular Vision of Life (Oxford: Oxford University Press, 1993)

E. F. Keller. Refiguring Life; Metaphors of Twentieth-Century Biology (New York: Columbia University Press, 1995)

D. J. Kevles, 'Renato Dulbecco and the New Animal Virology: Medicine, Methods, and Molecules', J. Hist. Biol., 26 (1993), 409-42

W. J. Kleinsmidt. J.C. Cline and E.B. Murphy. 'Interferon Production Induced by Statolon', Proc. Natl. Acad. of Sci. U.S. $52(1964), 741-4$

G. Kolata, 'Dilemma in Cancer Treatment', Science, 209 (1980)

K. Knorr-Cetina, The Manufacture of Knowledge (Oxford: Pergamon Press, 1981)

R. Kohler, Lords of the Fly: Drosophila Genetics and the Experimental Life (Chicago: Chicago University Press, 1994)

M. Krim, W. E. Stewart II, F. Sanders, L. Lin, 'Interferon Therapy'. New York Times, 17 June 1980, p. C5

S. Krimsky, Genetic Alchemy (Cambridge (MA): The MIT Press, 1982)

S. Krimsky, Biotechnics and Society (New York: Praeger, 1991)

S. Krown, 'Prospects for the Treatment of Cancer with Interferon', in J. Burchenal and H. Oettgen (eds.), Cancer; Achievements, Challengex, and Prospects for the 198\%'s (New York: Grune \& Stratton, 1981), 367-79

H. Lancaster, 'Potent Protein; Medical Researchers Say the Drug Interferon Holds Great Promise', The Wallstreet Journal, 6 december, 1979

G. Lampson, A. Tytell, M. Nemes and M. Hilleman, 'Purification and Characterixation of Chick Embryo Interferon'. P.S.E.B.M., $112(1963), 468-78$.

A. Landsborough Thomson, Half a Century of Medical Research Vol I ( London, Her Majesty's Stationary Office, 1973)

A. Landsborough Thomson. Half a Century of Medical Research Vol. II (London: Her Majesty's Stationary Office, 1975) 
B. Latour and S. Woolgar, Laboratory Life (London: Sage Publications, 1979)

B. Latour, Science in Action (Milton Keynes: Open University Press, 1987)

B. Latour, The Pasteurization of France (Cambridge (MA): Harvard University Press, 1988)

J. Law, 'On the Social Explanation of Technical Change: The Case of the Portugese Maritime Expansion', Technology and Culture 28 (1987), 227-52

J. Law, A Sociology of Monsters: Essays on Power, Technology and Domination (London: Routledge, 1991)

H. E. Le Grand (ed.), Experimental Enquiries: Historical, Philosophical and Social Studies of Experimentation in Science (Dordrecht: Kluwer Academic Publishers, 1990)

E. Lennette and H. Koprowski, 'Interference Between Viruses in Tissue Culture', J. Exp. Med., 83 (1945), $195-219$

E. Lennette, 'Interference Between Animal Viruses', Ann. Rev. Microb., 5 (1951), 277-94

H. van Lente, Promising Technology: The Dynamics of Expectations in Technological Developments (Delft, Ph.D. diss., Eburon, 1993)

A. J. Levine, Viruses (New York: Scientific American Library, W.H. Freeman and Company, 1992)

H. Levy, L. Law and A. Rabson, 'Inhibition of Tumor Growth by Polyinosinic-Polycytidylic acid', Proc. Nat. Acad. Sci. U.S.A., 62 (1996), 357-63

J. Liebenau, Medical Science and Medical Industry (Baltimore: The Johns Hopkins University Press, 1987)

J. Liebenau, The British Success with Penicillin', Social Studies of Science, 17 (1987), 69-86

J. Lindenmann, D. Burke and A. Isaacs, Studies on the Production, Mode of Action and Properties of Interferon', Brit. J. $\exp$. Path., 38 (1957), 551-62

J. Lindenmann und A. Isaacs, 'Versuche über Virus-Interferenz', Schweiz. Z Path. Bakt., 20 (1957), 640-6

J. Lindenmann, 'L'Interferon', Méd. et Hyg, 19 (1961), 945-46, p. 945

J. Lindenmann. 'Induction of Chick Interferon: Procedures of the Original Experiments', Methods in Enzymology, 78 (1981), 181-8

J. Lindenmann, The National Institute for Medical Research, Mill Hill: Personal Recollections from 1956/57, Arch. Virol. 140 (1995), 1687-91

J. Lindenmann, 'On Toine Pieters' 'Shaping a New Biological Factor'. Studies in History and Philosophy of Science, 29 (1998)

L. Löwy, 'Between Bench and Bedside' (Cambridge (Mass): Harvard University Press, 1996)

M. Lynch, Art and Artifact in Laboratory Science: A Study of Shop Work and Talk in a Research Laboratory (London: Routledge and Kegan Paul, 1985)

D. A. Mackenzie, Statistics in Britain: 1865-1930 (Edenburgh: Edenburgh University Press, 1981)

D. Mackenzie, Inventing Accuracy: A Historical Sociology of Nuclear Missile Guidance (Cambridge: The MrT Press. 1990)

C. McAdams, Interferon: The Penicillin of the Future?, American Journal of Nursing. April 1980, pp. 714-17 
E. De Maeyer and J. Enders, 'An Interferon Appearing In Cell Cultures Infected with Measles Virus', Proc. Soc. Exper. Biol. \& Med., 107 (1961), 573-78

E. De Maeyer, 'Interferon Twenty Years Later', Bulletin de L'Institut Pasteur, 76 (1978), 303-23

F. Magrassi, 'Studii sull'Infezione e sull'Immunita da Virus Erpetico. Nota I', Boll. Ist. Sieroter. Milan., 14 (1935), 773 821

F. Magrassi, 'Studii sull'Infezione e sull'Immunita da Virus Erpetico. Nota II and III, Z Hyg., 117 (1936), 501-28 and 573-620

J. T. Mahoney. The Merchants of Life. An Account of the American Pharmaceutical Industry (New York: Harper, 1959)

H. M. Marks, The Progress of Experiment; Science and Therapeutic Reform in the United States, 1900-1990 (Cambridge: Cambridge University Press, 1997)

H. M. Marks, 'Cortisone, 1949: A Year in the Political Life of a Drug, Bulletin of the History of Medicine, 66 (1992). 419-39

E. Martin, Flexible Bodies (Boston: Beacon Press, 1994)

J. L. Marx, Interferon (I): On the Threshold of Clinical Application, Science, 204 (1979), 1183-6; J.L Marx, Interferon (II): Learning About How it Works, Science, 204 (1979), 1293-95

L. G. Matthews, History of Pharmacy in Britain (London: E.\& S. Livingstone, 1962), pp. 330-3

T. Merigan, S. Reed, T. Hall, D. Tyrrell, 'Inhibition of Respiratory Virus Infection by locally applied Interferon, Lancet. i (1973), 563-7

T. Merigan, Human Interferon as a Therapeutic Agent, New. Engl. J. Med., 300 (1979), 42-3

M. De Mey. The Cognitive Paradigm (Chicago, The University of Chicago Press, 1992), pp. XVI-XVII, 89-93, $173-6$.

H. Mooser und J. Lindenmann, 'Homologe Interferenz Durch Hitzeinaktiviertes, an Erythrozyten Absorbiertes Influenza-B-virus', Experientia, XIII (1957), 147-8

R. W. Moss, The Cancer Industry (New York: Paragon House, 1989)

Y, Nagano and Y. Kojima, 'Inhibition de L'infection Vaccinale par un Facteur Liquide dans le Tissu Infecté par le Virus Homologue', Compt. Rend. Soc. Biol. Filiales, 152 (1958), 1627-29

P. Newmark, 'Interferon: Decline and Stall', Nature, 291 (1981), 105-6

T. Nickles, 'Good Science as Bad History: From order of Knowing to Order of Being', in E. McMullin (ed.), The Social Dimensions of Science (Indiana: The University of Notre Dame Press, 1992)

T. Nickles, 'Philosophy of Science and History of Science', Osiris, 10 (1995), 139-63

J. O'Malley and W. A. Carter, Human Interferons: Characterization of the Major Molecular Components', Journal of the ReticuloEndothelial Society, 23 (1978) 299-305

N. Oudshoorn. Beyond the Natural Body: An Archaeology of Sex Hormones (London: Routledge, 1994)

R. C. Olby, G.N. Cantor, J. R. Christie and M.J. Hodge (eds.), Companion to the History of Modern Science (London: Routledge, 1990) 
S. Panem and J. Vilcek, 'Will Interferon Ever Cure Cancer?', The Atlantic, December 1982

S. Panem, The Interferon Crusade (Washington, D.C.: The Brookings Institution, 1984)

J. Parascandola, 'The theoretical basis of Paul Ehrlich's chemotherapy, J. Hist. Med. 36 (1981), 19-43

R. Parker, Methods of Tissue Culture (New York: Paul B. Hoeber, Inc., 1950)

R. Parker. Methods of Tissue Culture (New York: Paul B. Hoeber, Inc., 1962)

D. Parkinson (ed.), The Expanding Role of Interferon-Alfa in the Treatment of Cancer., Seminars in Oncology, 21 (1994), 1-37

M. J. Parnham and J. Bruinvels (eds), Selections from Discoveries in Pharmacology (Amsterdam: Elsevier Science Publishers, 1987)

J. T. Patterson, The Dread Disease (Cambridge: Harvard University Press, 1987)

F. T. Perkins and R.H. Regamey (eds.), Symposia Series in Immunobiological Standardization vol. 14 (Basel: S. Karger, 1970)

J. C. Petersen and G. E. Markle, 'Expansion of conflict in cancer controversies', in L Kriesberg (ed.), Research in Social Movements, Conflicts and Change, 4, (1981)

H. C. Peyer, Roche: A Company History (Basel: Editiones Roche, 1996)

A. Pickering, Constructing Quarcks: A sociological History of Particle Physics (Chicago: Chicago University Press, 1984)

A. Pickering, (ed) Science as Practice and Culture (Chicago: The University of Chicago Press, 1992)

A. Pickering, The Mangle of Practice (Chicago: The University of Chicago Press, 1995)

T. Pinch, Confronting Nature: the Sociology of Solar-Neutrino Detection (Dordrecht: D. Reidel Publishing Company. 1986)

T. Pieters, 'Interferon and Its First Clinical Trial': Looking Behind the Scenes', Medical History, 37 (1993), $270-95$

T. Pieters, 'Shaping a New Biological Factor, 'The Interferon', in Room 215 of the National Institute for Medical Research, 1956/57', Studies in History and Philosophy of Science, 28 (1997), 27-73

T. Pieters, History of the Development of the Interferons: From Test-tube to Patient, in R. Stuart-Harris and R. Penny (eds.) The Clinical Applications of Interferons, (London: Chapman\&Hall, 1997)

T. Pieters, 'Managing Differences in Biomedical Research: The Case of Standardizing Interferons', Studies in History and Philosophy of Science, 29 (1998), 31-79

T. Pieters, Marketing Medicines Through Randomised Controlled Trials: the Case of Interferon, BMJ, 317 (1998), 1231-3

T. Pieters, 'About Media, Audiences and Marketing Medicines: the Interferons', in M. Gijswijt and T. Tansey (eds), (Forthcoming, Rodopi Press) 
T. Pieters, 'What Constitutes Therapeutic Success?: The Interferons', in S. Rand (ed.), (Forthcoming, Springer Verlag)

T. Pieters, 'New Molecules, Markets and Changing Drug Regulatory Practices at the End of the Twentieth Century', in J. Abrahams (ed.), (Forthcoming MacMillan Press)

S. Pocock, Clinical Trials (Chichester: John Wiley \& Sons, 1983)

M. Pollard (ed.) Perspectives in Virology: A Symposium, (Texas: The University of Texas Medical Branch, 1958)

M. Pollard (ed.), Perspectives in Virology (Minneapolis: Burgess Publishing, 1960)

R. Porter, The Greatest Benefit to Mankind; A Medical History of Humanity from Antiquity to the Present (London: Harper Collins Publishers, 1997)

T. M. Porter, Trust in Numbers: The Pursuit of Objectivity in Science and Public Life (Princeton: Princeton University Press, 1995)

T. Powledge, 'Interferon on Trial', Bio/technology, 2 (1984), 214-28

R. Proctor, Cancer Wars: How Politics Shapes What We Know \& Don't Know About Cancer (New York: Basic Books, 1995)

M. VandePutte, J. Delafonteyne, J. Billeau and P de Somer, 'Influence and Production of Interferon in Rauscher Virus Infected Mice', Arch. Ges. Virusforsch., 20 (1967), 235-45

J. Quesada, E. Hersh and J. Gutterman. 'Hairy Cell Leukemia: Induction of Remission with Alpha Interferon', Blood, 62 (1983), 207a

J. Quesada, J. Reuben, J. Manning, E. Hersh and J. Gutterman, 'Alpha Interferon for Induction of Remission in Hairy Cell Leukemia, N. Engl. J. Med., 310 (1984)

H. Radder, 'Experimental Reproducibility and the Experimenter's Regress', in D. Hull, M. Forbes and K. Okruklik (eds.) PSA, Volume I (East Lansing, Michigan: Philosophy of Science Association, 1992)

R. A. Rettig, Cancer Crusade, (Princeton: Princeton University Press, 1977)

H. J. Rheinberger, 'Experiment, Difference, and Writing: I. Tracing Protein Synthesis', Studies in History and Philolosophy of Science, 23 (1992), 305-31

H. J. Rheinberger, 'Experiment, Difference, and Writing: II. The Laboratory Production of Transfer RNA', Studies in History and Philosophy of Science, 23 (1992), 389-422

E. Richards, Vitamin C and Cancer: Medicine or Politics (New York: St. Martin's Press, 1991)

G. Rita (ed.), The Interferons (New York: Academic Press, 1968)

F. Robbins and J. Enders, 'Tissue Culture Techniques in the Study of Animal Viruses', Am. J. Med. Sci., 220 (1950), 316-38

G. Rosen et all, 'High Dose Methotrexate with Citrovorum Factor and Rescue and Adriamycin in Childhood Osteogenic Sarcoma', Cancer, 33 (1974), 1151-63

S. Rosenberg, The Transformed Cell (London: Phoenix, 1992) 
A. Rosenfeld, 'If IF works it could...', July, 1979, Life, 55-62

M. J. S. Rudwick, The Great Devonian Controversy (Chicago: The University of Chicago Press, 1985)

J. Sapp, Where the Truth Lies: Franz Moewus and the Origins of Molecular Biology (Cambridge: Cambridge University Press, 1990)

M. Sanders and E. H. Lennette, Medical and Applied Virology, Proc, Soc. Int. Symp. (St. Louis: Warren H. Green Inc., 1968)

H. Schellekens et al, 'Comparative Antiviral Efficiency of Leukocyte and Bacterially Produced Human Alpha Interferon in Rhesus Monkeys', Nature, 292 (1981), 775-6

G. Scott et al, 'Prevention of Rhinovirus Colds by Human Interferon Alpha-2 from Escherichia Coli', Lancet ii (1982), 186-7.

M. Sela (ed.), The Role of Non-Specific Immunity in the Prevention and Treatment of Cancer (Rome: Pontificia Academia Scientiarum, 1977)

R. E. Shope, 'Evolutionary Episodes in the Concept of Viral Oncogenesis', Persp. Bio. Med. 9 (1966), pp. 258-74

K. Sikora and H. Smedley, 'Interferon and Cancer', Br. Med. J., 286 (1983), 739-40

M. Silvermann and P.R. Lee, Pills Profits \& Politics (Berkely: California Press, 1974)

A. M. Silverstein, A History of Immunology (San Diego: Academic Press, Inc, 1989)

H. M. Schmeck, 'Interferon: Studies Put Cancer Use in Doubt', New York Times, 27 May 1980

J. S. Smith, Patenting the Sun; Polio and the Salk Vaccine (New York: William Morrow and Company, 1990)

W. Sneader, Drug Discovery: the Evolution of Modern Medicines (Chichester: John Wiley\&Sons, 1985)

W. Sneader, Drug Development: From Laboratory to Clinic (Chichester: John Wiley\&Sons, 1986)

D. Schwartzman, Innovation in the Pharmaceutical Industry (Baltimore: Johns Hopkins University Press, 1976)

S. Shapin and S. Schaffer, Leviathan and the Airpump; Hobbes, Boyle and the Experimental Life (Princeton: Princeton University Press, 1985)

M. Sun, Interferon: No Magic Bullet Against Cancer, Science, 212 (1981), 141-2

J. P. Swann, Academic scientists and the pharmaceutical industry (Baltimore: The Johns Hopkins University Press, 1988)

S. L. Star, Regions of the Mind: Brain Research and the Quest for Scientific Certainty (Stanford (CA): Stanford University Press, 1989)

W. E. Stewart II. The Interferon System (Wien: Springer-Verlag, 1979)

H. Strander and K. Cantell, 'Production of Interferon by Human Leukocytes in Vitro', Ann. Med. exp. Fenn, 44 (1966), 265-73

H. Strander, K. Cantell, G. Carlström, and P. Jakobsson, 'Clinical and Laboratory Investigations on Man: Systemic 
Administration of Potent Interferon in Man', J. Nat. Cancer. Inst., 51 (1973), 733-42

R. Stuart Harris and R. Penny, Clinical Applications of the Interferons (London: Chapman\&Hall Medical, 1997)

K. Studer and D. Chubin, The Cancer Mission: Social Contexts of Biomedical Research (Beverly Hills: Sage publications, 1980)

T. Taniguichi, M. Sakai, Y. Fujii-Kuriyama, M. Muramatsu, S. Kobayashi, T. Sudo, Construction and Identification of a Bacterial Plasmid Containing the Human Fibroblast Interferon Gene Sequence. Proc. Jpn. Acad. 55B (1979), 461-9

E. M. Tansey, Catterall P.P., Christie, D.A., Willhoft S.V. and Reynolds, L.A., Wellcome Witnesses to Twentieth Century Medicine Vol I (London: Wellcome Trust Occasional Publications, 1997)

G. Taubes, Use of Placebo Controls in Clinical Trials is Disputed, Science, 267 (1995), p. 25

J. Taylor, 'Inhibition of Interferon Action by Actinomycin', Biochem. Biophys. Res. Comm., 14 (1964), 447-51

R. Teitelman, Gene Dreams: Wall Street, Academia and the Rise of Biotechnology (New York: Basic Books, 1989)

P. Temin, Taking Your Medicine: Drug Regulation in the United States (Cambridge: Harvard University Press, 1980)

P. Thompson, The Voice of the Past (Oxford, Oxford University Press, 1988)

D. Tyrrell and I. Tamm, 'Prevention of Virus Interference by 2,5-Dimethylbenzimidazole', J. Immun., 75 (1955), 43-9

D. Tyrrell, 'Interferon Produced by Cultures of Calf Kidney Cells', Nature, 184 (1959), 452-3

D. Tyrrell, 'Personal memories of the early days', J. Interferon Res., 7 (1987), 443-4

D. Tyrrell, 'The Common Cold Unit 1946-1990: Farewell to a Much-Loved British Institution', PHLS Microbiology Digest, 6 (1991), 74-6.

J. Vilcek, Interferon (New-York, Springer-Verlag, 1969)

R. Vos, Drugs Looking for Diseases. Innovative Drug research and the development of the Beta Blockers and the Calcium Antagonists (Amsterdam: Kluwer Academic Publishers, 1991)

N. Wade, Cloning Gold Rush Turns Basic Biology into Big Business, Science, 208 (1980), pp. 688-92

R. R. Wagner, 'Viral Interference', Bacteriol. Rev,, 24 (1960), 151-66

R. R. Wagner, 'Biological Studies of Interferon', Virology, 13 (1961), 323-37

R. R. Wagner, 'The Interferons: Cellular Inhibitors of Viral Infection', Ann. Rev. Microbiol., 17 (1963), 285-94

R. R. Wagner, 'Inhibition of Interferon Biosynthesis by Actinomycin D', Nature, 204 (1964), 49-51

R. R. Wagner. 'Interferon; A Review and Analysis of Recent Observations'. American Journal of Medicine, 38 (1965), 726-737

R. R. Wagner, 'Reminiscences of a virologist wandering in Serendip', Arch. Virol., 141 (1996), 787-97

R. Walgate, 'Side Effect Scare Hits French Trials, Nature, 300 (1982), 97-8

M. Weatherall, In Search of a Cure (Oxford: Oxford University Press, 1990) 
C. Weissman, 'The Cloning of Interferon and Other Mistakes', in I. Gresser (ed.), Interferon 3 (London, Academic Press, 1981), pp. 101-134

F. E. Wheelock and J.H. Dingle, 'Observations on the Repeated Administration of Viruses to a Patient with Acute Leukemia', New. Eng. J. Med., 271 (1964) 645-51

E. F. Wheelock, Effect of Statolon on Friend Virus Leukemia in Mice, Proc. Soc. Exp. Biol. Med., 124 (1967), 855-8

E. F. Wheelock, and R. P. B. Larke, Efficacy of Interferon in the Treatment of Mice with Established Friend Virus Leukemia, P.S.E.B.M., 127 (1968), 230-8

D. Wilson, Penicillin in Perspective (London: Faber\& Faber, 1976)

G. E. Wolstenholme and M. O'Connor (eds.), Interferon (London: J\&A Churchill, 1968)

S. Wright, Molecular Politics: Developing American and British Regulatory Policy for Genetic Engineering 1972. 1982 (Chicago: Chicago University Press, 1994)

A. Wycke, 'Molecules and Markets', The Economist, 7 February 1987

B. Wynne, 'Unruly Technology: Practical Rules, Impractical Discourses and Public Understanding.' Social Studies of Science, 18 (1988), 147-67 


\section{SAMENVATTING}

De inzet van deze biografie over interferon-één van de eerste en meest spraakmakende therapeutische 'biomoleculen'-is tweeledig. In de eerste plaats beoogt dit proefschrift het historische ontwikkelingsproces van interferon als onderzoeksobject en als geneesmiddel te beschrijven en inzichtelijk te maken. Aandacht voor de aard en dynamiek van de wisselwerking tussen verschillende onderzoekspraktijken in de ontwikkeling van interferon staat voorop. Hierbij wordt afwisselend ingezoomd op de werkzaamheden van wetenschappers en clinici aan de laboratoriumtafel en in de behandelkamer en uitgezoomd naar de bredere maatschappelijke context waarin hun producten een weg vinden. Ten tweede laat dit boek aan de hand van de ontwikkelingsgeschiedenis van interferon zien hoe in de tweede helft van de twintigste eeuw de biomoleculaire wending in geneesmiddelonderzoek en -productie haar beslag krijgt.

Succesvolle geneesmiddelontwikkeling wordt doorgaans geassocieerd met grote farmaceutische firma's die in hun laboratoria naarstig op zoek zijn naar nieuwe medicijnen. Industriële onderzoekscentra zijn in deze optiek de stuwende kracht achter de continue stroom van innovatieve medicijnen (met een nieuwe of sterk verbeterde klinische werking) naar de therapeutische praktijk. Bij de bestendiging van dit beeld krijgen de 'marketers' van de farmaceutische industrie alle medewerking van media en wetenschappers. Deze studie betoogt dat in de gebruikelijke voorstelling van zaken weinig recht wordt gedaan aan het belang van de voortdurende wisselwerking tussen academische, geneeskundige en industriële onderzoekspraktijken voor de ontwikkeling van geneesmiddelen. Geheel ten onrechte wordt voorbij gegaan aan het historisch feit dat de meeste moderne geneesmiddelen hun oorsprong hebben op het grensvlak van het laboratorium en de kliniek.

Voortgekomen uit microbiologisch onderzoek in de jaren '30 en '40 van deze eeuw (Hoofdstuk1), kan de 'ontdekking' van interferon door de Engelse viroloog Alick Isaacs en de Zwitserse onderzoeker Jean Lindenmann in 1957 worden gedateerd. De scheppers van interferon worden in hoofdstuk 2 op de voet gevolgd in hun werkzaamheden bij het National Institute for Medical Research te Mill Hill (Londen). Kenmerkend voor hun gezamenlijke onderzoeksactiviteiten is de creatieve manier waarop zij omgaan met het beschikbare geheel aan technieken, onderzoeksmaterialen en hypothesen. Als onderdeel van dit dynamisch experimenteel systeem zijn onderzoeksvraag en onderzoeksobject voortdurend aan verandering onderhevig. Hierbij dwingen afwisselend materiële, sociale en cognitieve factoren in de onderzoeksomgeving aanpassingen af.

Als uitvloeisel van hun pogingen orde aan te brengen in hun onderzoeksresultaten en hypothesen menen Isaacs en Lindenmann een verklaring te hebben voor het zogenaamde 'virale interferentie' fenomeen, waarbij een organisme of celweefsel dat met één type virus is besmet, 
niet nog eens door een andere virussoort besmet kan worden. $\mathrm{Zij}$ claimen een voorheen onbekende substantie te hebben geïsoleerd uit de biologische soep afkomstig van specifieke weefselkweken waarin sprake is van virale interferentie. Celweefsels die met deze biologische stof, genaamd interferon, in contact worden gebracht, zijn beschermd tegen bijna alle soorten virussen.

Interferon wekt begin jaren zestig met name in Engeland hoge verwachtingen. Dat heeft tot gevolg dat Britse onderzoeksinstellingen en farmaceutische bedrijven gezamenlijke pogingen ondernemen om interferon te ontwikkelen als geneesmiddel tegen virusziekten. Terwijl in Engeland interferon enthousiast onthaald wordt als het nieuwe 'antivirale penicilline' verwerft het binnen de internationale academische context de dubieuze bijnaam 'misinterpreton'.

Aan de hand van historisch archiefonderzoek en 'oral history' volgt in hoofdstuk 3 een reconstructie van de lotgevallen van interferon als onderzoeksobject en als potentieel geneesmiddel tegen virusziekten in de jaren zestig. Hierbij ligt de nadruk op de verschillen in receptie van interferon tussen industrie, overheid en het algemene publiek in Engeland aan de ene kant en de internationale gemeenschap van virusonderzoekers aan de andere kant. Uitgebreide aandacht is er voor de transformatie van interferon als dubieus laboratoriumfenomeen tot nucleus van een nieuw onderzoeksgebied.

Hoofdstuk vier verhaalt in het bijzonder over hoe de Engelse overheid en farmaceutische industrie komen tot een formele vorm van samenwerking met als doel interferon te ontwikkelen tot geneesmiddel. Na een voortvarend begin krijgt het samenwerkingsverband te maken met een breed scala aan problemen. Aanhoudend treden cultuurverschillen tussen overheid en industrie aan het licht die overbrugd dienen te worden. Veelal is hierbij de precaire relatie tussen commercieel belang en wetenschappelijke attitude in het geding. Voorts gedraagt interferon zich niet conform de verwachtingen 'as just another penicilline or hormone' dat probleemloos inpasbaar is binnen het conventionele chemotherapeutisch geneesmiddelontwikkelingsmodel. Bij het onderzoek naar de 'grillige' biologische substantie interferon, bestaat continue spanning tussen het houvast zoeken in bestaande testpraktijken en pogingen om op ad-hoc basis nieuwe invulling te geven aan testprocedures. Als bij voortduring het referentiekader te kort schiet en nieuwe technische, organisatorische en logistieke problemen zich blijven voordoen, neemt de industriële belangstelling zienderogen af.

Het onderzoek naar interferon concentreert zich vervolgens in een internationaal vertakt netwerk van universitaire onderzoeksinstellingen. Na een vliegende start begin jaren zestig moeten de betrokken onderzoekers genoegen nemen met een marginale rol binnen het internationale veld van virusonderzoek. De onderzoeksagenda wordt beheerst door een aantal zich voortslepende problemen: de onvoldoende beschikbaarheid van interferon, het uitblijven van de chemische karakterisering en de soortspecificiteit van interferon. Dit gaat gepaard met een versnippering van het onderzoek dat gekenmerkt wordt door een brede waaier aan onderzoeksbenaderingen en hypothesen. 
Voor interferononderzoekers vormt de contextafhankelijkheid van onderzoeksresultaten ten gevolge van een grote variatie in experimentele systemen een voortdurende bron van conflicten. Deze problematiek wordt versterkt door het uitblijven van de chemische identificatie van interferon. Hoofdstuk vijf beschrijft in detail hoe onderzoekers en bedrijven de uitwisseling van kennis en onderzoeksmaterialen proberen te vergemakkelijken door informele en formele vormen van standaardisatie. Door het genereren van internationaal erkende interferon standaarden slagen zij er uiteindelijk in om de productie van context-onafhankelijke kennis met een universeel karakter te waarborgen. Interferon dat voorheen in hoge mate gebonden was aan een relatief klein netwerk van specifieke lokale laboratoriumpraktijken is nu verplaatsbaar naar elders.

Midden jaren zeventig krijgt het onderzoeksgebied een belangrijke nieuwe impuls. Scandinavische onderzoekers claimen niet alleen een veelbelovende methode ontwikkeld te hebben voor de productie van menselijk interferon maar menen ook een belangrijk nieuw therapeutisch effect waargenomen te hebben in kankerpatiënten. Met steun van de invloedrijke Amerikaanse lobbyiste Mathilde Krim weten zij met hun claim de aandacht te trekken van de media en van belangrijke onderzoekers en beleidsmakers op het gebied van kanker in Amerika.

De betekenisverschuiving van interferon als antivirale stof naar interferon als potentieel middel tegen kanker én virussen in de jaren zeventig, is het onderwerp van hoofdstuk 6. De aandacht gaat met name uit naar de dynamiek van wat retrospectief wordt aangeduid als de 'interferon crusade' in Amerika. Beschreven wordt hoe de claim van de anti-kankerwerking van interferon buiten het specifieke veld van onderzoek een eigen leven gaat leiden.

Hoewel vraagtekens geplaatst worden bij de wetenschappelijke toetsing van de geclaimde onderzoeksresultaten, zorgt de 'medialisering' van de belofte van interferon als niettoxisch lichaamseigen geneesmiddel tegen kanker voor een ware hausse aan verwachtingen. De aankondiging eind jaren zeventig van nieuwe biotechnologische bedrijven dat zij instaat zijn om met behulp van recombinant-DNA-technologie ongelimiteerde hoeveelheden van dit 'magische' eiwit te produceren, werkt op haar beurt als een vliegwiel. Wereldwijd verwerft interferon een kortdurende status als 'hét wondermiddel tegen kanker en virussen'. Echter, als bij voortduring de resultaten van klinisch onderzoek ver achterblijven bij de eerdere onderzoeksclaims en ernstige bijwerkingen blijken te kunnen optreden, neemt de belangstelling voor interferon in snel tempo af.

Opmerkelijk is de wijze waarop de wetenschappelijke en medische wereld opeenvolgend de hausse aan verwachtingen aanjagen als middel om geld te werven voor onderzoek en deze bestrijden als hun belangen in het geding komen. De massamedia fungeren hierbij als dynamisch platform voor de uitwisseling van informatie, verwachtingen en noties tussen wetenschappers, artsen, patiënten, politici, de industrie en het algemene publiek.

Ondanks het feit dat interferon begin jaren tachtig de dubieuze status verwerft van 'the miracle drug looking for a disease' verdwijnt het niet van het toneel. Voor een aantal van de 
betrokken wetenschappers en bedrijven blijkt 'het point of no return' te zijn bereikt. Zij volharden in hun zoektocht naar toepassingsmogelijkheden voor interferon. Hoofdstuk 7 laat zien hoe interferon als beloftevol prototype van een nieuwe generatie van therapeutische biomoleculen onderdeel wordt van de biotechnologisering van geneesmiddelontwikkeling en -productie. Tegelijkertijd brengt het in kaart de wijze waarop farmaceutische bedrijven interferon transformeren van een marginaal geneesmiddel tot een 'billion-dollar family of molecules'. Door strategisch gebruik te maken van 'clinical trials' als belangrijke schakel tussen laboratorium en kliniek, slagen zij erin om een herdefiniëring en verbreding van het therapeutisch profiel van interferon te bewerkstelligen. Als immuno-modulantia gaan interferonen (alpha, beta en gamma types) een veelgevraagd onderdeel vormen van combinatietherapie bij maligne en andersoortige aandoeningen in de klinisch therapeutische praktijk.

Het slothoofdstuk geeft een kritische analyse van de bevindingen en mogelijke implicaties van deze biografie van een 'wondermiddel'. Het laat zien dat tijdens de ontwikkeling van interferon sprake is van een verschuiving. Werd in de jaren vijftig nog een gouden toekomst toegedicht aan chemie bij de ontwikkeling van nieuwe en betere geneesmiddelen, aan de vooravond van de eenentwintigste eeuw heeft in het denken over medicijnen van de toekomst chemie plaats gemaakt voor moleculaire biologie. Dit verloopt synchroon met een overgang van proces- naar systeemdenken in biologie en geneeskunde. Hierbij gaat het om een verschuiving in het denken over het organisme als een complex geheel van biochemische processen naar het organisme als complex communicatie regel-systeem. Waar interferon als grillige therapeutische substantie in eerste instantie faalt bij het ontbreken van een geschikt wetenschappelijk en klinisch frame, weet het vervolgens met succes nieuwe ankerpunten te genereren als de biomoleculaire wending binnen geneesmiddelonderzoek en medische behandelpraktijk haar beslag krijgt.

Ook problematiseert dit proefschrift de verwevenheid van medisch-wetenschappelijke en medisch-therapeutische praktijken. Op de voorgrond treedt een voortdurend conflict tussen statistische significantie en klinische relevantie. Zo krijgen binnen de concrete context van ernstige onbehandelbare ziekten kwaliteiten als 'gering' en 'onzeker' een andere betekenis dan bij behandelbare aandoeningen. Deze studie maakt duidelijk dat bij de beoordeling van de werkzaamheid van een geneesmiddel sprake is van een afwegingsproces waarin industrie, wetenschappers, artsen en patiënten elkaar in een houdgreep van belofte en hoop houden. Gewekte verwachtingen over wat nieuwe generaties van therapeutische moleculen naar de toekomst toe vermogen bij het verlengen en verbeteren van 'het leven' blijken te interfereren met de beoordeling van de mogelijke gezondheidswinst van deze nieuwe therapieën in de actuele klinische setting. Hierbij moet het dilemma gezocht worden in de vervlechting van de dagelijkse praktijk van medische zorg, waarin de patiëntenstatus het referentiepunt is, met de praktijk van medisch onderzoek waarin de te verwachten kliniek van de toekomst als ijkpunt dient. 


\section{Curriculum Vitae}

Toine Pieters (1960) studied pharmacology and molecular biology at Utrecht University. After being awarded the degree of Msc (1986) he succeeded in registering as a pharmacist. However, in 1988 during a period of sabbatical leave in South-East Asia he decided to refocus his career, starting a PhD in the field of Science Studies at Maastricht University (1989). Four years later he was appointed as supervisor of the special project 'Teaching Social, Ethical and Legal Aspects of Biotechnology' at the Faculty of Philosophy of Groningen University. Subsequently in the autumn of 1998 he accepted his current position as an Assistant Professor of the History of Medicine at the 'Vrije Universiteit Amsterdam' School of Medicine. His teaching experience as a lecturer has covered a broad range of courses in the history and philosophy of the life sciences and in making modern biomedicine. 

